NISTIR 7338

\title{
Evaluation of Fire Models for Nuclear Power Plant Applications
}

\section{Benchmark Exercise \#3}

\author{
International Panel Report
}

Compiled by Kevin McGrattan 



\section{Evaluation of Fire Models for Nuclear Power Plant Applications}

\section{Benchmark Exercise \#3}

\section{International Panel Report}

Compiled by Kevin McGrattan

Fire Research Division

Building and Fire Research Laboratory

January 2007

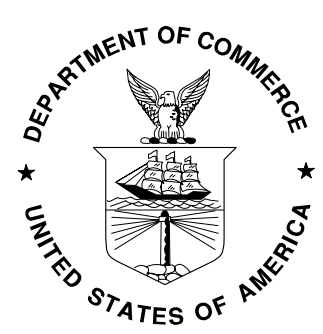

U.S. Department of Commerce Carlos M. Gutierrez, Secretary

Technology Administration Robert Cresanti, Under Secretary of Commerce for Technology

National Institute of Standards and Technology William Jeffrey, Director 



\begin{abstract}
A series of full-scale fire experiments was conducted at NIST in 2003 to provide data for an international benchmarking exercise to assess the accuracy of fire models over a range of operating conditions expected within a variety of compartments in a nuclear power plant. Seven organizations from France, Germany, Britain, and USA exercised seven fire models as part of the International Collaborative Fire Modeling Project (ICFMP). This report documents the results of the individual modeling studies.
\end{abstract}




\section{Content}

Introduction................................................................................1

$1.1 \quad$ Review of Previous NRC-Sponsored Experiments ......................... 1-2

1.1.1 Investigation of Twenty-Foot Separation Distance ............................... 1-2

1.1.2 Fire Tests in a Control Room Mock-Up .............................................. 1-2

1.1.3 Fire Test in a Cable Room Mock-Up ................................................. 1-3

$1.2 \quad$ Experimental Description of Benchmark Exercise \#3 …................. 1-3

$1.3 \quad$ The Benchmarking Process …..................................................

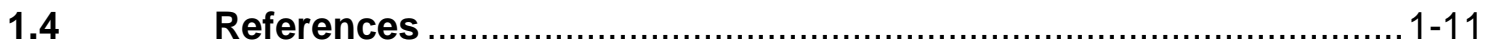

2 Simulations using Jasmine and CFAST (Stewart Miles, BRE) ............2-1

2.1 CFAST Input Parameters and Assumptions ................................. 2-1

2.2 JASMINE Input Parameters and Assumptions ..............................2-3

2.3 Comparison of CFAST Blind and Semi-Blind Predictions with Measurements ................................................................................

2.4 Comparison of JASMINE Blind and Semi-Blind Predictions with Measurements ......................................................................... 2-24

2.5 Conclusions and Recommendations for CFAST and JASMINE ......2-50

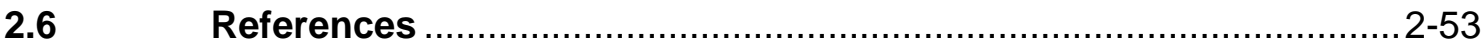

3 Open Simulations using FLAMME_S (Laurence Rigollet, IRSN) ........3-1

3.1 FLAMME_S Input Parameters and Assumptions ............................

3.2 Comparison of FLAMME_S with Measurements …...........................

3.2.1 Test 2 and 8: door closed and ventilation off.....................................

3.2.2 Tests 3 and 9: door opened and ventilation off ................................... 3-3

3.2.3 Tests 3 and 10: door closed and ventilation on .................................. $3-4$

3.2.4 Test 5: door opened and ventilation on .......................................... 3-5

3.3 Conclusions and Recommendations for FLAMME_S ..................... 3-6

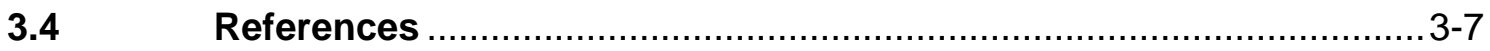

$4 \quad$ Open Simulations using FATE (Fauske and Associates) ...................4-1

4.1 FATE Input Parameters and Assumptions.................................... $4-2$

4.1.1 Room Geometry and Ventilation ..................................................... $4-2$

4.1.2 Cable Targets ................................................................................... $4-4$

4.1.3 Test Matrix and Fire Source ….................................................. 4

4.2 Comparison of FATE Predictions with Measurements ................... 4-5

4.2.1 BE-3 Case 1 FATE Open Benchmark Results ...................................4-5

4.2.2 BE-3 Case 2 FATE Open Benchmark Results ...................................4-20 
4.2.3 BE-3 Case 3 FATE Open Benchmark Results ................................4-36

4.2.4 BE-3 Case 4 FATE Open Benchmark Results .................................. 4-57

4.2.5 BE-3 Case 5 FATE Open Benchmark Results ...................................4-73

4.2.6 BE-3 Case 14, 15, and 18 FATE Open Benchmark Results .................4-73

4.3 Conclusions and Recommendations for FATE ........................... $4-96$

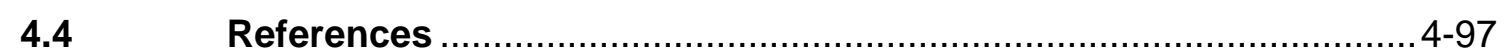

$5 \quad$ Simulations using COCOSYS (Walter Klein-Hessling, GRS) .............5-1

5.1 COCOSYS Input Parameters and Assumptions .................................5-1

5.2 Comparison of COCOSYS Predictions with Measurements .............5-7

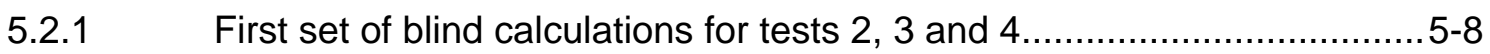

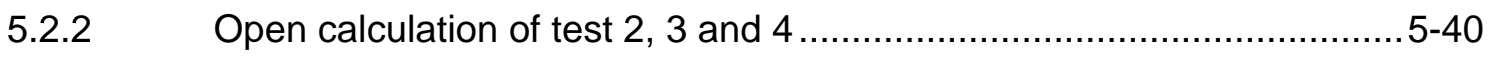

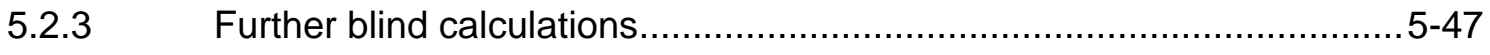

5.3 Conclusions and Recommendations for COCOSYS ....................5-68

$6 \quad$ Open Simulations using CFX (Matthias Heitsch, GRS) ..................... 6-1

6.1 CFX Input Parameters and Assumptions ................................. 6-1

6.2 Comparison of CFX Predictions with Measurements ........................ 6-3

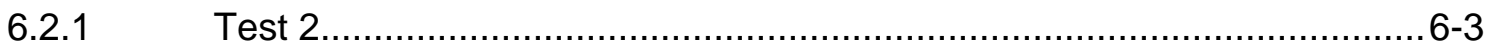

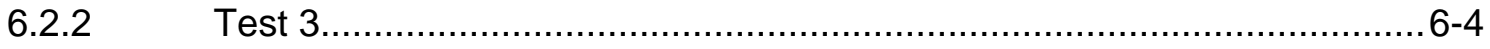

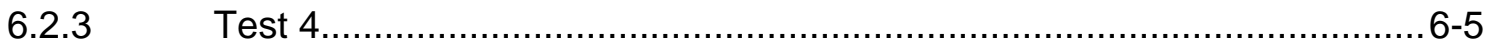

$6.3 \quad$ Conclusions and Recommendations for CFX ........................... 6-7

$7 \quad$ Open Simulations using FDS (Kevin McGrattan, NIST) ..................... 7-1

7.1 FDS Input Parameters and Assumptions ..................................

7.1.1 Compartment Geometry ...........................................................

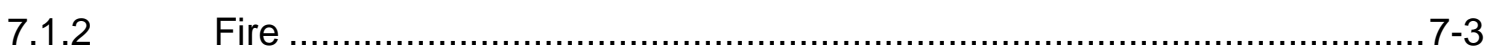

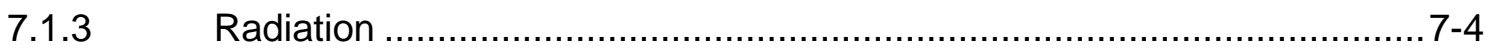

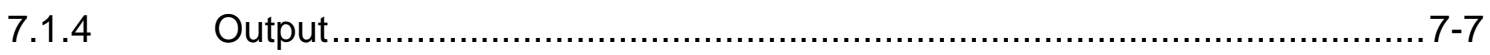

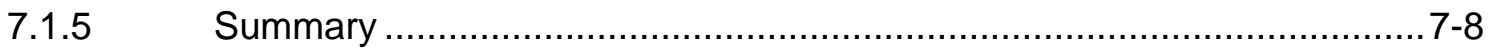

7.2 Comparison of FDS Open Predictions with Measurements .............. 7-9

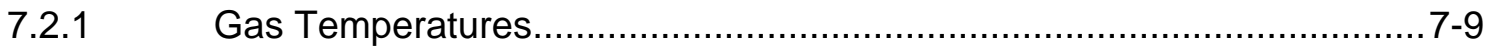

7.2.2 Heat Flux and Surface Temperature at the Walls, Ceiling and Floor ..... 7-22

7.2.3 Heat Flux and Surface Temperature at Cable Targets.......................... 7-34

7.2.4 Gas Species Concentrations ..................................................... $7-57$

7.2.5 Smoke Concentration ..................................................................

7.2.6 Compartment Pressure ….............................................................

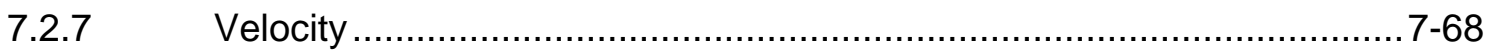

7.2.8 Energy Budget....................................................................... 
7.3 FDS Sensitivity Analysis .........................................................

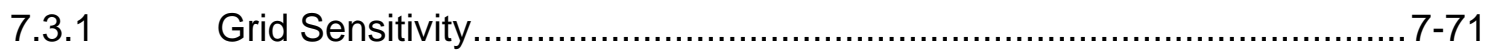

7.3.2 Sensitivity to Wall Material Properties ............................................ $7-72$

7.3.3 Radiation Parameters ......................................................... $7-74$

7.4 Conclusions and Recommendations for FDS …........................ 7-75

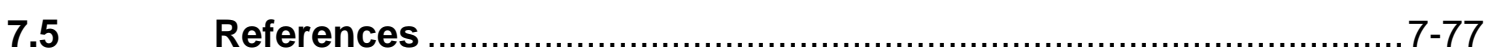

8 Summary of Conclusions from Individual Contributions .................... 8-1

$8.1 \quad$ Modeling the Fire ..................................................................................

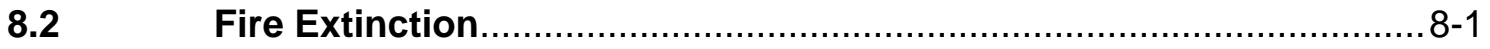

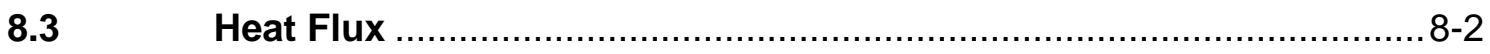

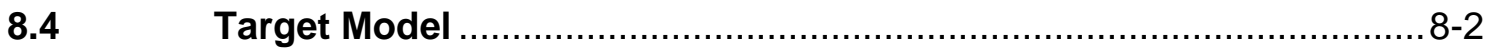

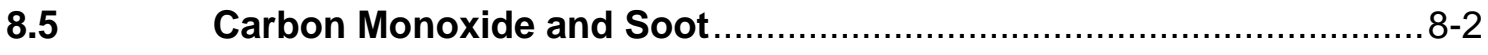

8.6 Mechanical Ventilation .......................................................... 8

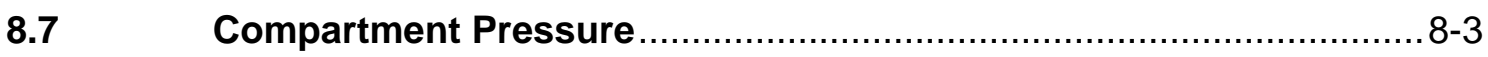




\section{Foreword}

This report documents the results of fire model calculations of Benchmark Exercise \#3 of the International Collaborative Fire Model Project. The objective of this study was to provide participants in the project an opportunity to test their respective fire models, and to evaluate the capabilities of the fire models for fire safety analyses in nuclear power plants. The work of each organization participating in the exercise is included as a chapter in the main report. These chapters document the analyses conducted by the participating organizations, their assessment of the accuracy and capabilities of their models, and the insights gained from the validation exercise. These studies are the product of each organization. The chapters also discuss the considerations for developing input parameters and assumptions for the models, and a summary of the conclusions from the individual studies. No attempt has been made to provide code to code comparisons, or assess the performance of each model used. Each organization participating in the exercise will assess the performance of the models it exercised, and determine their specific applicability for the organization's own use and benefit. This document is not intended to provide guidance to users of fire models. Guidance on the use of fire models is currently being developed by several national and international standards organizations, industry groups, and utilities. This document is intended to be a source and reference for technical information and insights gained through the analyses conducted, and provided by the experts participating in this project. This information may be beneficial to users of fire models, and developers of guidance documents or standards for the use of fire models in nuclear power plant applications. 


\section{Acknowledgments}

In addition to the analysts identified in the table of contents who performed the calculations for the benchmark exercise, several experts from various organizations contributed to this benchmark exercise in many ways. The test series used for this benchmark exercise was co-sponsored by the US Nuclear Regulatory Commission and the National Institute of Standards and Technology (NIST). Anthony Hamins and Alex Maranghides of NIST conducted the test series. Jonathan Barnett at Worcester Polytechnic Institute, USA, Olavi KeskiRahkonen and Simo Hostika at VTT, Finland, and James Oldham and Doug Brandes (retired) at Duke Energy, USA hosted the $7_{\text {th }}$, th $_{\text {th }}$ and $9^{\text {th }}$ ICFMP meetings where the benchmark exercise was planned and discussed, and also provided peer reviews and technical support. Marina Roewekamp at GRS, Germany and Chantal Casselman at IRSN, France provided project management and technical support to the ICFMP group. Alan Coutts at WSMS, USA, Jason Floyd at Hughes Associates, USA, Bijan Najafi and Francisco Joglar-Billoch at SAIC/EPRI, USA, Bernard Gautier and Laurent Gay at EdF, France, Peter Rew at WS Atkins Consultants, UK, Olaf Riese at iBMB, Germany, Jurgen Will at HHPBraunschweig, Germany, Walter Jones at NIST, USA, Bob Kassawara at EPRI, USA, Naeem Iqbal at NRC/NRR, USA, and Alan Wylie and Geoff Jones at HSE/NII, UK all contributed to this benchmark exercise either by providing peer comments, participating in meetings to discuss the test plan and results, or indirectly by sponsoring participants for the project. This benchmark exercise and document would not have been possible without the help and expertise of all the above. 


\section{Acronyms}

$\begin{array}{ll}\text { BFS } & \text { Bundesamt fur Strahlenschutz } \\ \text { BRE } & \text { Building Research Establishment } \\ \text { CFAST } & \text { Consolidated Model for Fire and Smoke Transport } \\ \text { CFD } & \text { Computational Fluid Dynamics } \\ \text { COCOSYS } & \text { Containment Code System } \\ \text { EdF } & \text { Electricite de France } \\ \text { EPRI } & \text { Electric Power Research Institute } \\ \text { FATE } & \text { Facility Flow, Aerosol, Thermal, and Explosion Model } \\ \text { FDS } & \text { Fire Dynamics Simulator } \\ \text { GRS } & \text { Gesellschaft fuer Anlagen-und Reaktorsicherheit } \\ \text { HGL } & \text { Hot Gas Layer } \\ \text { HRR } & \text { Heat Release Rate } \\ \text { HSE } & \text { Health and Safety Executive } \\ \text { ICFMP } & \text { International Collaborative Fire Model Project } \\ \text { iBMB } & \text { Institut fuer Baustoffe, Massivbau und Brandschutz } \\ \text { IRSN } & \text { Institut de Radioprotection et de Surete Nucleaire } \\ \text { JASMINE } & \text { Analysis of Smoke Movement in Enclosures } \\ \text { LOL } & \text { Lower Oxygen Limit } \\ \text { NII } & \text { Nuclear Installations Inspectorate } \\ \text { NIST } & \text { National Institute of Standards and Technology } \\ \text { NPP } & \text { Nuclear Power Plant } \\ \text { NRC } & \text { Nuclear Regulatory Commission } \\ \text { PRA } & \text { Probabilistic Risk Analysis } \\ \text { SAIC } & \text { Science Applications International Corporation } \\ \text { VTT } & \text { Valtion Teknillinen Tutkimuskeskus } \\ \text { WPI } & \text { Worcester Polytechnic Institute } \\ \text { WSMS } & \text { Westinghouse Safety Management Solutions } \\ & \\ \text { HRP } & \end{array}$




\section{1}

Introduction

The US Nuclear Regulatory Commission (USNRC) has co-organized an International Collaborative Fire Model Project (ICFMP) to evaluate fire models for Nuclear Power Plant (NPP) applications. Five benchmarking exercises have been conducted as part of the ICFMP to evaluate the predictive capability and limitations of fire models (both zone and CFD). This report documents numerical simulations by ICFMP participants of a set of full-scale compartment fire experiments that were conducted at the National Institute of Standards and Technology (NIST) in 2003. These experiments are referred to throughout this document as ICFMP Benchmark Exercise (BE) \#3. The other benchmarking exercises are:

ICFMP BE \#1: Hypothetical Cable Tray Fire Scenarios (no experiments) [Dey, 2002]

ICFMP BE \#2: Pool Fires in Large Halls [Miles, 2004]

ICFMP BE \#4: Large Fires in Compartments [Klein-Hessling, 2005]

ICFMP BE \#5: Flame Spread in Cable Tray Fires [Riese, 2005]

The details of the BE \#3 experiments are documented in Hamins et al. [2005]. This report provides a brief discussion of the design of the experiments and previous experiments.

This report includes contributions from the BRE in the U.K. (Chapter 2), IRSN in France (Chapter 3), Fauske and Associates, a U.S. consulting firm specializing in nuclear issues (Chapter 4), GRS in Germany (Chapters 5 and 6), and NIST in the U.S. (Chapter 7). A chapter was originally submitted by the US NRC, but was withdrawn because it did not satisfy that organization's internal review process. The models that were used in the withdrawn chapter, CFAST and FDS, are nevertheless applied in Chapters 2 and 7, respectively. In all, seven fire models are reviewed: the zone models CFAST, FLAMME_S, and FATE; the CFD models JASMINE, CFX, and FDS; and one "hybrid" model COCOSYS. 


\subsection{Review of Previous NRC-Sponsored Experiments}

In the past, the US NRC sponsored three large-scale fire test series. Sandia National Laboratories (SNL) conducted an "Investigation of Twenty Foot Separation Distance as a Fire Protection Method as Specified in 10 CFR 50 Appendix R" [NRC, 1983]. SNL conducted two additional test series for the NRC; "Enclosure Environment Characterization Testing for the Base Line Validation of Computer Fire Simulation Codes" [NRC, 1987], and "An Experimental Investigation of Internally Ignited Fires in Nuclear Power Plant Control Cabinets" [NRC, 1988]. The Laboratory on Research and Modeling of Fires, Institut de Radioprotection et de Sûreté Nucléaire, France, also conducted a single test. The test and results are described in "Probability Study on Fire Safety" [IRSN, 1997]. A brief description of each test series follows.

\subsubsection{Investigation of Twenty-Foot Separation Distance}

The tests were conducted at Underwriters Laboratories and the work reported in 1983 . The primary purpose of these tests was to evaluate the effectiveness of the fire protection afforded by the separation of redundant safety related cables by a horizontal distance of $6.1 \mathrm{~m}$ (20 ft) with no intervening combustibles or hazards. The full-scale test compartment was 7.6 $\mathrm{m} \times 4.3 \mathrm{~m} \times 3.0 \mathrm{~m}(25 \times 14 \times 10 \mathrm{ft})$. Construction was hollow core concrete blocks laid with mortar. The ceiling was of $12 \mathrm{~mm}$ inorganic board fastened to steel form and coated with a cementious mixture. There was a $4 \mathrm{ft} \times 8 \mathrm{ft}$ opening. The fire was a $1 \mathrm{ft} \times 5 \mathrm{ft}$ steel pan filled with five gallons of heptane. The pan was placed against the wall. The compartment contained two vertical trays with 43 cables, corresponding to a $12.5 \%$ fill. The vertical trays were directly above the heptane pan. Two horizontal cable trays, also filled at $12.5 \%$, were located $20 \mathrm{ft}$ from the fire. The horizontal trays were energized. Ventilation was free convective movement through the compartment opening.

\subsubsection{Fire Tests in a Control Room Mock-Up}

A series of fire experiments were conducted by researchers from Sandia National Laboratories (SNL), New Mexico, at the Factory Mutual Research Center (FMRC) in Rhode Island. The results were reported in March 1987 (US NRC, 1987, 1988). The primary purpose of these tests was to provide data for fire model validation. The test compartment was $18.3 \mathrm{~m} \mathrm{x}$ $12.2 \mathrm{~m} \times 6.1 \mathrm{~m}(60 \times 40 \times 20 \mathrm{ft})$. Interior ceiling and walls were lined with marinate, a fireresistant insulating material often used in fire testing. The floor was a concrete slab. Fuels 
used were a gas burner, heptane pool, methanol pool, and solid polymethylmethacrylate (PMMA) with fires ranging from $500 \mathrm{~kW}$ to $2000 \mathrm{~kW}$. Some of the tests were conducted in an empty enclosure; some with a control room mock-up. Forced ventilation was used during the tests ranging form one to ten room air changes per hour. Test variables included fire intensity, enclosure ventilation rate, and fire location.

\subsubsection{Fire Test in a Cable Room Mock-Up}

A single test was conducted at the Laboratory on Research and Modeling of Fires, IRSN, Cadarache, France. The objectives of this test were to provide quantitative information to safety analysts and contribute data to the qualification of the FLAMME_S fire model. The test was conducted on March 28, 1996 and reported in April 1997. The test was designed to simulate a cable room fire. Compartment construction was reinforced concrete with walls $0.25 \mathrm{~m}$ thick. Compartment dimensions were $9 \mathrm{~m} \times 6 \mathrm{~m} \times 7.5 \mathrm{~m}$. A forced ventilation system consisted of a floor blower, and an extractor located high on the wall. The ventilation flow rate was set at five air changes per hour. The compartment contained five bundles of 20 cables mounted on a ladder-like support. Four were set horizontally with voltage supplied to them (two near the ceiling and two near the fire). One was vertical without any voltage applied. One electrical cabinet was also included ( $1 \mathrm{~m} \times 0.3 \mathrm{~m} \times 1.2 \mathrm{~m})$ with metal plates inside to represent electrical equipment. One hundred liters of MOBIL DTE medium oil preheated to $250^{\circ} \mathrm{C}$ were used for the fire that yielded a peak heat release rate of $940 \mathrm{~kW}$. Temperature measurements were made along each cable run on either side of the bundle using $2 \mathrm{~mm}$ diameter type $\mathrm{K}$ thermocouples.

\subsection{Experimental Description of Benchmark Exercise \#3}

The experiments for Benchmark Exercise \#3 of the International Collaborative Fire Model Project (ICFMP) were designed by staff members from the US NRC and NIST. A draft specification of the experiments was issued to participants on September 6, 2002, to solicit comments, further ideas, and suggestions. Written comments on the draft specification were received from participants. The draft specification was also presented at the 6th ICFMP meeting at British Research Establishment (BRE), UK on October 10-11, 2002, when verbal comments from participants in the meeting were received and documented. Appendix $A$ of Hamins et al. [2005] provides the written and verbal comments received on the draft specification of the ICFMP Benchmark Exercise \#3, including the resolution and disposition of the comments. 
The design of Benchmark Exercise \#3 incorporated several lessons learned from previous NRC-sponsored large-scale fire tests described in the previous section. Most importantly, a considerable emphasis was placed on obtaining material properties, ventilation flow rates, leakage rates, and enough measurements to estimate the integrated heat losses through the walls and compartment opening. This information is vital for a model validation exercise.

Benchmark Exercise \#3 consisted of 15 large-scale experiments performed at NIST in June 2003. Numerous measurements (350 per test) were made including gas and surface temperatures, heat fluxes and gas velocities. The experiments are documented in Hamins et al. [2005]. Only a brief description of the experiments is included here.

The test compartment dimensions were $21.7 \mathrm{~m} \times 7.1 \mathrm{~m} \times 3.8 \mathrm{~m}$ high. An overview of the compartment is shown in Figure 1-1. The walls and ceiling were lined with two layers of marinate boards; the floor was covered with one layer of gypsum board on top of one layer of plywood. Thermo-physical properties of the marinite and other materials were provided to the participants. The compartment had one $2 \mathrm{~m}$ by $2 \mathrm{~m}$ door and a mechanical air injection and extraction system. Some of the tests were conducted with the door closed and no mechanical ventilation (Tests $2,7,8,13$, and 17), and in those tests the measured compartment leakage was an important consideration. Hamins et al. [2005] reports leakage area based on measurements performed prior to Tests $1,2,7,8$, and 13.

The mechanical ventilation was used during Tests $4,5,10$, and 16, providing about 5 air changes per hour. The door was closed during Test 4 and open during Tests 5, 10, and 16. The supply duct was positioned on the south wall, about $2 \mathrm{~m}$ off the floor. An exhaust duct of equal area to the supply duct was positioned on the opposite wall at a comparable location. The flow rates through the supply and exhaust ducts were measured in detail during breaks in the testing, in the absence of a fire.

Ventilation conditions, the fire size, and fire location were the key parameters varied in the test series. Table 1-1 lists the fuel type, pan position and duration of the ramp-up, rampdown and steady burn periods. The fire pan was located at floor level in the center of the compartment for most of the tests (Tests 1-13, 16, and 17). In Test 14, the fire was centered $1.8 \mathrm{~m}$ from the North wall. In Test 15, the fire was centered $1.25 \mathrm{~m}$ from the South wall. In Test 18, the fire was centered $1.55 \mathrm{~m}$ from the South wall. The fuel pan was $2 \mathrm{~m}$ long $\mathrm{x} 1 \mathrm{~m}$ wide and $0.1 \mathrm{~m}$ deep. The fuel used in 14 of the tests was heptane, while toluene was used for one test (Test 17). The HRR was determined using both the estimated fuel flow rate and 
oxygen consumption calorimetry. The uncertainty in the HRR measurement is described in Hamins et al. [2005]. The recommended uncertainty values were $17 \%$ for all of the tests. 

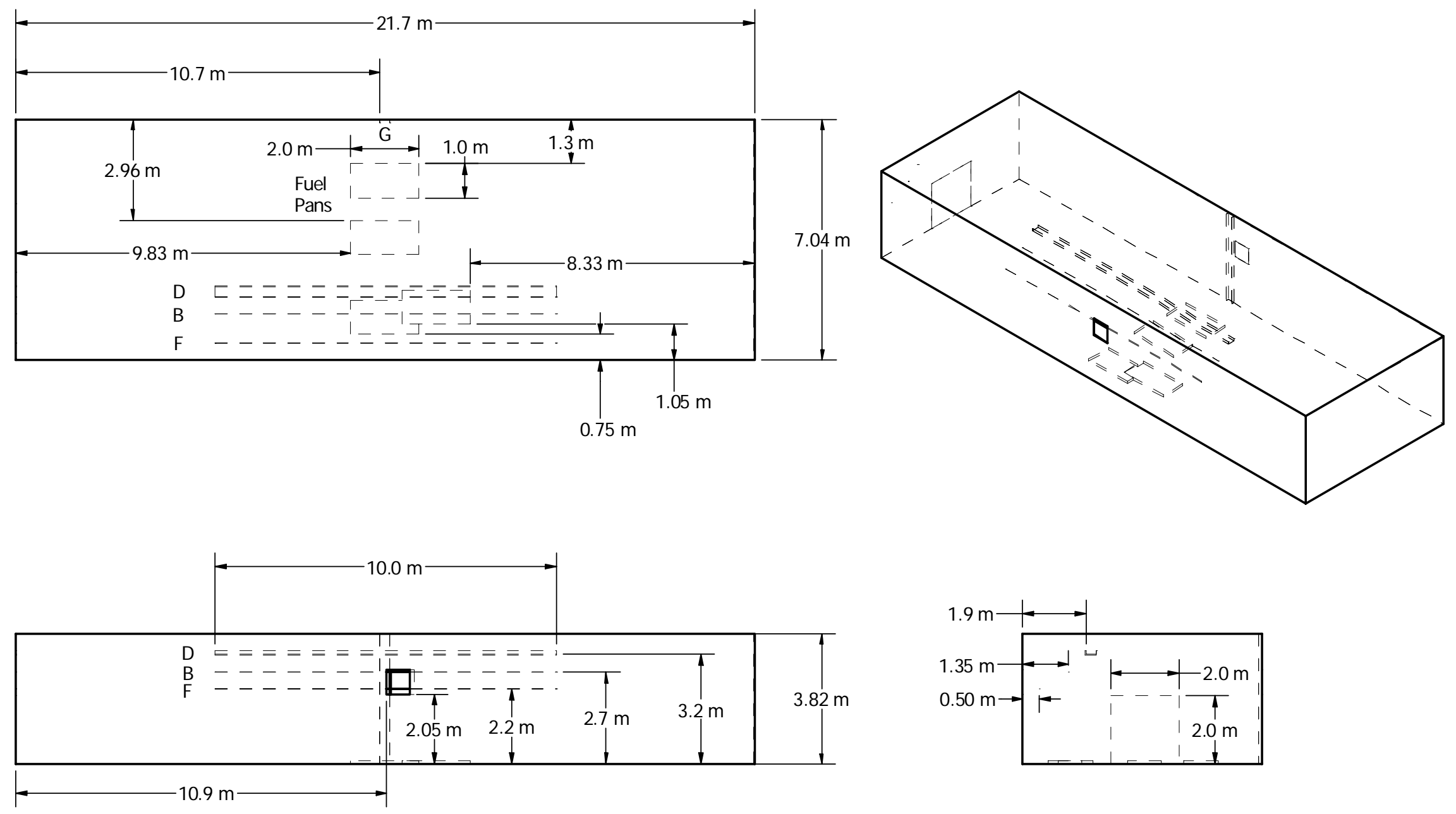

Figure 1-1. Plan, side and perspective schematic drawings of the ICFMP BE \#3 experimental arrangement. The north side of the compartment is at the top of the plan view. 
Table 1-1 Test matrix, including fuel type, pan position and duration of the ramp-up, ramp-down and steady burn period.

\begin{tabular}{|c|c|c|c|c|c|c|c|}
\hline \multirow[b]{2}{*}{ Test } & \multirow[b]{2}{*}{ Fuel } & \multirow[b]{2}{*}{$\begin{array}{l}\text { Fuel Pan } \\
\text { Position }\end{array}$} & \multicolumn{2}{|c|}{ Steady HRR (kW) } & \multirow{2}{*}{$\begin{array}{l}\text { Ramp-up } \\
\text { (s) }\end{array}$} & \multirow{2}{*}{$\begin{array}{c}\text { Steady Period } \\
\text { (s) }\end{array}$} & \multirow{2}{*}{$\begin{array}{c}\text { Ramp Down } \\
\text { (s) }\end{array}$} \\
\hline & & & $\begin{array}{c}\text { Based on } \\
\text { Fuel Flow } \\
\text { (NISTIR 1013) } \\
\end{array}$ & $\begin{array}{c}\text { Based on } \\
\text { Calorimetry } \\
\text { (NISTIR 1013-1) }\end{array}$ & & & \\
\hline 1 & Heptane & Center & 390 & 410 & 148 & 1202 & 150 \\
\hline 2 & Heptane & Center & 1140 & 1190 & 180 & 445 & 1 \\
\hline 3 & Heptane & Center & 1150 & 1190 & 178 & 1201 & 183 \\
\hline 4 & Heptane & Center & 1150 & 1200 & 178 & 636 & 1 \\
\hline 5 & Heptane & Center & 1150 & 1190 & 178 & 1201 & 183 \\
\hline 6 & \multicolumn{7}{|c|}{ test not conducted } \\
\hline 7 & Heptane & Center & 380 & 400 & 129 & 1203 & 128 \\
\hline 8 & Heptane & Center & 1140 & 1190 & 176 & 434 & 1 \\
\hline 9 & Heptane & Center & 1120 & 1170 & 175 & 1201 & 184 \\
\hline 10 & Heptane & Center & 1130 & 1190 & 176 & 650 & 1 \\
\hline 11 & \multicolumn{7}{|c|}{ test not conducted } \\
\hline 12 & \multicolumn{7}{|c|}{ test not conducted } \\
\hline 13 & Heptane & Center & 2220 & 2330 & 177 & 187 & 1 \\
\hline 14 & Heptane & North & 1130 & 1180 & 176 & 1205 & 186 \\
\hline 15 & Heptane & South & 1130 & 1180 & 180 & 1200 & 187 \\
\hline 16 & Heptane & Center & 2200 & 2300 & 177 & 205 & 1 \\
\hline 17 & Toluene & Center & 1070 & 1160 & 181 & 91 & 1 \\
\hline 18 & Heptane & South-East & 1130 & 1180 & 178 & 1202 & 187 \\
\hline
\end{tabular}




\subsection{The Benchmarking Process}

Participants in the modeling exercise were invited to submit predictions for Tests 2, 3 and 4 prior to the experiments to a non-participating third party. These results are referred to as "blind" throughout this report ${ }^{1}$. The specified heat release rate for Tests 2, 3 and 4 was $1000 \mathrm{~kW}$. This HRR was to be achieved by calibrating the fuel flow rate into the pan prior to the tests. No flow meter was installed on the fuel line, but oxygen consumption calorimetry was performed during the tests. Following the tests, the originally targeted HRR of $1000 \mathrm{~kW}$ was adjusted to roughly $1150 \mathrm{~kW}$ based on the actual fuel flow to the pan, and these results were published in NISTIR 1013, January 2004. Those using the adjusted HRR have referred to their calculations as "semi-blind" because they were given the HRR, but not the gas phase measurements within the compartment. In 2005, the HRR estimate was re-evaluated based on the results of a study of the large fire exhaust hood at NIST, in which several of the BE \#3 tests were repeated to reassess the HRR. The new estimate was a small increase over the older one (for example $1150 \mathrm{~kW}$ was increased to $1190 \mathrm{~kW}$ ). The NIST report was re-issued as NISTIR 1013-1 and is cited here as Hamins et al. [2005]. All of the modeling studies described in this report were completed prior to the release of NISTIR 1013-1, and the difference between the HRR cited in NISTIR 1013 and NISTIR 1013-1 is well within the reported uncertainty of this measurement (17\%).

The "blind" results reported in chapters 2 and 5 are based on the original specified HRR of $1000 \mathrm{~kW}$. These chapters also contain so-called "semi-blind" results. Chapters 3, 4, 6, and 7 contain only "semi-blind," or "open" results. Semi-blind calculations are those in which the modeler had knowledge of the measured HRR but not the measured compartment temperatures, heat fluxes, etc. The semi-blind set of calculations, along with those considered "open," are based on the revised HRR estimates (1130 kW to $1150 \mathrm{~kW}$ for most

\footnotetext{
${ }^{1}$ The use of the terms "blind" and "semi-blind" in this report differ from terms used in ASTM E 135505a, "Standard Guide for Evaluating the Predictive Capability of Deterministic Fire Models." According to the ASTM standard, "blind" means that the modeler is provided with "a basic description of the scenario," and that the modeler "is responsible for developing appropriate model inputs, ... including additional details of the geometry, material properties, and fire description, as appropriate." For the "blind" calculations described in this report, the modelers were given as much detail as was available, which according to the ASTM standard would more appropriately be characterized as "specified."
} 
tests), the most appropriate HRR for comparing models with experiments. None of the studies included in this report made use of the calorimetry-based HRR values reported by Hamins et al. [2005] because the calculations documented in this report were done prior to the release of the final HRR values. The final HRR values are only slightly greater than the 1130-1150 kW estimates. The two sets of HRR values are within the reported uncertainty of $17 \%$ and are shown in Table 1-1.

To put this into perspective, consider the set of graphs shown in Figure 1-2. Tests 2, 3 and 4 (the original blind simulation cases) were re-run with the CFD model FDS using HRR values of $1000 \mathrm{~kW}, 1150 \mathrm{~kW}$, and $1190 \mathrm{~kW}$. Notice that the HRR has little effect on the HGL (Hot Gas Layer) Height predictions, but it does have a noticeable effect on the HGL Temperature predictions. The difference between the $1150 \mathrm{~kW}$ and $1190 \mathrm{~kW}$ simulations is fairly small, and well within the uncertainty bounds of the HRR measurement itself. However, the $1000 \mathrm{~kW}$ simulations noticeably under-predict the HGL temperature. This is not surprising, because the HGL Temperature Rise is proportional to the two-thirds power of the HRR, according to the well-established MQH (McCaffrey, Harkleroad, Quintiere) correlation. Thus, a $15 \%$ change in the input HRR will yield a $10 \%$ change in the HGL Temperature Rise.

The decision by NIST staff to increase the HRR estimates from the values reported in NISTIR 1013 to the values reported in NISTIR 1013-1 was not based on the predictions of FDS (the NIST CFD model) but rather a re-assessment of the intrumentation and procedures used during the 2003 experiments in the Large Fire Laboratory at NIST. In fact, the FDS predictions are slightly closer to the measurements, on average, when using the intermediate values of the HRR reported in NISTIR 1013, the same values used by the ICFMP participants and reported in this report. The goal of the experimentalists at NIST is to make the most accurate measurements possible, not to match fire model predictions. Neither the experiments nor the model was tweaked or tuned to improve agreement. 

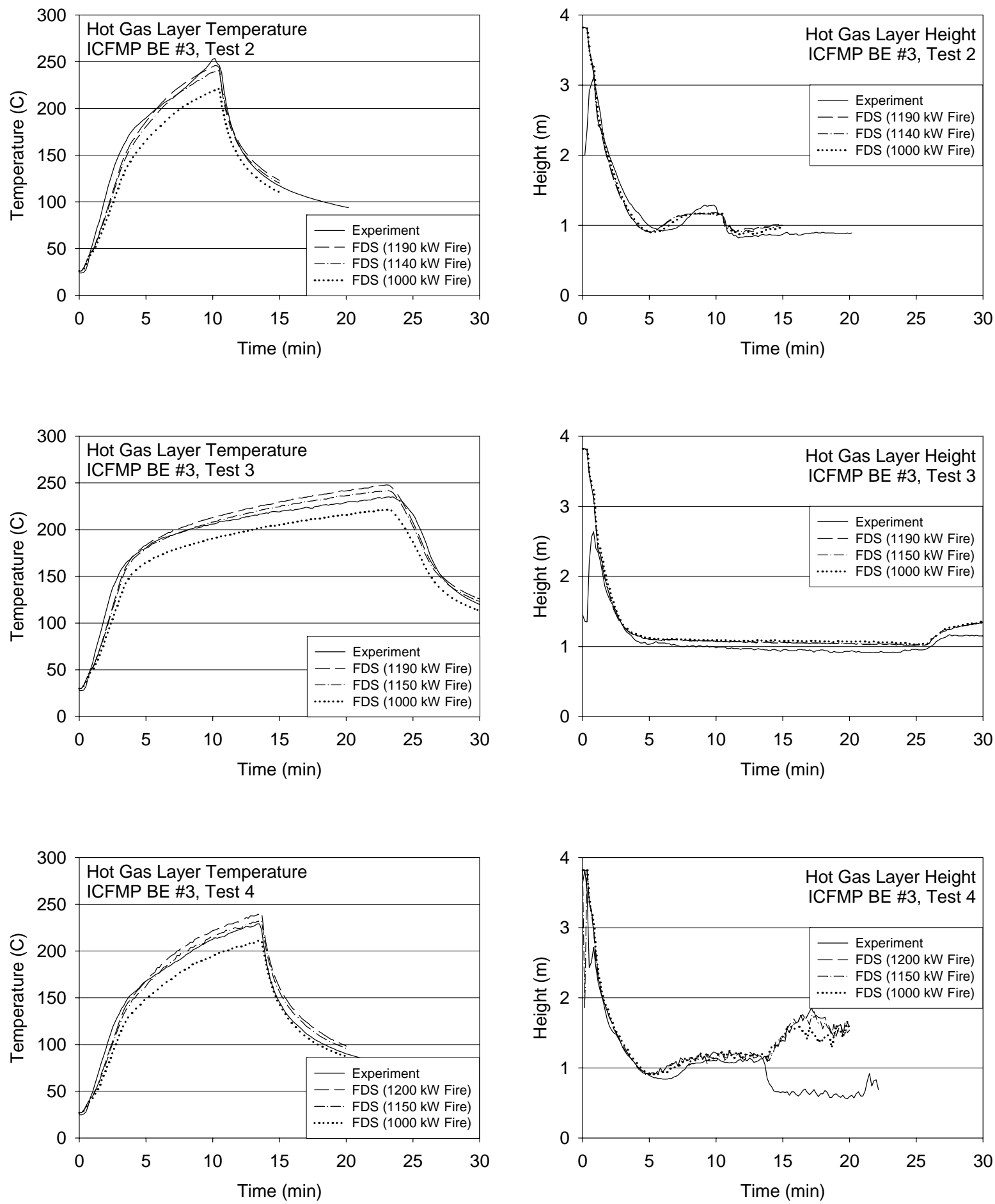

Figure 1-2. Hot Gas Layer Temperature and Height for Tests 2, 3 and 4, showing the experimental measurements and three model predictions with three different heat release rates. 


\section{$1.4 \quad$ References}

Dey, M., Ed., "International Collaborative Project to Evaluate Fire Models for Nuclear Power Plant Applications: International Panel Report on Benchmark Exercise \# 1, Cable Tray Fires," U.S. Nuclear Regulatory Commission, NUREG-1758, June 2002.

Klein-Hessling, W., M. Roewekamp and O. Riese, "Evaluation of Fire Models for Nuclear Power Plant Applications - Large Fires in Compartments, Benchmark Exercise \#4, International Panel Report," Gesellschaft fur Anlagen-und Reaktorsicherheit (GRS) Report Number GRS-213, ISBN-Nr.: 3-931995-80-1, 2006.

Hamins, A. et al. "Report of Experimental Results for the International Fire Model Benchmarking and Validation Exercise \#3," NIST Special Publication 1013-1, National Institute of Standards and Technology, Gaithersburg, Maryland, November 2003. Revised as 1013-1, June 2005.

Institut de Radioprotection et de Sûreté Nucléaire (IRSN), France, "Probability Study Program on Fire Safety," Laboratory on Research and Modeling of Fires, J. M. Such EF.30.15.R/96.442, April 1997.

Miles, S., "International Collaborative Project to Evaluate Fire Models for Nuclear Power Plant Applications: International Panel Report on Benchmark Exercise \# 2, Pool Fires in Large Halls," Building Research Establishment Ltd., BRE Report Number 212214, May 2004.

Riese, O., D. Hosser and M. Roewekamp, "Evaluation of Fire Models for Nuclear Power Plant Applications - Flame Spread in Cable Tray Fires, Benchmark Exercise \#5, International Panel Report," Gesellschaft fur Anlagen-und Reaktorsicherheit (GRS) Report Number GRS-214, ISBN-Nr.: 3-931995-81-X, 2006.

Riesemann, James M. Chavez, NUREG/CR-3192, SAND83-0306, RP, October 1983

U.S. Nuclear Regulatory Commission, "An Experimental Investigation of Internally Ignited Fires in Nuclear Power Plant Control Cabinets," J. M.Chavez, S. P. Nowlen, NUREG/CR-4527, SAND86-0336, Vol 2, November 1988.

U.S. Nuclear Regulatory Commission, "Enclosure Environment Characterization Testing for the Base Line Validation of Computer Fire Simulation Codes," S. P. Nowlen, NUREG/CR 4681, SAND86-1296, RP, March 1987. 
U.S. Nuclear Regulatory Commission, "Investigation of Twenty-Foot Separation Distance as a Fire Protection Method as Specified in 10 CFR 50, Appendix R," Douglas D. Cline, Walter A von 
Blind, i.e. fully-blind (prior to the release of fuel mass loss and mechanical ventilation flow rates), and semi-blind (with knowledge of the above rates) calculations were performed with CFAST (zone model) version 3.1.6 and Jasmine (CFD model) version 3.2.3. These are also referred to as 'stage 1' and 'stage 2' calculations respectively. Tests 2, 3 and 4 were studied in both the CFAST and JASMINE work.

For the blind calculations the geometry of the experimental rig was represented as closely a possible to that given in the problem specification [Maranghides et al, 2003] and the two subsequent small addendum documents. In the semi-blind calculations the experimentally measured fuel release and mechanical ventilation rates, and the estimated infiltration ventilation area (for the door-closed tests), [Hamins et. al., 2003a] were used. Comparisons between calculated results and the experimental measurements [Hamins et. al., 2003b] were made and are reported here.

\subsection{CFAST Input Parameters and Assumptions}

CFAST is a widely used zone model, available from the National Institute of Standards and Technology (NIST). It is a multi-room zone model, with the capability to model multiple fires and targets. The fuel pyrolysis rate is a pre-defined input, and the burning in the compartment is then modeled to generate heat release and allow species concentrations to be calculated. For most applications CFAST is used as a conventional two-zone model, whereby each compartment is divided into a hot gas upper layer and a cold lower layer. In the presence of fire, a plume zone/model transports heat and mass from the lower to upper layer making use of an empirical correlation [McCaffrey, 1983]. Flows through vents and doorways are determined from correlations derived from the Bernoulli equation. Radiation heat transfer between the fire plume, upper \& lower layers and the compartment boundaries is included using an algorithm derived from other published work [Siegel and Howell, 1981]. Other features of CFAST relevant to the benchmark exercise include a one-dimensional solid phase heat conduction algorithm employed at compartment walls and targets and a network flow model for mechanical ventilation. 
For an in-depth description of CFAST, including its scientific and engineering background, the solved equations and the physical sub-models see the reference [Jones et al., 2000] and the later reference accompanying CFAST version 5 [Jones et al., 2004]. A summary of validation exercises that have been undertaken for the CFAST model is also included in [Jones et. al., 2004]. The panel report [Miles, 2004] and accompanying technical annexes for the second benchmark exercise include a number of examples where CFAST calculations have been compared to experimental measurement data for smoke filling inside a large hall.

Information relevant to the application of CFAST to this benchmark exercise is given below.

- Main geometry and thermal boundary conditions. Dimensions of room, and also the door in Test 3, were modeled as in the problem specification. Thermal properties of the walls, floor and ceiling were represented by creating a new user defined material with the required properties. Conduction losses to the room walls, ceiling and floor were included, using the one-dimensional heat conduction sub-model.

- Leakage area for closed-door tests. A leakage area (vent) of width $0.17 \mathrm{~m}$ and height $0.17 \mathrm{~m}$, located at floor level, was used in the blind calculations. Some parametric calculations were undertaken using increased leakage vent dimensions of $0.34 \mathrm{~m}$ by $0.34 \mathrm{~m}$. By contrast, for the semi-blind calculations the dimensions were fixed at $0.289 \mathrm{~m}$ by $0.289 \mathrm{~m}$. Note that as a consequence of the zone modeling assumptions employed there is no positional information attached to leakage vent location.

- Mechanical ventilation. In Test 4 mechanical ventilation was defined. In CFAST, and zone models in general, the height of the vents above floor level is specified, but no further geometrical information is included, i.e. supply and extraction is to/from either the lower or upper layer, which by definition extends across the entire width and length of the room. However, initial attempts to specify a mechanical supply and a mechanical extract failed to produce converged solutions. The problem was by-passed by specifying a mechanical extract vent and a vertical natural vent (window), with the same dimensions and height above the floor. The natural vent allowed the inflow of replacement air for the mechanical extraction.

- Fire specification. This was treated as a constrained fire (i.e. oxygen controlled) with area $2 \mathrm{~m}^{2}$. The fuel was modeled as heptane, using a heat of combustion of $4.5 \times 10^{7} \mathrm{~J} \mathrm{~kg}^{-1}$. The pyrolysis rate was then set such that in the blind simulations the chemical energy release was exactly $1 \times 10^{6} \mathrm{~W}$ and in the semi-blind simulations was 
adjusted to the suggested actual value. In all calculations a two-layer gas assumption was assumed inside the room and the 'ceiling jet' option was active.

In the blind calculations the radiative fraction was set initially to 0.35 , and then adjusted to 0.0 and 0.5 in various parametric analyses. For the semi-blind calculations the value was fixed at 0.44 . In all calculations the lower oxygen limit (LOL) was fixed at $10 \%$. However, the test was considered to be over once the oxygen concentration in the lower layer (where the measurement location was positioned) dropped to $15 \%$.

The choice of radiative fraction and heat of combustion requires careful scientific/engineering judgment. An appropriate choice of the radiative fraction in particular may have a significant bearing on the calculations for upper layer temperature. For a discussion of these parameters, see the panel report of the second benchmark exercise [Miles, 2004], where the terms and their meaning are reviewed.

- Targets. A required approximation in CFAST is that cable targets are treated as rectangular slabs. Furthermore, heat conduction is modeled one-dimensionally, i.e. in the direction of the surface normal. In the calculations the cable targets were orientated such as to be exposed to the maximum thermal load. The appropriate choice of target location and orientation is highly important in a hazard analysis for applications such as nuclear power plant safety cases where cable damage is an important factor.

\subsection{JASMINE Input Parameters and Assumptions}

JASMINE [Cox \& Kumar, 1987] solves the Reynolds-Averaged Navier Stokes (RANS) equations of fluid flow on a single-block Cartesian grid. The coupled set of equations for each of the three Cartesian velocity components, enthalpy (heat) and other scalars required by the various sub-models (e.g. fuel mass and mixture fractions for combustion) is approximated as a system of algebraic equations that are solved numerically on a discrete grid. This generates a solution value for each variable at each grid location. JASMINE uses the finite volume method, where the differential equations are first transformed into an integral form and then discretized on the control volumes (or cells) defined by the numerical grid. This solution procedure is coupled with a variant of the SIMPLE pressure-correction scheme [Patankar, 1980].

In solving the RANS equations, the fluctuating components of the turbulent motion are not solved directly, but instead the effect of turbulence is included by means of a separate sub- 
model. This saves computational effort, and for many industrial applications, including fire modelling, has been shown to produce reliable solutions provided a suitable turbulence mode is used. JASMINE employs a standard $\kappa-\varepsilon$ turbulence model [Launder \& Spalding, 1974] with additional buoyancy source terms. An "effective turbulent viscosity" is calculated at each control volume, which can be considered as an increased viscosity to incorporate the effects of turbulence. Standard wall functions for enthalpy and momentum [Launder \& Spalding, 1974] describe the turbulent boundary layer adjacent to solid surfaces. Transient solutions are generated by a first-order, fully-implicit scheme.

The fire combustion process is generally modelled using an eddy break-up assumption [Magnussen \& Hjertager, 1976] in which the fuel pyrolysis rate is specified as a boundary condition. Combustion is then calculated at all control volumes as a function of fuel concentration, oxygen concentration and the local turbulent time-scale (provided by the $\kappa-\varepsilon$ model). A simple one-step, infinitely fast chemical reaction is assumed. The eddy break-up model is appropriate for turbulent diffusion flames characteristic of fire, where the rate of reaction is controlled by the comparatively slow mixing of fuel with oxygen. Complete oxidation of the fuel is assumed when sufficient oxygen is available.

Radiant heat transfer is modelled with either the six-flux model [Gosman \& Lockwood, 1973], which assumes that radiant transfer is normal to the co-ordinate directions or potentially more accurate discrete transfer method [Lockwood \& Shah, 1981]. Local absorptionemission properties are computed using a mixed grey-gas model [Truelove, 1976], which calculates the local absorption coefficient as a function of temperature and gas species concentrations.

Thermal conduction into solid boundaries may be included by means of a quasi-steady, semi-infinite, one-dimensional assumption, which is appropriate for many smoke movement applications. Alternatively, the solution of the one-dimensional heat conduction equation into the solid is also available. In both methods, the purpose of the solid-phase conduction calculation is to generate the solid surface temperature, at each control volume adjacent to a solid boundary at each time step, by balancing the convection, radiation and conduction heat fluxes. The surface temperature then provides the (thermal) boundary condition for the next time-step of the CFD solution.

JASMINE has been successfully used to simulate fire and smoke movement in a wide variety of construction projects (e.g. atria, hospitals, sport stadia, warehouses, tunnels, etc.), as well as in other applications such as a nuclear containment cell, transportation vehicles and 
marine ships. The references [Cox et al., 1987], [Miles, 2000] and [Miles, 2001] provide some examples of JASMINE validation exercises. The panel report [Miles, 2004] and the BRE technical annex (Appendix $\mathrm{H}$ ) for the second benchmark exercise report the comparison of JASMINE calculations and measurement data for the smoke filling of a large hall.

Information relevant to the application of JASMINE to this benchmark exercise is given below.

- Main geometry and thermal boundary conditions. Dimensions of room, and also the door in Test 3, were modeled exactly as in the problem specification. The thermal properties of the walls, floor and ceiling were included as specified. Conduction losses to the room walls, ceiling and floor were included, using the one-dimensional heat conduction submodel available in JASMINE. Radiative and convective fluxes from the radiation/gas fields were calculated at each solid surface cell face at each numerical time step.

- Leakage area for closed-door tests. A leakage area (vent) of width $0.17 \mathrm{~m}$ and height $0.17 \mathrm{~m}$, located at floor level, was used in the blind calculations. For the semi-blind calculations the dimensions were increased to $0.289 \mathrm{~m}$ by $0.289 \mathrm{~m}$, and the leakage vent was again located at floor level.

- Mechanical ventilation. In Test 4 mechanical ventilation was included, with the supply and extract vents located as specified. For the blind calculations a fixed mass flow rate was imposed, corresponding to 5 air changes per hour at ambient temperature. Note here that as the gas temperature inside the room increases the air changes per hour will also increase according to the reduction in density. The mechanical ventilation rate was adjusted in the semi-blind calculations to $1.157 \mathrm{~kg} \mathrm{~s}^{-1}$ according to the information supplied [Hamins, 2003a].

In the blind calculations the air supply was directed normal to the wall surface. However, for the semi-blind calculations the supply angle was adjusted to $45^{\circ}$ as indicated in the information supplied [Hamins, 2003a].

- Fire specification. Combustion was included using the eddy break-up sub-model, assuming a heptane fuel source and a simple one-step chemical reaction to yield $\mathrm{CO}_{2}$ and $\mathrm{H}_{2} \mathrm{O}$. As in the CFAST calculations the heat of combustion to $4.5 \times 10^{7} \mathrm{~J} \mathrm{~kg}^{-1}$. Again, in the blind predictions the chemical energy release rate was exactly $1 \times 10^{6} \mathrm{~W}$ and in the semi-blind simulations was adjusted to the suggested actual value. The heptane was as- 
sumed to pyrolyse as a pool fire with area $2 \mathrm{~m}^{2}$, meaning that the fuel spray itself was not modeled.

Oxygen limitation was not applied in the calculations. However, the oxygen concentration at the measurement location was 'tracked' and the experiment was assumed to terminate once the level had reduced to $17 \%$ by mass (assumed to be approximately equivalent to $15 \%$ by volume), which occurred only in Tests 2 and 4 .

A main limitation of the fire modeling adopted is the absence of a soot generation and subsequent radiation process. For the blind calculations $30 \%$ of the heat was removed at the source to represent that which could be expected to be 'lost' by radiation from the sooty flame region above the fuel source. The remainder of this heat is assumed to be convected into the rest of the compartment or, as a relatively small fraction, by radiation from the plume region due to $\mathrm{CO}_{2}$ and $\mathrm{H}_{2} \mathrm{O}$. Note also that of the heat convected into the compartment, some of this may subsequently be radiated from the smoke gases (due to $\mathrm{CO}_{2}$ and $\mathrm{H}_{2} \mathrm{O}$ ). In some parametric calculations the heat 'lost' by radiation from the sooty flame region was reduced to zero. In the semi-blind calculations the figure used was either $35 \%$ and $0 \%$.

The note made above under CFAST regarding the choice of radiative fraction (here the heat 'lost' by radiation) and heat of combustion applies equally to CFD modeling.

- Targets. The response at the target gauges 3, 4, 5, 6, 9 and 10 was included. Here local gas temperature and incident radiation fluxes were calculated. Cable target temperature calculations were not performed with JASMINE. However, comparisons of predicted and measured gas temperatures at locations in close vicinity to the cable target measurement locations were performed.

- Radiation. The six-flux radiation model was employed throughout. This provides a reasonable and efficient treatment for radiation exchange between the hot smoke gases and the compartment boundaries. Its primary limitation with respect to this benchmark exercise is that the radiation intensities are modeled in the Cartesian axial directions, and so important off-axis directional information may be missed. This may have a consequence for the target heating calculations.

Emission/absorption due to the presence of the primary products of combustion, i.e. $\mathrm{CO}_{2}$ and $\mathrm{H}_{2} \mathrm{O}$, was included, but not soot (which was not explicitly included in the JASMINE calculations). 
- Other settings. A numerical mesh of approximately 100,000 cells was employed, with a limited grid sensitivity for Test 2 using 800,000 cells (times 2 in each direction). The numerical time-step was fixed at $2 \mathrm{~s}$ throughout. For all calculations the $\kappa-\varepsilon$ turbulence model with buoyancy modifications was employed.

\subsection{Comparison of CFAST Blind and Semi-Blind Predictions with Measurements}

Results are presented in this section primarily as graphs that directly compare calculated quantities (blind and semi-blind predictions) against measurements made in the experimental program (Tests 2,3 and 4). In the various figures, the terms 'stg1' (i.e. stage 1) and 'stg2' (i.e. stage 2) have been adopted for blind the semi-blind calculations respectively. In some cases the prediction data has been plotted only until the fire was predicted to have been terminated (due to the oxygen concentration dropping below $15 \%$ by volume). Likewise, the experimental measurement data in Tests 2 and 4 is plotted until the fuel supply was terminated for safety reasons (oxygen concentration at O2-2 dropping below $15 \%$ ).

A number of parametric calculations (runs) were performed for each test in the blind (stg1) stage and a couple of additional ones in the semi-blind (stg2) phase. Table 2-1 summarizes the main features of each of these calculations.

Table 2-1 CFAST calculation runs

\begin{tabular}{|c|c|c|c|c|}
\hline Test No. & \multicolumn{2}{|c|}{ Blind or semi-blind and Run No. } & $\begin{array}{l}\text { Leakage vent size } \\
\text { (Tests } 2 \text { \& 4) }\end{array}$ & Radiative fraction \\
\hline 2 & $(\operatorname{stg} 1)$ & run 1 & $0.17 \mathrm{~m} \times 0.17 \mathrm{~m}$ & 0.35 \\
\hline 2 & blind $\quad(\operatorname{stg} 1)$ & run 2 & $0.17 \mathrm{~m} \times 0.17 \mathrm{~m}$ & 0 \\
\hline 2 & blind $\quad(\operatorname{stg} 1)$ & run 3 & $0.17 \mathrm{~m} \times 0.17 \mathrm{~m}$ & 0.5 \\
\hline 2 & $(\operatorname{stg} 1)$ & run 4 & $0.34 \mathrm{~m} \times 0.34 \mathrm{~m}$ & 0.35 \\
\hline 2 & semi-blind (stg2) & run 1 & $0.289 \mathrm{~m} \times 0.289 \mathrm{~m}$ & 0.44 \\
\hline 3 & $(\operatorname{stg} 1)$ & run 1 & $\mathrm{n} / \mathrm{a}$ & 0.35 \\
\hline 3 & blind $\quad(\operatorname{stg} 1)$ & run 2 & $\mathrm{n} / \mathrm{a}$ & 0 \\
\hline 3 & blind $\quad(\operatorname{stg} 1)$ & run 3 & $\mathrm{n} / \mathrm{a}$ & 0.5 \\
\hline 3 & semi-blind (stg2) & run 1 & $\mathrm{n} / \mathrm{a}$ & 0.44 \\
\hline 4 & blind & run 1 & $0.17 \mathrm{~m} \times 0.17 \mathrm{~m}$ & 0.35 \\
\hline 4 & blind & run 2 & $0.17 \mathrm{~m} \times 0.17 \mathrm{~m}$ & 0 \\
\hline 4 & blind $\quad(\operatorname{stg} 1)$ & run 3 & $0.17 \mathrm{~m} \times 0.17 \mathrm{~m}$ & 0.5 \\
\hline
\end{tabular}


Figs. 2-1 to 2-5 compare the predicted and measured upper and lower layer gas temperatures, layer heights, pressures, species concentrations and target incident radiation fluxes for Test 2. In the plots of target incident radiation flux at gauge 3 CFAST values are provided in both the downward direction (as in the measurement) and for comparative purposes also in the upward direction.

Fig. 2-6 compares he predicted and measured surface temperatures at various cable target locations for run 1 of the blind and semi-blind stages. As CFAST models the targets a rectangular 'slabs' with a one-dimensional heat conduction treatment at each face, the figure includes the surface temperature as calculates assuming various slab orientations. This is important when performing a hazard analysis in order that the most critical direction(s) are accounted for.

Figs. 2-7 to 2-11 repeat the above for Test 3, except that lower gas layer temperature, pressure and oxygen concentration are not plotted as these are not significant hazard variables with the door open. Finally, Figs. 2-12 to 2-17 provide the information for Test 4.

The main observations and findings from the CFAST calculations are summarized below:

- While the size of the infiltration opening for Test 2 had a major effect on the pressure inside the compartment (see Fig. 2-3), it had little influence on other calculated quantities. CFAST calculations suggest that the infiltration leakage area in the experiment may have been just above a critical value, below which pressure inside the compartment may have been expected to rise significantly, i.e. the experimental leakage area was just above this critical value, and so the measured pressures were relatively low.

- Tests 2, 3 and 4 all suggest that a radiative fraction of at lease 0.35 is appropriate. Using a value of zero consistently resulted in hot gas layer temperatures being too high. The results actually suggest a value closer to 0.5 . However, care should be required in reaching such a conclusion as the influence of the heat of combustion, fuel supply rate and heat losses to the boundaries all have a bearing too.

- The layer height predictions were judged to be good. The apparent discrepancy in Tests 2 and 4, where the measurement data suggests that the layer did not descend to the floor, whereas CFAST calculations indicate that it did, is most likely due to the numerical data reduction method employed in calculating layer height from the measured temperature data. 
- In Test 3, where the open door condition provided a 'classical' room fire scenario, the CFAST predictions for gas temperatures, gas species and incident radiant fluxes and door flows were judged to be in good agreement with the experimental measurements. See the remark above about the appropriate choice of radiative fraction. Note also that given the correct gas temperature, the incident radiant fluxes should be reasonable.

- Apart from CO, the gas species predictions were in reasonable agreement to the measured values. Not being a major product of combustion, calculation of $\mathrm{CO}$ concentration is significantly more difficult than for $\mathrm{CO}_{2}$, which is a major product.

- A notable feature of the CFAST predictions was that the calculated incident radiant fluxes were, in each Test, largely independent of location or orientation. However, in Tests 2 and 4 the measurements indicate a significant location and/or orientation dependency on the incident fluxes. Hence the agreement between predicted and measured incident radiant flux was quite variable for Tests 2 and 4. One cause of discrepancy is the fact zone models such as CFAST do not model most of the three dimensional effects of a compartment fire.

- Given the level of discrepancy between the predicted and measured incident fluxes, the target surface temperature predictions were judged to be in reasonable agreement with the measured values.

- The method used to implement mechanical ventilation in Test 4 (mechanical extract vent and a vertical natural vent for make-up air) seemed to have no significant implication for the quality of the CFAST predictions. An under prediction of compartment pressure may, however, be one consequence.

- In Tests 2 and 4 CFAST consistently predicted that the test would terminate (lower layer oxygen concentration dropping below 15\%) earlier than was the case in the experiment. 

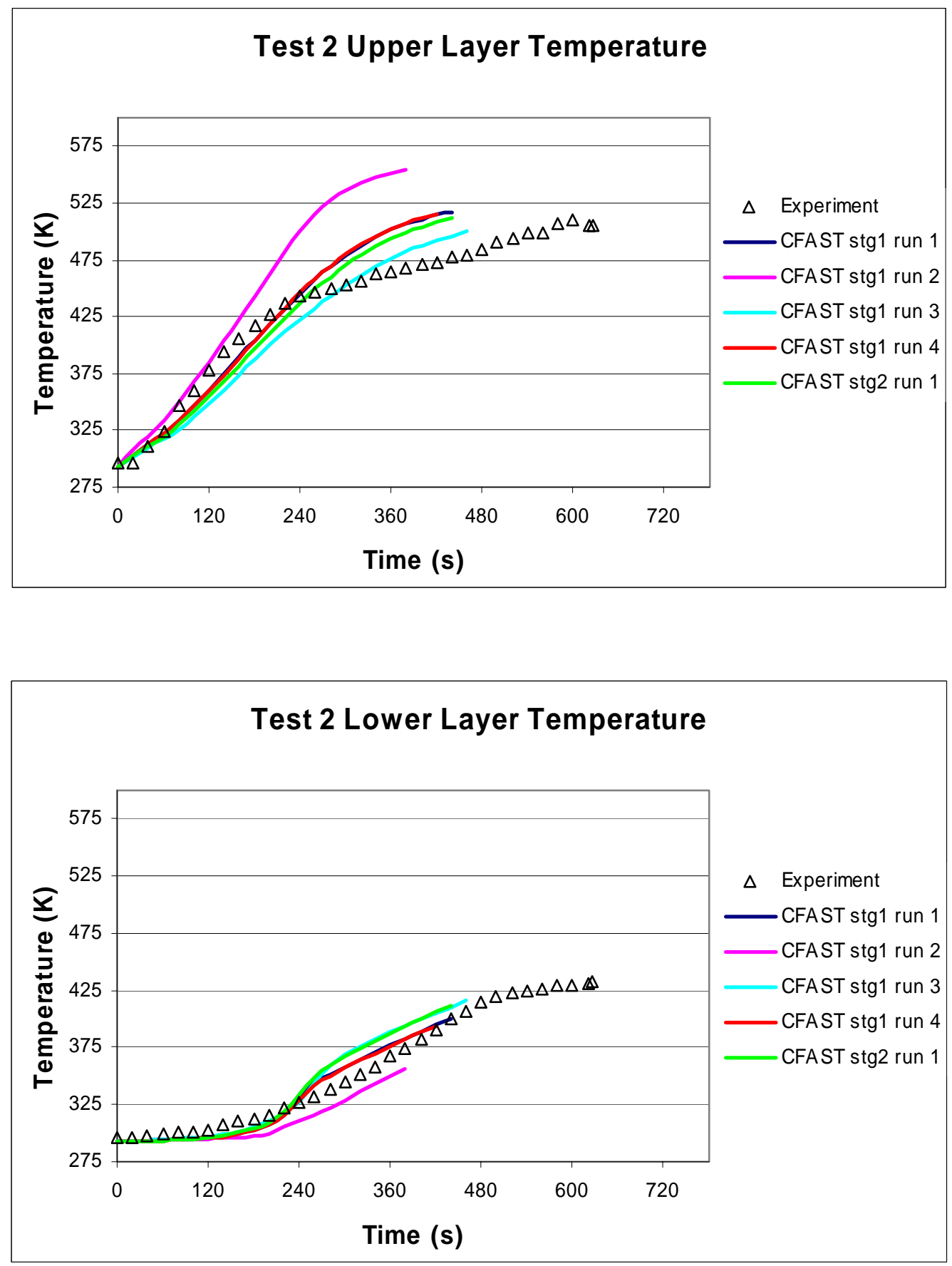

Fig. 2-1 Measured and CFAST predicted gas layer temperatures for Test 2 


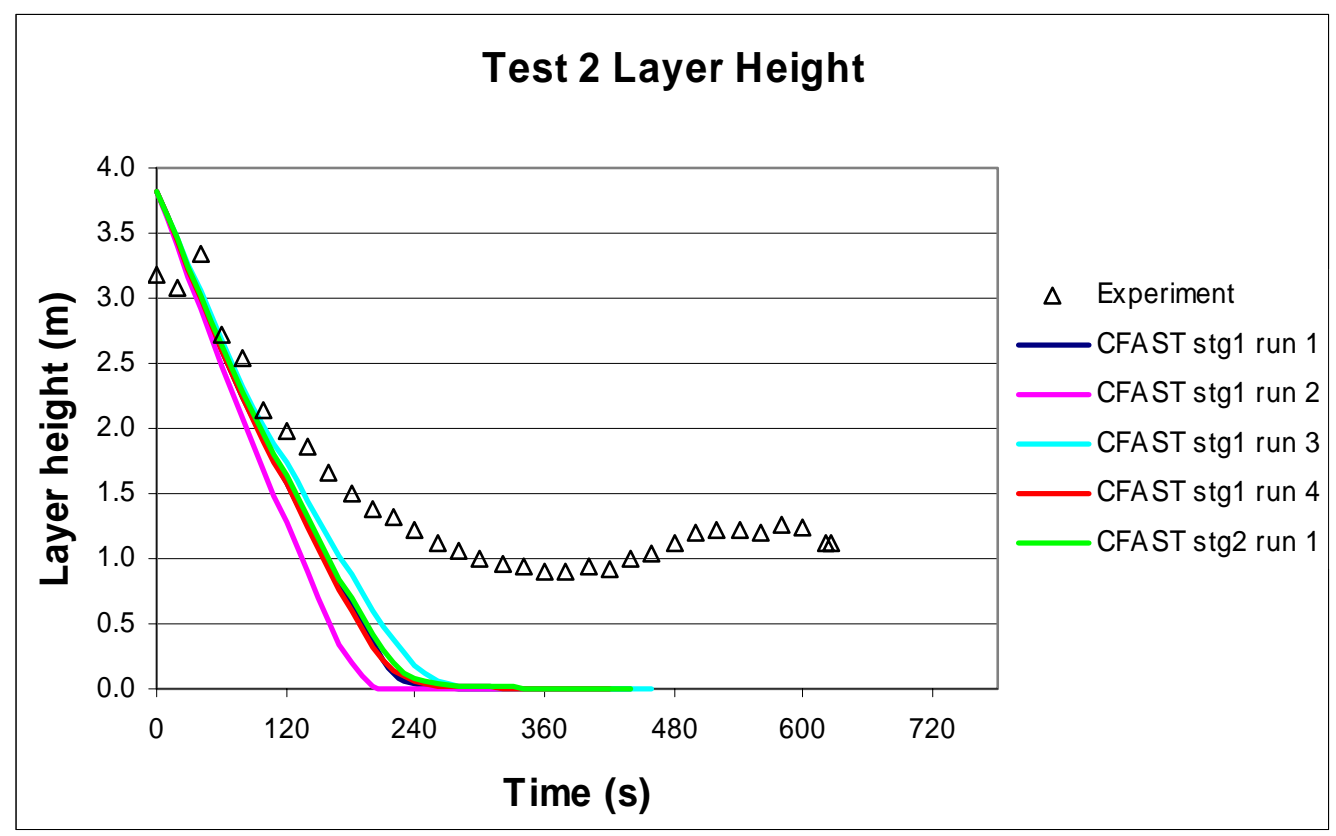

Fig. 2-2 Measured and CFAST predicted hot gas layer height for Test 2

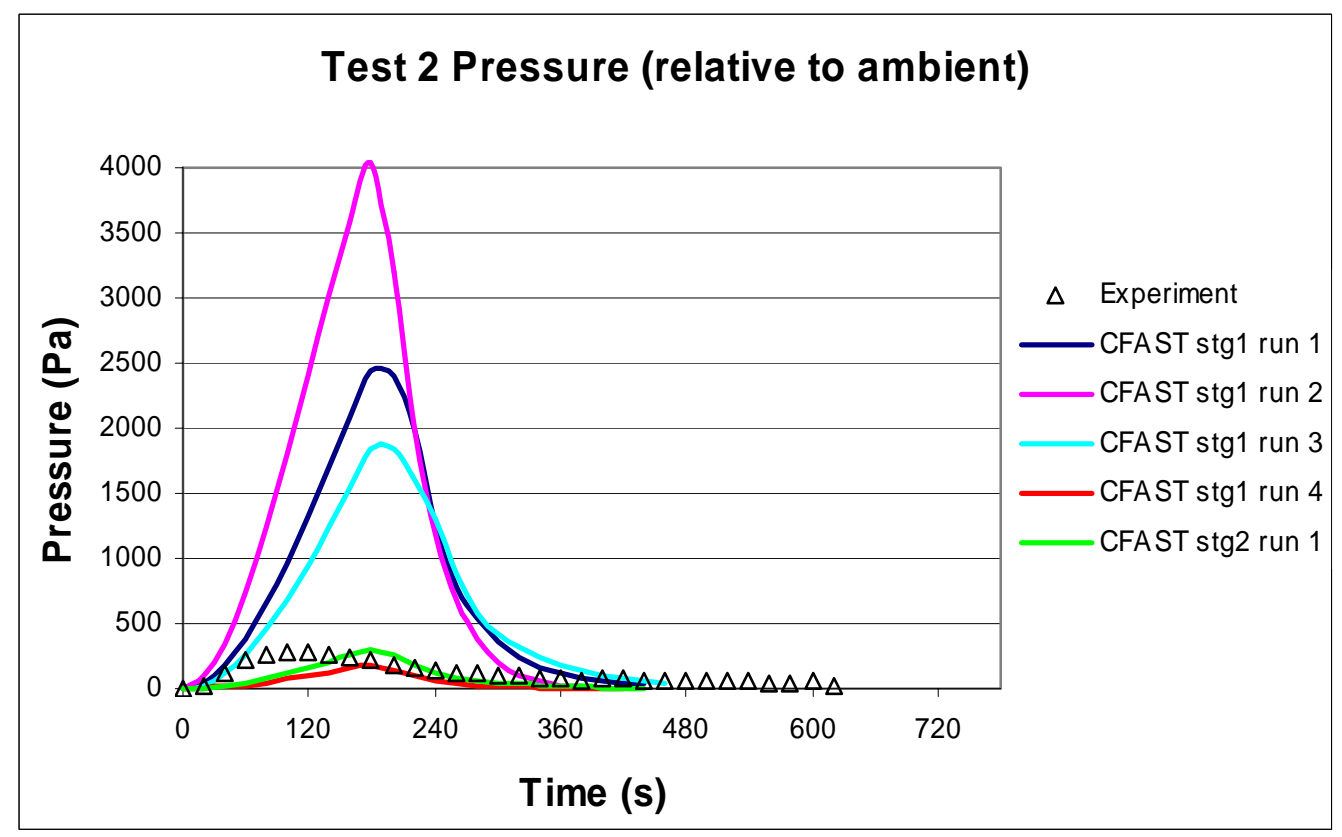

Fig. 2-3 Measured and CFAST predicted static pressure for Test 2 

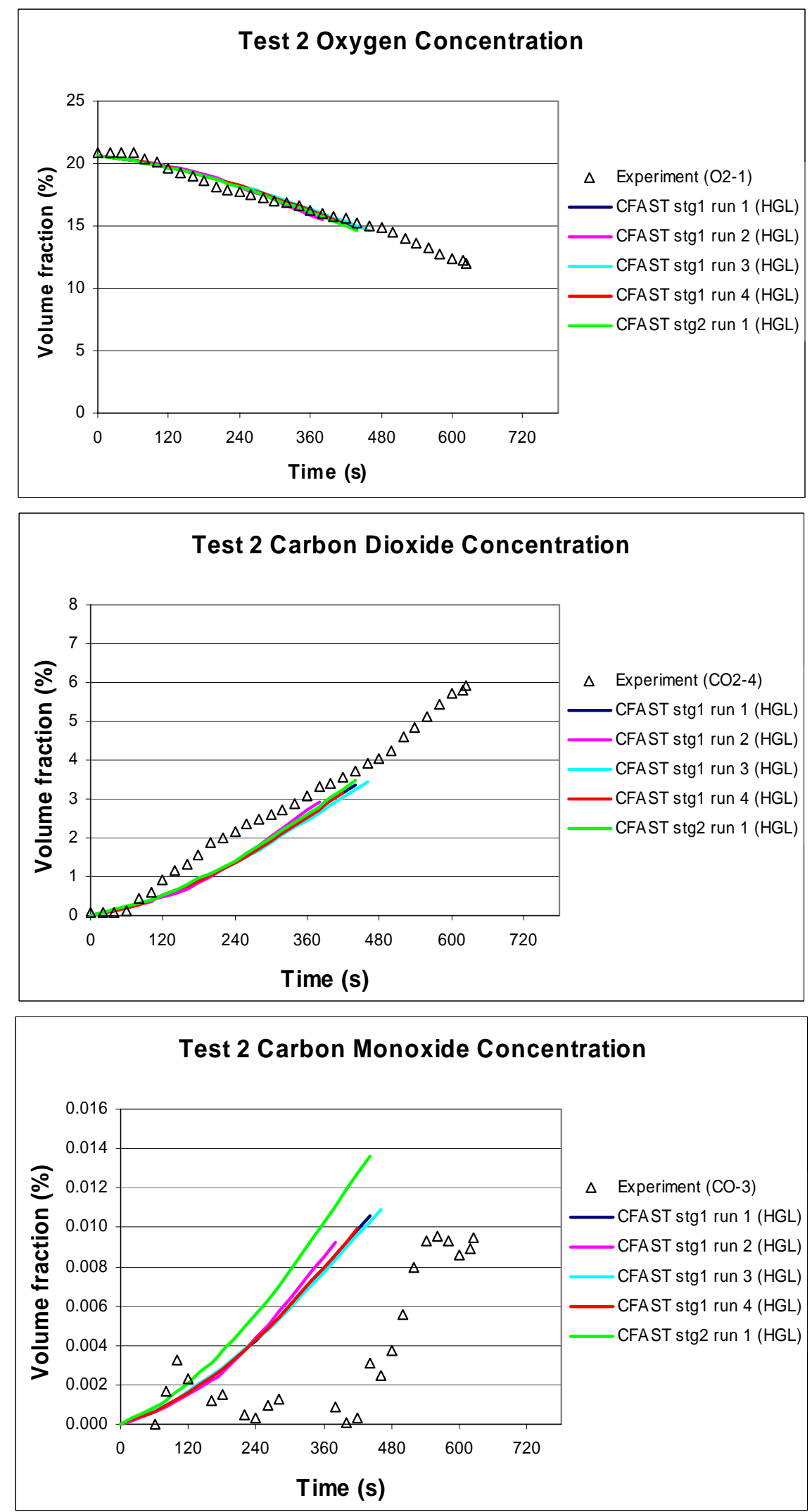

Fig. 2-4 Measured and CFAST predicted gas concentrations for Test 2 

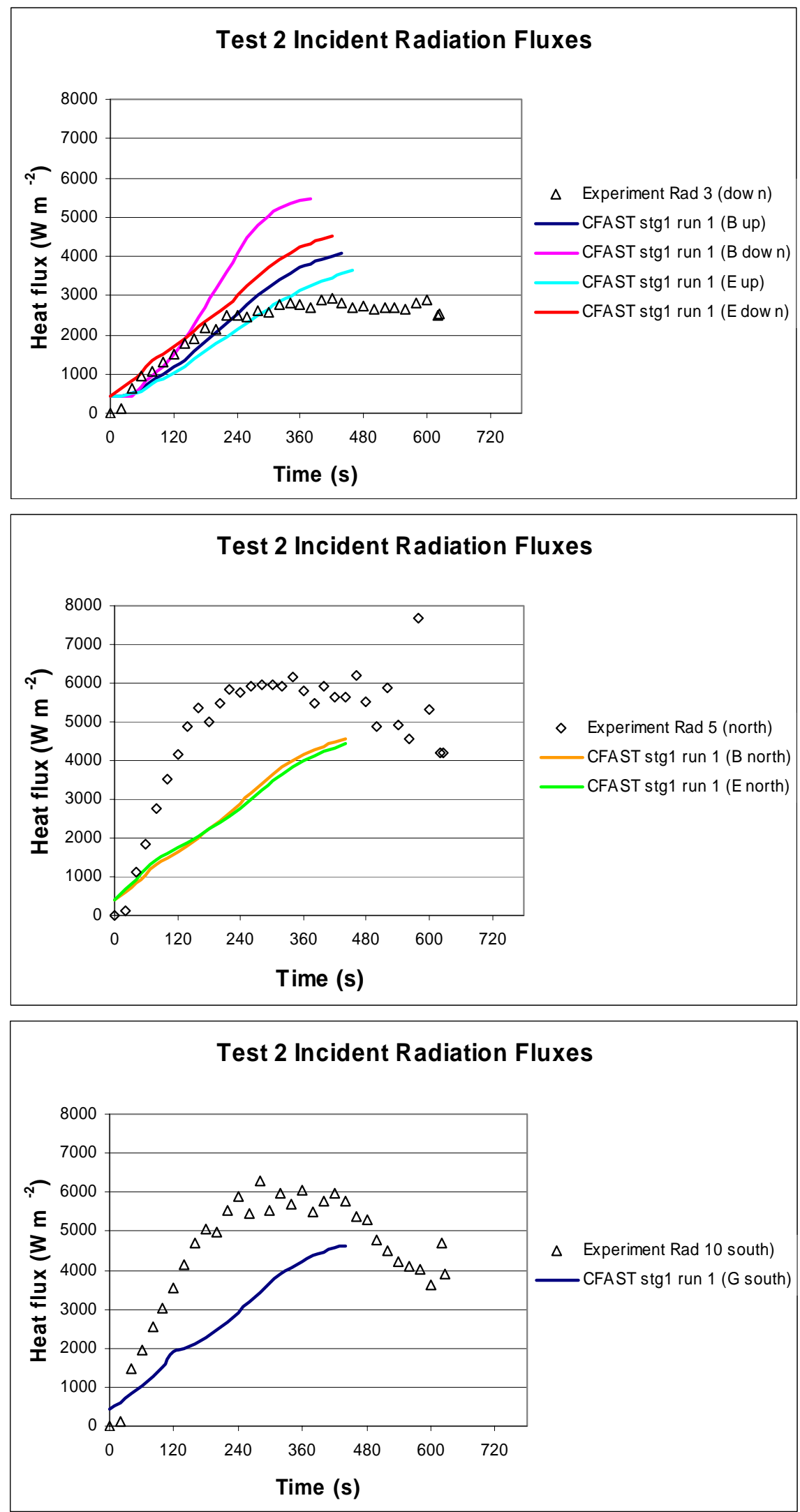

Fig. 2-5 Measured and CFAST predicted incicent fluxes for Test 2. 

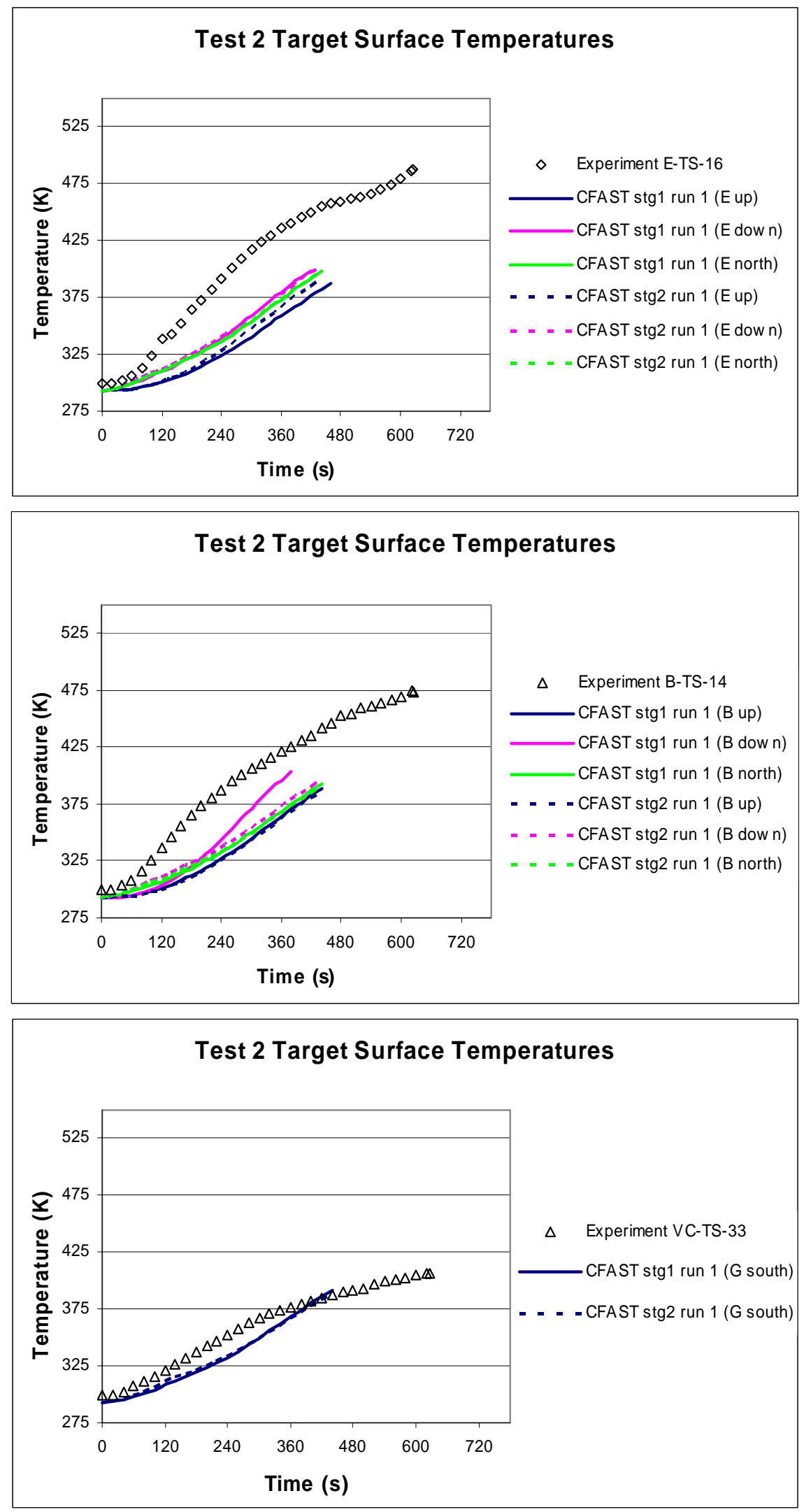

Fig. 2-6 Measured and CFAST predicted target surface temperatures for Test 2 


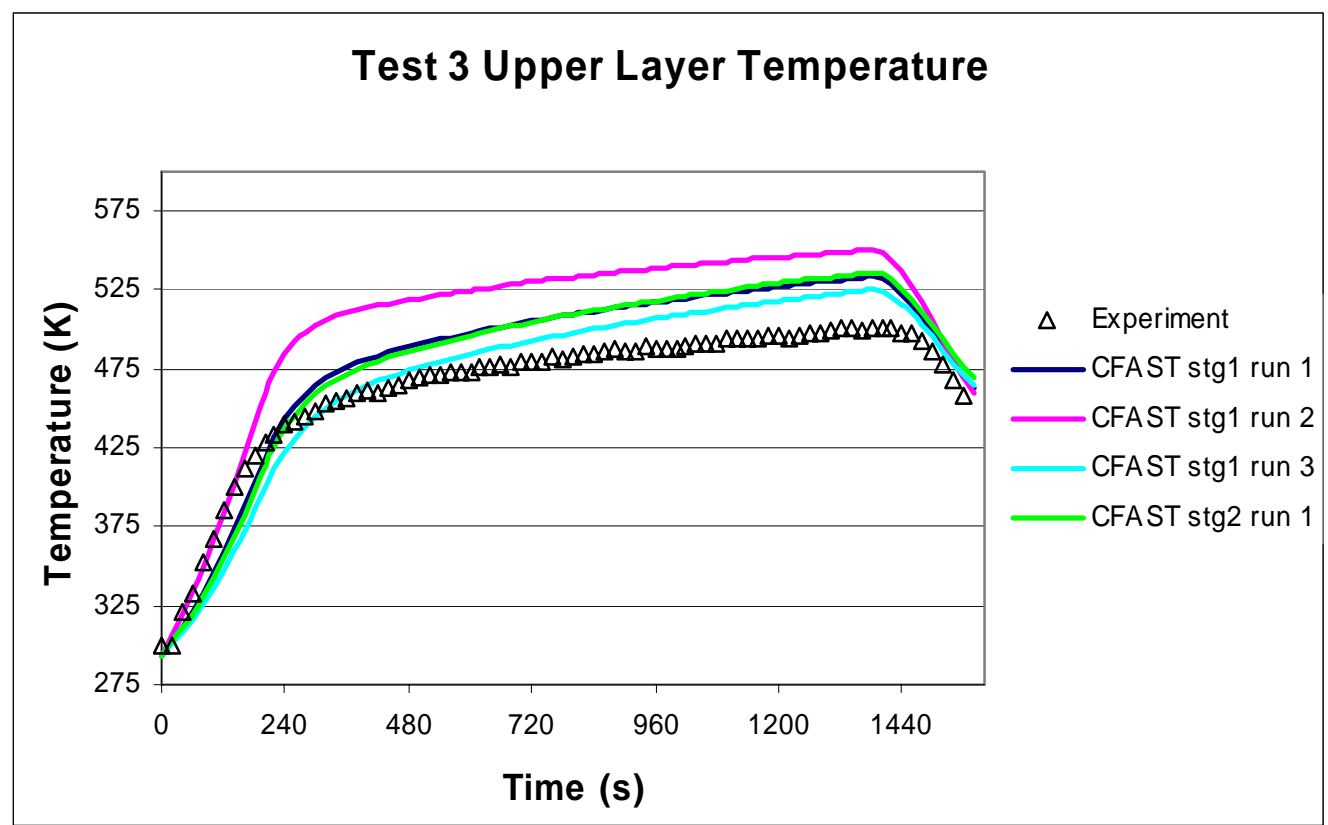

Fig. 2-7 Measured and CFAST predicted upper gas layer temperatures for Test 3

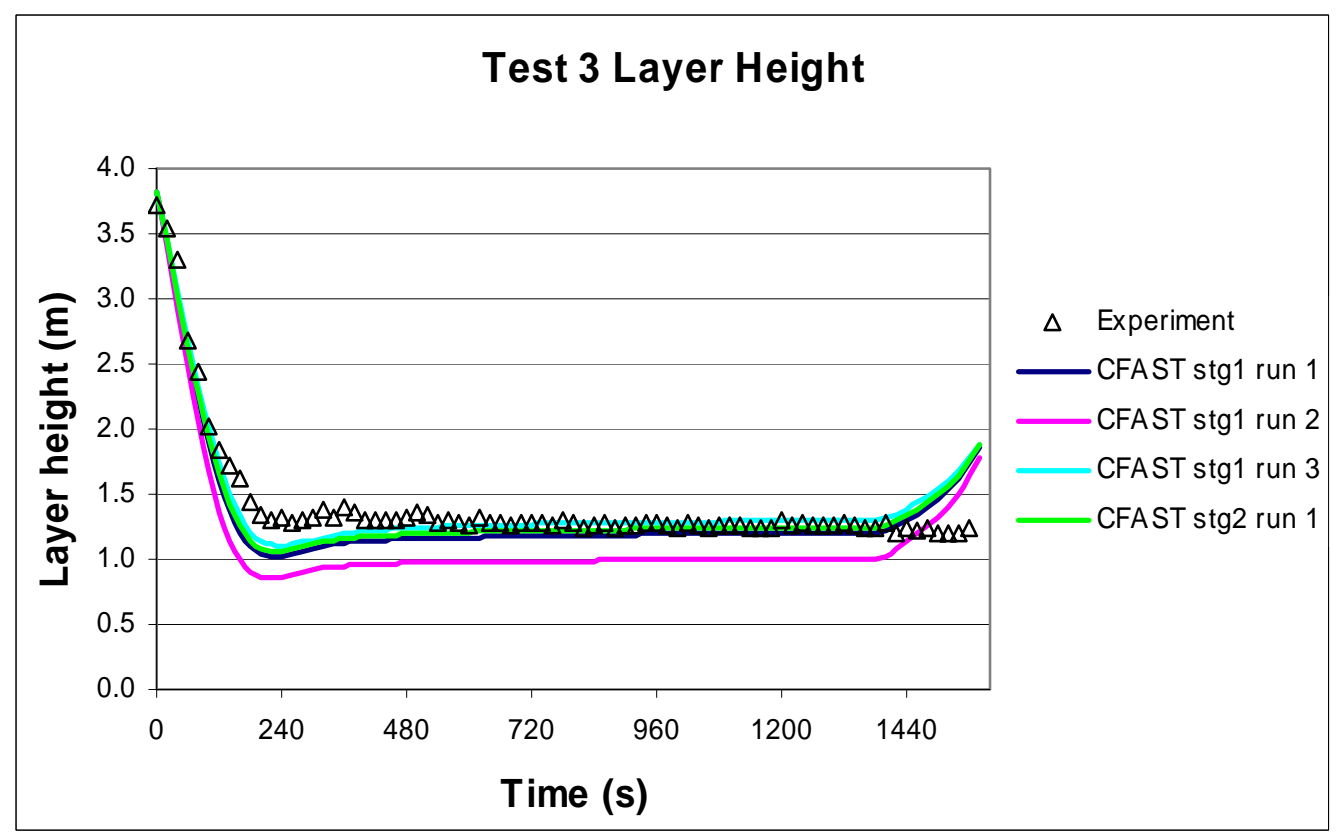

Fig. 2-8 Measured and CFAST predicted hot gas layer height for Test 3 

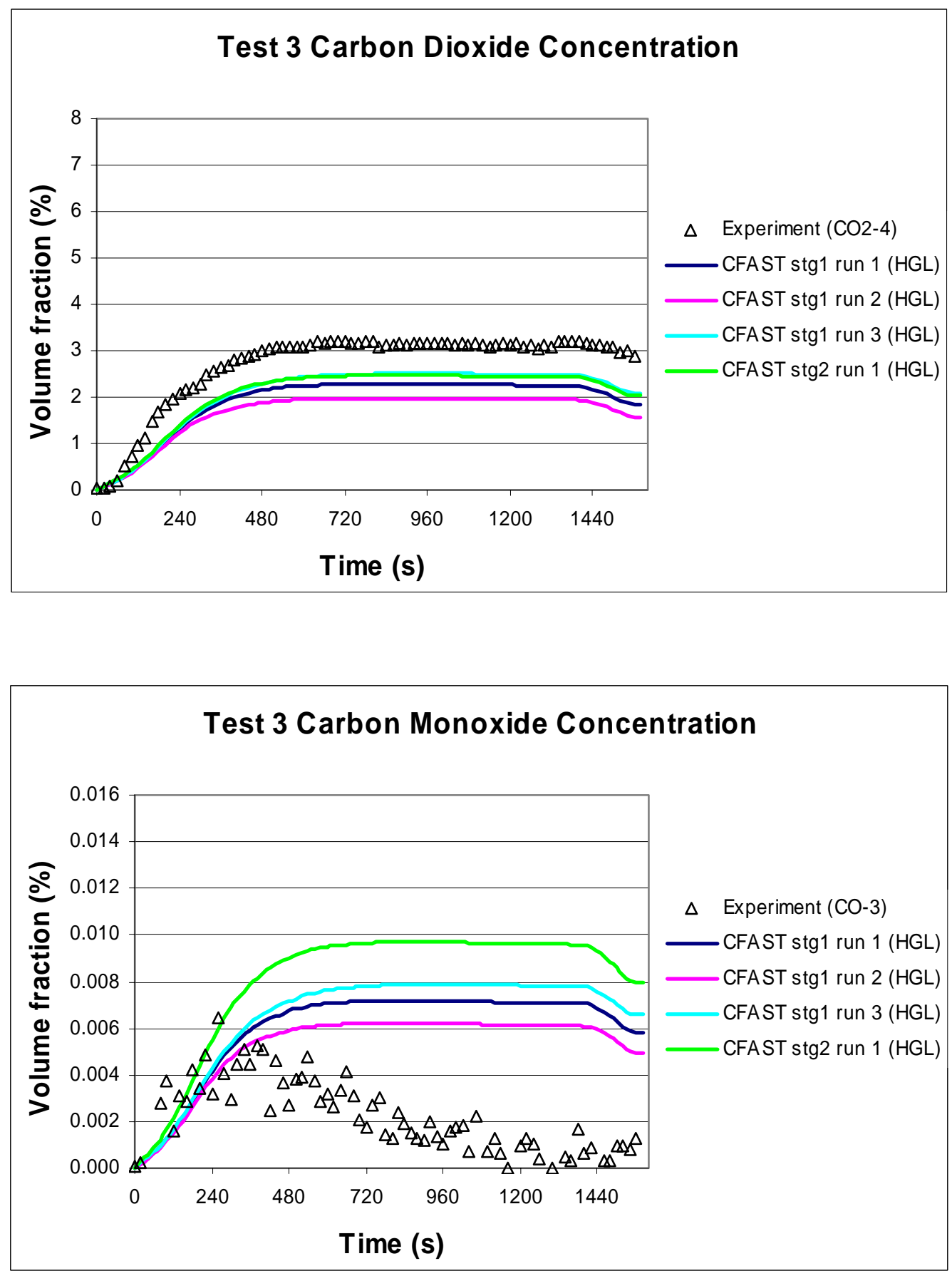

Fig. 2-9 Measured and CFAST predicted gas concentrations for Test 3 

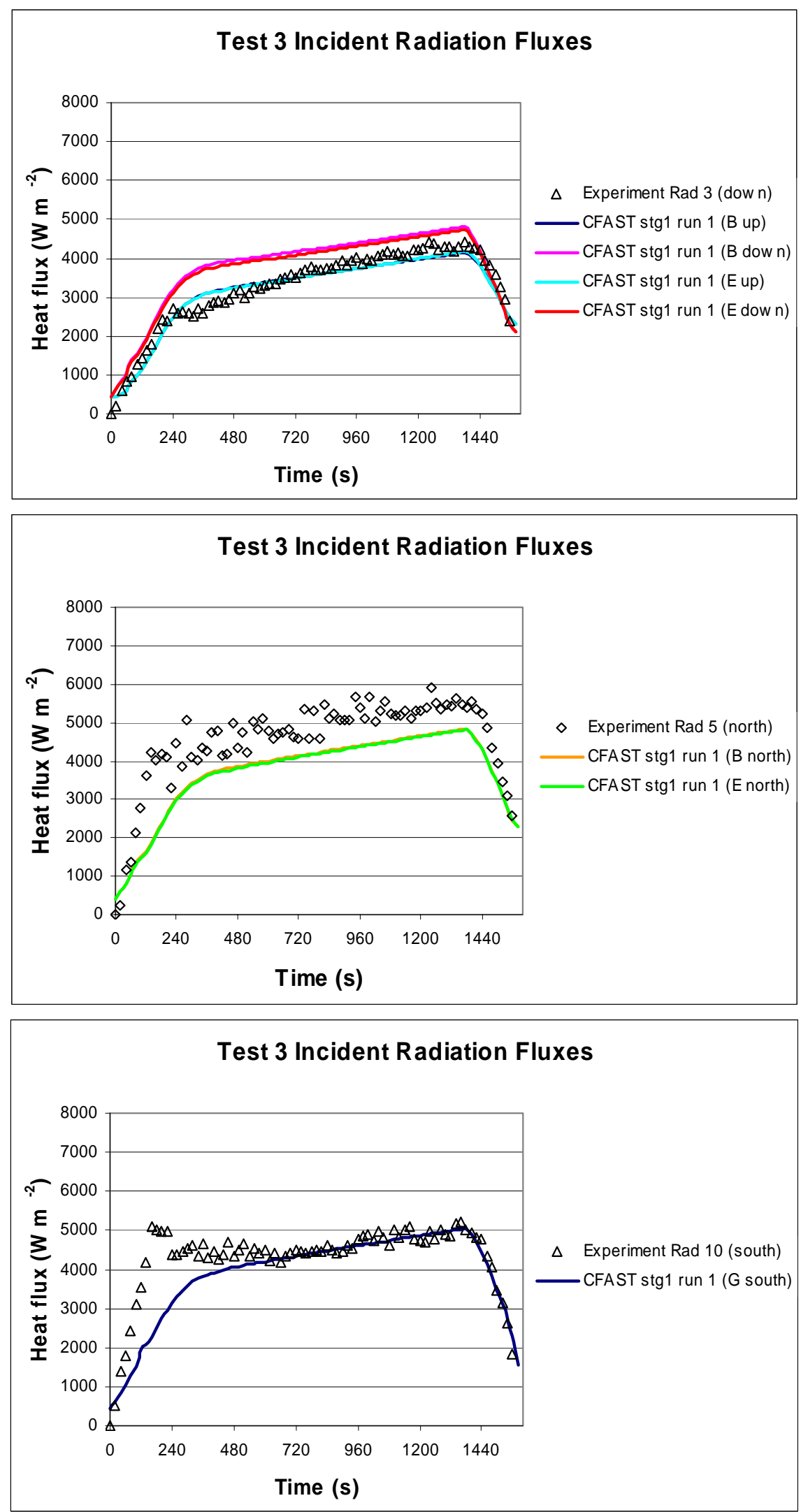

Fig. 2-10 Measured and CFAST predicted incicent fluxes for Test 3 

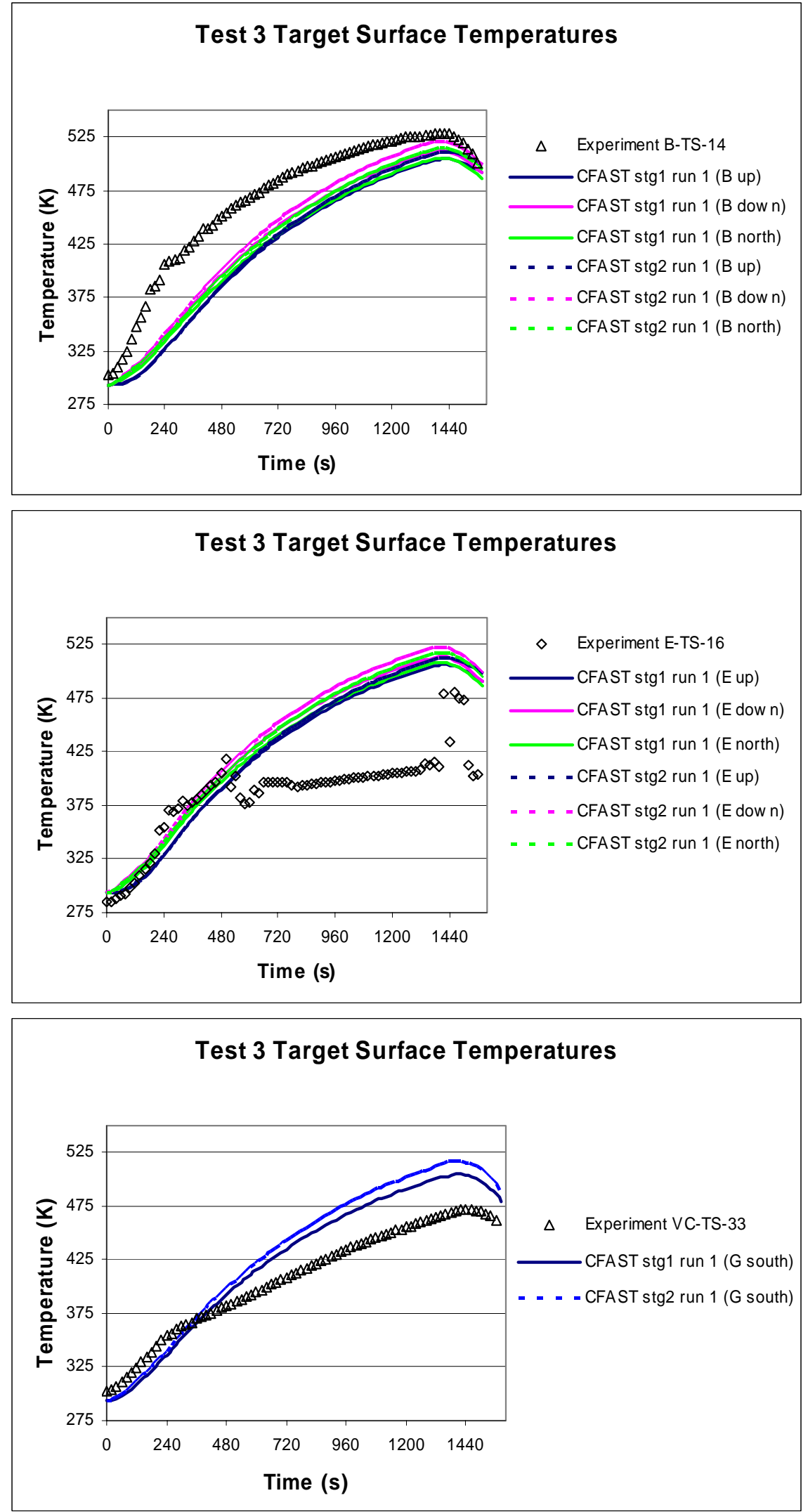

Fig. 2-11 Measured and CFAST predicted target surface temperatures for Test 3 

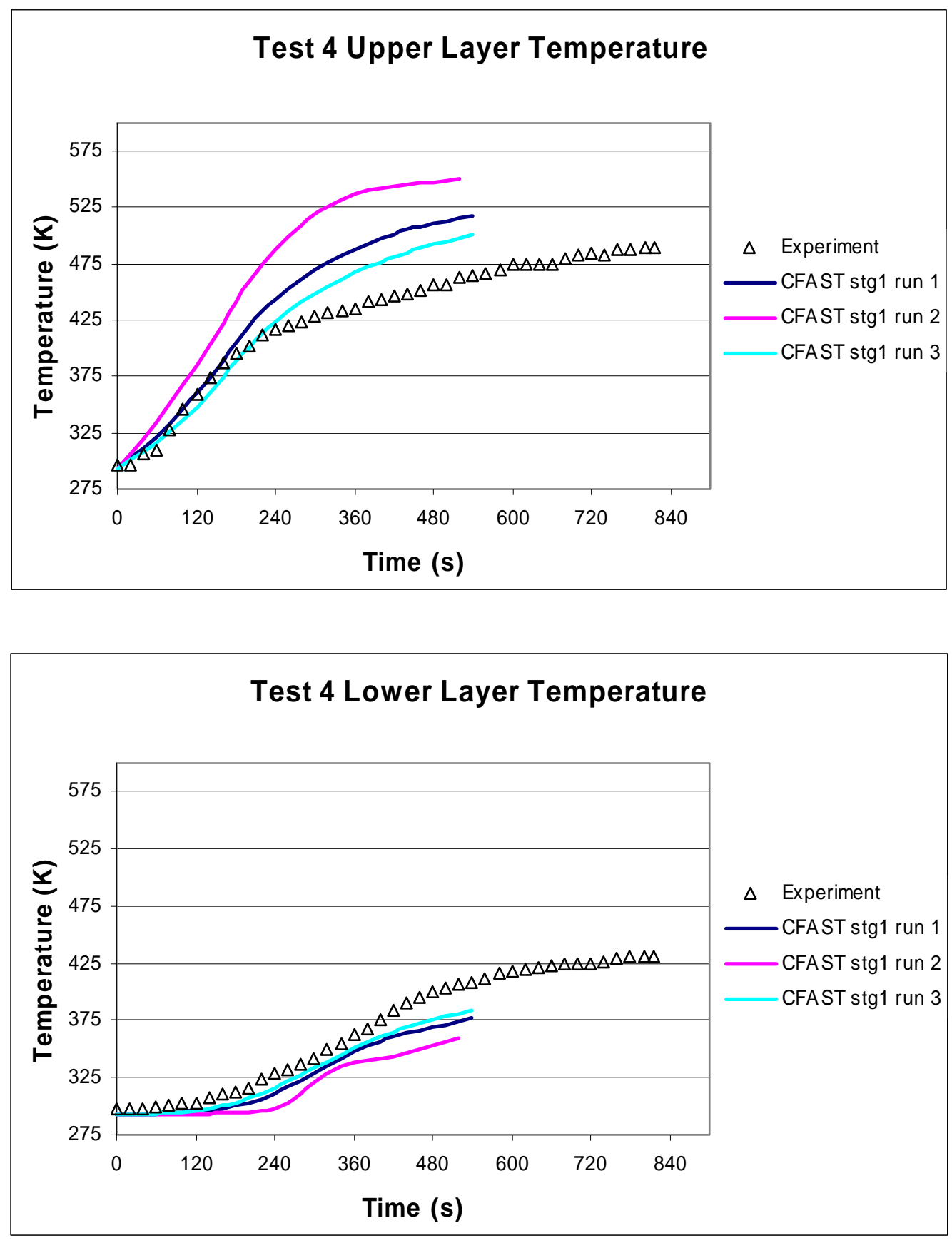

Fig. 2-12 Measured and CFAST predicted gas layer temperatures for Test 4 


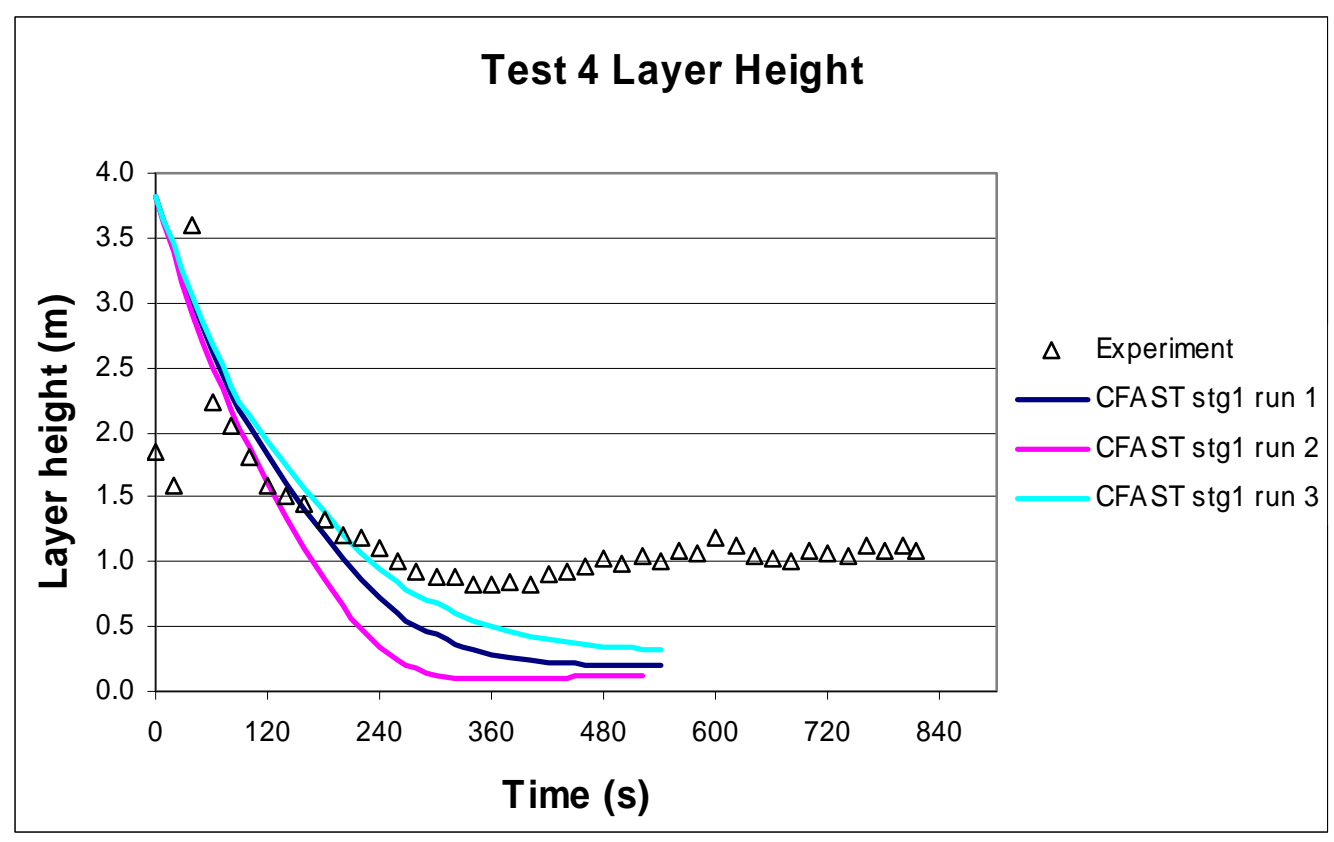

Fig. 2-13 Measured and CFAST predicted hot gas layer height for Test 4

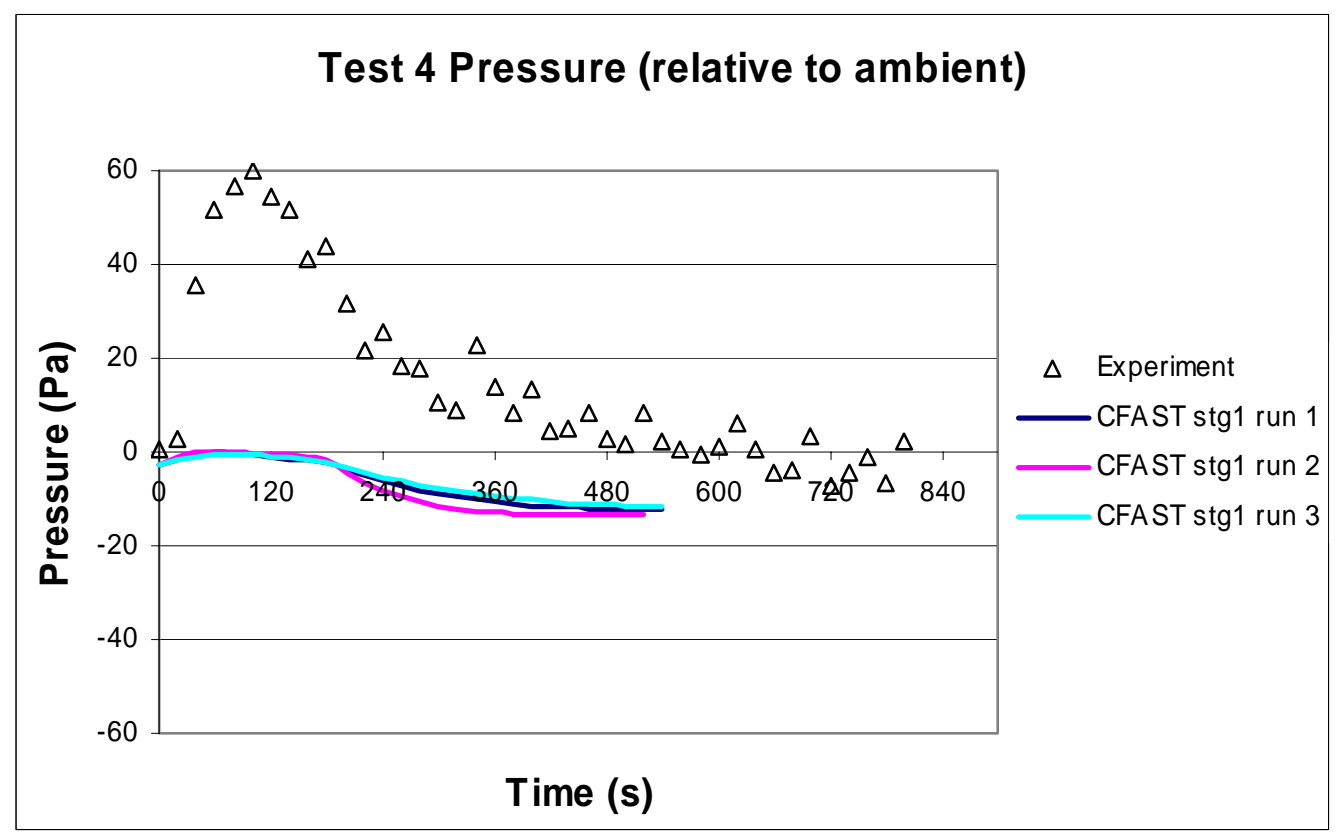

Fig. 2-14 Measured and CFAST predicted static pressure for Test 4 

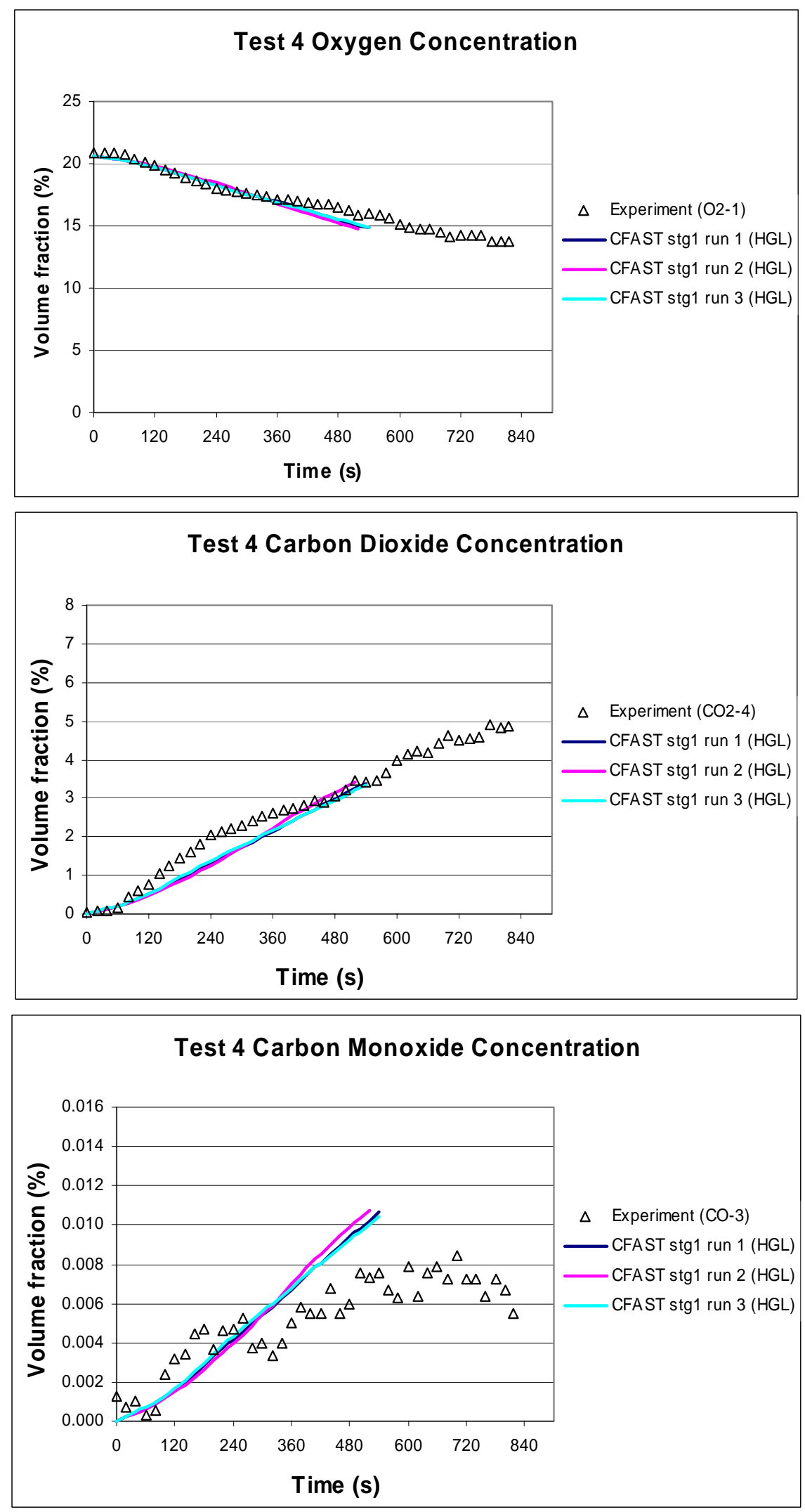

Fig. 2-15 Measured and CFAST predicted gas concentrations for Test 4 

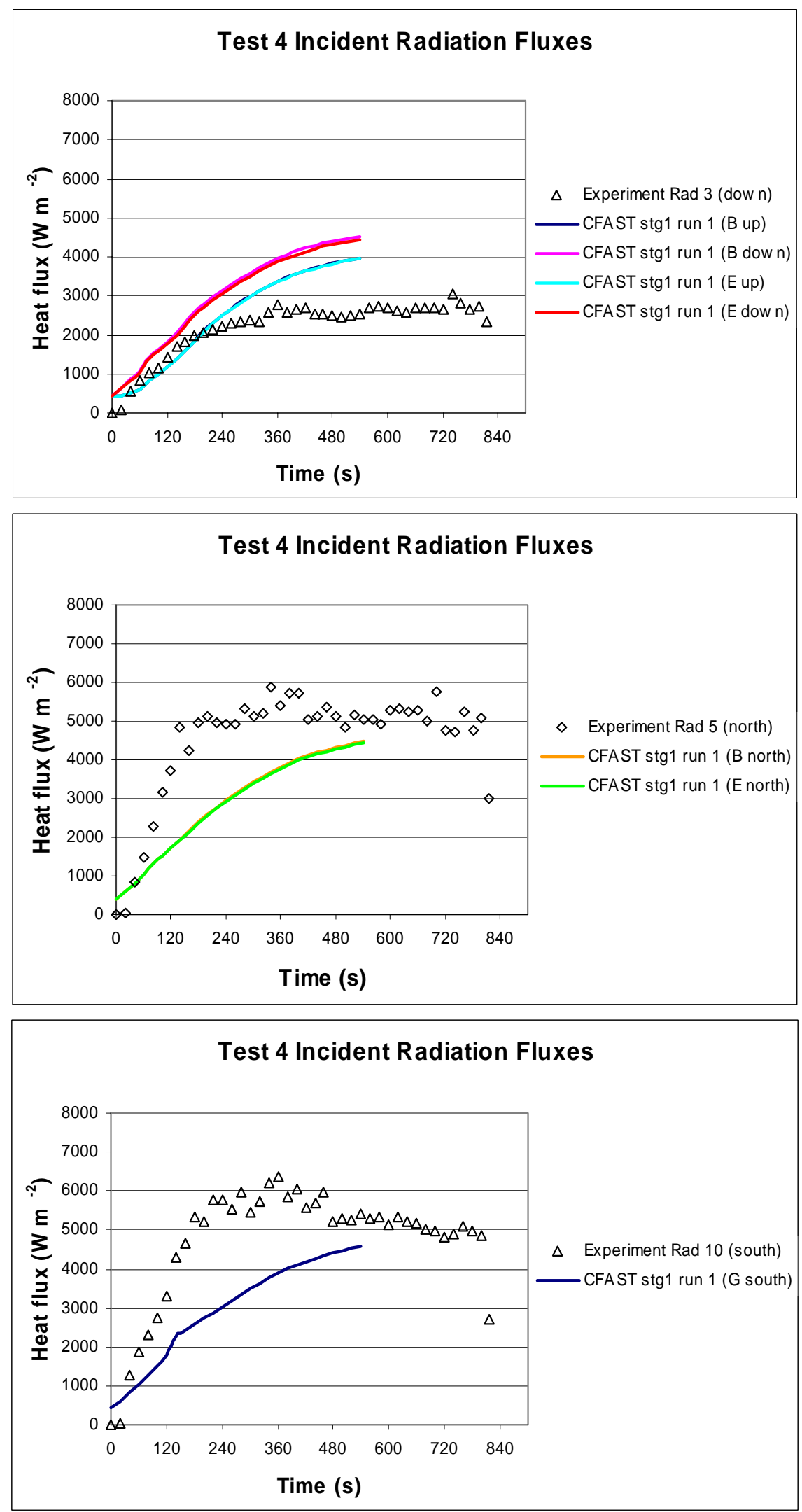

Fig. 2-16 Measured and CFAST predicted incicent fluxes for Test 4 

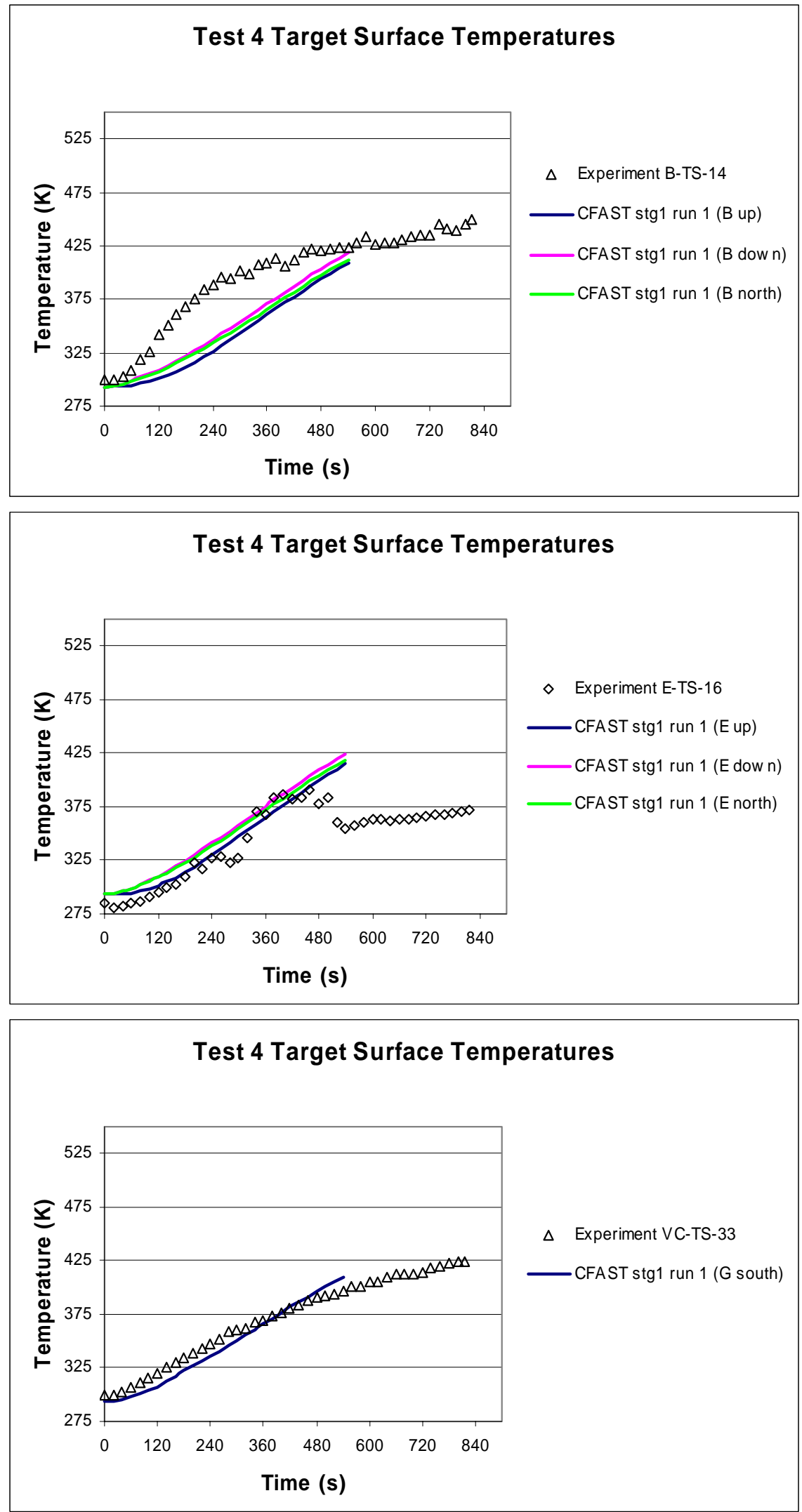

Fig. 2-17 Measured and CFAST predicted target surface temperatures for Test 4 


\subsection{Comparison of JASMINE Blind and Semi-Blind Predictions with Measure- ments}

A number of parametric calculations (runs) were performed for each test in the blind (stg1) stage, followed by some additional runs in the semi-blind (stg2) phase. Table 2-2 summarizes the main features of each of these calculations.

Table 2-2 JASMINE calculation runs

\begin{tabular}{|c|c|c|c|}
\hline Test No. & Blind or semi-blind and Run No. & $\begin{array}{l}\text { Leakage vent size } \\
\text { (Tests } 2 \& 4 \text { ) }\end{array}$ & $\begin{array}{l}\text { Heat lost by soot } \\
\text { radiation }\end{array}$ \\
\hline 2 & blind $\quad(\operatorname{stg} 1)$ run 1 & $0.17 \mathrm{~m} \times 0.17 \mathrm{~m}$ & $30 \%$ \\
\hline 2 & blind $\quad$ (stg1) run 2 & $0.17 \mathrm{~m} \times 0.17 \mathrm{~m}$ & 0 \\
\hline 2 & semi-blind (stg2) run 1 & $0.289 \mathrm{~m} \times 0.289 \mathrm{~m}$ & $35 \%$ \\
\hline 2 & semi-blind (stg2) run 2 & $0.289 \mathrm{~m} \times 0.289 \mathrm{~m}$ & 0 \\
\hline 3 & (stg1) run 1 & $\mathrm{n} / \mathrm{a}$ & $30 \%$ \\
\hline 3 & blind $\quad(\operatorname{stg} 1)$ run 2 & $\mathrm{n} / \mathrm{a}$ & 0 \\
\hline 4 & $(\operatorname{stg} 1)$ run 1 & $0.17 \mathrm{~m} \times 0.17 \mathrm{~m}$ & $30 \%$ \\
\hline 4 & blind $\quad(\operatorname{stg} 1)$ run 2 & $0.17 \mathrm{~m} \times 0.17 \mathrm{~m}$ & 0 \\
\hline 4 & semi-blind (stg2) run 1 & $0.289 \mathrm{~m} \times 0.289 \mathrm{~m}$ & $35 \%$ \\
\hline 4 & semi-blind (stg2) run 2 & $0.289 \mathrm{~m} \times 0.289 \mathrm{~m}$ & 0 \\
\hline
\end{tabular}

Fig. 2-18 illustrates the single-block, Cartesian grid used in the JAMSINE calculations, which included approximately 100,000 cells. Note that the grid is refined at the fire source and vents, towards the walls and ceiling and at the locations of the target cables. The minimum cell dimensions were $0.075 \mathrm{~m}$ in the $\mathrm{x}$ direction (see Fig. 2-18), $0.04 \mathrm{~m}$ in the $\mathrm{y}$ direction and $0.0425 \mathrm{~m}$ in the $\mathrm{z}$ direction. The maximum cell dimension inside the compartment was $0.2 \mathrm{~m}$ in each direction.

Figs. 2-19 to 2-21 illustrate the development of gas temperatures over the first 12 minutes in the JASMINE calculations for Tests 2, 3 and 4 (stg1 run 1 in each case).

Figs. 2-22 to 2-26 compare the predicted and measured gas temperatures (at selected measurement locations), pressures, species concentrations and target incident radiation fluxes for Test 2 . In the plots of target incident radiation flux, predicted values are given for 
fluxes onto a surface with a normal in the specified direction and also, for comparative purposes, from in the opposite direction (identified in the captions as 'opp rad 3' etc), e.g. for cable radiation gauge 3 the specified direction is 'pointing down' so that the 'opp rad 3' surface normal is therefore 'pointing up'.

While JASMINE calculations for the target cable temperatures were not performed, Fig. 2-27 compares the predicted and measured gas temperatures of various cable target locations (actually in close vicinity to). Finally, Fig. 2-28 shows the principle calculated heat losses for a blind calculation (run 1) for Test 2. Here the convected heat to the solids is the heat transfer to the walls etc and the transfer to radiation is the heat removed from the gas phase to the radiation field, from where it is transferred mainly to the walls etc.

Figs. 2-29 to 2-34 repeat the above for Test 3, except that pressure is not plotted as it is not a significant hazard variable with the door open. Also, the predicted and measured door mass flow rates are compared and the graphs showing the principle heat losses include a plot of the convected heat that passes through the open door. Finally, Figs. 2-35 to 2-41 provide the information for Test 4.

The main observations and findings from the JASMINE calculations are summarized below:

- As in the CFAST work, the assumed size of the leakage vent in Test 2 was found to be very sensitive (see Fig. 2-24). Furthermore, the calculations exhibited some convergence difficulties once the compartment pressure had increased significantly. In Test 4, increasing the area of the leakage vent to that suggested for the semi-blind calculations had a significant effect on the predicted pressure, bringing the value close to the measured value. It seems perhaps obvious that specifying a small opening to represent the total leakage/infiltration area has limitations. A better approach may be to distribute the leakage area over a entire wall (or walls) in some way. This requires further development.

- The predicted quantities in Test 3 (with the open door) were, in the main, in reasonable agreement with the measured values. The gas temperatures were accurately captured in the run where $30 \%$ of the heat release rate was assumed to be removed due to soot radiation from the burning region. Where no heat removal was assumed the gas temperatures were then too high.

The door flow rates were captured accurately in Test 3. There was, however, a notable discrepancy between predicted and measured incident radiant fluxes, with JASMINE tending to under predict the values. This is quite likely due to the six-flux radiation model 
used, which considers radiation transport in the three Cartesian directions. The more accurate discrete transfer radiation model, which traces rays in many directions, could be expected to provide more accurate predictions for target incident flux in scenarios such as the tests in this benchmark exercise.

- In Tests 2 and 4 the predicted gas temperatures were, in general, in closer agreement to the measured values when $30 \%$ to $35 \%$ of the heat release rate was assumed to be removed due to soot radiation from the burning region (compared to where no heat was removed).

While the gas temperatures were judged to be reasonable, there was a noticeable collapse of the thermal gradient (cooler lower down and hotter above) in the JASMINE predictions later in the simulations where there was a significant increase in compartment pressure. This was observed to a much lesser extent in the experiments. Note that in the semi-blind run for Test 4 , where the pressure did not increase significantly, the thermal gradient did not collapse.

- Gas species predictions were, in general, in reasonable agreement with the measured values. Furthermore, for Tests 2 and 4 the time predicted for the test to terminate (due to the lower layer oxygen concentration dropping below 15\%) was generally similar to that in the experiment. However, in the runs for Test 4 where $30 \%$ to $35 \%$ of the heat release rate was assumed to be removed due to soot radiation the test was predicted to continue for its full duration, i.e. the oxygen level remained above the critical value.

- As for Test 3, the predicted incident radiant fluxes in Tests 2 and 4 were generally lower than those measured. Again, a likely reason is the choice of the radiation sub-model.

- The energy 'budgets' shown in Figs. 2-28, 2-34 and 2-41 are quite informative. For Test 3 the JASMINE calculations indicate that approximately as much heat is 'lost' to radiation (due to the presence of $\mathrm{CO}_{2}$ and $\mathrm{H}_{2} \mathrm{O}$ ) as is convected through the open door. Most of this radiated heat is subsequently transferred to the walls, ceiling and floor. The amount of heat transferred to the walls etc by convection is significantly less.

- A general note from the JASMINE work is that the presence of soot, and the subsequent radiation absorption and emission, may have an important bearing on the gas temperatures predicted. This in turn influences the radiation fluxes significantly (bearing in mind the $\mathrm{T}^{4}$ dependency on radiant intensity), which then has a major influence on the conduction into the walls and targets and their temperature rise. 


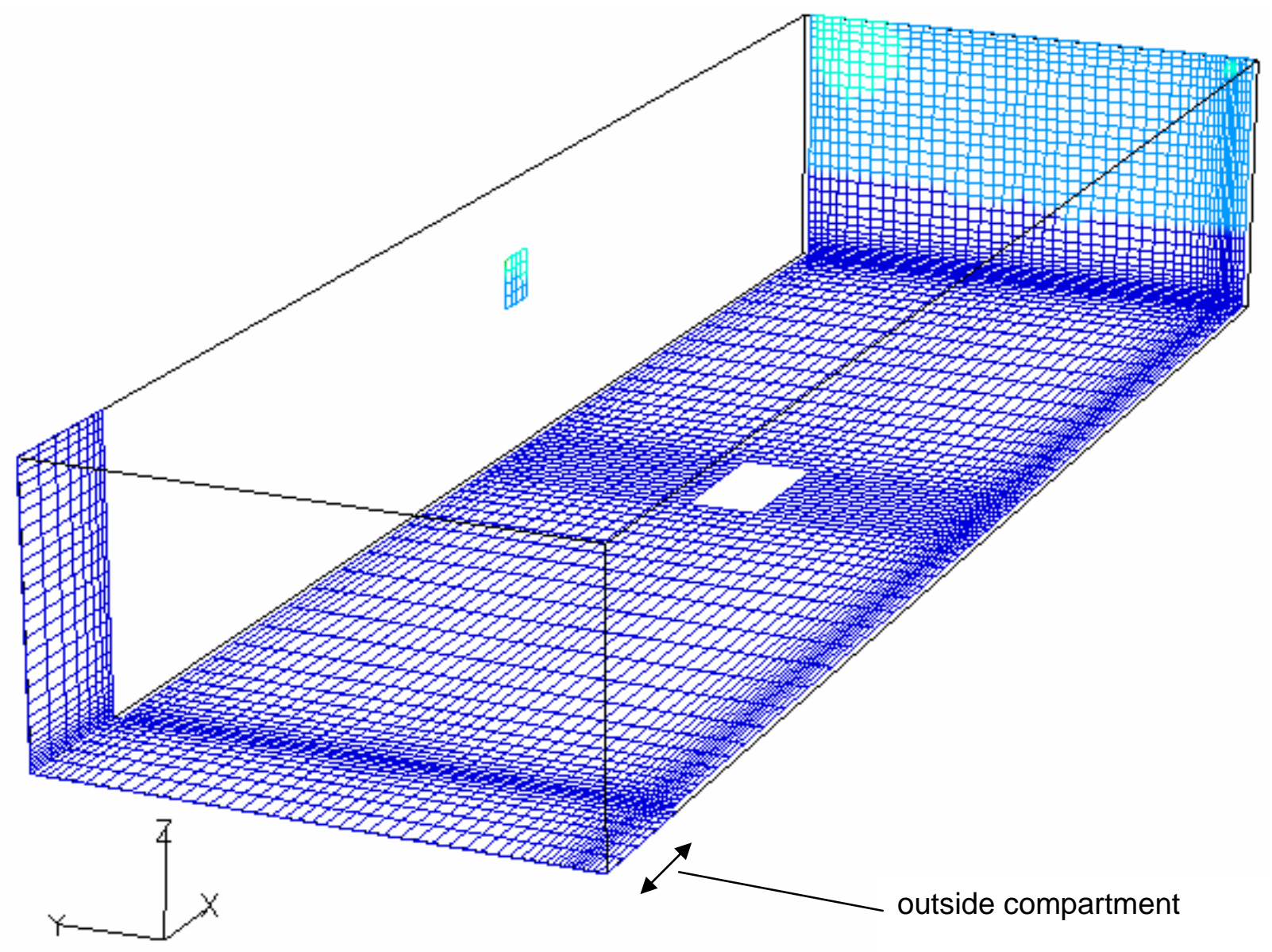

Fig. 2-18 JASMINE CFD grid 

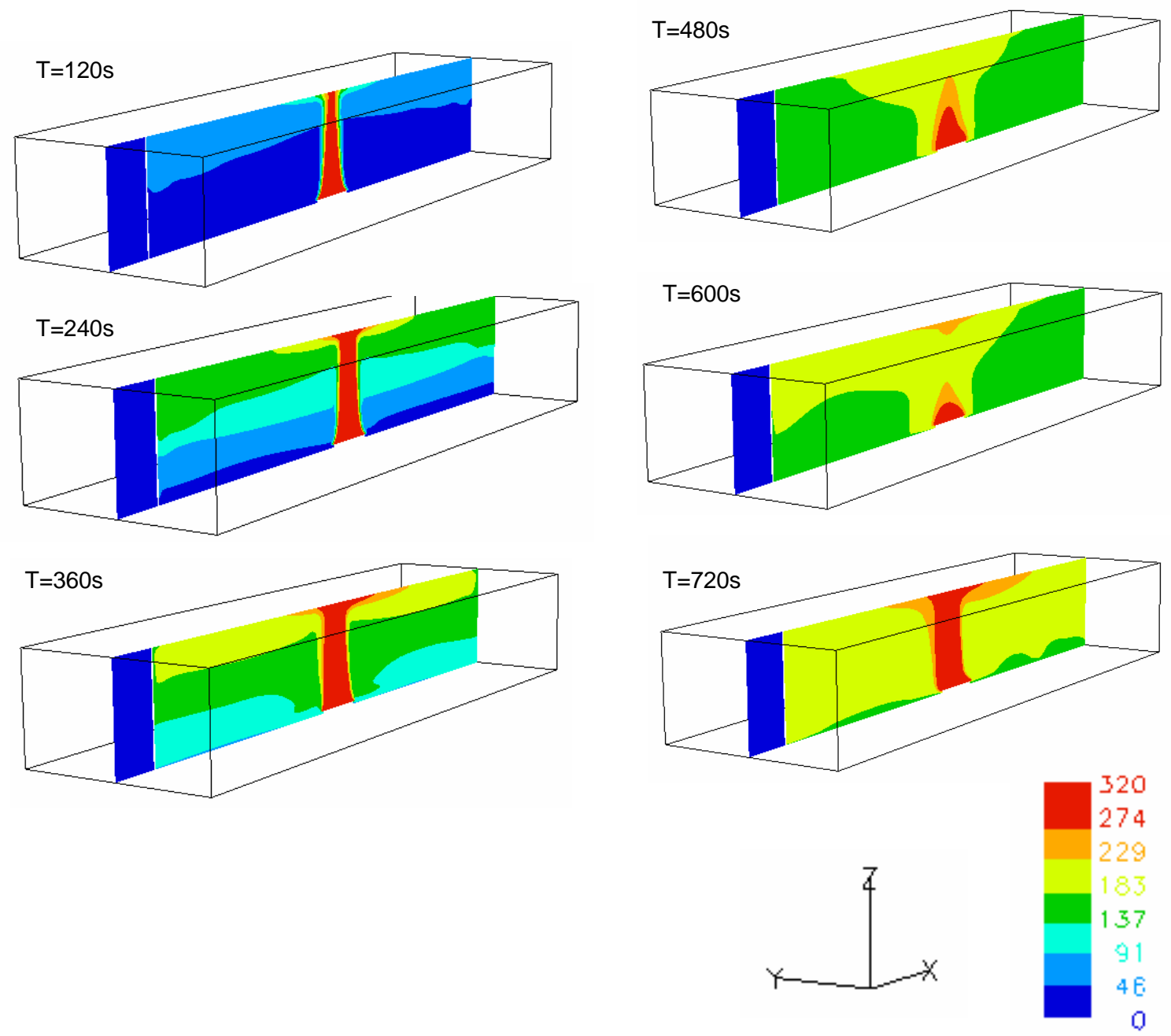

Fig. 2-19 JASMINE calculated temperature rise for Test 2, run 1 

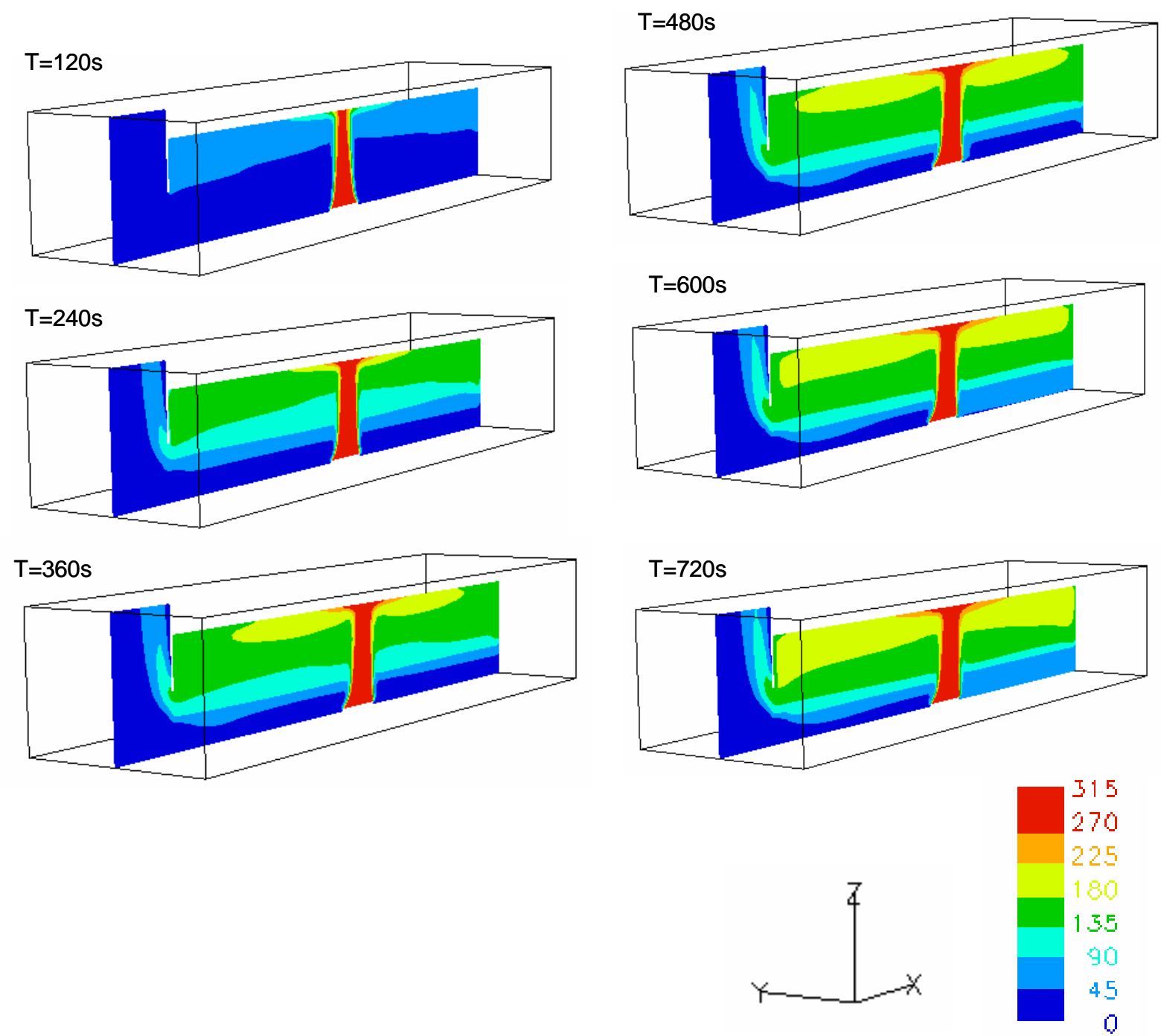

Fig. 2-20 JASMINE calculated temperature rise for Test 3, run 1 

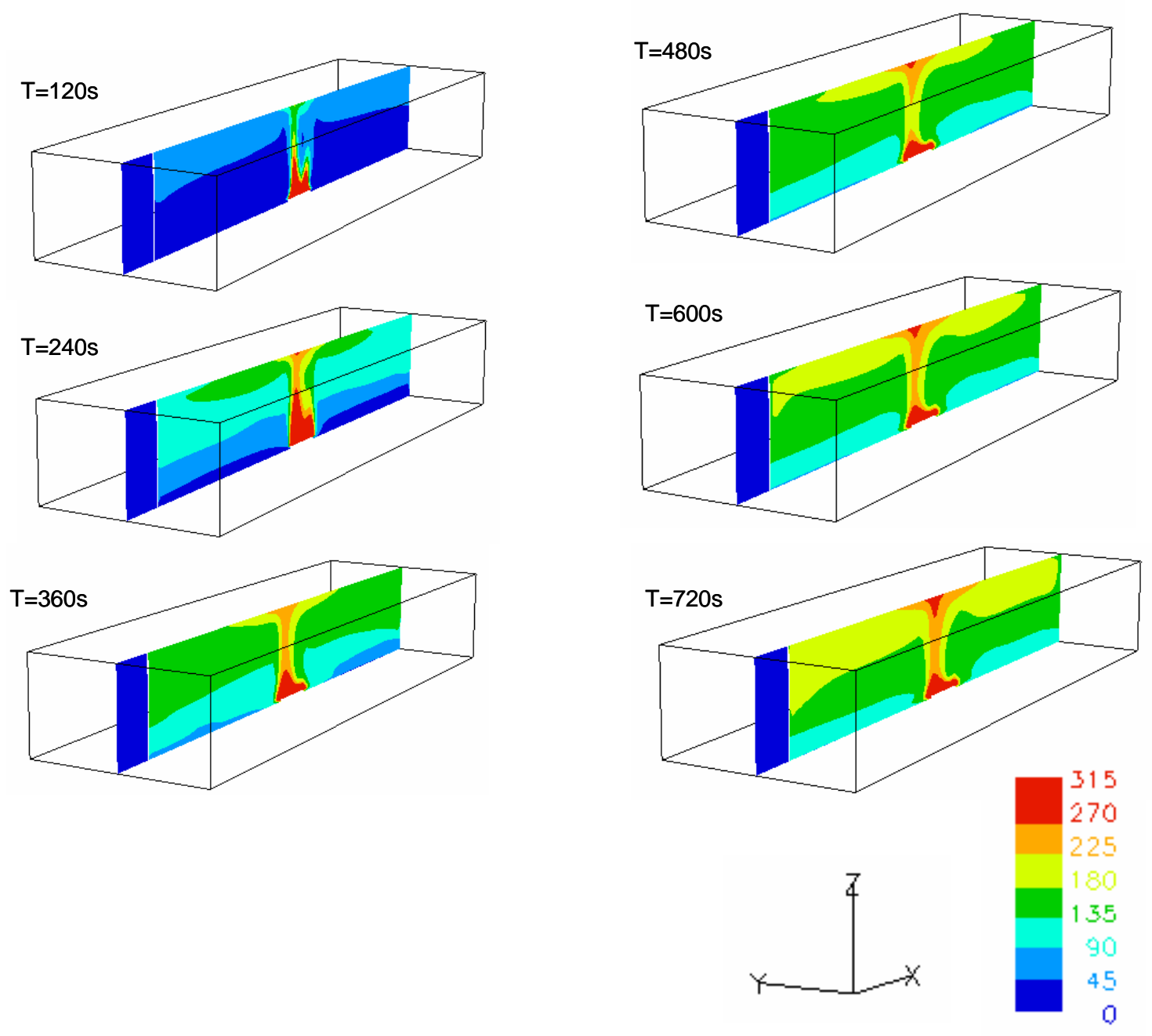

Fig. 2-21 JASMINE calculated temperature rise for Test 4, run 1 

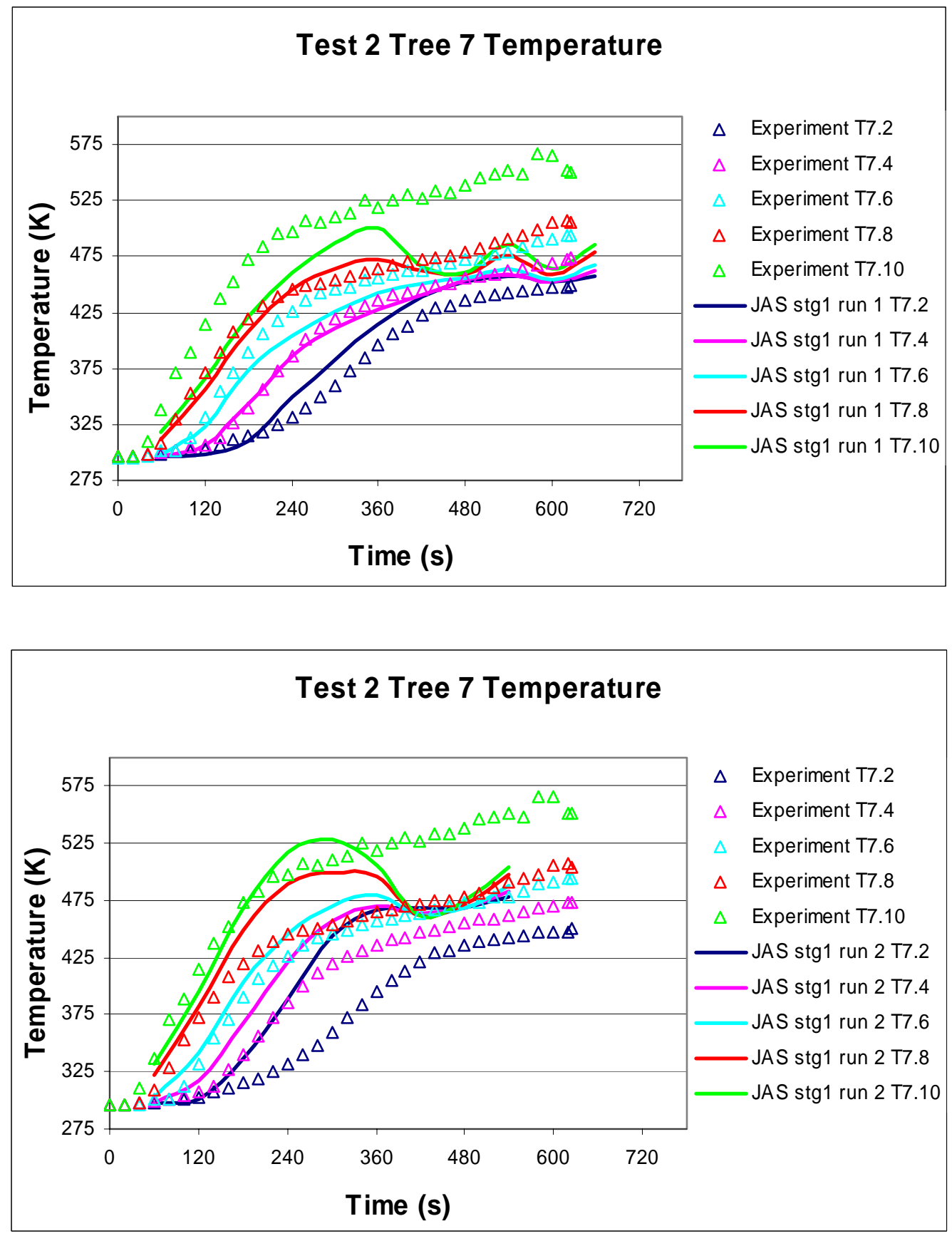

Fig. 2-22 Measured and JASMINE predicted (blind) gas temperatures for Test 2 at Tree 7 

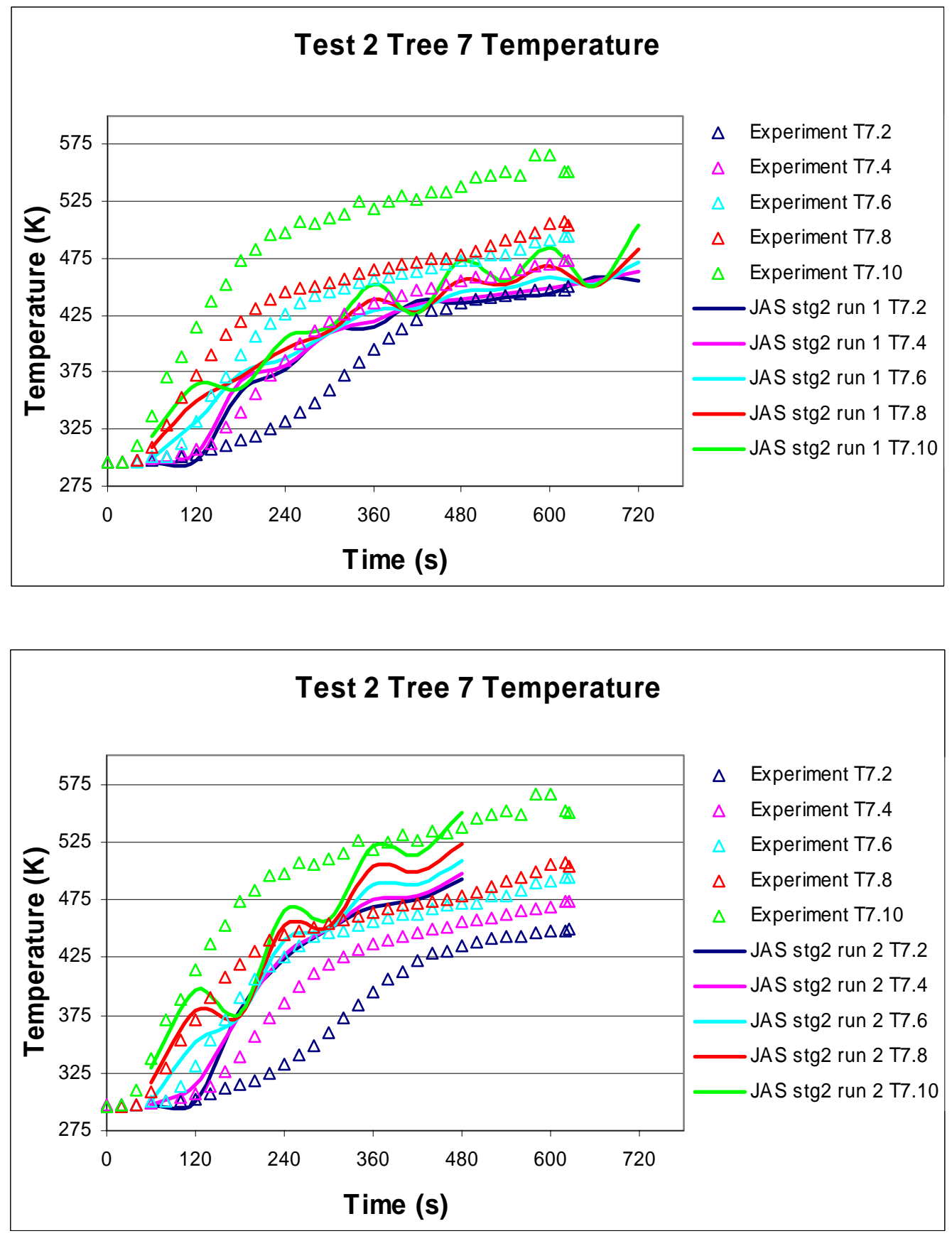

Fig. 2-23 Measured and JASMINE (semi-blind) gas temperatures for Test 2 at Tree 7 
Note that JASMINE exhibited some convergence difficulties due to pressure build up

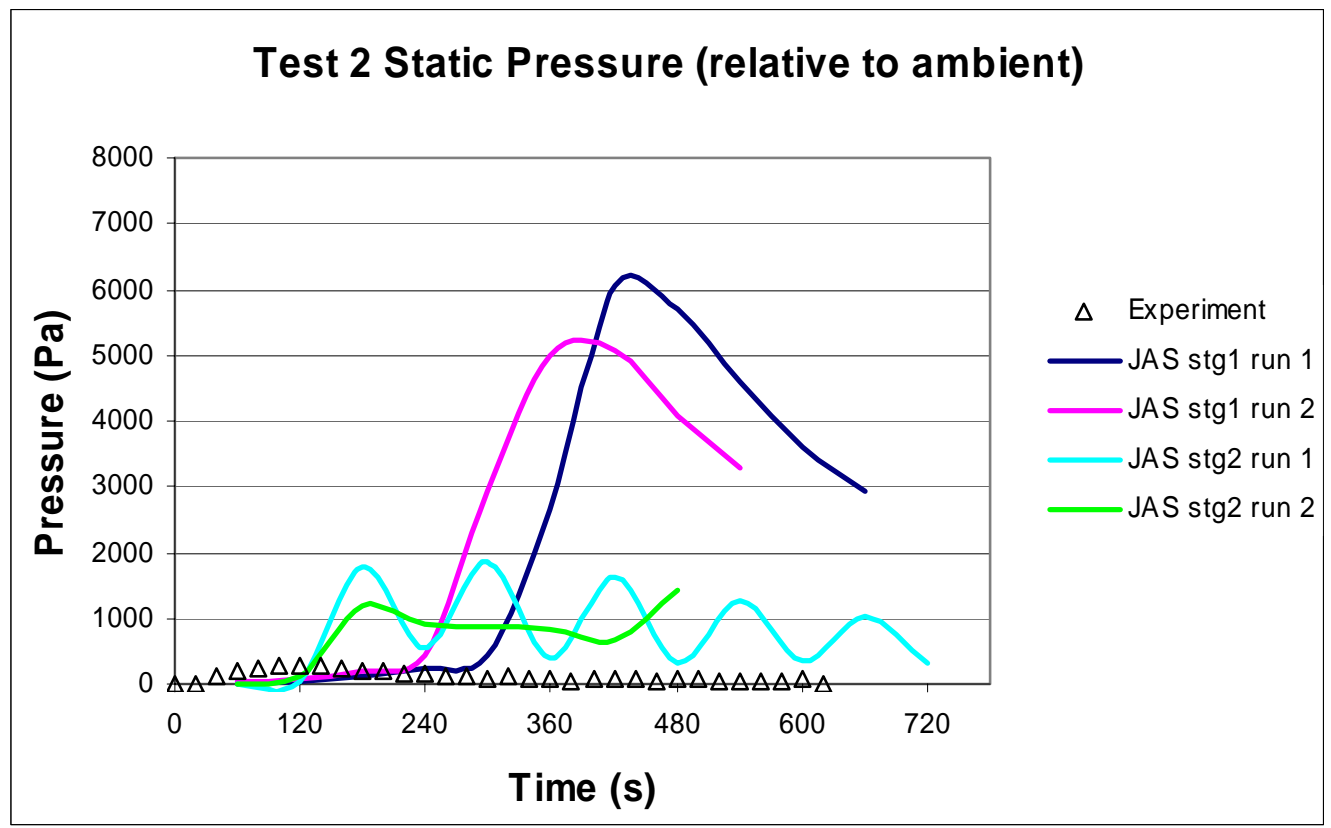

Fig. 2-24 Measured and JASMINE predicted static pressure for Test 2 
Note this is a plot at O2-1 (upper layer) and that JASMINE data is a percentage by mass
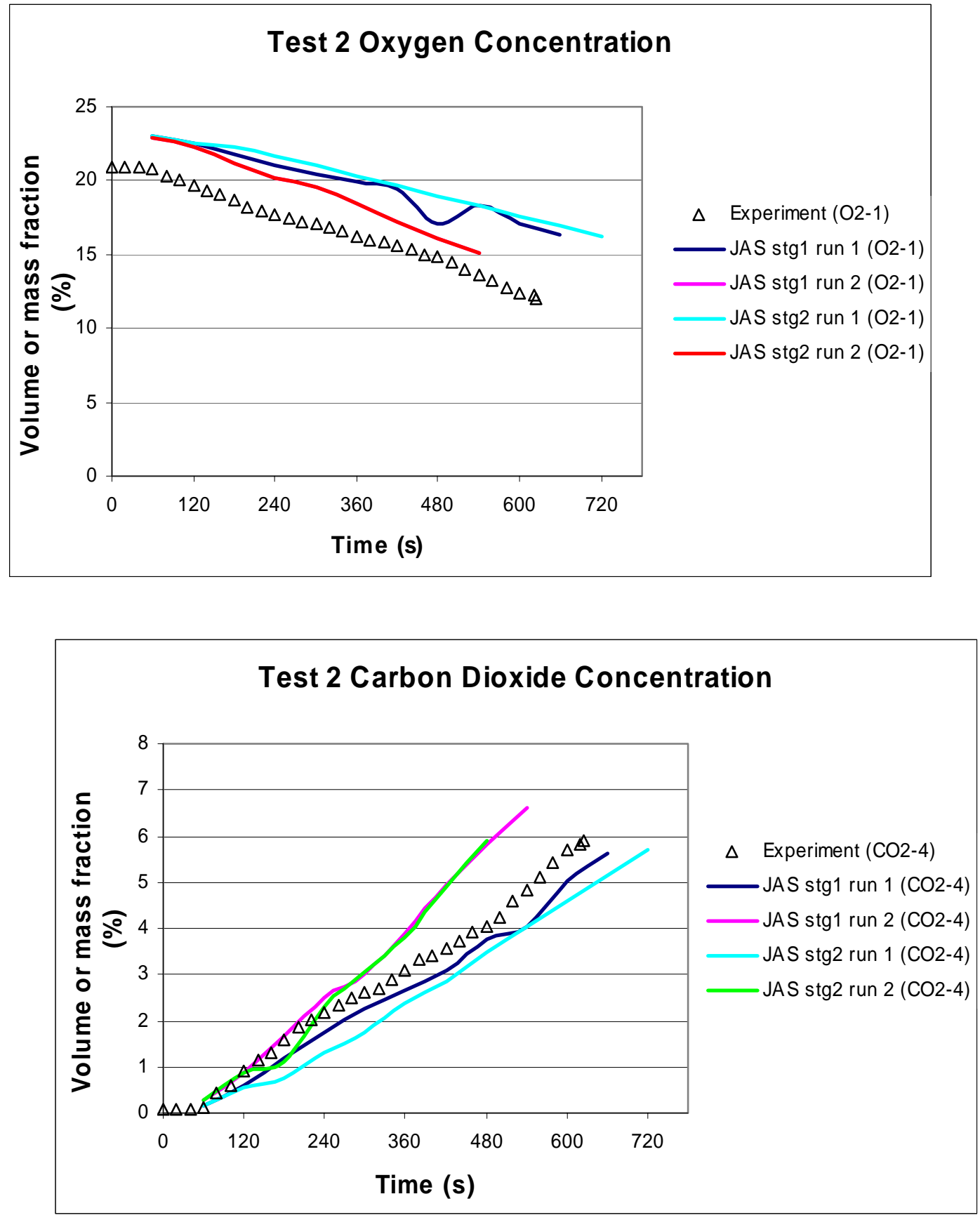

Fig. 2-25 Measured and JASMINE predicted gas concentrations for Test 2 

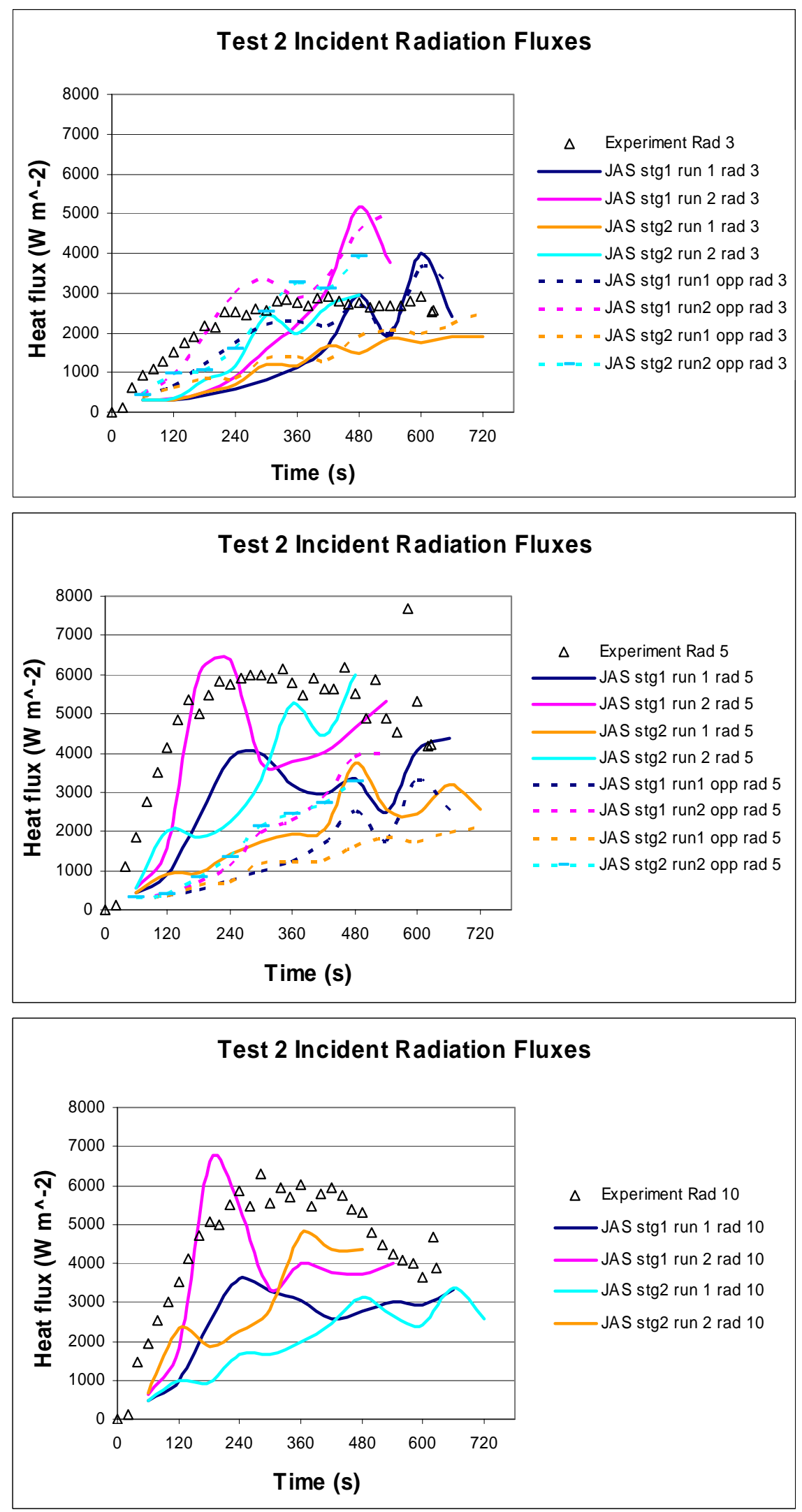

Fig. 2-26 Measured and JASMINE predicted incicent fluxes for Test 2 

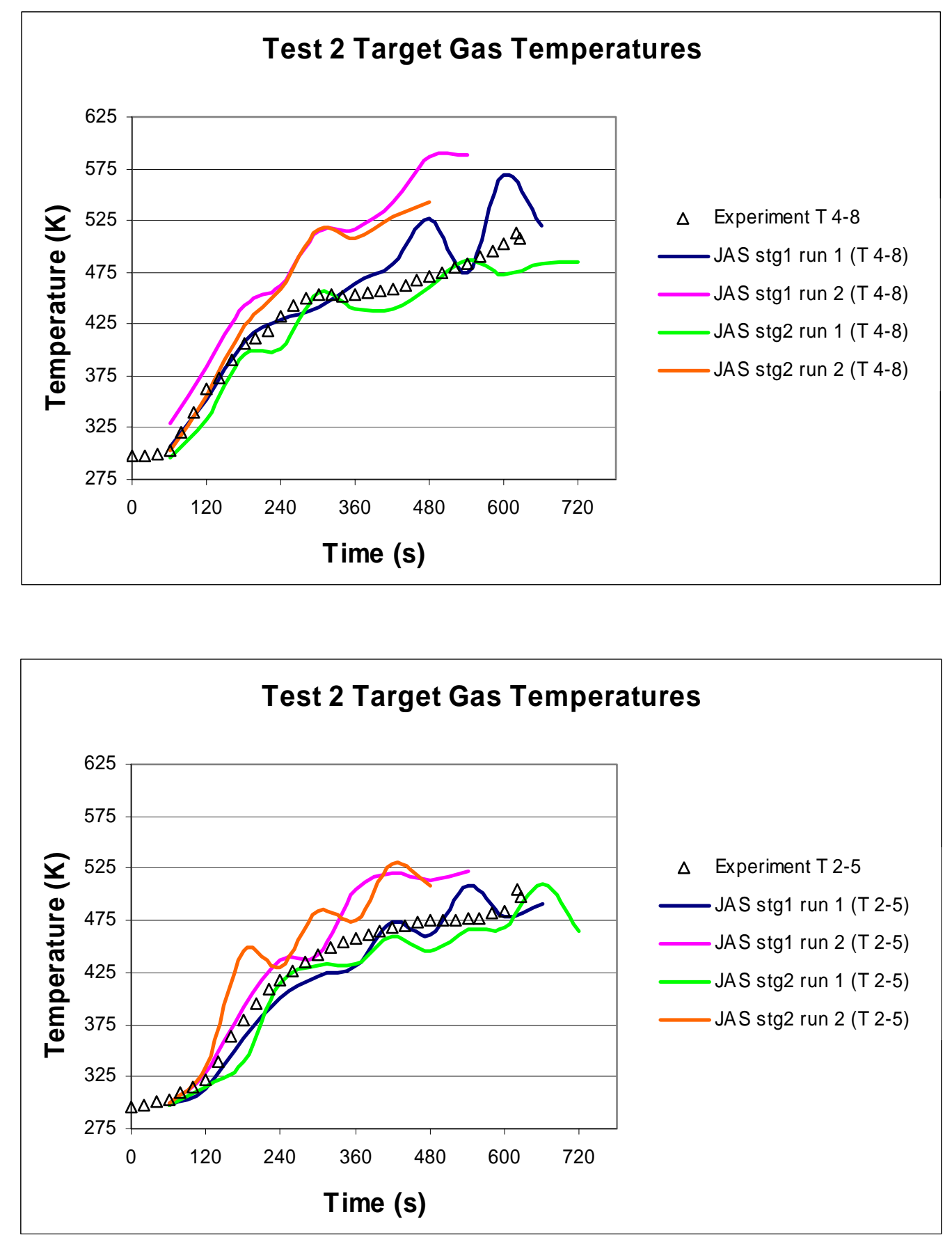

Fig. 2-27 Measured and JASMINE predicted gas temperatures near cable targets for Test 2 


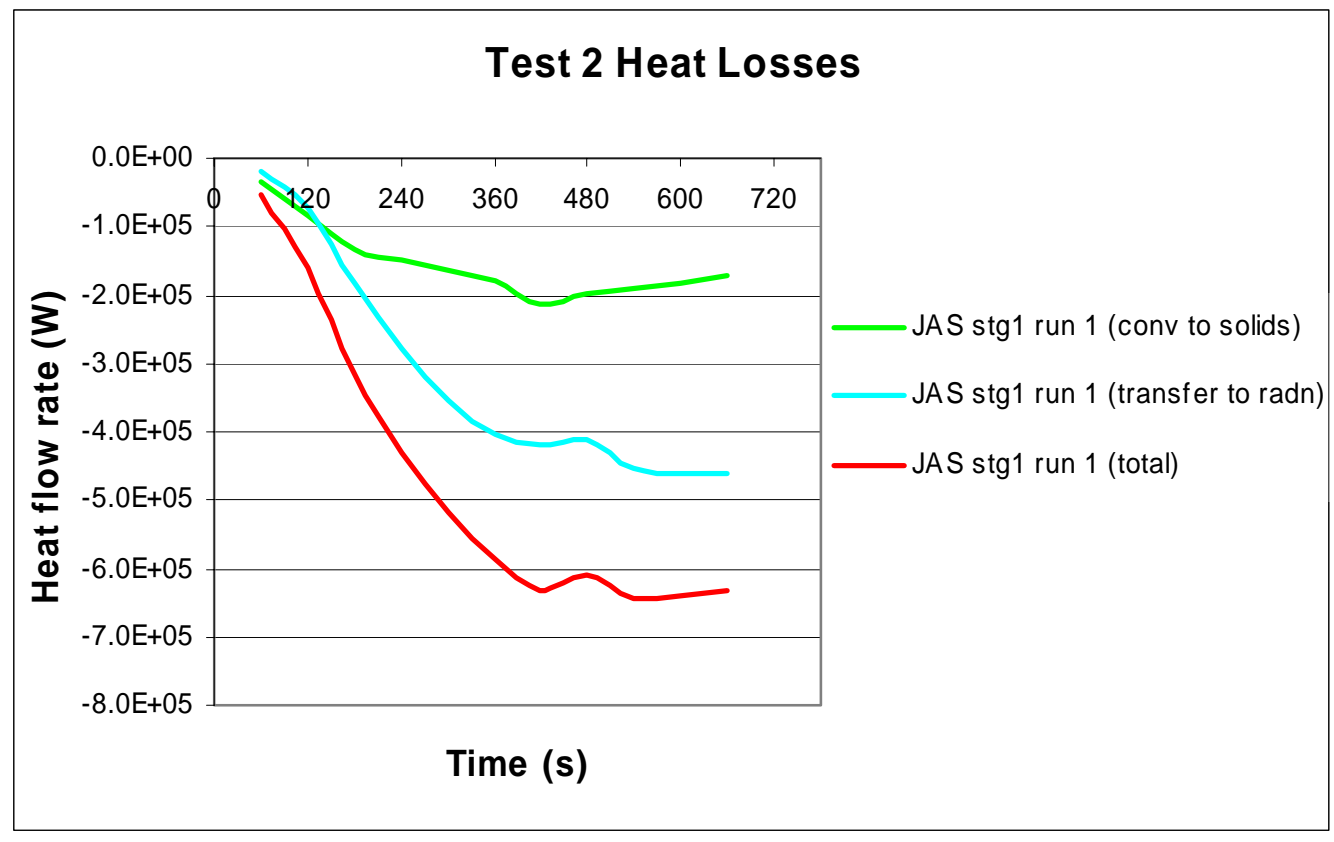

Fig. 2-28 JASMINE predicted heat losses for Test 2 

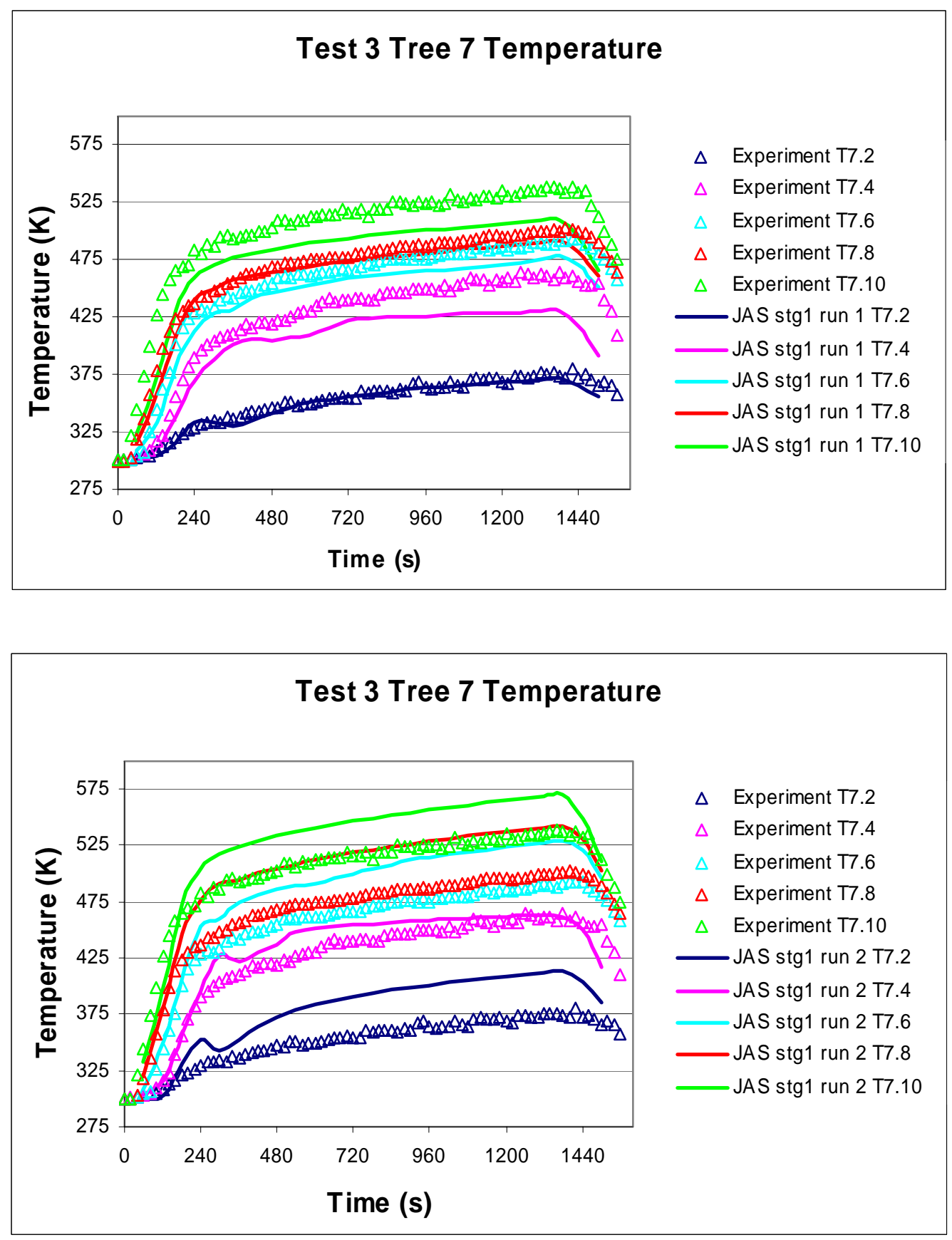

Fig. 2-29 Measured and JASMINE predicted (blind) gas temperatures for Test 3 at Tree 7 


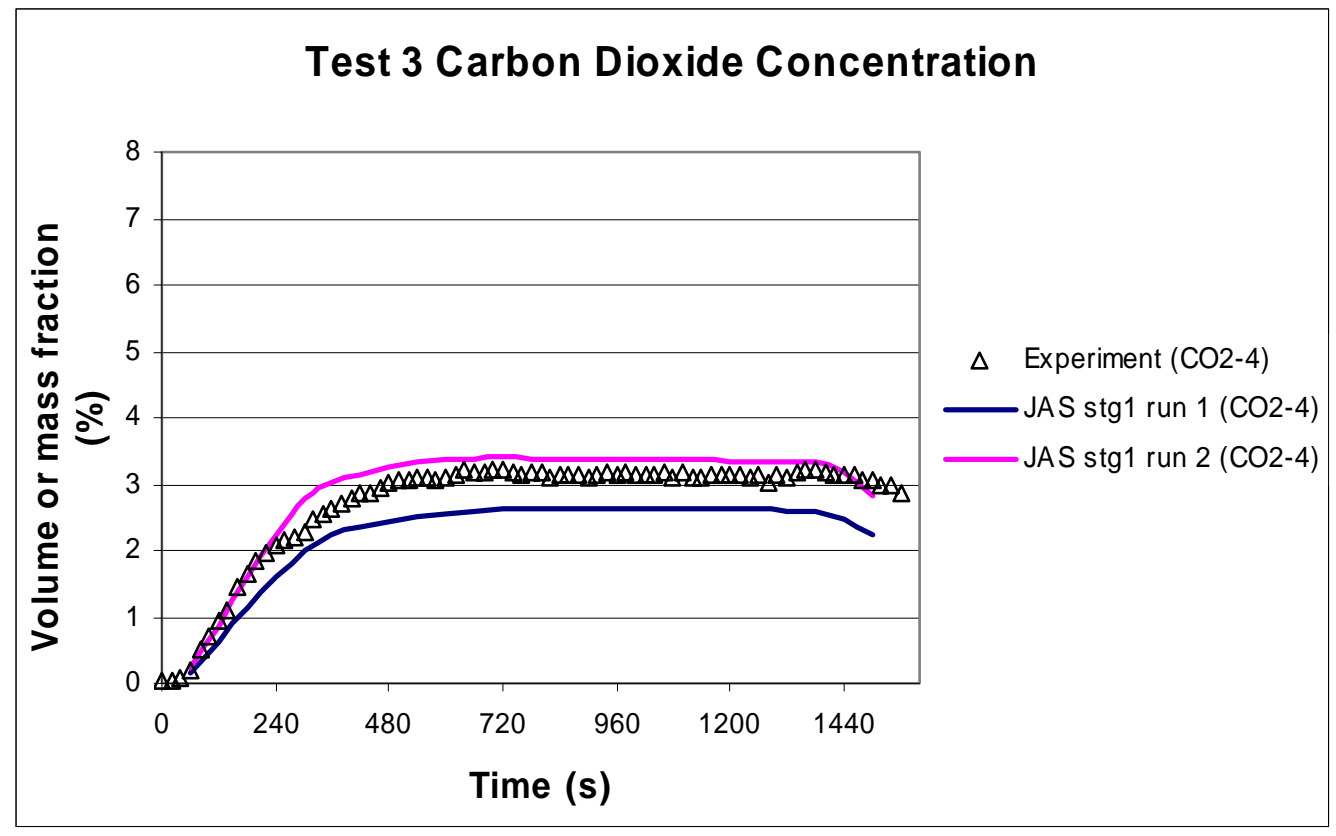

Fig. 2-30 Measured and JASMINE predicted $\mathrm{CO}_{2}$ concentrations for Test 3

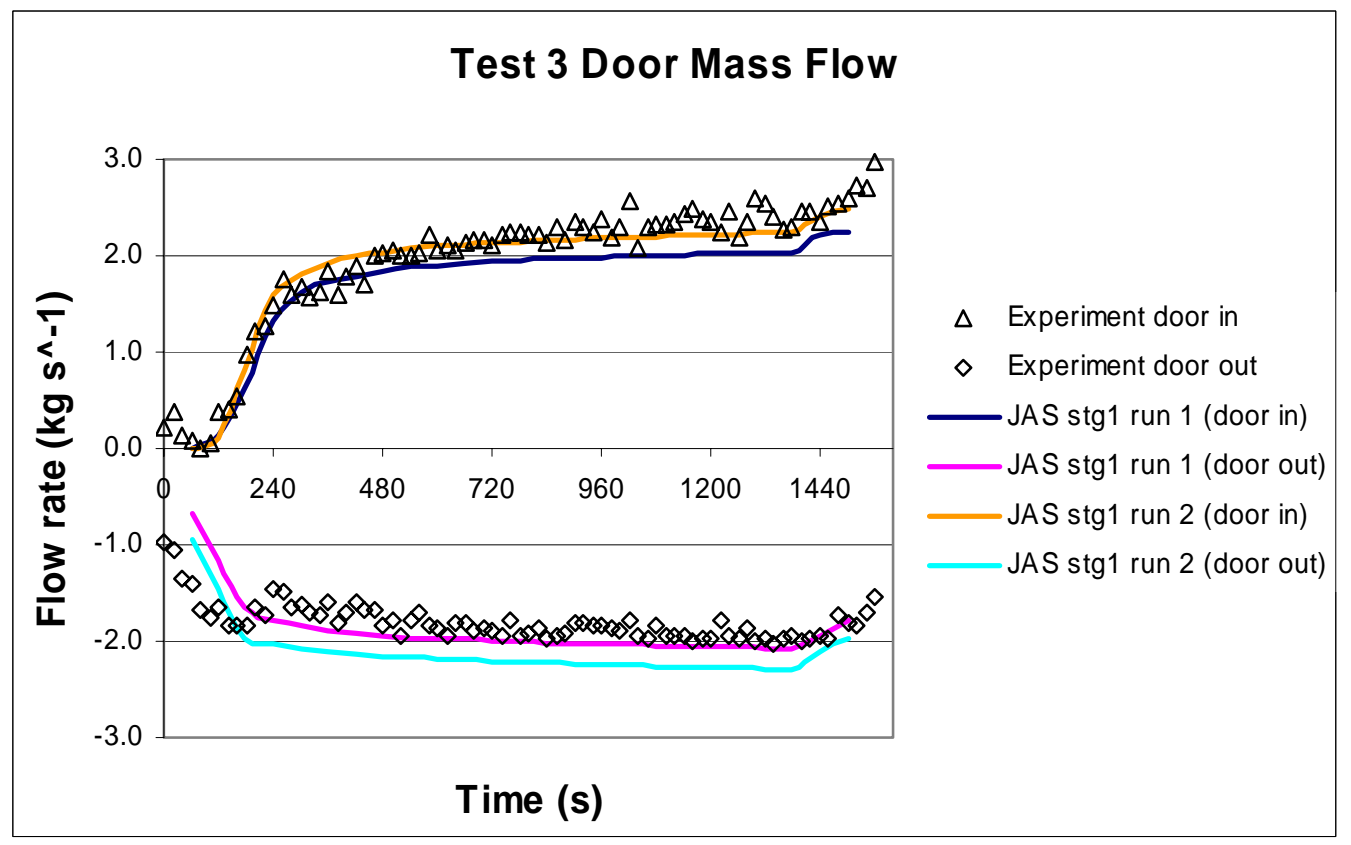

Fig. 2-31 Measured and JASMINE door mass fkow rates for Test 3 

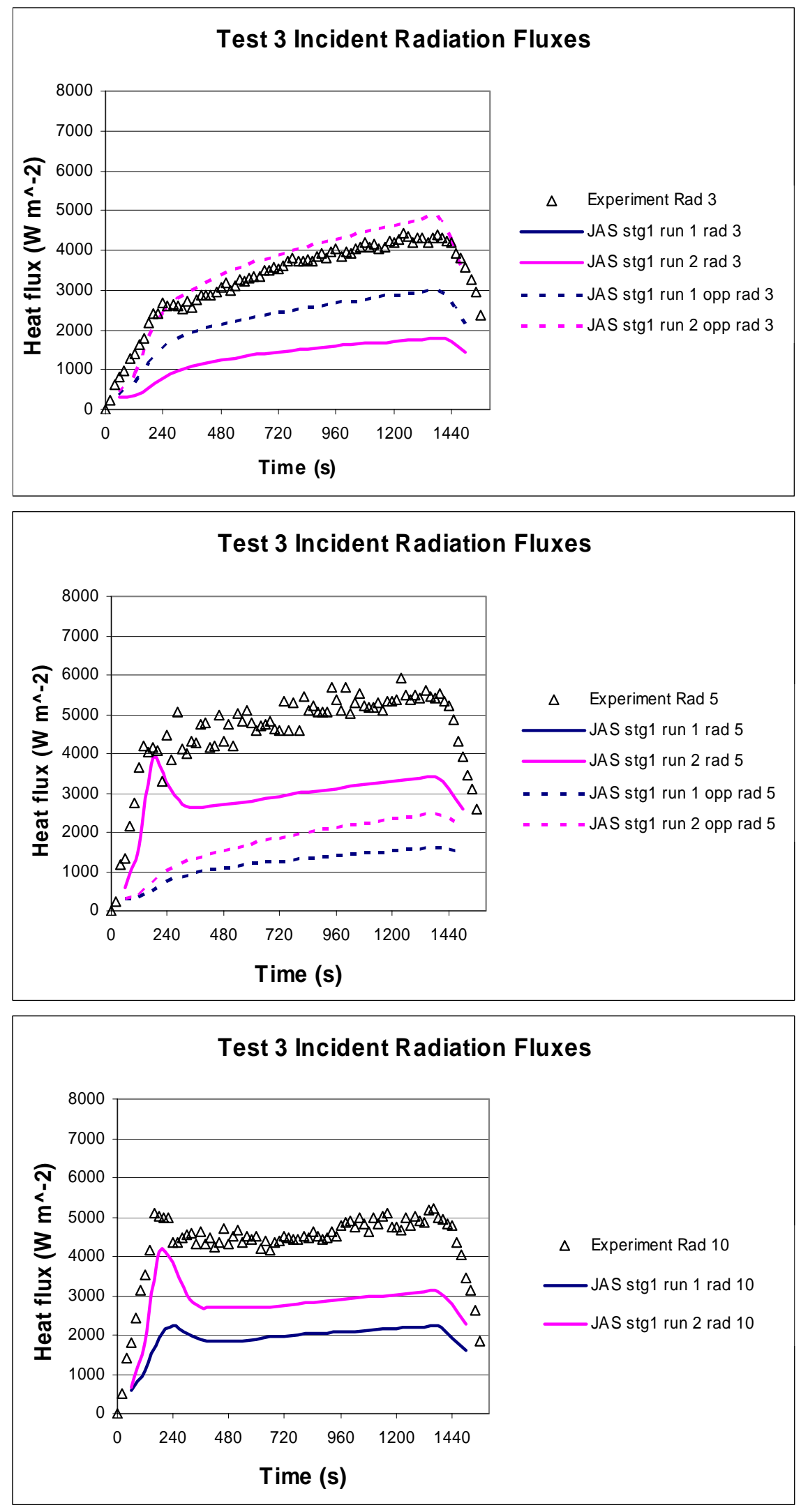

Fig. 2-32 Measured and JASMINE predicted incicent fluxes for Test 3 

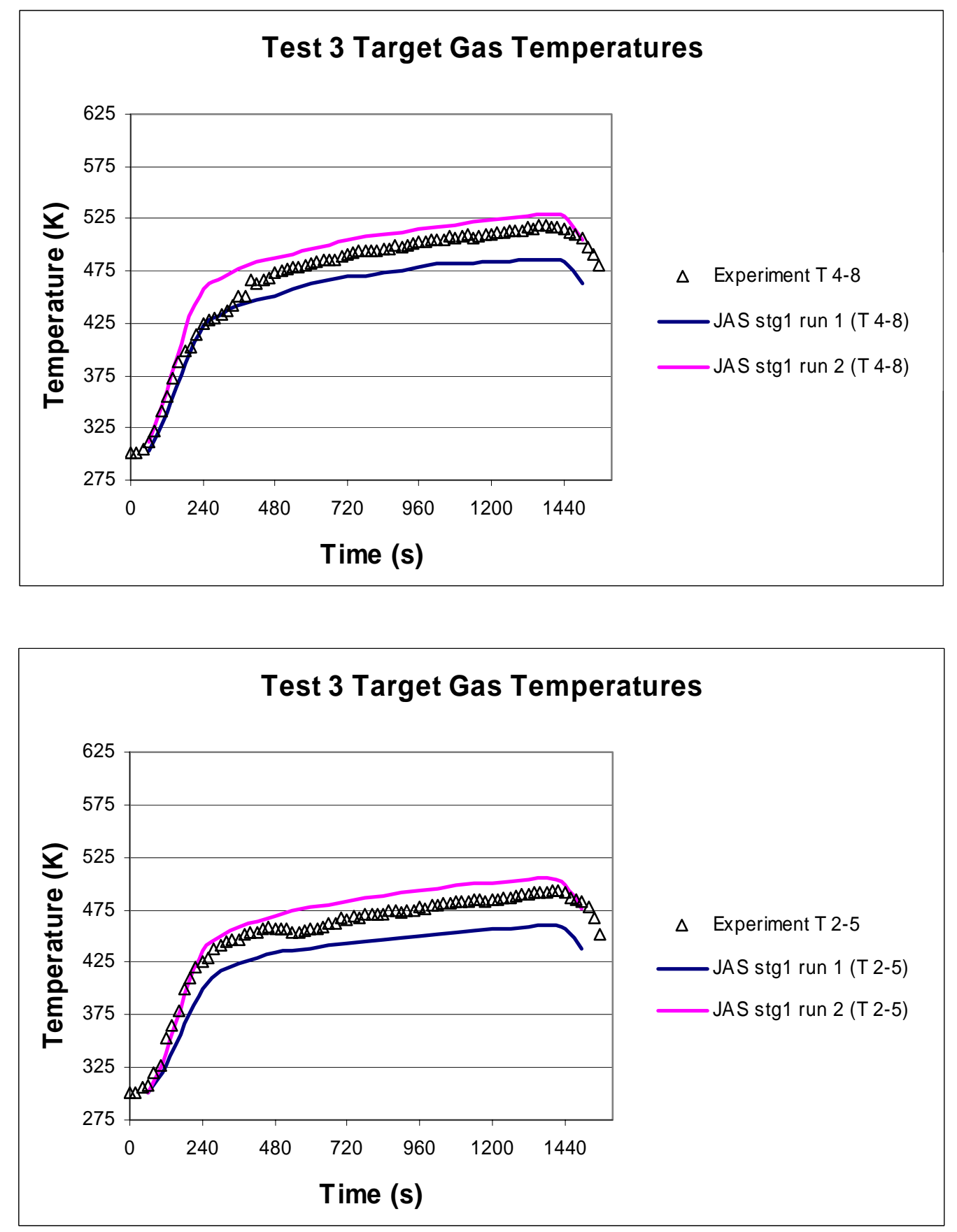

Fig. 2-33 Measured and JASMINE predicted gas temperatures near cable targets for Test 3 

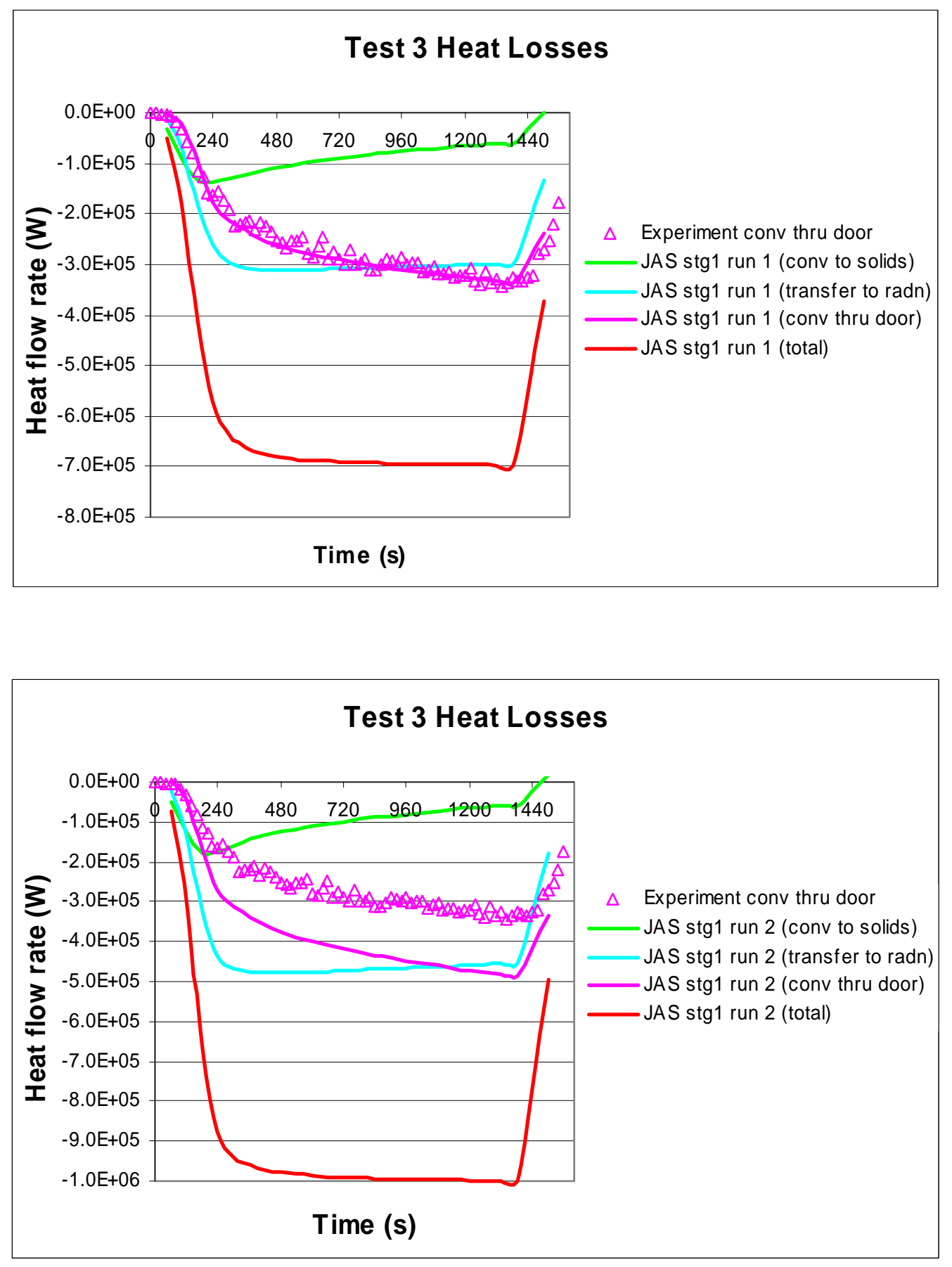

Fig. 2-34 JASMINE predicted heat losses for Test 3 

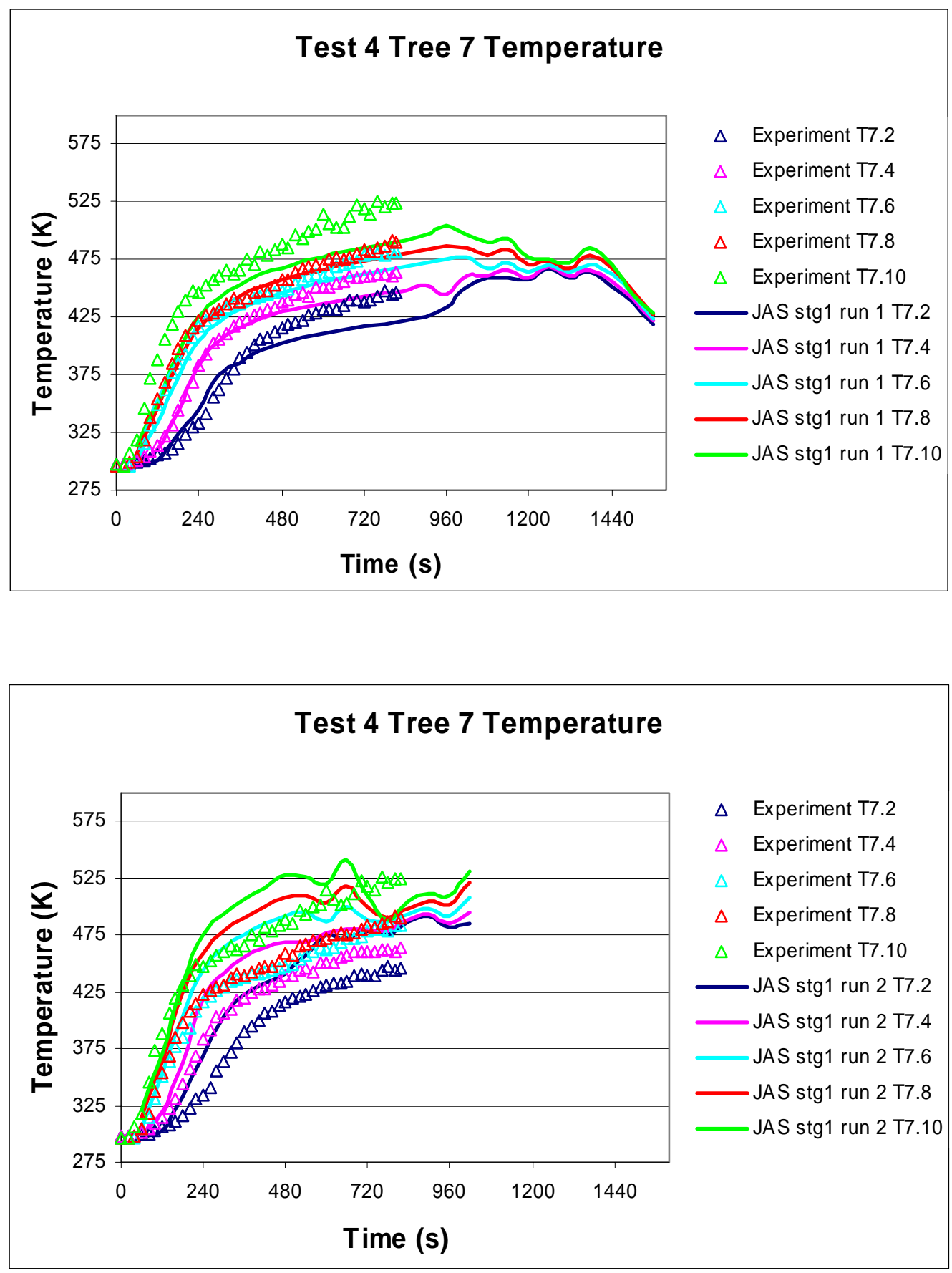

Fig. 2-35 Measured and JASMINE predicted (blind) gas temperatures for Test 4 at Tree 7 

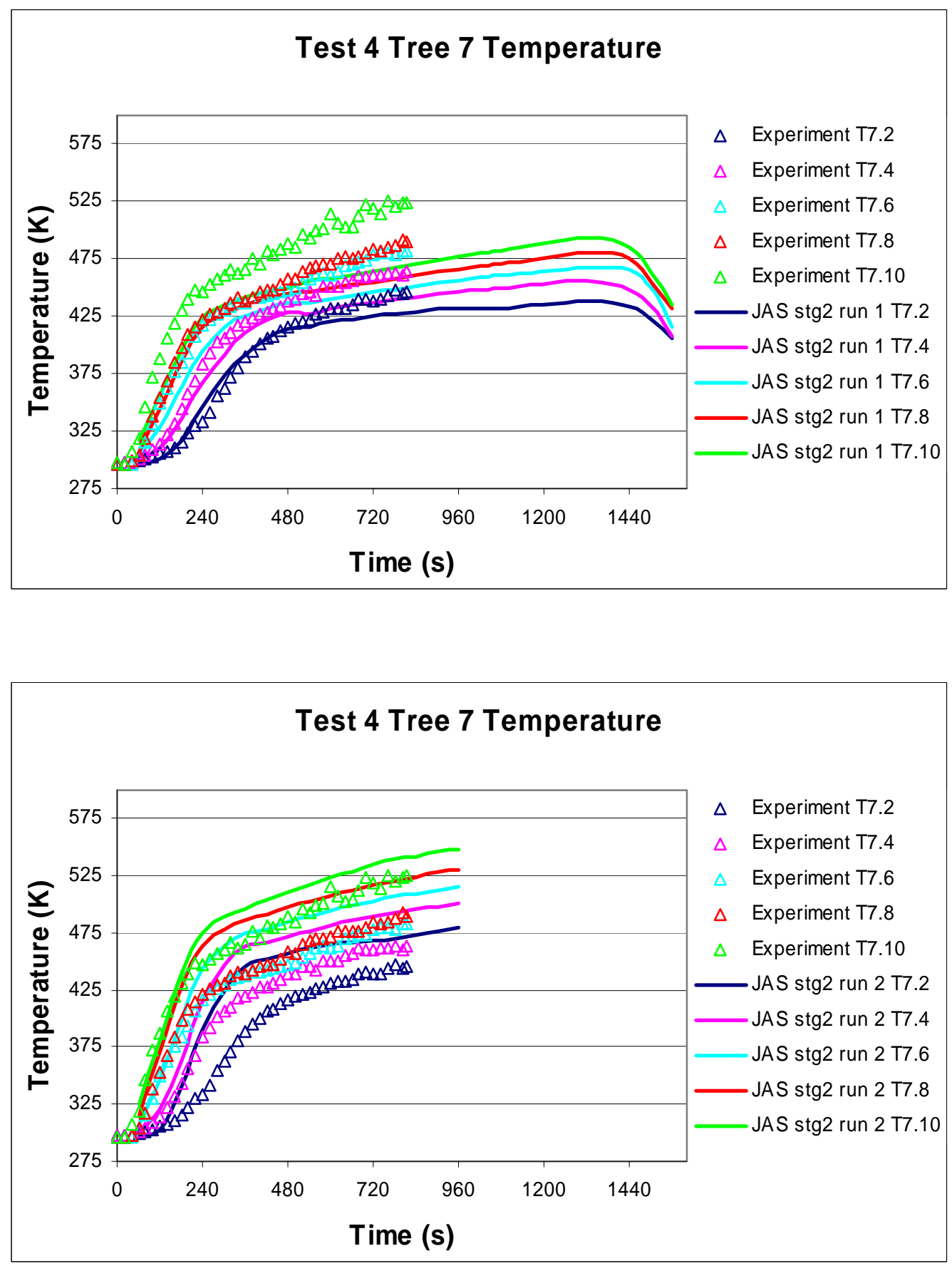

Fig. 2-36 Measured and JASMINE predicted (semi-blind) gas temperatures, Test 4 at Tree 


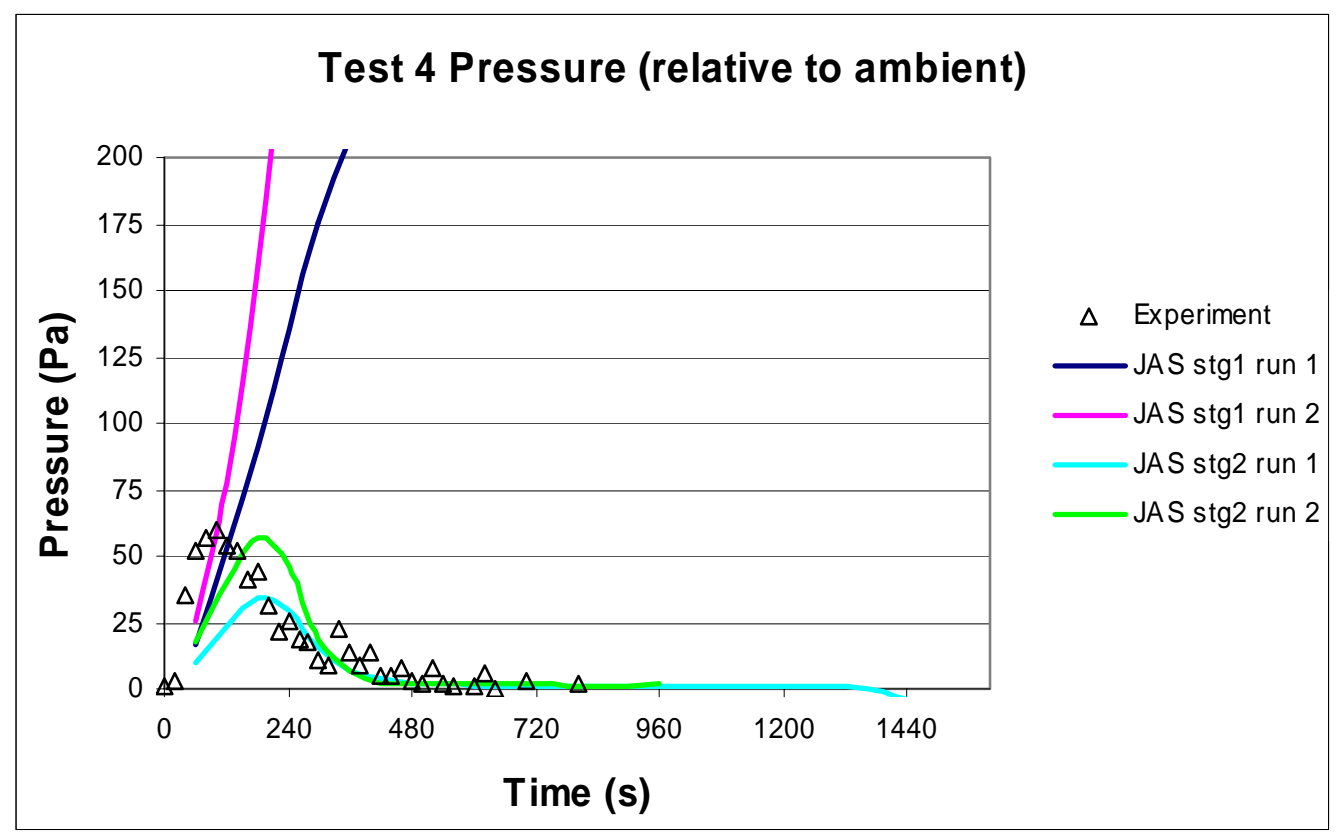

Fig. 2-37 Measured and JASMINE predicted static pressure for Test 4 
Note this is a plot at O2-1 (upper layer) and that JASMINE data is a percentage by mass
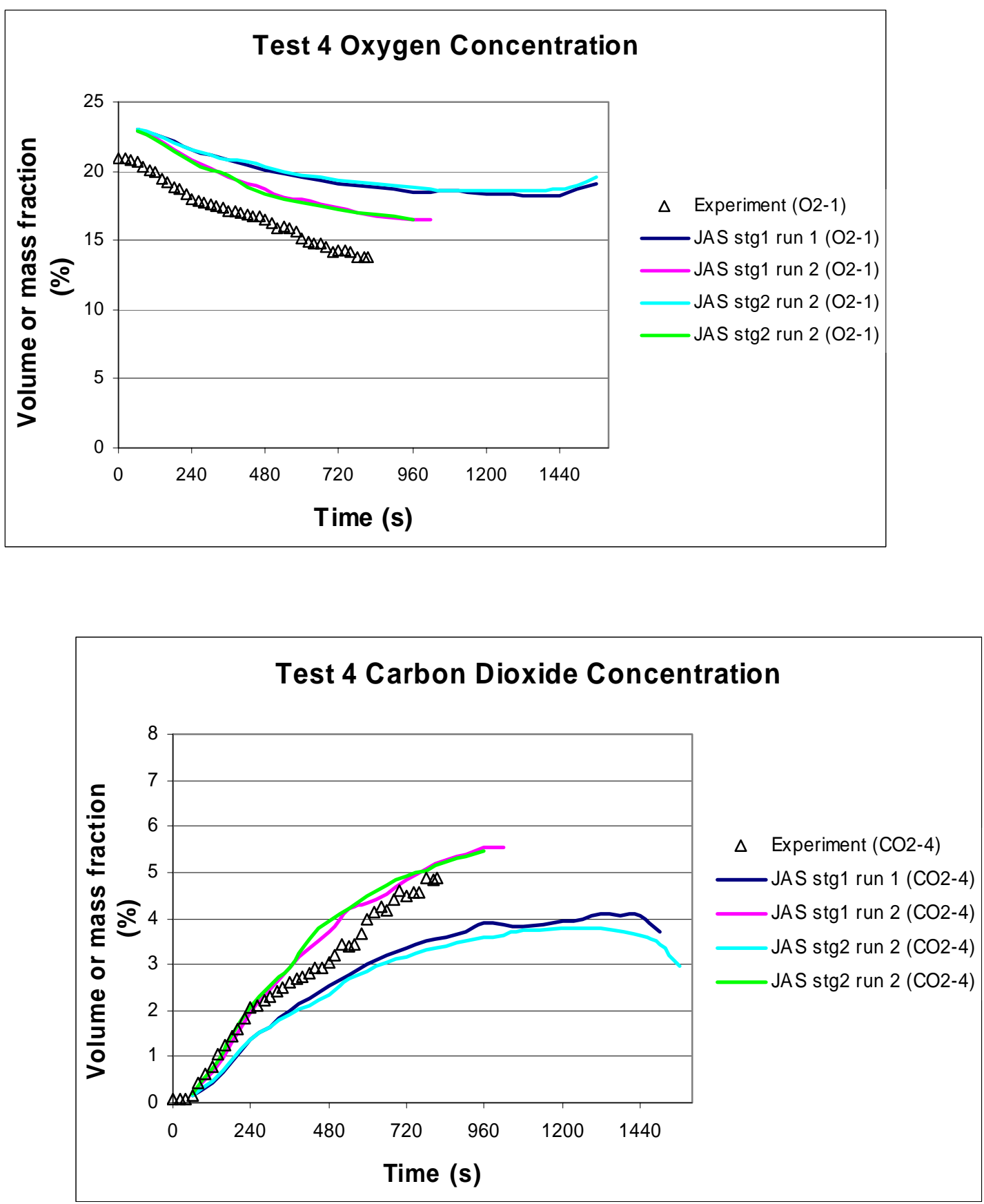

Fig. 2-38 Measured and JASMINE predicted gas concentrations for Test 4 

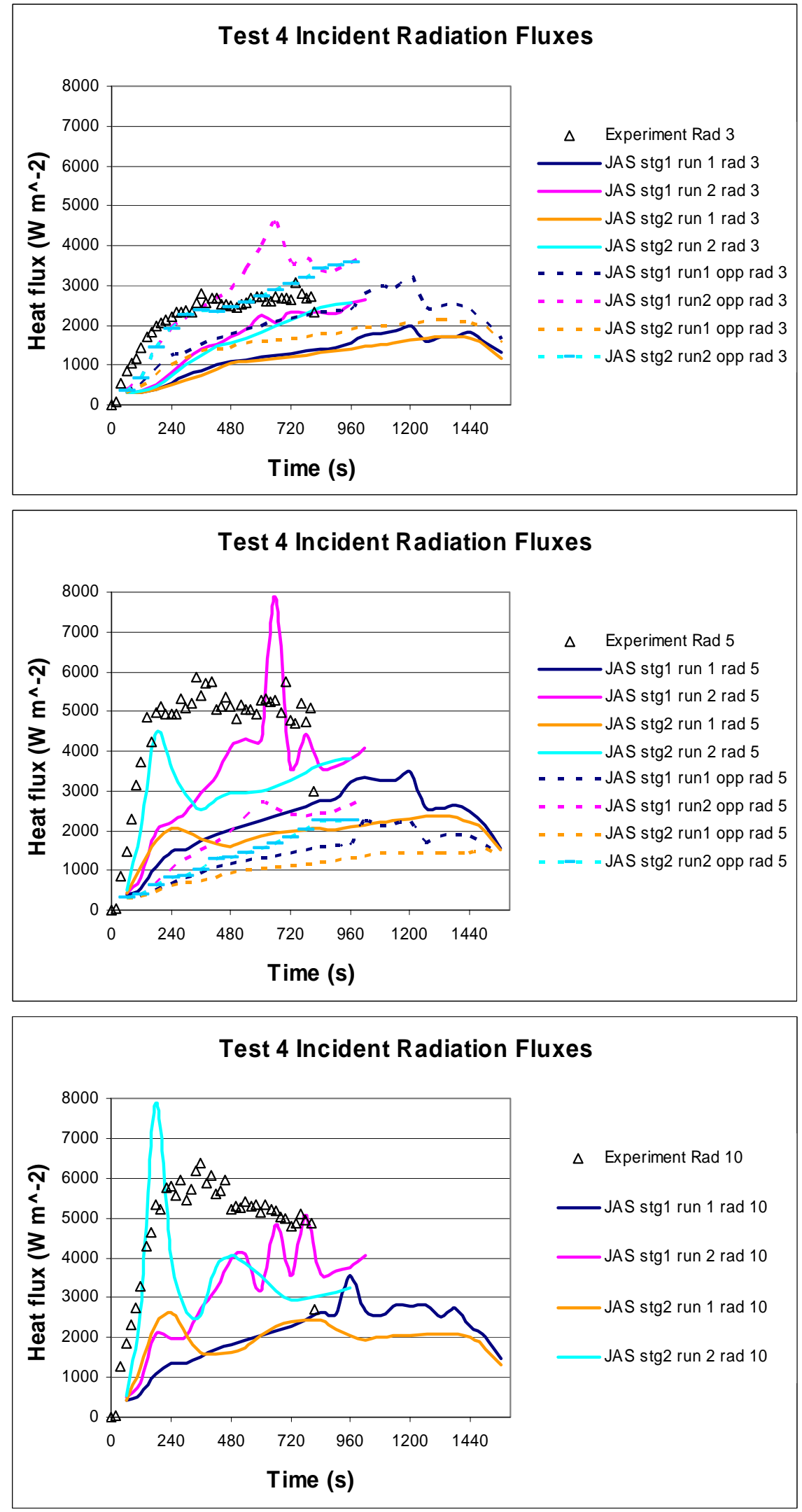

Fig. 2-39 Measured and JASMINE predicted incicent fluxes for Test 4 

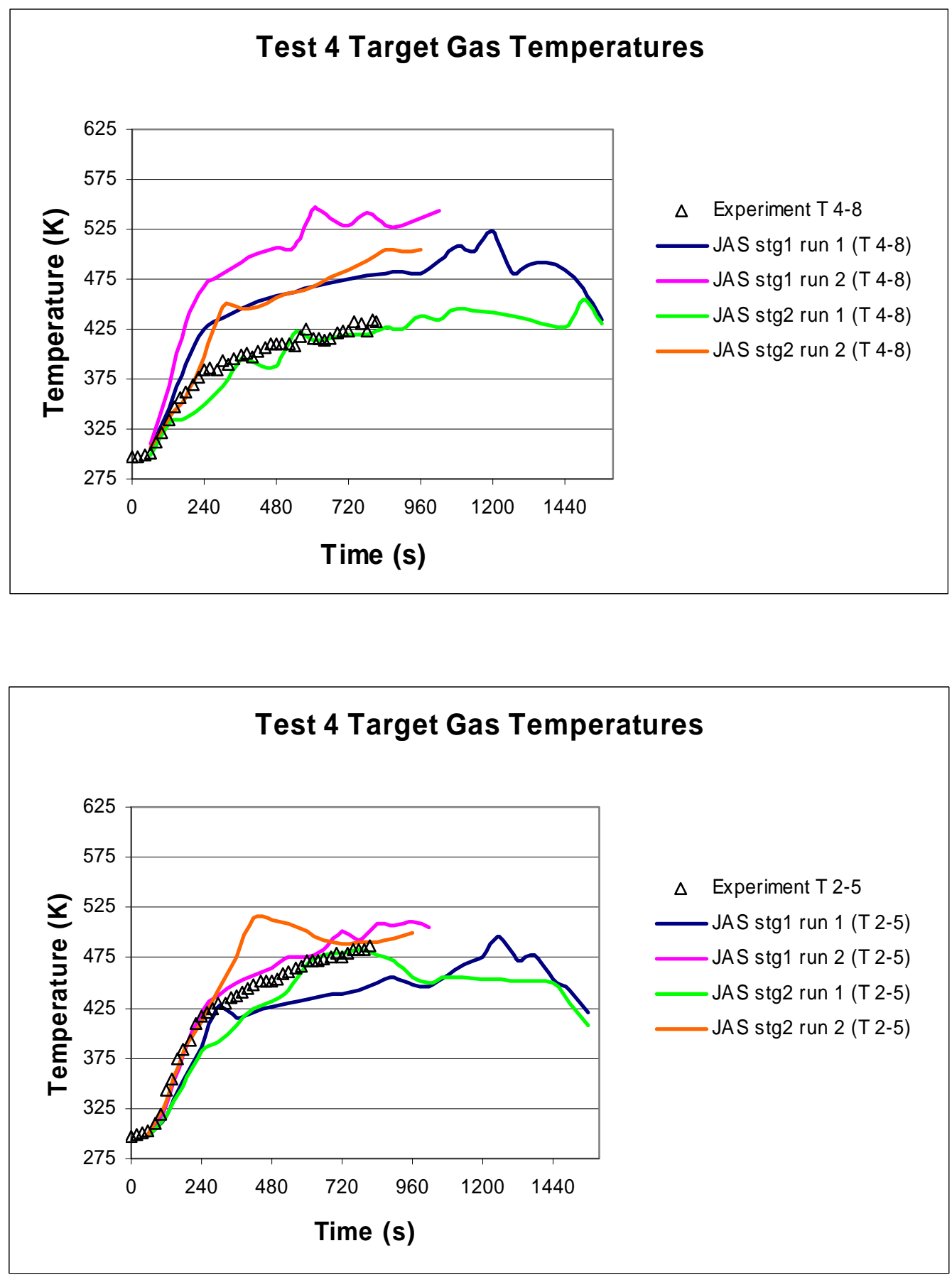

Fig. 2-40 Measured and JASMINE predicted gas temperatures near cable targets for Test 4 


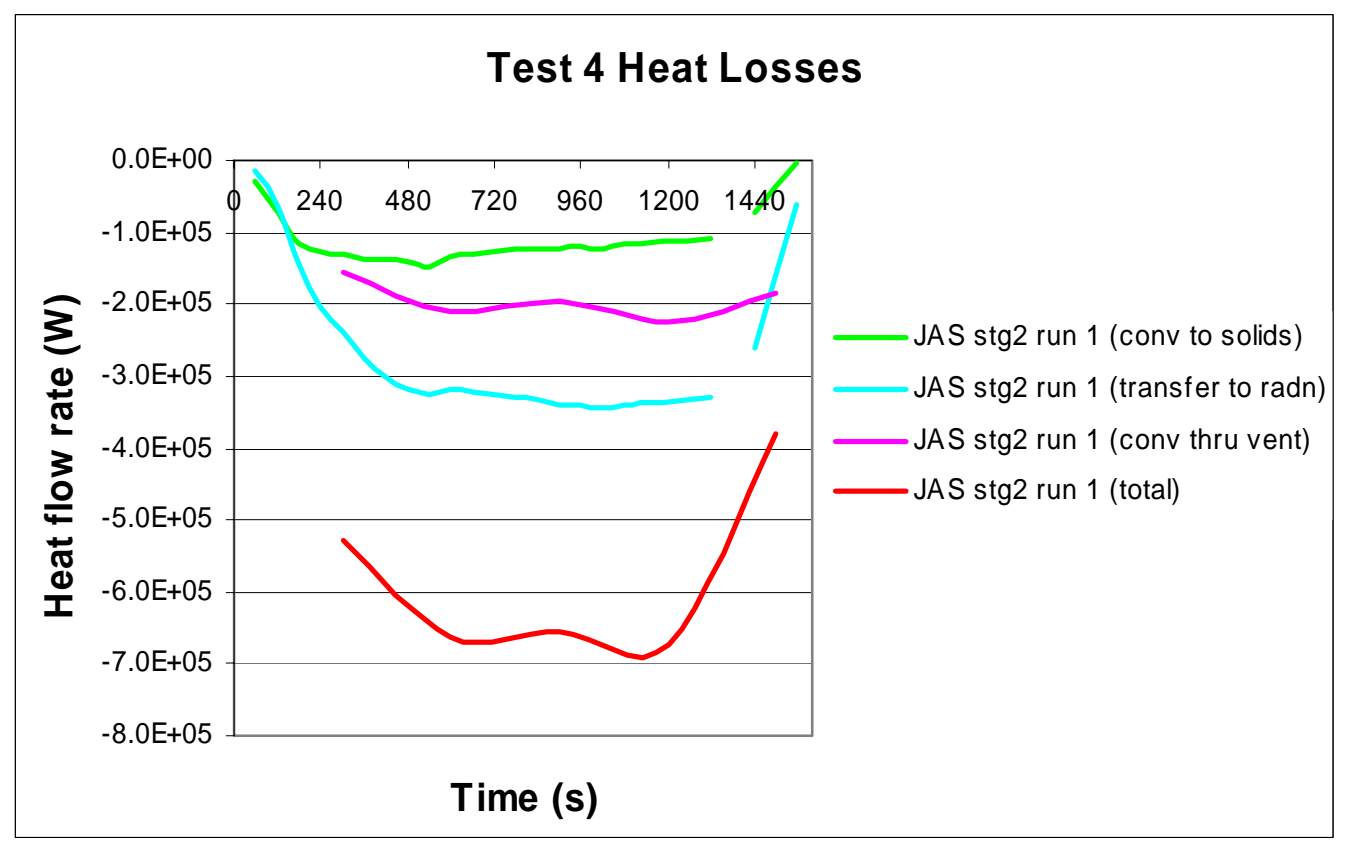

Fig. 2-41 JASMINE predicted heat losses for Test 4 


\subsection{Conclusions and Recommendations for CFAST and JASMINE}

The main findings from both the CFAST and JASMINE work were reported in the previous sections. The main points raised, and some recommendations, are given below:

- For both CFAST and JASMINE the results were judged to be encouraging when interpreted correctly and the limitations of the various modeling assumptions acknowledged. Test 3, with the open door, was relatively easy to model with both the zone and CFD approach. However, due to the ventilation restrictions, Tests 2 and 4 (closed compartment) were more difficult with both models and care was required when performing the calculations.

- The predicted pressure rise, in Tests 2 and 4, was very sensitive to the size of infiltration opening used in the calculation. It was concluded that using a single opening to represent all the leakage paths may not be the best approach. It is clear that where a fire compartment is closed and the calculation of pressure is important that a sensitivity analysis is performed as the initial choice of leakage area may be close to the critical value in respect to any significant pressure effects. The level of numerical convergence needs to be considered when modeling closed compartments. This may be especially true of CFD models.

- A radiative fraction of at least 0.3 in CFAST was found appropriate. A lower value resulted in calculated gas temperatures being too high. Indeed, a value of 0.5 provided the closest agreement between calculated and measured gas temperature. However, it should be recalled that the choice of the heat of combustion, the fuel supply rate and the heat losses to the boundaries may all have a significant bearing too.

For JASMINE it was found that assigning $30 \%$ to $35 \%$ of the heat release to radiation from soot particles (in the 'plume') was an appropriate choice. This was borne out especially in Test 3 where a choice of $30 \%$ yielded accurate gas temperature predictions. It should be noted that while radiation absorption and emission due to the presence of $\mathrm{CO}_{2}$ and $\mathrm{H}_{2} \mathrm{O}$ was included in the JASMINE calculations, and also the subsequent radiation heat transfer to the solid boundaries, the contribution due to soot particles was neglected. This may be important for target response predictions.

As in previous benchmark exercises, see [US NRC, 2002] and [Miles, 2004], the appropriate choice of radiative fraction, or soot alternative modeling methodology, is consid- 
ered to be a critical parameter for a reliable hazard analysis. For CFD models such as JASMINE the implementation of a reasonably simple, yet effective, soot modeling and radiation treatment is recommended. Earlier work in this area was performed by Magnussen and coworkers, e.g. [Magnussen et. al., 1979], here extending the concept of the eddy breakup combustion model. Some work on a more empirical approach to soot modeling for CFD applications has been reported recently [Lautenberger et. al., 2005].

- The choice of lower oxygen limit (LOL) in CFAST is another combustion related parameter that can have an important bearing on the hazard development. However, as the experiments were effectively terminated once the oxygen level had dropped below 15\%, there was only limited scope to address this parameter in the current benchmark exercise. However, for Tests 2 and 4 CFAST predicted time to termination of the test (fuel supply) was notably earlier than in the experiment. By comparison, JASMINE tended to either predict about the right time for the duration of the experiment or a significantly longer time, e.g. in Test 4 with 30 to $35 \%$ of the heat release rate removed due to soot radiation the calculations indicated, in contrast to the actual experiment, that the test would not terminate.

- The value in reducing the compartment space into an upper and lower gas layer is perhaps questionable for scenarios such as Tests 2 and 4 where there is significant mixing. Nonetheless, CFAST calculated that the layer interface height dropped to floor level within the first few minutes for both tests, and this can probably be interpreted as a correct behavior.

While for Test 3 JASMINE predicted approximately the correct degree of thermal stratification, for Tests 2 and 4 it tended to under-predict the level of stratification, i.e. the calculated level of mixing was greater than that observed. This was particularly apparent in the later stage in those cases where the compartment pressure was predicted to rise significantly, where once the calculated pressure started to rise strongly the compartment became well mixed, i.e. no stratification. This may be due, in part at least, to numerical nonconvergence. The effect was not observed in the semi-blind calculations for Test 4 where the leakage area was increased to the recommended value.

- Carbon monoxide predictions, using CFAST, were similar to the measurements only in the early stages of the tests. As each test proceeded the predicted level of CO was notably higher than that measured. This may have been due in part to the difficultly associated in modeling $\mathrm{CO}$ generation compared to the major products of combustion $\left(\mathrm{CO}_{2}\right.$ and $\mathrm{H}_{2} \mathrm{O}$ ). CO predictions were not made with JASMINE. 
- The method used to implement mechanical ventilation in the CFAST calculation for Test 4 (mechanical extract vent and a vertical natural vent for make-up air) seemed to have no significant implication in terms of for the quality of the predictions. An under-prediction of compartment pressure may, however, be one consequence.

- For the CFAST predictions the calculated incident radiant fluxes were, in each test, largely independent of location or orientation. However, in Tests 2 and 4 the measurements indicate a significant location and/or orientation dependency on the incident fluxes and so the agreement between predicted and measured incident radiant flux was quite variable for these tests. One cause of discrepancy is the fact zone models such as CFAST do not model most of the three dimensional effects of a compartment fire.

JASMINE tended to under-predict incident radiant flux in all tests. This is likely due, in part at least, to the use of the six-flux radiation model which biases the radiant flux information in the three Cartesian directions. It may be due also to the absence of an explicit soot treatment.

For both CFAST and JASMINE, the surface temperature calculations were judged to be reasonable given the accuracy of the incident fluxes. It can be argued that given reasonable incident flux predictions the surface (and interior) target temperatures may be expected to be reasonable provided the target structure is not too complicated. As in the previous benchmark exercises, it is recommended that further consideration be given to the most appropriate way to treat target response assessment, e.g. purely as a postprocess outside the primary fire model or more integrated into the fire simulation. 


\section{$2.6 \quad$ References}

Cox G., Kumar S. \& Markatos N.C. Some field model validation studies. Fire Safety Science - Proceedings of the First International Symposium, Hemisphere Publishing, pp. $159-171,1986$.

Cox G. \& Kumar S. Field modelling of fire in forced ventilated enclosures. Combustion. Science and Technology, vol. 52, pp. 7-23, 1987.

Gosman A.D. \& Lockwood F.C. Incorporation of a flux model for radiation into a finite difference procedure for furnace calculations. In Proceedings of the 14th Symposium (International) on Combustion, The Combustion Institute, pp. 661-671, 1973.

Hamins, A. et. al. Report of test on experimental conditions and preliminary results for the International Fire Model Benchmarking and Validation Exercise \# 3. July 2003a.

Hamins, A. et. al. Report of experimental results for Tests 2,3 and 4 of the International Fire Model Benchmarking and Validation Exercise \# 3. July 2003b.

Jones, W.W. et. al. A Technical Reference for CFAST: An Engineering Tool for Estimating Fire and Smoke Transport. NIST TN 1431. April 2003.

Jones, W.W. et. al. CFAST - Consolidated Model of Fire Growth and Smoke Transport (Version 5): Technical Reference Guide. NIST Special Publication 1030. October 2004.

Launder B.E. \& Spalding D.B. The numerical computation of turbulent flows. Computer Methods in Applied Mechanics and Engineering, vol. 3, pp. 269-289, 1974.

Lautenberger C.W. et. al. A simplified model for soot formation and oxidation in CFD simulation of non-premixed hydrocarbon flames. Fire Safety Journal, vol. 40, pp. 141176, 2005.

Lockwood F.C. \& Shah N.G. A new radiation solution method for incorporation in general combustion prediction procedures. In Proceedings of the 18th Symposium (International) on Combustion, The Combustion Institute, pp. 1405-1414, 1981. 
Magnussen B.F. \& Hjertager B.H. On mathematical modelling of turbulent combustion with special emphasis on soot formation and combustion. In Proceedings of the 16th Symposium (International) on Combustion, The Combustion Institute, pp. 719 729, 1976.

Magnussen B.F. et, al. Effects of turbulent structure and local concentrations on soot formation and combustion in $\mathrm{C} 2 \mathrm{H} 2$ diffusion flames. In Proceedings of the 17th Symposium (International) on Combustion, The Combustion Institute, pp. 1383 1393, 1976.

Maranghides, A. et al. Test plan for international fire model benchmarking and validation exercise \# 3. 11 March 2003.

McCaffrey B.J. Momentum implications for buoyant diffusion flames. Combustion and Flame, vol. 52, p. 149, 1983.

Miles S.D., Kumar S. \& Cox G. Comparisons of 'blind predictions' of a CFD model with experimental data. Fire Safety Science - Proceedings of the Sixth International Symposium, IAFSS, pp. 543-554, 2000.

Miles S. The predictive capability of CFD for fully developed fires. Fire and Explosion Hazards - Proceedings Third International Seminar, Windermere, UK, pp. 229-240, 2001.

Miles, S. International Panel Report: Benchmark Exercise \# 2 - Pool Fires in Large Halls. May 2004.

Patankar S.V. Numerical heat transfer and fluid flow. Hemisphere Publishing Co., 1980.

Siegel R. \& Howell J.R. Thermal Radiation Heat Transfer. Hemisphere Publishing Corporation, New York, 2nd ed., 1981.

Truelove J.S. Mixed grey gas model for flame radiation. UK Atomic Energy Authority Report AERE HL 76/3448, 1976.

U.S. Nuclear Regulatory Commission. International Collaborative Project to Evaluate Fire Models for Nuclear Power Plant Applications: Cable Tray Fires. Compiled by Dey, M., NUREG-1758, Washington, DC, June 2000. 
The two-zone fire model FLAMME_S v.2.3.2, developed by IRSN, was used for open simulations of Tests 2, 3, 4 and 5 . The influence of the fire on the targets was not studied because the model is not accurate enough for this problem.

\subsection{FLAMME_S Input Parameters and Assumptions}

Most of input data were taken from the specifications. Some specific assumptions were:

- The emissivity of all surfaces was assumed to be 0.95 .

- The convective heat transfer was assumed to be $10 \mathrm{~W} \cdot \mathrm{m}^{-2} \cdot \mathrm{K}^{-1}$ inside the compartment and $6 \mathrm{~W} \cdot \mathrm{m}^{-2} \cdot \mathrm{K}^{-1}$ outside.

- The fire source was a pool of heptanes located at the center of the room with a heat release rate around $1 \mathrm{MW}$.

- $\quad$ Most of the heptane properties are given in the SFPE handbook 0.

- Complete combustion was assumed: $\mathrm{C}_{7} \mathrm{H}_{16}+11 \mathrm{O}_{2} \rightarrow 8 \mathrm{H}_{2} \mathrm{O}+7 \mathrm{CO}_{2}$

- The pyrolysis rate was deduced from the measured heat release rate, specified in the report of experimental results.

\subsection{Comparison of FLAMME_S with Measurements}

\subsubsection{Test 2 and 8: door closed and ventilation off}

This scenario was designed to determine the predictive capability of models for underventilated conditions. The nominal peak HRR was $1 \mathrm{MW}$ with a heptane fire located in the center of the compartment. The door was closed during these tests and the mechanical ventilation was turned off with the supply and exhaust vents sealed. Several calculations were 
performed with varying input data like heat release rate and the area of leakage. Final results, presented in this report, used a maximum heat release rate of $1.3 \mathrm{MW}$ corresponding to the upper bound of the experimental uncertainty, and with an opening simulating compartment leakage of $729 \mathrm{~cm}^{2}$, which corresponds to an average value of experimental measurements 0 .

The FLAMME_S code calculates the fire extinguishment earlier than what was observed experimentally (see Figure 3-1). In this case, the fire extinguishes through lack of oxygen, for a value of the oxygen concentration around $12 \%$. But the experimental decrease of oxygen consumption is slower four minutes after the fire ignition than the calculated one (see Figure 3-2). The difference between code and experiments may be due to two physical models: either the chemical reaction of combustion, which is imposed in the code, or the estimation of the flow rate through the leakage. In the input data, a complete reaction of combustion is supposed with only production of water and carbon dioxide. With this hypothesis of chemical reaction, the production of carbon dioxide seems to be well estimated by the code (see Figure 3-3). In order to verify if the flow rate through the leakage is well estimated by the code, the pressure measured during tests was compared to the code results (see Figure 3-4). The calculated pressure shows that there is perhaps another oxygen contribution which is not modeled. In fact, the experimental pressure difference of the two tests is never equal to zero after the over-pressure which follows the fire ignition.

The calculated layer height falls rapidly contrary to the one measured experimentally, during the two tests, which is stabilized around $0.8 \mathrm{~m}$ above the floor (see Figure 3-5). So, there is rapidly only one gas zone modeled.

Four minutes after the fire ignition, the calculated gas temperature does not increase like the measured temperatures (see Figure 3-6). The difference between the code and experiments can be due to the uncertainties on the heat release rate or to an over-estimation of the heat transfer between gas and walls.

For the two experiments 2 and 8 , the heat release rate is not measured but it is estimated to be equal to $1140 \mathrm{~kW} \pm 15 \% 0$. The calculations were performed with the maximum value of the heat release rate, i.e. 1.3 MW (see Figure 3-1). Even with this upper value, the gas temperature is under-estimated. The fire energy which heats the compartment surfaces is estimated experimentally (see Figure 3-7). The rate of heat loss to surfaces increases during the fire and reaches $1.3 \mathrm{MW}$. So if we consider that the energy loss to the walls surfaces repre- 
sents $70 \%$ or $80 \%$ of the fire energy, the experimental heat release rate may be experimentally under-estimated.

Calculated and measured energy loss to compartment surfaces was compared. The calculated energy loss to the side walls (north/south; east/west - see Figure 3-8) and to the ceiling (see Figure 3-9) are in good agreement with experimental estimations. But the calculated energy loss to the floor is overestimated (see Figure 3-9). In this case, the heat transfer is between the one gas zone modeled and the floor, and the calculated temperature is greater than the experimental gas temperature of the lower layer (see Figure 3-6).

This configuration shows that a model that assumes well-ventilated conditions may not work well with under-ventilation conditions. In particular, the mass transfer between the lower and upper layers via the Heskestad correlation may lead to an over-estimate in the decrease in layer height

\subsubsection{Tests 3 and 9: door opened and ventilation off}

This scenario was designed to determine the predictive capability of models for wellventilated conditions. The nominal peak of HRR was $1 \mathrm{MW}$ with a heptane fire located in the center of the compartment. The door was open during these tests and the mechanical ventilation was turned off with the supply and exhaust vents sealed.

For this configuration, the calculated parameters are overall in good agreement with experimental results.

The experimental heat release rate was used directly as input data of FLAMME_S (see Figure 3-10) so the fire did not extinguish through lack of oxygen but by the stopping of the fuel delivery system.

The calculated height of the gas layer is in good agreement with the one estimated experimentally (see Figure 3-11). The calculated gas temperature of the upper layer is underestimated, but the relative error is less than $20 \%$ (see Figure 3-12). The calculated gas temperature of the lower layer is also under-estimated by more than $20 \%$.

The comparison of the molar fractions of oxygen and carbon dioxide shows that the hypothesis of a complete reaction of combustion is correct (see Figure 3-13 and Figure 3-14). 
The energy losses to side walls are in good agreement with experimental results (see Figure 3-15). On the other hand, the energy loss to the ceiling is over-estimated and the energy loss to the floor is under-estimated (see Figure 3-16). The under-estimation of the loss to the floor may be due to the under-estimation of the gas temperature of the lower layer. The overestimation of the loss to the ceiling seems to be due to the heat transfer between the plume and the ceiling instead of the use of an option which prevent this heat exchange.

In spite of these important differences on the losses to the ceiling and to the floor, the total energy loss calculated by the code is within $20 \%$ of the measurements (see Figure 3-17).

The prediction of the mass flow through the door is in good agreement with experimental results (see Figure 3-18).

\subsubsection{Tests 3 and 10: door closed and ventilation on}

This scenario was designed as a variation of test 2 for providing data to determine the predictive capability of models for closed door scenarios with the mechanical ventilation system on. The nominal peak of HRR was $1 \mathrm{MW}$ with a heptane fire located in the center of the compartment. The door was closed during these tests and the mechanical ventilation was turned on.

For this configuration, the heat release rate, specified as input data in the simulations, is the one mentioned in the report of experimental results 0 , with a ramp up, a steady state and a ramp down (see Figure 3-19).

The calculated layer height falls rapidly contrary to the layer height estimated experimentally which is stabilized around $1 \mathrm{~m}$ above the floor (see Figure 3-20). So, in the simulation, $6 \mathrm{~min}$ $30 \mathrm{~s}$ after the fire ignition there is only one gas zone modeled. The average gas temperature, calculated by the code, is, in a first time, closed to the experimental temperature of the upper layer and then it is under-estimated (see Figure 3-21). The over-estimation of the gas temperature in the first minutes may be due to a bad estimation of the experimental heat release rate.

The disappearance of the lower layer leads that the heat exchange between the gas and the floor are over-estimated (see Figure 3-22), contrary to the others heat exchanges which are in good agreement with experimental results (see Figure 3-23). 
The hypothesis of a complete reaction of combustion gives good results concerning the molar fractions (see Figure 3-24 and Figure 3-25).

The pressure is in good agreement with experimental results except the over-pressure after fire ignition (see Figure 3-26). This result may be due to the over-estimation of the heat release rate in the first minutes (see Figure 3-19) and not to the mass flows through the exhaust and the supply vents which are well calculated (see Figure 3-27 and Figure 3-28).

\subsubsection{Test 5: door opened and ventilation on}

This scenario was designed as a variation of test 3 for fires in well ventilated conditions with natural and mechanical ventilation. The nominal peak of HRR was $1 \mathrm{MW}$ with a heptane fire located in the center of the compartment. The door was opened during these tests and the mechanical ventilation was turned on.

The heat release rate specified as input data in the simulations is the one mentioned in the report of experimental results 0 , with a ramp up, a steady state and a ramp down (see Figure 3-29).

In this case, the layer height is in good agreement with the experimental estimation (see Figure 3-30). The gas temperature of the upper layer is well calculated and the one of the lower layer is colder than the experimental data (see Figure 3-31).

The loss to the walls is over-estimated except the heat loss to the floor (see Figure 3-32 and Figure 3-33).

The hypothesis of a complete reaction of combustion gives good results concerning the molar fractions (see Figure 3-34 and Figure 3-35).

The pressure is weak because the fire is well-ventilated (see Figure 3-36). The order of magnitude of the mass flows through the door and through the vents is good (see Figure 3-37 and Figure 3-38). 


\subsection{Conclusions and Recommendations for FLAMME_S}

The two-zone fire model FLAMME_S has been applied to perform calculations for the benchmark exercise \#3, to simulate heptane pool fires inside a compartment with different ventilation conditions. The calculated results are compared to experimental measurements of seven experiments.

This exercise highlights that fires in a well-ventilated compartment are simulated adequately with the code and models used in a two-zone code are limited in the case of fires in underventilated conditions.

The comparisons with experimental results show that the calculated height of the gas layer falls rapidly when the door is closed. These results may be due the plume model used which has been established under well ventilated conditions.

The heat loss to the side wall is well predicted, but the heat exchange with the floor and with the ceiling is not. This problem is linked to the layer height:

When the lower layer disappears, in under ventilated conditions, the loss to the floor is overestimated because the calculated gas temperature is higher than the experimental temperature;

Under well ventilated condition, the loss to the floor is under estimated because the calculated temperature of the lower gas layer is always lower than the one which is estimated experimentally.

Cases with door opened are those which are better simulated with the code and the calculated results are in good agreement with experimental results.

In all cases, the calculated mass flows through the ventilation or through the door are in good agreement with experimental results. 


\subsection{References}

Maranghides A., Hamins A., Bryant R., Johnson E., Mulholland G., Widdman J. (NIST), Test plan for International Fire Model Benchmarking and validation exercise \#3, NIST, March 2003

Hamins A., Maranghides A., Johnson R., Donnelly M., Yang J., Mulholland G., Anleitner R., Report of experimental results for the International Fire Model Benchmarking and validation exercise \#3, NIST Special Publication 1013, January 2004

The SFPE Handbook of Fire Protection Engineering, 1995 


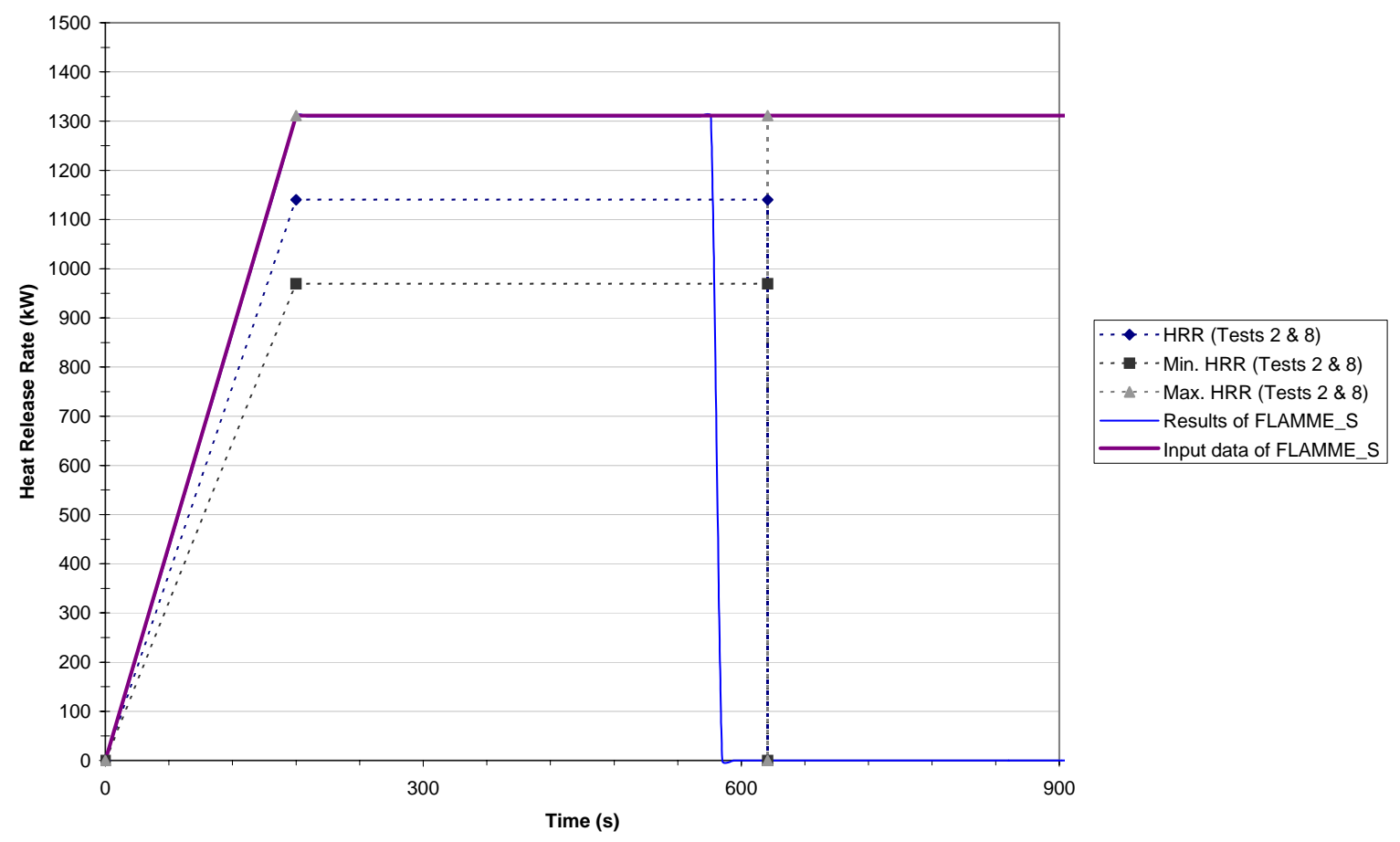

Figure 3-1: Heat release rate of the first configuration

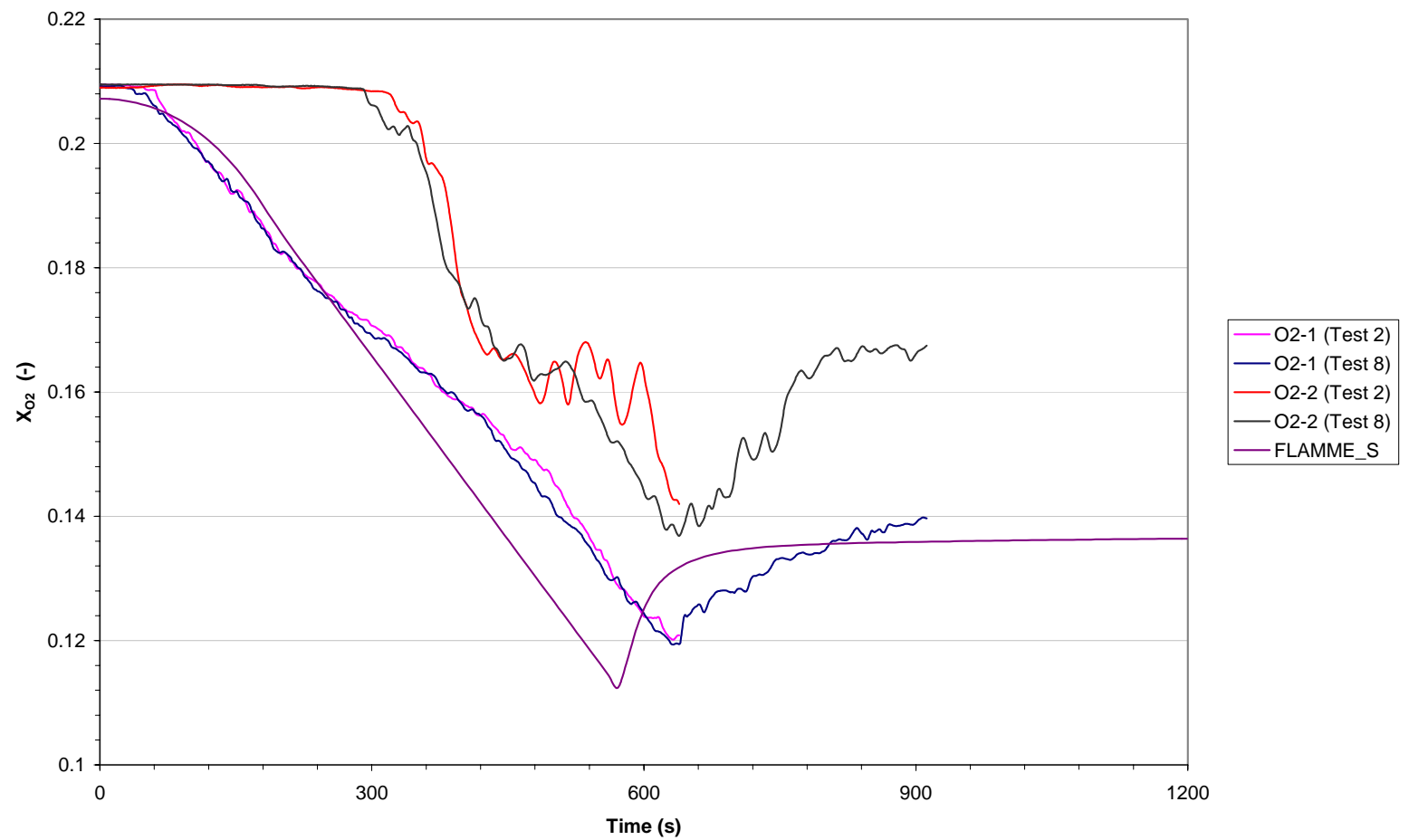

Figure 3-2: Molar fraction of oxygen 


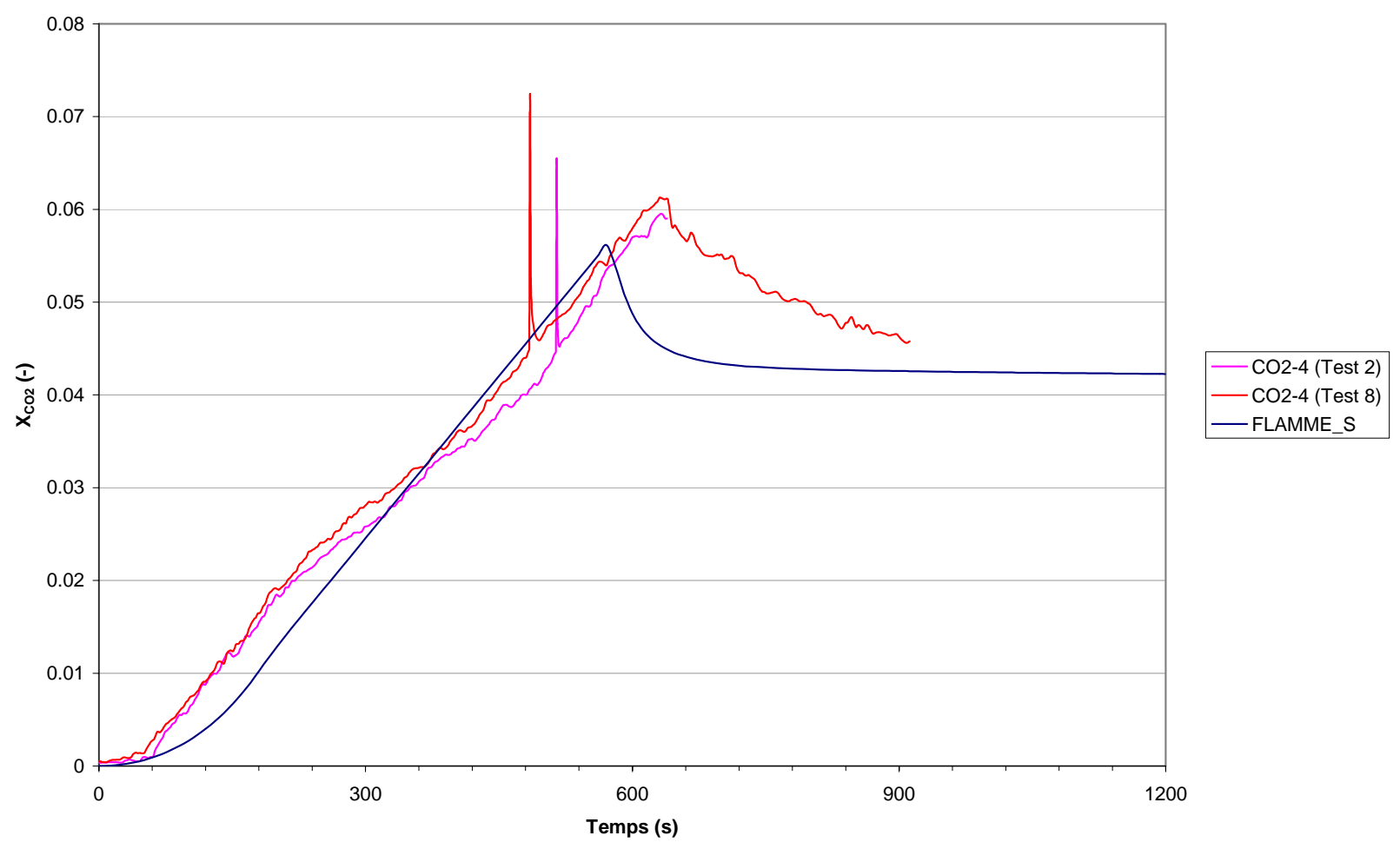

Figure 3-3: Molar fraction of carbon dioxide

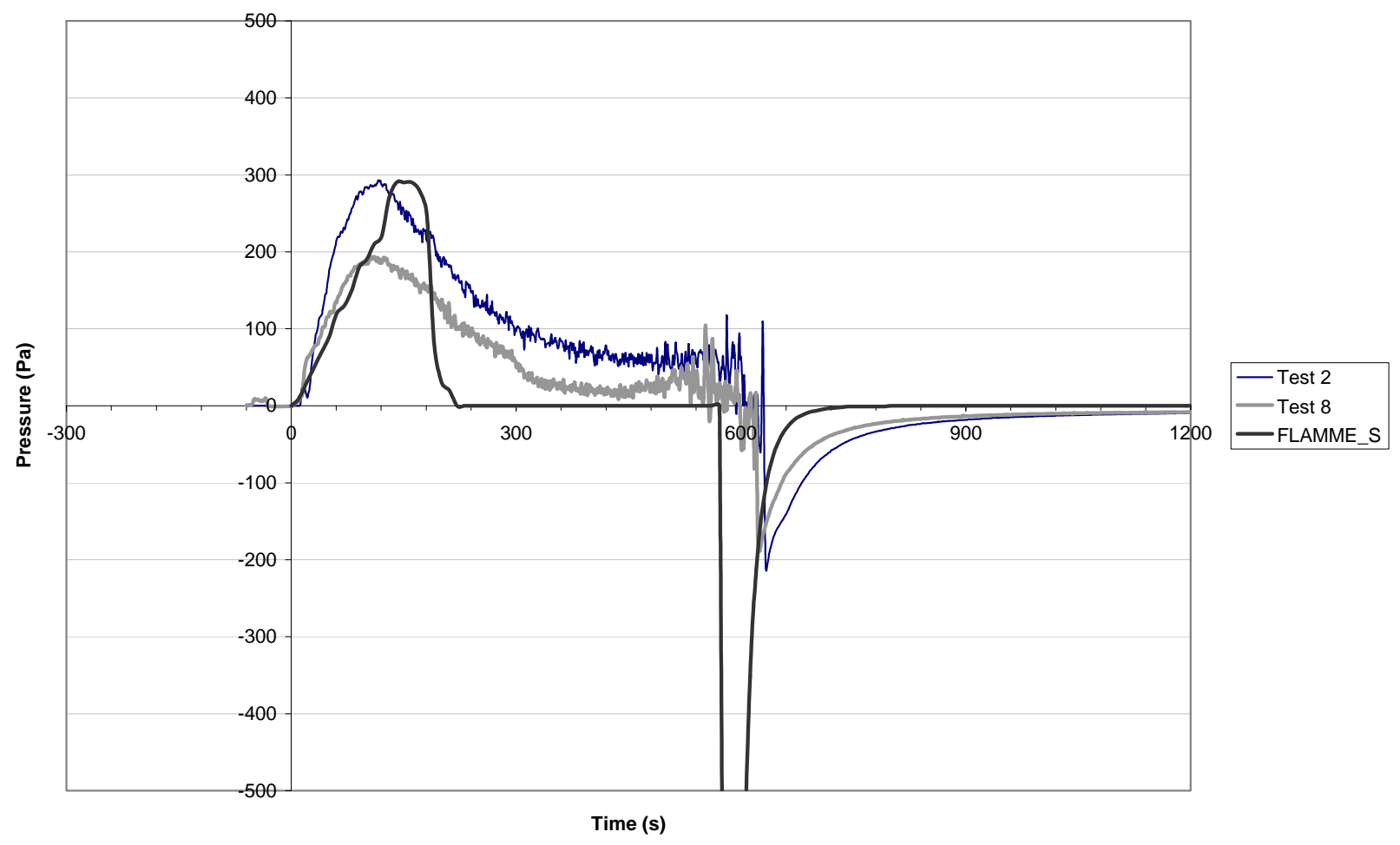

Figure 3-4: Pressure of the compartment 


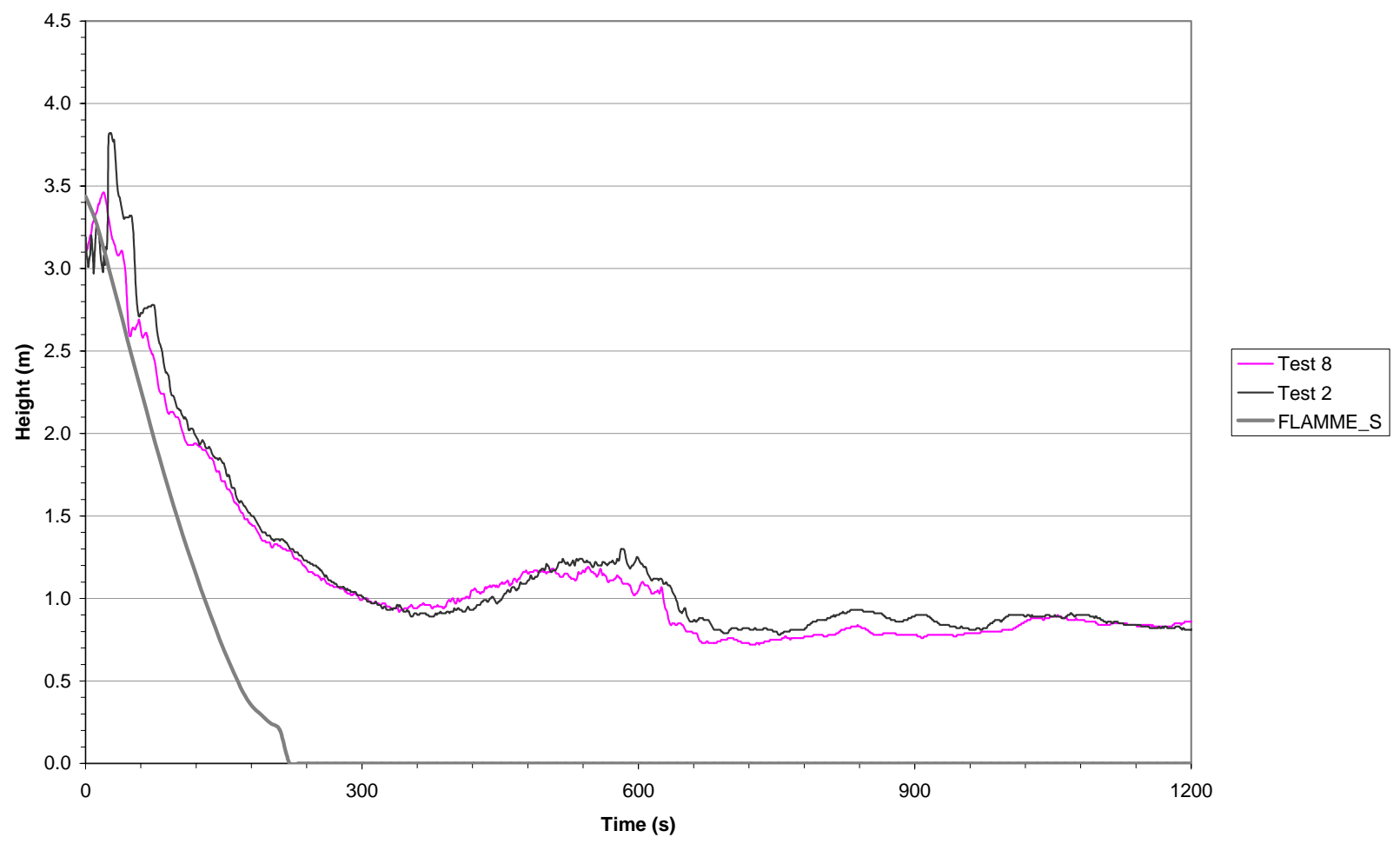

Figure 3-5: Layer height

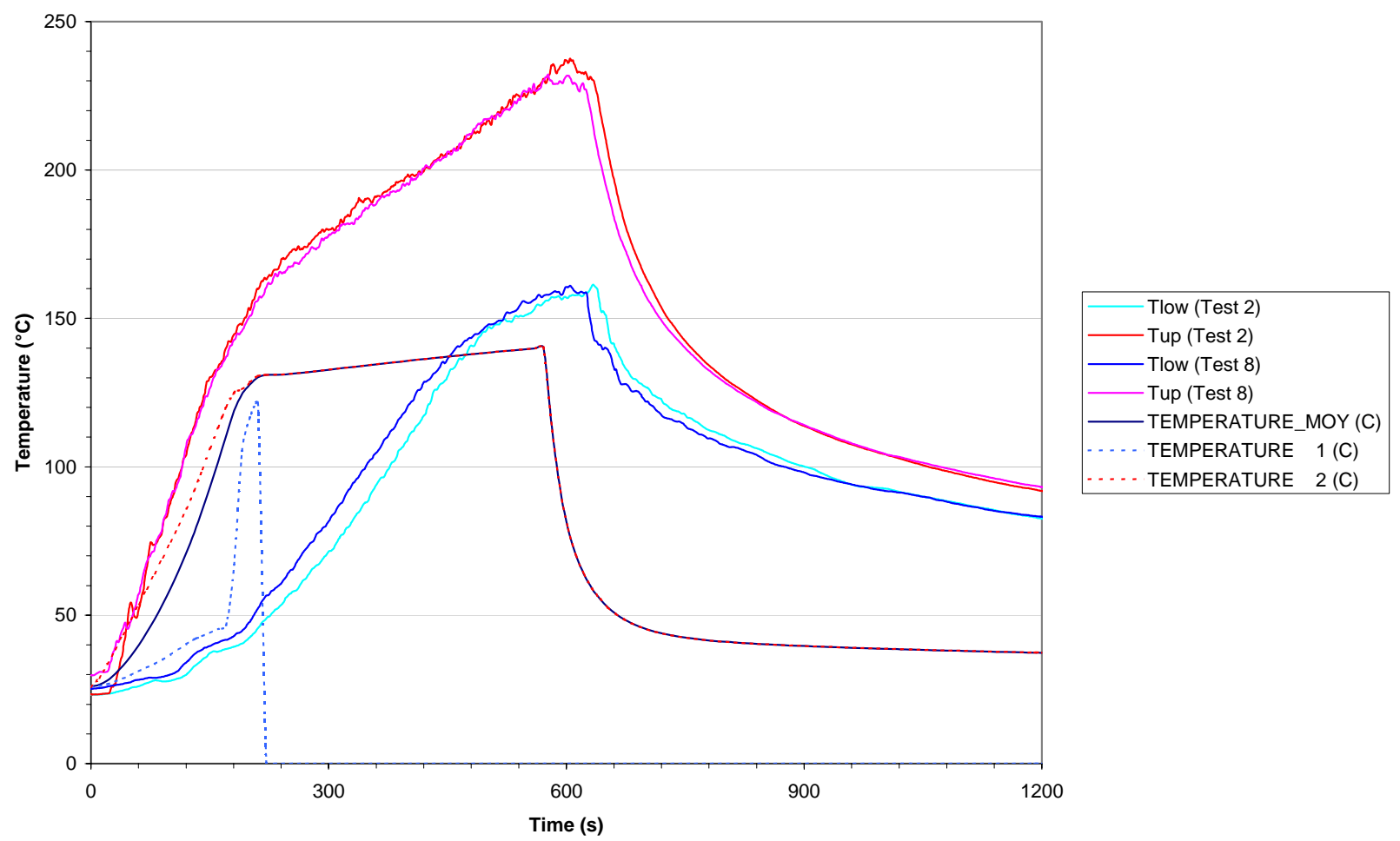

Figure 3-6: Gas temperature in the compartment 


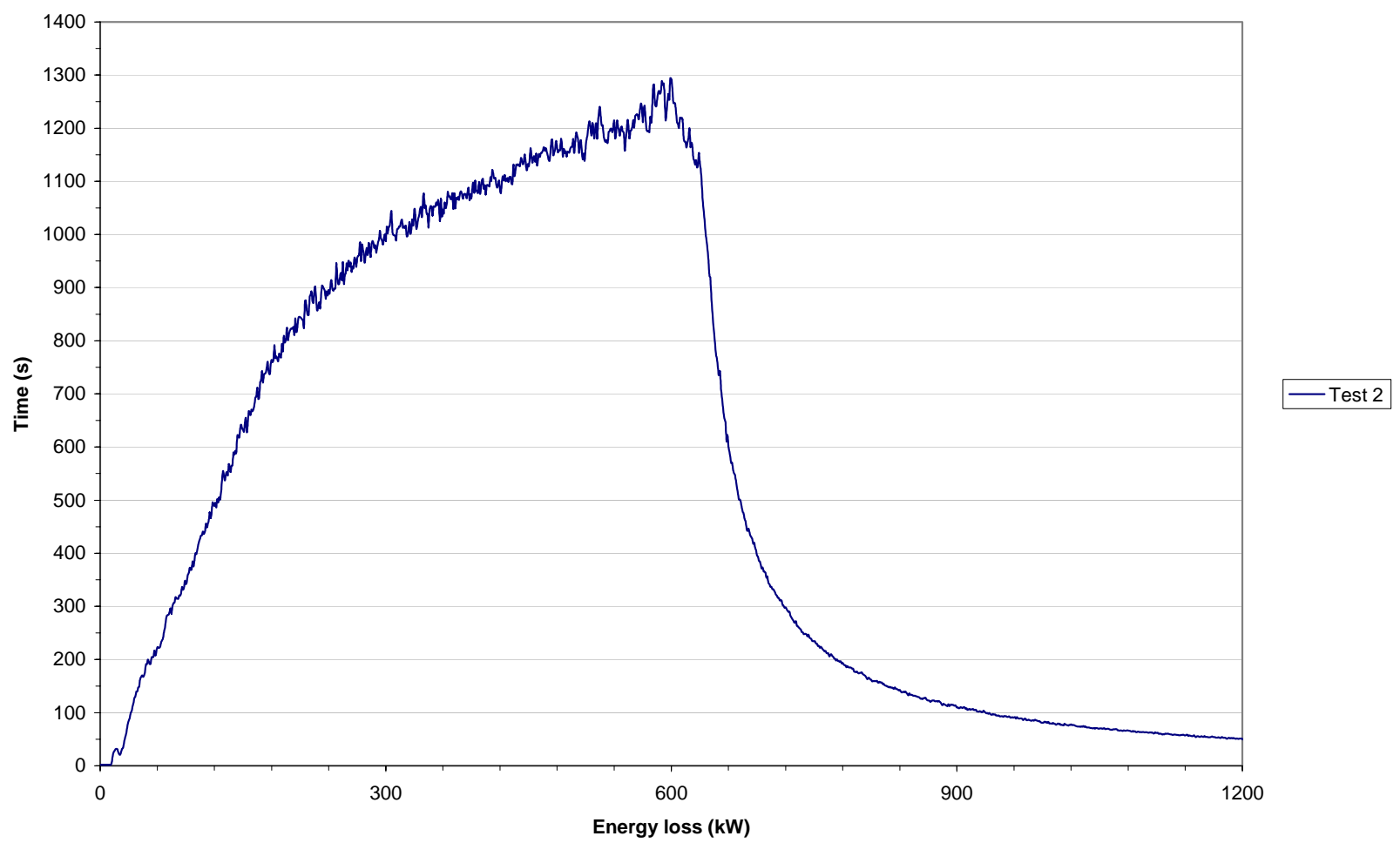

Figure 3-7: Total energy loss by all surfaces of the compartment
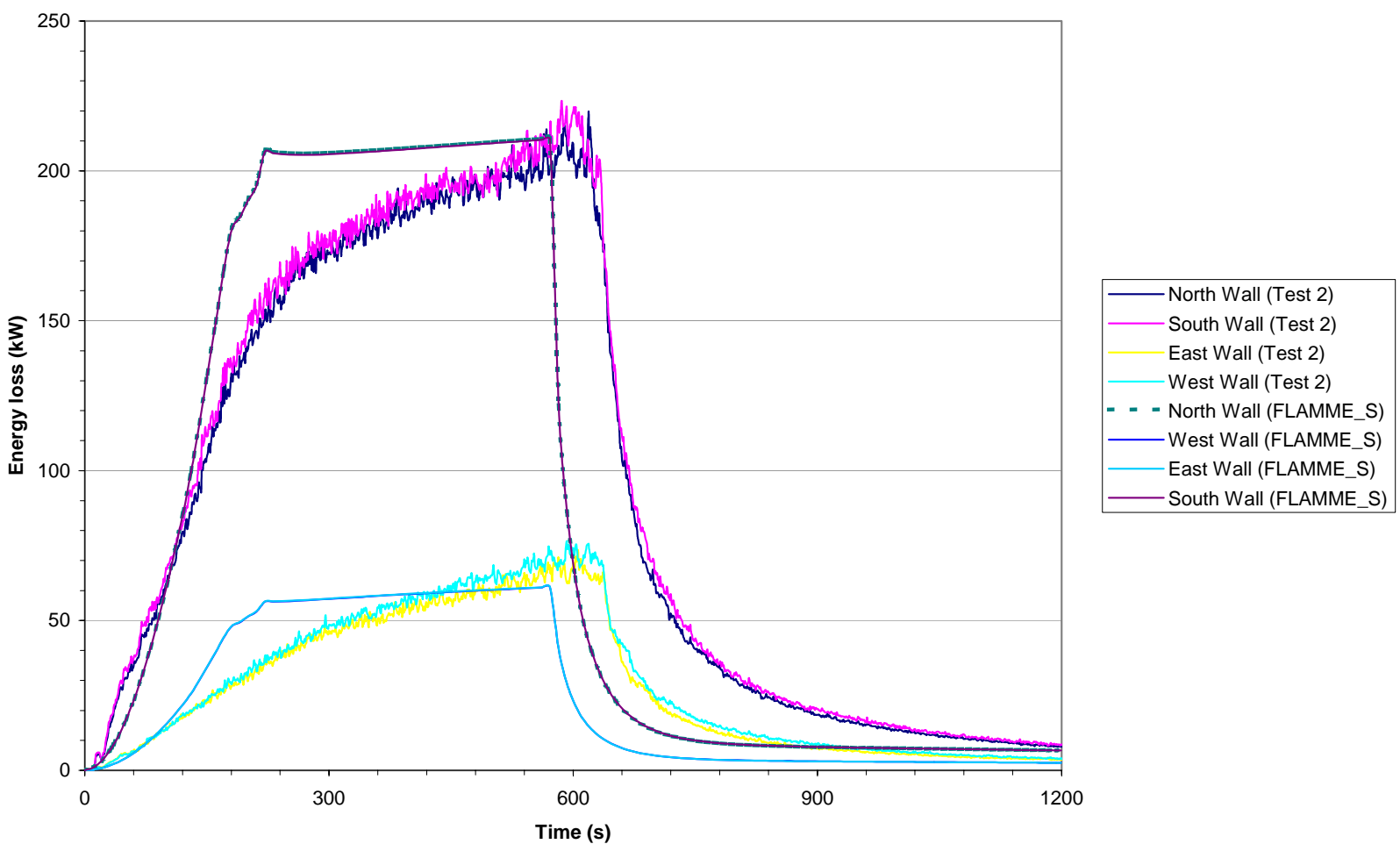

Figure 3-8: Energy loss to the side walls 


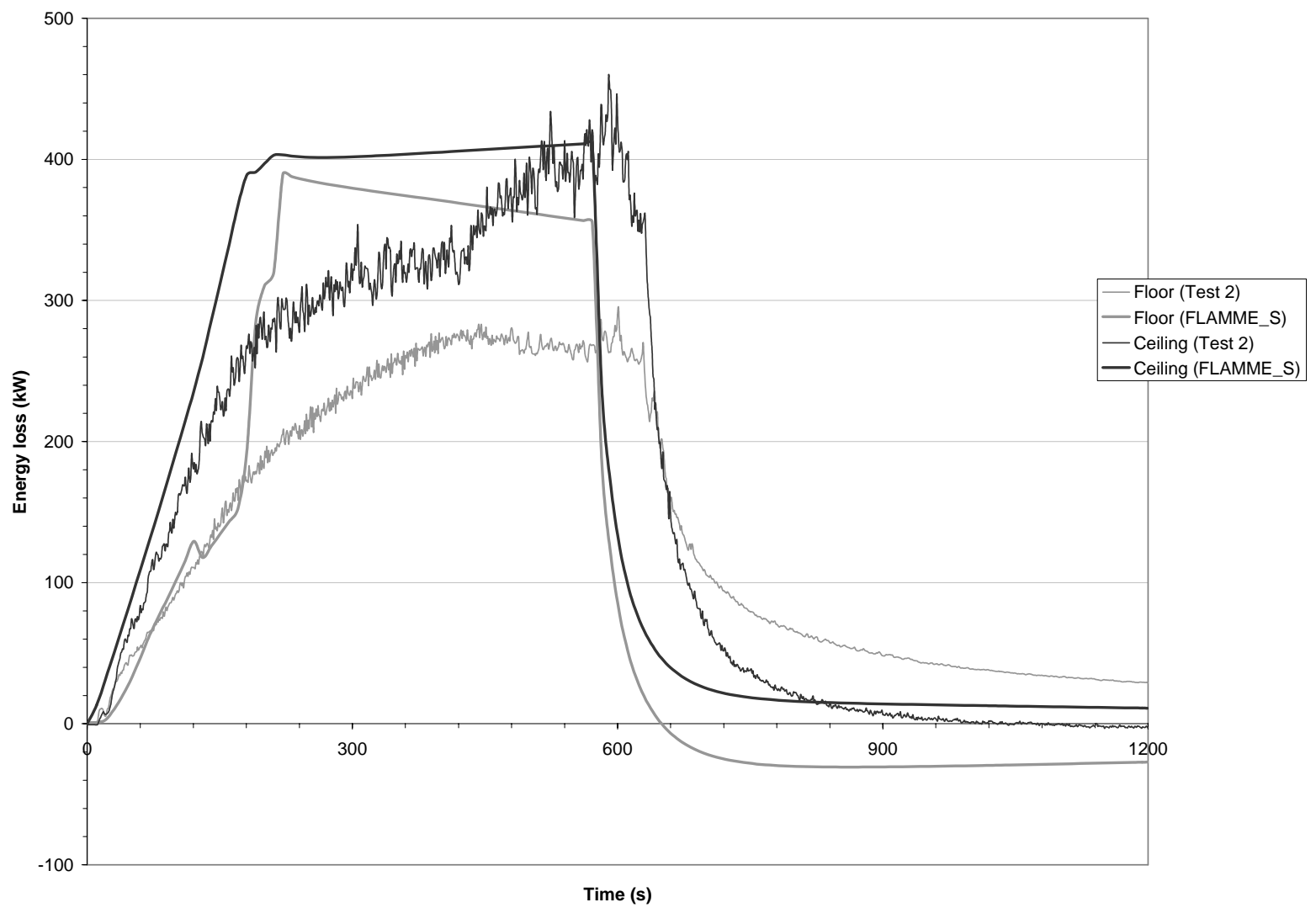

Figure 3-9: Energy loss to the ceiling and to the floor 


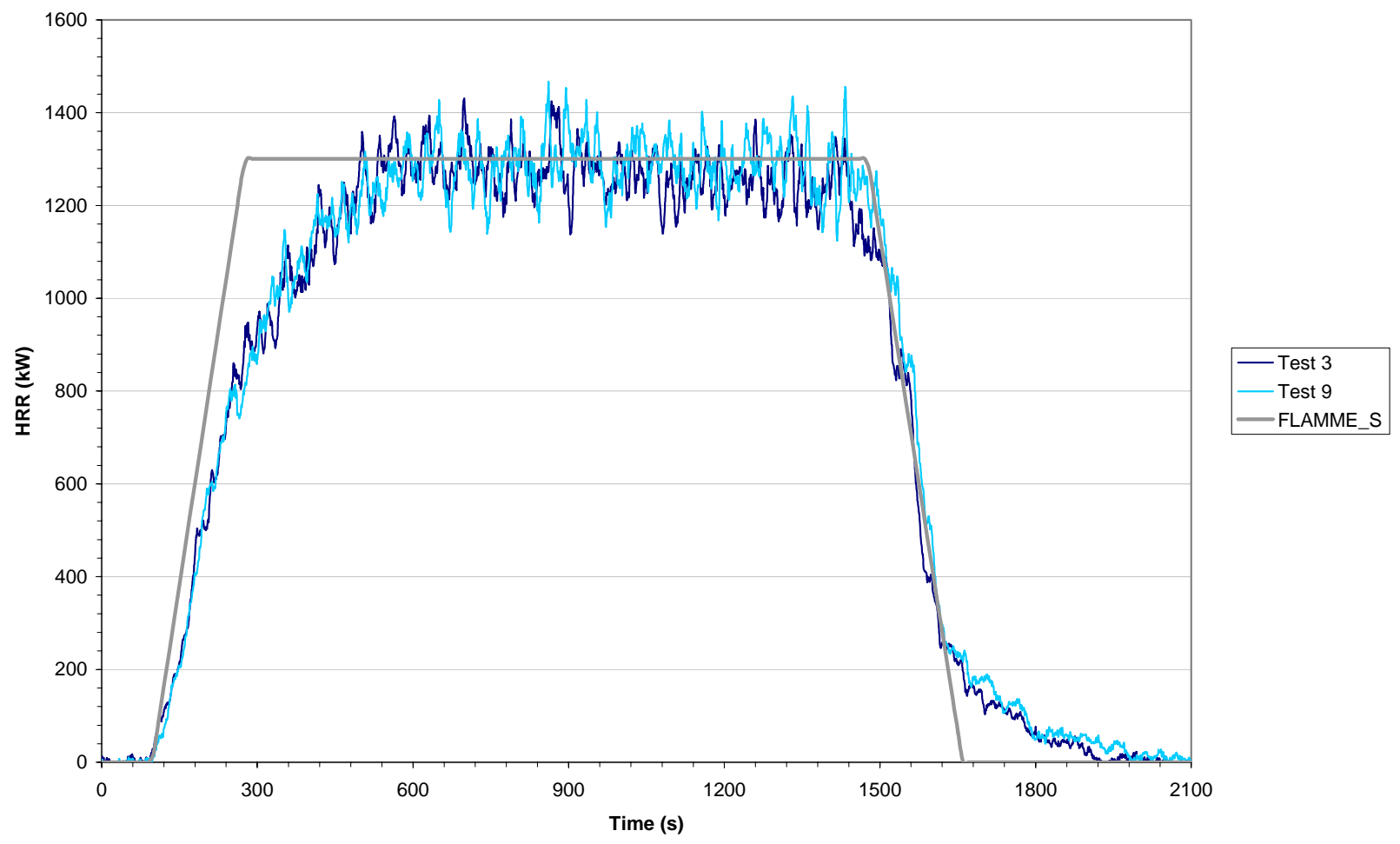

Figure 3-10: Heat release rate

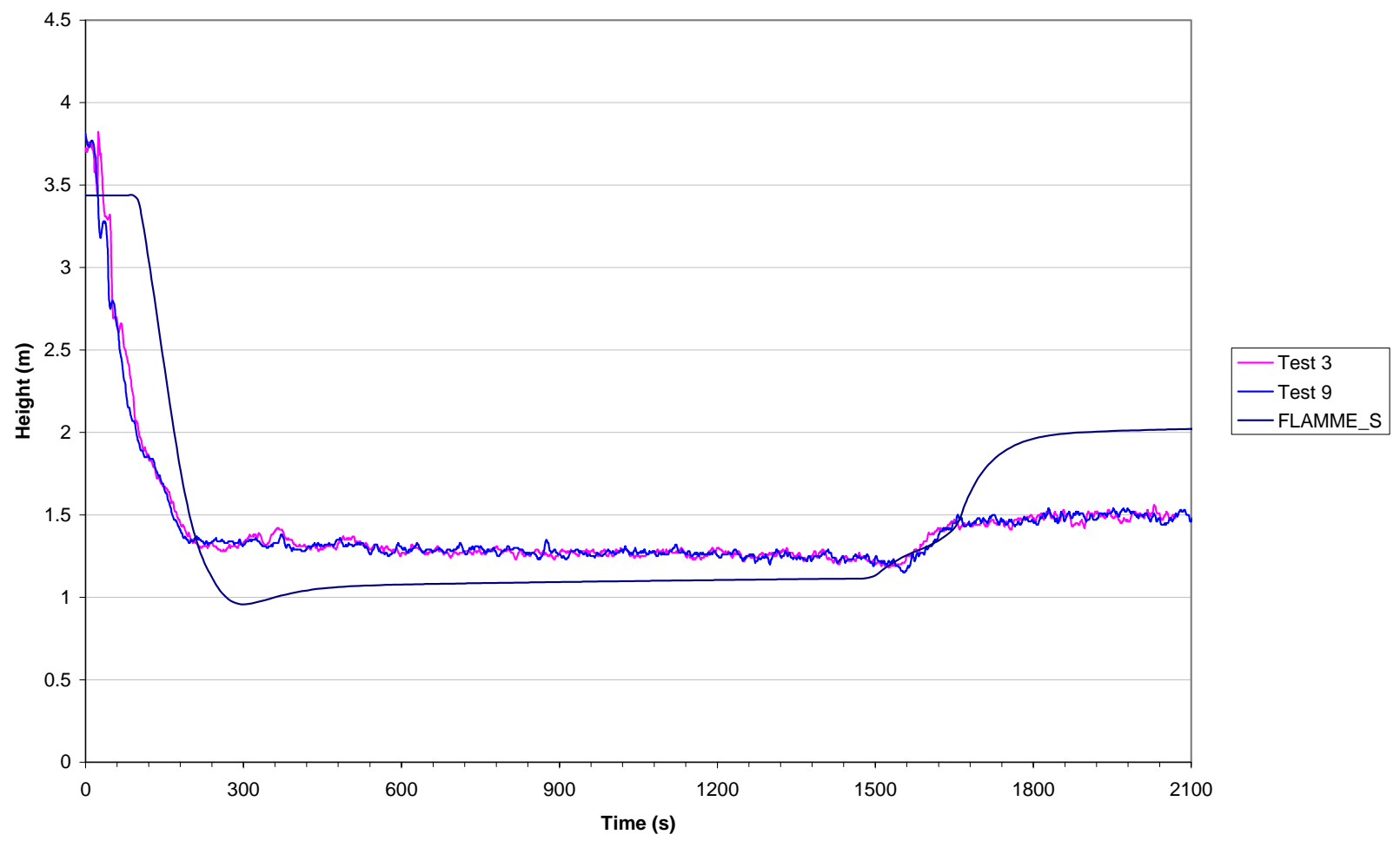

Figure 3-11: Layer height 


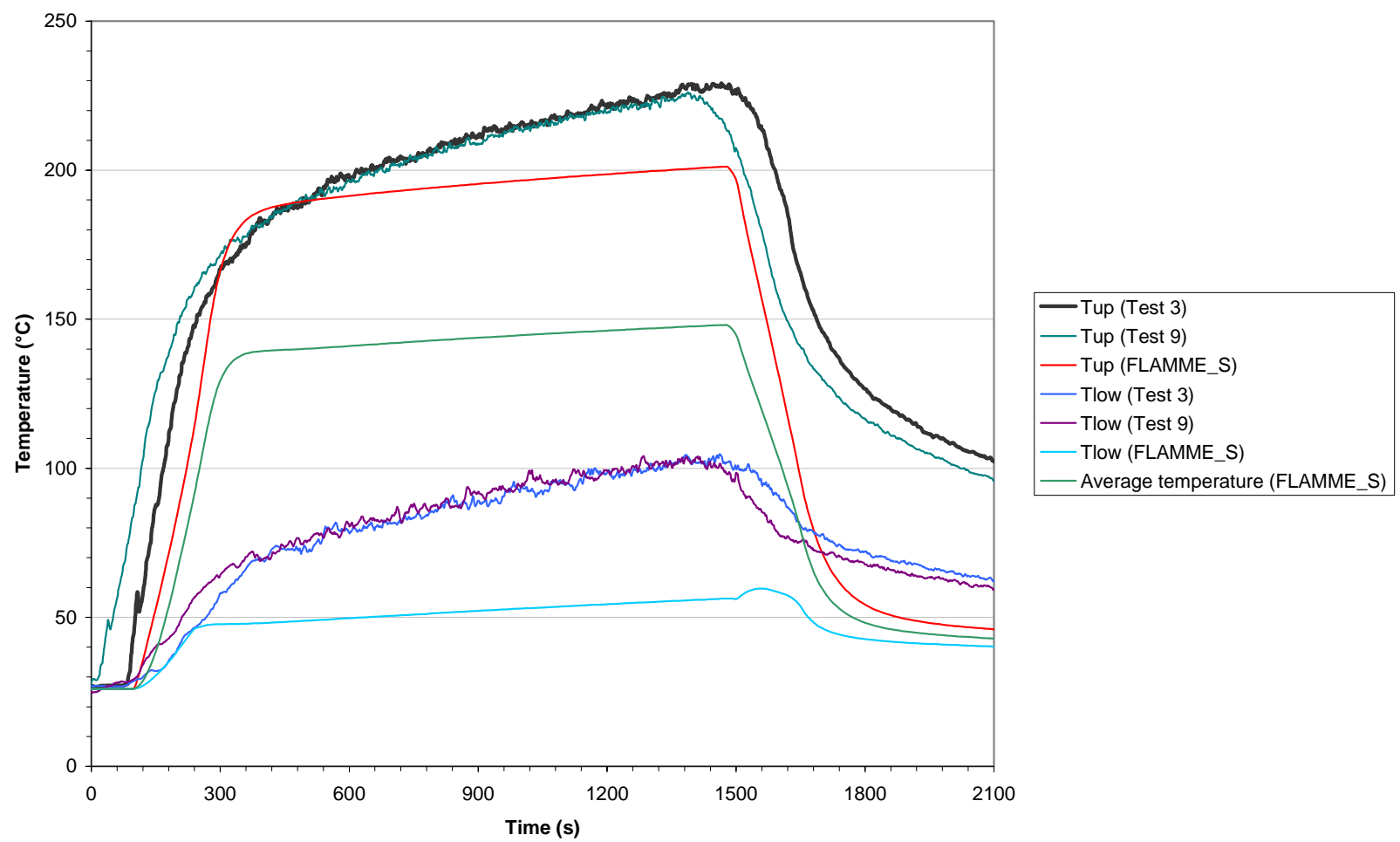

Figure 3-12: Gas temperature of the compartment

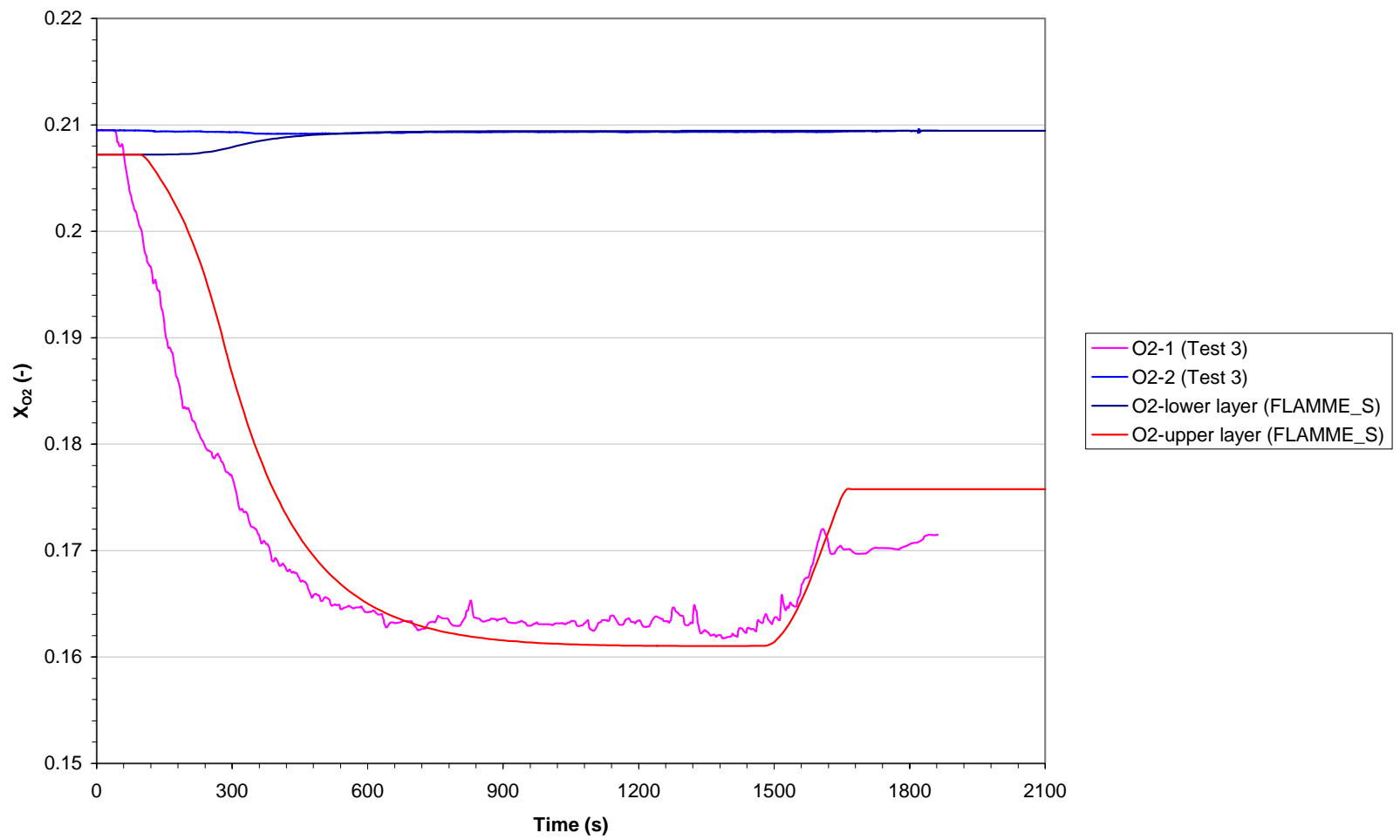

Figure 3-13: Molar fraction of oxygen 


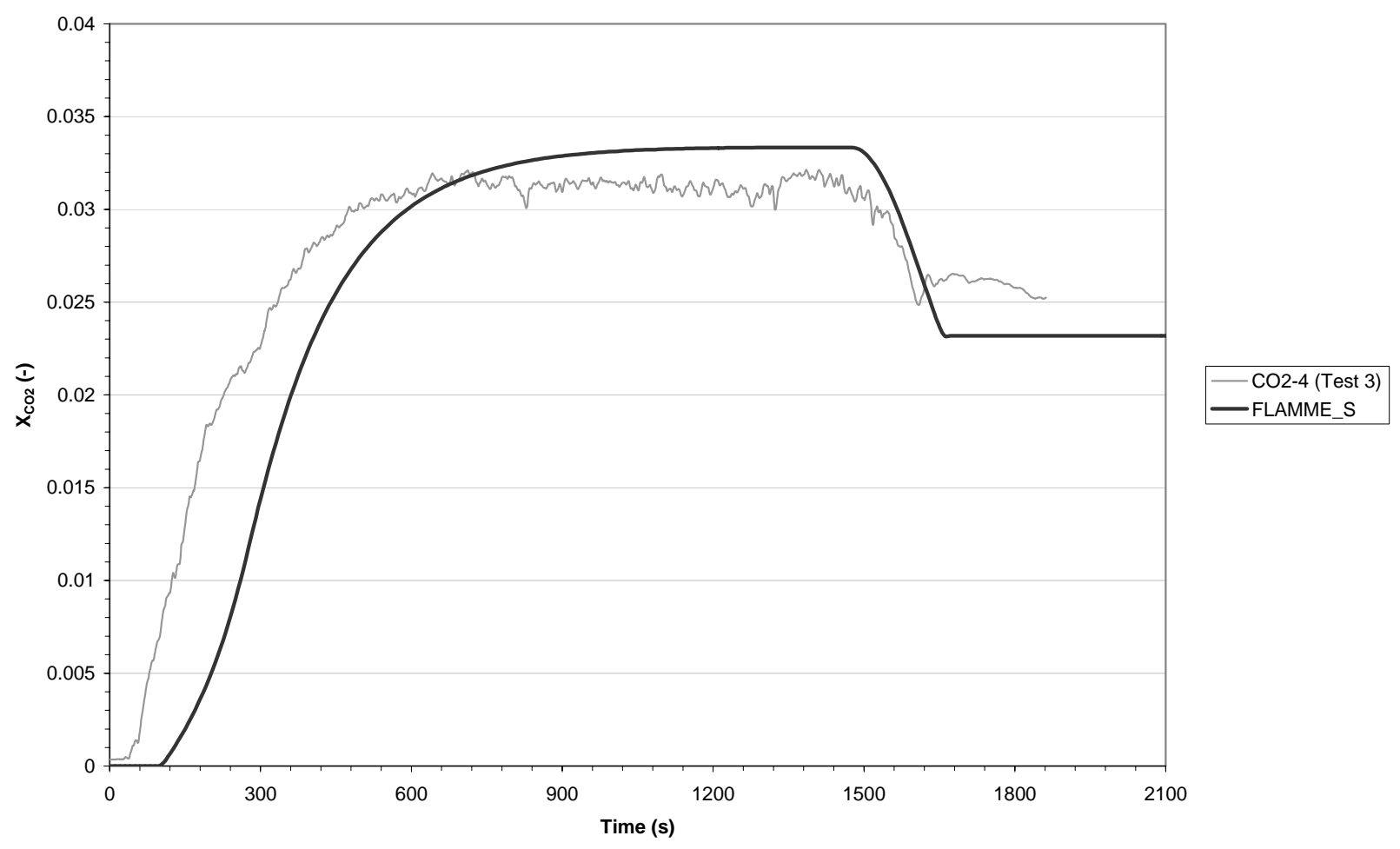

Figure 3-14: Molar fraction of carbon dioxide

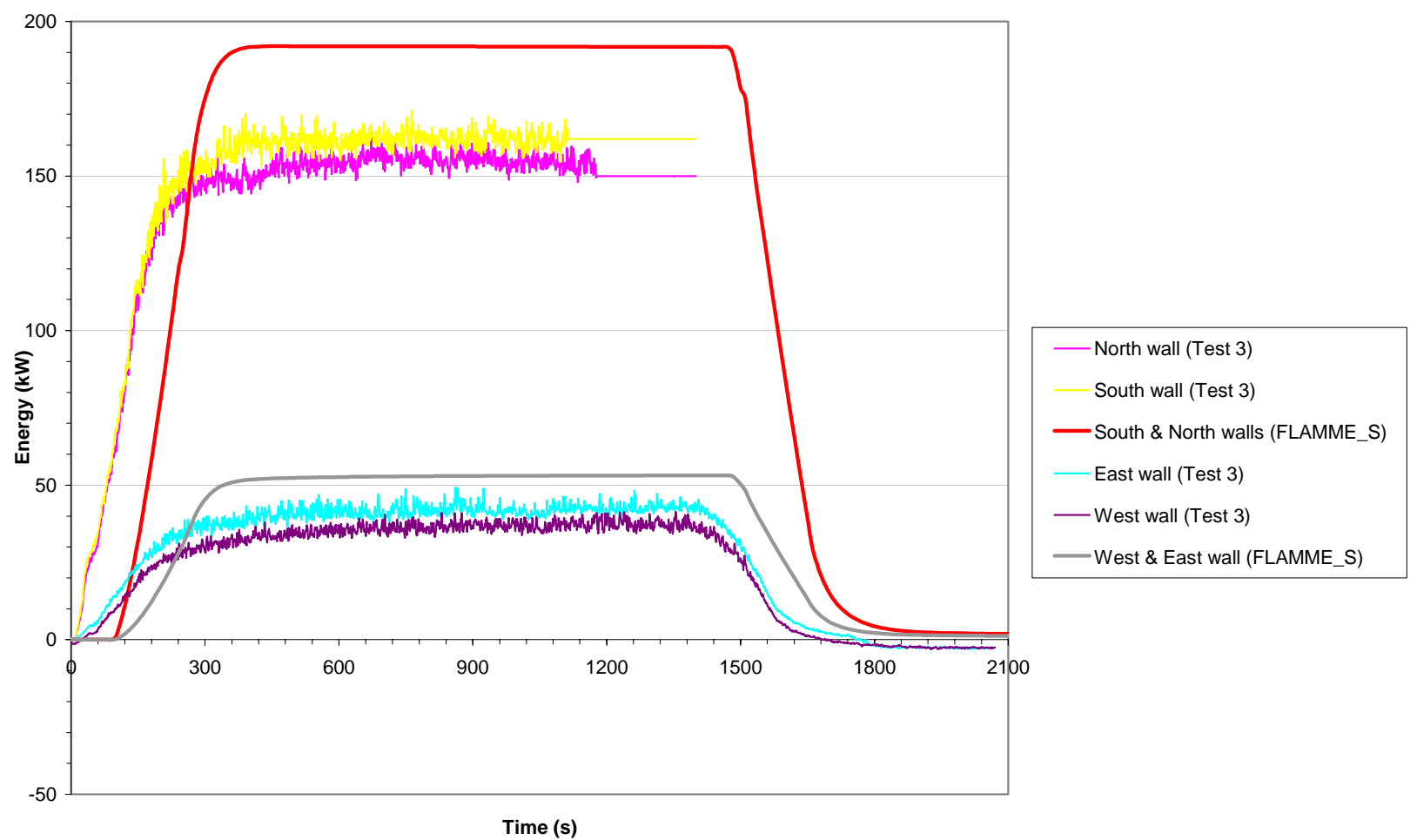

Figure 3-15: Energy loss to the side walls 


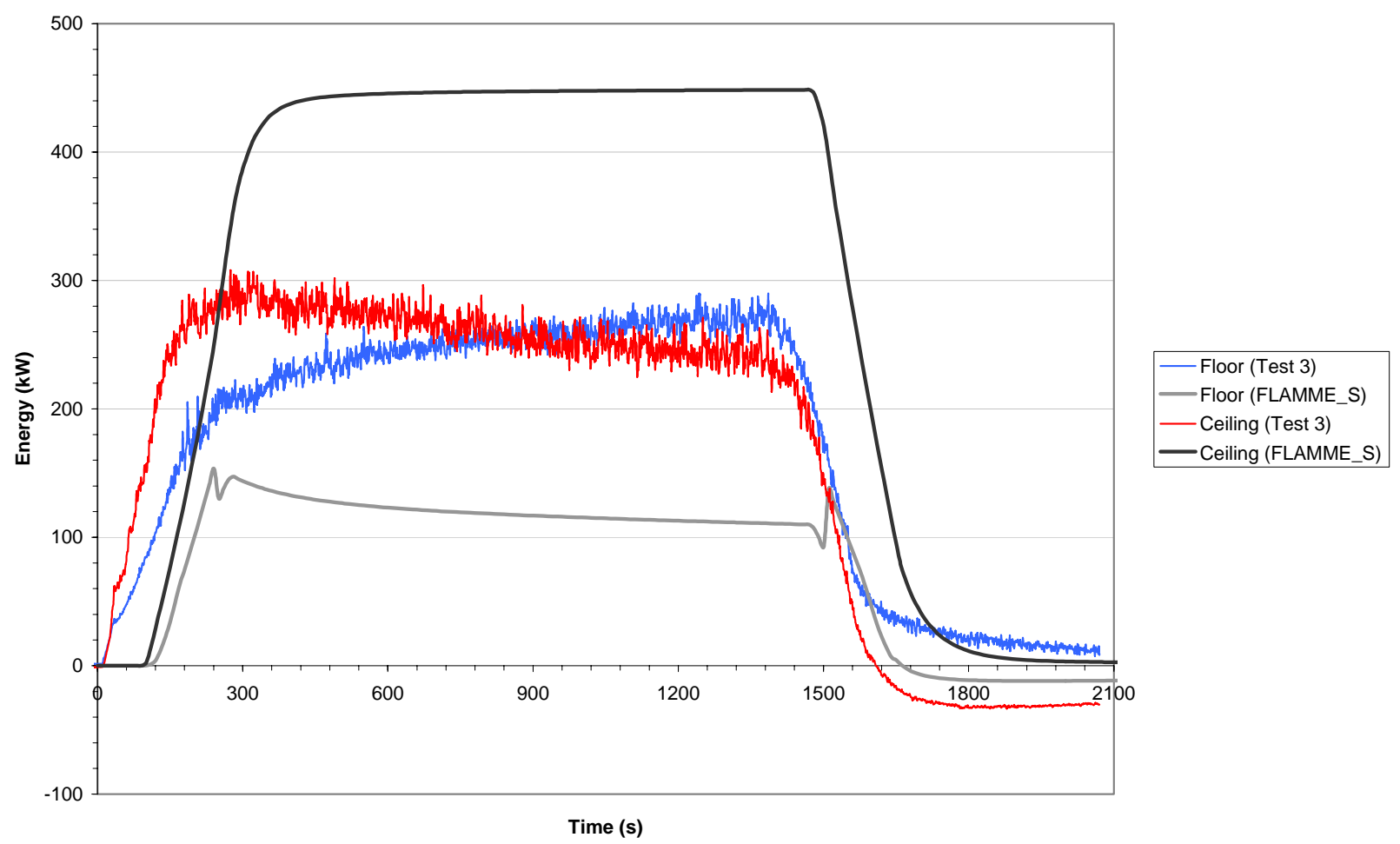

Figure 3-16: Energy loss to the ceiling and to the floor

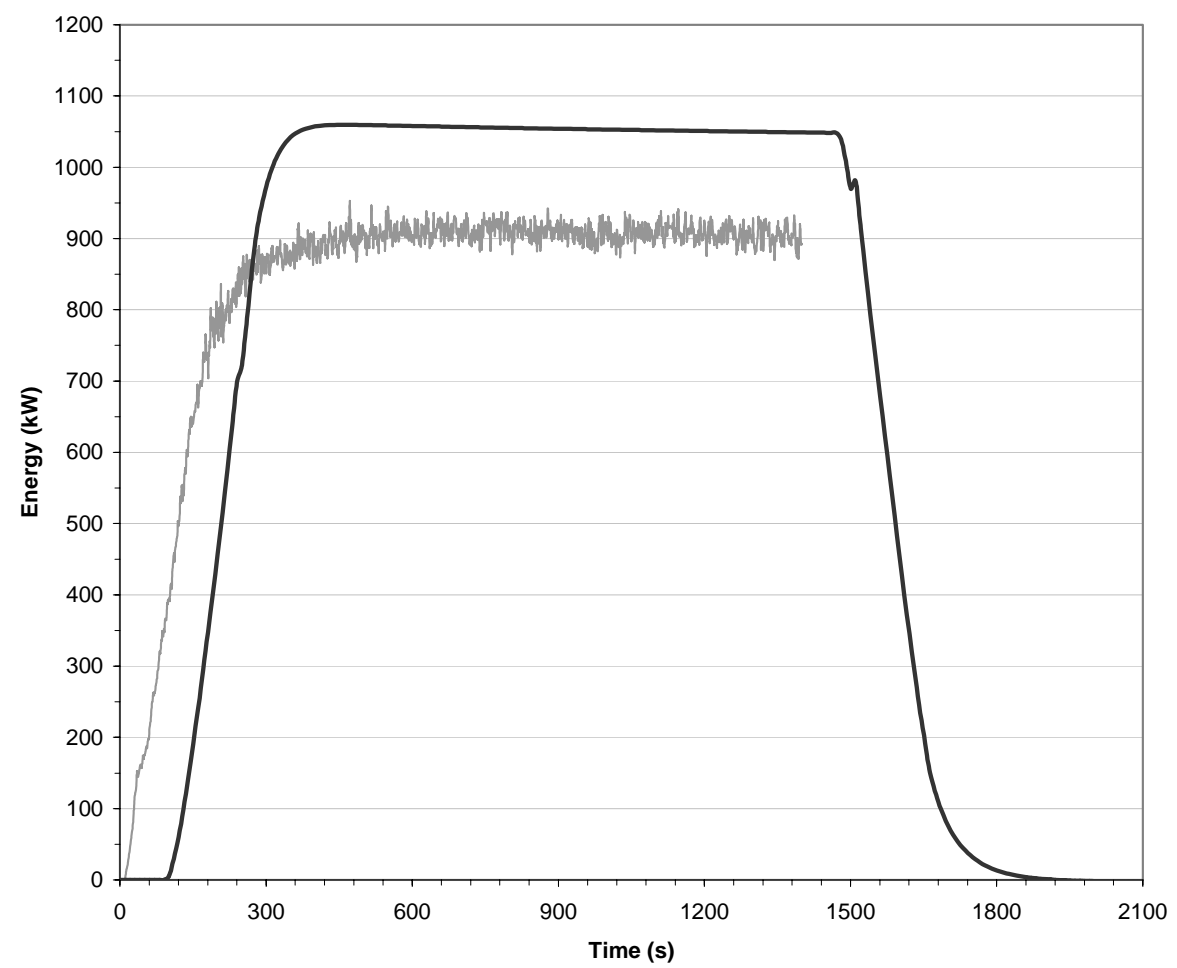

Figure 3-17: Total energy loss 


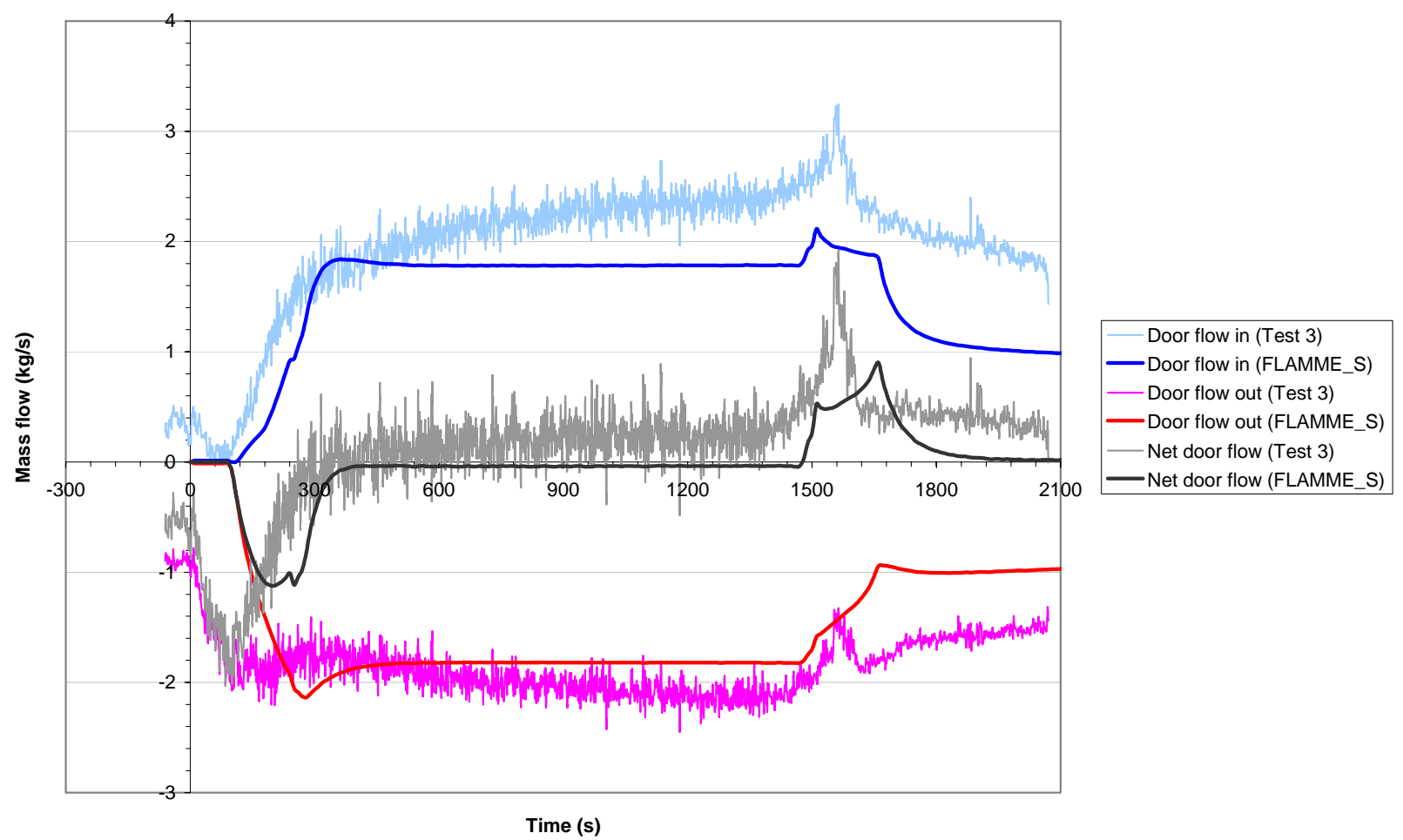

Figure 3-18: Mass flow through the door 


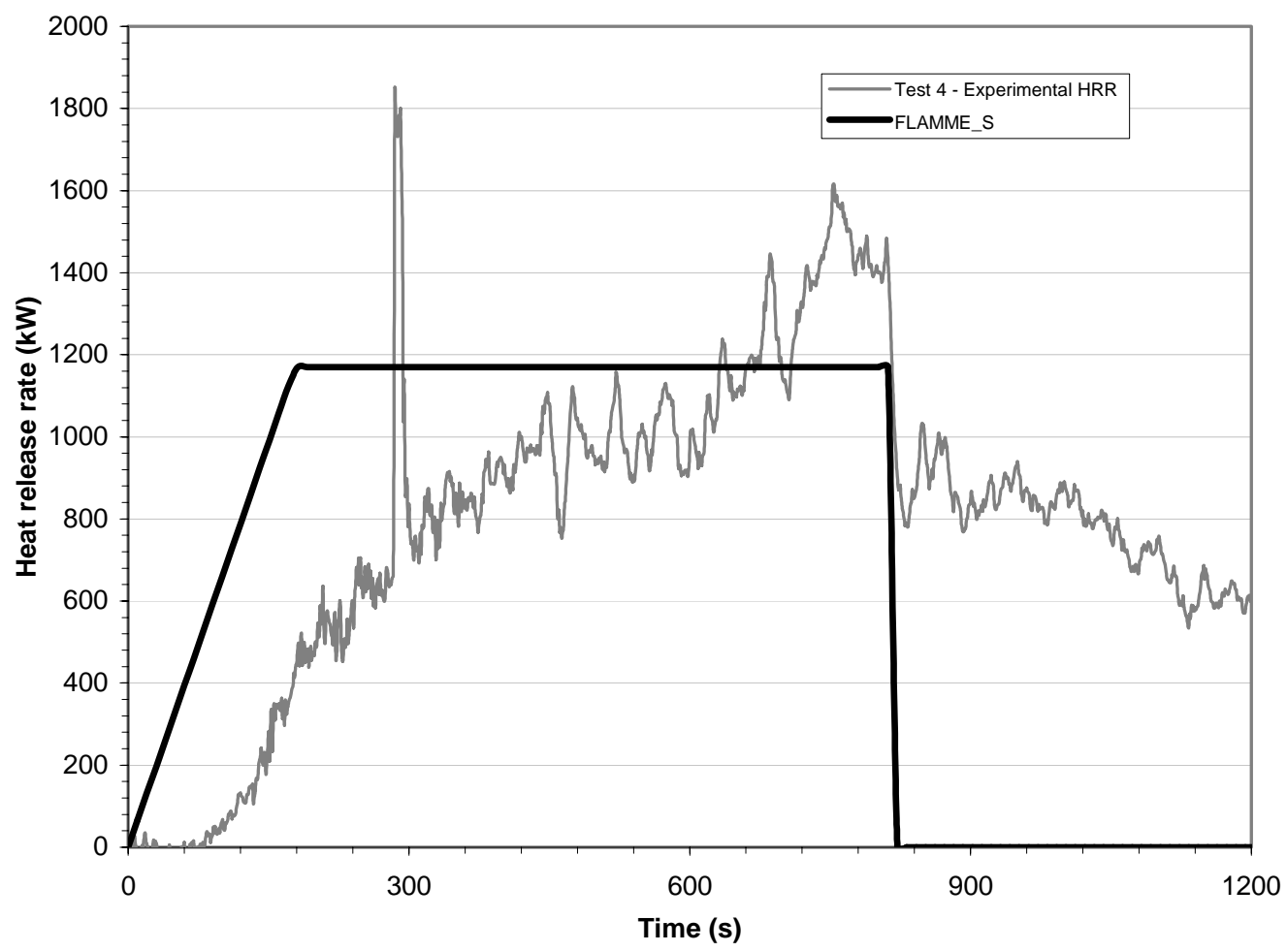

Figure 3-19: Heat release rate of the third configuration

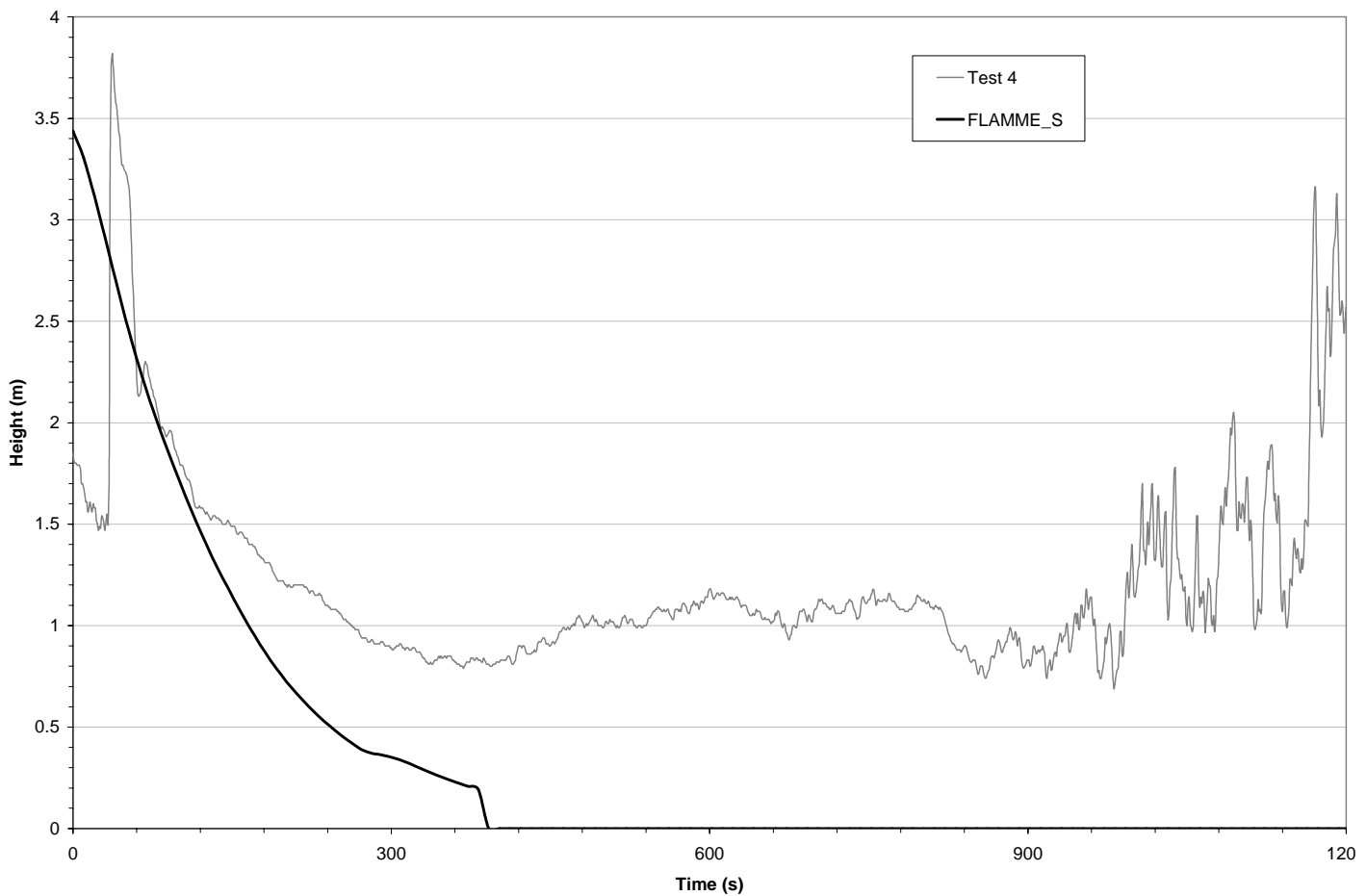

Figure 3-20: Layer height 


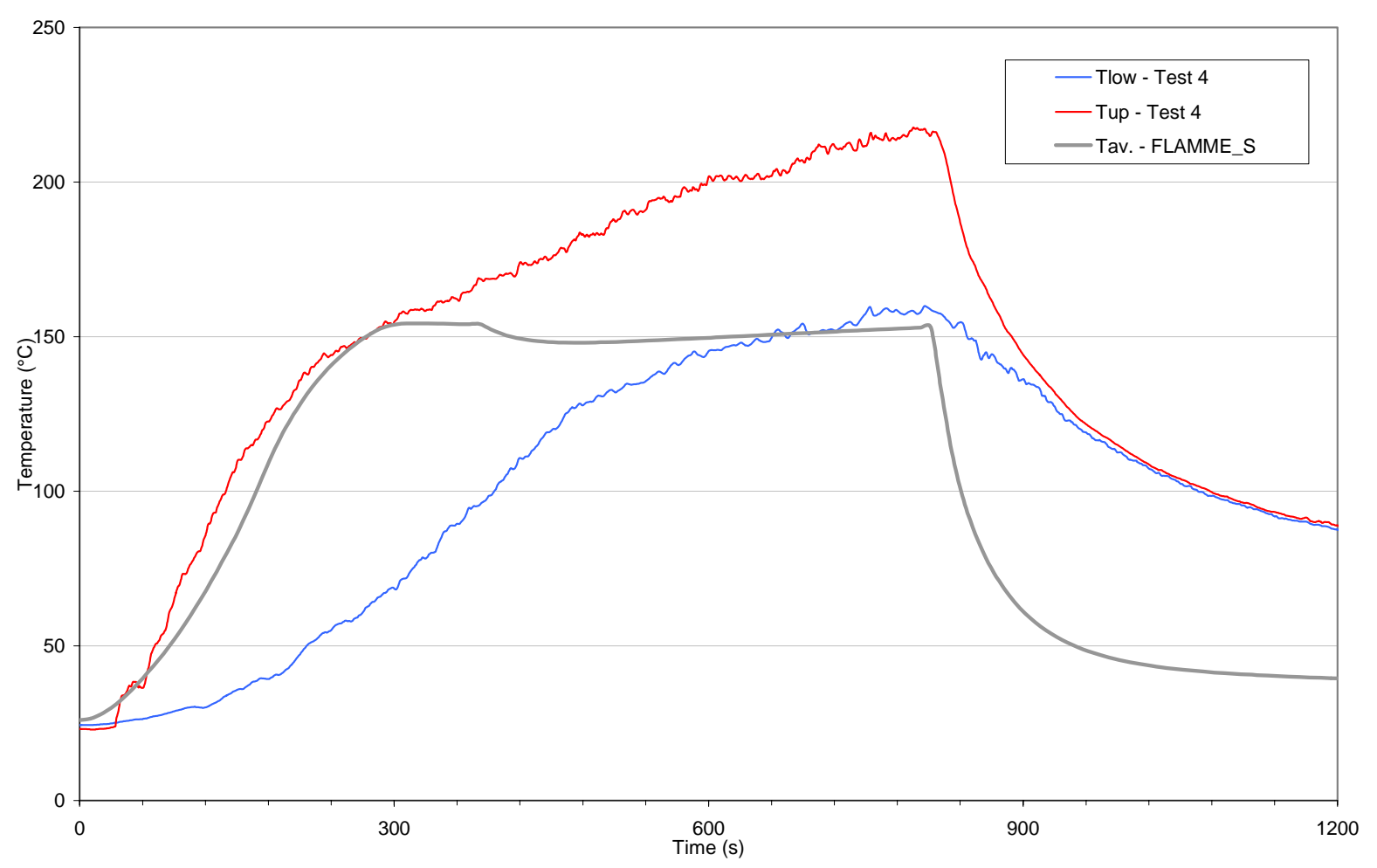

Figure 3-21: Gas temperature

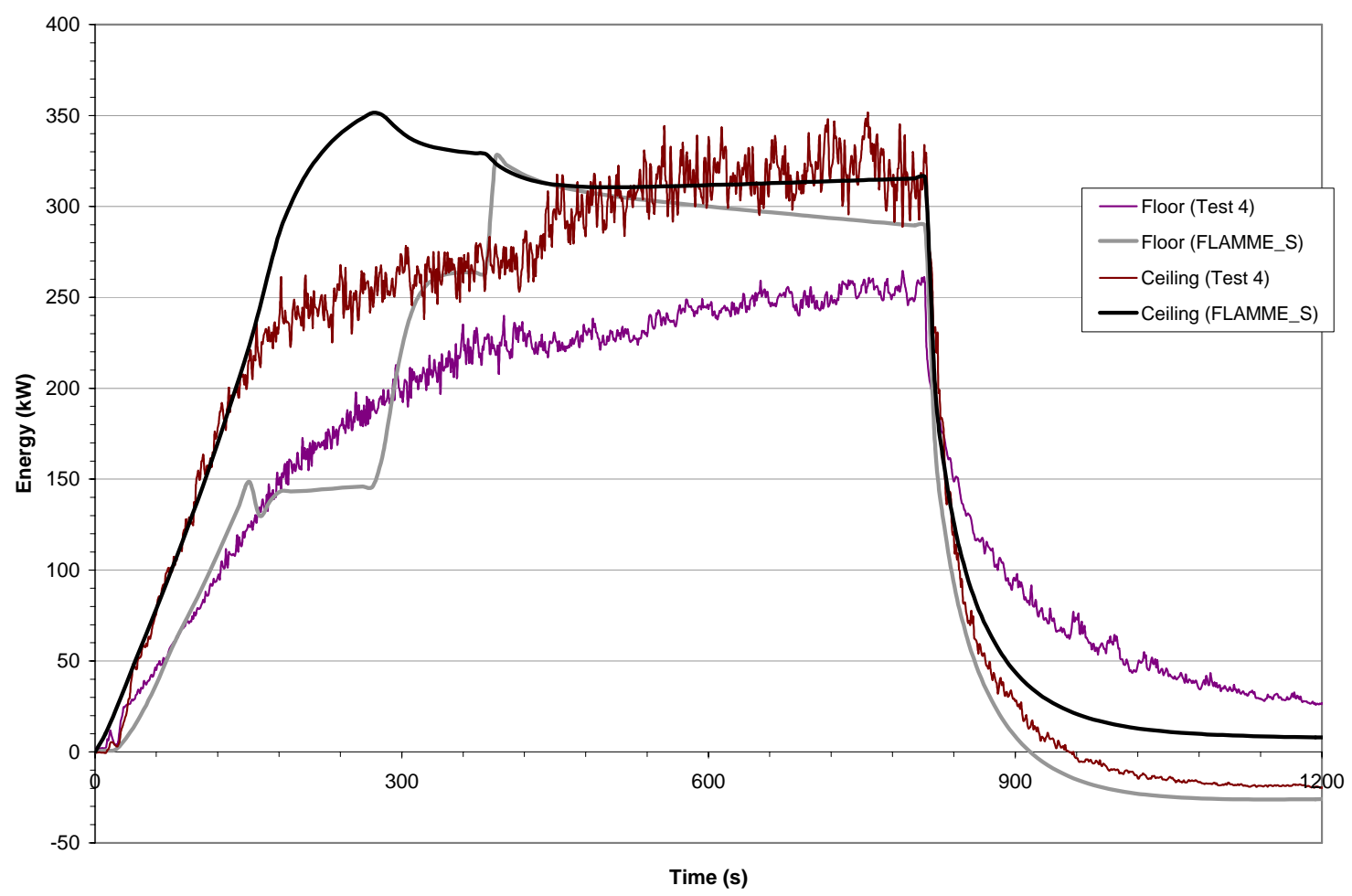

Figure 3-22: Energy loss to the floor and to the ceiling 


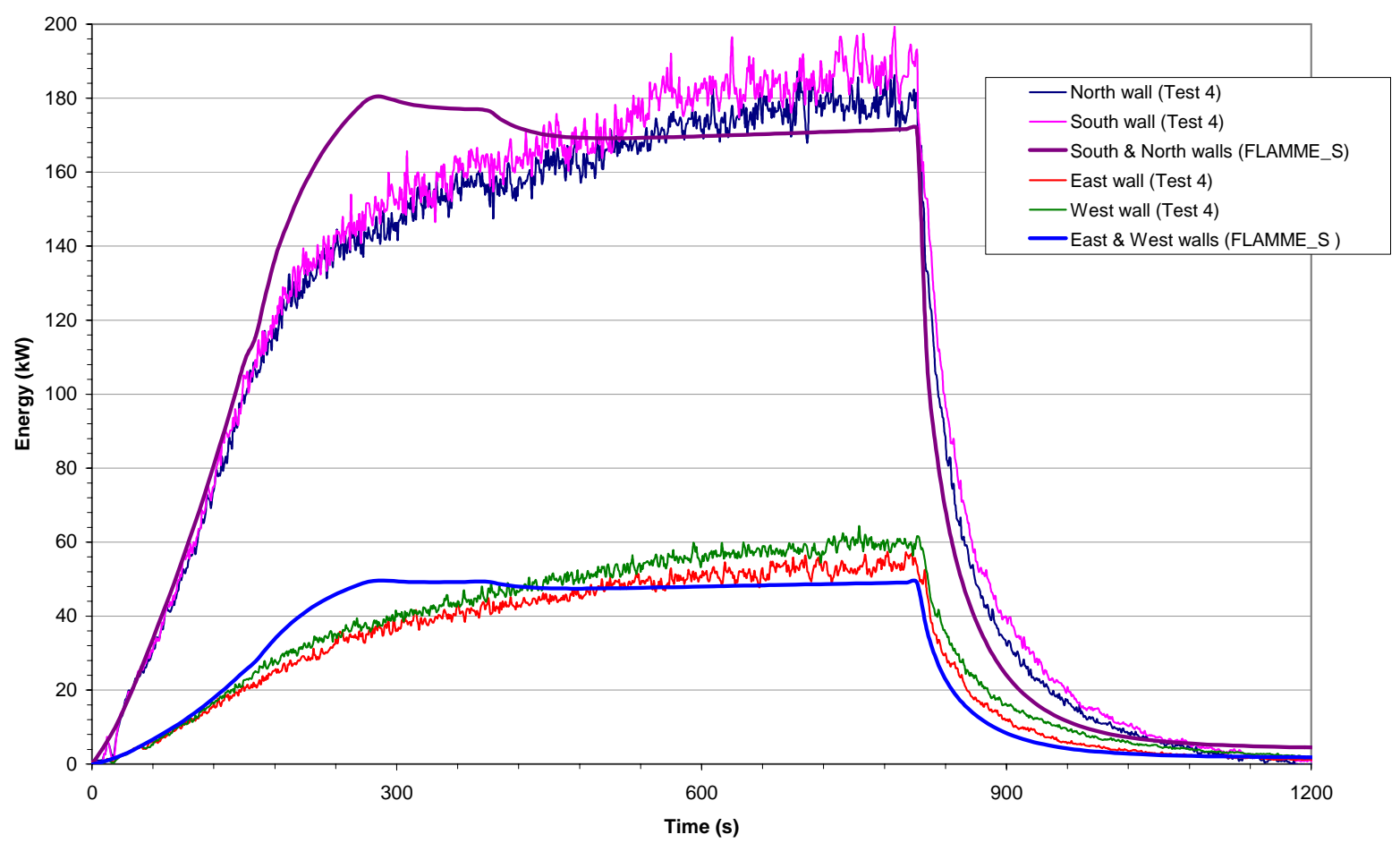

Figure 3-23: Energy loss to side walls

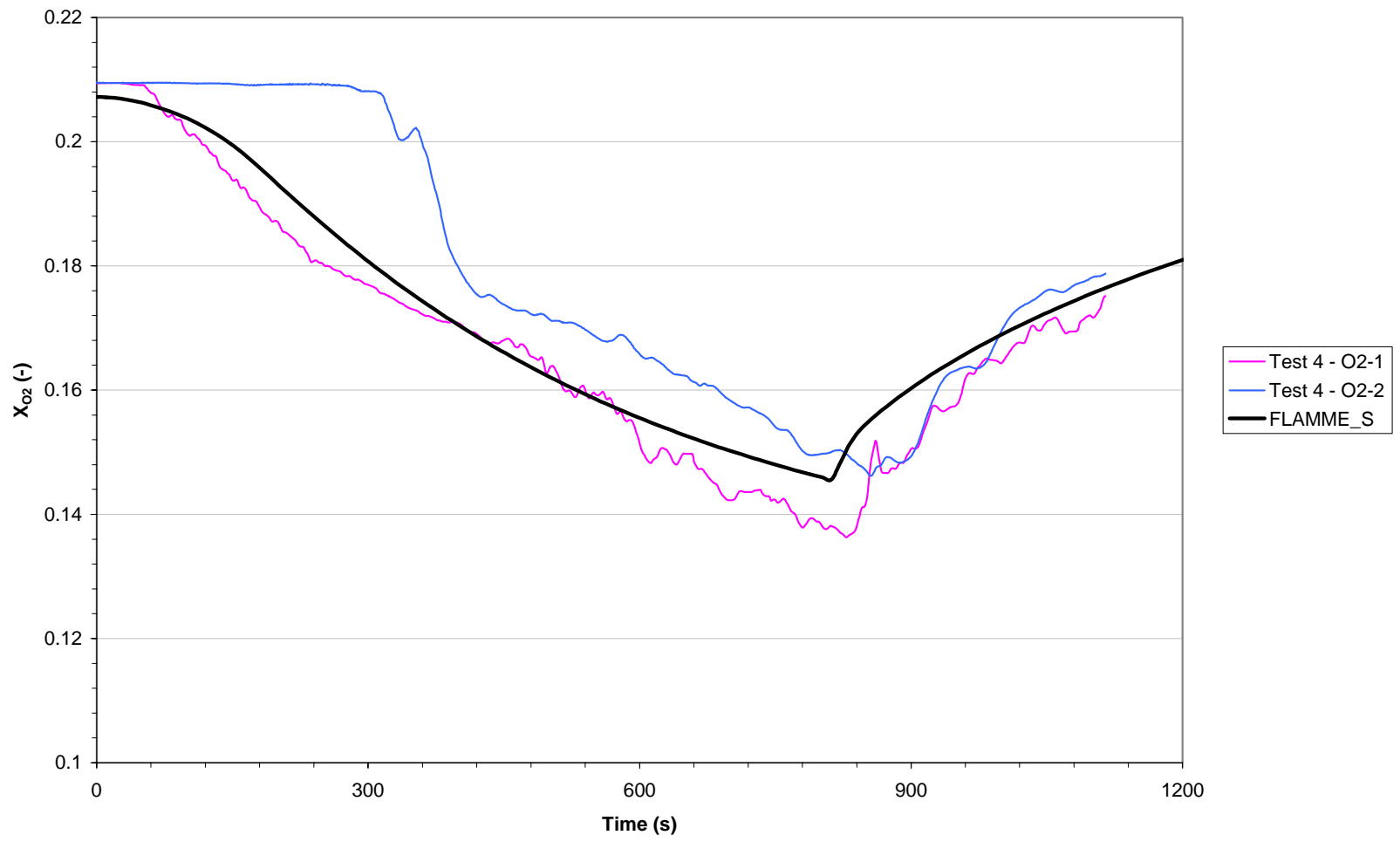

Figure 3-24: Molar fraction of oxygen 


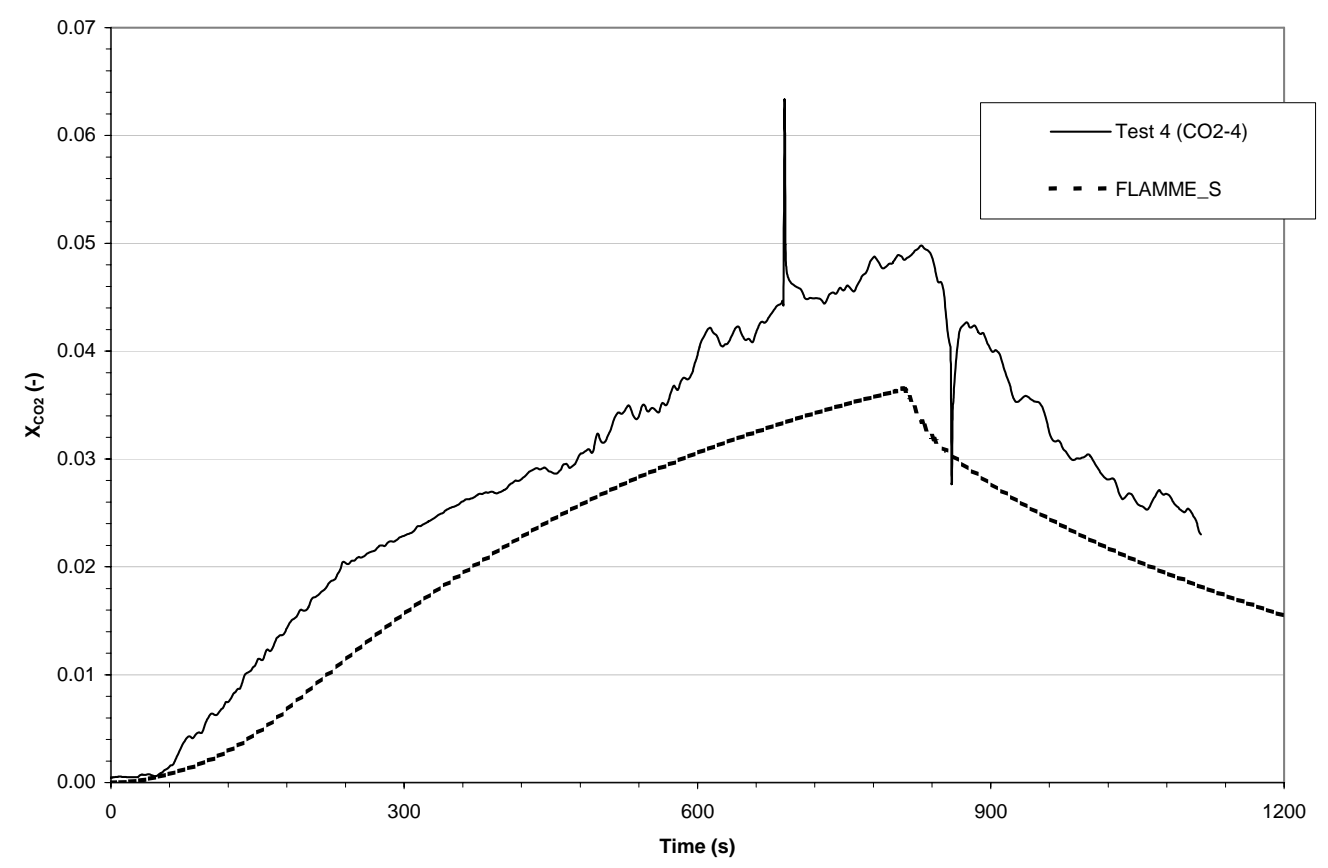

Figure 3-25: Fraction molar of carbon dioxide

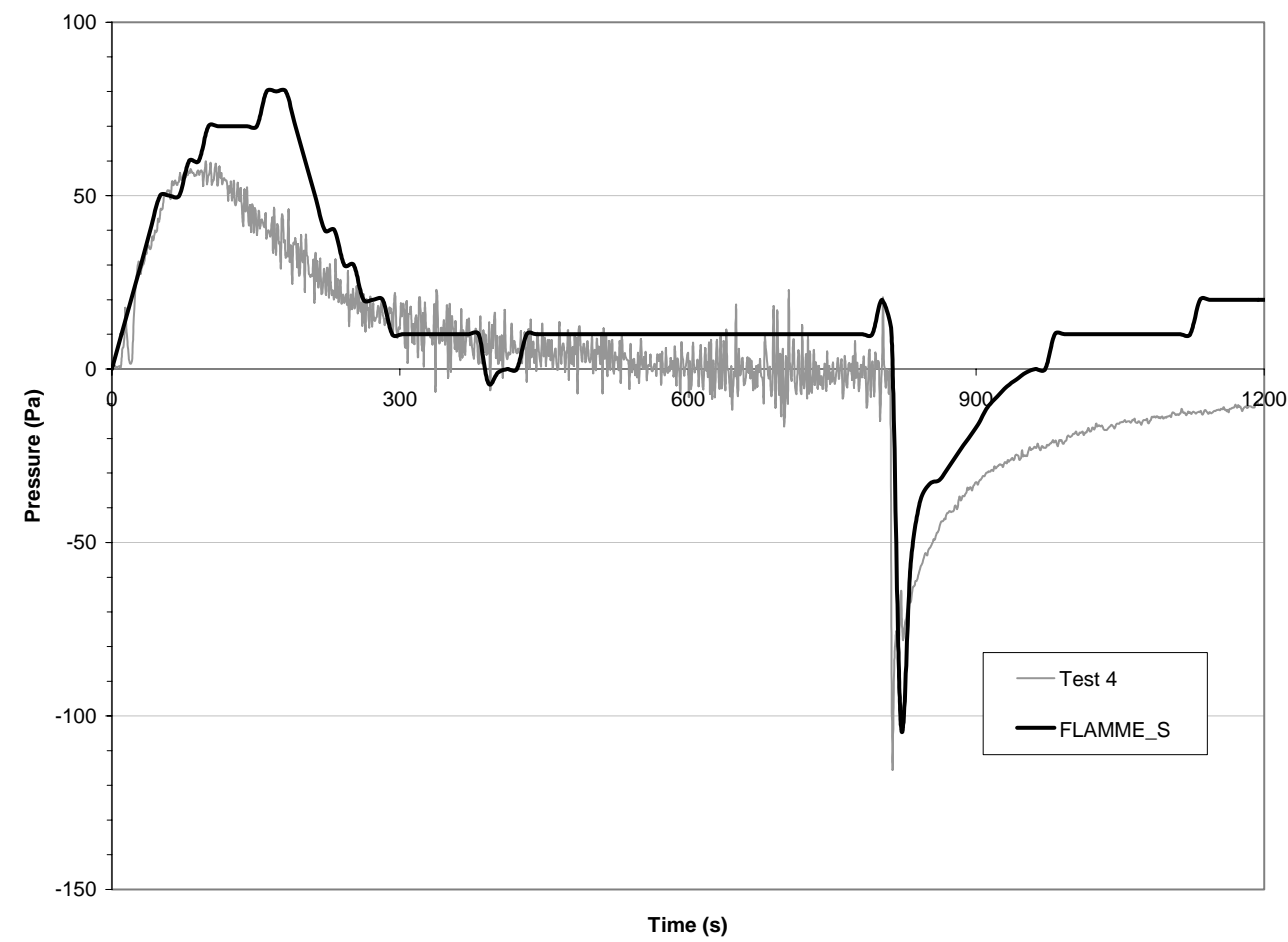

Figure 3-26: Pressure 


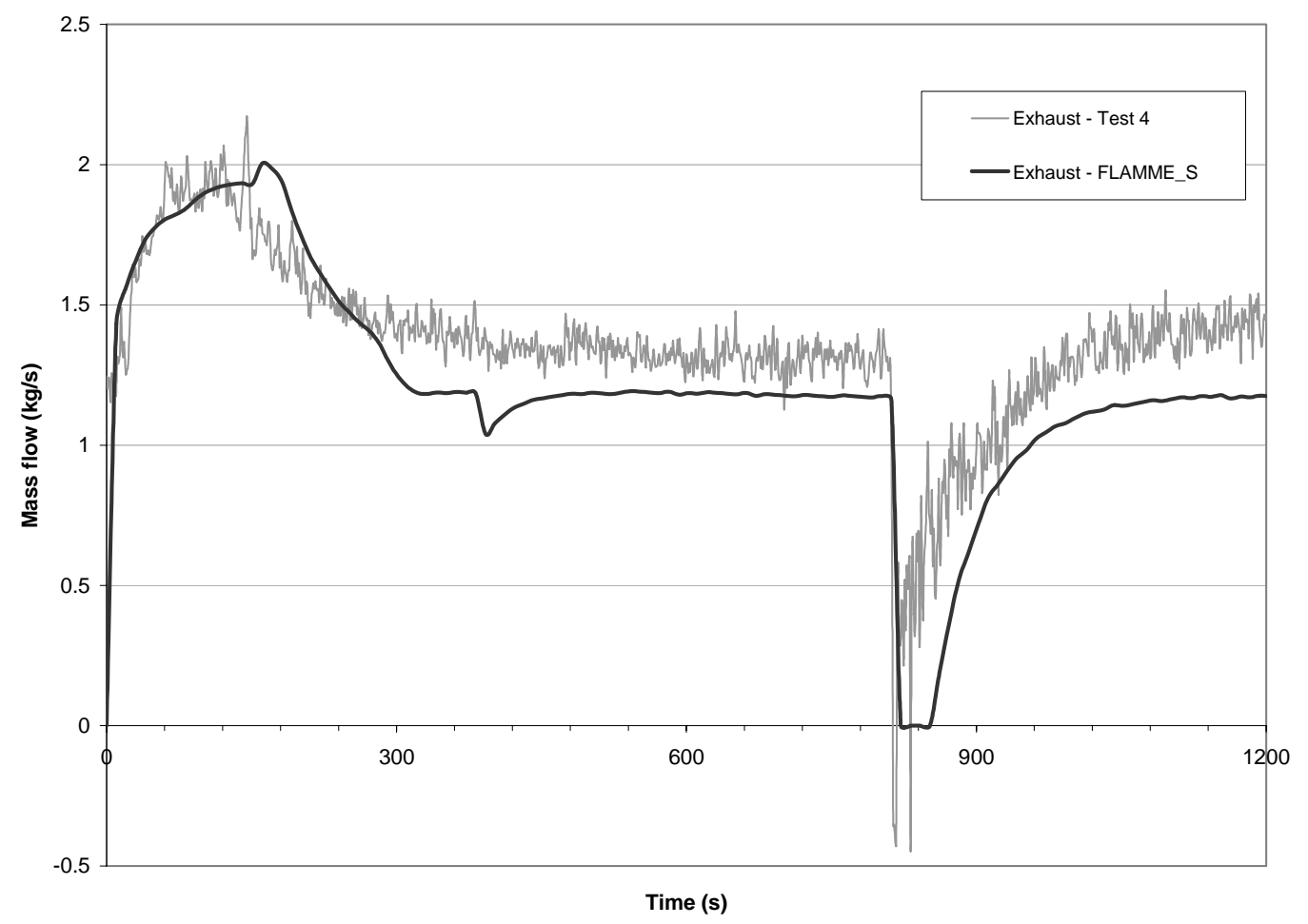

Figure 3-27: Mass flow through the exhaust vent

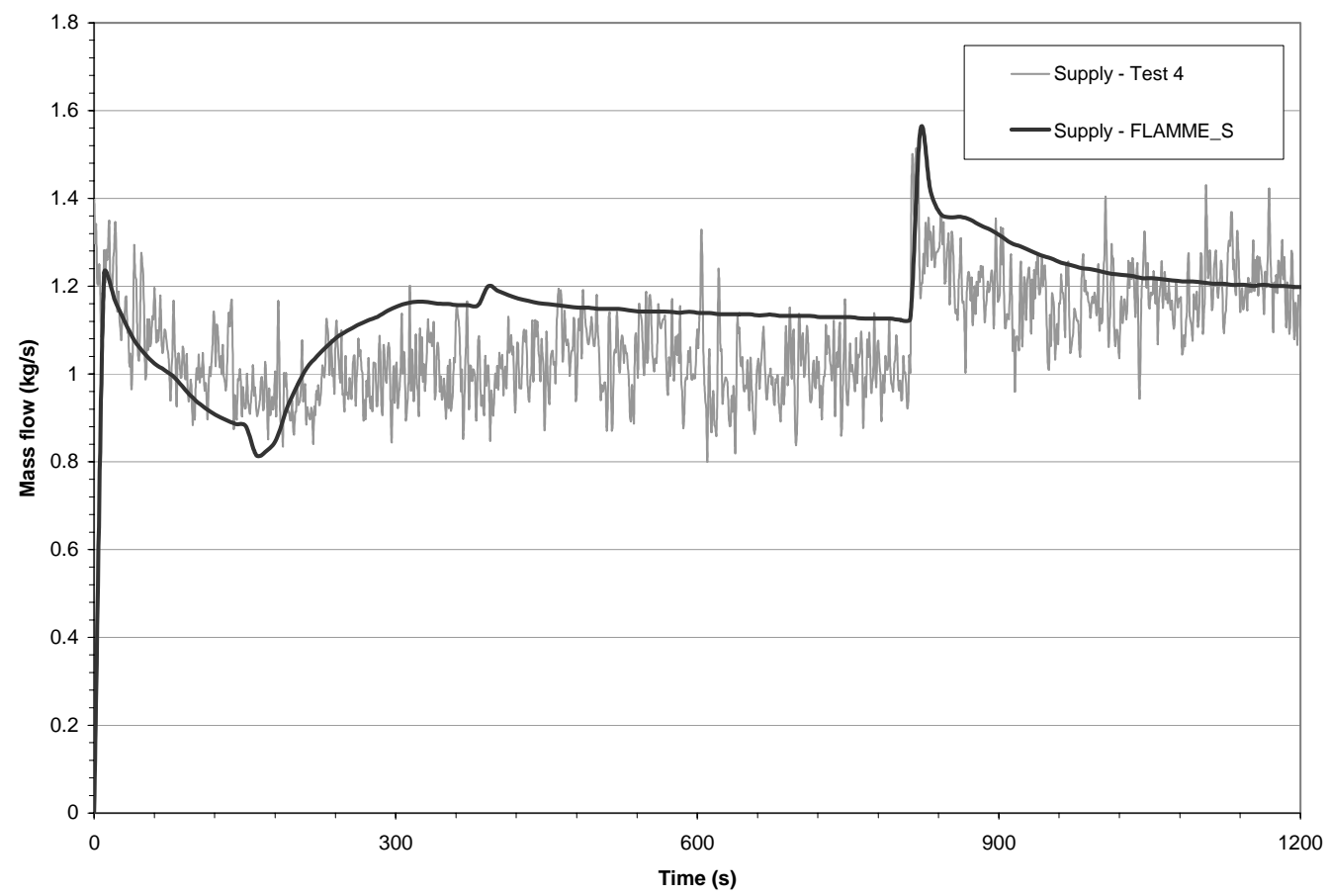

Figure 3-28: Mass flow through the supply vent 


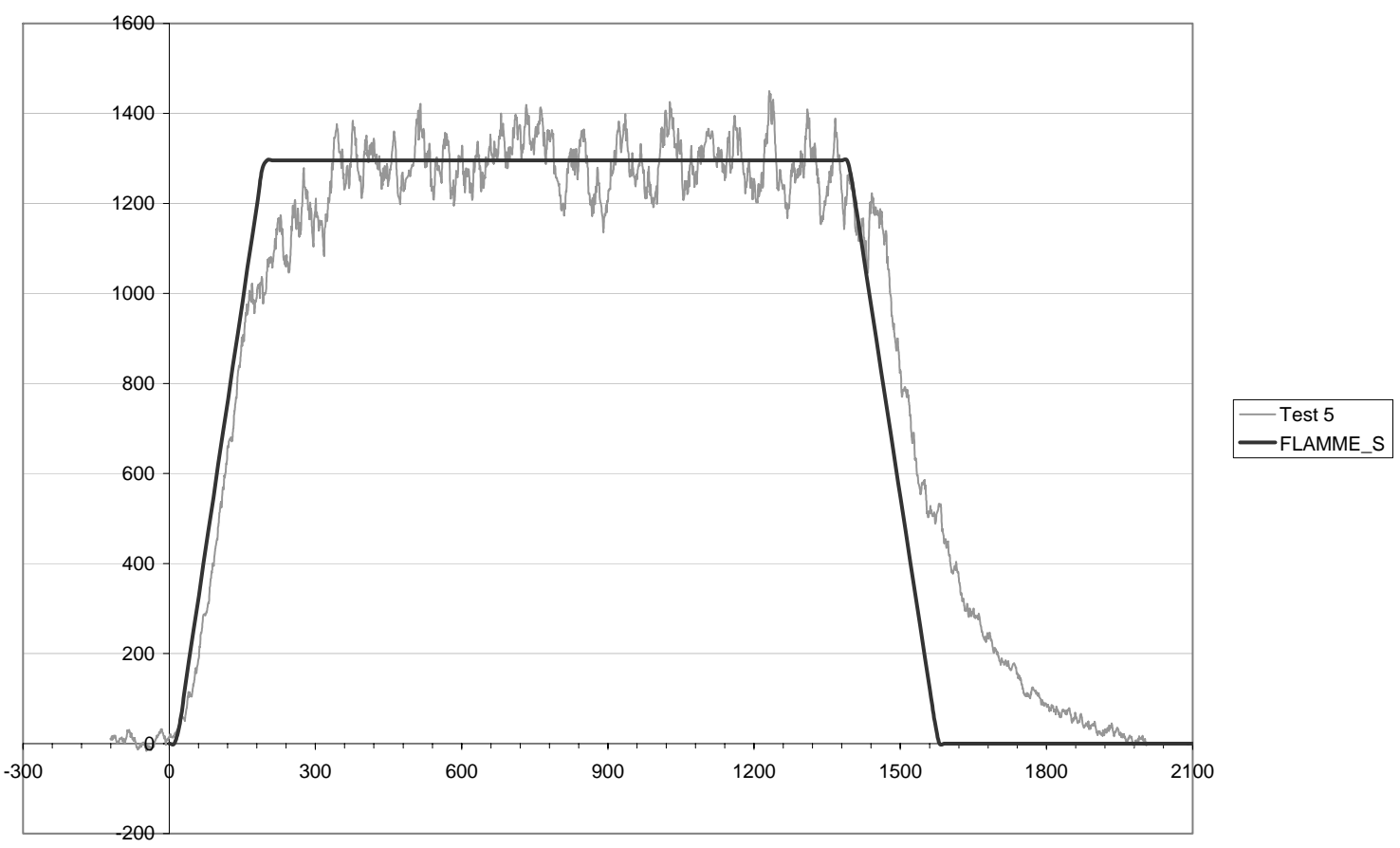

Figure 3-29: Heat release rate

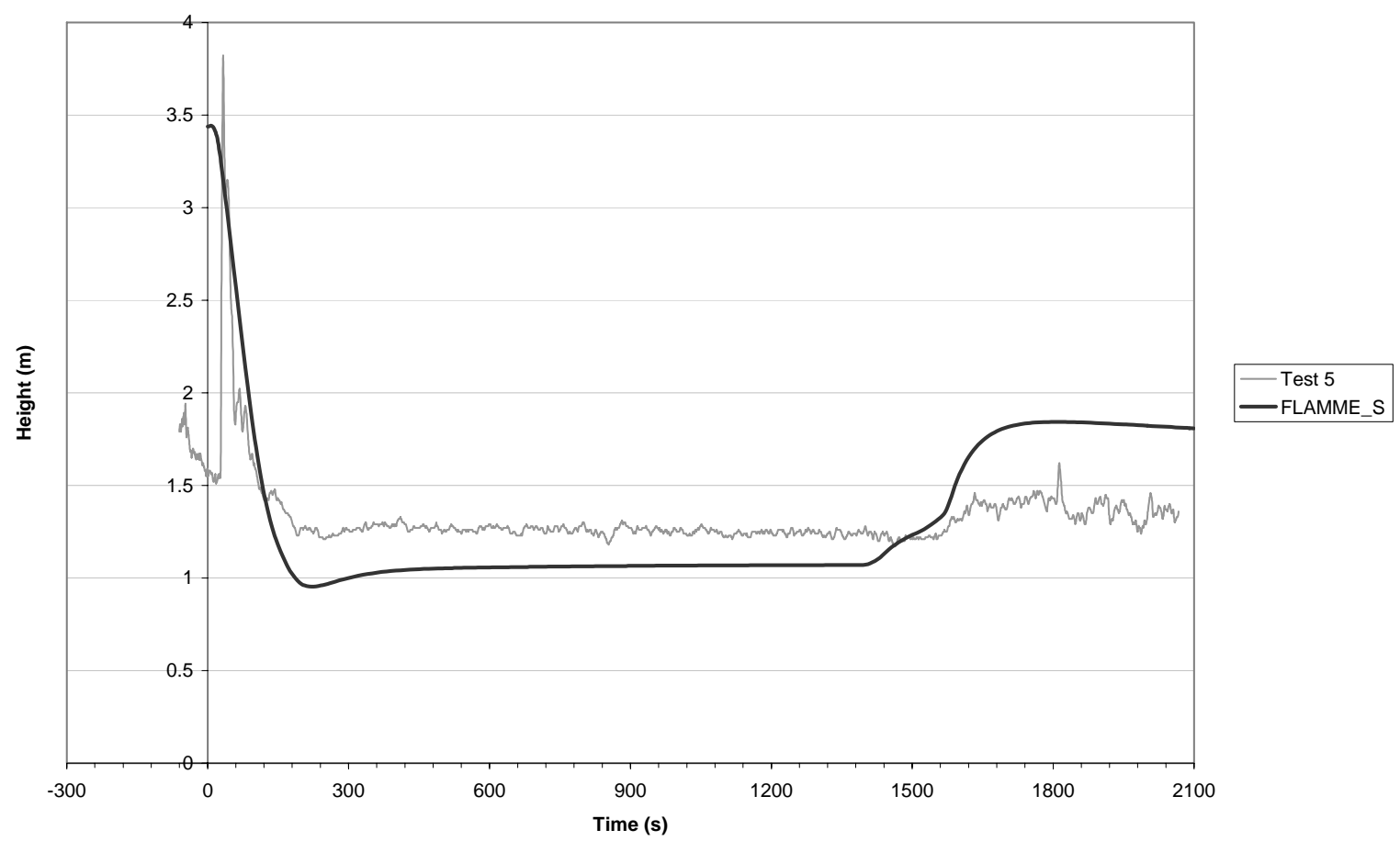

Figure 3-30: Layer height 


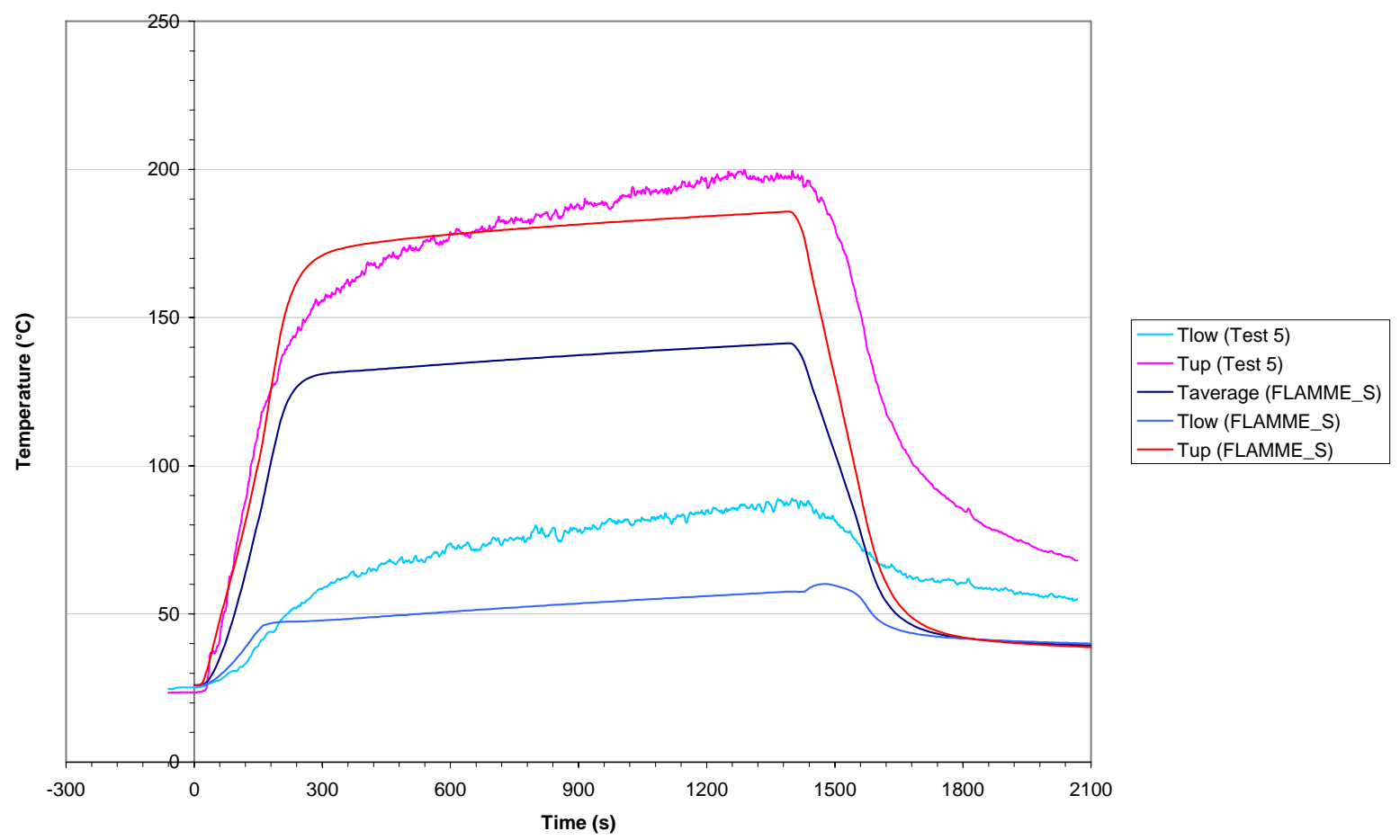

Figure 3-31: Gas temperature

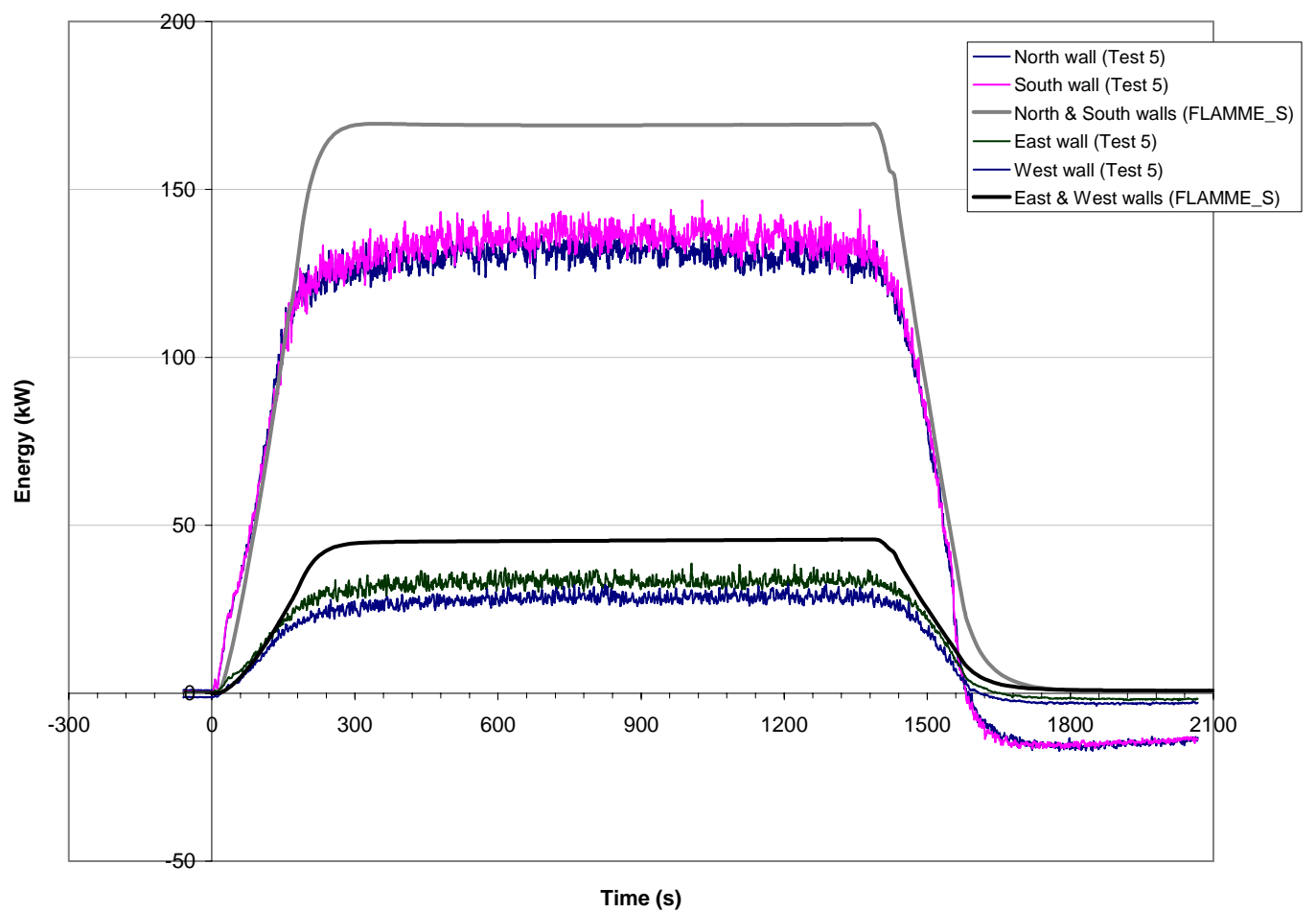

Figure 3-32: Energy loss to the side walls 


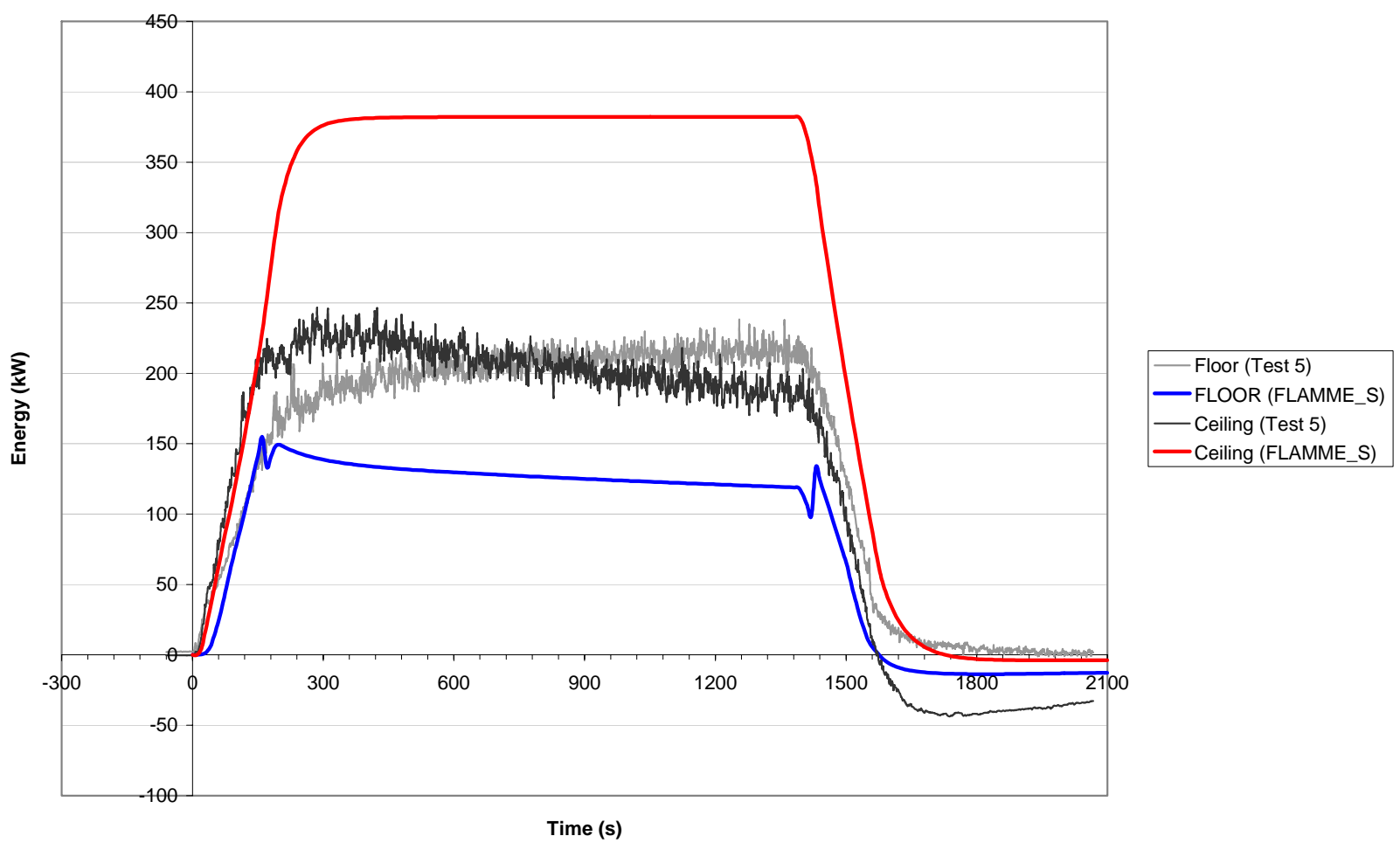

Figure 3-33: Energy loss to the ceiling and to the floor

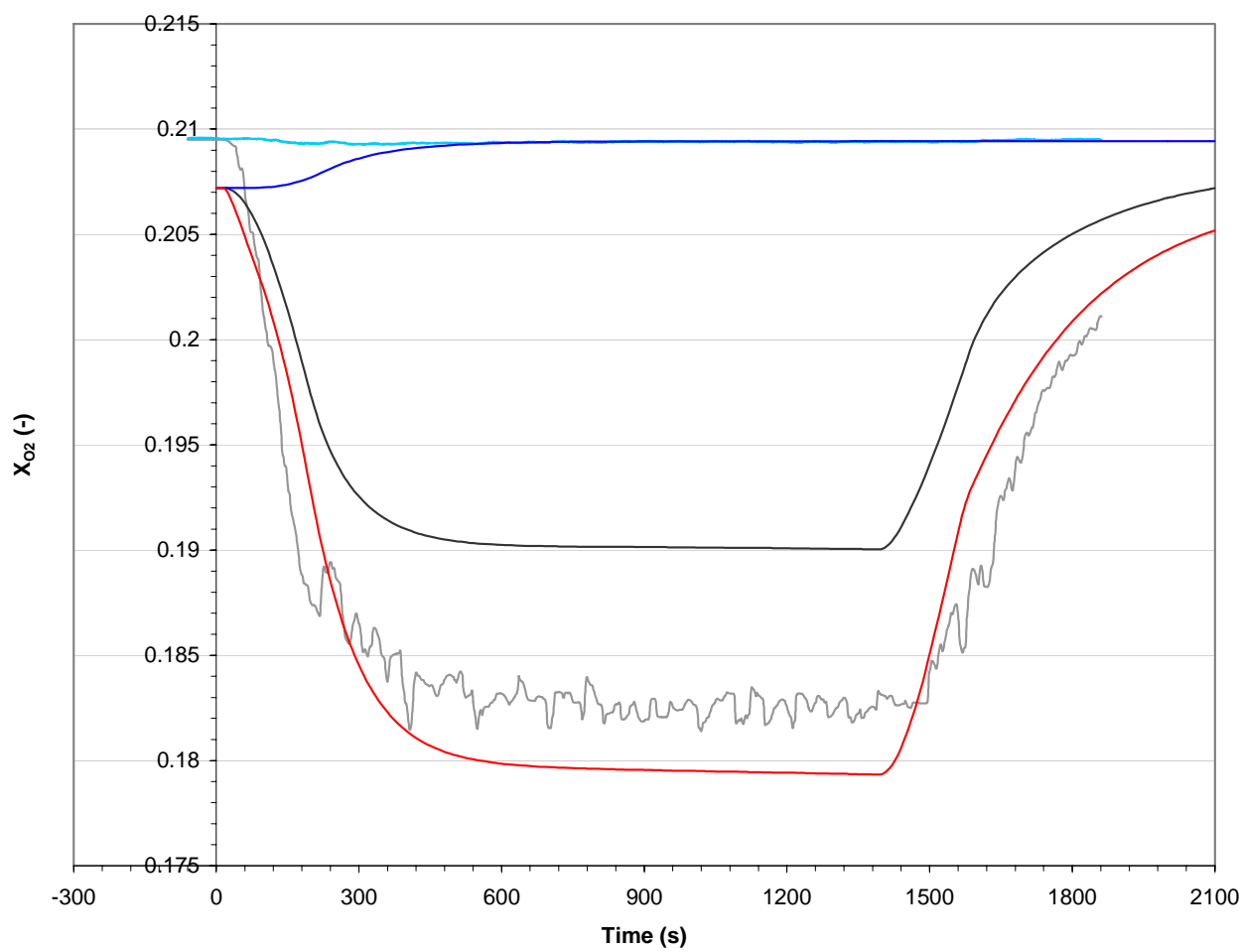

\begin{tabular}{|l}
\hline O2-1 (Test 5) \\
O2-2 (Test 5) \\
O2 Average (FLAMME_S) \\
O2 low (FLAMME_S) \\
O2 up (FLAMME_S)
\end{tabular}

Figure 3-34: Molar fraction of oxygen 


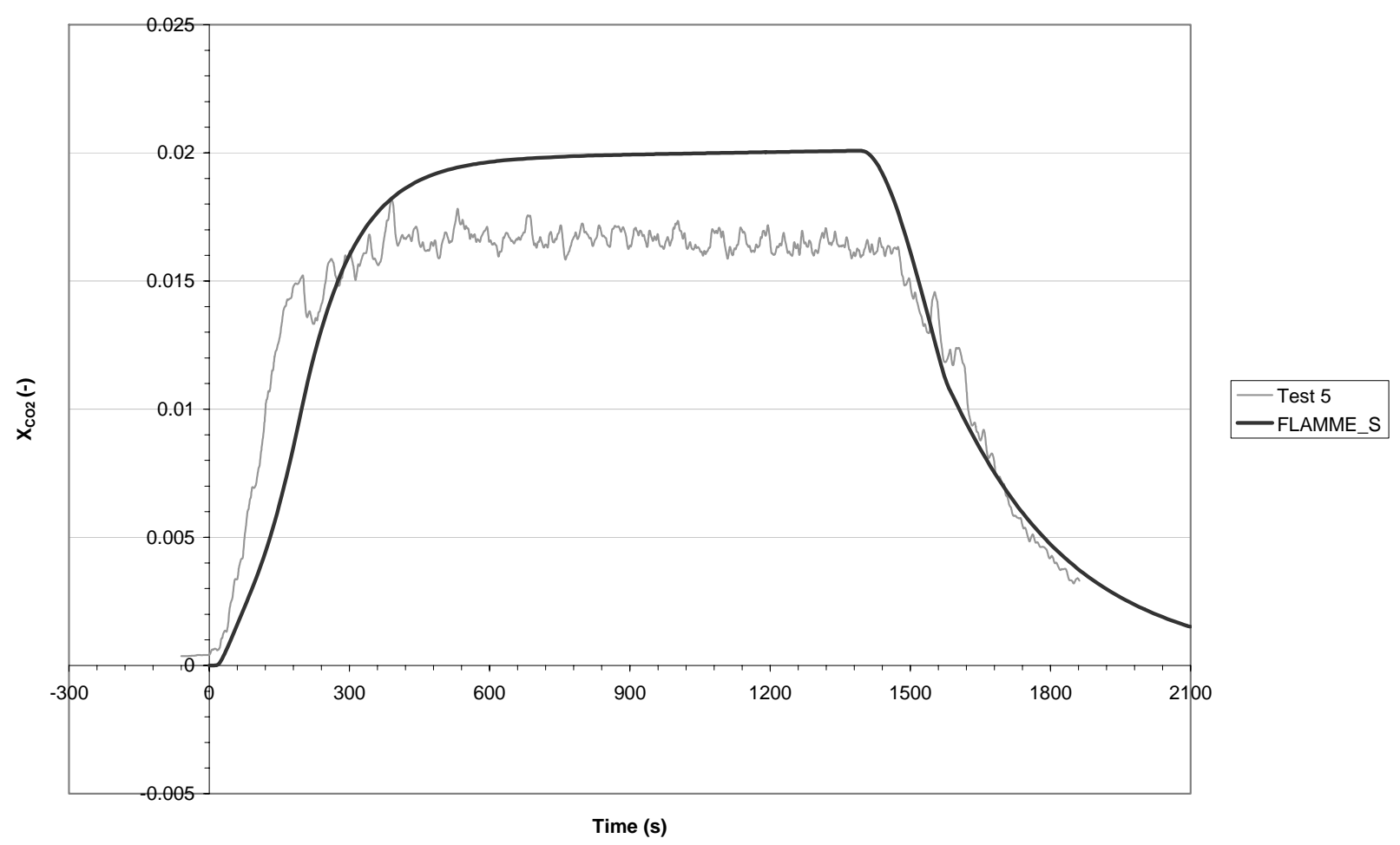

Figure 3-35: Molar fraction of carbon dioxide

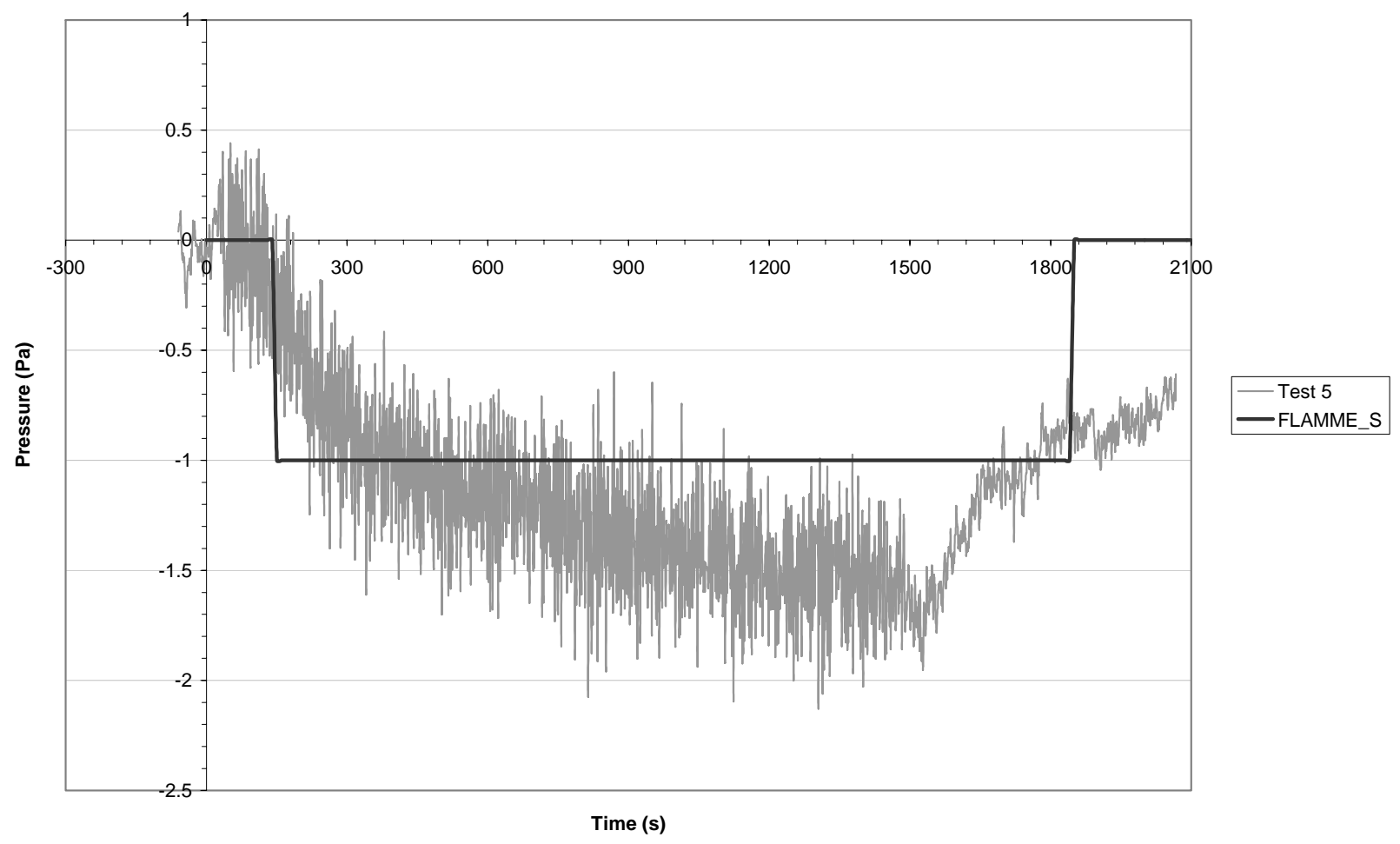

Figure 3-36: Pressure 


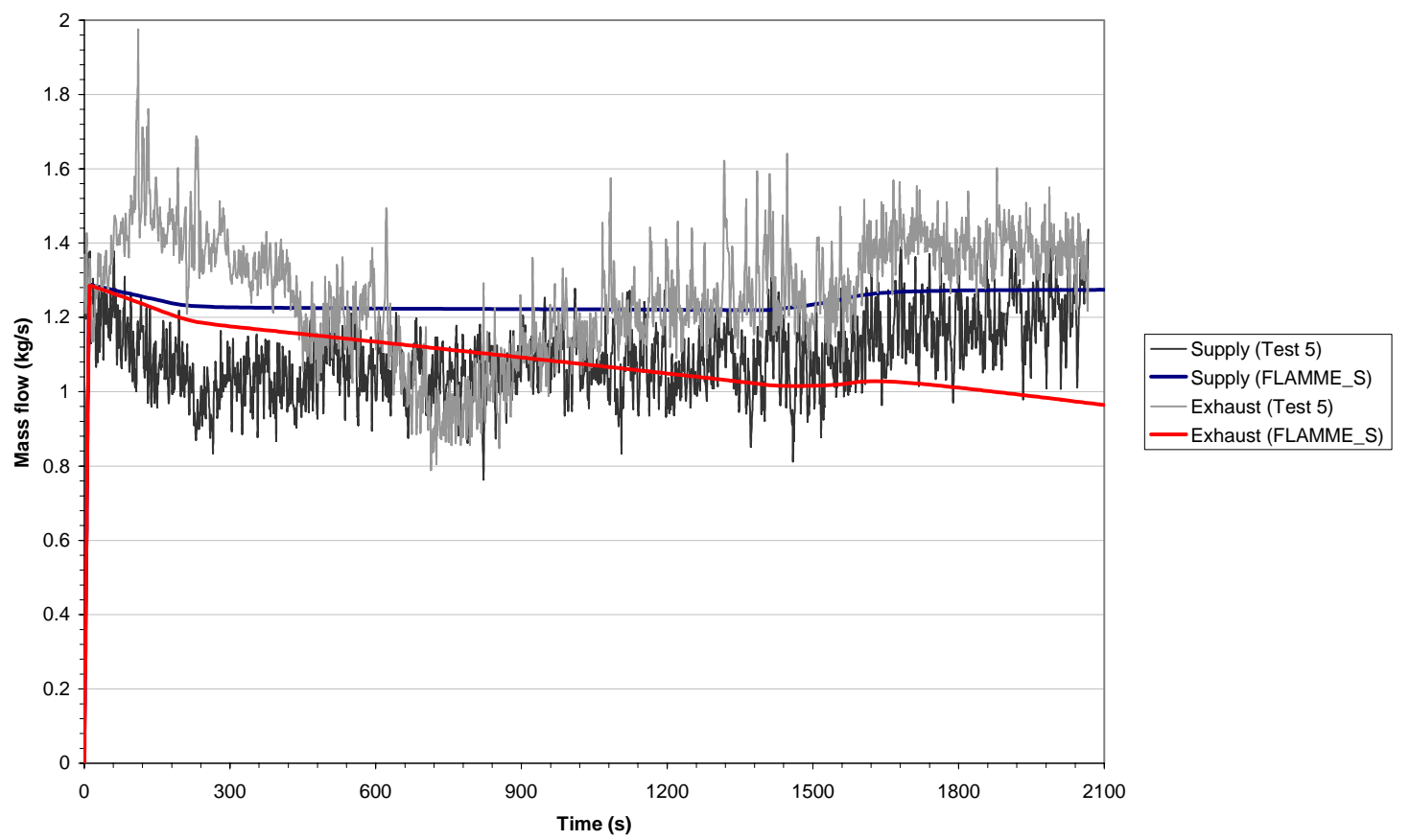

Figure 3-37: Mass flow through the supply vent and through the exhaust vent

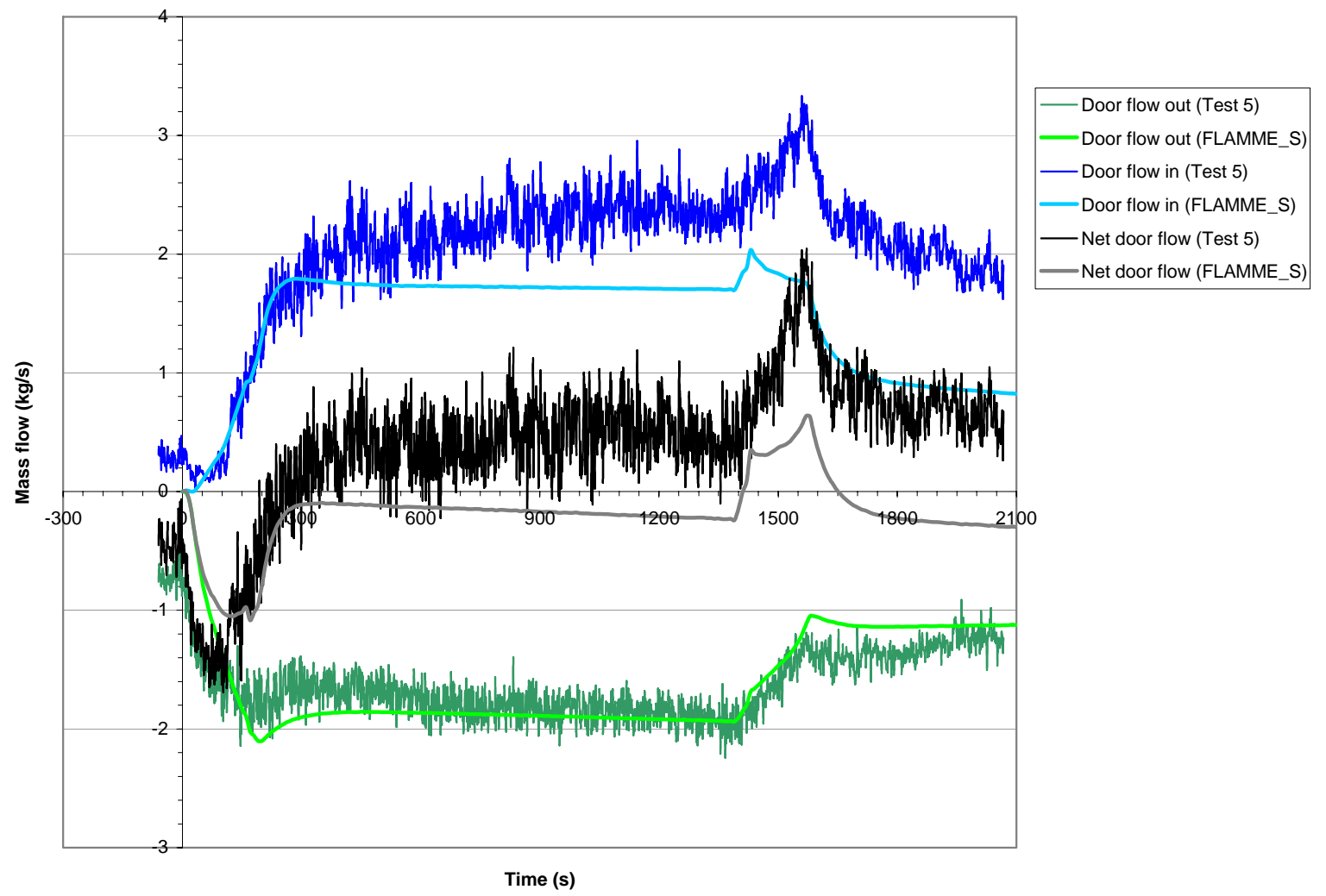

Figure 3-38: Mass flow through the door 


\section{$4 \quad$ Open Simulations using FATE (Fauske and Associates)}

The FATE ${ }^{\text {TM }}$ Computer Code is used for this analysis. FATE is the successor to HADCRT, which was used in previous ICFMP benchmarks. FATE version 2.11 contains upgrades to address fire modeling issues. FATE stands for Facility Flow, Aerosol, Thermal, and Explosion Model, for PCs and workstations [Plys and Lee, 2004]. FATE is used for design, offnormal, and accident analyses of nuclear and chemical facilities.

General capabilities of FATE 2.11 include:

- Fire model: Define burn rate and yields; Smoky layer model; Propagation of smoke in stratified layers throughout facility; Aerosol transport with smoky layers; Aerosol settling from smoky layer to lower layer and embedded surfaces.

- Multiple-compartment thermodynamics and general species: Facility rooms have separate pressure, temperature, and composition; Compound property libraries are input; Tracking of condensed, gaseous, and aerosol species.

- Facility nodalization and flow: Compartments are connected in arbitrary topology by flow paths; Flows are pressure-driven, density-driven counter-current, and diffusional.

- Aerosol behavior: Aerosol coagulation, sedimentation, transport with flow; Deposition on bends and filters; Deposition by condensation; Aerosol formation by boiling and fog.

- Heat transfer: Convection of liquids and gases to structures with internal temperature distributions; Linking for 2D and 3D heat transfer; Condensation.

- Flammability and combustion of gases, vapors, and aerosols: Any input compound may participate; examples include solvent vapors, hydrogen, and $U$ metal or hydride aerosols.

- Entrainment of deposits to form aerosols: Powder, liquid, and sludge waste may be entrained by combustion or flow.

- Thermal radiation networks: View factor models and automatic network balancing.

- Event-oriented simulation: Intervention criteria and actions for scenario evolution.

- Sources and time-dependent conditions. Prescribe liquid, gas, and aerosol source histories and environmental conditions with time. 
- Nuclear fuel and sludge models including chemical reactions.

FATE $^{\text {TM }}$ is owned and licensed by Fauske and Associates, LLC (FAI) and FATE is a registered trademark of FAI. FATE is created and maintained under the FAI Quality Assurance Program (10CFR50 App. B \& ISO 9001 compliant).

\subsection{FATE Input Parameters and Assumptions}

\subsubsection{Room Geometry and Ventilation}

The Benchmark Exercise 3 (BE-3) base model is based on a standardized room model successfully developed during Benchmark Exercise 1 (BE \#1) (NISTIR-6986). The BE \#1 effort demonstrated that the rectangular room geometry can be accurately modeled with a single region and 14 heat sinks to represent floor, ceiling, and walls, and a radiation network containing the heat sinks, fire source, and smoky layer. In addition the BE \#1 effort provides an effective methodology for modeling forced ventilation, natural circulation through open doors, and intrinsic leakage through the use of five flow paths. Ventilation in the FATE standardized room model is refined in the current effort.

Table 4-1 summarizes the heat sinks used to define the region boundaries, while Table 4-2 summarizes the flow paths. The wall heat sinks are modeled as "strips" that circumscribe the room perimeter and extend vertically between the indicated elevations. The heat sinks are then subdivided in the FATE heat transfer model into 20 slabs for 1-dimensional conduction through the heat sink. The four side walls are modeled as a series of 12 stacked wall strips. The advantage to this approach is input and computational simplicity, while the limitation is that a single surface temperature is reported for the North, East, South, and West walls at each elevation.

As will be discussed, calculation results proved to be sensitive to modeling of intrinsic leakage. The leak centerline elevation, leak area, and leak orientation are all shown to influence the smoky layer elevation and oxygen content of the room, even in the cases where the main door is open or the ventilation fan is on. Based on results from multiple sensitivity runs, a best match of the test data can be obtained by modeling intrinsic leakage only through assumed gaps in the closed door, and assumed gaps in the supply and exhaust duct louvers (for cases without forced ventilation). Table 4-2 shows an additional leak path (junction 5), but this is only used in sensitivity calculations. Junctions 2 and 3 are modified to model in- 
trinsic leakage for cases without forced ventilation by assuming a $0.0054 \mathrm{~m}$ (0.21") gap around the vent perimeter for a total leakage area of $0.015 \mathrm{~m}^{2}$ for each junction. The door gap is assumed to be $0.0032 \mathrm{~m} \mathrm{(1/8")}$ around the perimeter of a set of double doors (2 doors $2 \mathrm{~m} \times 1 \mathrm{~m}$, each), for a total leakage area of $0.032 \mathrm{~m}^{2}$. Finally, for cases with forced ventilation, a constant fan volumetric flow of $1.06 \mathrm{~m}^{3} / \mathrm{s}$ is imposed across junction 2 .

The leak path parameters and the open door loss coefficient were adjusted based on comparisons against BE-3 tests 1 (fans off, doors closed) and 3 (fans off, door open). The derived path parameters were then used for the remainder of the tests. The door loss coefficient was set at one-half of the recommended value, $\mathrm{K}_{\text {door }}=0.54$.

Table 4-1 FATE BE-3 Base Model Heat Sink Summary Table

\begin{tabular}{|c|c|c|c|c|c|c|}
\hline $\begin{array}{c}\text { Heat } \\
\text { Sink } \\
\#\end{array}$ & Label & Type & $\begin{array}{l}\text { Bottom El. } \\
\text { (m) }\end{array}$ & $\begin{array}{c}\text { Top El. } \\
\text { (m) }\end{array}$ & Material & $\begin{array}{l}\text { Thickness } \\
\text { (m) }\end{array}$ \\
\hline 1 & FLOOR & $\begin{array}{l}\text { Upward facing } \\
\text { rectangle }\end{array}$ & -2.54E-02 & $0.00 E+00$ & Gypsum & 0.0254 \\
\hline 2 & WALL-1 & $\begin{array}{l}\text { Rectangular } \\
\text { enclosure strip }\end{array}$ & $0.00 \mathrm{E}+00$ & 3.18E-01 & Marinite & 0.0254 \\
\hline 3 & WALL-2 & $\begin{array}{l}\text { Rectangular } \\
\text { enclosure strip }\end{array}$ & 3.18E-01 & 6.37E-01 & Marinite & 0.0254 \\
\hline 4 & WALL-3 & $\begin{array}{l}\text { Rectangular } \\
\text { enclosure strip }\end{array}$ & 6.37E-01 & 9.55E-01 & Marinite & 0.0254 \\
\hline 5 & WALL-4 & $\begin{array}{l}\text { Rectangular } \\
\text { enclosure strip }\end{array}$ & 9.55E-01 & $1.27 \mathrm{E}+00$ & Marinite & 0.0254 \\
\hline 6 & WALL-5 & $\begin{array}{l}\text { Rectangular } \\
\text { enclosure strip }\end{array}$ & $1.27 \mathrm{E}+00$ & $1.59 \mathrm{E}+00$ & Marinite & 0.0254 \\
\hline 7 & WALL-6 & $\begin{array}{l}\text { Rectangular } \\
\text { enclosure strip }\end{array}$ & $1.59 \mathrm{E}+00$ & $1.91 \mathrm{E}+00$ & Marinite & 0.0254 \\
\hline 8 & WALL-7 & $\begin{array}{l}\text { Rectangular } \\
\text { enclosure strip }\end{array}$ & $1.91 \mathrm{E}+00$ & $2.23 \mathrm{E}+00$ & Marinite & 0.0254 \\
\hline 9 & WALL-8 & $\begin{array}{l}\text { Rectangular } \\
\text { enclosure strip }\end{array}$ & $2.23 \mathrm{E}+00$ & $2.55 E+00$ & Marinite & 0.0254 \\
\hline 10 & WALL-9 & $\begin{array}{l}\text { Rectangular } \\
\text { enclosure strip }\end{array}$ & $2.55 \mathrm{E}+00$ & $2.87 \mathrm{E}+00$ & Marinite & 0.0254 \\
\hline 11 & WALL-10 & $\begin{array}{l}\text { Rectangular } \\
\text { enclosure strip }\end{array}$ & $2.87 \mathrm{E}+00$ & $3.18 \mathrm{E}+00$ & Marinite & 0.0254 \\
\hline 12 & WALL-11 & $\begin{array}{l}\text { Rectangular } \\
\text { enclosure strip }\end{array}$ & $3.18 \mathrm{E}+00$ & $3.50 \mathrm{E}+00$ & Marinite & 0.0254 \\
\hline 13 & WALL-12 & $\begin{array}{l}\text { Rectangular } \\
\text { enclosure strip }\end{array}$ & $3.50 \mathrm{E}+00$ & $3.82 \mathrm{E}+00$ & Marinite & 0.0254 \\
\hline 14 & CEILING & $\begin{array}{l}\text { Upward facing } \\
\text { rectangle }\end{array}$ & $3.82 E+00$ & $3.85 E+00$ & Marinite & 0.0254 \\
\hline
\end{tabular}


Table 4-2 FATE BE-3 Base Model Flow Path Summary

\begin{tabular}{|c|c|c|c|}
\hline Junction \# & Description & $\begin{array}{l}\text { Centerline el. } \\
\text { (m) }\end{array}$ & Area $\left(m^{2}\right)$ \\
\hline 1 & DOOR & 1 & $4, K=0.54$ \\
\hline \multirow[t]{2}{*}{2} & FAN (fan on) & 2.4 & 0.49 \\
\hline & FAN (fan off) & 2.4 & 0.015 (gap leak 0.0054 m) \\
\hline \multirow[t]{2}{*}{3} & EXHAUST (fan on) & 2.4 & $0.49, K=1.08$ \\
\hline & EXHAUST (fan off) & 2.4 & 0.015 (gap leak 0.0054 m) \\
\hline 4 & HIGH LEAK & 3.3 & $\mathrm{~N} / \mathrm{A}$ \\
\hline 5 & & & $\begin{array}{l}\text { Perimeter of double door }(2 \mathrm{~m} \times \\
2 \mathrm{~m}) \times \text { assumed gap width of }\end{array}$ \\
\hline & DOOR GAP LEAK & 1.2 & $0.0032 \mathrm{~m} ;$ total area $=0.032 \mathrm{~m}^{2}$ \\
\hline
\end{tabular}

\subsubsection{Cable Targets}

A single cable target is included in the radiation network to model cable heating. A representative cable, based on the 3-conductor power cable with a hypalon jacket, is used as the basis for the target cable. The target is then modeled as a cylindrical composite heat sink with a $0.25 \mathrm{~cm}$ thick outer hypalon jacket (10 radial nodes) and a $0.13 \mathrm{~cm}$ layer of XLP insulation (5 radial nodes). The copper conductor and other complexities of the cable morphology are not considered. This simple cable target model allows prediction of the jacket outer surface and insulation inner surface temperatures, which is consistent with available experimental measurements. The cable target is then included in the radiation network. Axial conduction along the length of the cable is not considered, however the cable is divided azimuthally into three sections to consider variations in temperature around the cable surface. This approach allows the cable target to be placed at any arbitrary location within the room to represent a particular section of cable.

Thermal properties of Hypalon are not provided as part of the standard test specification, therefore typical properties for high molecular weight polyethylene are used in the FATE model (www.er6s1.eng.ohio-state.edu; www.bpsolvaype.com ):

$$
\rho=930 \mathrm{~kg} / \mathrm{m}^{3}
$$




$$
\begin{aligned}
& \mathrm{k}=0.50 \mathrm{~W} / \mathrm{m} / \mathrm{C} \\
& c_{p}=1850 \mathrm{~J} / \mathrm{kg} / \mathrm{C}
\end{aligned}
$$

\subsubsection{Test Matrix and Fire Source}

Tests 1 through 5, 14, 15, and 18 have been selected for simulation by FATE. Two sets of pilot fire inputs will vary from test case to test case. The first set of fire parameters is the definition of the fire surface for the radiation network. The fire is located in the room center for most cases (FATE input coordinates XRVF $=3.52$, YRVF $=10.83$ ). For tests 14,15 , and 18 the fire location is as follows:

$\begin{array}{lll}\text { Test } & \text { XRVF } & \text { YRVF } \\ 14 & 5.24 & 10.83 \\ 15 & 1.25 & 10.83 \\ 18 & 1.55 & 10.83\end{array}$

The second set of inputs is for the fire burn flux as a function of time. The test specification provides the peak fire heat rate $(\mathrm{kW})$, linear ramp up and ramp down times, and time at peak heat rate, while FATE requires the fuel burn mass flux $\left(\mathrm{kg} / \mathrm{m}^{2} / \mathrm{s}\right)$ as a function of time. The fuel burn mass flux can be calculated as, $\mathrm{G}=\mathrm{Q} / \mathrm{Hc} / \mathrm{A}$, where $\mathrm{Q}$ is the heat rate, $\mathrm{Hc}$ is the heat of combustion (45 MJ/kg for heptanes and $40.3 \mathrm{MJ} / \mathrm{kg}$ for Toluene), and $A$ is the fuel flow area. The fuel flow area is specified in input as $A=0.60 \mathrm{~m}^{2}$.

\subsection{Comparison of FATE Predictions with Measurements}

FATE open calculations are presented below for BE-3 Tests 1, 2, 3, 4, 5, 14, 15, and 18. Calculations are performed on a personal computer with a $1.39 \mathrm{GHz}$ AMD Athlon processor and a DOS/Windows XP operating environment. A typical run time for the model is 139 seconds of CPU time for a 2500 second simulation, or 18 times faster than real time.

\subsubsection{BE-3 Case 1 FATE Open Benchmark Results}

Case 1 is characterized by a heptanes fuel fire with a peak power of $390 \mathrm{~kW}$ and no ventilation -- the main door is closed and ventilation system is off. Thus, this test case provides insights into the influence of normal room leakage on the room response and fire behavior. FATE calculations for BE-3 Case 1 are presented in Figure 4-1 through Figure 4-11. 
The FATE smoky layer height shows reasonable agreement with the test data with two notable exceptions. First, the FATE smoky layer minimum height is lower than the test data, and second, following fire termination, the FATE smoky layer recedes faster than indicated in the test data. These two results are typical of all the FATE calculations and indicate that either the leak path elevations or reverse flow characteristics may not be in perfect agreement with the test facility conditions. Furthermore, sensitivity cases indicate that the flow split of the air inflow between the smoky and lower layers influences both the smoky layer minimum elevation and the rat at which the smoky layer recedes one the fire is terminated.

Base case results are obtained by assuming that all door leakage inflow is split such that half of the inflow goes to the smoky layer and half of the inflow goes to the lower layer. This flow split is non-mechanistic, however calculations for this case and the others to follow, suggest that there is a flow split that play a dominant role in the smoky layer behavior.

Figure 4-2 shows the influence of door gap leakage on the calculated results. All FATE calculations presented in this figure place the air inflow into the lower layer, only. As shown in the figure, if all inflow goes to the lower layer, then the smoky layer recedes faster than if the inflow is split between the smoky and lower layers. Also, when leakage is increased, the smoky layer minimum elevation is consistent with the leak path centerline elevation (sensitivities $a$ and $b$ ). If the door gap leakage is replaced with an equivalent leak area at a higher elevation (sensitivity $c$ ), the smoky layer descends to the floor elevation. If counter current flow is disabled through the door leak (sensitivity $\mathrm{d}$ ), the smoke behavior is similar to case $\mathrm{c}$ where there is no door leak. These sensitivities in conjunction with the test data suggest that there is an influential leak path in the test near the $1 \mathrm{~m}$ elevation and that in general seemingly small leak paths can have a strong influence on smoky layer behavior. The sensitivity calculations also indicate that counter current flow is an important mechanism when considering small leaks through door gaps.

Figure 4-4 compares average gas temperatures in the upper and lower layers. The average gas temperature is an indicator of the overall energy balance. The good agreement between the FATE calculations and the test data validates the FATE approach of modeling the room with one region specified through input and divided into two sub-regions by the fire submodels (a hot smoky layer and a relatively cool lower layer). This comparison also lends validation to the sub-models for plume behavior and radiant heat transfer (including automatic, continuous view factor calculation). 
Figure 4-5 through Figure 4-10 present comparisons of heat sink calculations. Points of interest from this set of figures are:

- FATE represents the floor as s single heat sink with 20 slabs, or nodes, for onedimensional heat transfer through the floor thickness. Therefore, FATE presents an average heat sink surface temperature which is comparable to the reported temperatures taken at various locations across the test facility floor. The peak temperature in FATE is also consistent with the peak lower layer gas temperature.

- The measured wall temperatures reflect a vertical temperature stratification within the smoky layer. Since FATE uses a single temperature to represent the smoky layer, FATE cannot distinguish this level of detail. However, the heat sink temperatures in the smoky and lower layers generally bound the test data temperature stratification. For example, in Figure 4-6, measurements are taken at an elevation of $1.49 \mathrm{~m}$ which is near the bottom of the smoky layer (1.2 m elevation). In FATE, the smoky layer descends to the 0.2 $\mathrm{m}$ elevation, and as each wall strip becomes submerged in the smoky layer, its surface temperature increases due to convective heat transfer with the hot gas. These wall strips all exhibit temperatures in excess of the measured wall temperature. The single strip remaining in the FATE lower level (heat sink 2 at elevation $0.16 \mathrm{~m}$ ) bounds the measured wall temperatures on the low side. Figure 4-8 compares wall temperatures above the $3.69 \mathrm{~m}$ elevation. As shown, the FATE calculation shows better agreement with the test data at this elevation because of the temperature stratification in the test.

- As discussed above, even though FATE uses "strips" to represent all four sidewalls at a particular elevation, this representation provides reasonable agreement with the test data. The most influential factor in accurately determining wall temperatures is whether or not the wall section is submerged in the smoky layer.

- Overall the good comparison of wall surface temperatures with test data validates the approach to modeling the side walls as a series of wall strips. Furthermore, the level of detail in the FATE standardized room model (12 wall strips to represent the entire wall height) is sufficiently detailed to accurately reflect the test conditions.

One of the most important capabilities of a fire model is the ability to predict target response to a postulated fire. Figure 4-11 presents one such comparison of the FATE code against power cable F mid-plane temperatures for BE-3 Test 1 . As shown, the peak cable temperature is accurately predicted. The time dependent behavior of the cable deviates slightly from the measured performance. This may be caused by the confluence of several factors includ- 
ing, modeling of the fire power profile, smoky layer behavior, cable material properties, leak path behavior, and interaction between the fire, gas, and cable surface.

Generally good agreement has been obtained between FATE and BE-3 Test 1 results, and it is noted that the FATE calculations are influenced by assumptions regarding the leakage flow paths. 


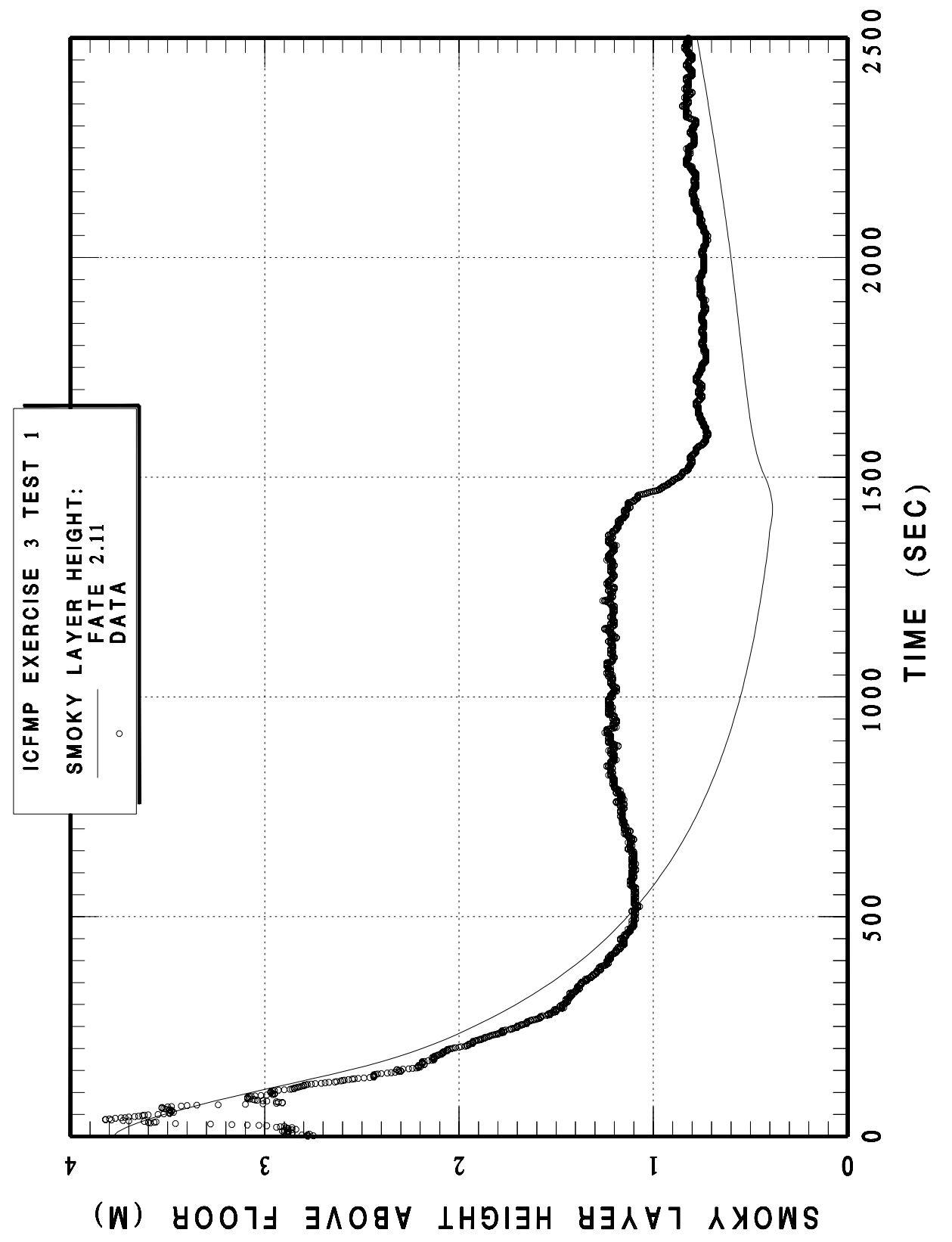

Figure 4-1 BE 3 CASE1 Smoky layer height. Air in leakage through the door is split equally between the smoky and lower layers. 


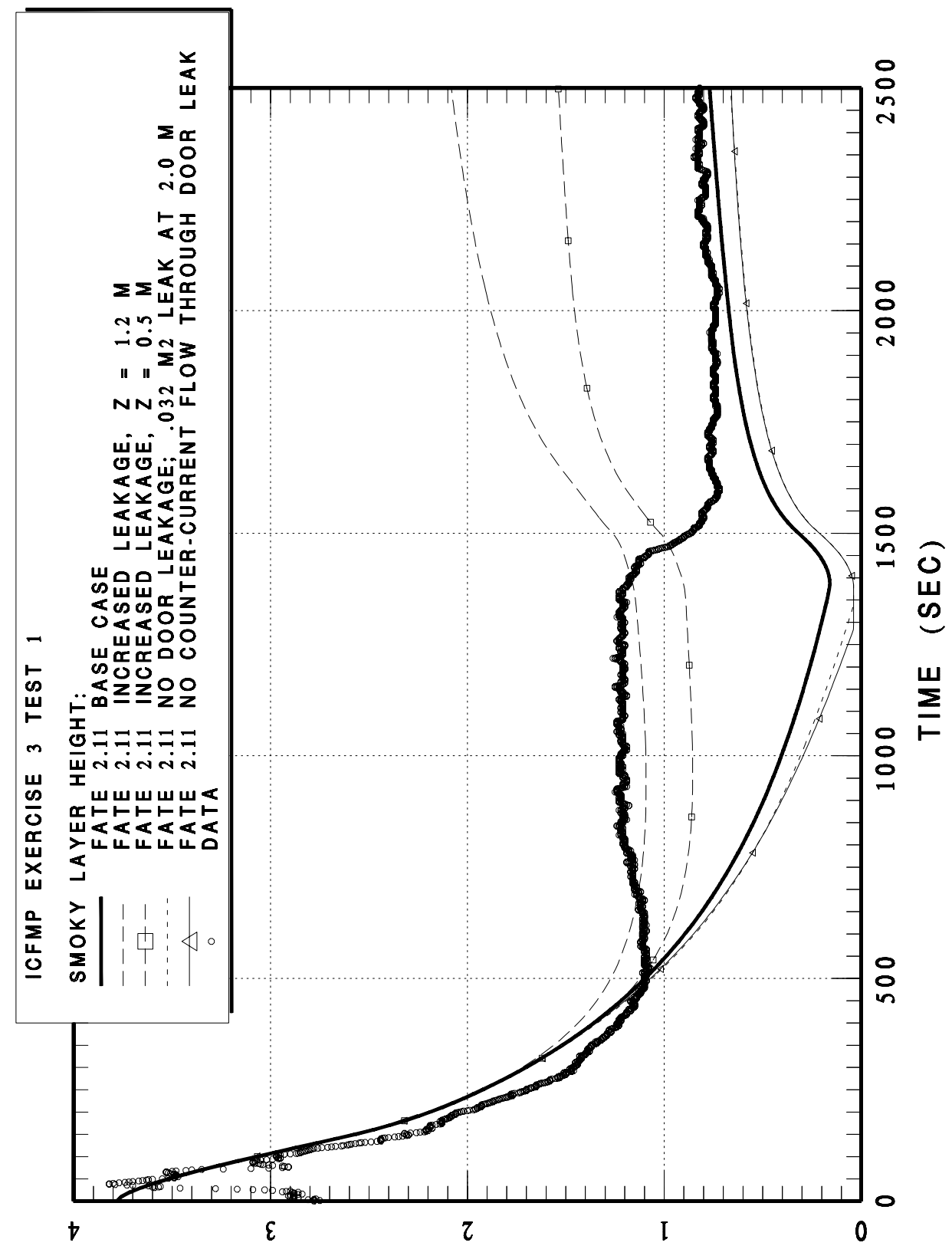

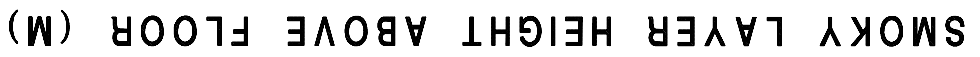

Figure 4-2 BE 3 CASE1 Smoky layer height sensitivity calculations. All air in leakage through the door goes into the lower layer. 


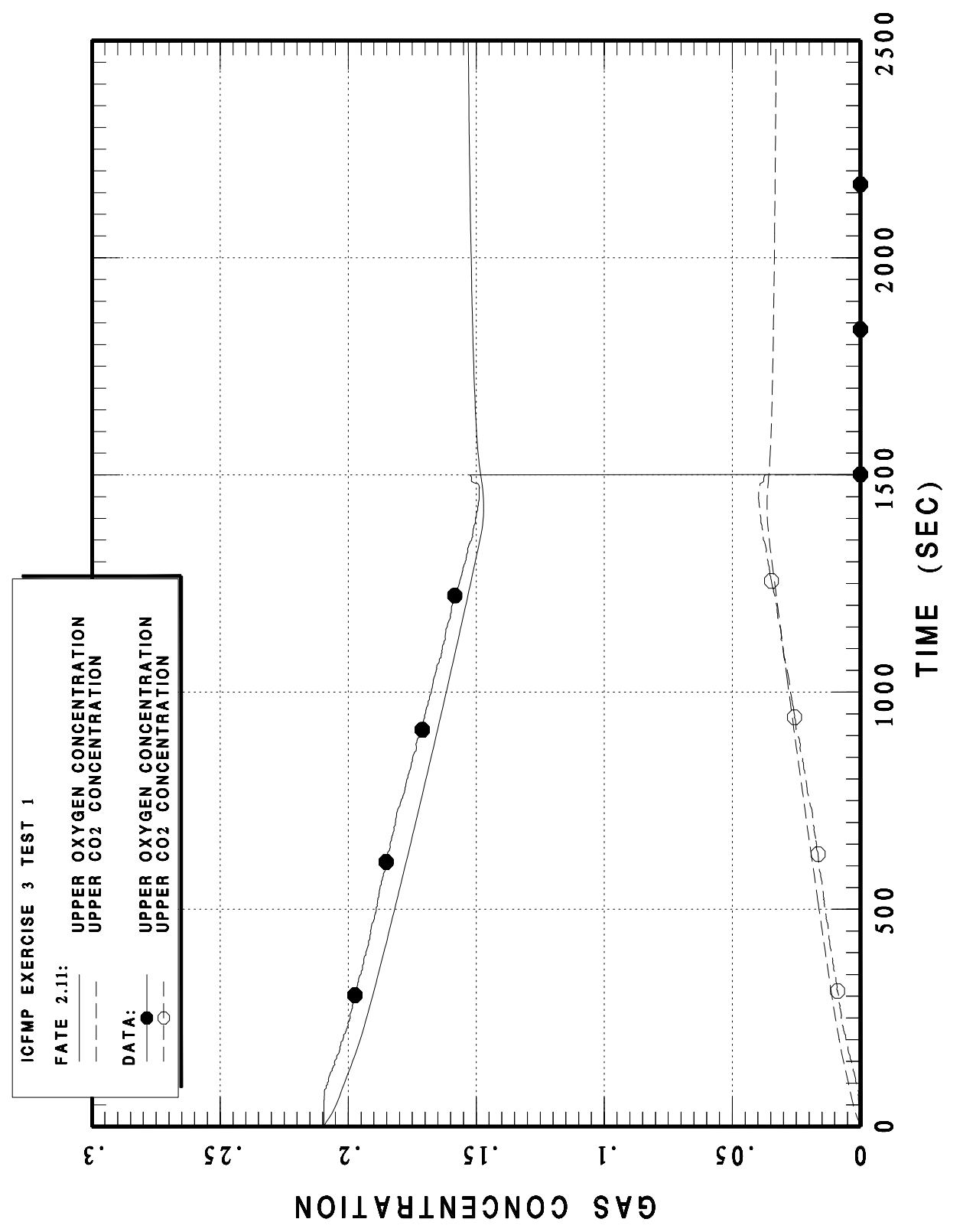

Figure 4-3 BE 3 CASE1 upper room gas concentrations 


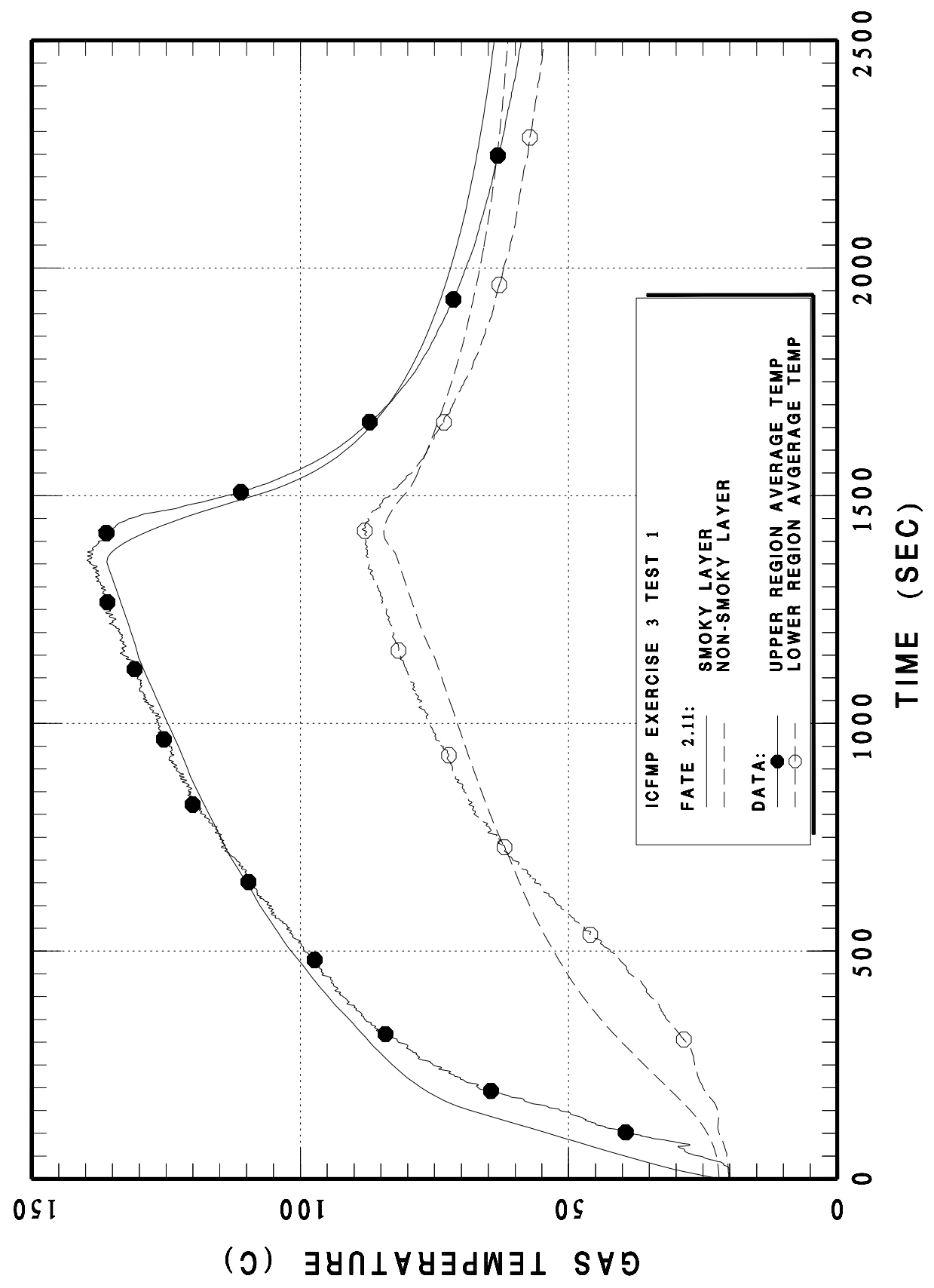

Figure 4-4 BE 3 CASE1 upper and lower layer average gas temperatures 


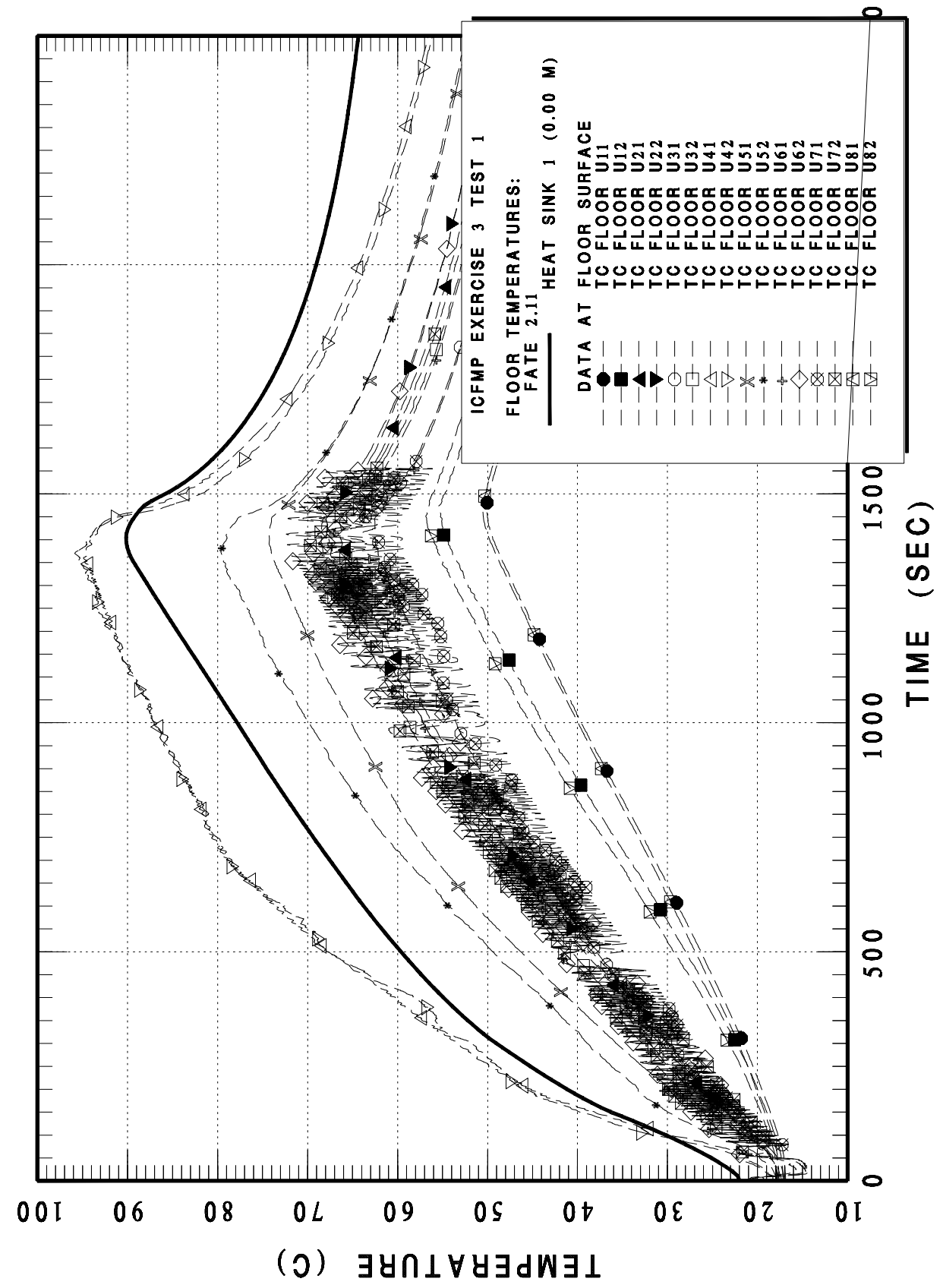

Figure 4-5 BE 3 CASE1 floor surface temperatures 


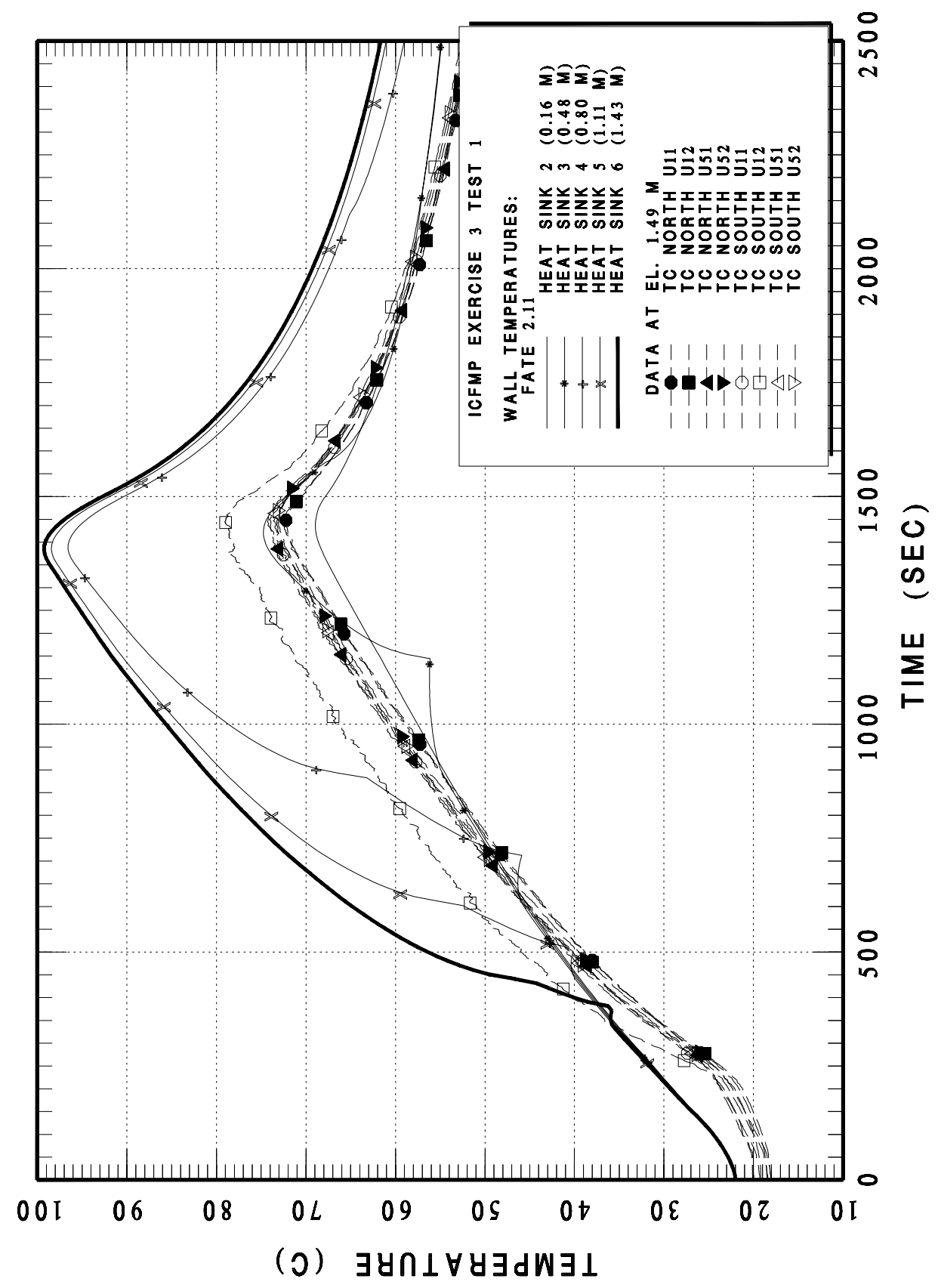

Figure 4-6 BE 3 CASE1 North and South wall temperatures at the 1.49 m elevation 


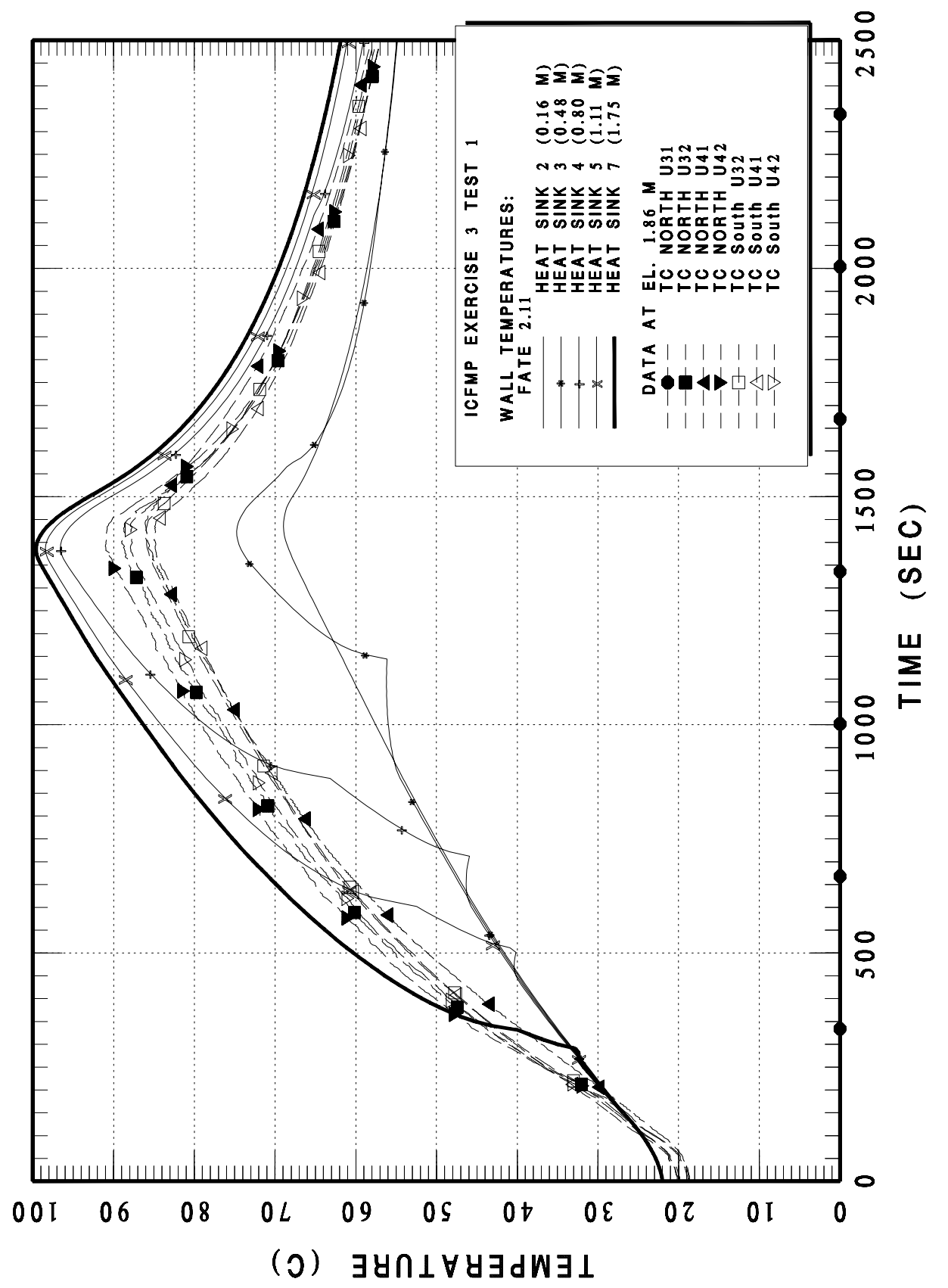

Figure 4-7 BE 3 CASE1 North and South wall temperatures at the 1.86 m elevation 


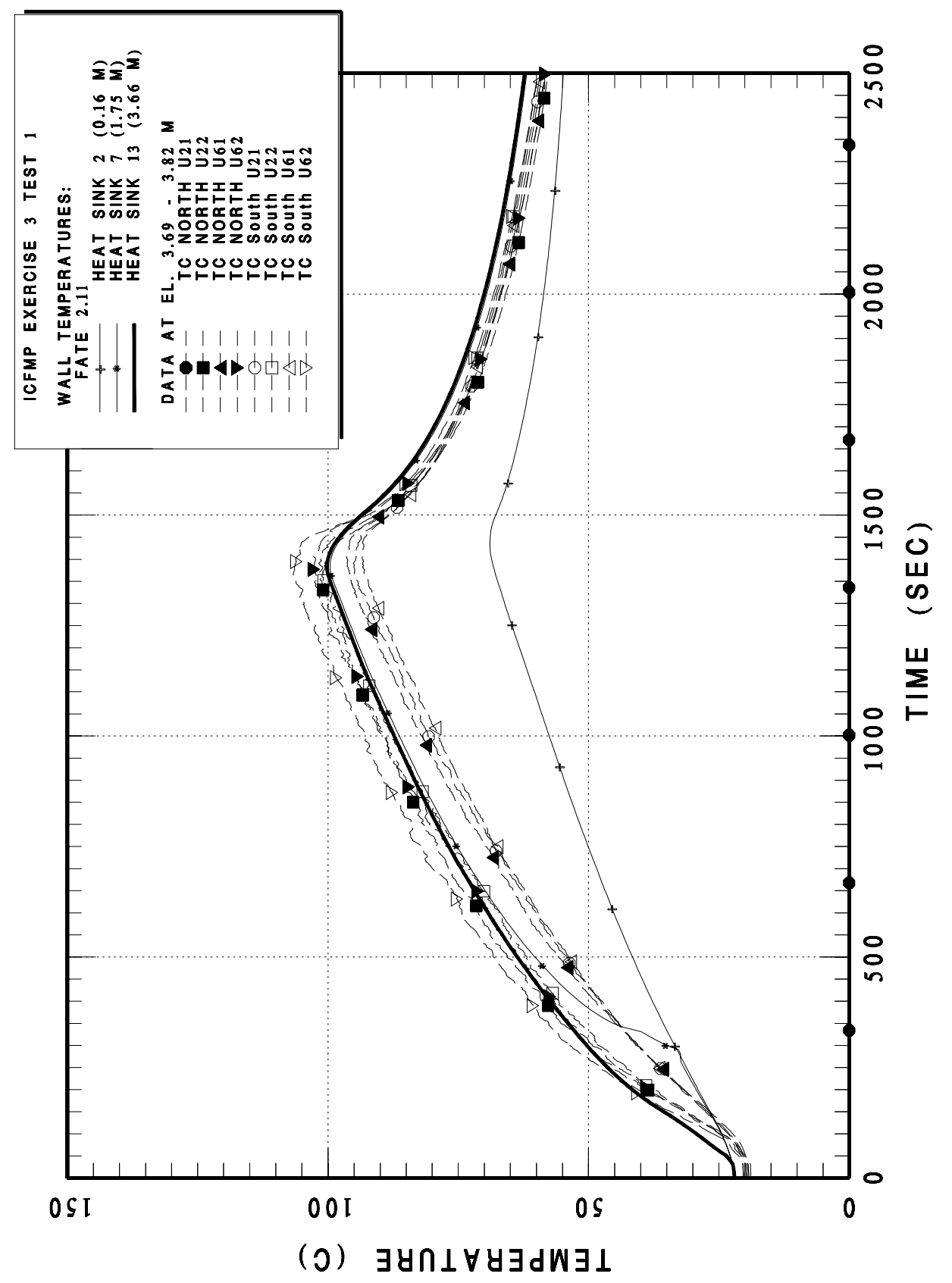

Figure 4-8 BE 3 CASE1 North and South wall temperatures between the 3.69 and $3.82 \mathrm{~m}$ elevations 


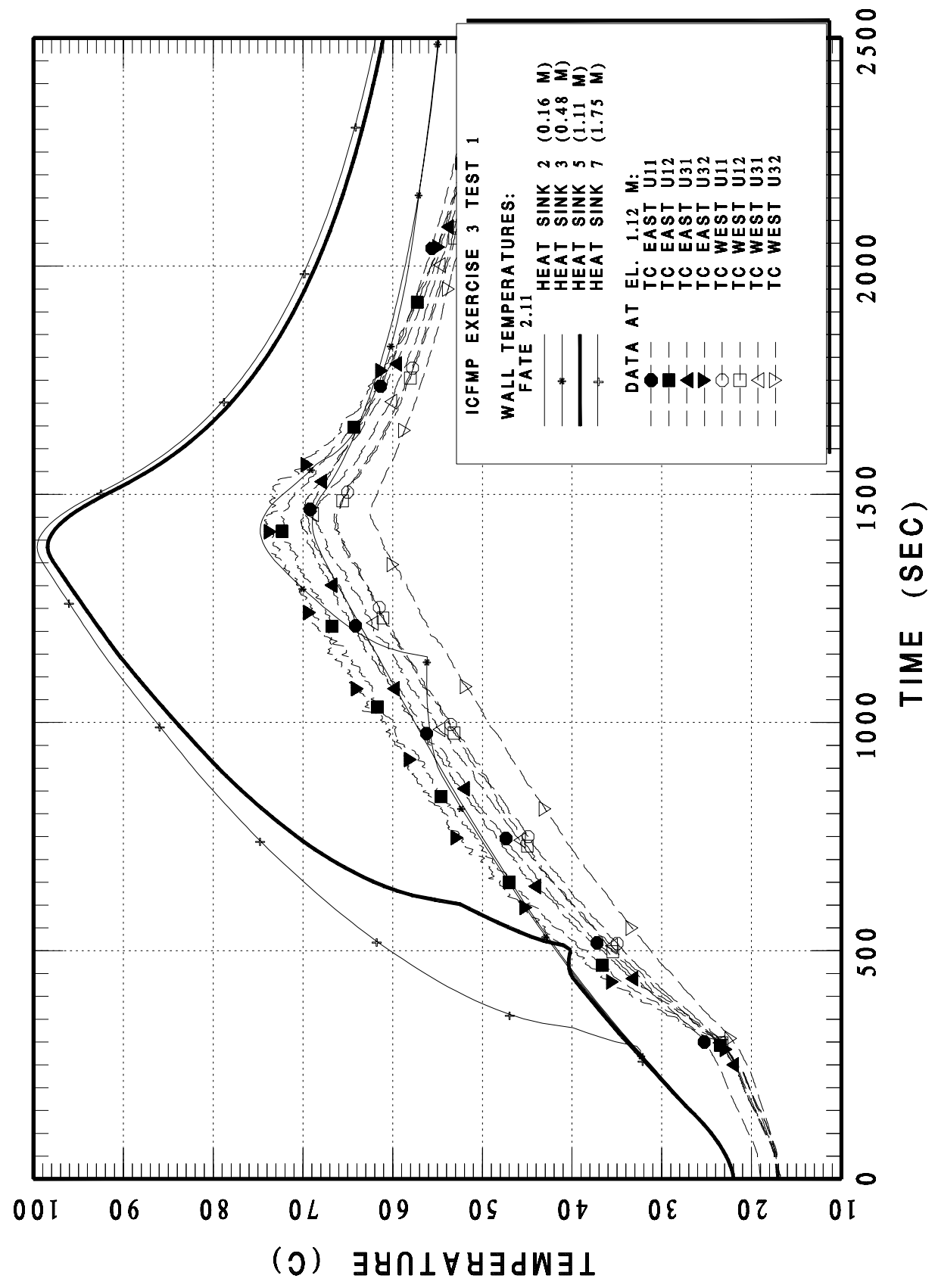

Figure 4-9 BE 3 CASE1 East and West wall temperatures at the 1.12 m elevation 


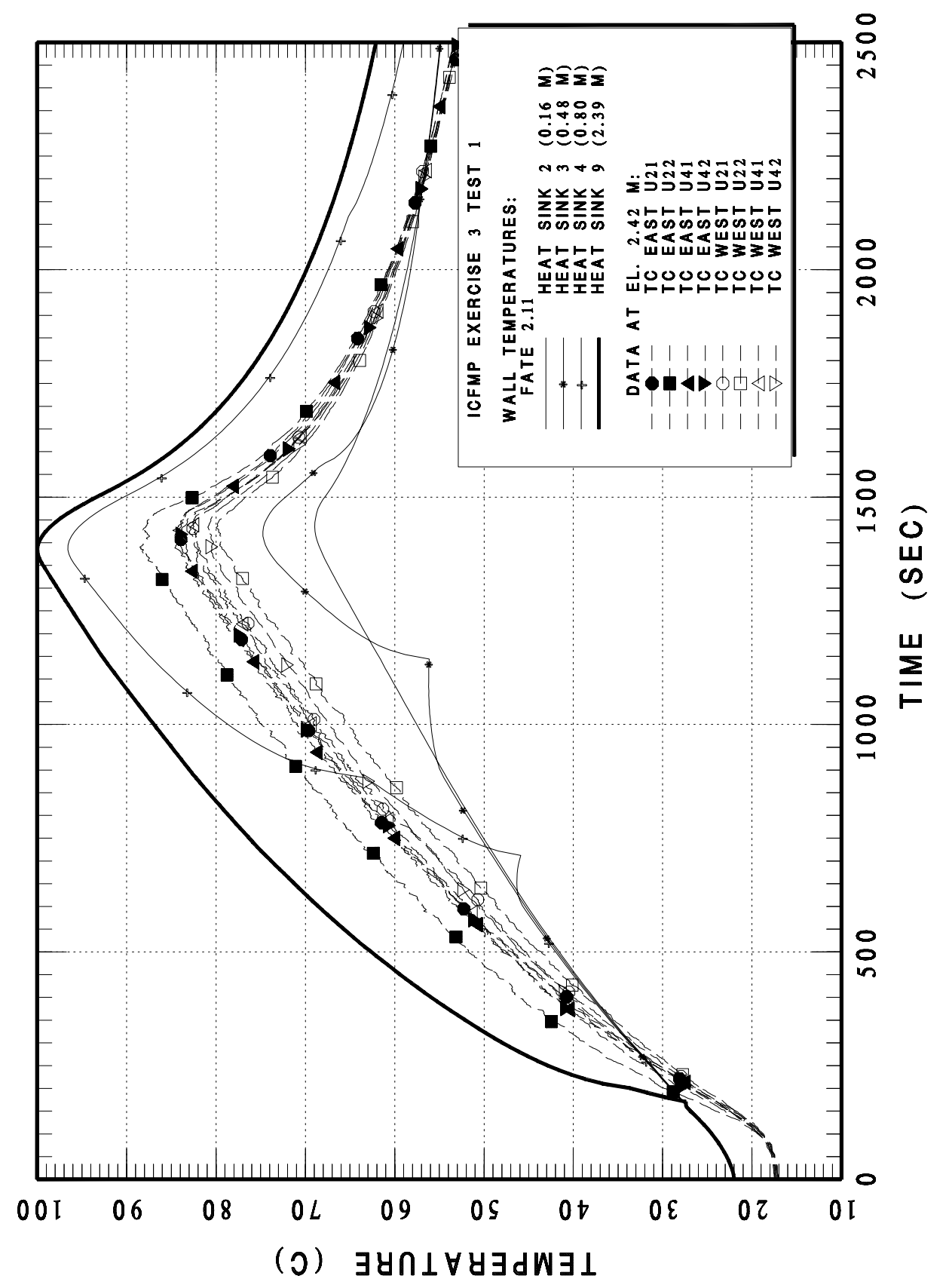

Figure 4-10 BE 3 CASE1 East and West wall temperatures at the 2.42 m elevation 


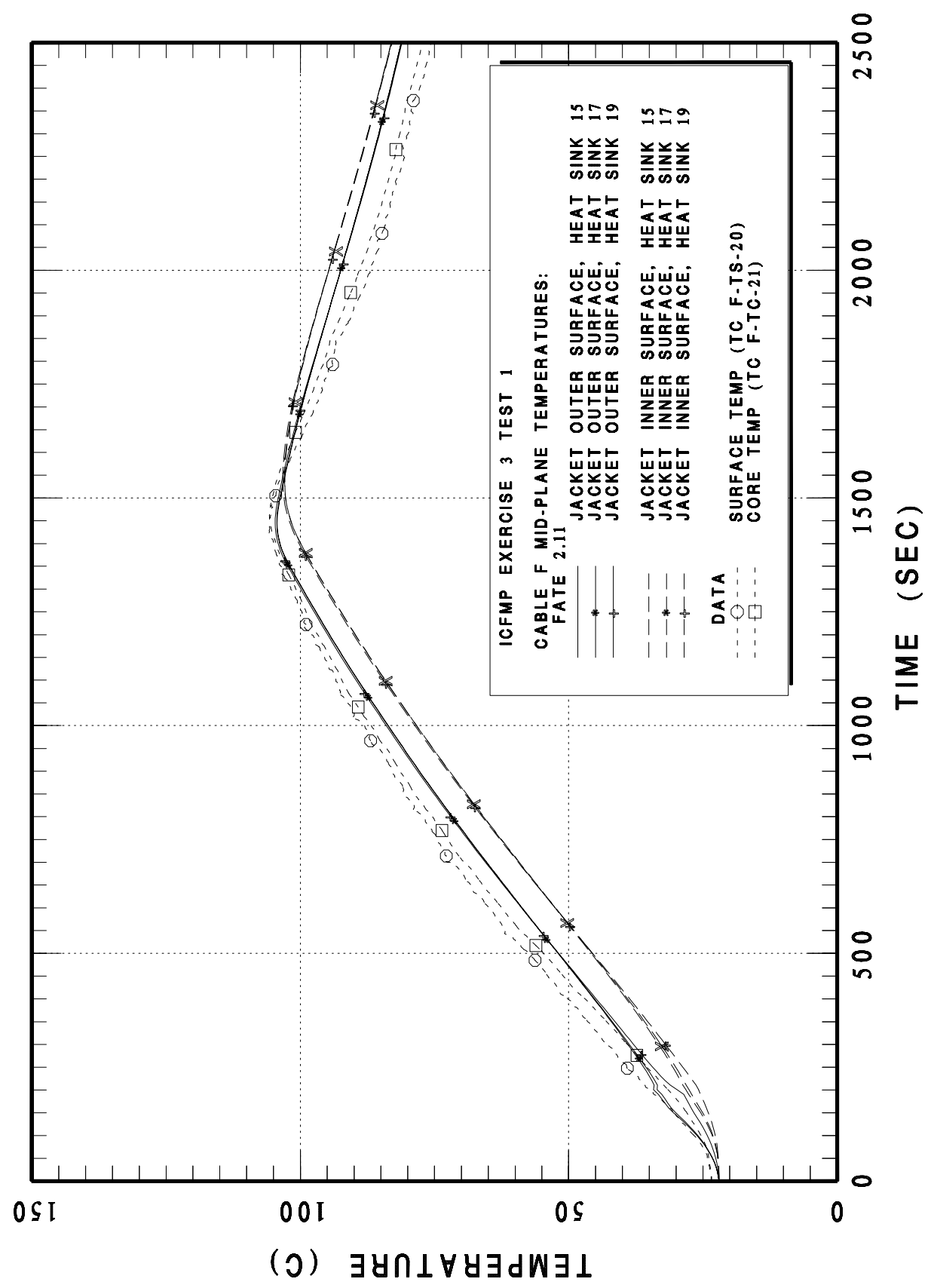

Figure 4-11 BE 3 CASE1 Cable F midplane temperatures 


\subsection{2}

BE-3 Case 2 FATE Open Benchmark Results

Case 2 is characterized by a heptanes fuel fire with a peak power of $1140 \mathrm{~kW}$ and no ventilation -- the main door is closed and ventilation system is off. This test case is similar to Case 1 except the fire power is significantly higher. Whereas case 1 calculations were used to set the leak path parameters, this test case will verify the applicability of these parameters when subjected to a stronger fire. Furthermore, data is available regarding heat sink heat losses for the current case, so the current case will provide additional insights into wall, floor, and ceiling modeling assumptions. FATE calculations for BE-3 Case 2 are presented in Figure 4-12 through Figure 4-26.

Many of the results for the current case show the same trends observed in Case 1. For instance, FATE calculates a smoky layer in the unventilated room that is lower than observed experimentally. Also, as with Case 1, the FATE calculations of gas concentrations and gas layer temperatures show good agreement with the test data. The floor and wall surface temperatures also show similar trends. Namely, the controlling factor in surface temperature is the heat sink location relative to the smoky layer. The FATE calculations generally bound the test data.

Figure 4-15 through Figure 4-25 compare measured heat losses to the FATE calculated heat losses. The calculated ceiling and wall heat losses are significantly higher than observed. The overestimate of the wall heat loss may be due to the additional coverage of the walls by the smoky layer in the FATE calculation. For example, when the heat loss to the walls below $1.0 \mathrm{~m}$ is not included in the FATE calculation significantly better agreement with the test data is obtained. Presumably since these wall elevations are submerged in the smoky layer in the FATE calculation but not in the test, the FATE heat transfer is overstated relative to the test. The over prediction of ceiling heat loss is a trend observed in other cases as well. Further investigation of code calculations and models is warranted. Overall, the predicted heat losses exceed the experimentally reported values, however general trends are predicted by the code.

Finally, Figure 4-26 compares the cable $\mathrm{F}$ target temperatures. For the higher fire power, even though FATE shows good agreement with the test data for the smoky layer temperature, the target temperatures are under predicted. The FATE heating of the target lags behind the test data and the higher heating rate for a shorter duration seems to have exacerbated the target temperature differences. This may be due to FATE assumptions regarding radiation heat transfer from the fire to the target (i.e., cylindrical fire geometry with radiation 
from cylinder sides, only; no radiation heat transfer once the target is submerged in the smoky layer) or convective heat transfer coefficients (i.e., the higher fire power may have resulted in more turbulent mixing at the cable surface leading to higher heat transfer coefficients) and requires further investigation.

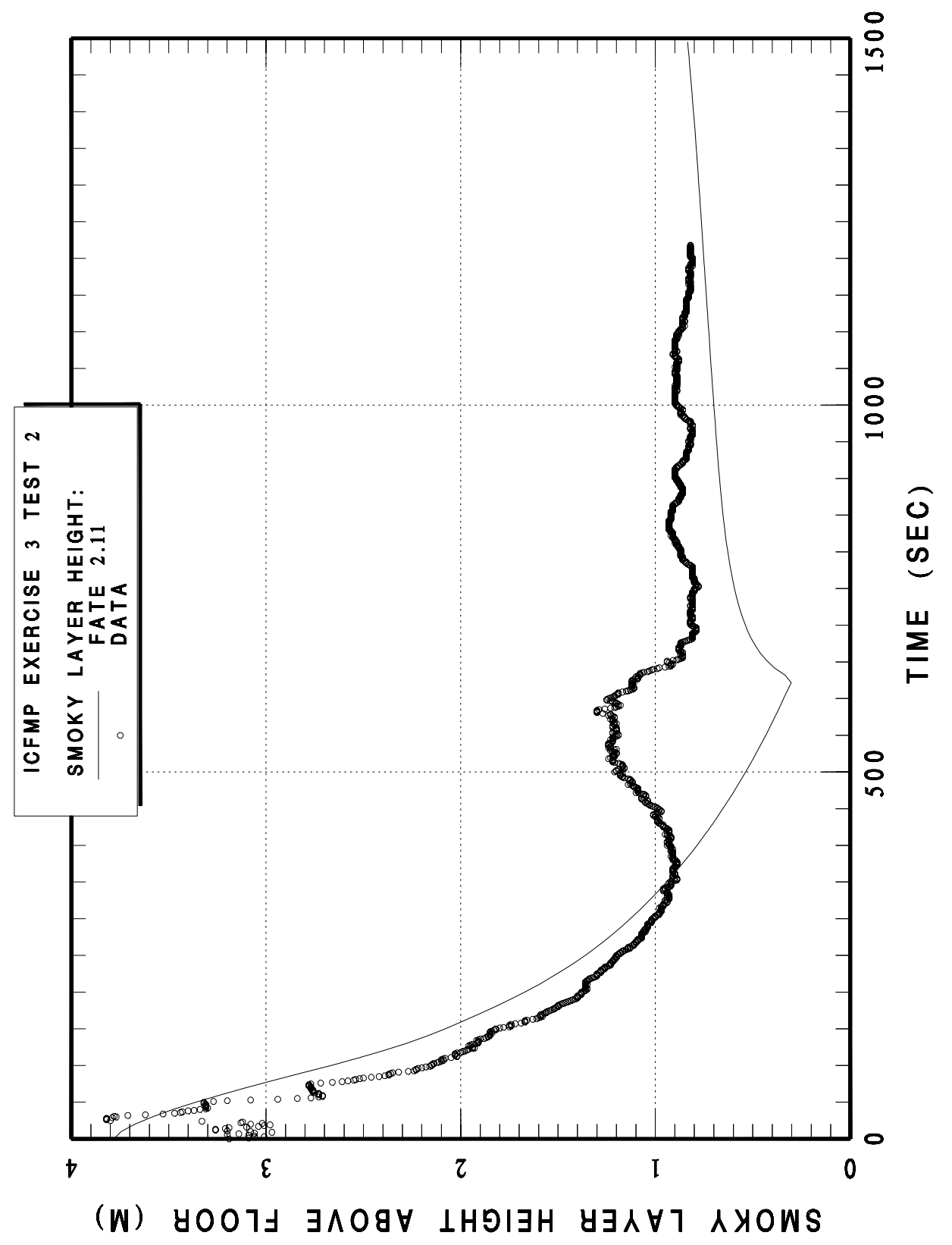

Figure 4-12 BE 3 CASE2 Smoky layer height 


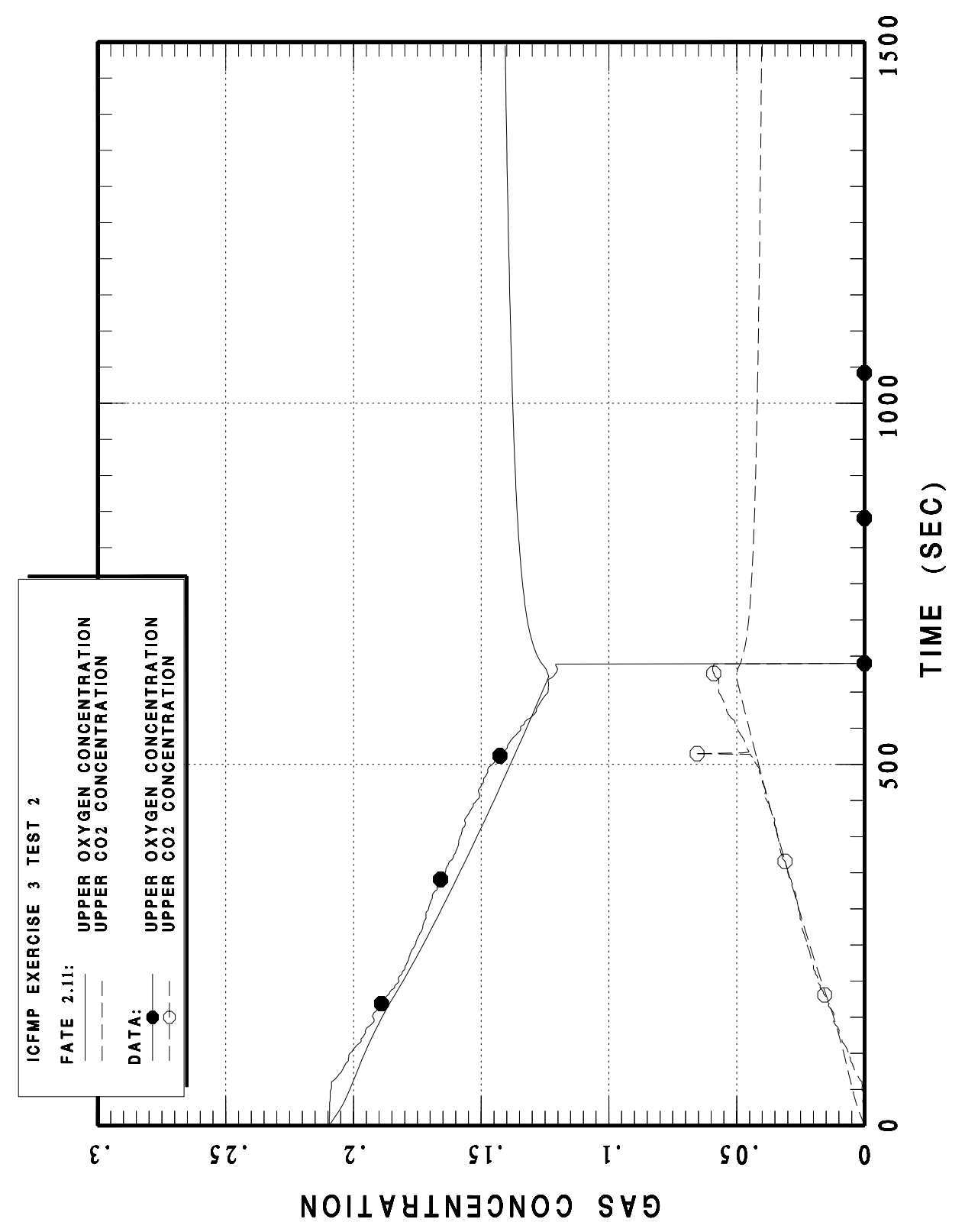

Figure 4-13 BE 3 CASE2 upper room gas concentrations 


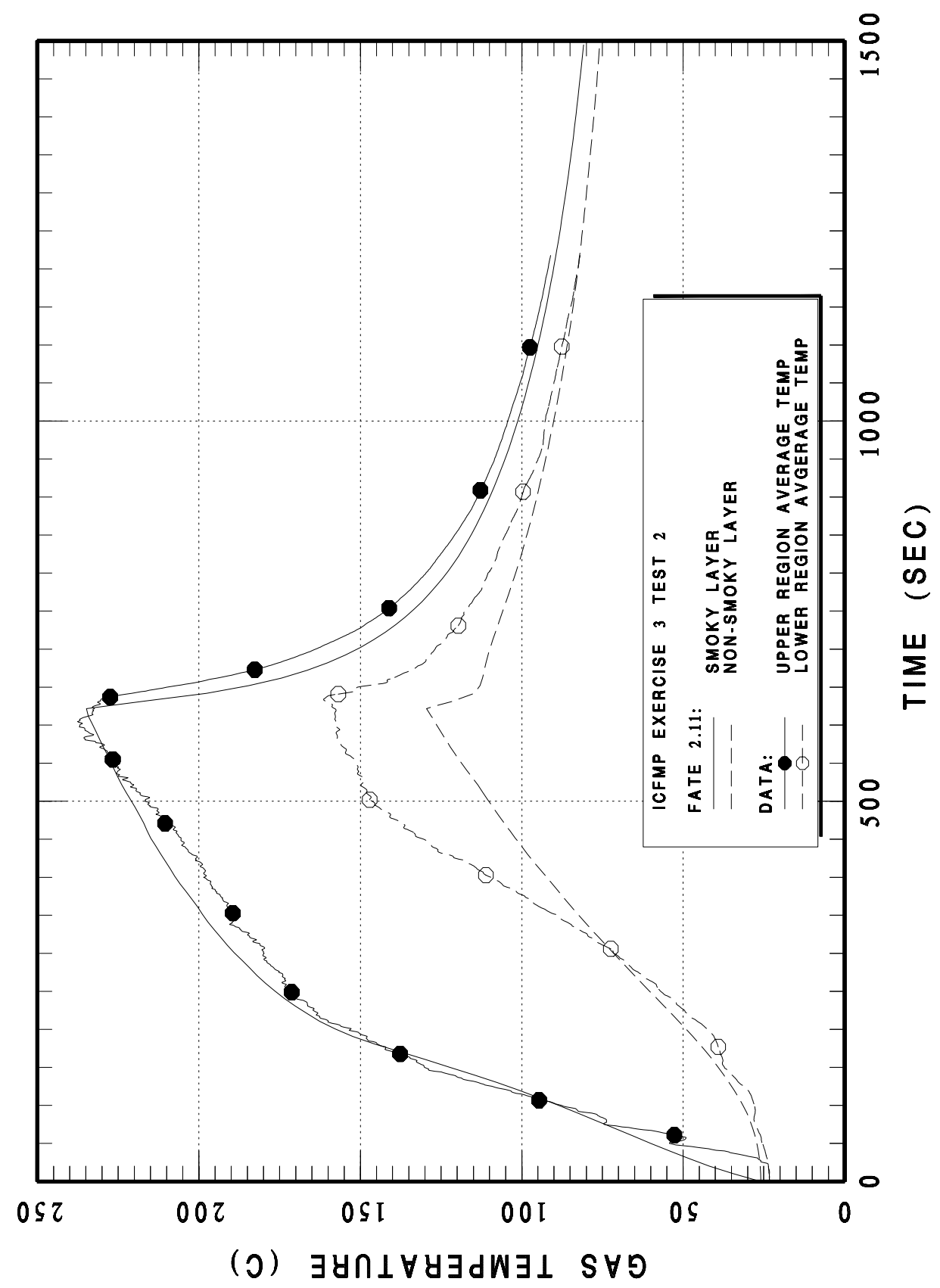

Figure 4-14 BE 3 CASE2 upper and lower layer average gas temperatures 


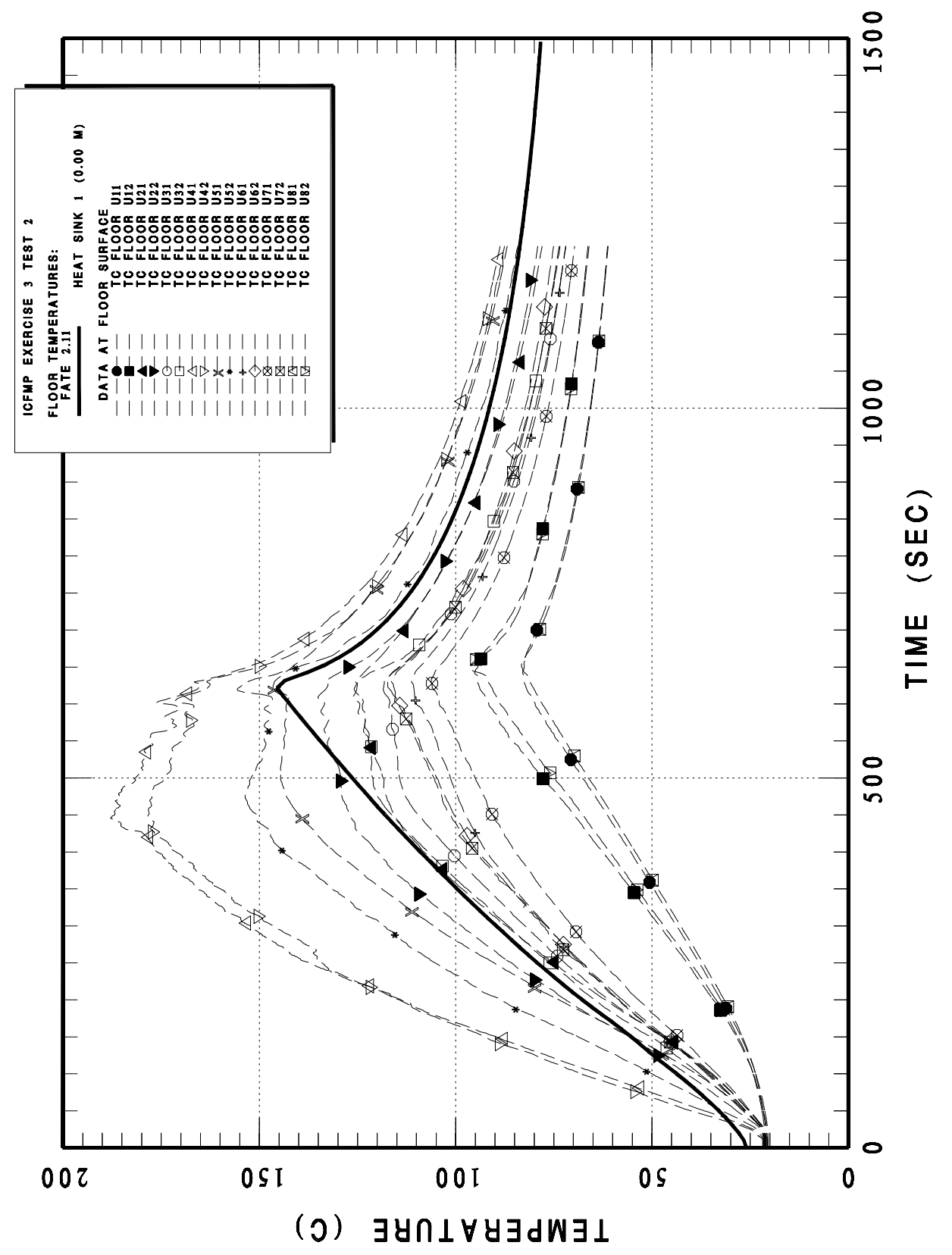

Figure 4-15 BE 3 CASE2 floor surface temperatures 


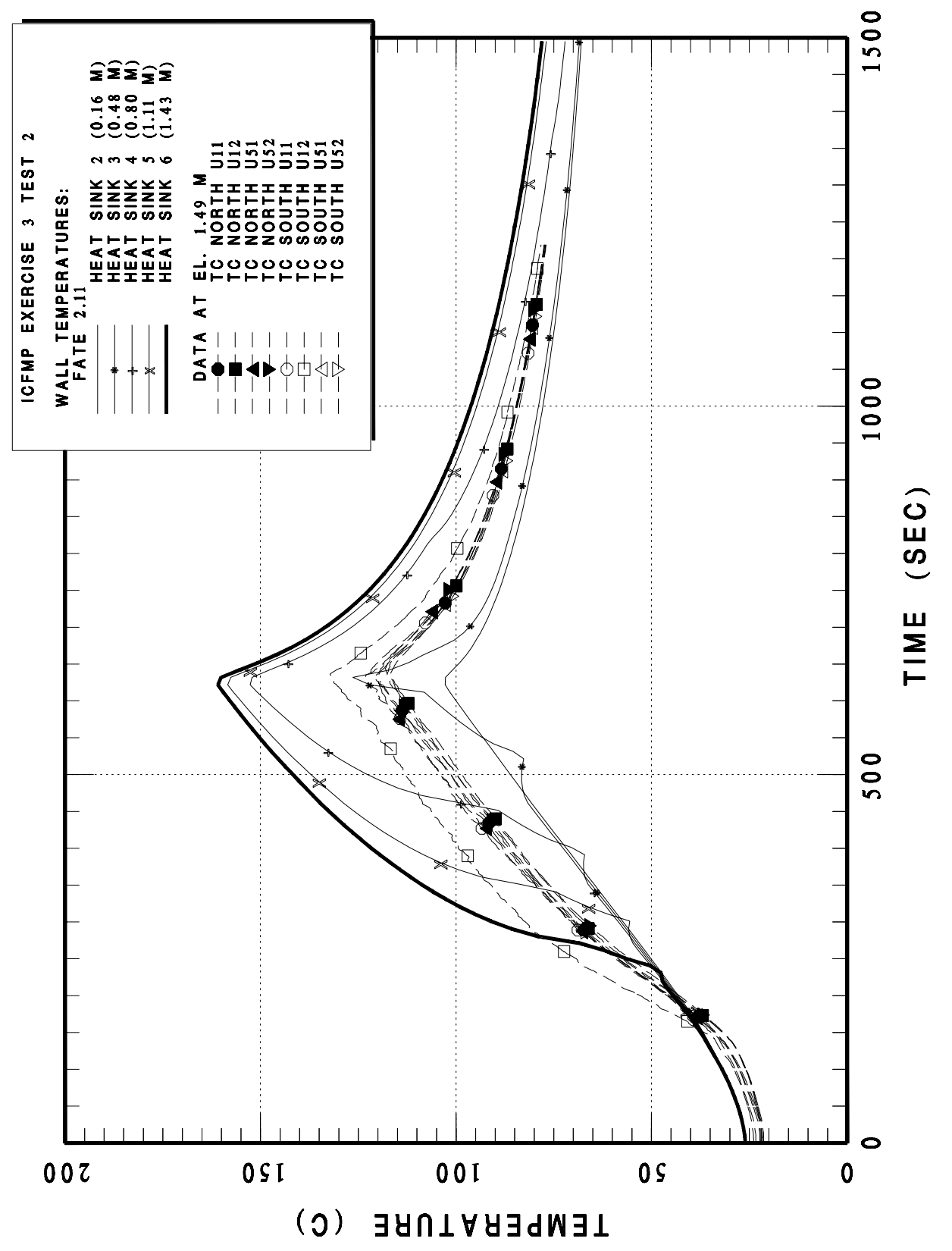

Figure 4-16 BE 3 CASE2 North and South wall temperatures at the 1.49 m elevation 


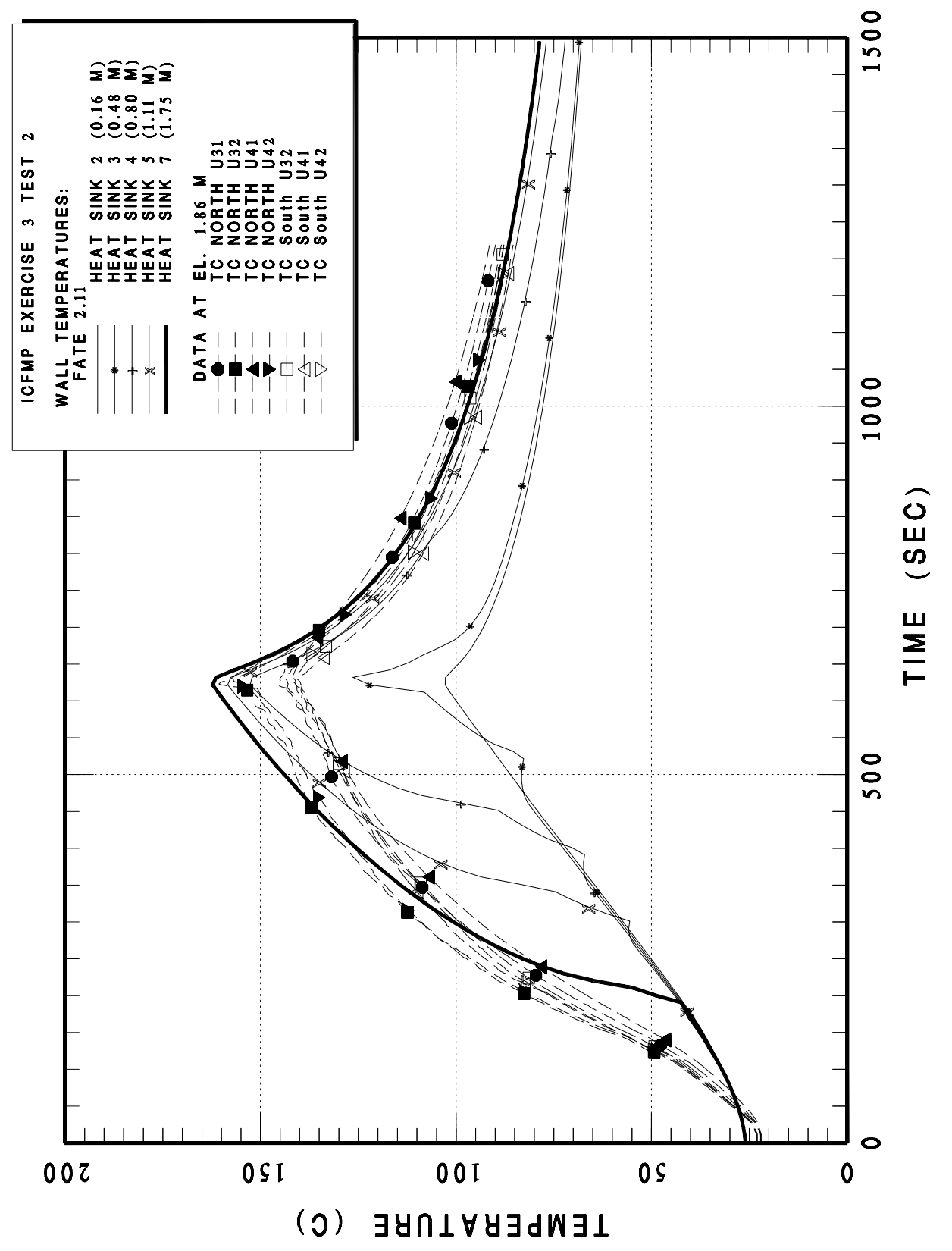

Figure 4-17 BE 3 CASE2 North and South wall temperatures at the 1.86 m elevation 


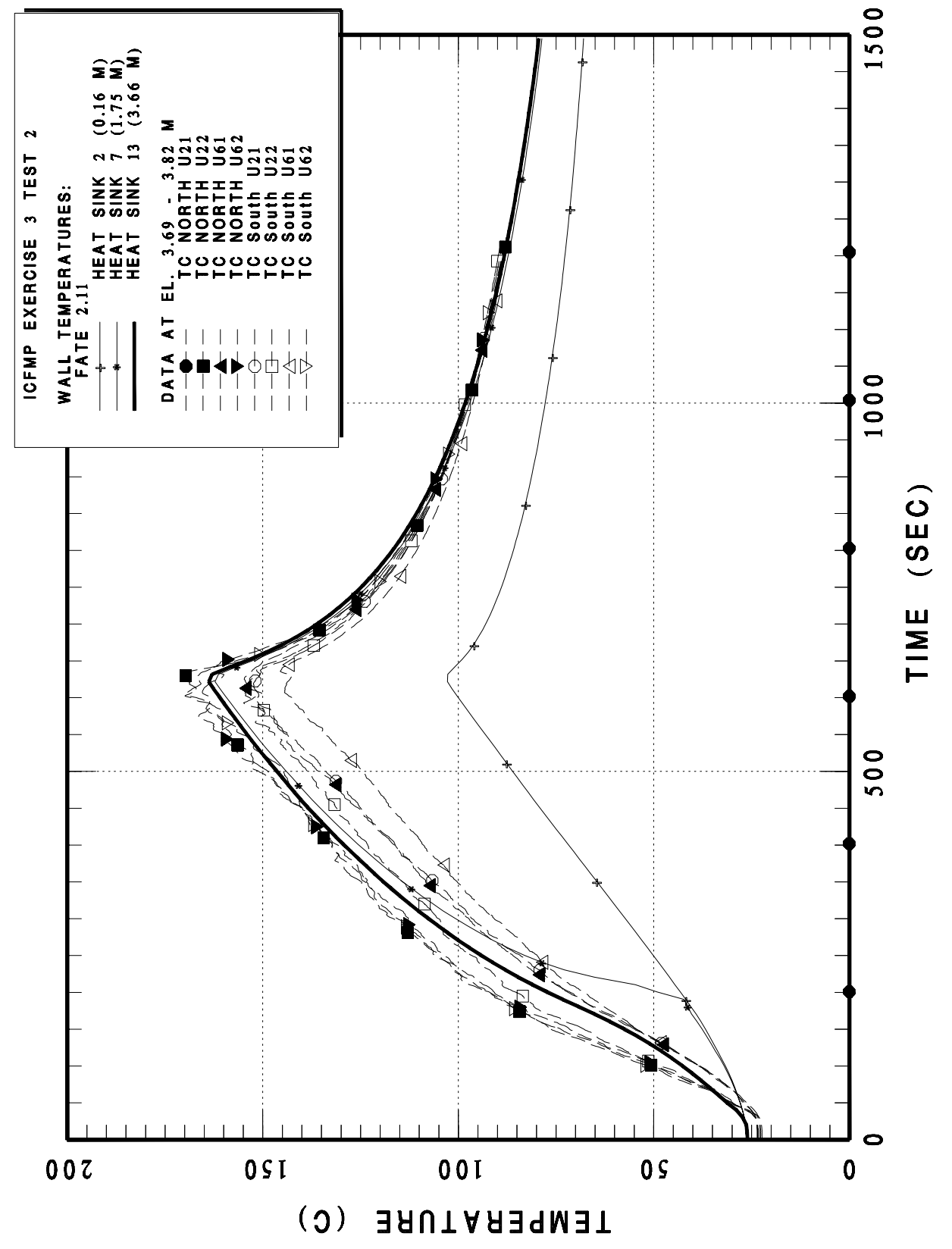

Figure 4-18 BE 3 CASE2 North and South wall temperatures between the 3.69 and $3.82 \mathrm{~m}$ elevations 


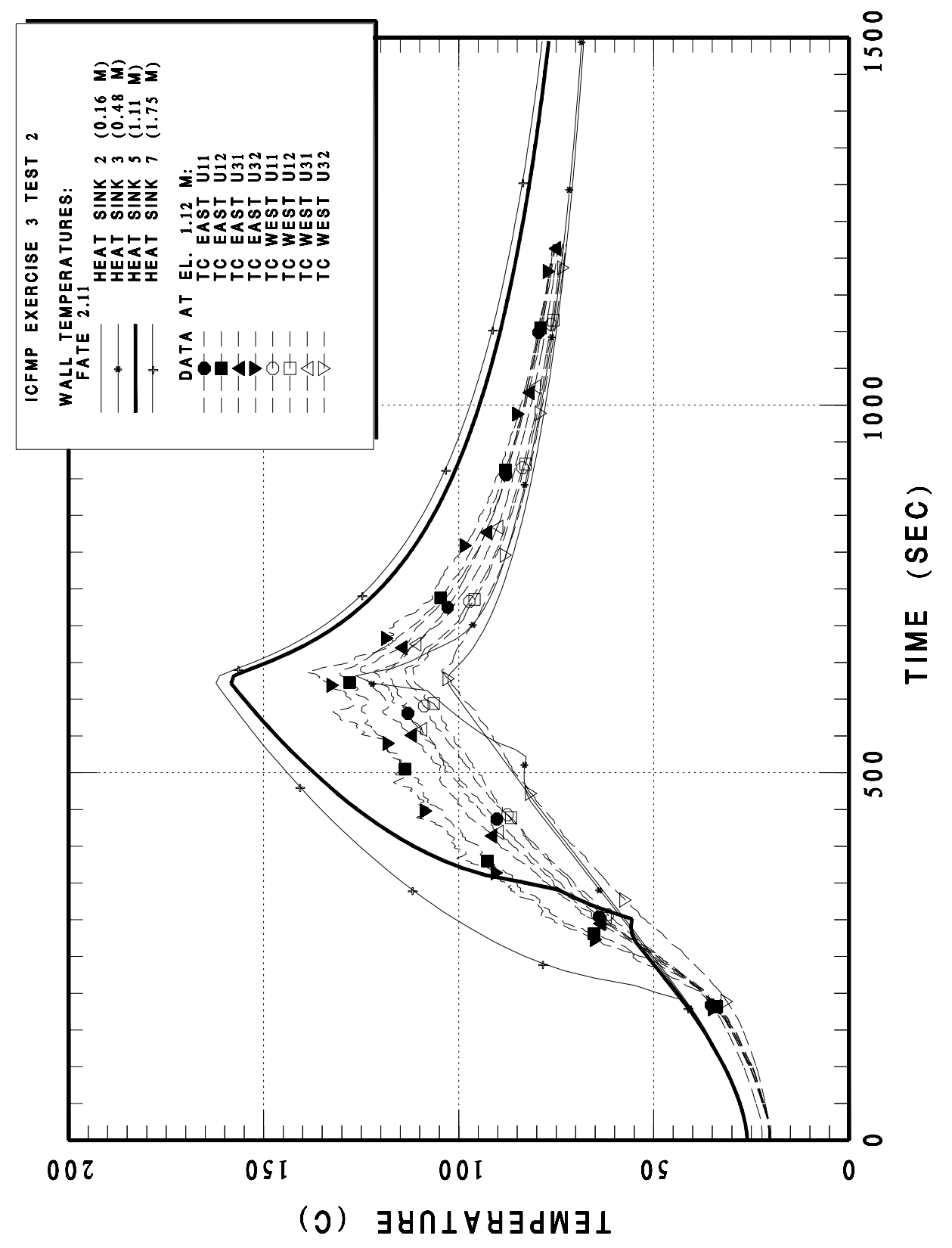

Figure 4-19 BE 3 CASE2 East and West wall temperatures at the $1.12 \mathrm{~m}$ elevation 


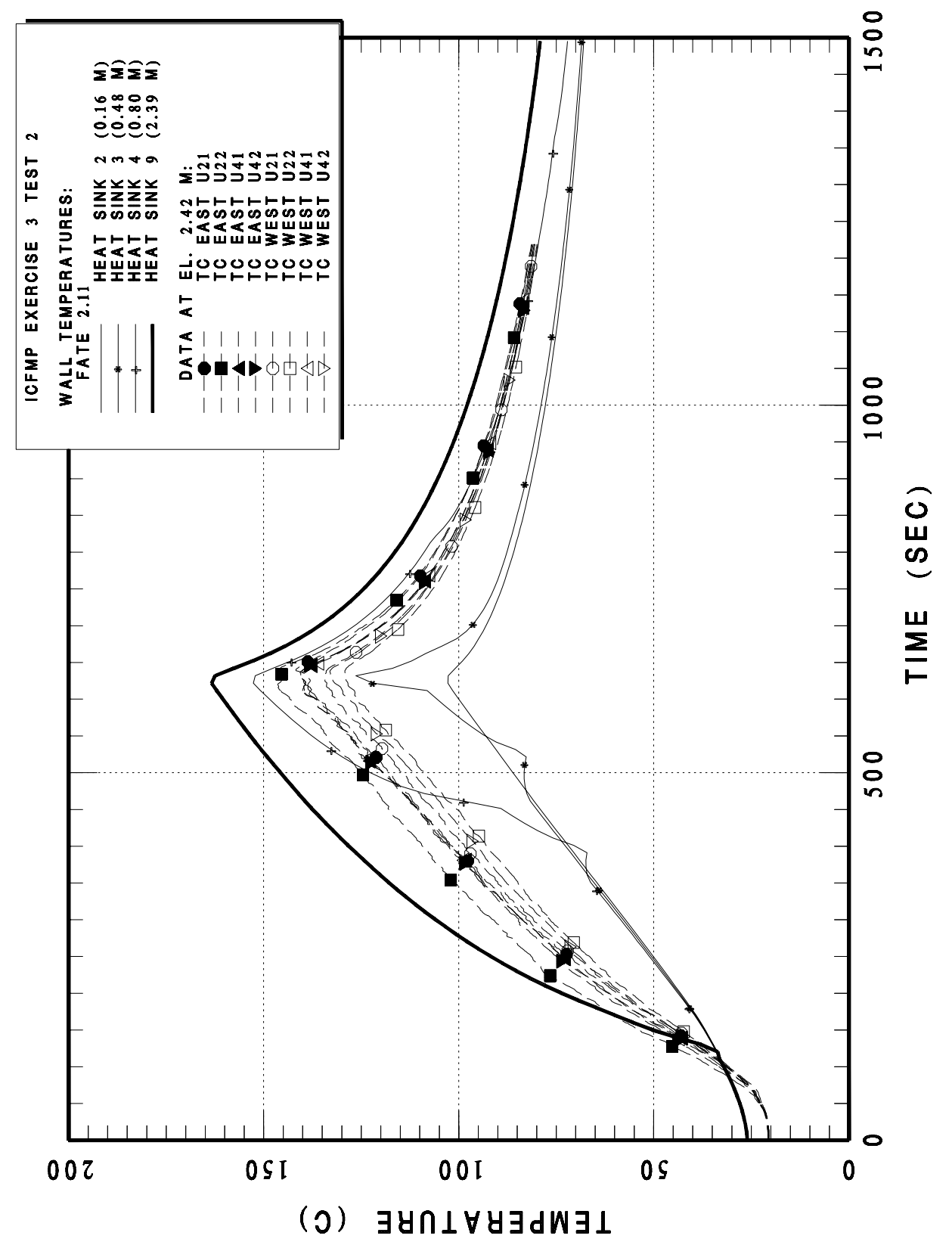

Figure 4-20 BE 3 CASE2 East and West wall temperatures at the $2.42 \mathrm{~m}$ elevation 


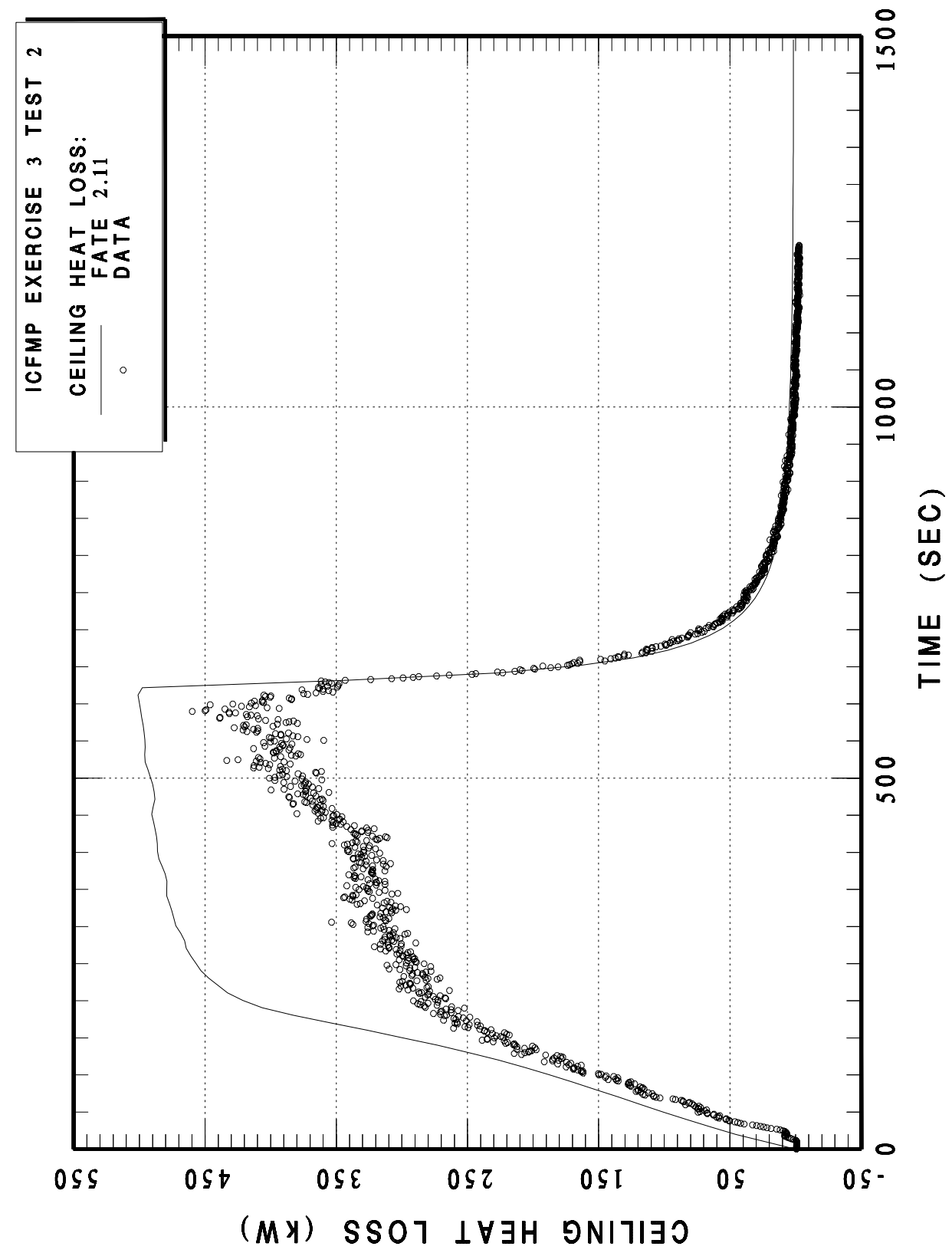

Figure 4-21 BE 3 CASE2 ceiling heat loss (convective and radiative) 


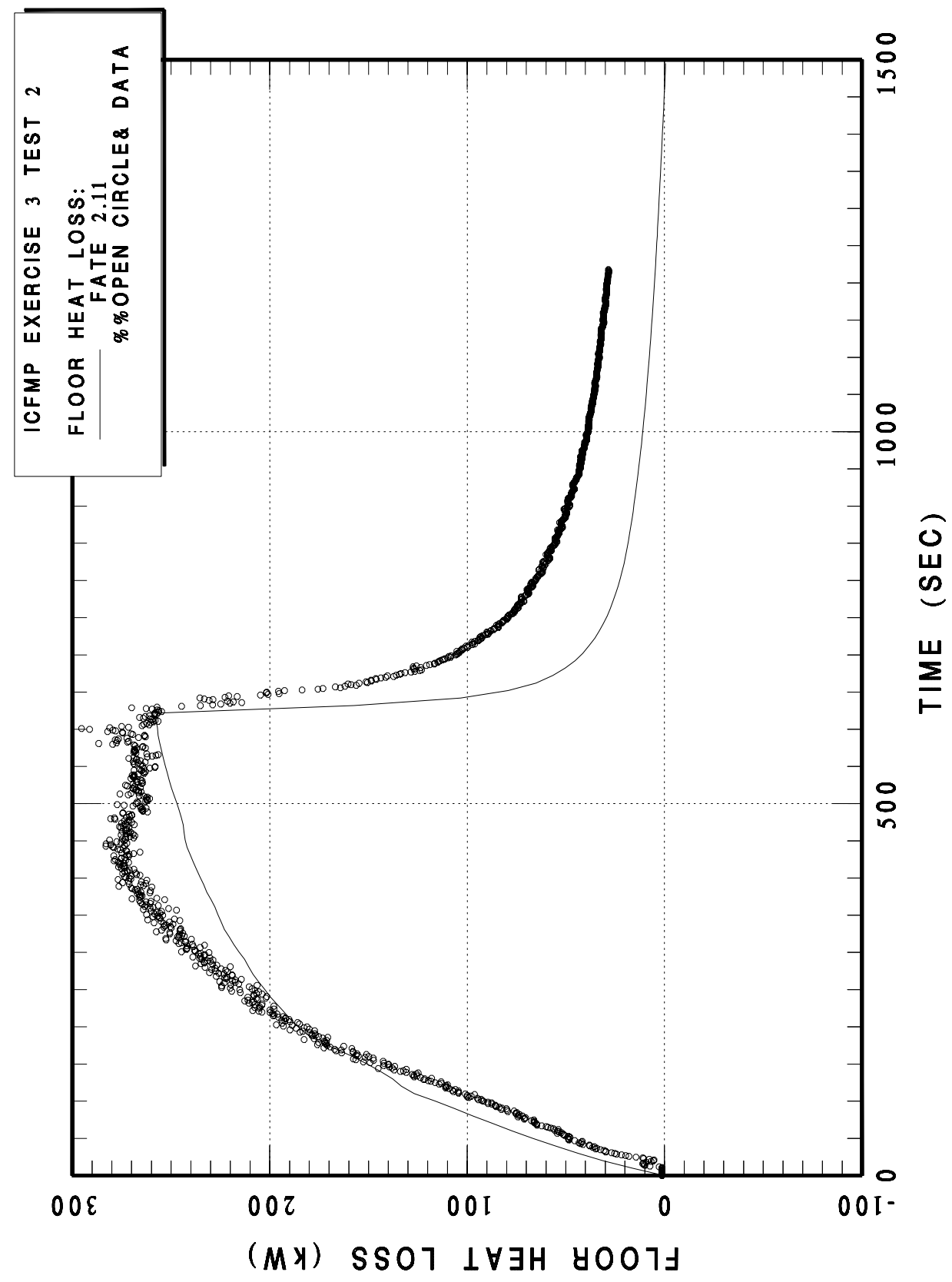

Figure 4-22 BE 3 CASE2 floor heat loss (convective and radiative) 


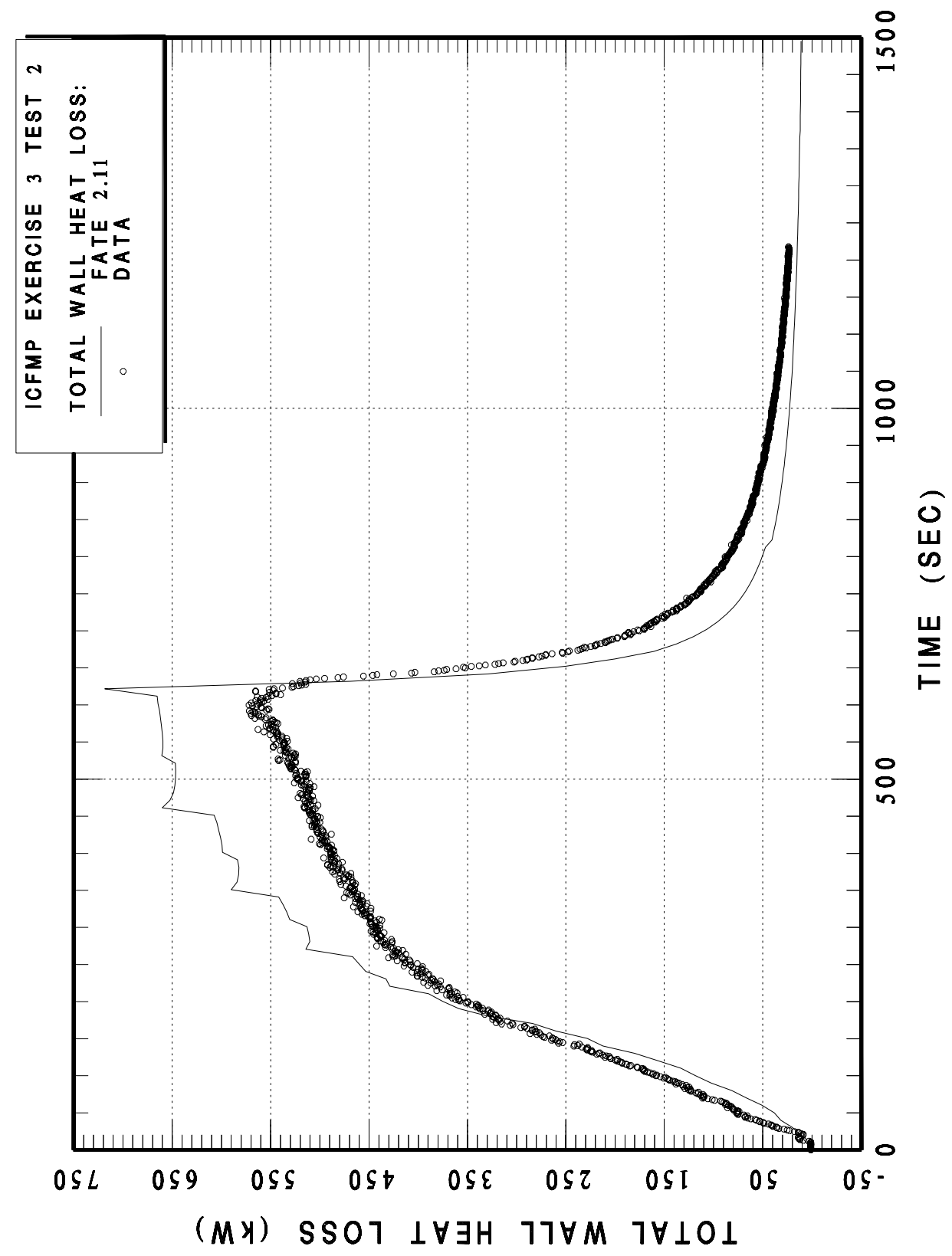

Figure 4-23 BE 3 CASE2 side wall heat loss (convective and radiative) 


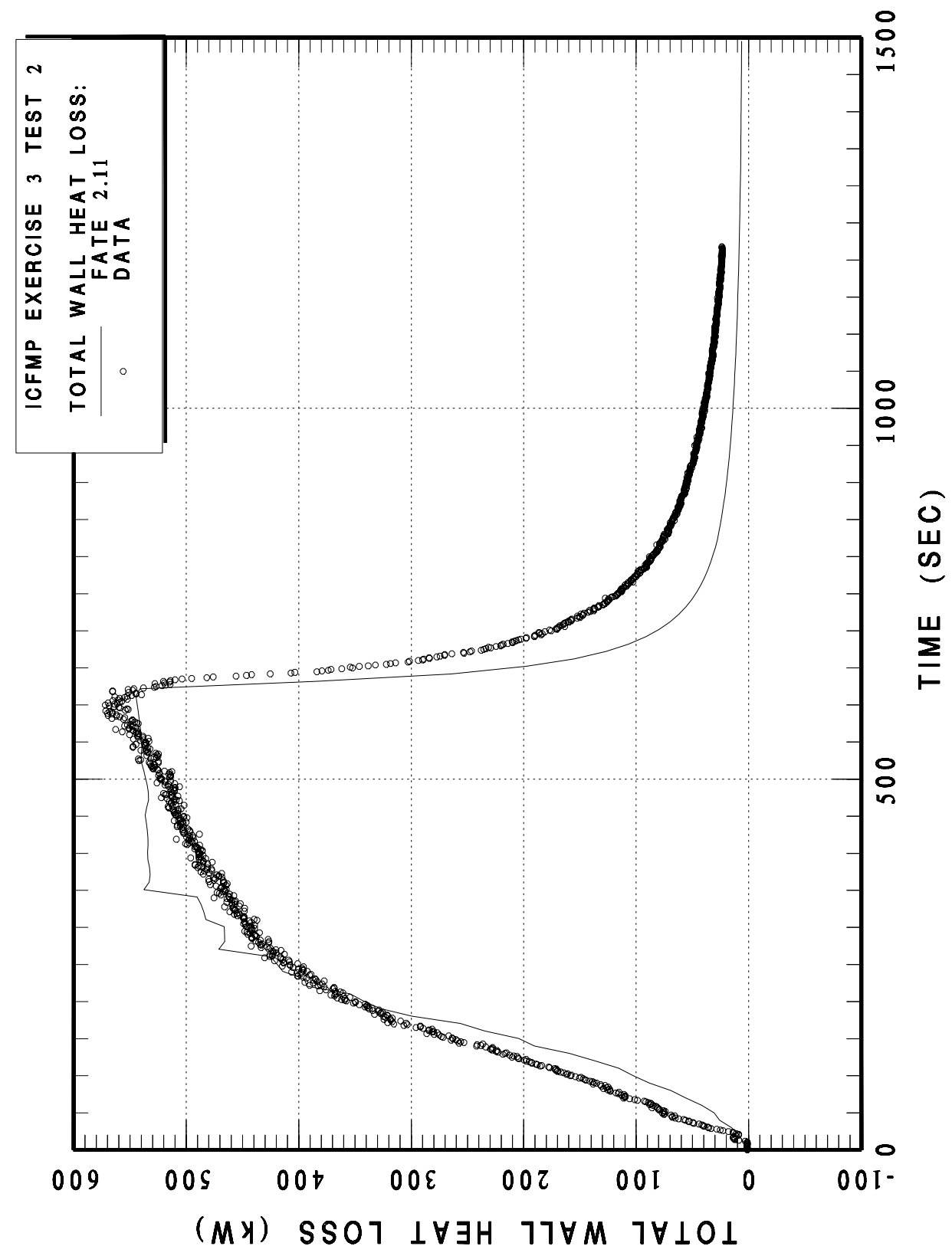

Figure 4-24 BE 3 CASE2 side wall heat loss not including convective heat loss in FATE for wall strips below $1.0 \mathrm{~m}$ (convective and radiative) 


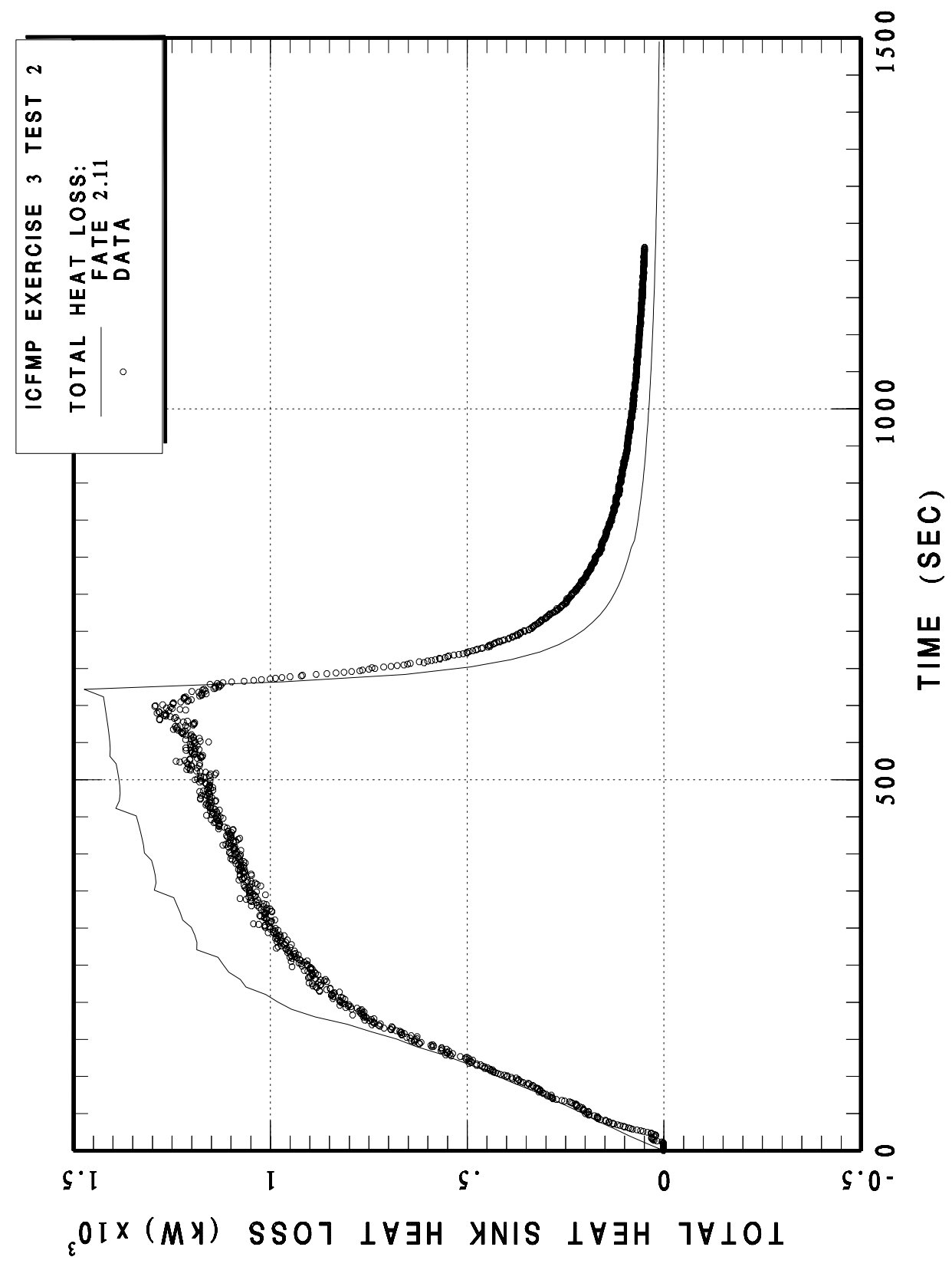

Figure 4-25 BE 3 CASE2 total heat loss to ceiling, floor, and walls (convective and radiative) 


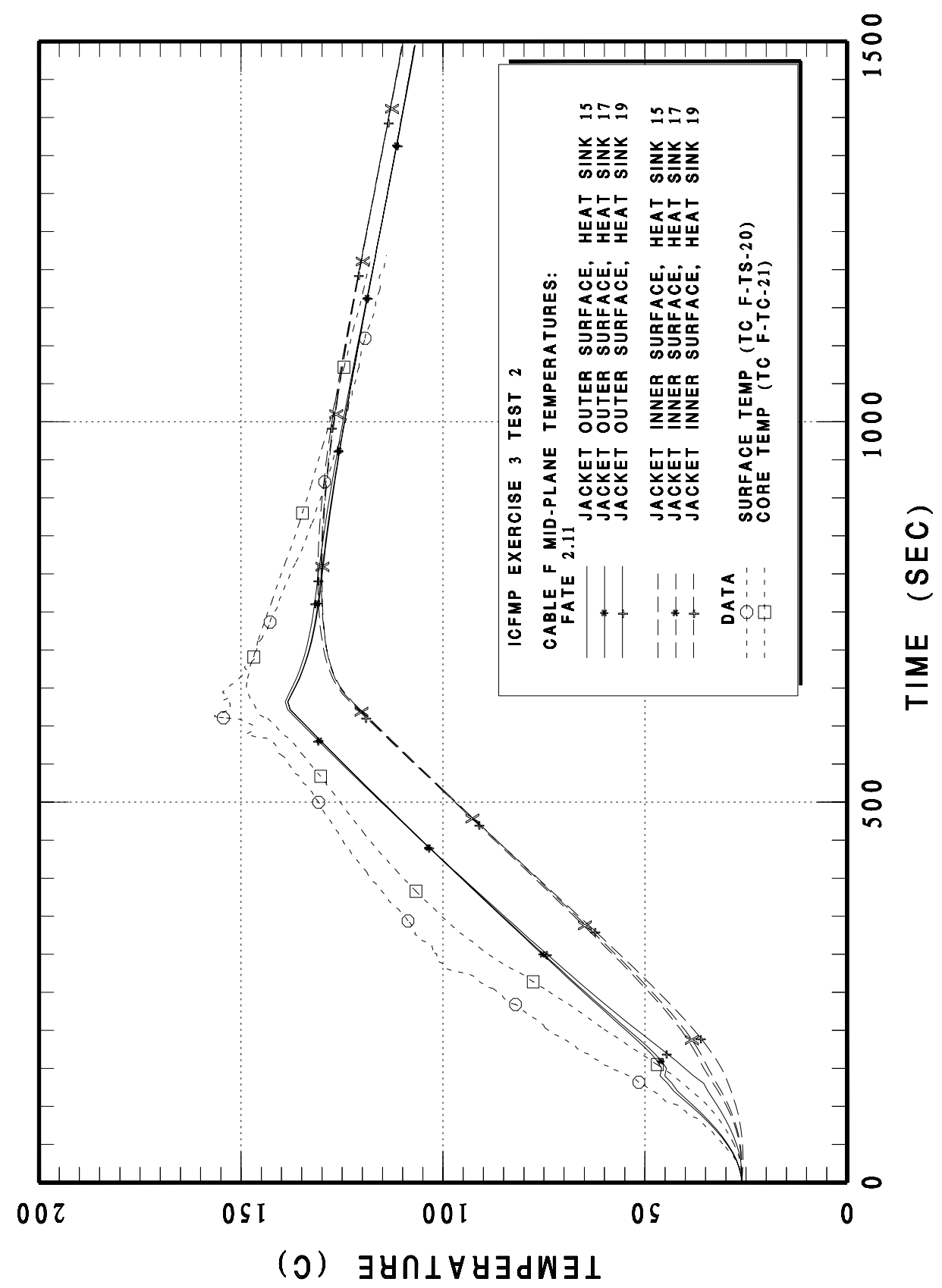

Figure 4-26 BE 3 CASE2 Cable F midplane temperatures 


\subsection{3}

BE-3 Case 3 FATE Open Benchmark Results

Case 3 is characterized by a heptanes fuel fire with a peak power of $1150 \mathrm{~kW}$ and natural ventilation through the opened $2 \mathrm{~m} \times 2 \mathrm{~m}$ doorway. However, there is no forced ventilation as the fan is off. FATE calculations for this case use the same vent supply and exhaust leak path assumptions as are used in Cases 1 and 2 and add the open doorway as an additional flow path. As with Cases 1 and 2, it is assumed that inflow through the doorway is split equally between the smoky and lower layers. As will be shown, the relatively low flow leak paths are influential in the smoky layer behavior and assumptions regarding flow split are also influential. In addition to providing heat transfer validation, this case also validates models and assumptions regarding counter-current flow through an open doorway when the doorway is partially submerged in the smoky layer. FATE calculations for BE-3 Case 3 are presented in Figure 4-27 through Figure 4-45.

It is interesting to note that even with the door open, the smoky layer reported from the test data behaves similarly to Cases 1 and 2 where the door was closed. That is, the minimum elevation is about $1 \mathrm{~m}$ and following fire termination, the smoky layer does not recede or recedes only slightly and remains below the top of the door for the duration of the 2000 second comparison. FATE calculations show similar behavior if the door inflow is assumed to be split equally between the smoky and lower layers. In contrast, if the door inflow is assumed to go into the lower layer only, the FATE calculated smoky layer stops near the door mid-plane elevation and then recedes to the top of the ventilation ducts where the presumed leakage is modeled. Finally, if the door inflow is assumed to flow only into the smoky layer, then the FATE calculations closely follow the test data and the smoky layer recedes only slightly following fire termination. These sensitivity cases illustrate the importance crediting a flow split of all inflows between the smoky and lower layers and suggest that a mechanistic model for flow splitting is necessary. As a result of this benchmark exercise, a mechanistic model for flow splitting is now being developed for FATE.

Additional FATE sensitivity calculations, presented in Figure 4-30, suggest that the smoky layer behavior is also influenced by counter current flow through the door and the leak path elevation. As shown, when doorway counter-current flow is stopped (artificially), the smoky layer descends to $0.5 \mathrm{~m}$ before receding to the vent elevation. If the leak top elevation is reduced to $1.5 \mathrm{~m}$ and counter-current flow is restored, then the smoky layer recedes to 2.1 which is corresponds to the top of the doorway. This insight adds to that gained from the Case 1 comparison - counter-current flow and room leakage play a prominent role in the behavior of the smoky layer. 
The comparisons of layer temperatures and gas concentrations suggest that the fire power is higher in the FATE calculation than actually experienced in the test case. The presumably higher power level in the calculation contributes to higher floor and wall temperatures and higher heat transfer rates. Once again, the prediction of ceiling heat transfer is significantly higher than indicated by the test data.

Finally, FATE provides a good prediction of the peak cable temperature. However the initial heat up rate lags behind the test data.

Additional data is provided for this test to measure uni-directional and counter-current flow through the door. Generally good agreement is indicated between FATE and the test data. The door loss coefficient was reduced to 0.54 (one-half the recommended value) to obtain the agreement indicated in the figures. 


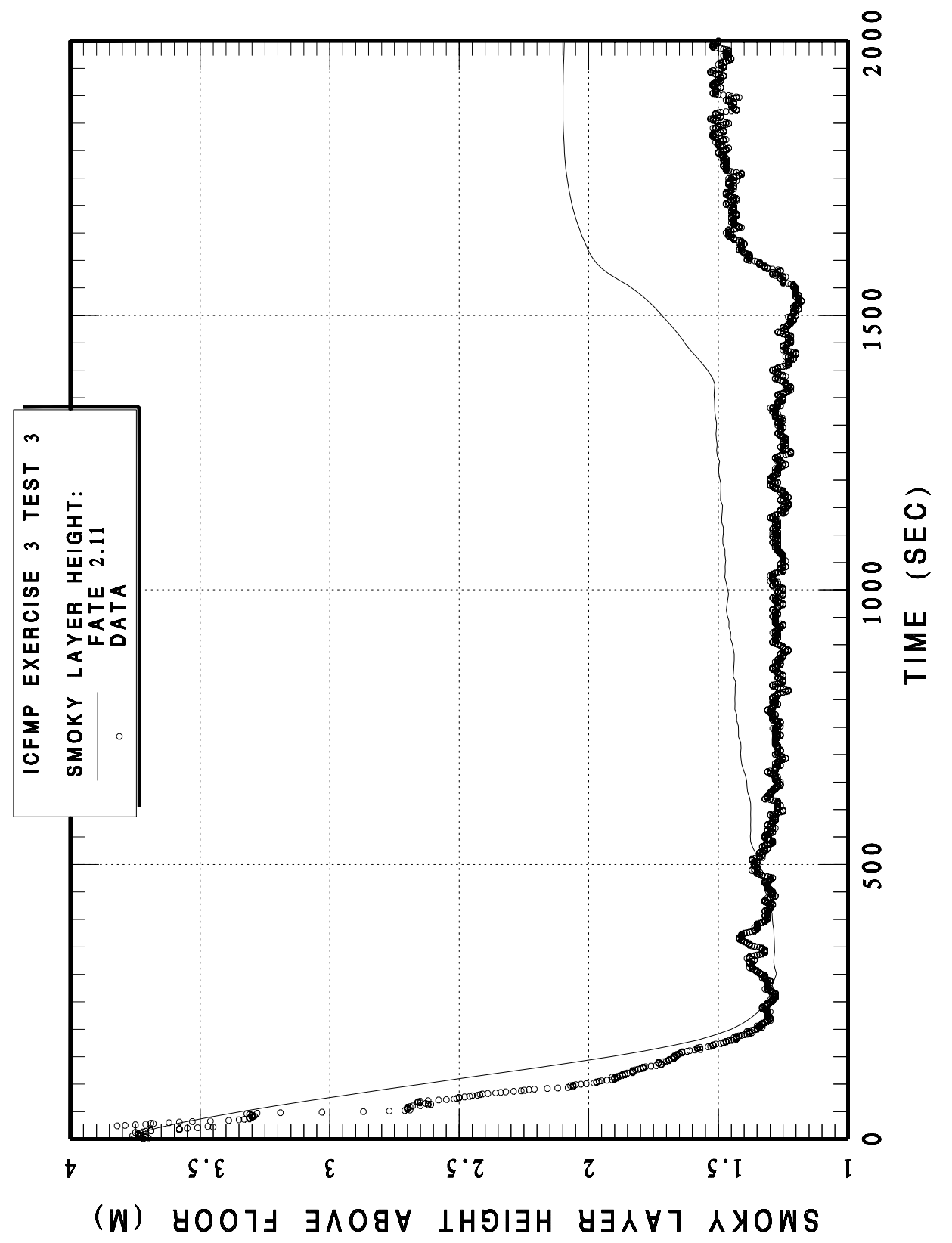

Figure 4-27 BE 3 CASE3 Smoky layer height. Door inflow is split equally between the smoky and lower layers. 


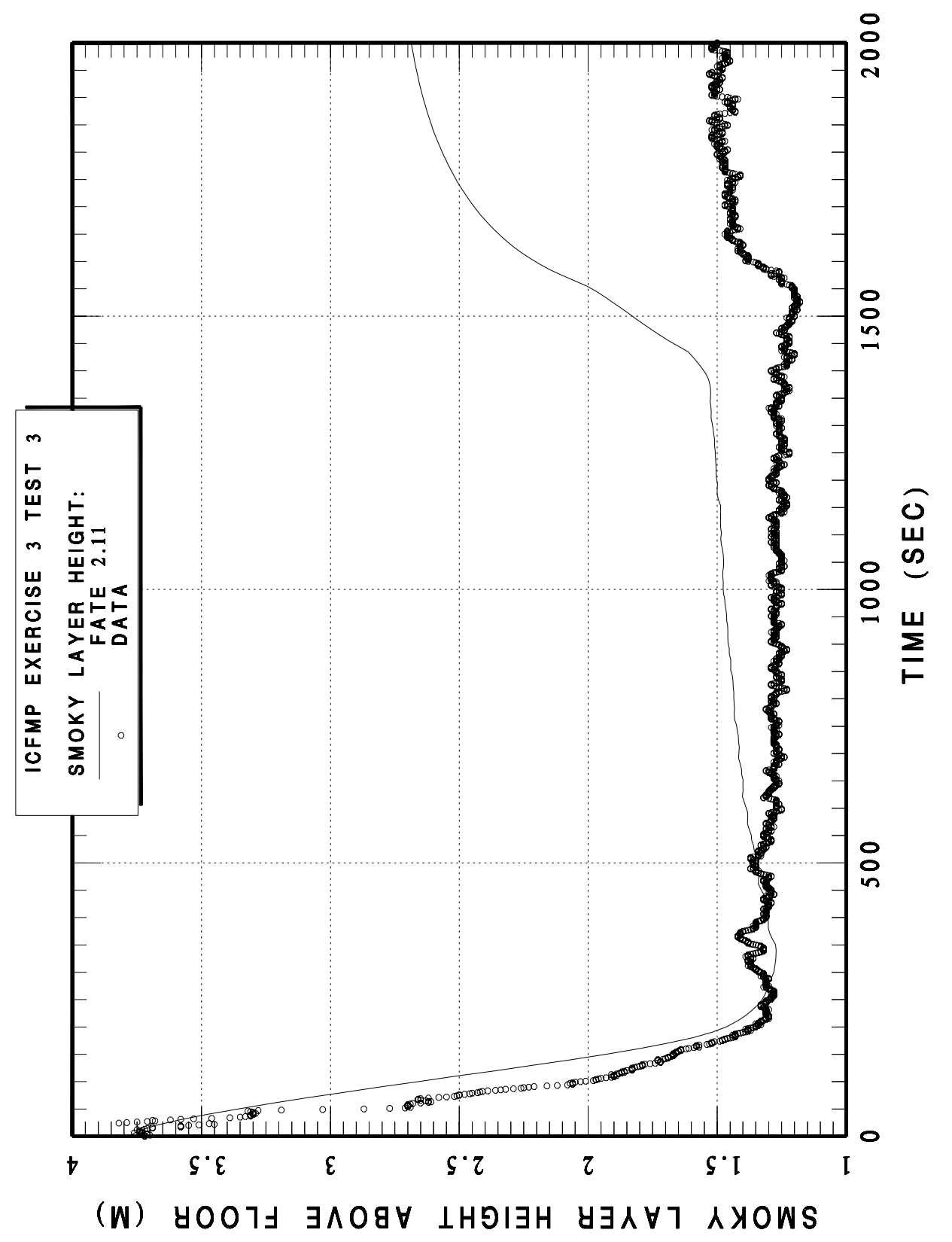

Figure 4-28 BE 3 CASE3 Smoky layer height. All door inflow goes to the lower layer. 


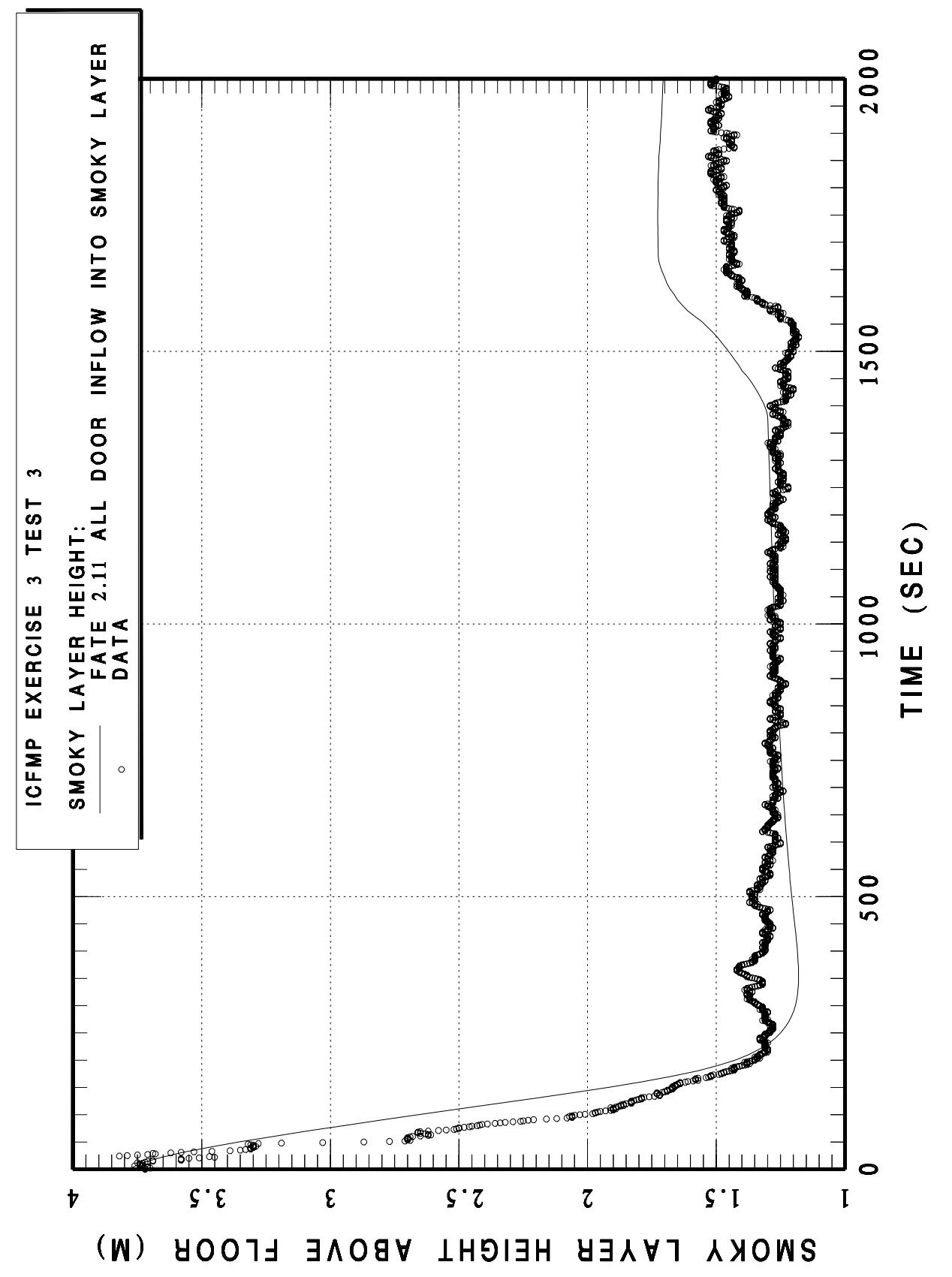

Figure 4-29 BE 3 CASE3 Smoky layer height. All door inflow goes to the smoky layer. 


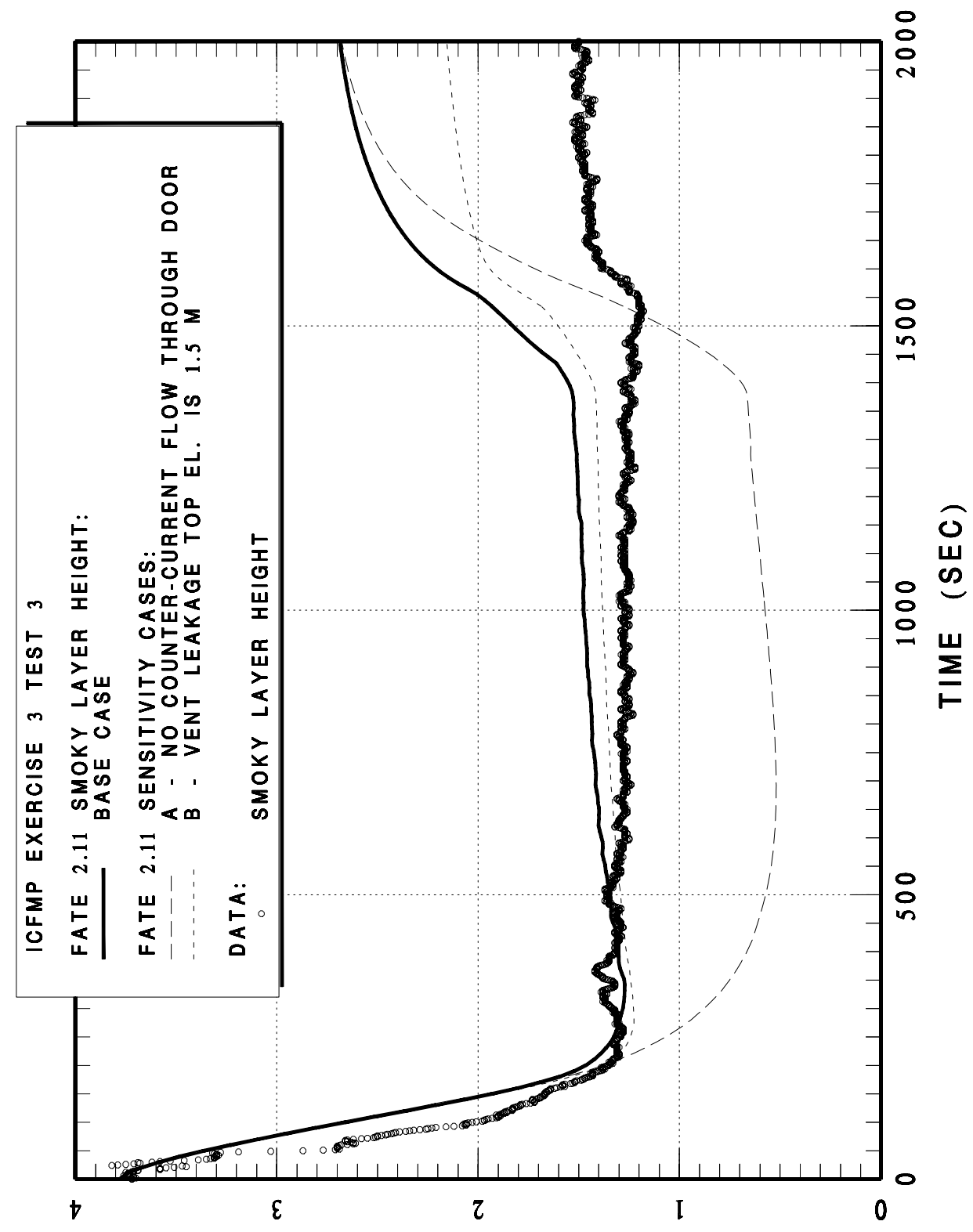

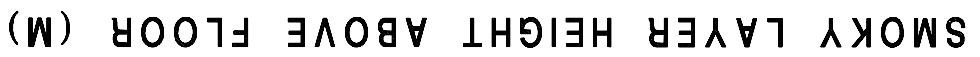

Figure 4-30 BE 3 CASE3 Smoky layer height sensitivity calculations with all door inflow going to the lower layer. 


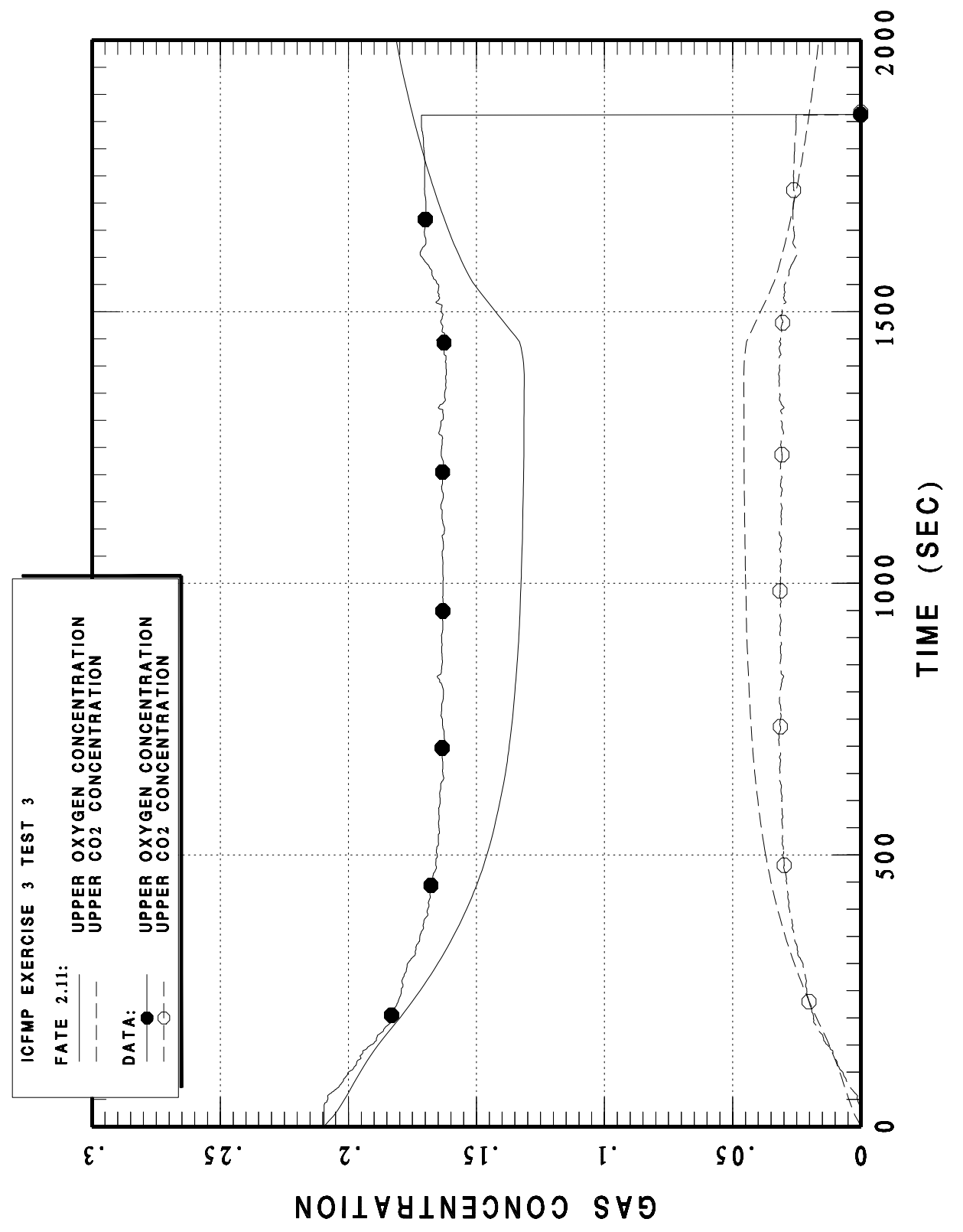

Figure 4-31 BE 3 CASE3 upper room gas concentrations 


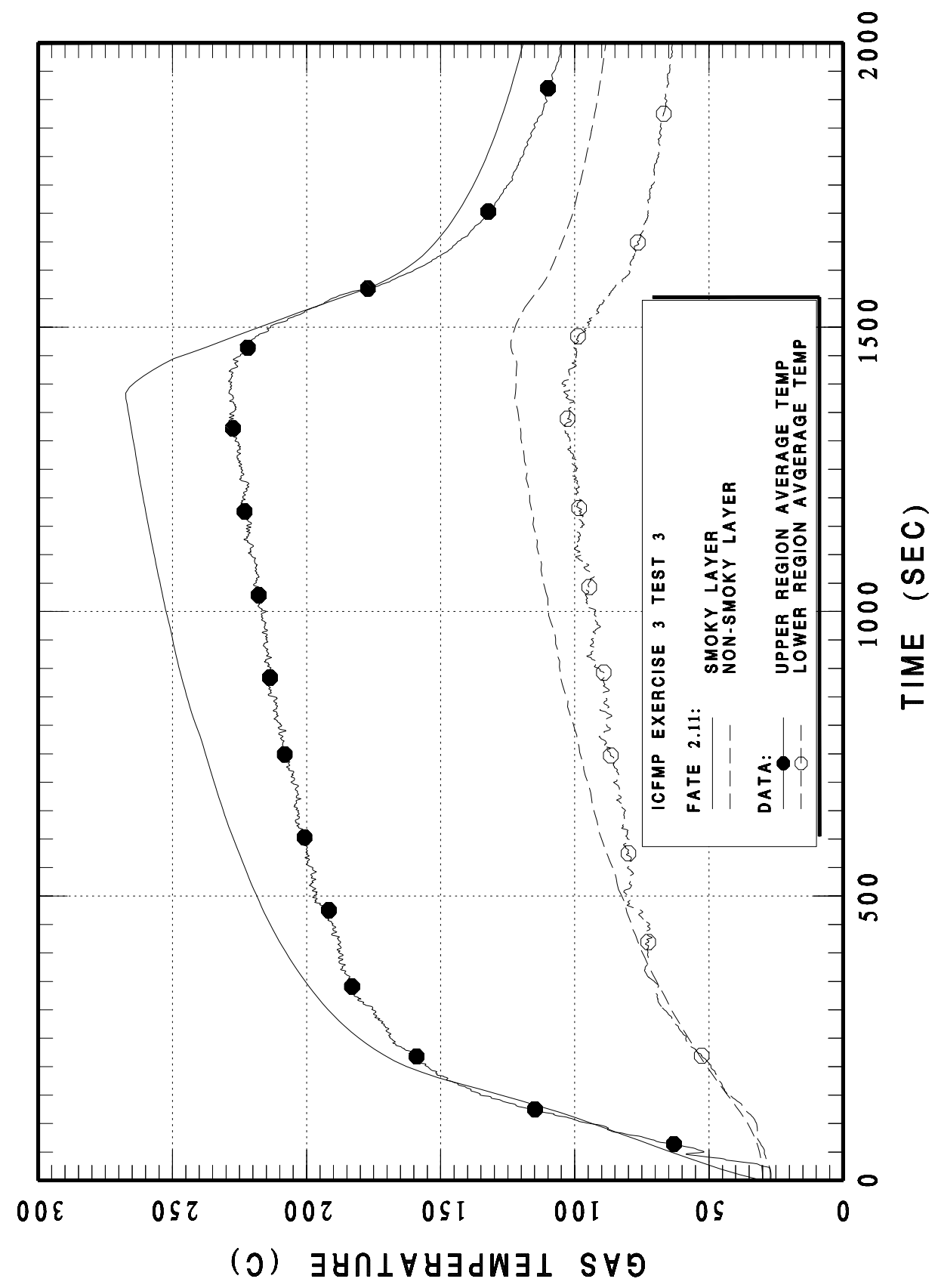

Figure 4-32 BE 3 CASE3 upper and lower layer average gas temperatures 


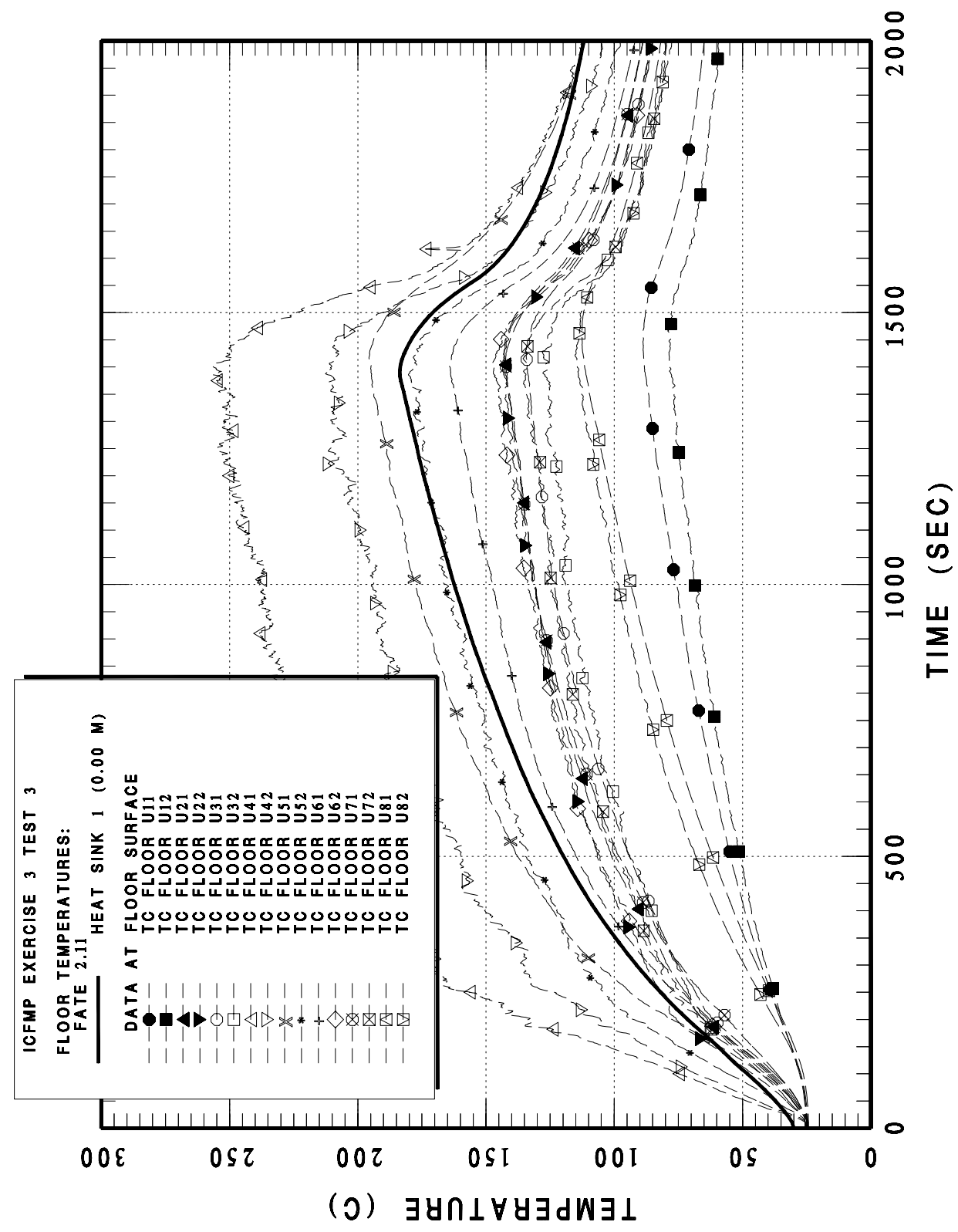

Figure 4-33 BE 3 CASE3 floor surface temperatures 


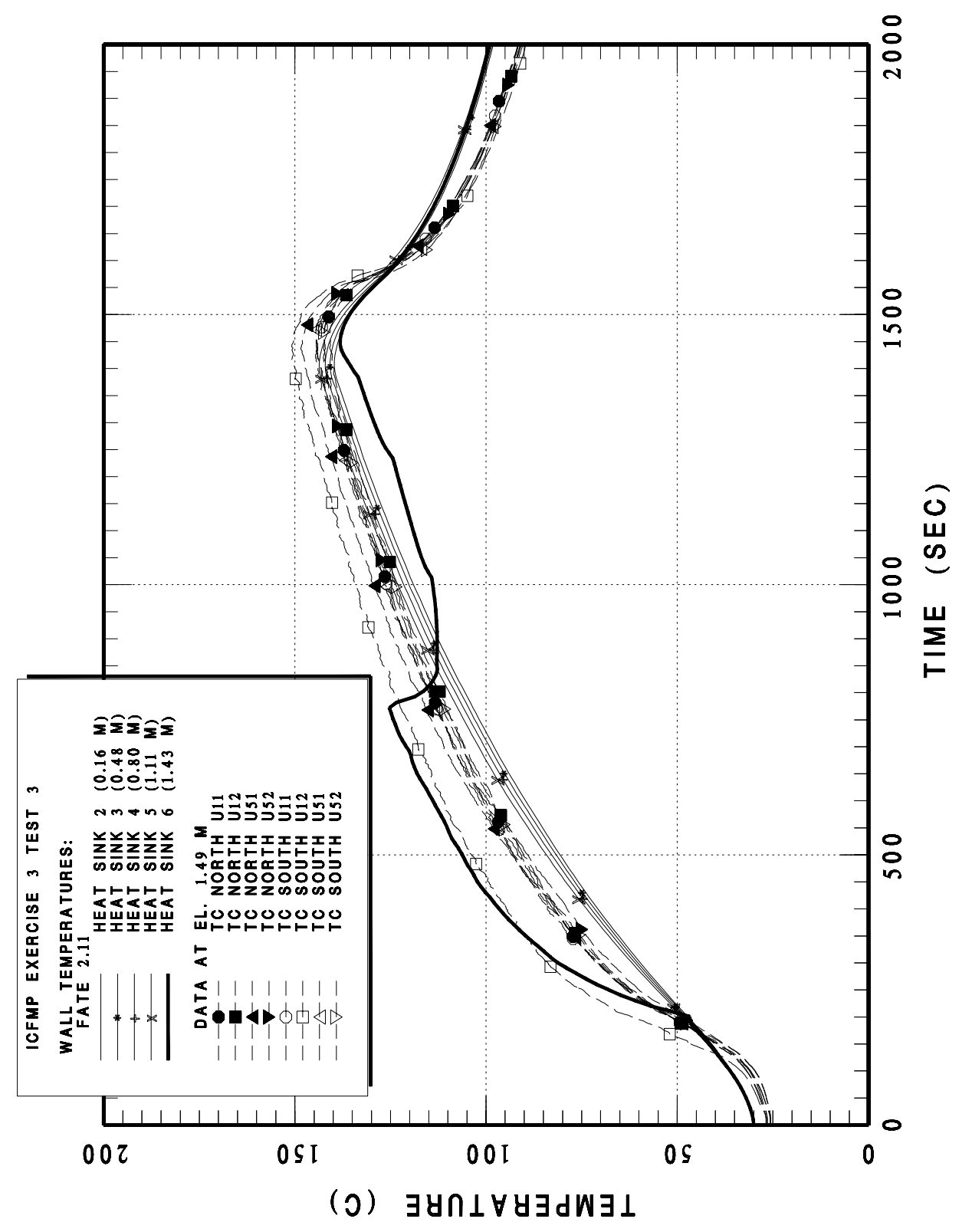

Figure 4-34 BE 3 CASE3 North and South wall temperatures at the $1.49 \mathrm{~m}$ elevation 


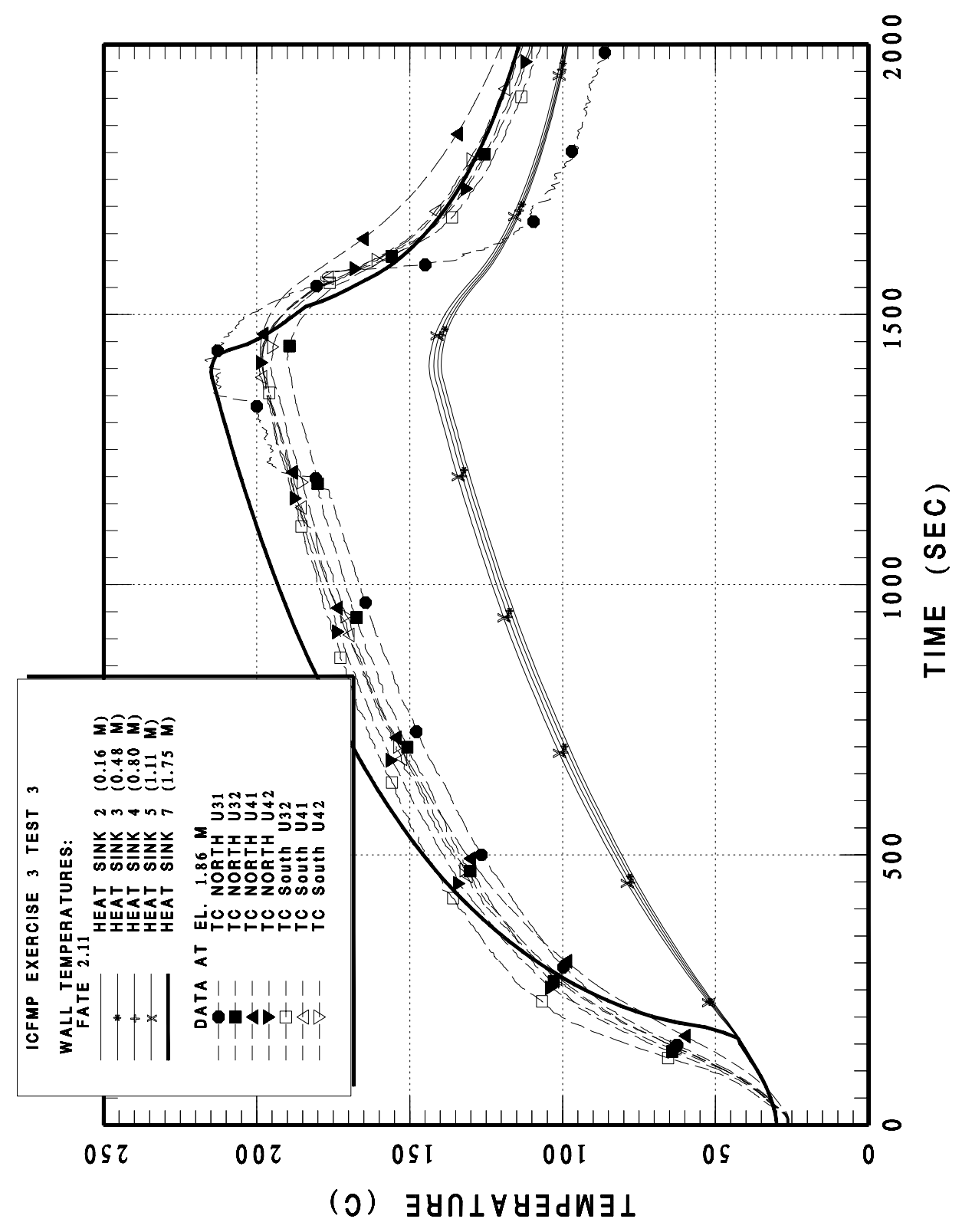

Figure 4-35 BE 3 CASE3 North and South wall temperatures at the $1.86 \mathrm{~m}$ elevation 


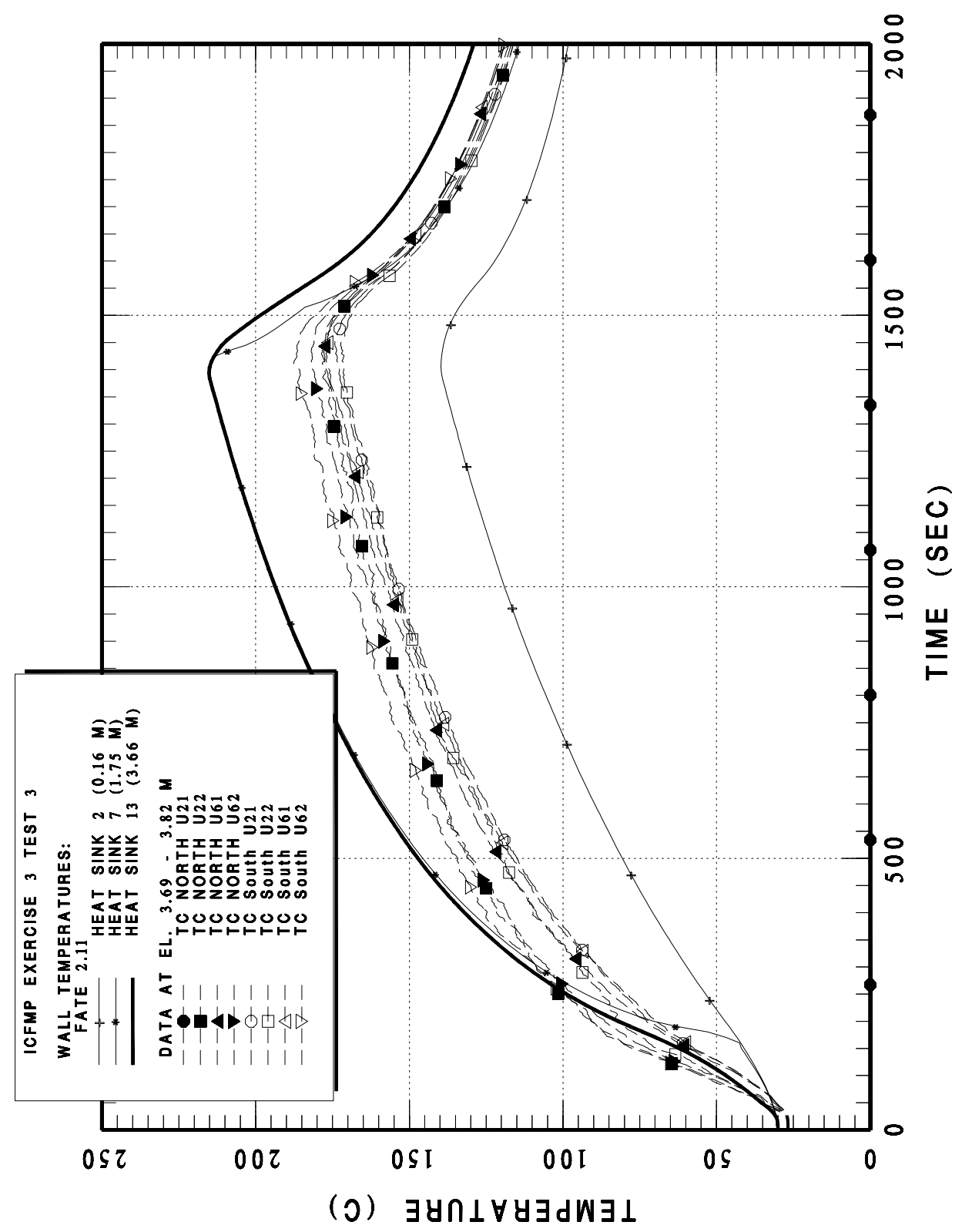

Figure 4-36 BE 3 CASE3 North and South wall temperatures between the 3.69 and $3.82 \mathrm{~m}$ elevations 


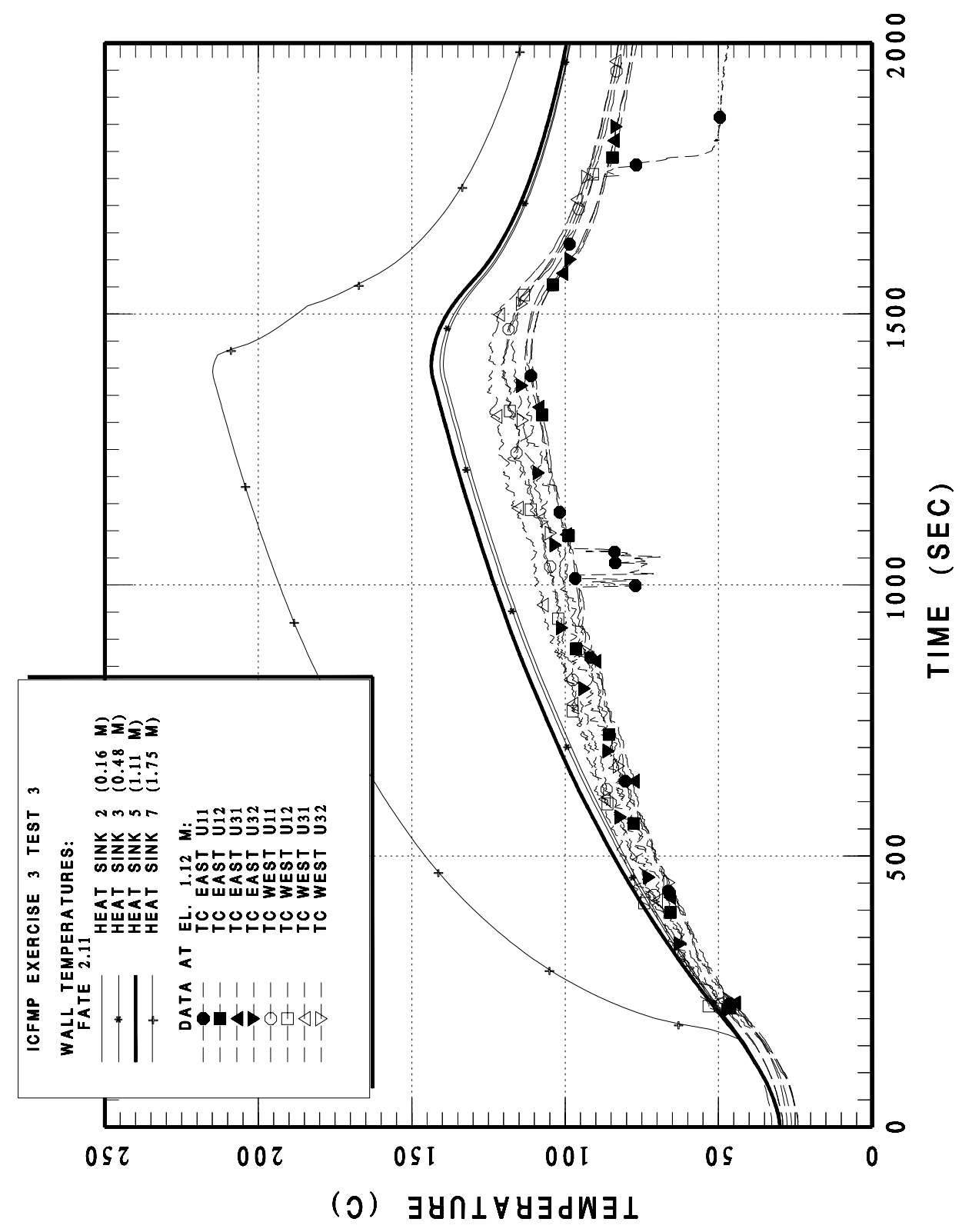

Figure 4-37 BE 3 CASE3 East and West wall temperatures at the $1.12 \mathrm{~m}$ elevation 


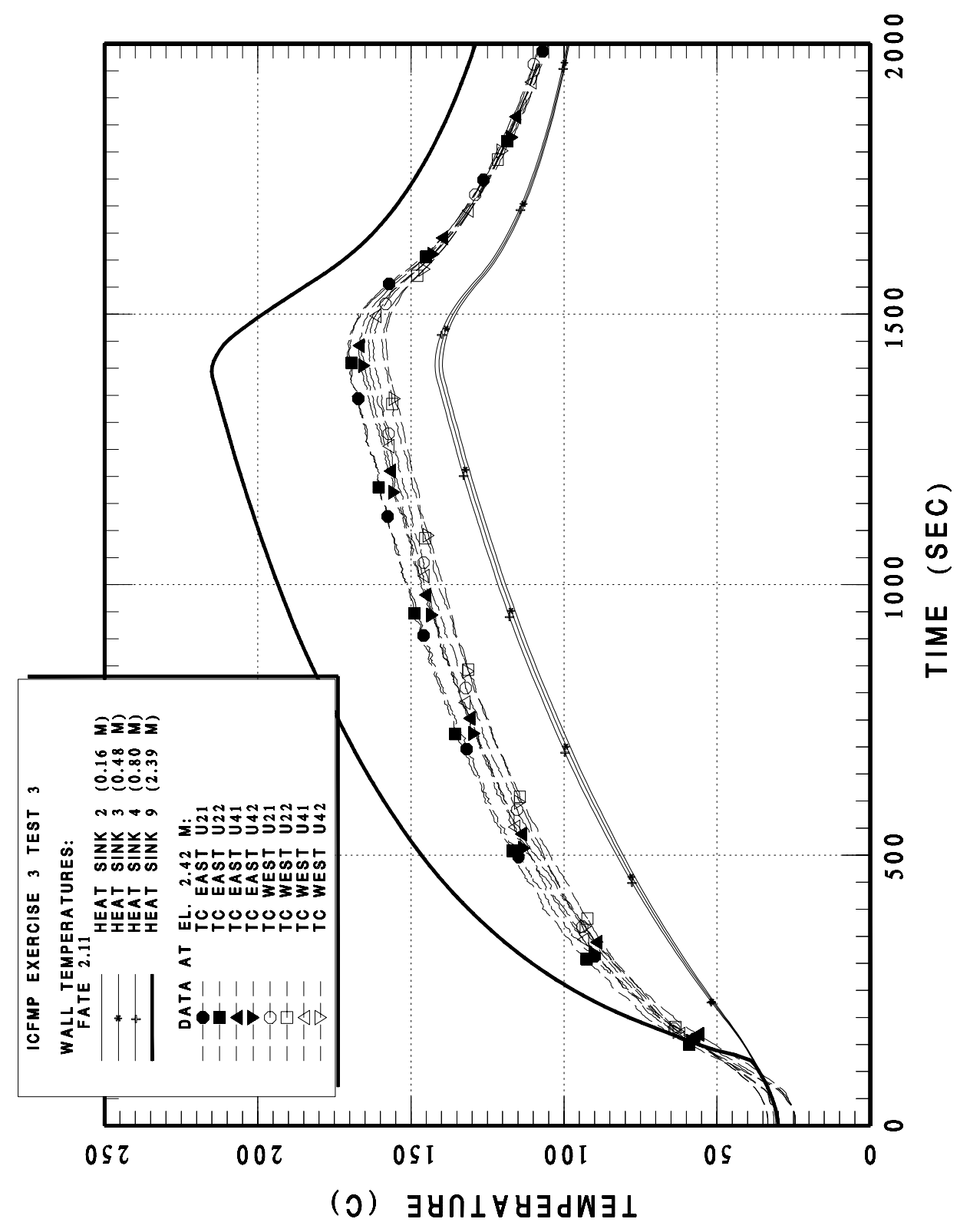

Figure 4-38 BE 3 CASE3 East and West wall temperatures at the 2.42 m elevation 


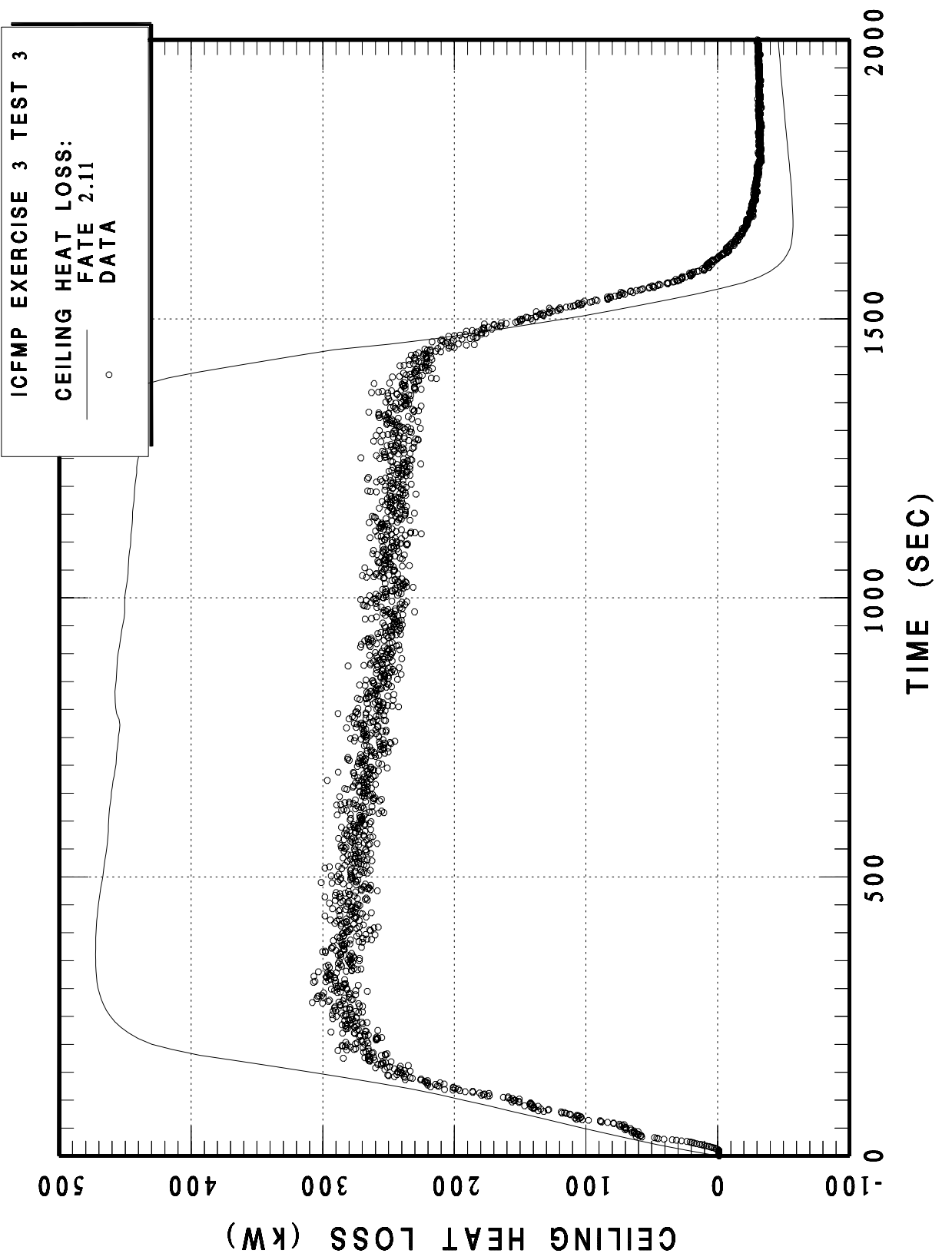

Figure 4-39 BE 3 CASE3 ceiling heat loss (convective and radiative) 


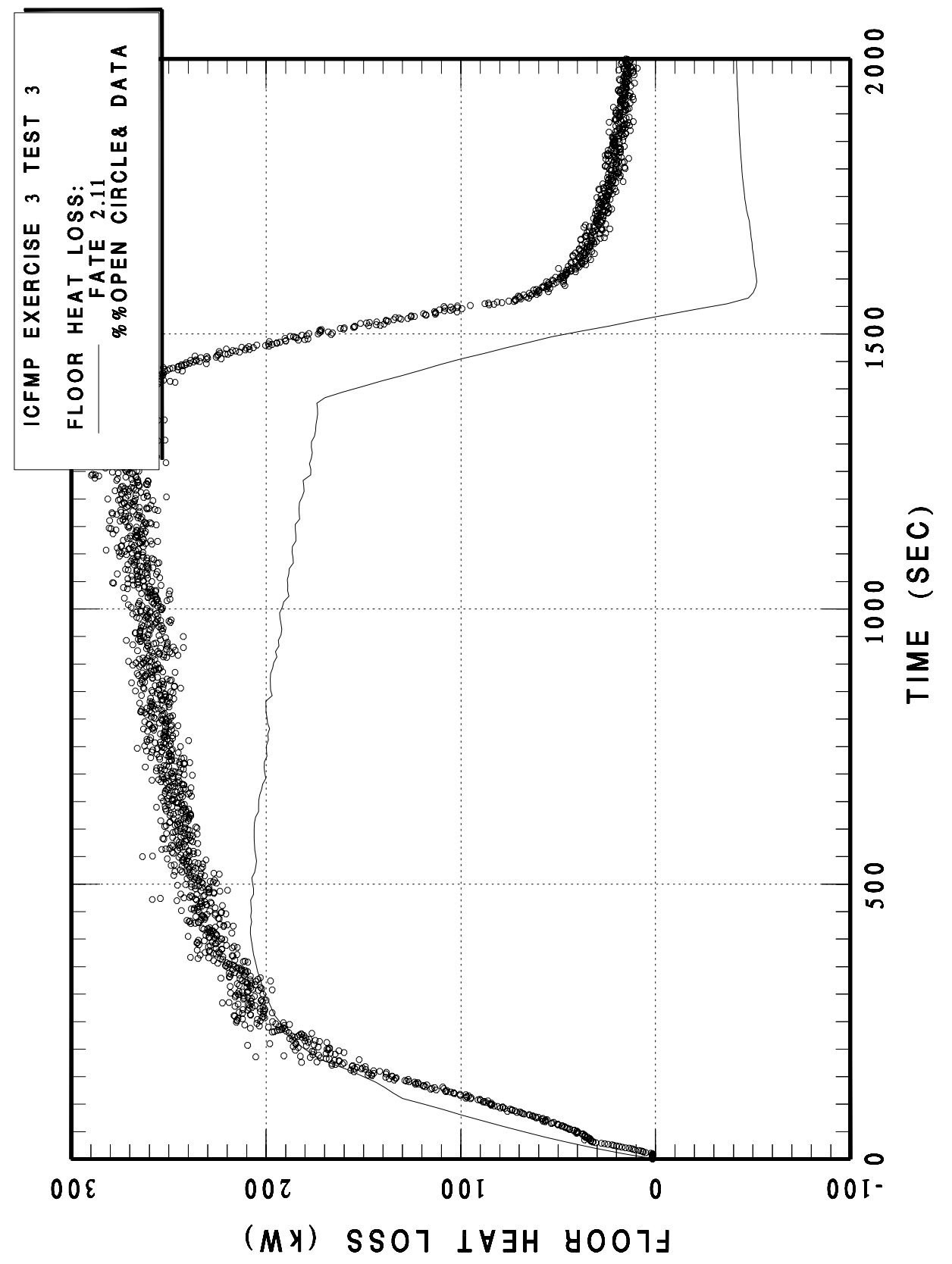

Figure 4-40 BE 3 CASE3 floor heat loss (convective and radiative) 


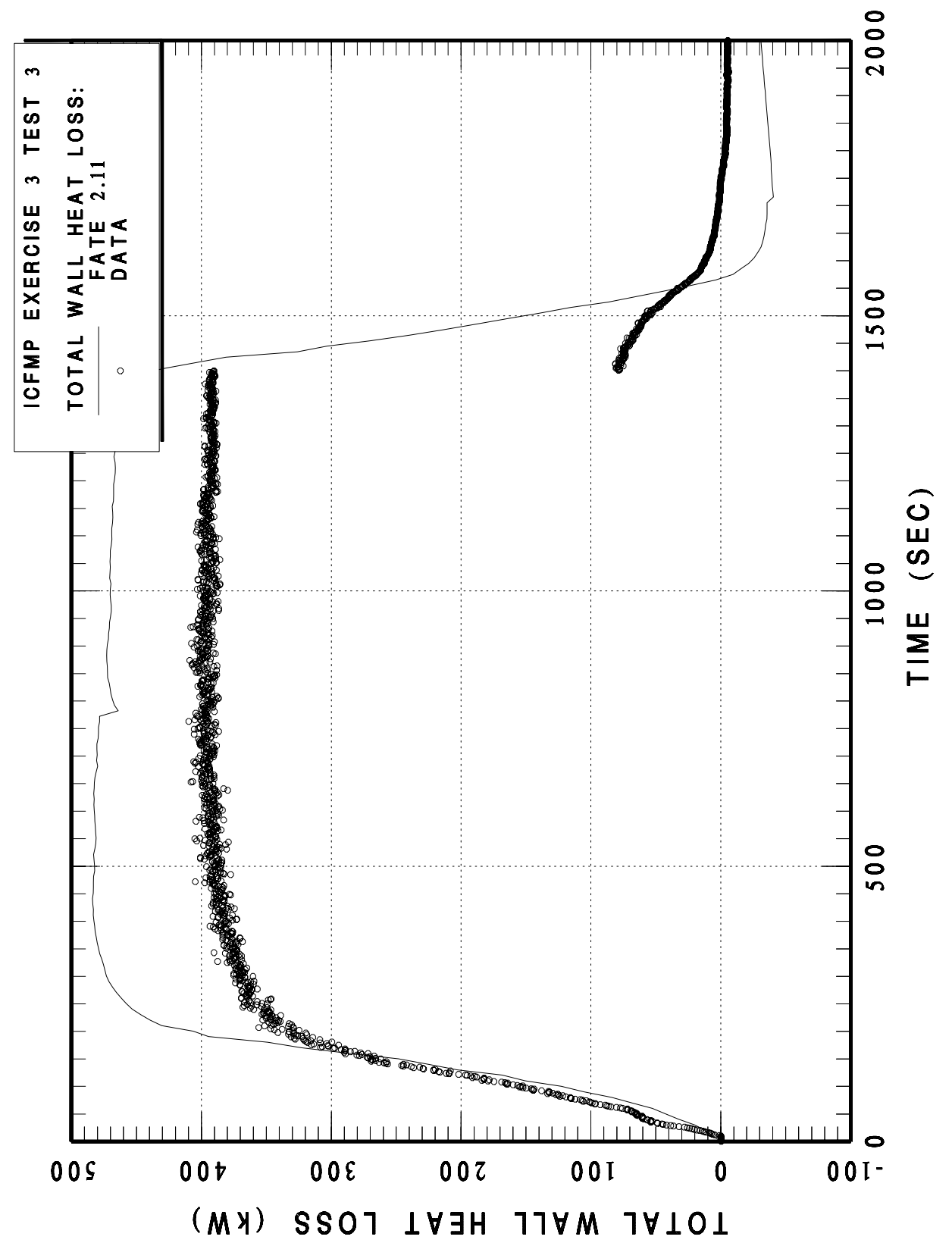

Figure 4-41 BE 3 CASE3 side wall heat loss (convective and radiative) 


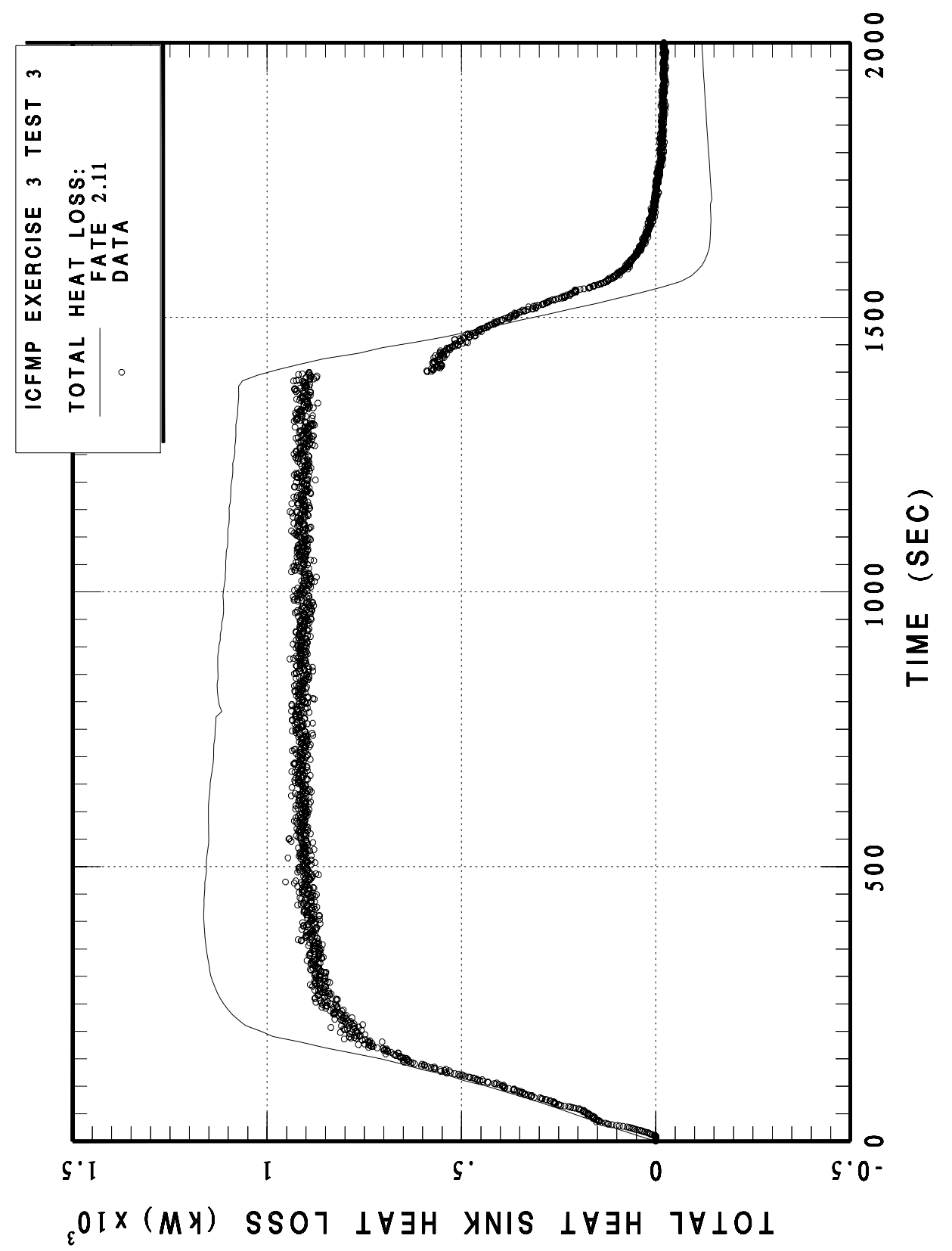

Figure 4-42 BE 3 CASE3 total heat loss to ceiling, floor, and walls (convective and radiative) 


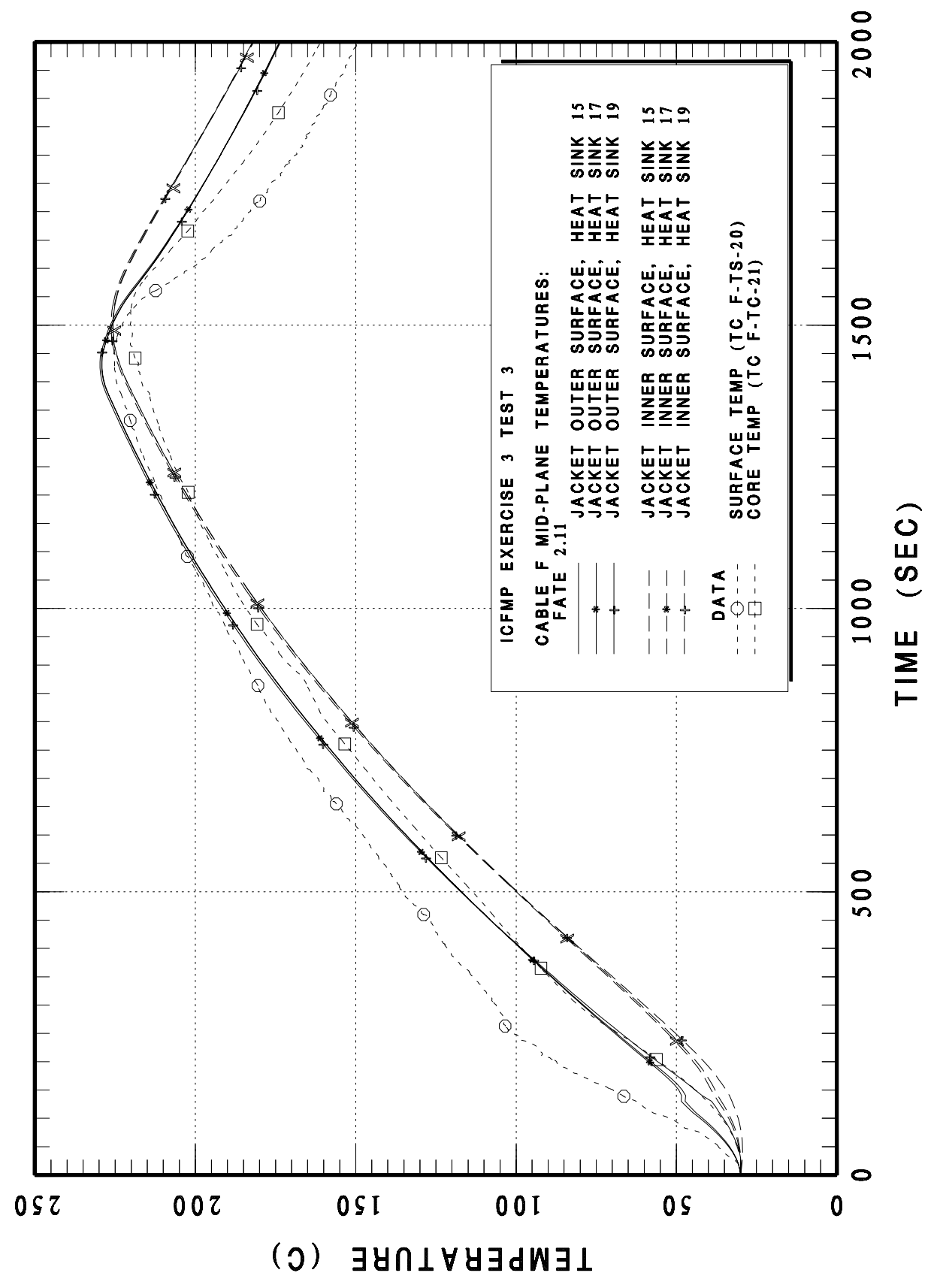

Figure 4-43 BE 3 CASE3 Cable F midplane temperatures 


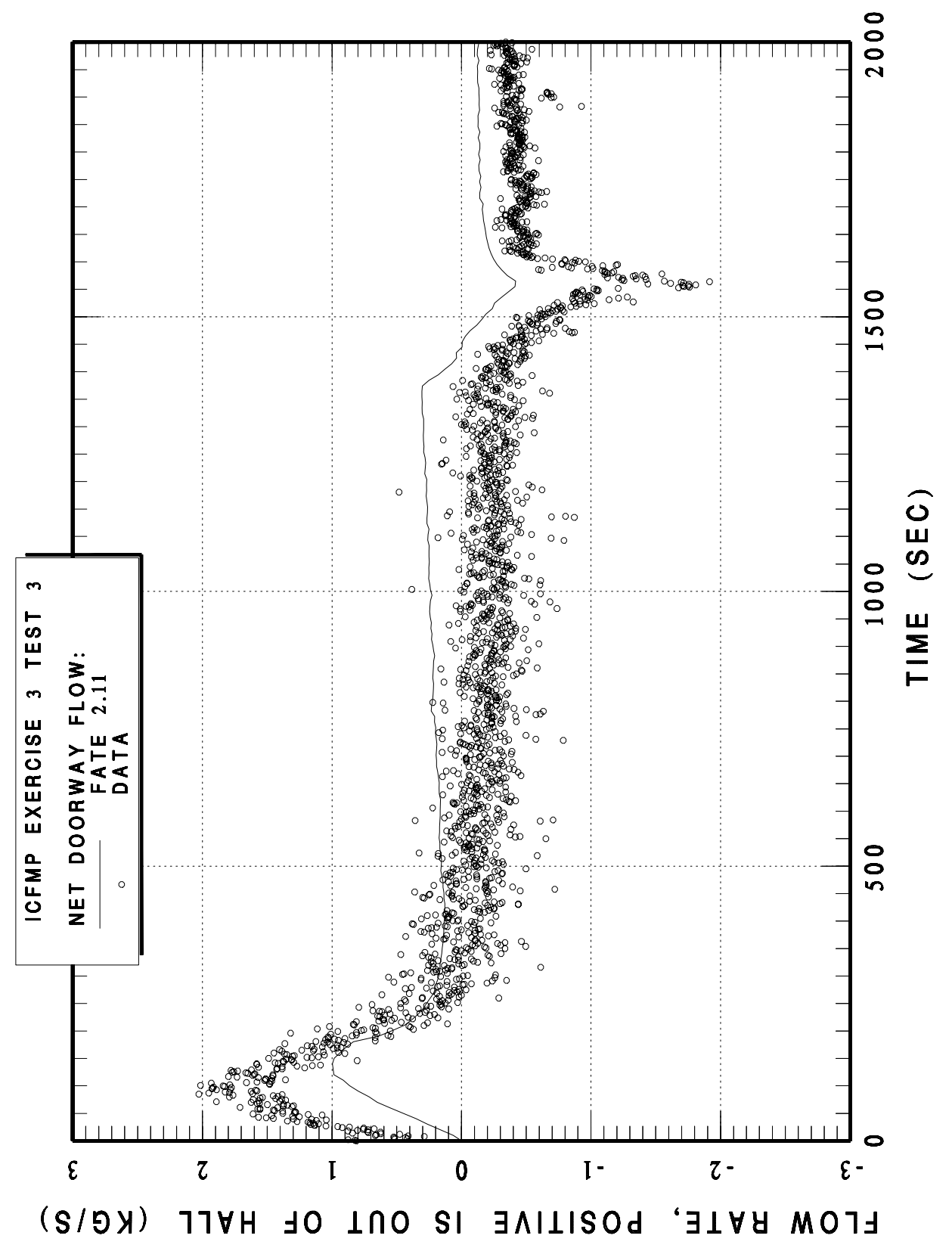

Figure 4-44 BE 3 CASE3 uni-direction flow through the doorway 


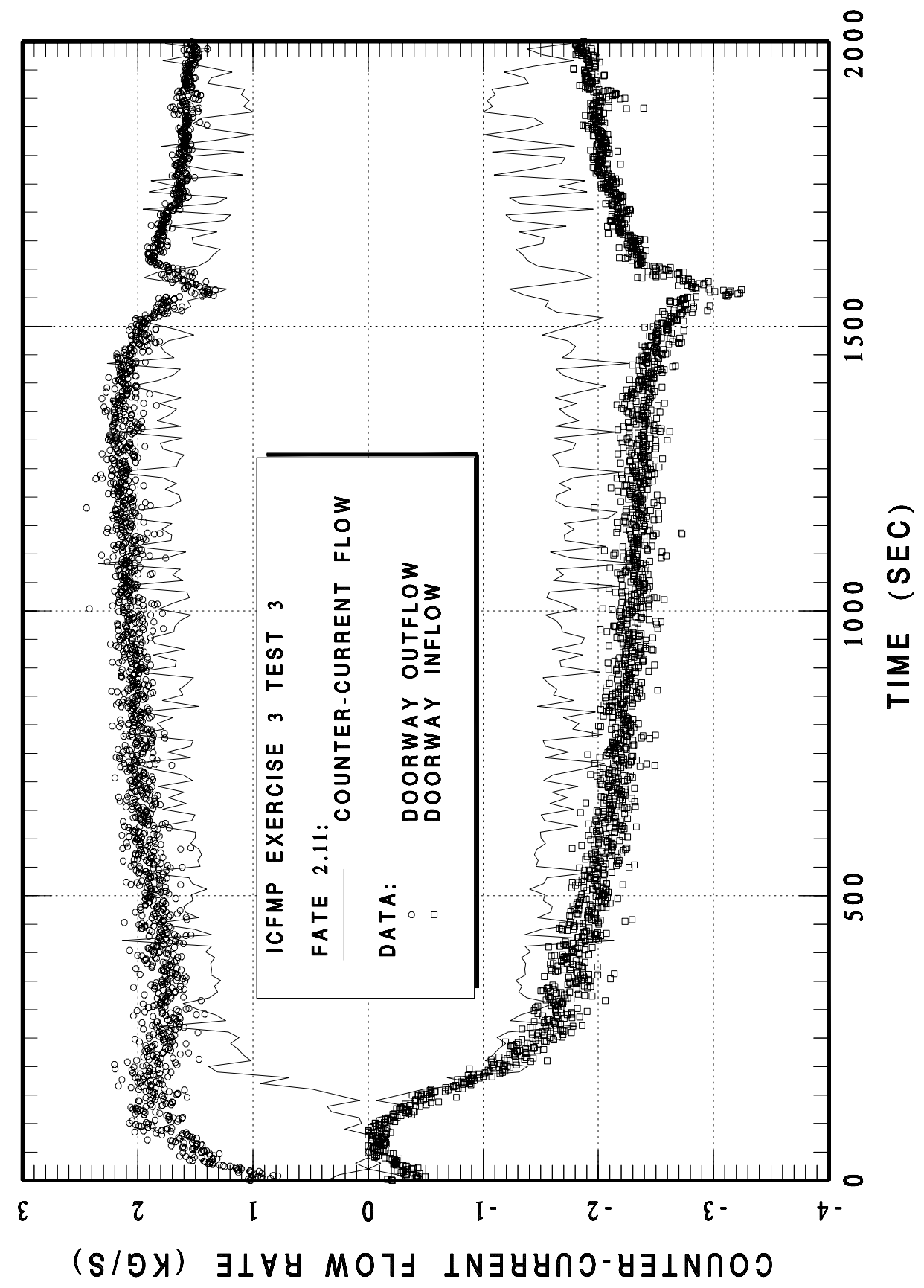

Figure 4-45 BE 3 CASE3 counter-current flow through the doorway 


\subsubsection{BE-3 Case 4 FATE Open Benchmark Results}

Case 4 is characterized by a heptanes fuel fire with a peak power of $1150 \mathrm{~kW}$ and forced circulation due to operation of the fan supply and exhaust. A constant fan flow of $1.06 \mathrm{~m}^{3} / \mathrm{sec}(1.2 \mathrm{~kg} / \mathrm{s})$ is assumed for the duration of the run. The main doors are closed, however leakage around presumed door gaps is modeled. FATE calculations for BE-3 Case 4 are presented in Figure 4-46 through Figure 4-60.

As with all previous cases, test data indicates that the smoky layer descends to about $1.0 \mathrm{~m}$. When the FATE calculation assumes a fan supply flow split equally between the smoky and lower regions, good agreement with the test data is obtained. If the fan flow is assumed to go to the smoky layer, only, then the smoky layer descends all the way to the floor and remains near the floor even after fire termination. Finally, if a best estimate fan flow during the fire period of $1 \mathrm{~kg} / \mathrm{s}$ is assumed, then the calculated smoky layer elevation shows slightly better agreement with the data.

The sensitivity to fan performance is also evident in the plot of gas concentration. When a best estimate fan flow of $1.0 \mathrm{~kg} / \mathrm{s}$ in conjunction with a flow split equally between the smoky and lower regions is used, the FATE calculations closely follow the test data.

The remaining calculations of gas and heat sink temperatures presented show similar levels of agreement as obtained in the preceding tests cases. 


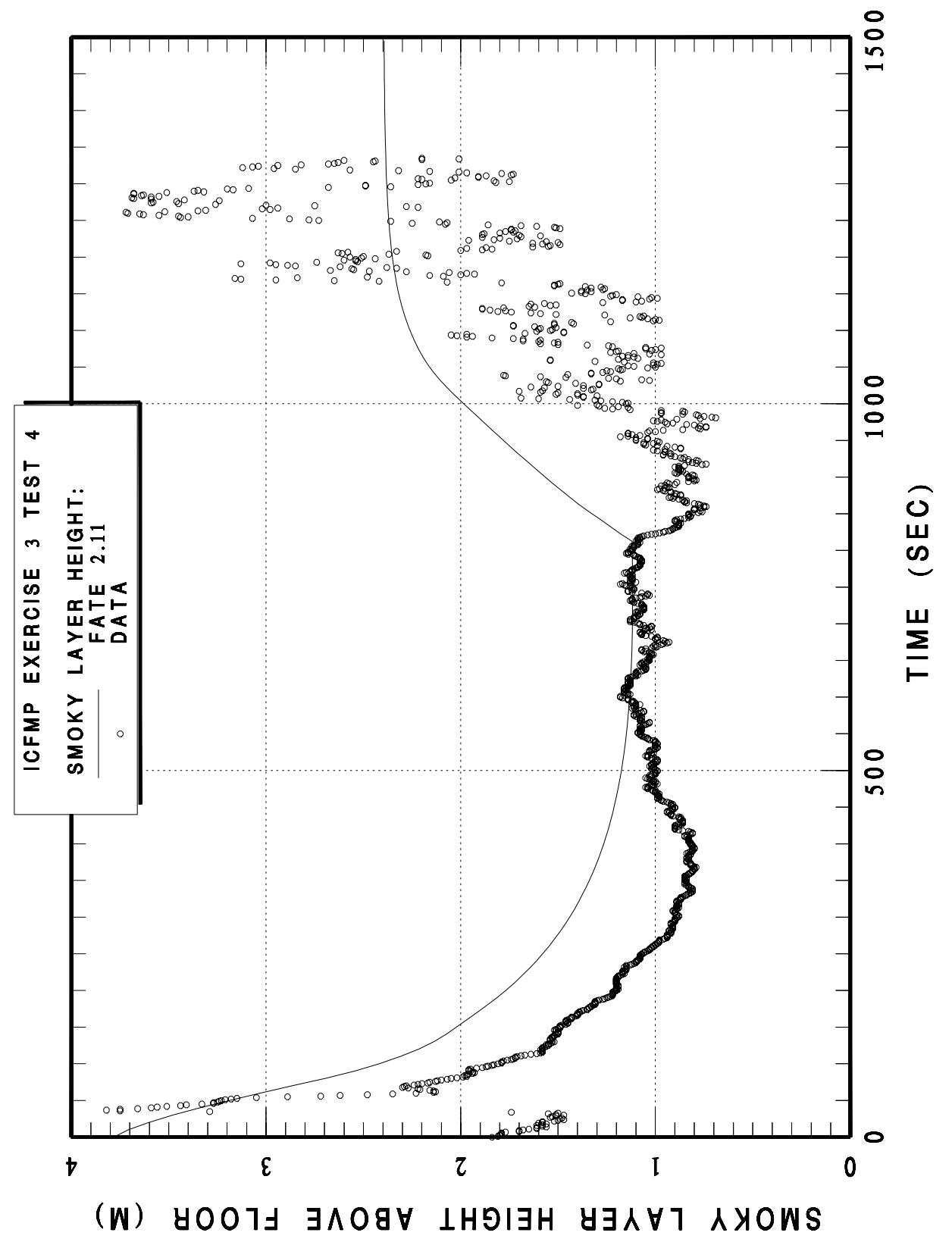

Figure 4-46 BE 3 CASE4 Smoky layer height 


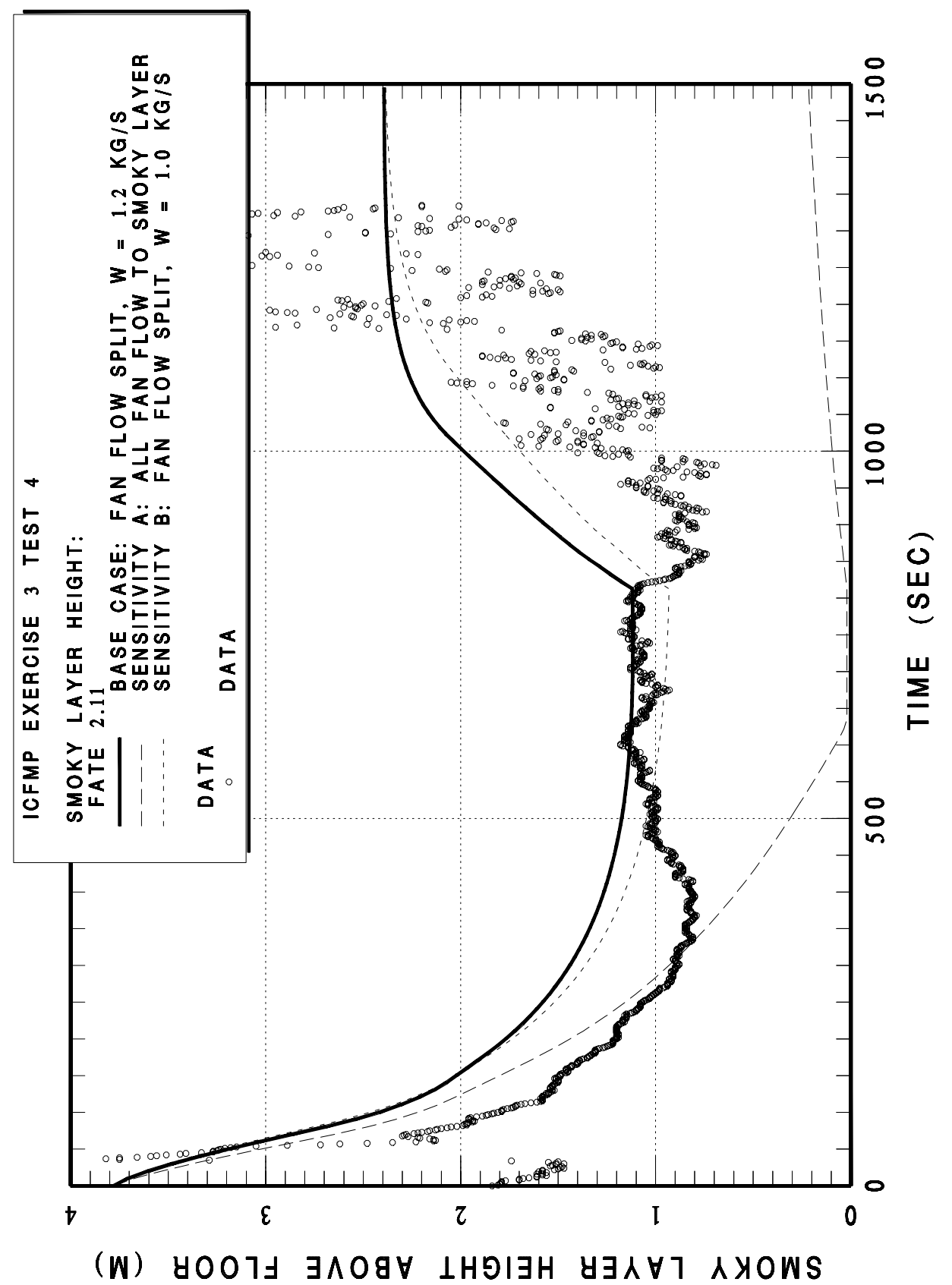

Figure 4-47 BE 3 CASE4 Smoky layer height sensitivity calculations 


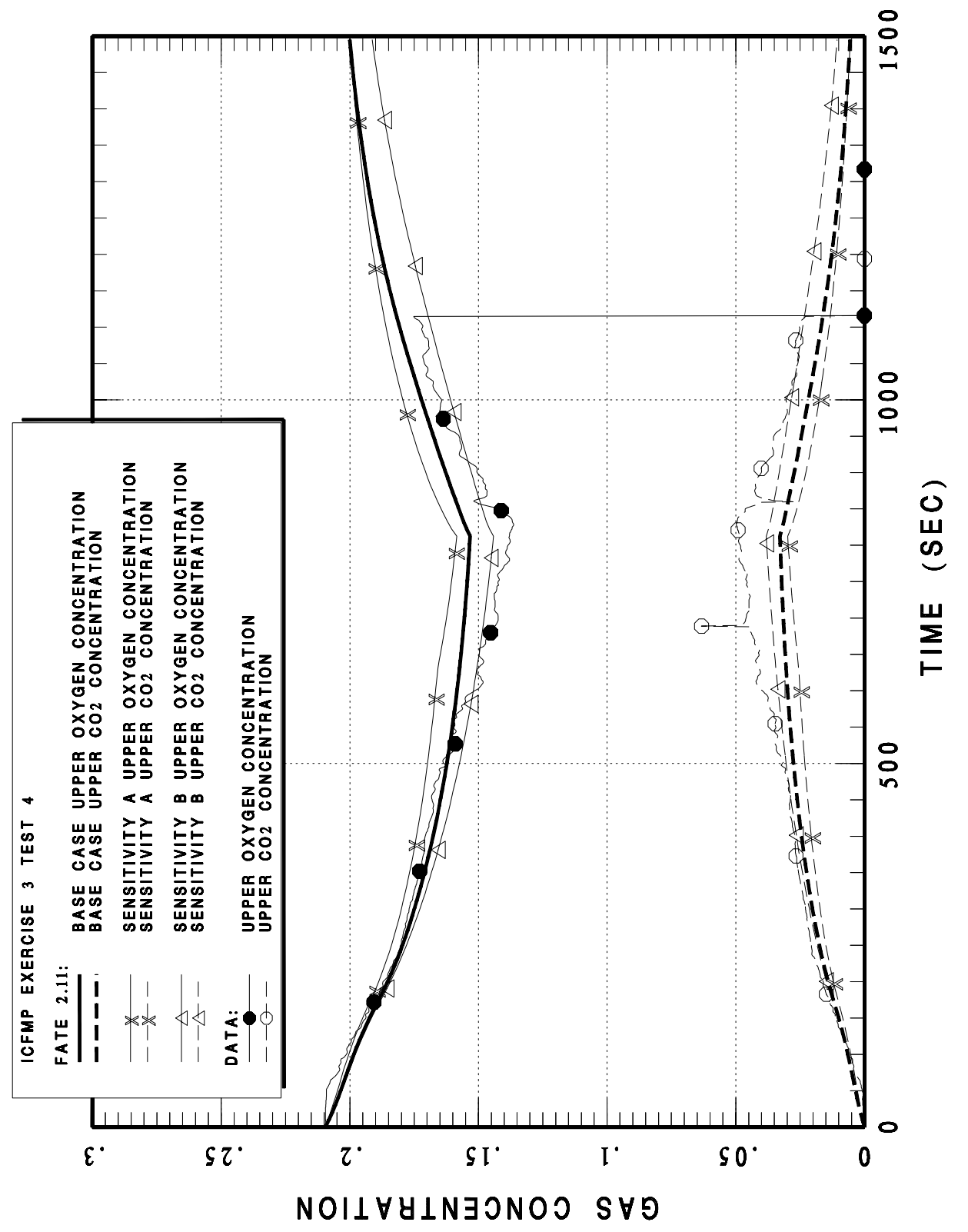

Figure 4-48 BE 3 CASE4 upper room gas concentrations 


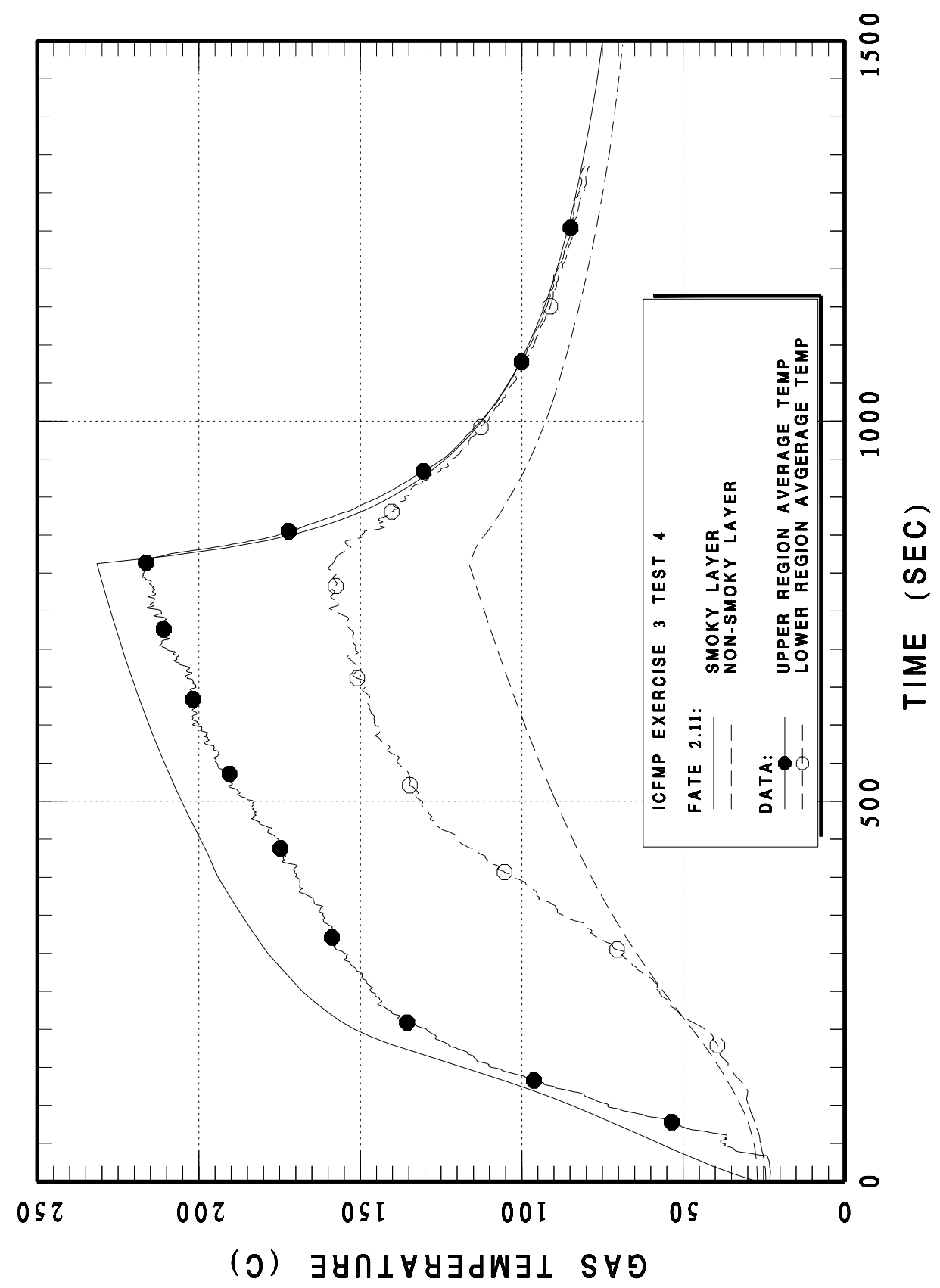

Figure 4-49 BE 3 CASE4 upper and lower layer average gas temperatures 


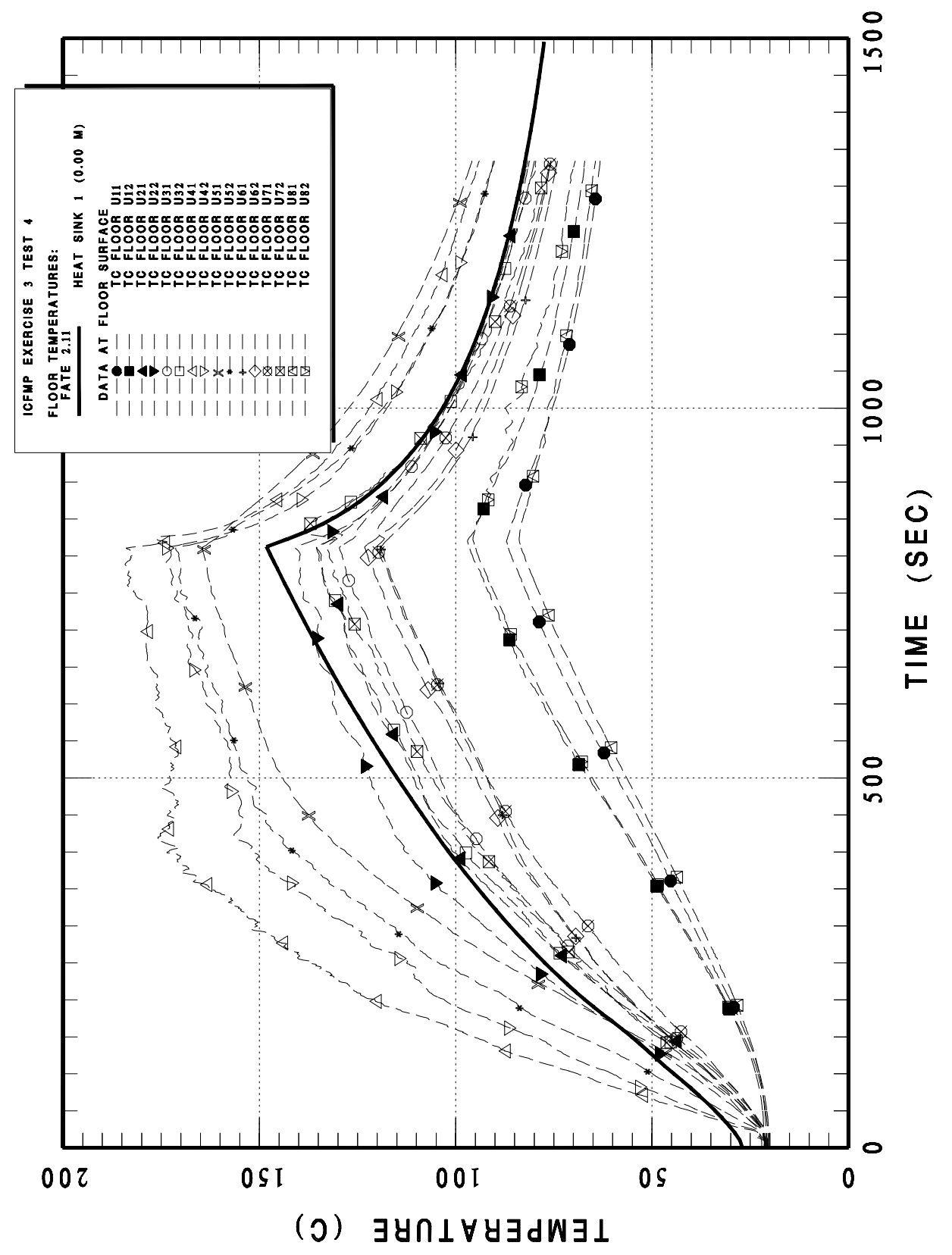

Figure 4-50 BE 3 CASE4 floor surface temperatures 


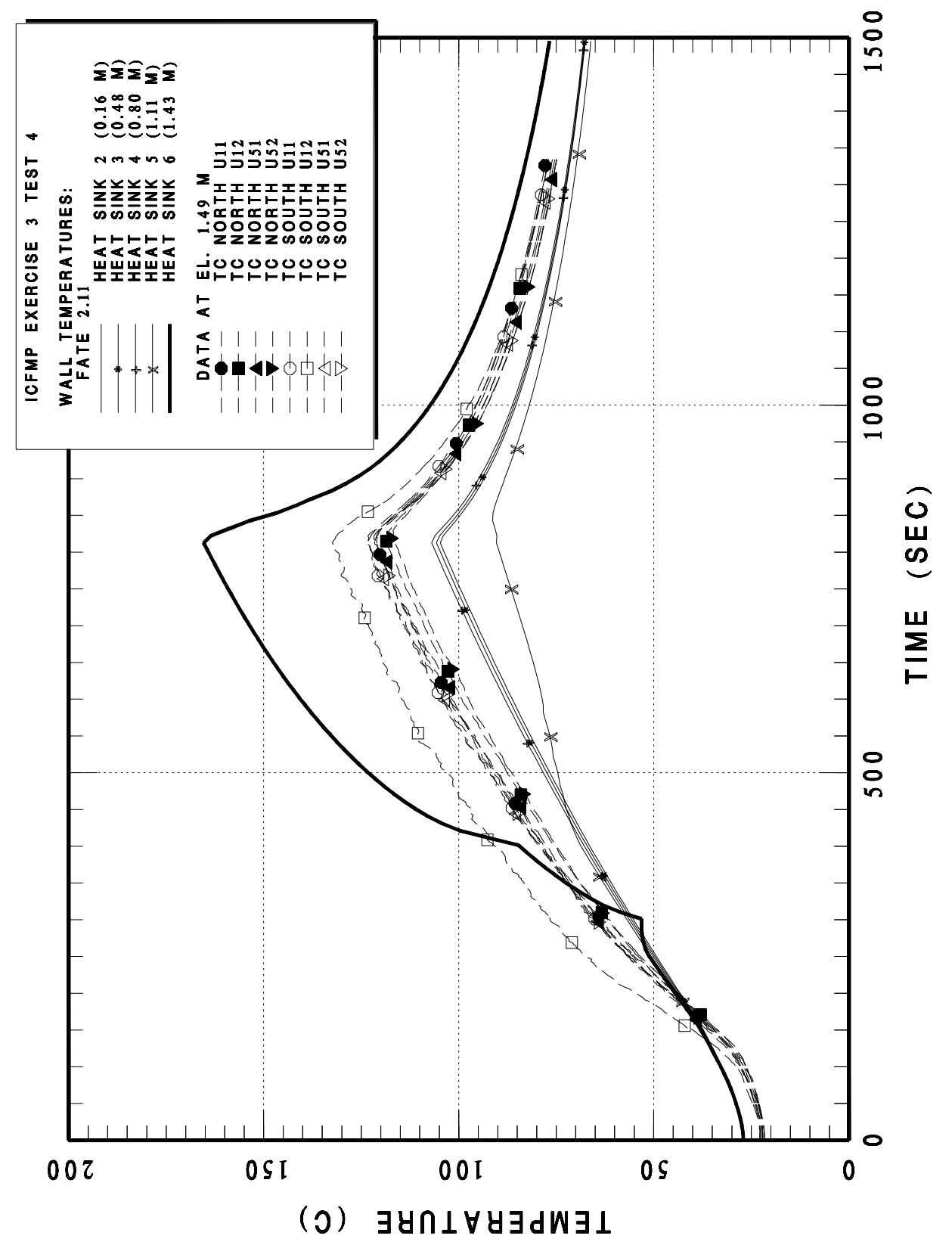

Figure 4-51 BE 3 CASE4 North and South wall temperatures at the $1.49 \mathrm{~m}$ elevation 


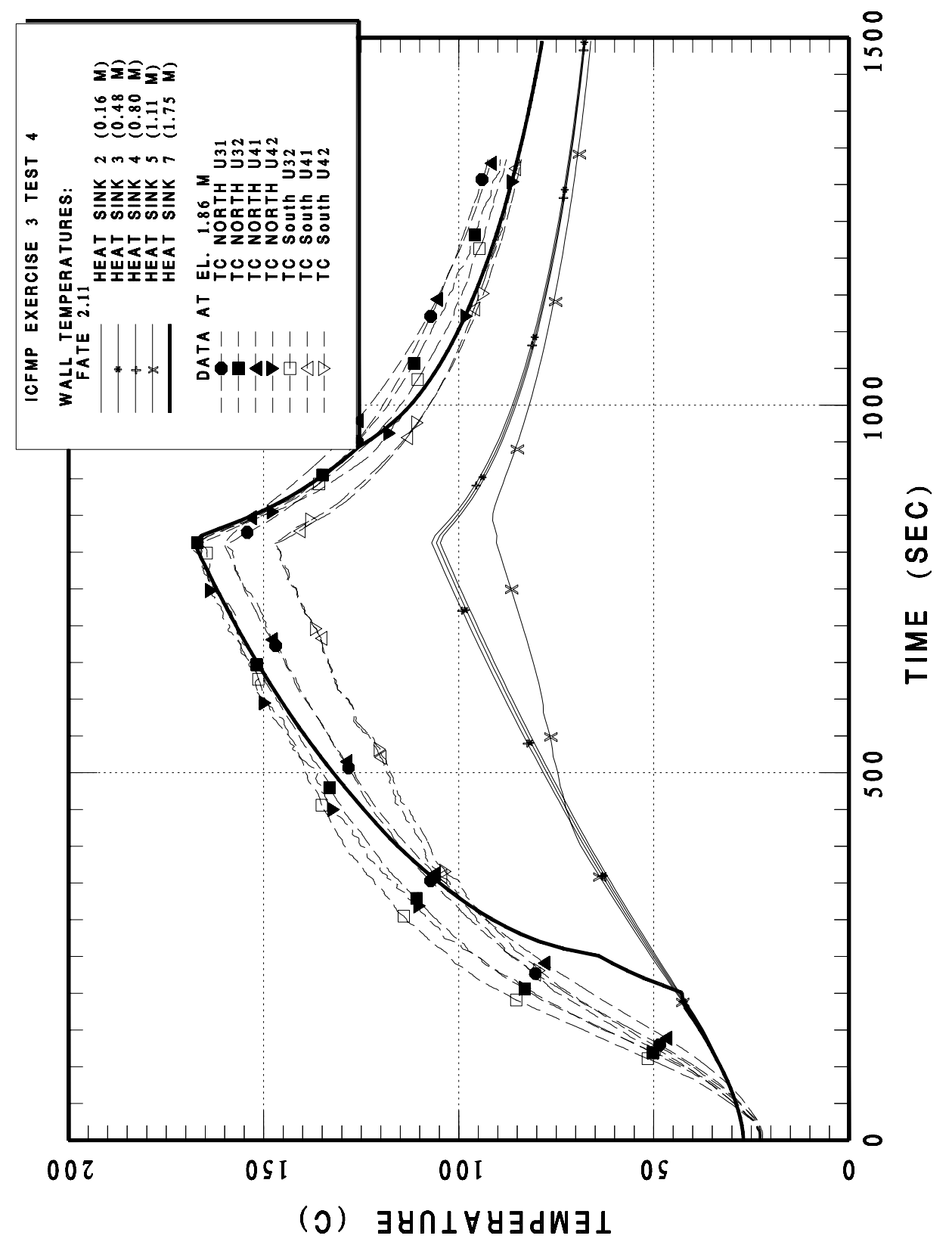

Figure 4-52 BE 3 CASE4 North and South wall temperatures at the $1.86 \mathrm{~m}$ elevation 


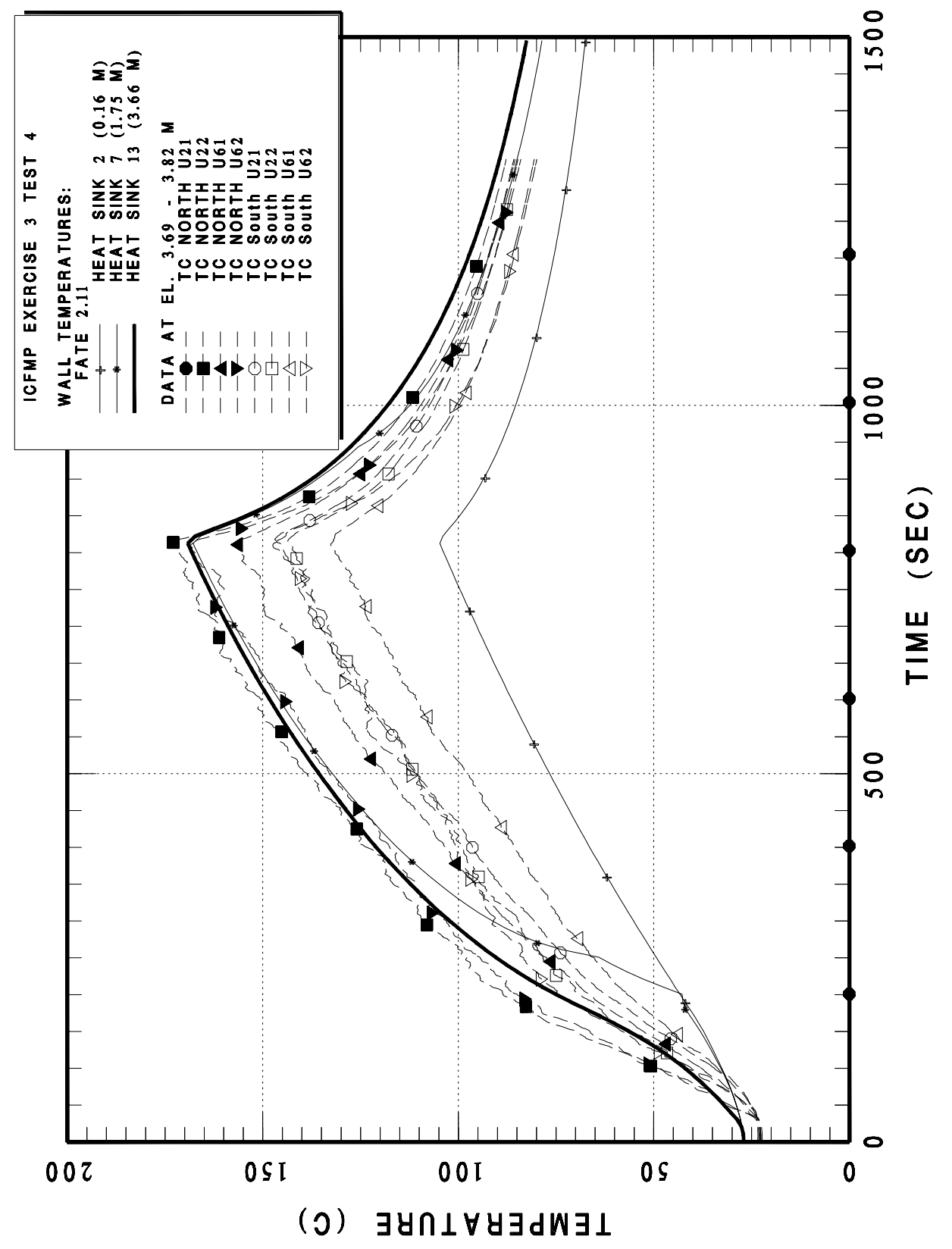

Figure 4-53 BE 3 CASE4 North and South wall temperatures between the 3.69 and $3.82 \mathrm{~m}$ elevations 


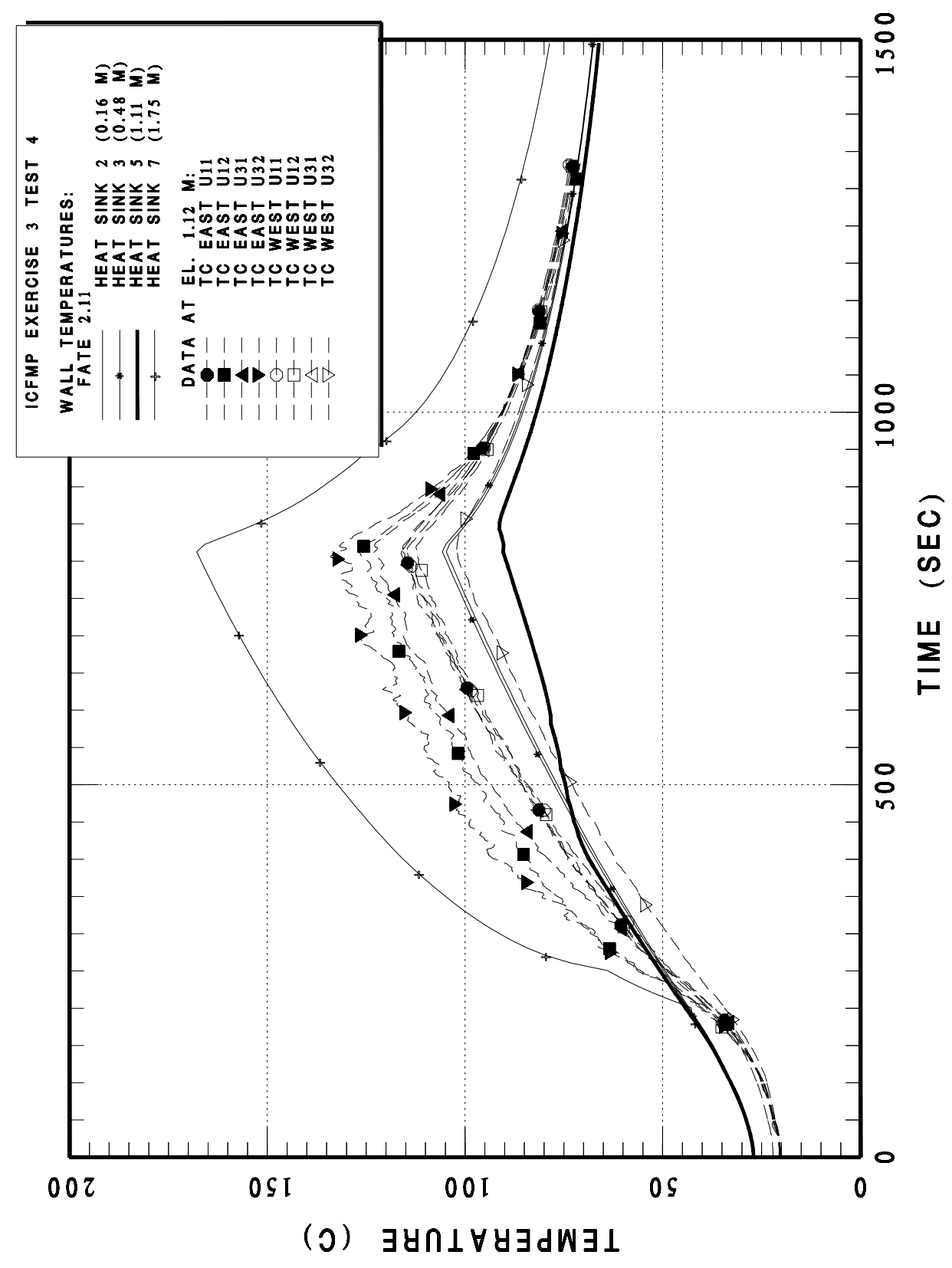

Figure 4-54 BE 3 CASE4 East and West wall temperatures at the 1.12 m elevation 


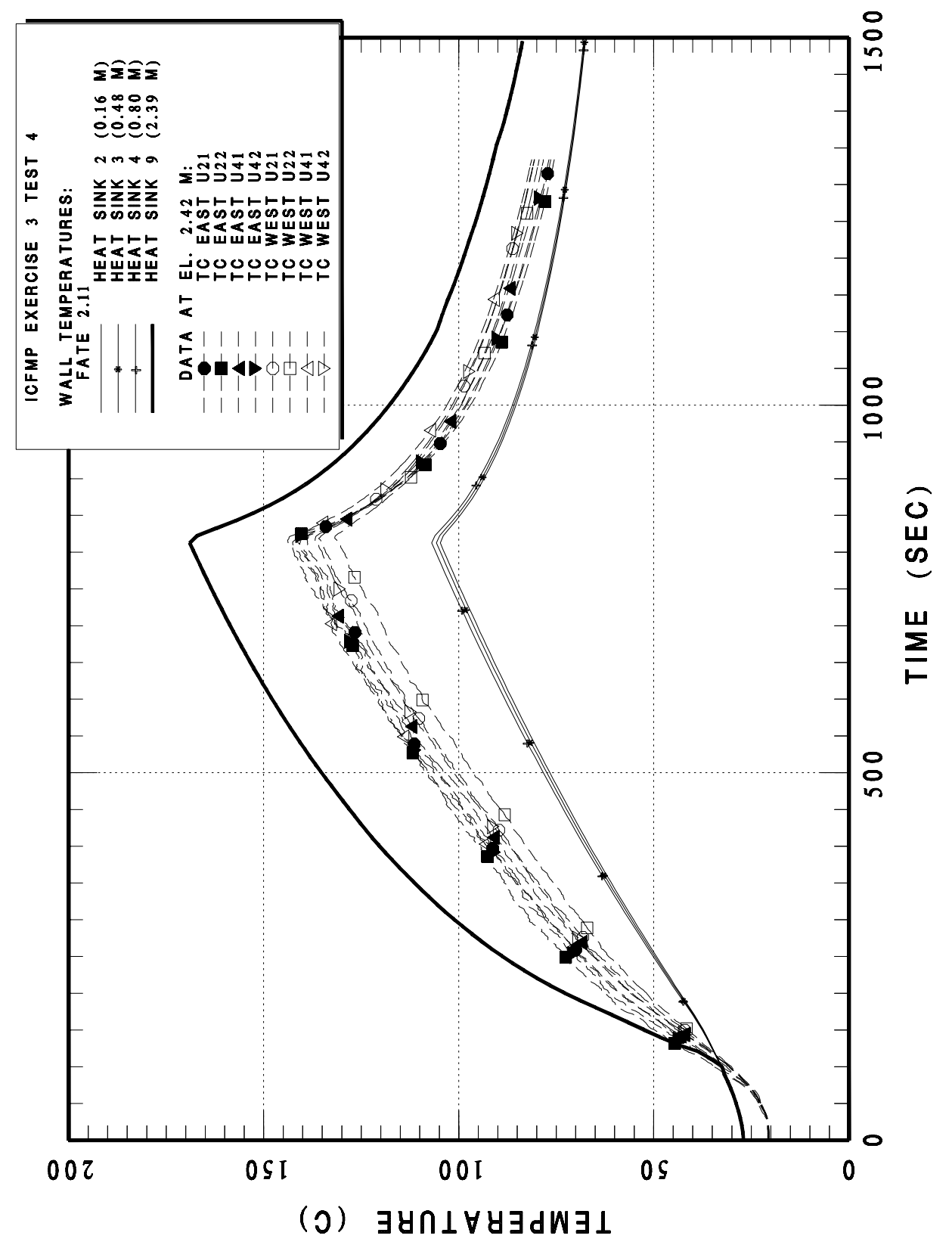

Figure 4-55 BE 3 CASE4 East and West wall temperatures at the $2.42 \mathrm{~m}$ elevation 


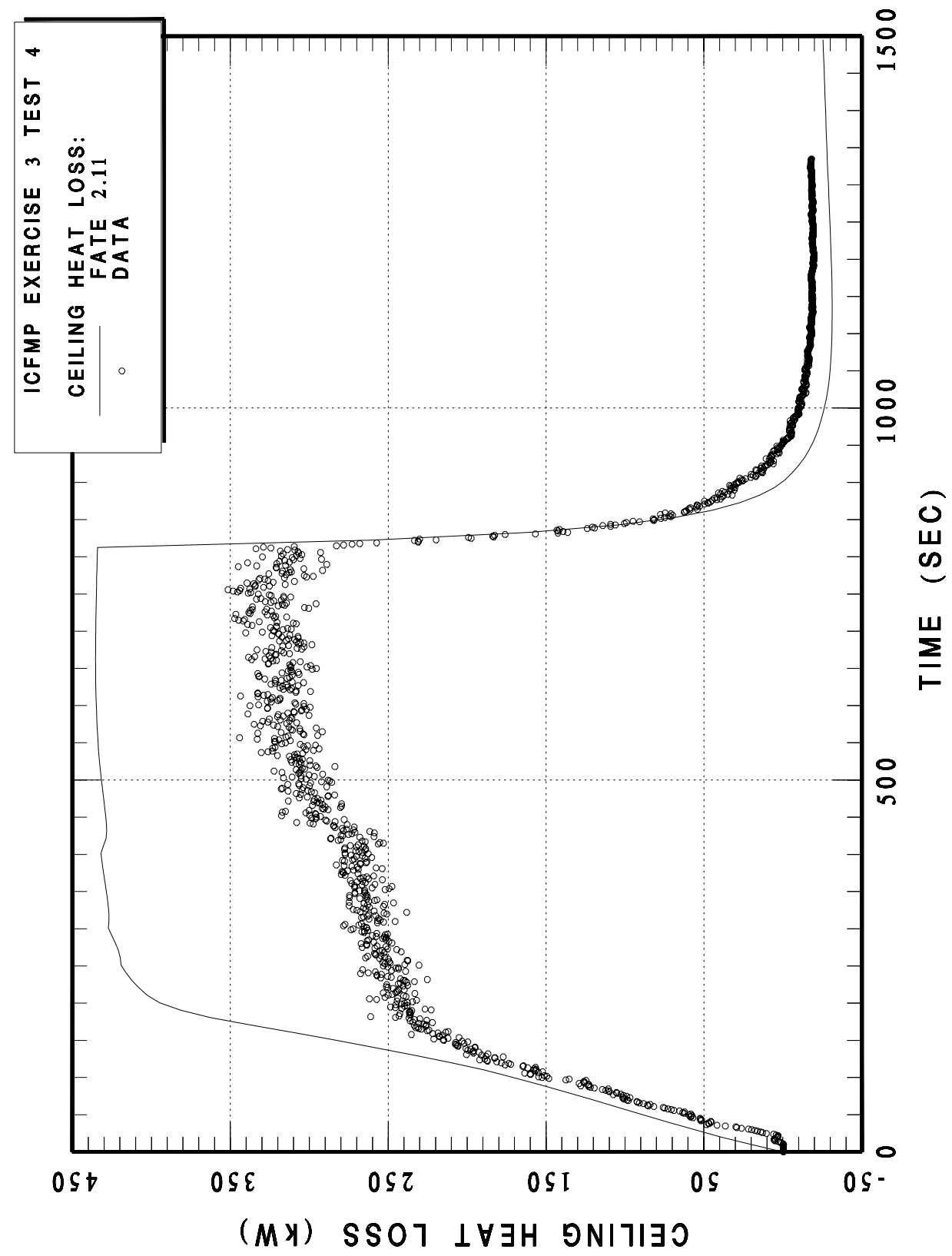

Figure 4-56 BE 3 CASE4 ceiling heat loss (convective and radiative) 


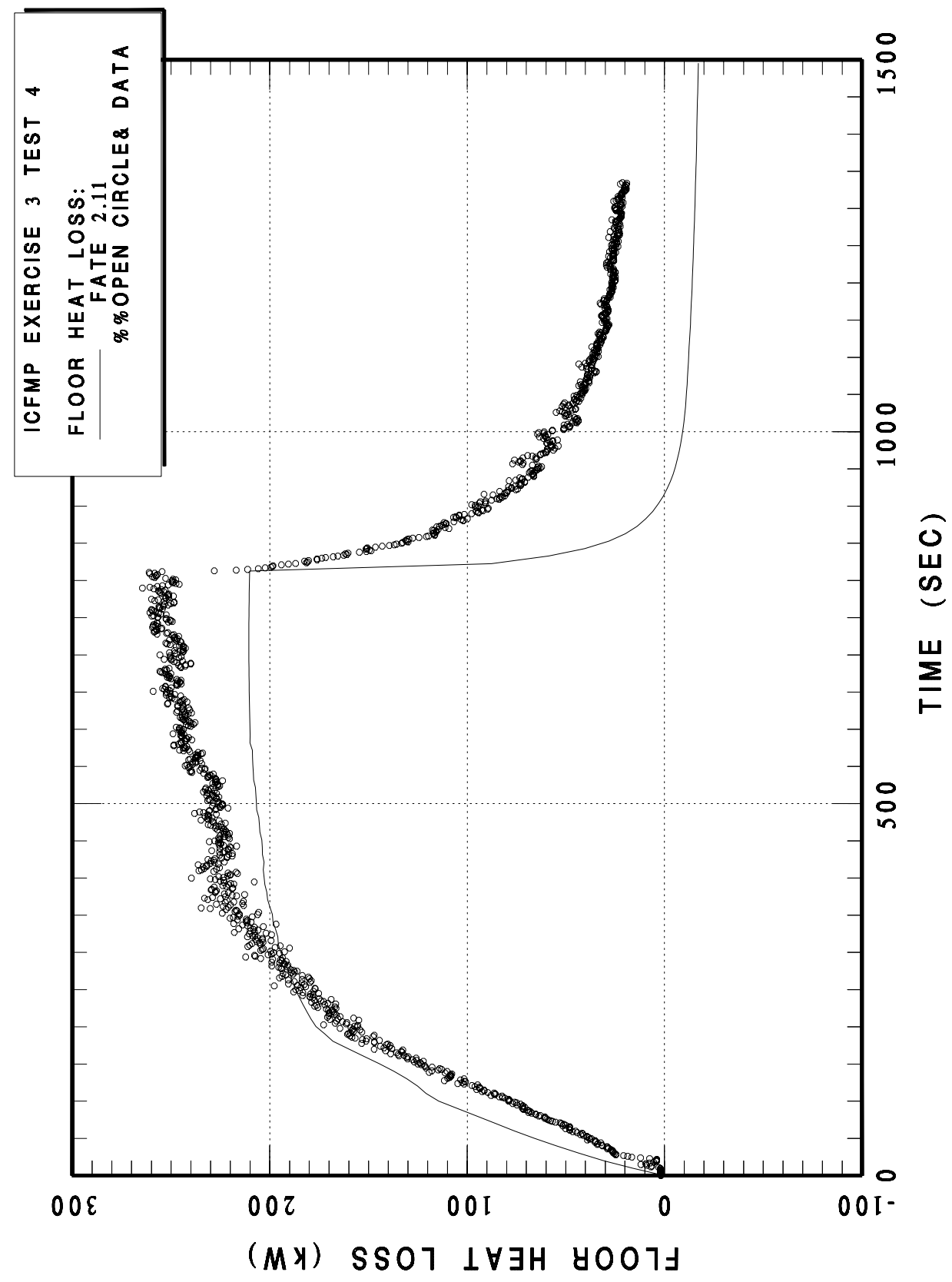

Figure 4-57 BE 3 CASE4 floor heat loss (convective and radiative) 


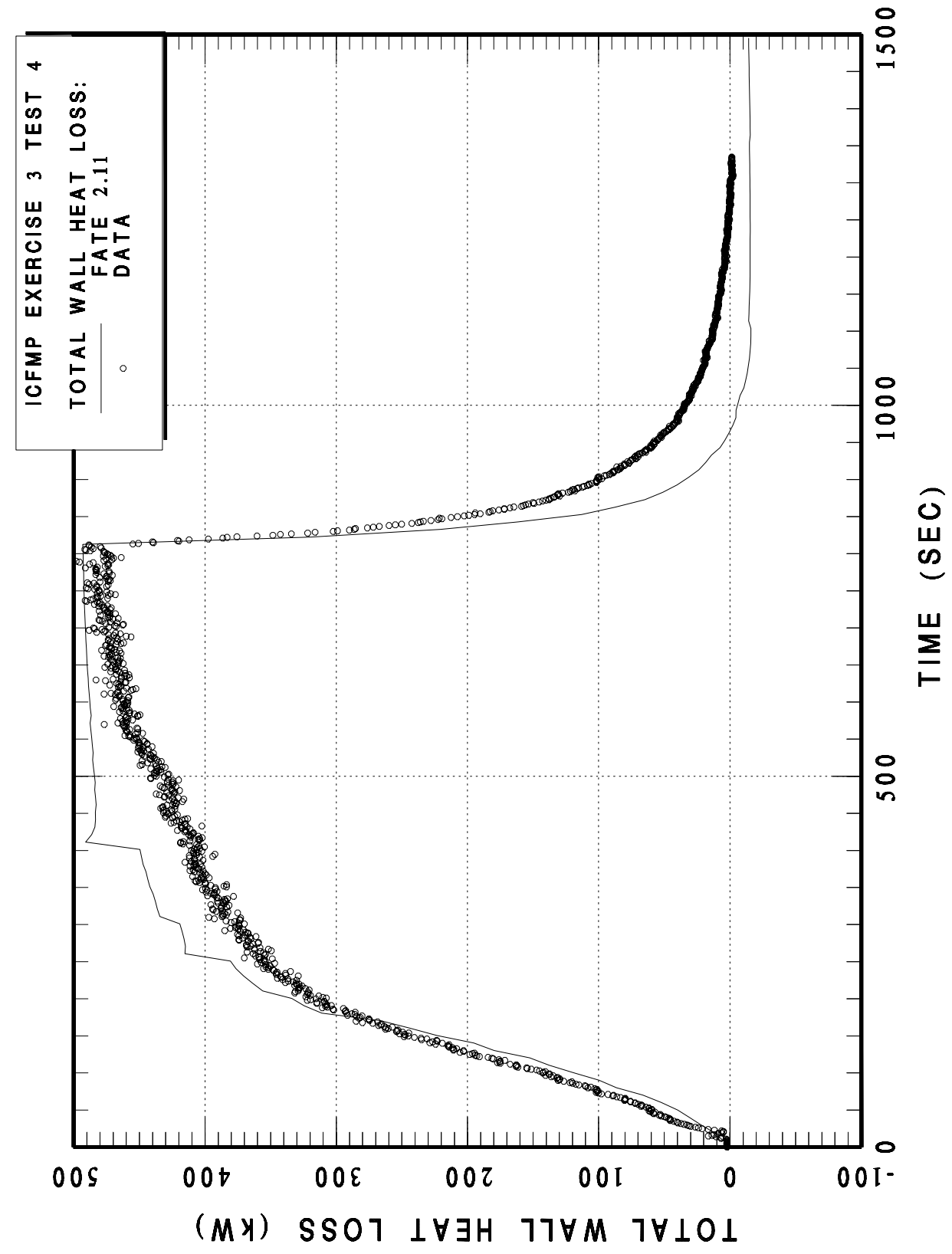

Figure 4-58 BE 3 CASE4 side wall heat loss (convective and radiative) 


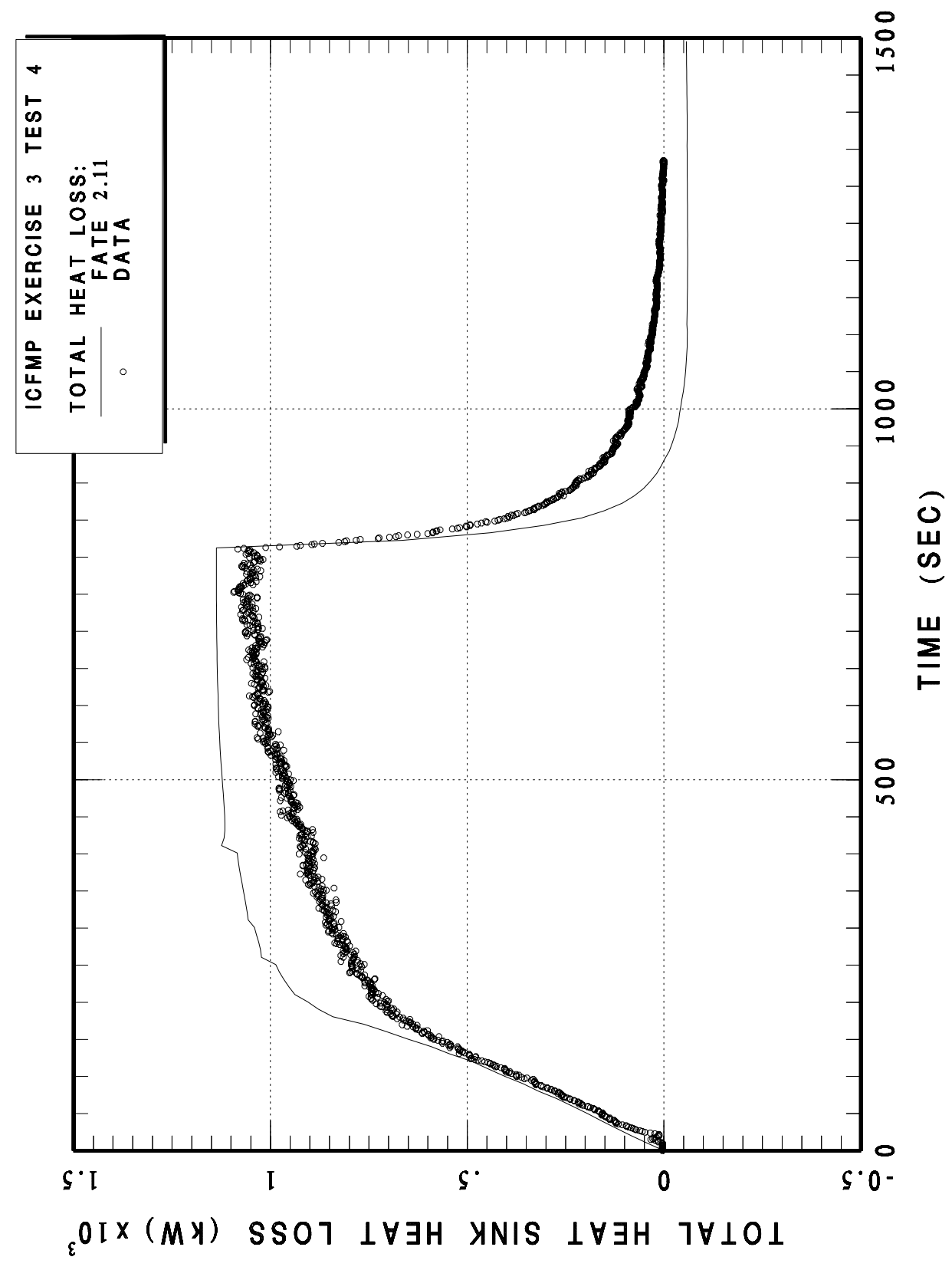

Figure 4-59 BE 3 CASE4 total heat loss to ceiling, floor, and walls (convective and radiative) 


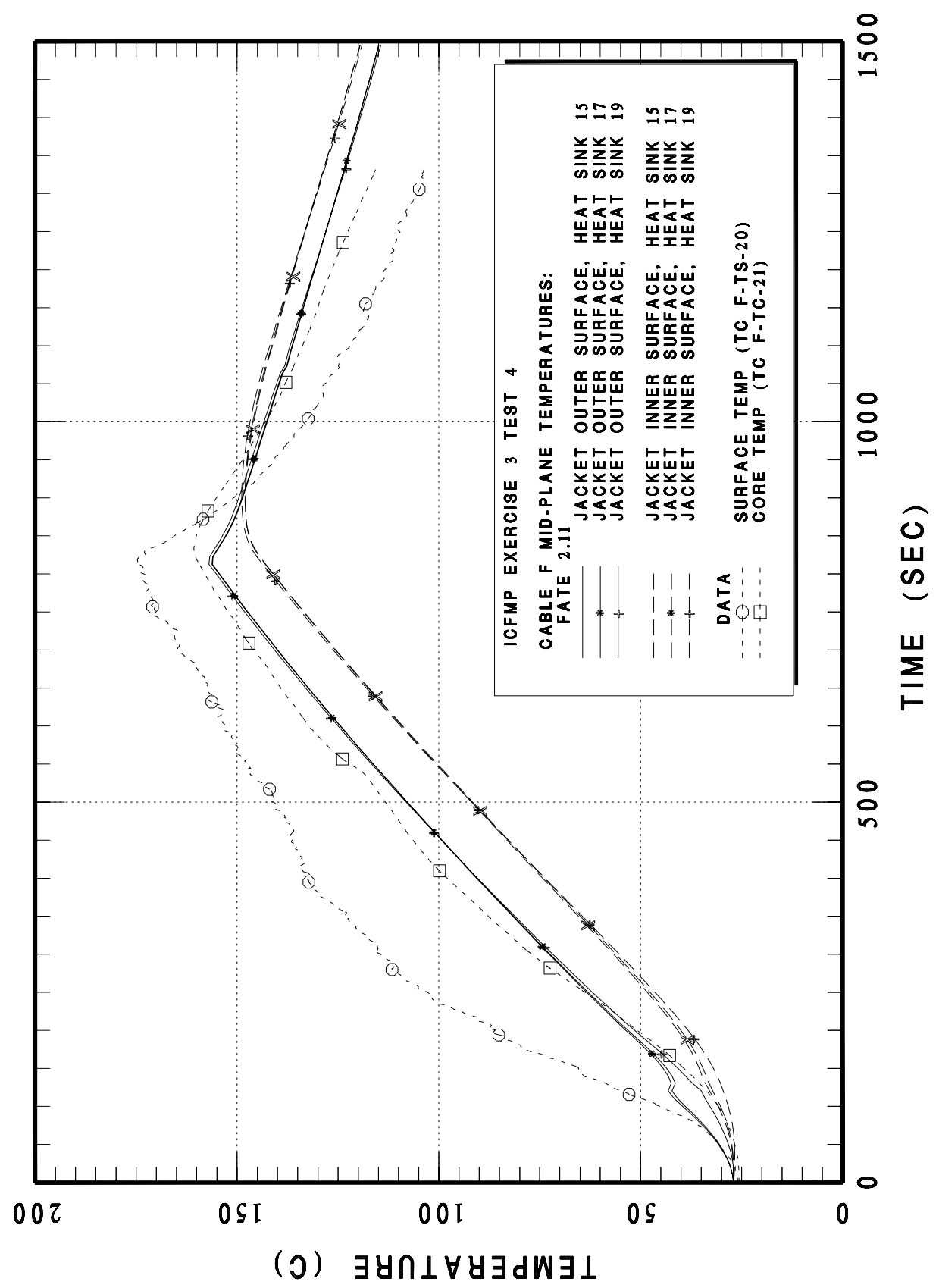

Figure 4-60 BE 3 CASE4 Cable F midplane temperatures 


\subsubsection{BE-3 Case 5 FATE Open Benchmark Results}

Case 5 is a heptane fire with a peak power of $1150 \mathrm{~kW}$ and both forced circulation due to operation of the fan supply and exhaust and natural circulation through the open doors. A constant fan flow of $1.06 \mathrm{~m}^{3} / \mathrm{sec}(1.2 \mathrm{~kg} / \mathrm{s})$ is assumed for the duration of the run. The fan inflow is assumed to go only into the smoky layer, while the door inflow is split equally between the lower and smoky layers. No new insights are obtained from this test case, however previous insights are confirmed. FATE calculations for BE-3 Case 4 are presented in Figure 4-61 through Figure 4-66.

\subsubsection{BE-3 Case 14, 15, and 18 FATE Open Benchmark Results}

Cases 14,15 , and 18 are similar to previously analyzed Case 3, except the fire pan is moved to an off center location. In all cases the fire is nominally $1130 \mathrm{~kW}$ and the door is open but the fan is off.

No new insights are obtained from these three test cases, however previous insights are confirmed. Comparisons of FATE calculations and test data for a subset of the measured quantities are presented in Figure 4-67 through Figure 4-82. 


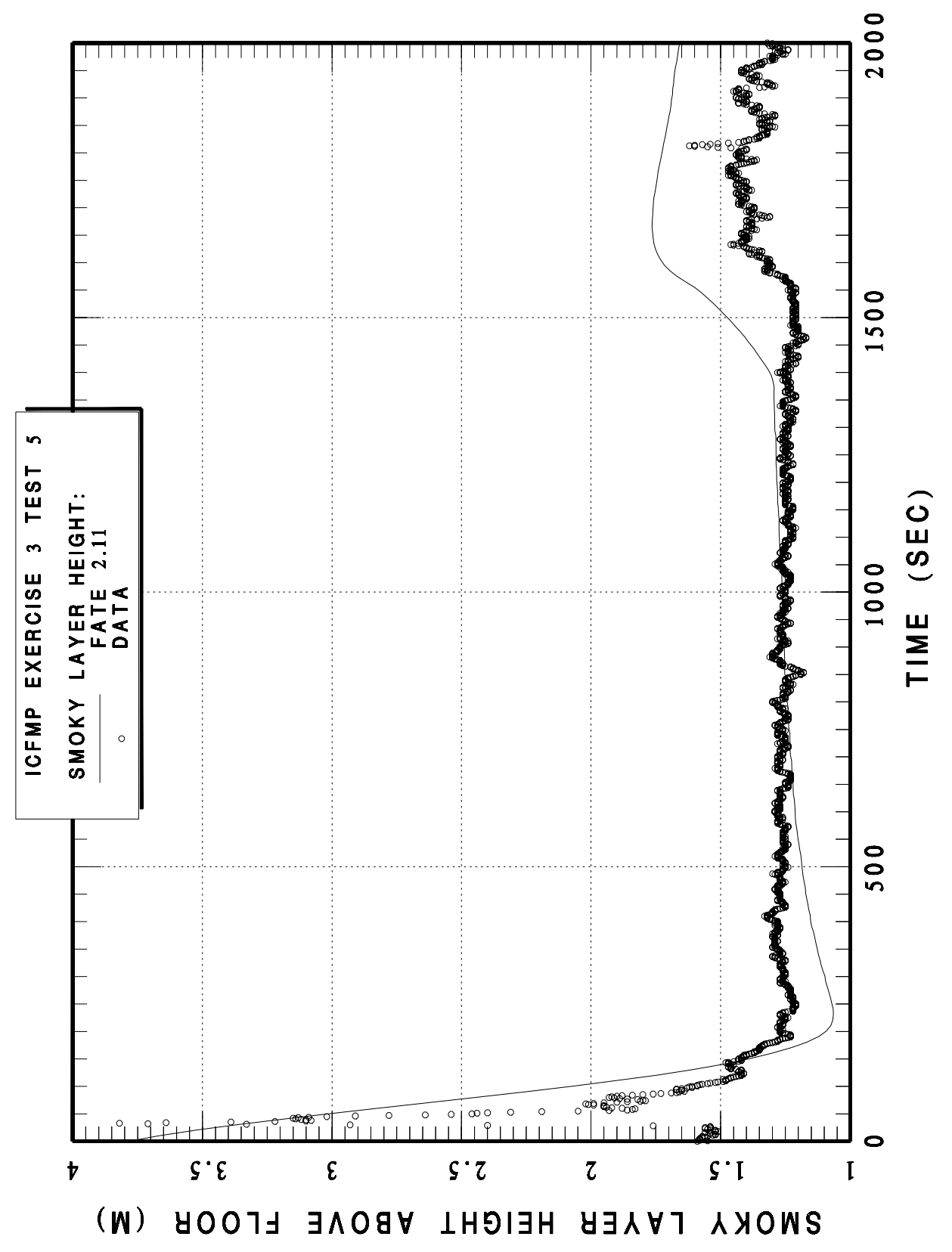

Figure 4-61 BE 3 CASE5 Smoky layer height 


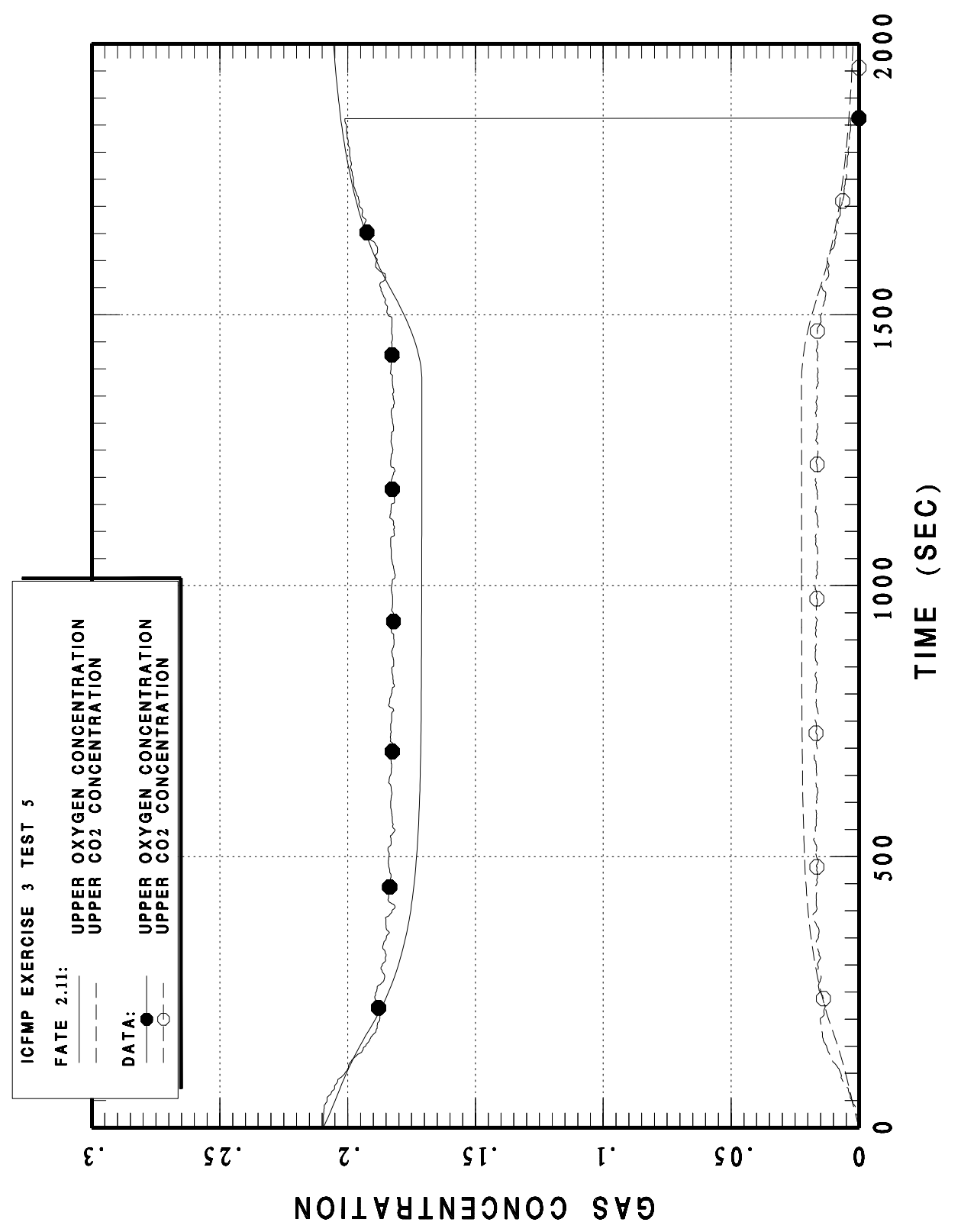

Figure 4-62 BE 3 CASE5 upper room gas concentrations 


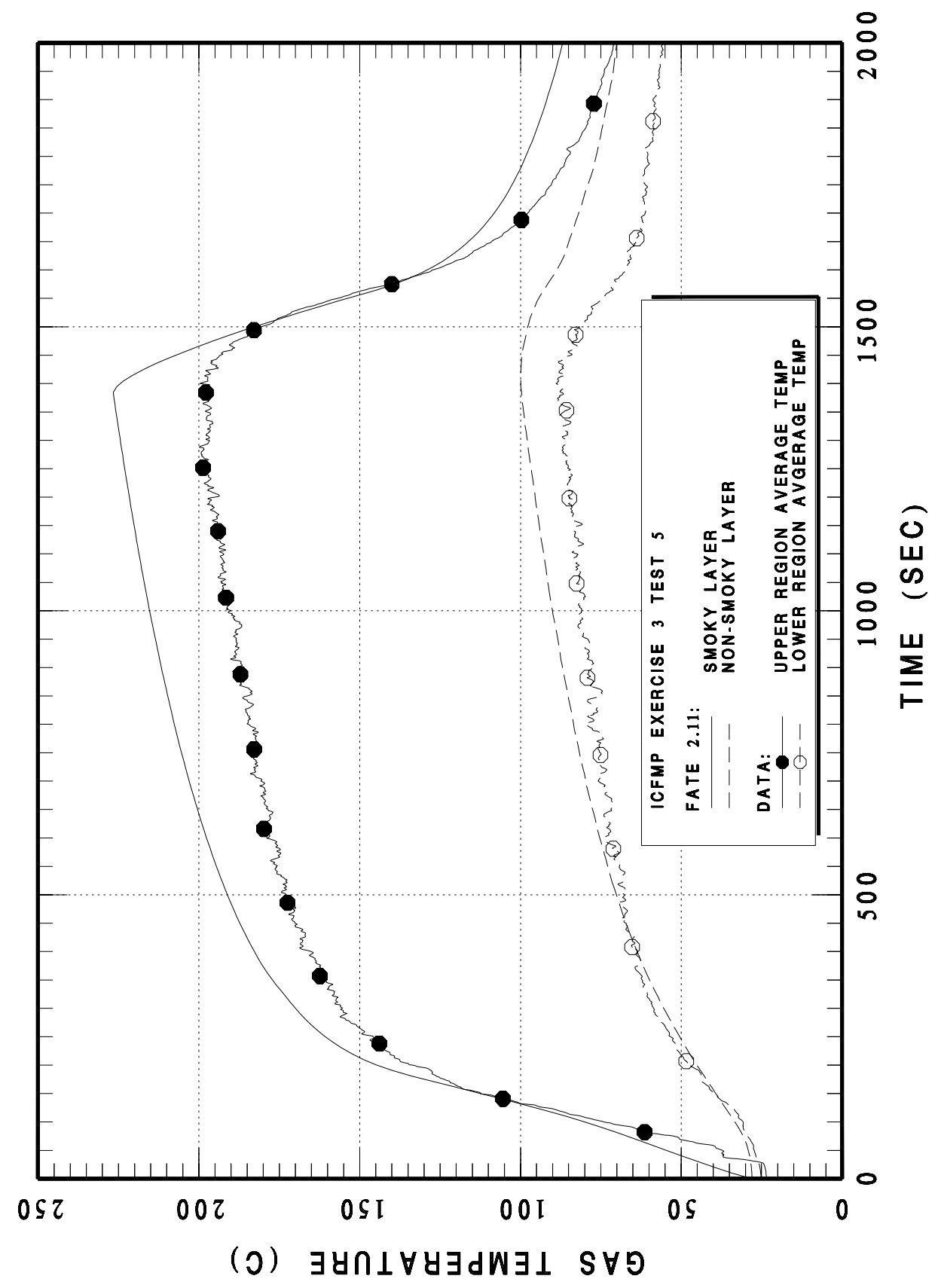

Figure 4-63 BE 3 CASE5 upper and lower layer average gas temperatures 


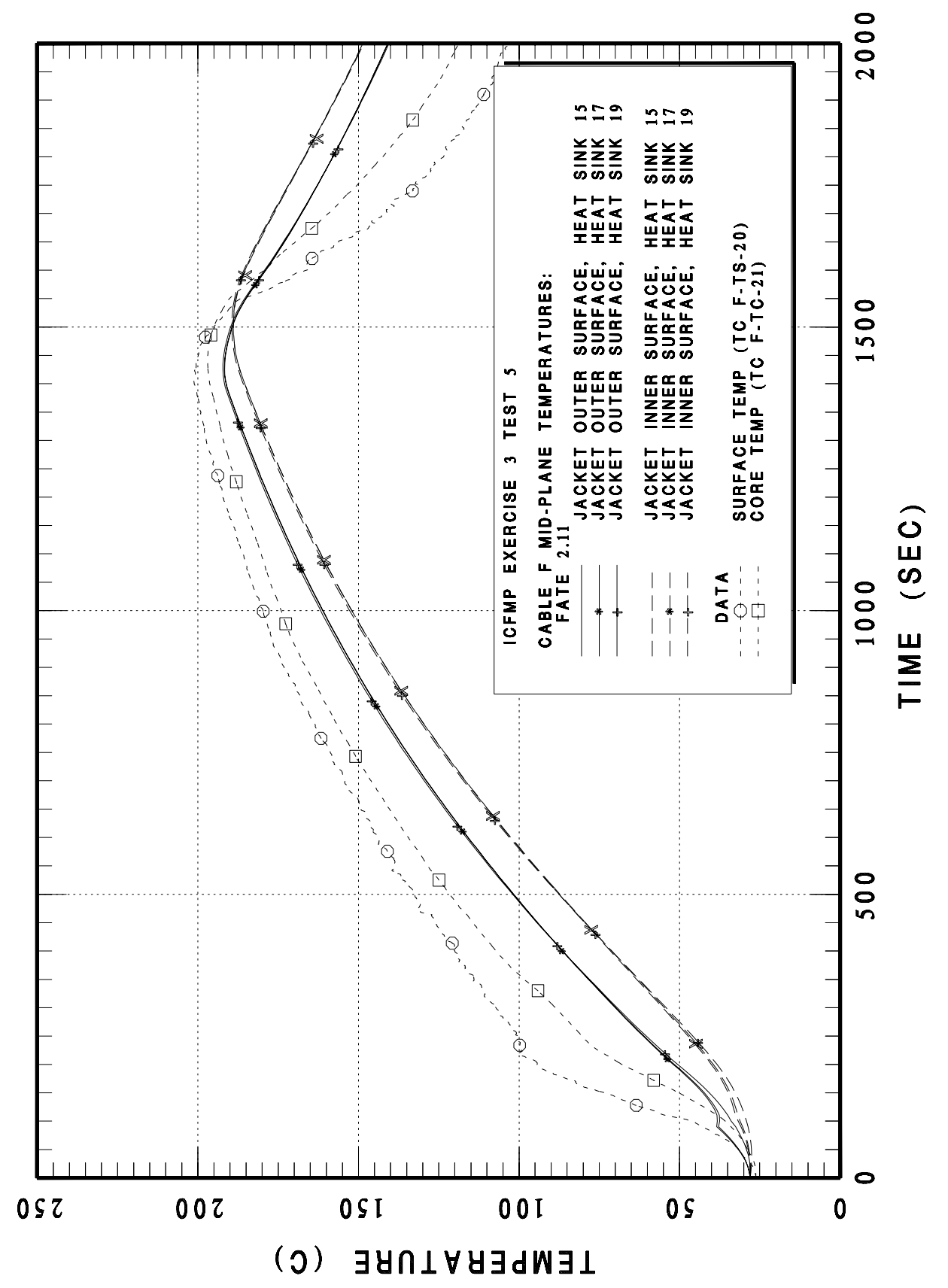

Figure 4-64 BE 3 CASE5 Cable F midplane temperatures 


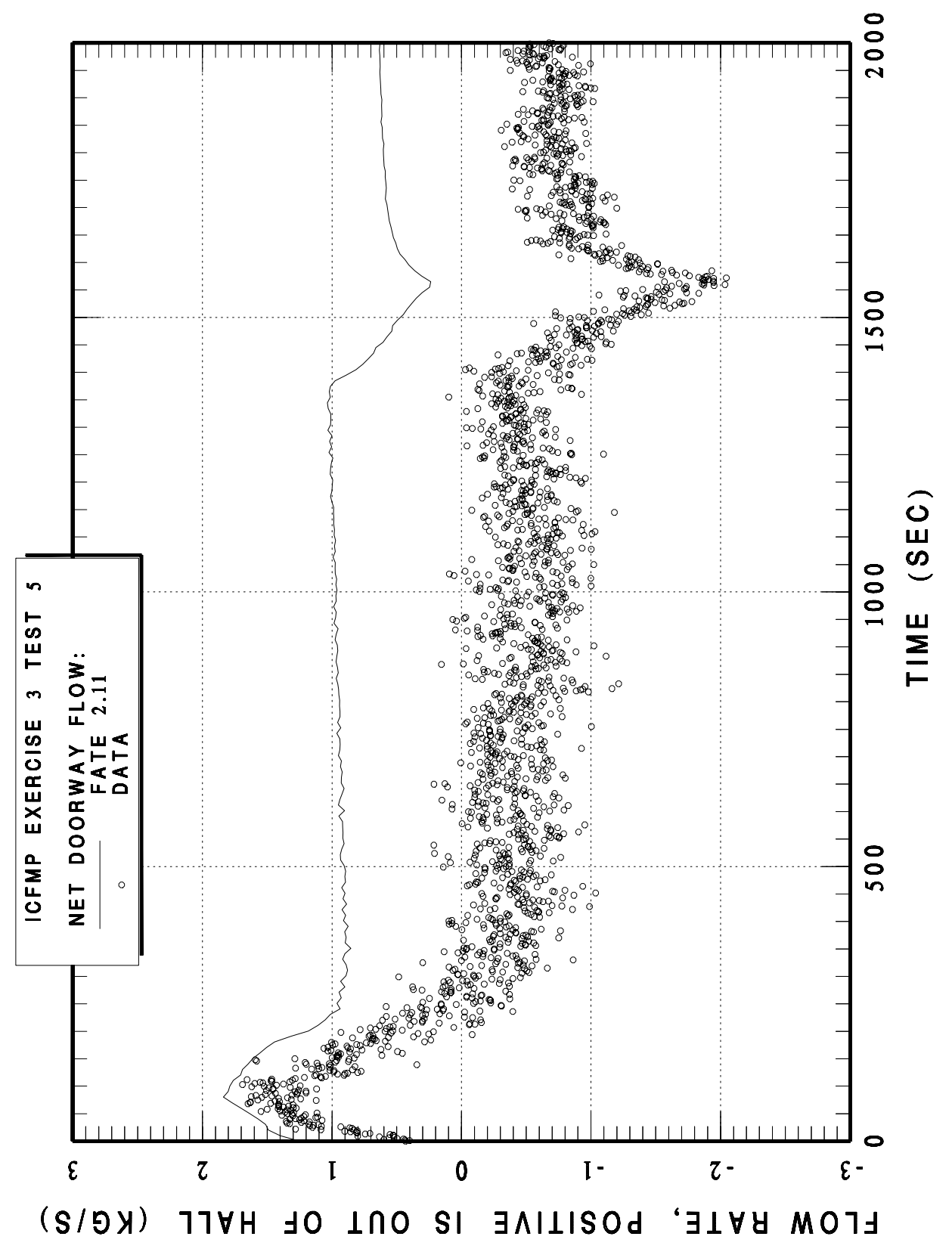

Figure 4-65 BE 3 CASE5 uni-direction flow through the doorway 


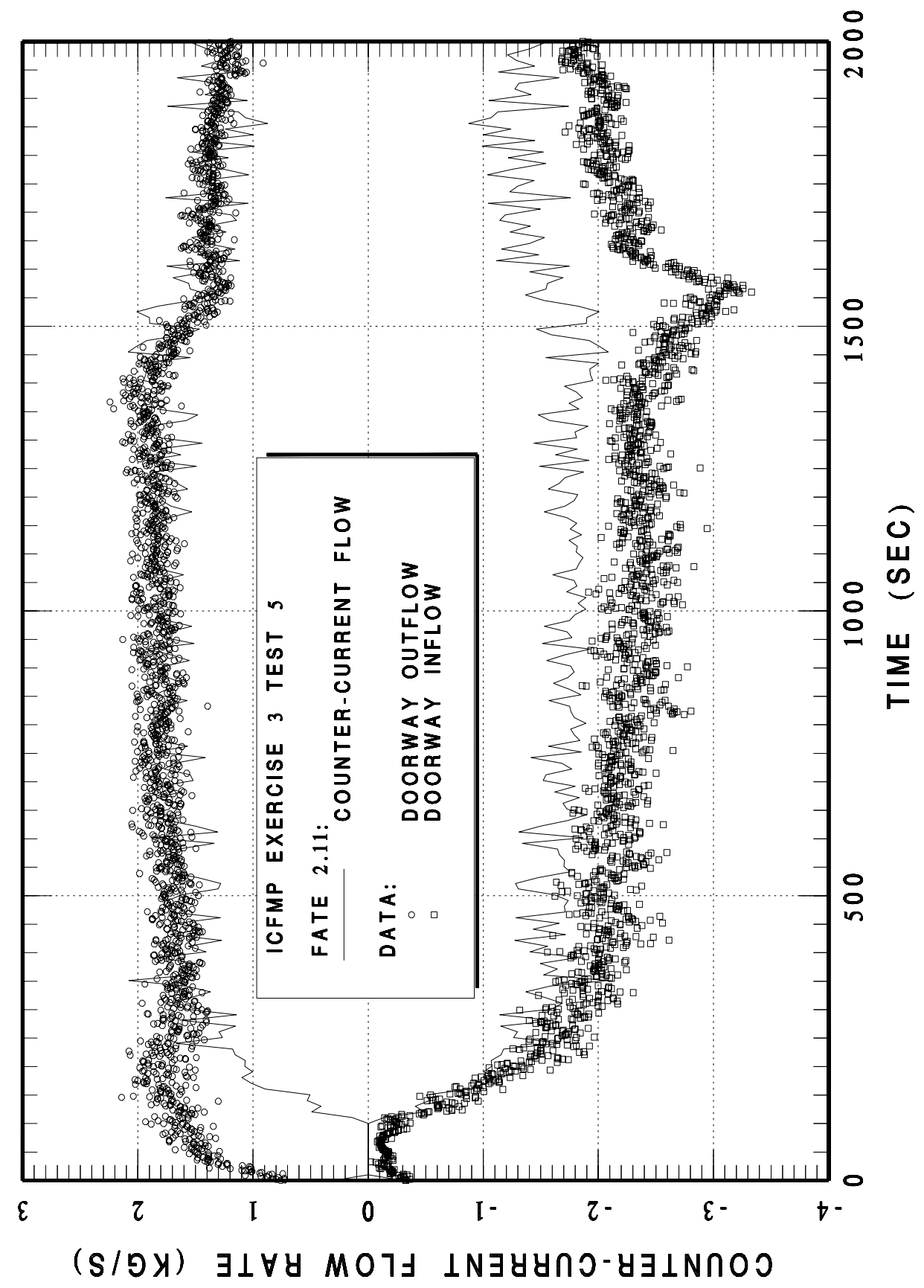

Figure 4-66 BE 3 CASE5 counter-current flow through the doorway 


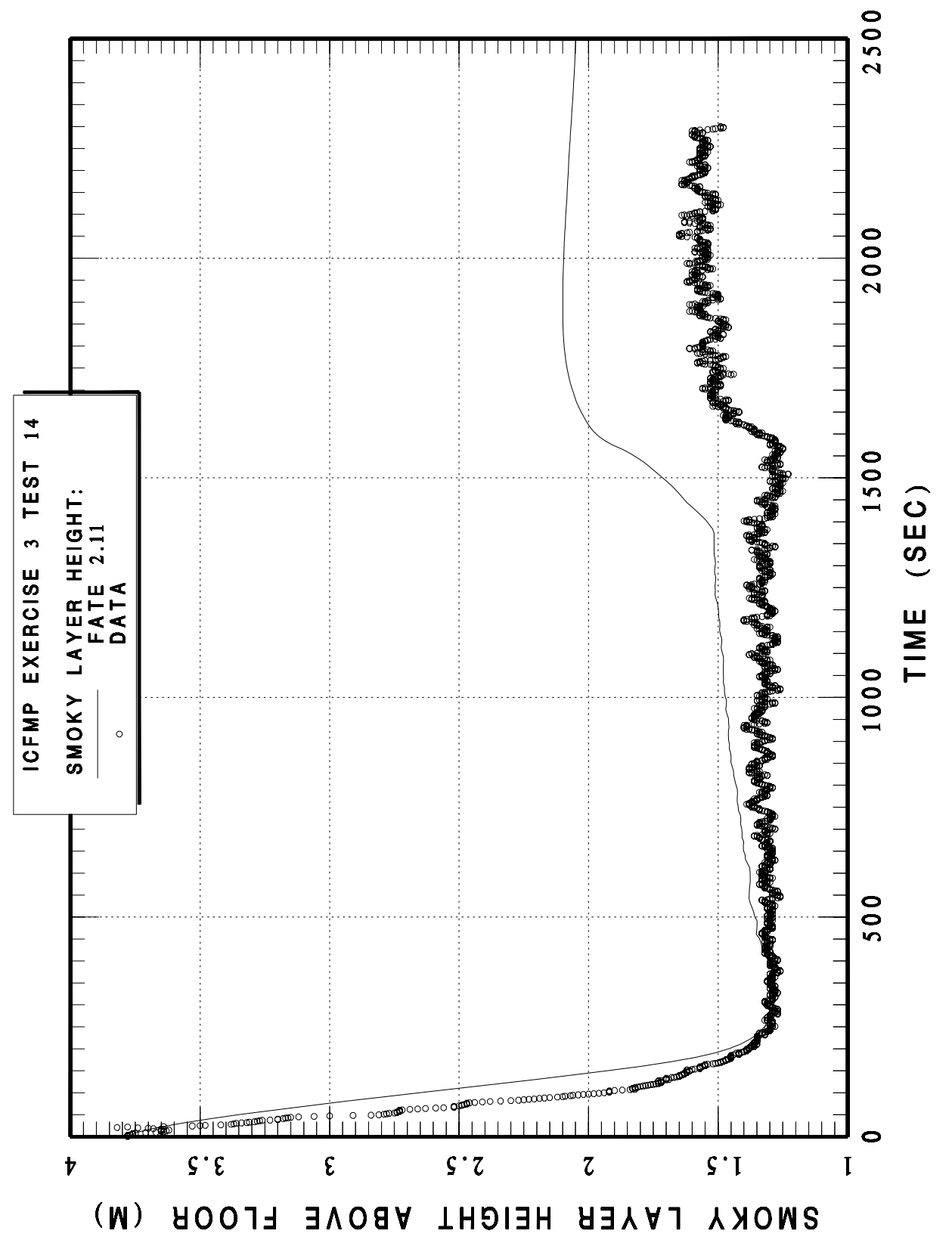

Figure 4-67 BE 3 CASE14 Smoky layer height 


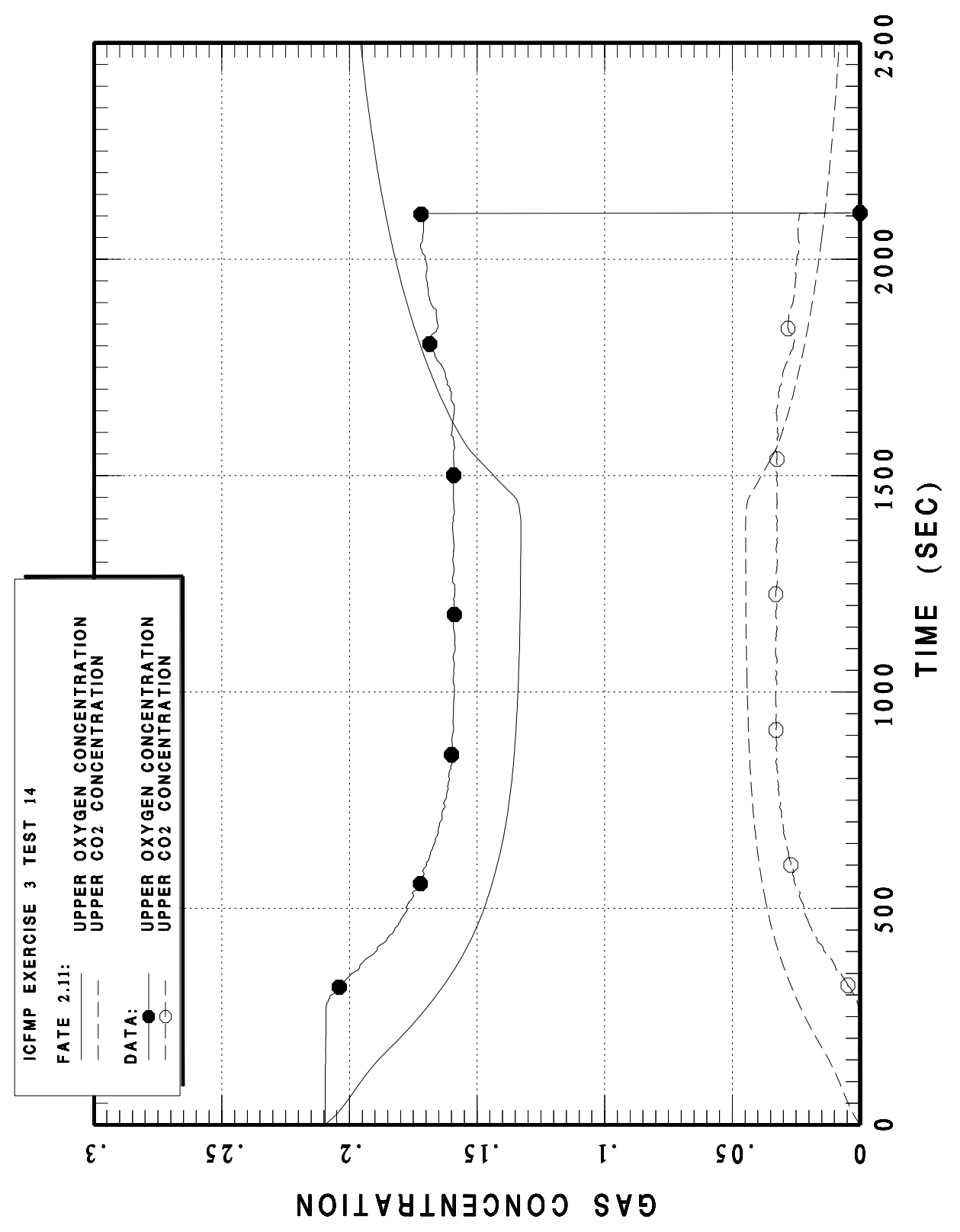

Figure 4-68 BE 3 CASE14 upper room gas concentrations 


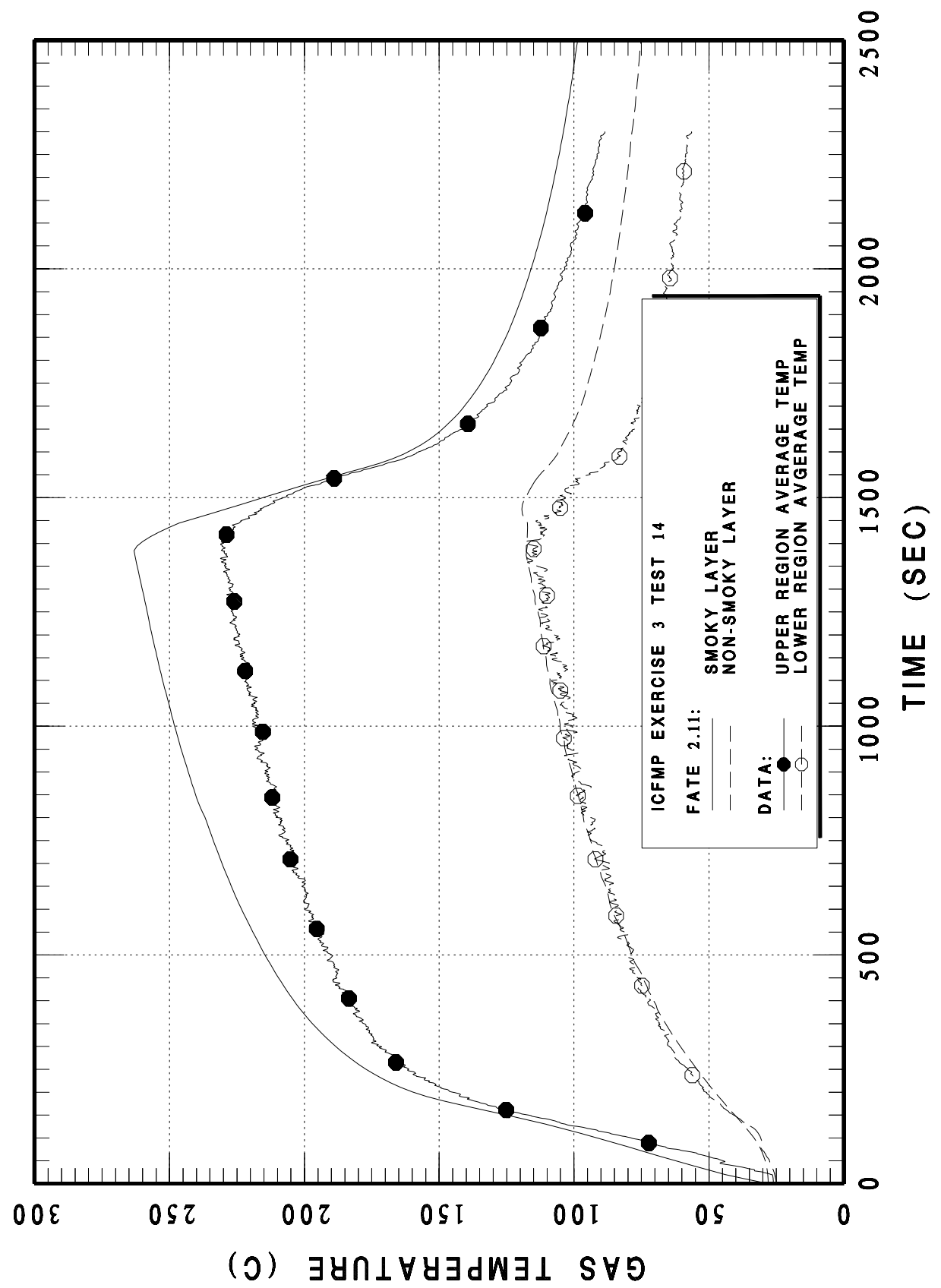

Figure 4-69 BE 3 CASE14 upper and lower layer average gas temperatures 


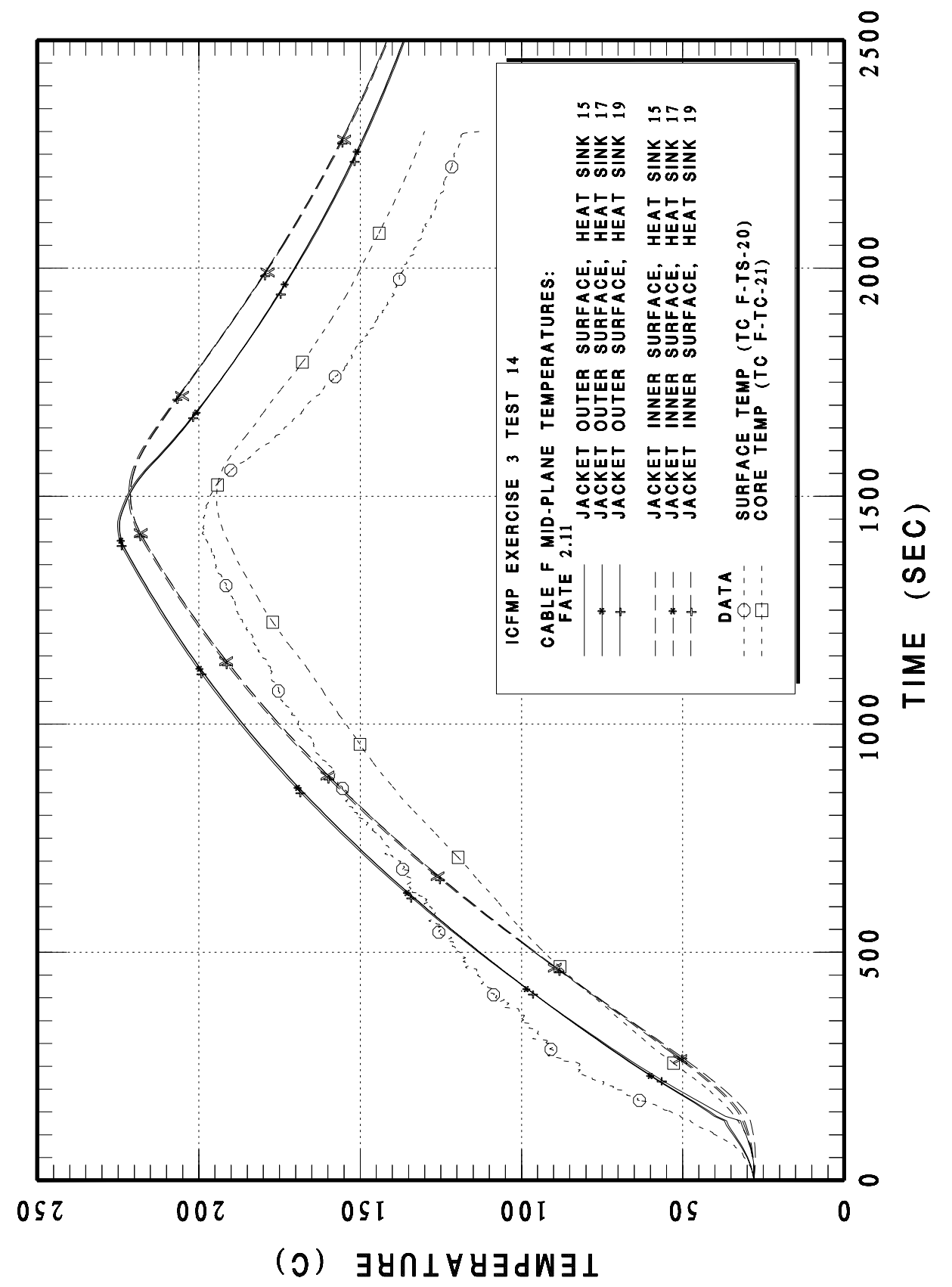

Figure 4-70 BE 3 CASE14 Cable F midplane temperatures 


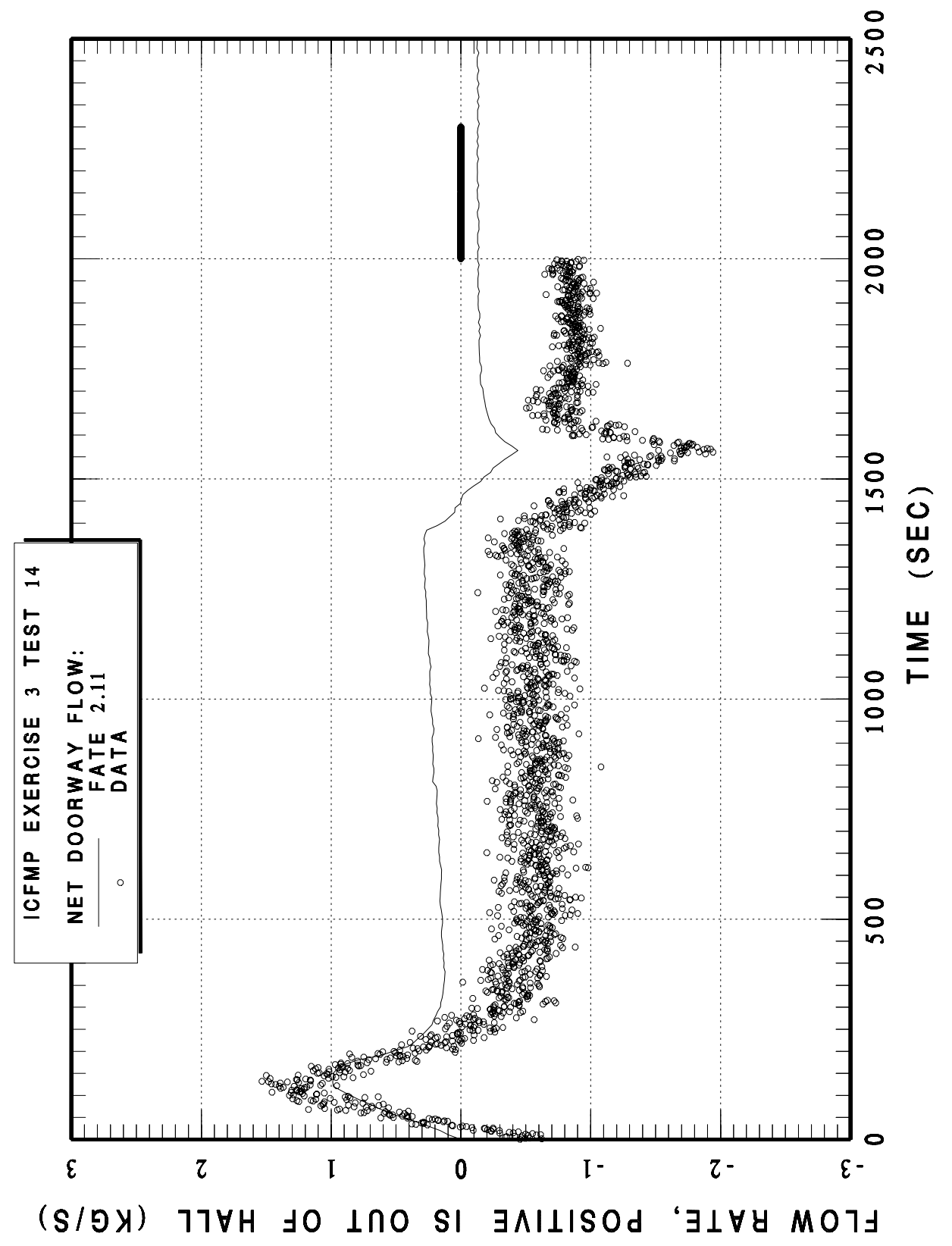

Figure 4-71 BE 3 CASE14 uni-direction flow through the doorway 


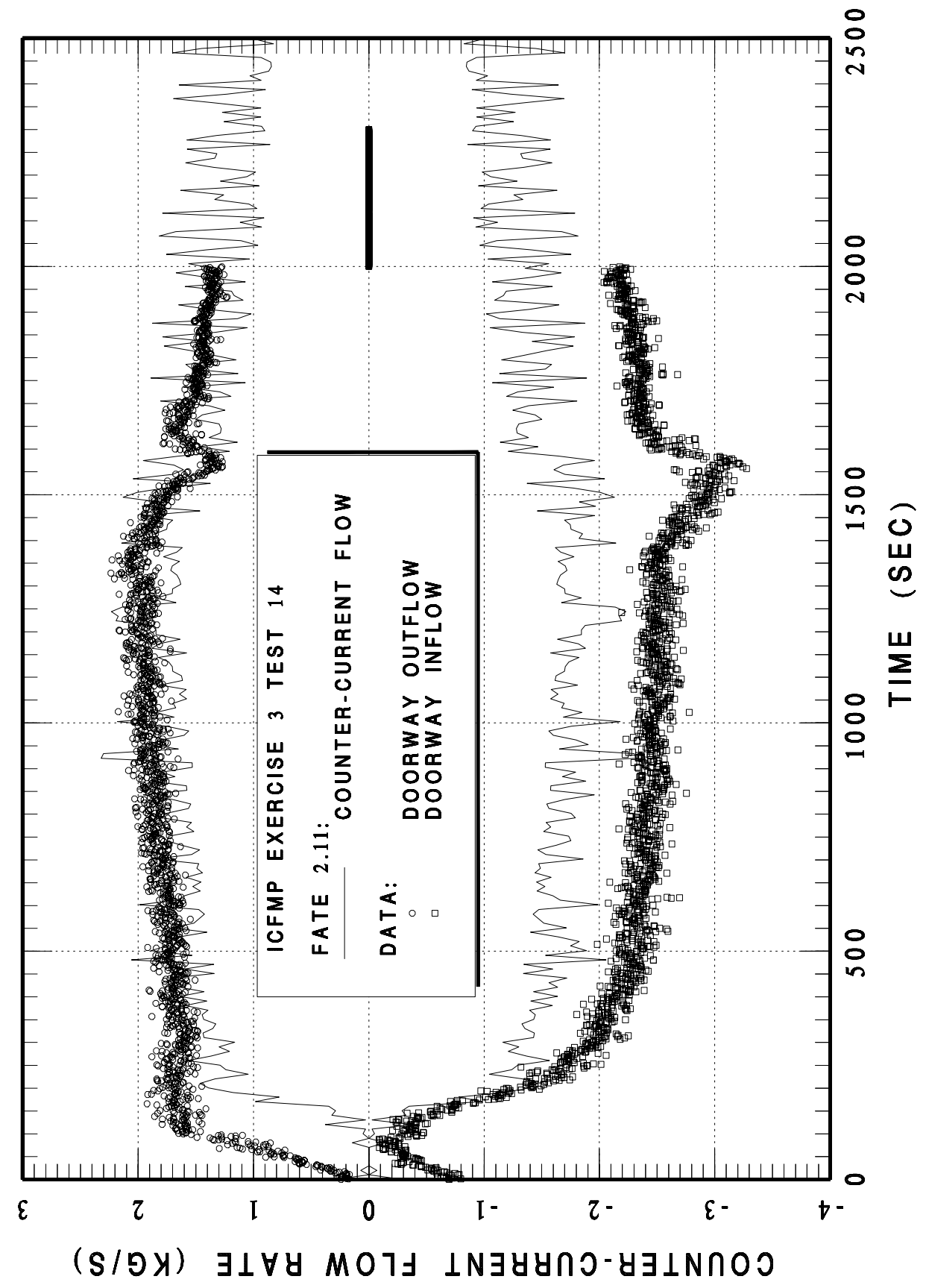

Figure 4-72 BE 3 CASE14 counter-current flow through the doorway 


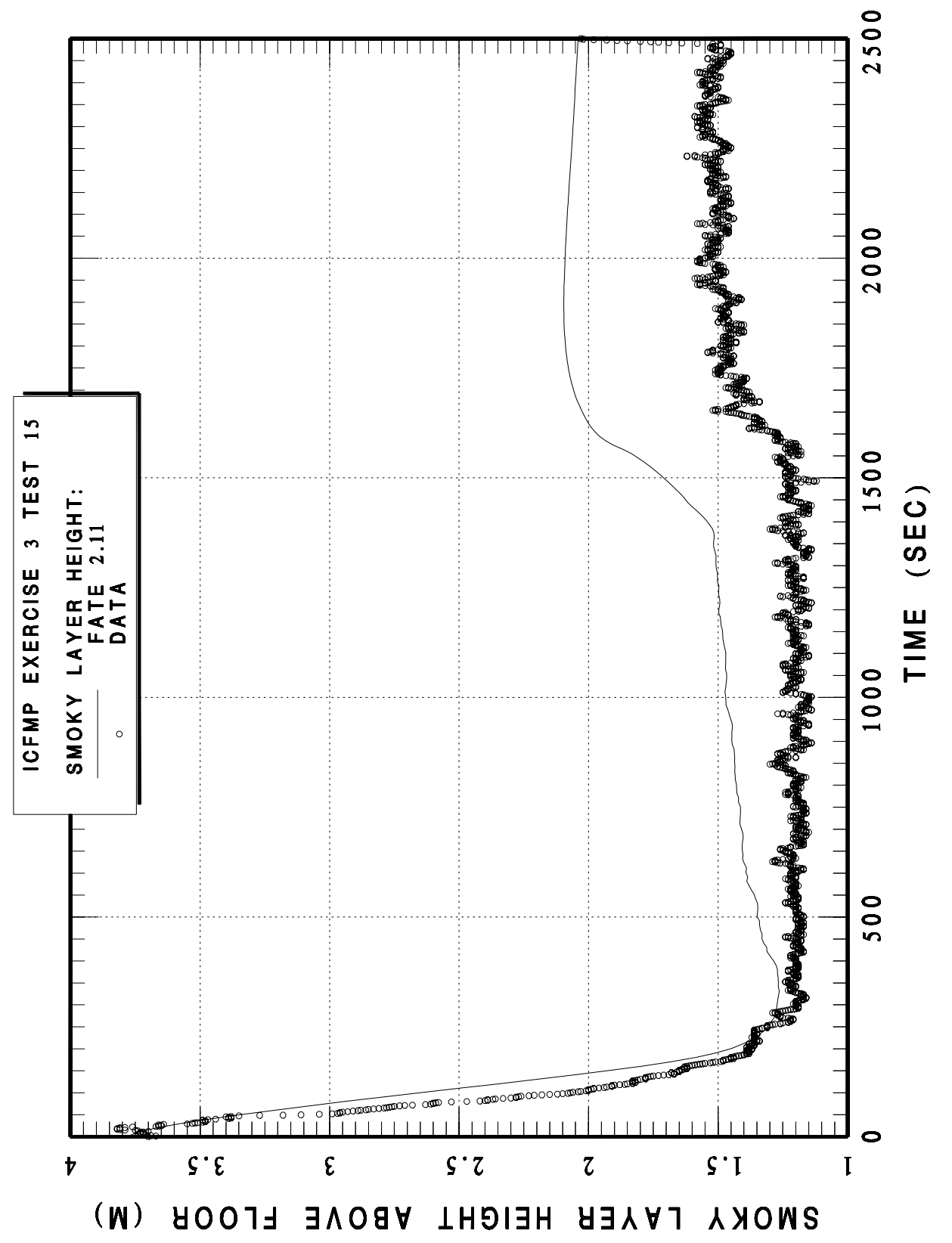

Figure 4-73 BE 3 CASE15 Smoky layer height 


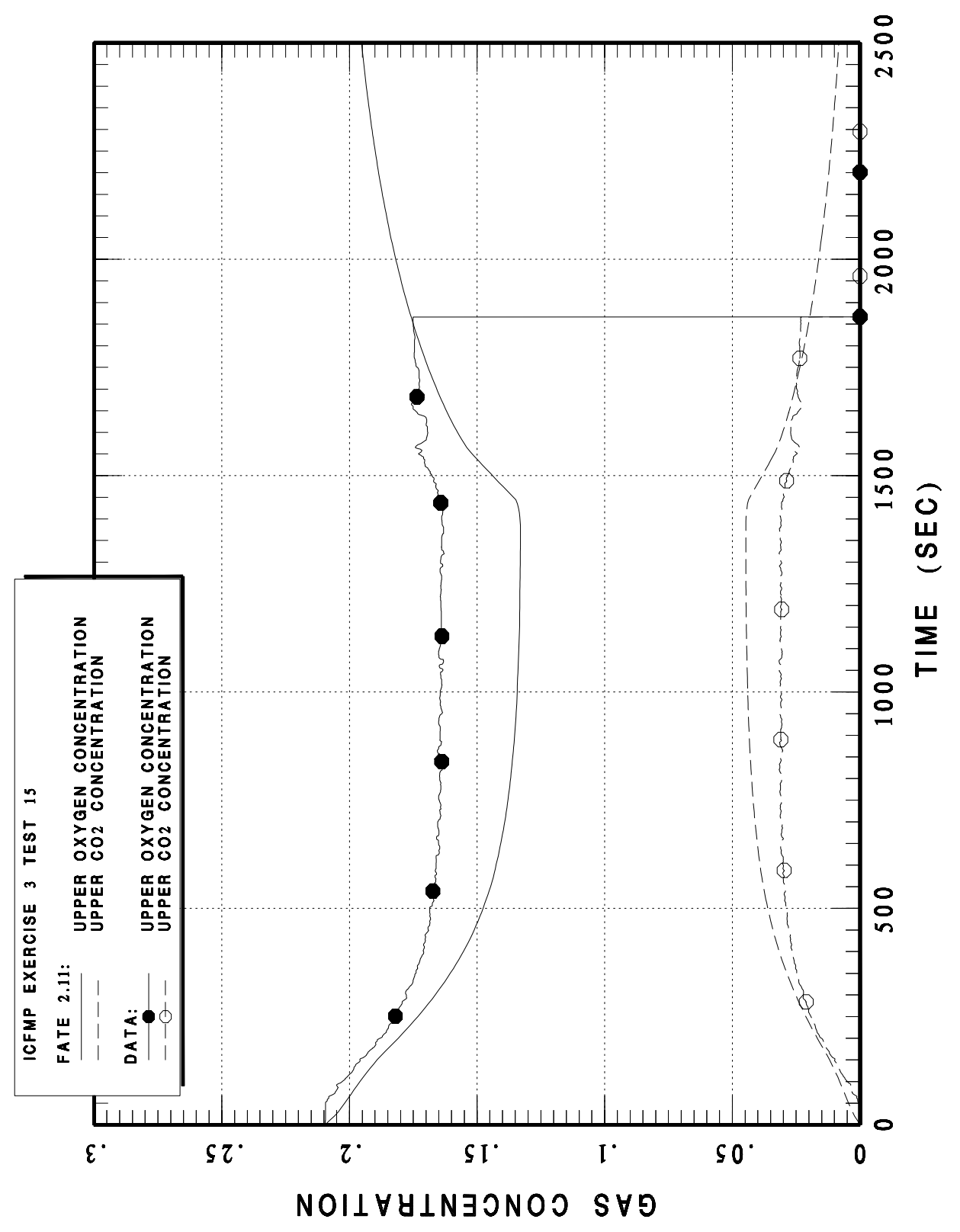

Figure 4-74 BE 3 CASE15 upper room gas concentrations 


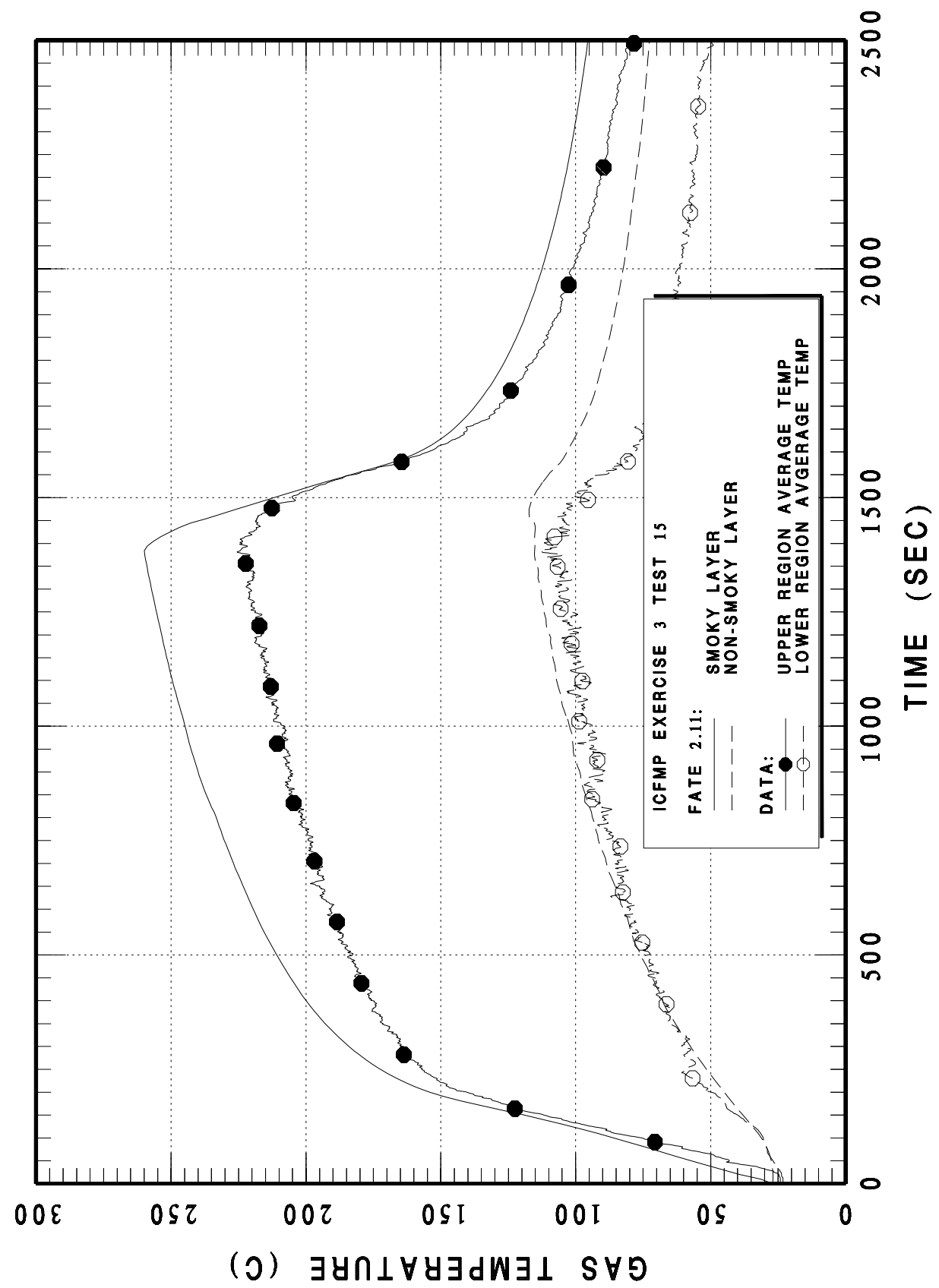

Figure 4-75 BE 3 CASE15 upper and lower layer average gas temperatures 


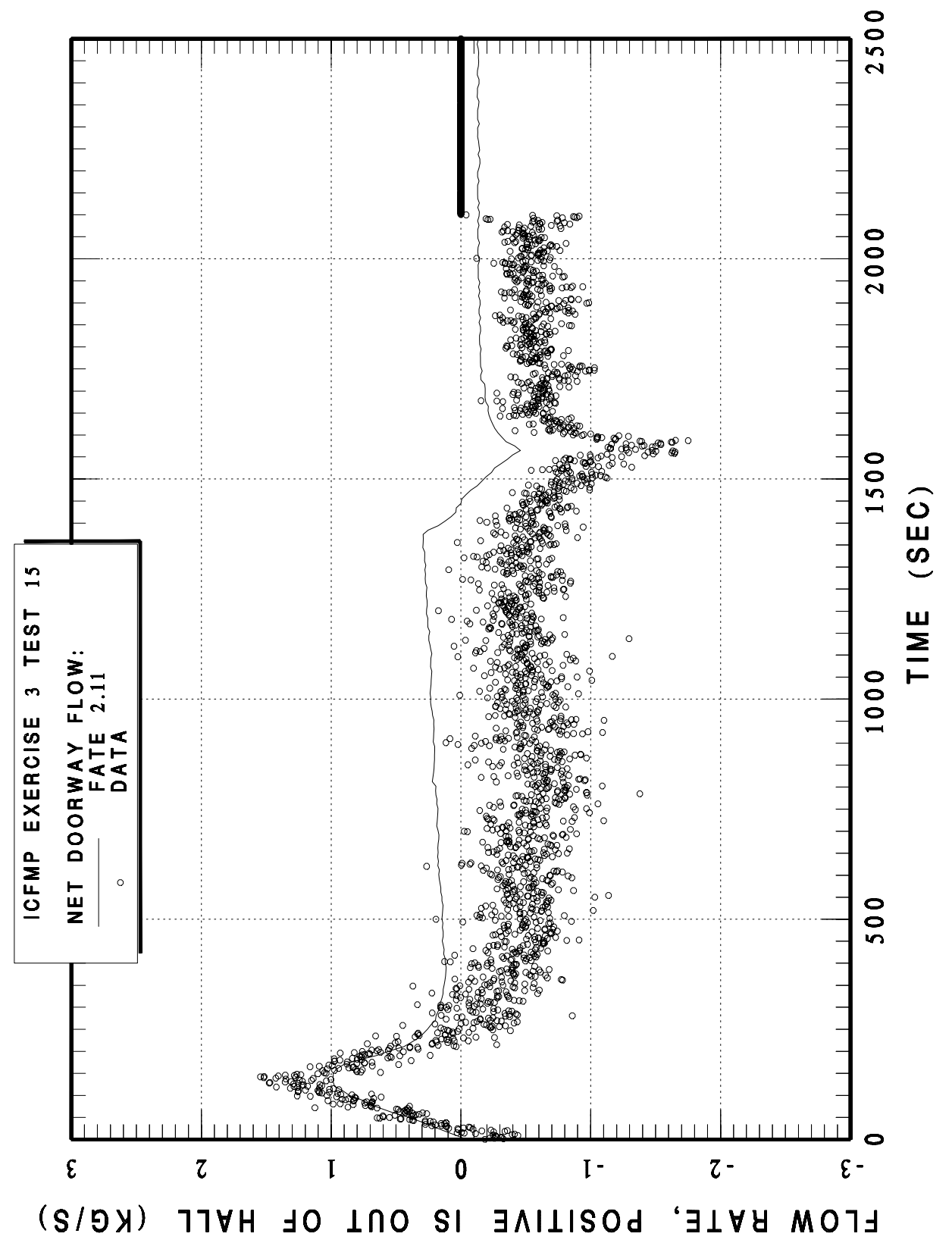

Figure 4-76 BE 3 CASE15 uni-direction flow through the doorway 


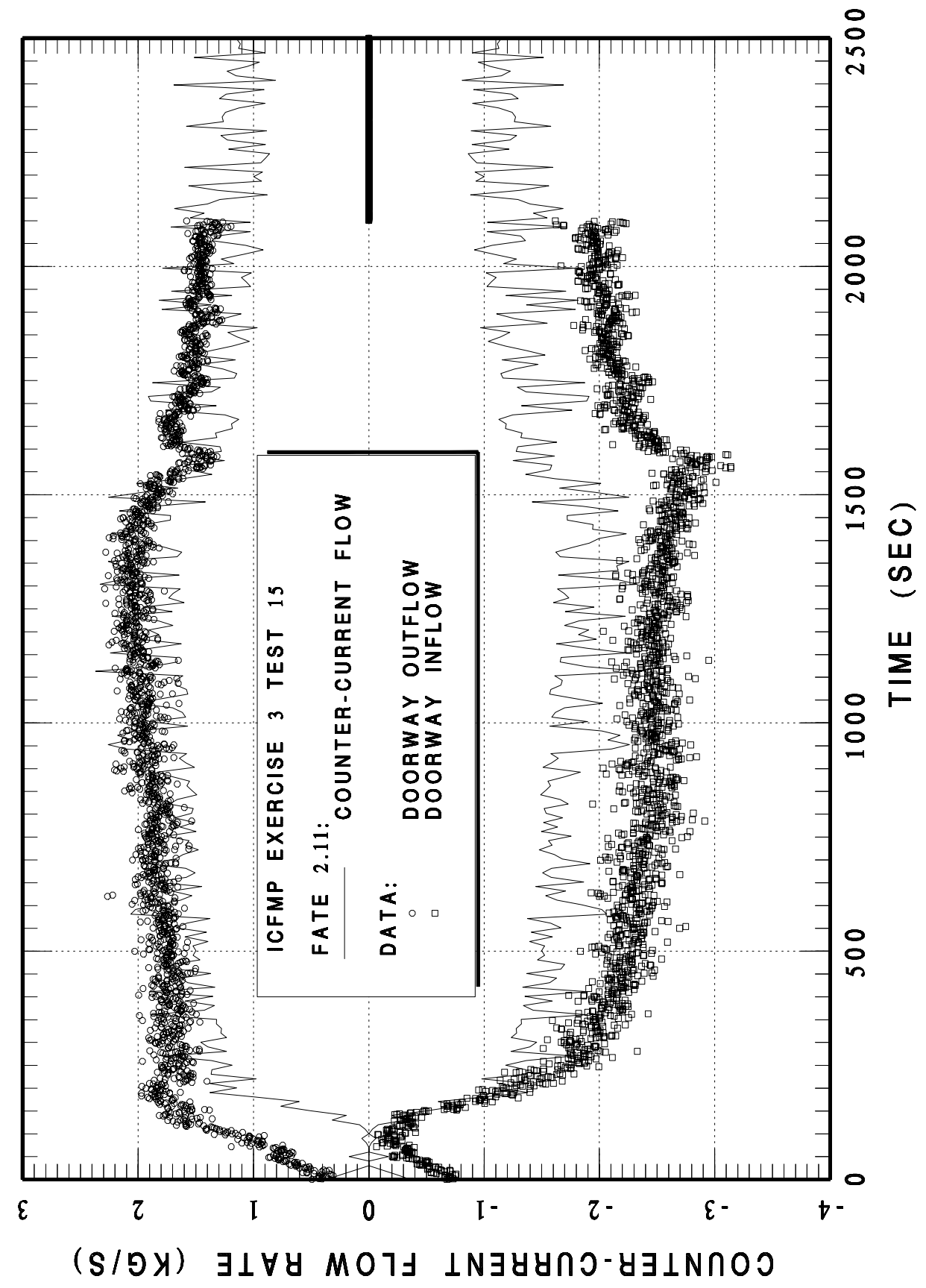

Figure 4-77 BE 3 CASE15 counter-current flow through the doorway 


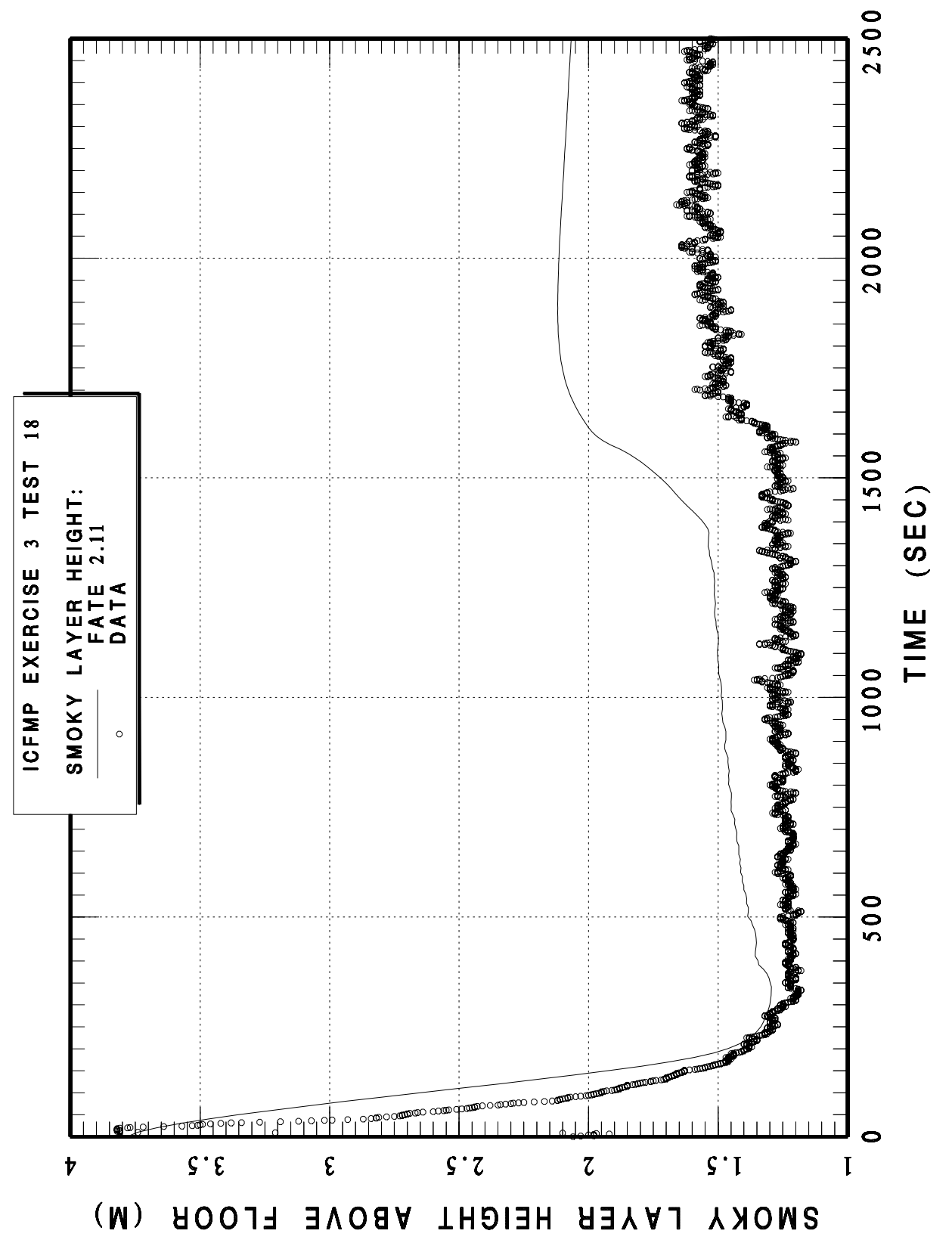

Figure 4-78 BE 3 CASE18 Smoky layer height 


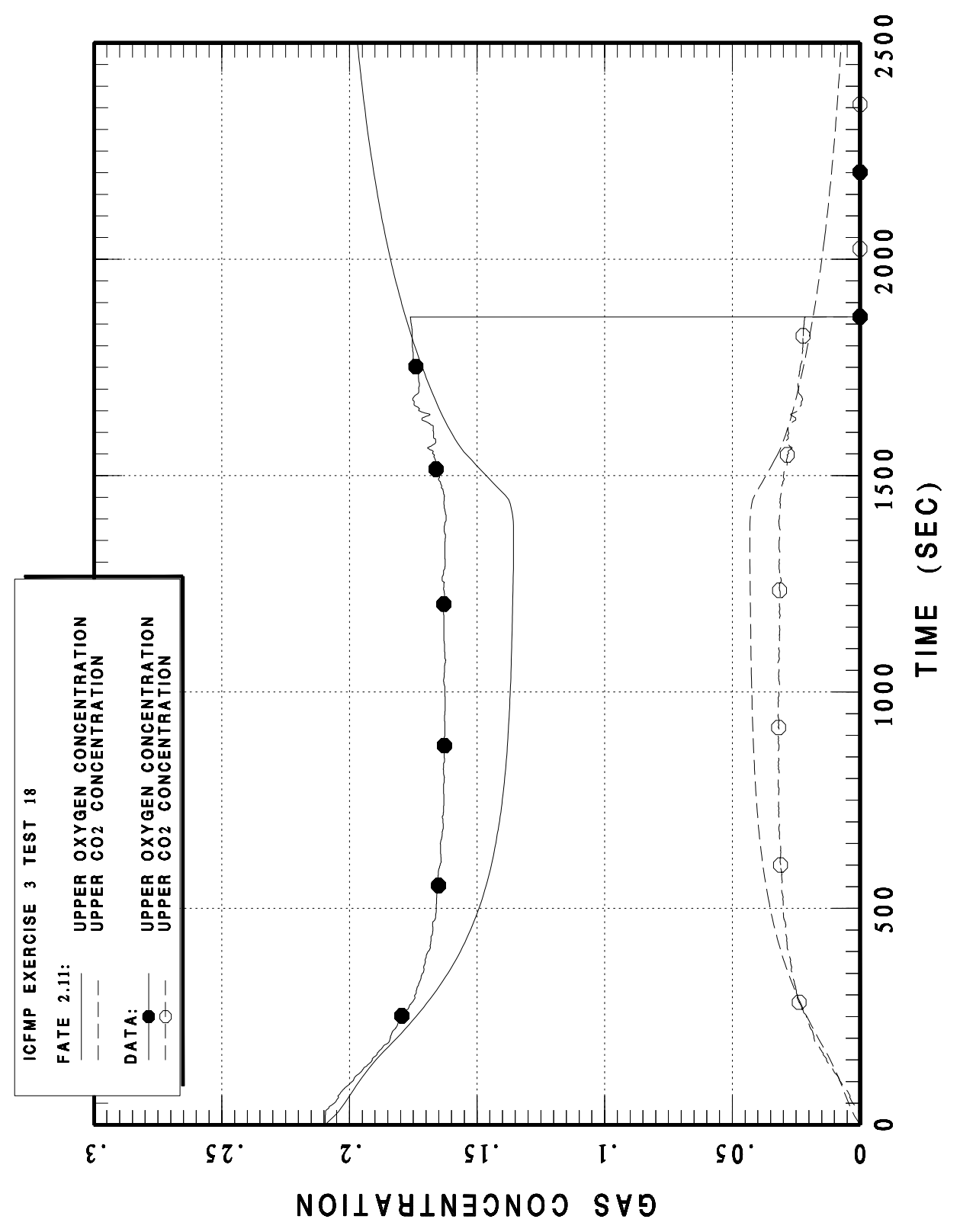

Figure 4-79 BE 3 CASE18 upper room gas concentrations 


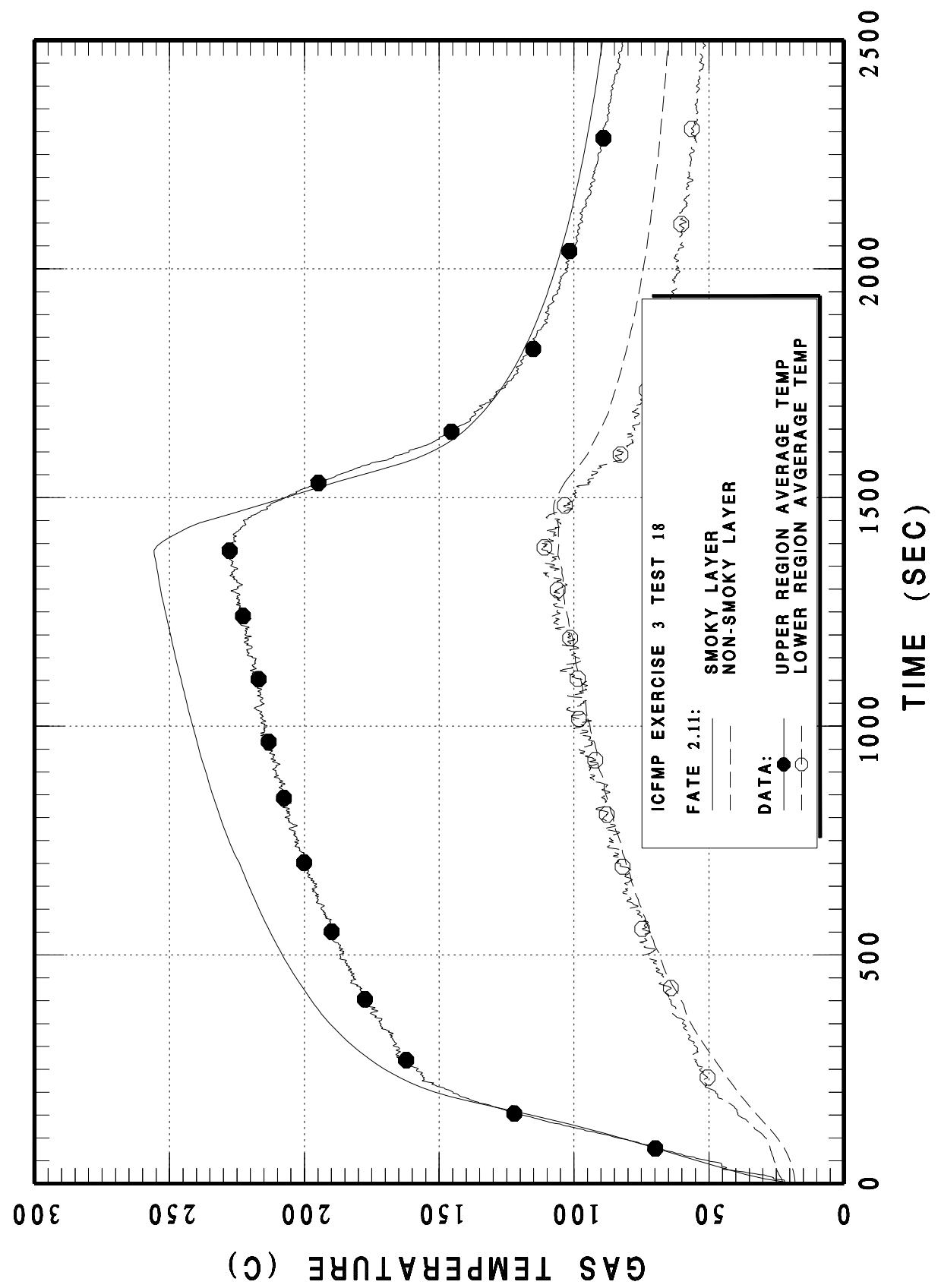

Figure 4-80 BE 3 CASE18 upper and lower layer average gas temperatures 


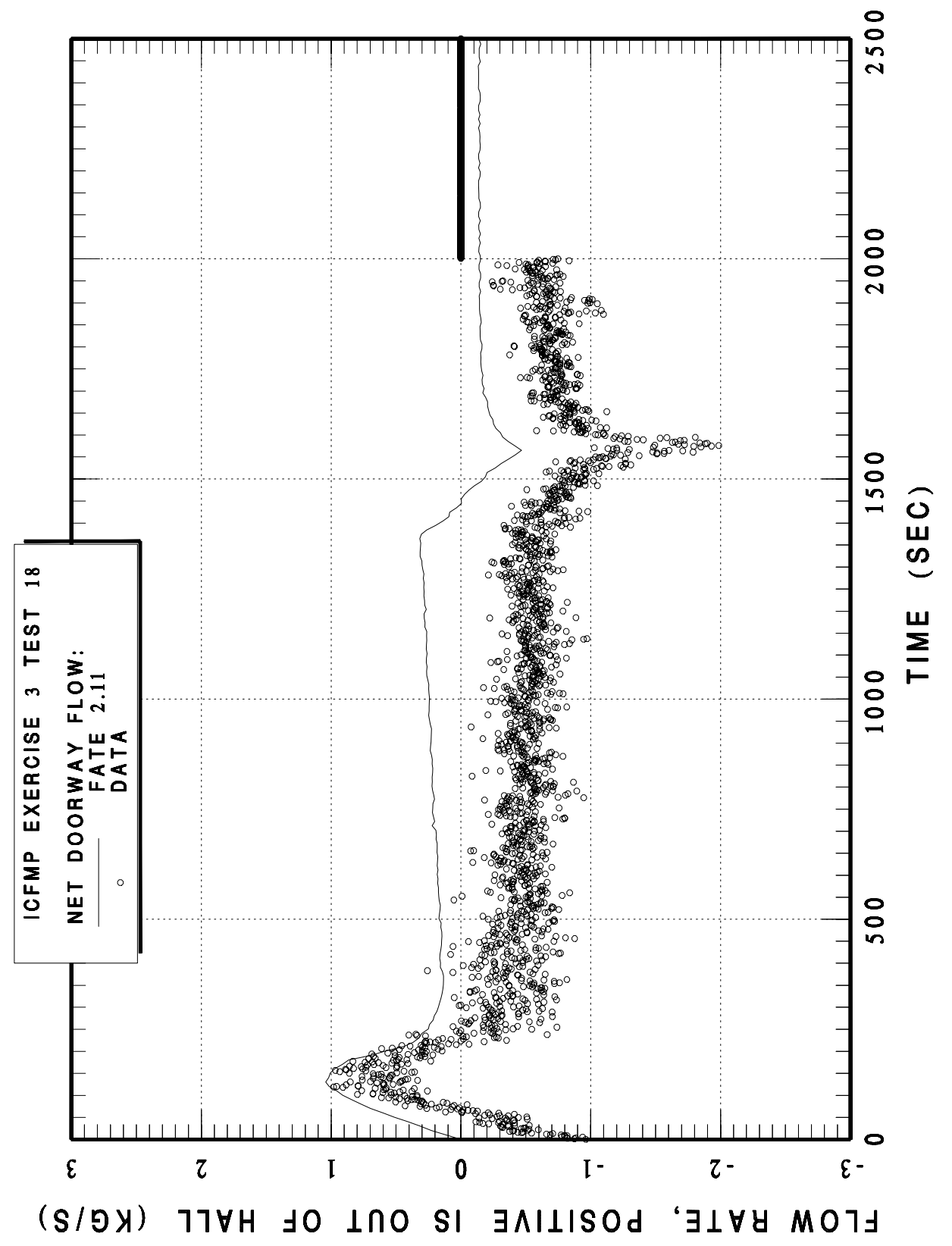

Figure 4-81 BE 3 CASE18 uni-direction flow through the doorway 


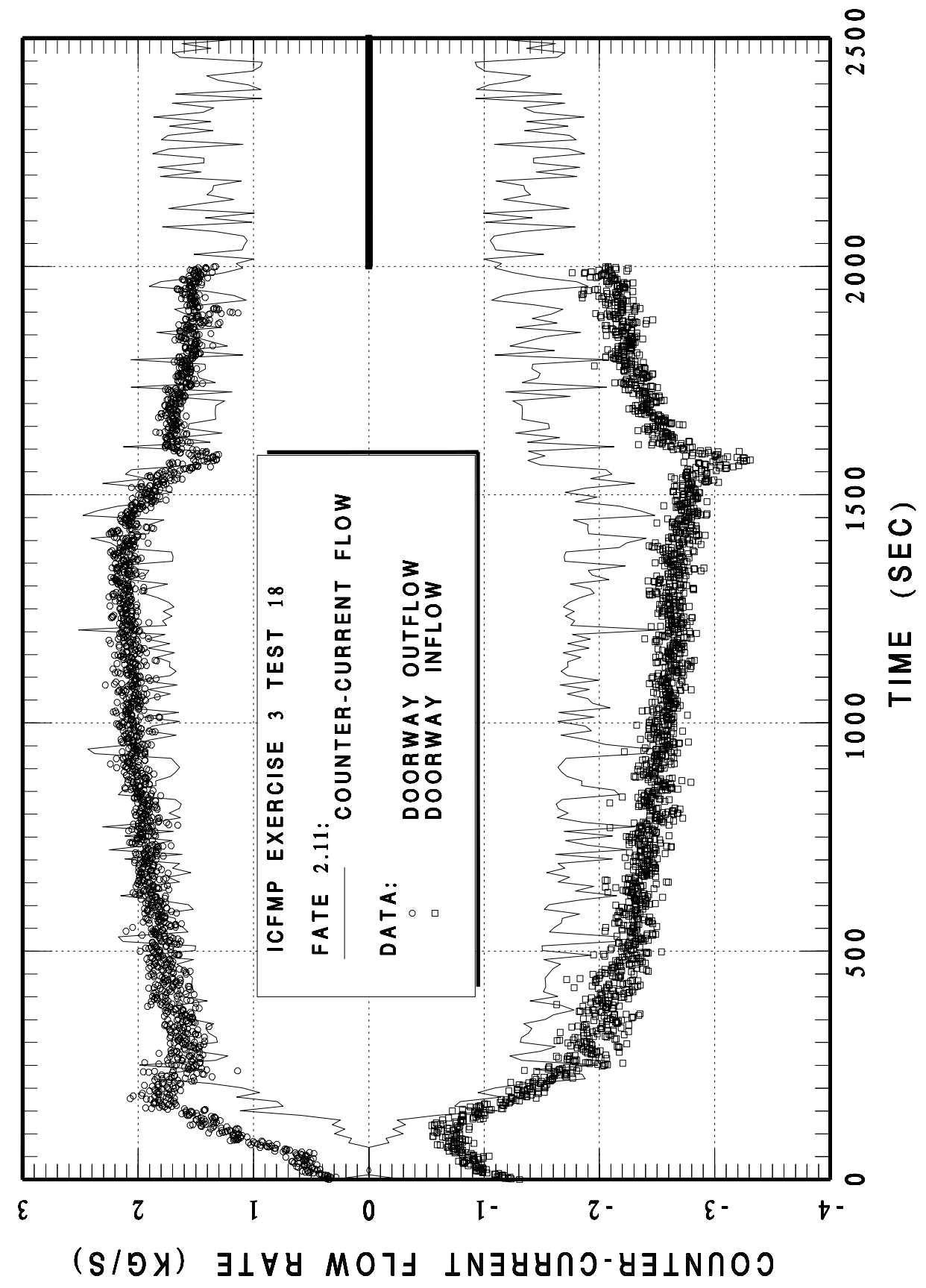

Figure 4-82 BE 3 CASE18 counter-current flow through the doorway 


\section{3}

Conclusions and Recommendations for FATE

Open benchmark results based on the FATE computer program have been presented for Benchmark Exercise 3 Cases 1 through 5, 14, 15, and 18. Key conclusions from this benchmark effort are:

1. Room leakage, even with doors open or fans on, plays an important role in smoky layer height and $\mathrm{O} 2$ concentration. Furthermore, counter current flow (through door for door open cases or through door gaps for door closed cases) is essential to obtaining reasonable estimates of smoke behavior.

2. FATE calculations indicate that air inflow to the room, whether through open doorways, operating fans, or leak paths, mixes with both the smoky layer and lower layer. Proper behavior of the smoky layer was obtained when the inflow was split between the two layers. Development of a mechanistic flow split model is under consideration.

3. The FATE standard room model consisting of a single region (subdivided into a smoky and lower region) with 14 heat sinks to represent floor, ceiling, and wall enclosure strips, and 5 junctions to represent door, fan supply and exhaust, miscellaneous leakage, and door gap leakage has proven to be effective in modeling prototypical cable room fires.

4. The standard room model also relies on a radiation network containing all heat sinks, targets, the smoky layer, and fire, and automatically calculates view factors between the members of the radiation network. This benchmark exercise validated the radiation network approach used in the room model.

5. In addition to the standard room model, a simplified cable model has also proven to be an effective means for evaluating target temperatures. The cable model simplifies the complex cable morphology into a 2 layer heat sink (outer jacket, inner cable insulation; typically the outer jacket encloses multiple cables with fill material in between the cables), neglects axial conduction along the cable, and divides the cable surface into 3 components azimuthally. As shown in the benchmark, there is no azimuthal variation in temperature prediction by FATE, so future cable models can consider only 1 azimuthal component.

Shortcomings in the FATE calculations are as follows:

1. Heat loss to ceiling is consistently higher in FATE than indicated in test data. 
2. Cable temperatures are often under-predicted. This is likely due to calculating too low of a value for the cable surface heat transfer coefficient.

\subsection{References}

Plys, Martin G., and Sung Jin Lee, 2004, FATE ${ }^{\text {TM }}$ 2.0: Facility Flow, Aerosol, Thermal, and Explosion Model (Improved and Combined HANSF and HADCRT Models), FAI/04-71, SNF-23281, Two Volumes, November.

NIST, 2003, Special Publication, "Report of Experimental Results for the International Fire Model Benchmarking and Validation Exercise \#3,"Anthony Hamins, Alexander Maranghides, Rik Johnsson, Michelle Donnelly, Jiann Yang, George Mulholland, Robert L. Anleitner, Building and Fire Research Laboratory (November, 2003).

NIST, 2004, "Test Plan For International Fire Model Benchmarking and Validation Exercise \#3," Alexander Maranghides, Anthony Hamins, Rodney Bryant, Erik Johnson, George Mulholland, John Widman (December 19, 2004).

NISTIR 6986, "International Collaborative Project to Evaluate Fire Models for Nuclear Power Plant Applications: Summary of $5^{\text {th }}$ Meeting," Dey, M., Hamins, A., Miles, S. (September, 2003). 
COCOSYS is a so-called lumped-parameter code. To simulate the local conditions (natural convection, temperature stratification) the fire compartment has been divided into a relatively large number of control volumes. The idea is to have for each temperature measurement a separate control volume, and to have separate control volumes around the fire plume, the ventilation system, and the doors and openings.

For the simulation a 3-D grid has been created using the grid generator GRIDGEN. With a specific interface routine a COCOSYS input file has been created. This interface routine calculates the view factors between different walls and from the control volumes to the walls using a Monte-Carlo simulation method. By this it is possible to simulate the radiation flux to the different targets in a realistic way.

Blind calculations for tests 2, 3 and 4 were performed before the experiments were conducted. After the release of the experimental data for tests 2, 3 and 4, the calculations were improved and presented as open calculations. Finally, using the specified input parameters from the test documents (like the fire's radiative fraction), the remainder of the tests were calculated without knowledge of the experimental results.

\subsection{COCOSYS Input Parameters and Assumptions}

COCOSYS is a so-called lumped-parameter code, dividing the compartments into several control volumes, where the mass and energy balance is solved. The main difference to usual CFD codes (like CFX or FDS) is that the momentum balance is not considered. The nodalization is presented in Fig. 5-1 and Fig. 5-2. So far as possible each measurement point has its own control volume. The nodalization has been created with the commercial tool GRIDGEN, and with an interface program the COCOSYS input was created. The door was sub-divided into four levels to allow counter-current flow. The cable targets were represented as structures. Therefore they were placed between the zone levels. As seen in Fig. 5-2 the nodalization is more detailed near the fire and the cable targets. In total, 274 zones (control volumes) were created (Fig. 5-3). 


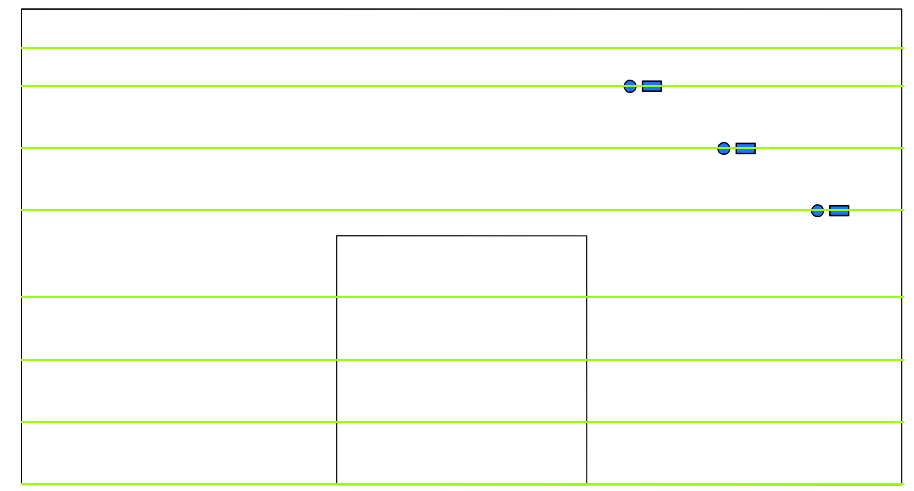

Fig. 5-1 Side view of COCOSYS model

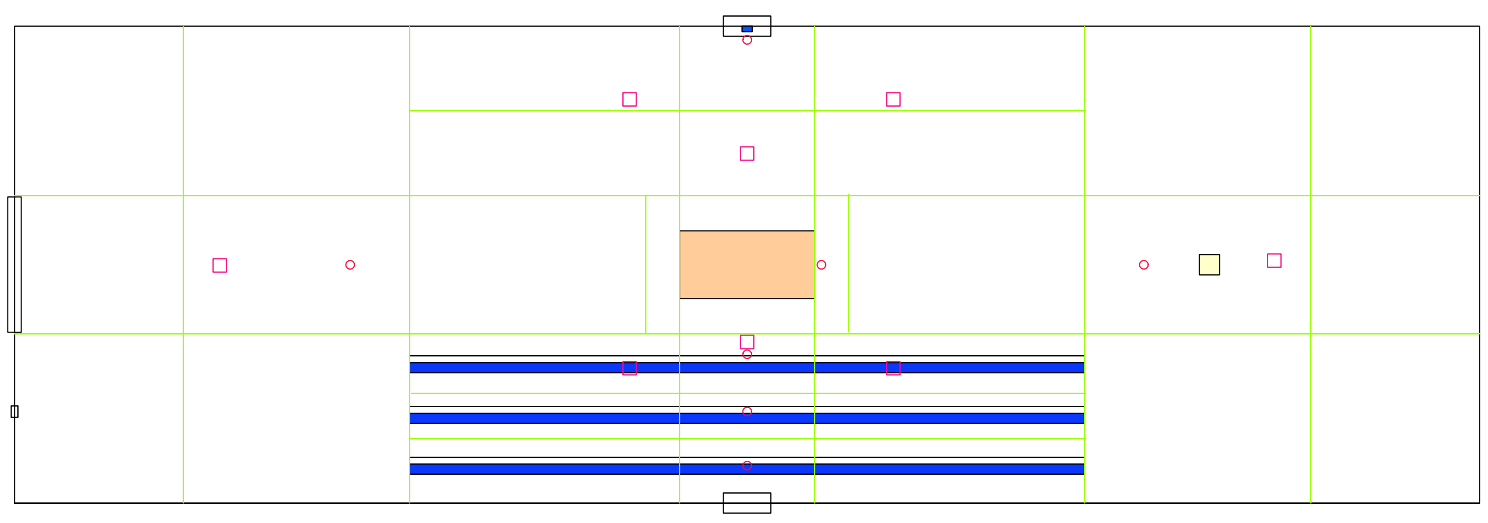

Fig. 5-2 Top view of COCOSYS model 


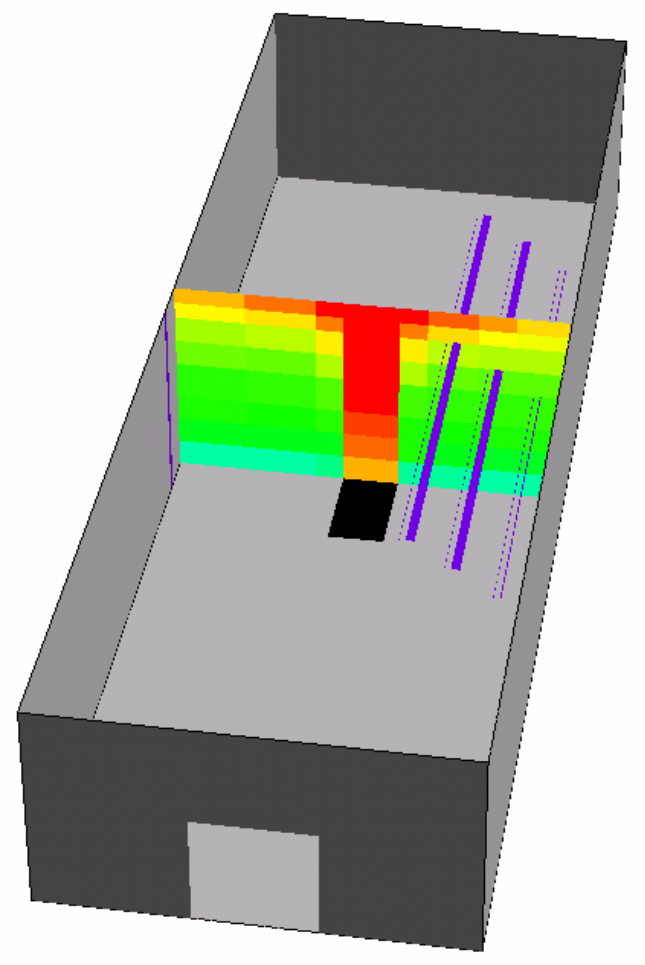

Fig. 5-3 3-D view of COCOSYS model

1-D heat conduction through the $2.54 \mathrm{~cm}$ thick outside walls was performed. The leak area was assumed to be $0.0289 \mathrm{~m}^{2}$ in the first blind calculation (test 2, 3 and 4) as given in the specification. According to the experimental results this value was increased to $0.058 \mathrm{~m}^{2}$ (value for test 2) for the open calculations and further blind calculations. Although specific leakage areas were given for each experiment, this value was not changed in the cocosys input.

For the simulation of the heptane fire, injection tables were used. The flow rate was estimated using the specified heat of combustion of $45 \mathrm{MJ} / \mathrm{kg}$. In the first blind calculations (test 2, 3 and 4) the fuel flow rates were based on the specified heat release rate of $1 \mathrm{MW}$. In the following open and blind calculations, the measured values were used. For the first blind calculations a radiative fraction of $35 \%$ was assumed. In the following calculations this value was increased to $44 \%$ according to the documented value. In Tab. 5-1 the parameters for combustion in the first blind and the following open/blind tests are presented. In the open and further blind calculations the heat release rate was increased. In the first blind calculations the $\mathrm{CO}$ reaction was under-estimated, so the $\mathrm{CO}$ reaction rate was increased. In the same way the combustion stoichiometry was adjusted (Tab. 5-2). 
In COCOSYS it is possible to stop the calculation if the oxygen concentration in a certain control volume falls below a given value. This option was used in the blind calculations. After the stop, the calculation was restarted without a fire. In the open calculations the times of fuel supply were used directly.

In the calculations for tests 4, 5, 10 and 16, an air supply and exhaust system was used. This was modeled with the fan system model in COCOSYS. The fan characteristics are shown in Tab. 5-3. These characteristics were used for all calculations. Only the flow rate was increased from $0.75 \mathrm{~m}^{3} / \mathrm{s}$ to $1.1 \mathrm{~m}^{3} / \mathrm{s}$ in the open and further blind calculations.

The cable targets are simulated by plate-type wall structures (Fig. 5-4). These structures are composed of the outside insulation material with an inner metal core. The area of the plate corresponds to the projection of the cable or cable tray. The thickness is estimated to the existing cross sections of wires and insulation material. It has to kept in mind, that the interaction with the environment is only via the plate surface. Especially for small cable objects some differences may be expected.

The junction box was simulated by an additional control volume BOX connected via a feraloy structure to the fire compartment and to the ceiling via a combined feraloy-marinite structure. Because this additional volume was not created by GRIDGEN, the junction box structure was not considered in the view factor calculation and no wall-to-wall radiation was considered. That means only convective and radiative heat transfer from the connected control volume of the fire compartment. 
Tab. 5-1 Combustion Parameters

\begin{tabular}{|l|l|l|l|}
\hline Parameter & Comment & First blind & Open/blind \\
\hline PYRMIX & Mixing velocity $[\mathrm{m} / \mathrm{s}]$ & 2.0 & 0.5 \\
\hline TIGNMX & Ignition temperature $\left[{ }^{\circ} \mathrm{C}\right]$ & 280. & 300. \\
\hline TIGNMN & Min. burning temperature $\left[{ }^{\circ} \mathrm{C}\right]$ & 70. & 70. \\
\hline RLIM_CHX & $\mathrm{CH}_{X}$ ignition conc. $[\mathrm{Vol}-\%]$ & 0.05 & 0.24 \\
\hline RIGN_CO & $\mathrm{CO}$ ignition conc. $[$ Vol-\%] & 0.5 & 0.1 \\
\hline DLIM_O2 & Lower oxygen limit [Vol-\%] & $0.0+2.0$ & $0.0+2.0$ \\
\hline FEFF & Burning efficiency $[-]$ & 0.99 & 0.99 \\
\hline
\end{tabular}

Tab. 5-2 Combustion Stoichiometry

\begin{tabular}{|c|c|c|c|c|}
\hline & \multicolumn{3}{l|}{ First blind } & \multicolumn{2}{l|}{ Open/blind } \\
\hline O2 conc. [VOL-\%] & \multicolumn{1}{|l|}{ FCHX } & FCO & FCHX & FCO \\
\hline 0. & 0. & 0. & 0. & 0. \\
\hline 4. & 0.1 & 0.1 & & \\
\hline 4.84 & & & 0.07 & 0.85 \\
\hline 11.15 & & & 0.39 & 0.97 \\
\hline 12. & 0.1 & 0.3 & & \\
\hline 15. & 0.8 & 0.8 & 0.78 & 1.0 \\
\hline 17. & & & 0.9 & 1.0 \\
\hline 21. & 1.0 & 1.0 & 1.0 & 1.0 \\
\hline 25. & 1.0 & 1.0 & 1.0 & 1.0 \\
\hline
\end{tabular}


Tab. 5-3 Fan characteristics
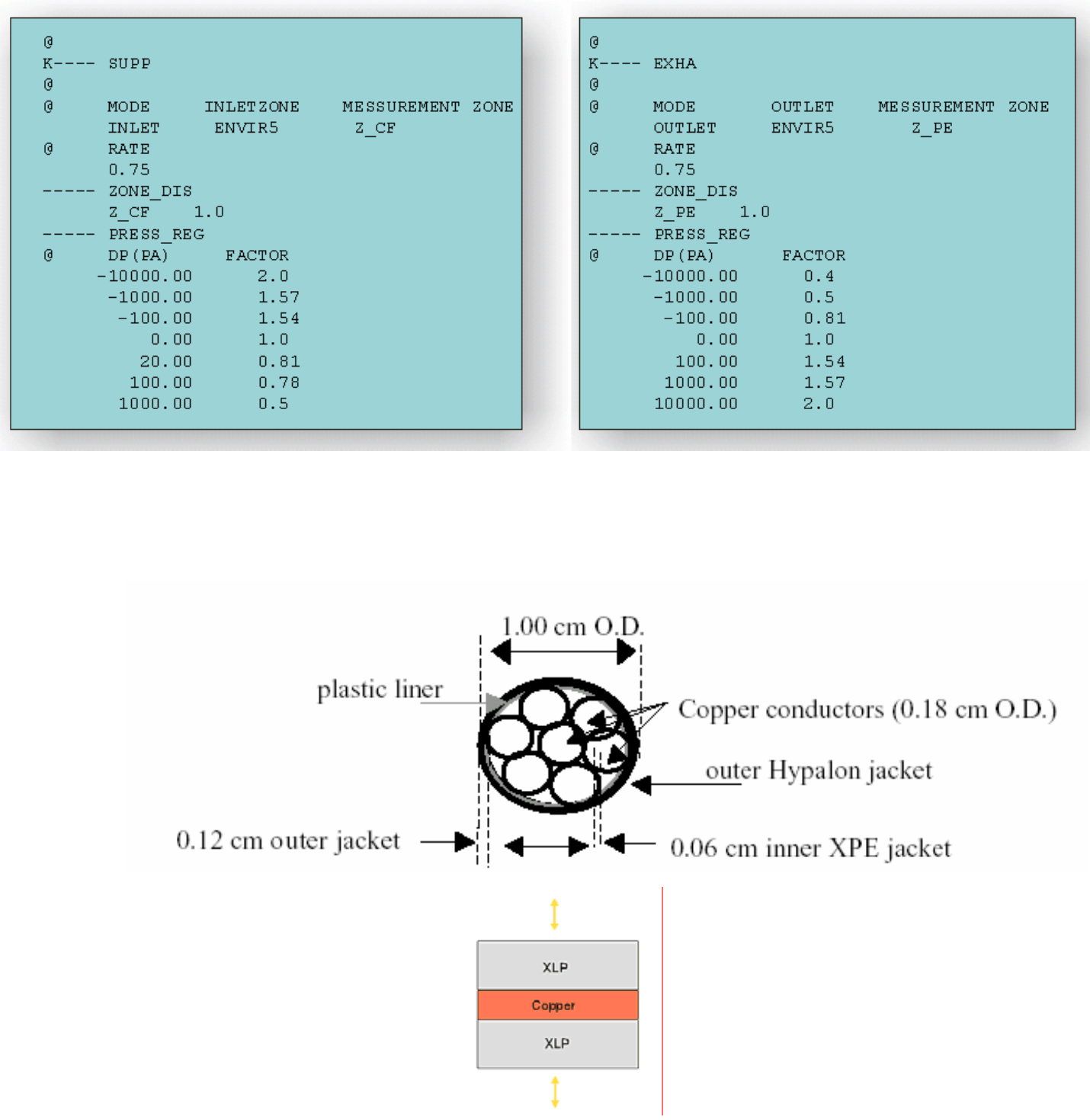

Fig. 5-4 Simulation of cable targets with plate-type wall structures 


\subsection{Comparison of COCOSYS Predictions with Measurements}

In the following the results of all performed calculations will be presented. Due to the high number of calculations it is somewhat difficult to discuss each point in detail. On the other hand it is important to present all results at least as figures. Therefore not all figures may be commented.

Fig. 5-1 presents the list of calculations performed (green shaded). The blind calculations of tests 2,3 and 4 are indicated by blue text. Tests $1,5,7,10,13,16,17$ and 18 were calculated blind using the measured HRR. Tests 8 and 9 were identical to 2 and 3, respectively, and were not calculated. In tests 14 and 15 the fire location was changed. But this change did not conform to the nodalization used and were therefore not calculated.

\begin{tabular}{|c|c|c|c|c|c|c|}
\hline Test & HRR & Cable & Fuel & Location & Door & Vent. \\
\hline 1 & 0.35 & XPE & Heptane & center & closed & off \\
\hline 2 & 1.00 & $\mathrm{XPE}$ & Heptane & center & closed & off \\
\hline 3 & 1.00 & $X P E$ & Heptane & center & open & off \\
\hline 4 & 1.00 & XPE & Heptane & center & closed & on \\
\hline 5 & 1.00 & $X P E$ & Heptane & center & open & on \\
\hline 7 & & PVC & \multicolumn{4}{|c|}{ Replicate 1} \\
\hline 8 & & $X P E$ & \multicolumn{4}{|c|}{ Replicate 2} \\
\hline 9 & & XPE & \multicolumn{4}{|c|}{ Replicate 3} \\
\hline 10 & & PVC & \multicolumn{4}{|c|}{ Replicate 4} \\
\hline 13 & 2.00 & XPE & Heptane & center & closed & off \\
\hline 14 & 1.00 & $\mathrm{XPE}$ & Heptane & $1.8 \mathrm{~m}$ from $\mathrm{N}$ & open & off \\
\hline 15 & 1.00 & PVC & Heptane & $1.25 \mathrm{~m}$ from $\mathrm{s}$ & open & off \\
\hline 16 & 2.00 & XPE & Heptane & center & closed & on \\
\hline 17 & 1.00 & PVC & Toluene & Center & closed & off \\
\hline 18 & 1.00 & XPE & Heptane & $0.5 \mathrm{~m}$ from $\mathrm{s}$ & open & off \\
\hline
\end{tabular}

Fig. 5-5 Calculation Matrix 


\subsubsection{First set of blind calculations for tests 2, 3 and 4}

Inside this section the results of first blind calculations of test 2, 3 and 4 will be presented.

\section{- Gas temperatures at Tree 1}

Fig. 5-6 to Fig. 5-8 shows the comparison for the temperature Tree 1 . The temperature spreading between the lowest and highest position is simulated quite well for test 1 (closed conditions). Only in the inner positions the maximum deviations are larger and about $50 \mathrm{~K}$. The time behavior shows that the fuel supply has been stopped about $100 \mathrm{~s}$ too early. The deviations in test 3 are somewhat larger. The maximum temperature below the ceiling is calculated about $20 \mathrm{~K}$ too low. Also the temperatures at the lowest position 2 and 3 are under-estimated by about $25 \mathrm{~K}$. The temperatures at position $3(1.05 \mathrm{~m})$ and $4(1.40 \mathrm{~m})$ are quite different. In the COCOSYS simulation these positions belong to the same control volume (level between 1.0 and $1.5 \mathrm{~m}$ ). Therefore the separation between hot and cold gas layer is sometimes not well-resolved in the COCOSYS simulation. For test 4 the temperatures below the ceiling are over-estimated by about $50 \mathrm{~K}$. The main reason seems to be the under-estimate of air flow from the ventilation system.

\section{- Temperature profiles}

Fig. 5-9 to Fig. 5-11 show the temperature profiles at all trees at certain times close to the maximum temperatures of the corresponding test. For test 2 the deviations are about $50 \mathrm{~K}$ and somewhat larger as in the lower part. One has to keep in mind that this kind of deviation includes deviations in time and in values. For test 3 the temperature of tree 3 is underestimated for all positions. The experimental measurement at tree 6 is possibly flawed. All other values are quite good. In test 4 the deviations in the upper part are larger also. This is valid especially for trees 3 to 5 close to the ventilation supply. Due to the missing momentum balance the simulation is limited close to such systems. But on the other side the ventilation flow rate is too small here, leading to higher gas temperatures.

\section{- Wall temperatures}

The temperatures on the floor are shown in Fig. 5-12 to Fig. 5-14. In the tests 2 and 3 the calculated values are somewhat lower than in the experiment. Only for test 4 do the calculated temperatures reach the right level. The measured value at position $\mathrm{U} 4$ seems to be too high. The corresponding symmetric value at position U5 is much lower. The radiation to the floor seems to be under-estimated in the COCOSYS calculation. It has to be kept in mind 
that the fuel spray is not simulated. The flame height in COCOSYS (control volumes with energy release) may be too low.

Fig. 5-15 to Fig. 5-17 shows the wall temperatures at the west side. The deviations are about $20 \mathrm{~K}$ in test 2 and 3 and about $50 \mathrm{~K}$ for test 4 . The direction of deviation corresponds to the calculated atmospheric temperatures. The deviations are somewhat higher for the south wall (Fig. 5-18 to Fig. 5-20) in test 2. These are for the upper positions about $40 \mathrm{~K}$. In test 3 the correspondence is much better, whereas in test 4 the calculated values are too high.

The ceiling temperatures (Fig. 5-21 to Fig. 5-23) are not so strongly influenced by a possible wrong calculation of stratification. Therefore the temperatures are calculated quite well for test 2 . For test 3 the deviations are somewhat larger $(50 \mathrm{~K})$ and the temperatures are overestimated for test 4 . The difference between position U4 and U5 show the influence of the ventilation system. This could not be simulated in COCOSYS.

\section{- Concentrations}

Fig. 5-24 to Fig. 5-26 shows the comparison for the gas concentrations. The lower oxygen concentration in tests 2 and 4 decreases rapidly when the hot gas layer reaches the measurement position. Due to the fixed grid and the resulting numerical diffusion, the calculated decrease is less rapid. Regardless, the time to fuel stop is calculated quite well. After the fuel stop the concentrations became more or less equal in the experiment. This is somewhat underestimated in the COCOSYS calculation. Further, the $\mathrm{CO}_{2}$ concentration is underestimated as well in tests 2 and 3 . Due to the low air supply in test 4 the $\mathrm{CO}_{2}$ concentration is much better here. But this is a compensating effect.

\section{- Tray temperatures}

One main objective of these experiments was the evaluation of temperature loads on cable trays of different size and distance to the fire source. Due to the high number of temperature measurements not all results could be discussed, but all comparisons are shown in the Fig. 5-27 to Fig. 5-50. Mostly the temperature inside and on the cable surface are somewhat under-estimated in COCOSYS (partly up to $50 \mathrm{~K}$ ). It has to be kept in mind, that in the blind calculations a radiation fraction of $35 \%$ is used, which is lower as observed in other experiments. The differences between experiment and calculation is much less for control cable $A$ compared to the power cable F (see Fig. 5-31, Fig. 5-39 and Fig. 5-47) In test 4 the cable temperatures close to the ventilation system may be cooled by the air flow. Therefore the deviation between calculation and experiment is much larger (Fig. 5-46). The deviations at 
position 22 and 23 are much larger compared to the positions 8 and 9 . The main reason is the COCOSYS results, which is about $50 \mathrm{~K}$ higher. The reason for this asymmetric behavior is not clear.

\section{- Junction box}

The results for the junction box are unlike the other temperature results (Fig. 5-51 to Fig. 5-53). The reason is not clear, because due to the missing radiation term between fire source and box in the heat transfer correlation the temperatures should be lower compared to the experiment. Also the trend in test 3 and 4 is not consistent with the experimental results. Further investigations are necessary.

\section{- Temperatures and velocity at door in test 3}

In test 3 the door to the environment was open. The temperature results are presented in Fig. 5-54 to Fig. 5-57 and the velocity in Fig. 5-58 to Fig. 5-61. The use of four levels of control volumes allows the simulation of counter-current flow structures, but the resolution is somewhat limited. The calculated results are taken from control volumes close to the door but inside the fire compartment. Therefore the temperatures in the lower part are too high. Overall the quality of the results is quite good. The experiment shows that the flow direction changes in level 2 (Fig. 5-59). Therefore, the COCOSYS results are qualitatively correct, but it is not possible to provide a more quantitative result.

\section{- Ventilation in test 4}

Fig. 5-62 shows the comparison of ventilation flow rate. The calculated supply flow rate is by a factor of two too low compared to the experiment. This could explain the high gas temperatures in test 4 . The difference at $t=0 \mathrm{~s}$ between the measured inlet and outlet flow should be explained in more detail. The time history looks quite similar between experiment and calculation. 
Test 2 (blind) :Temperature Tree 1

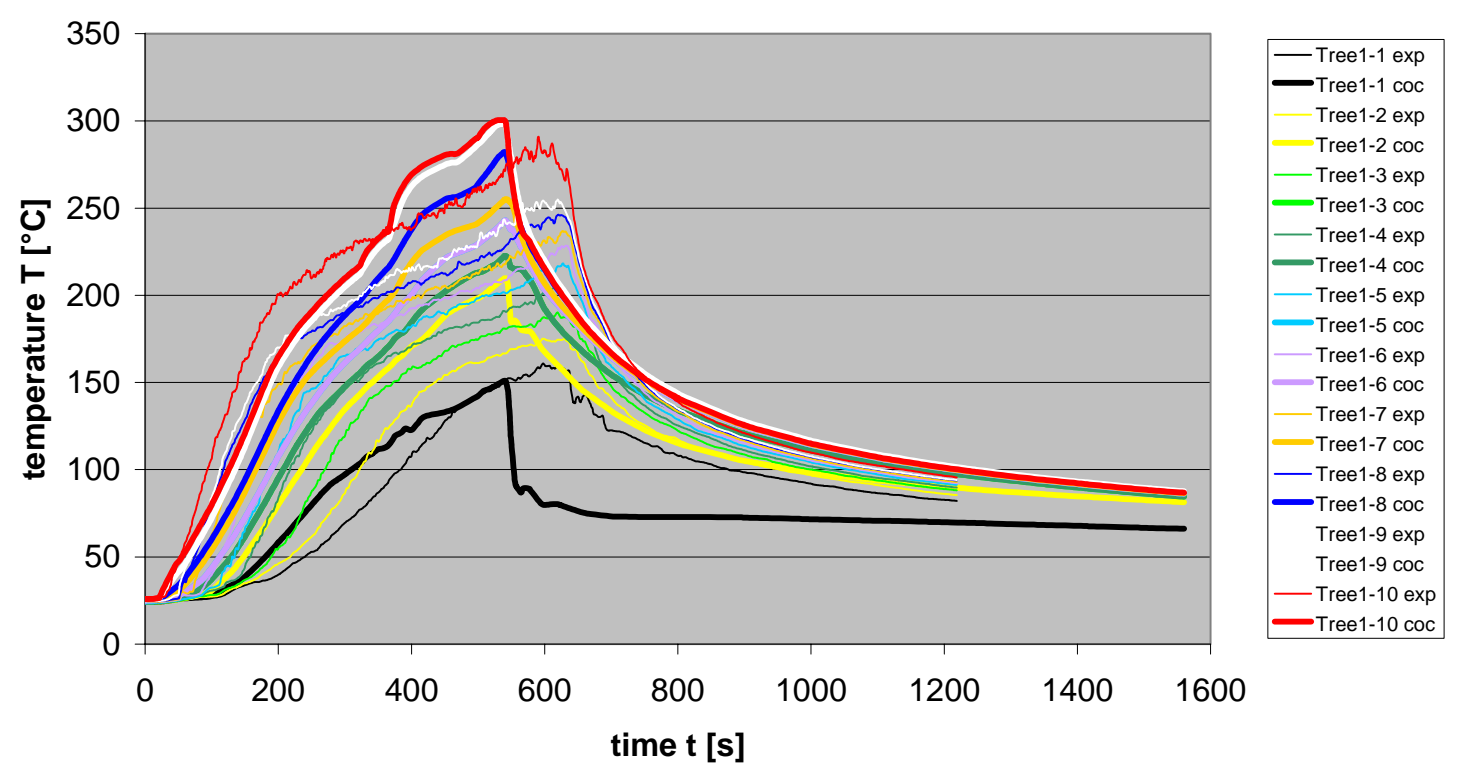

Fig. 5-6 Test 2: Temperatures at Tree 1

Test 3 (blind) :Temperature Tree 1

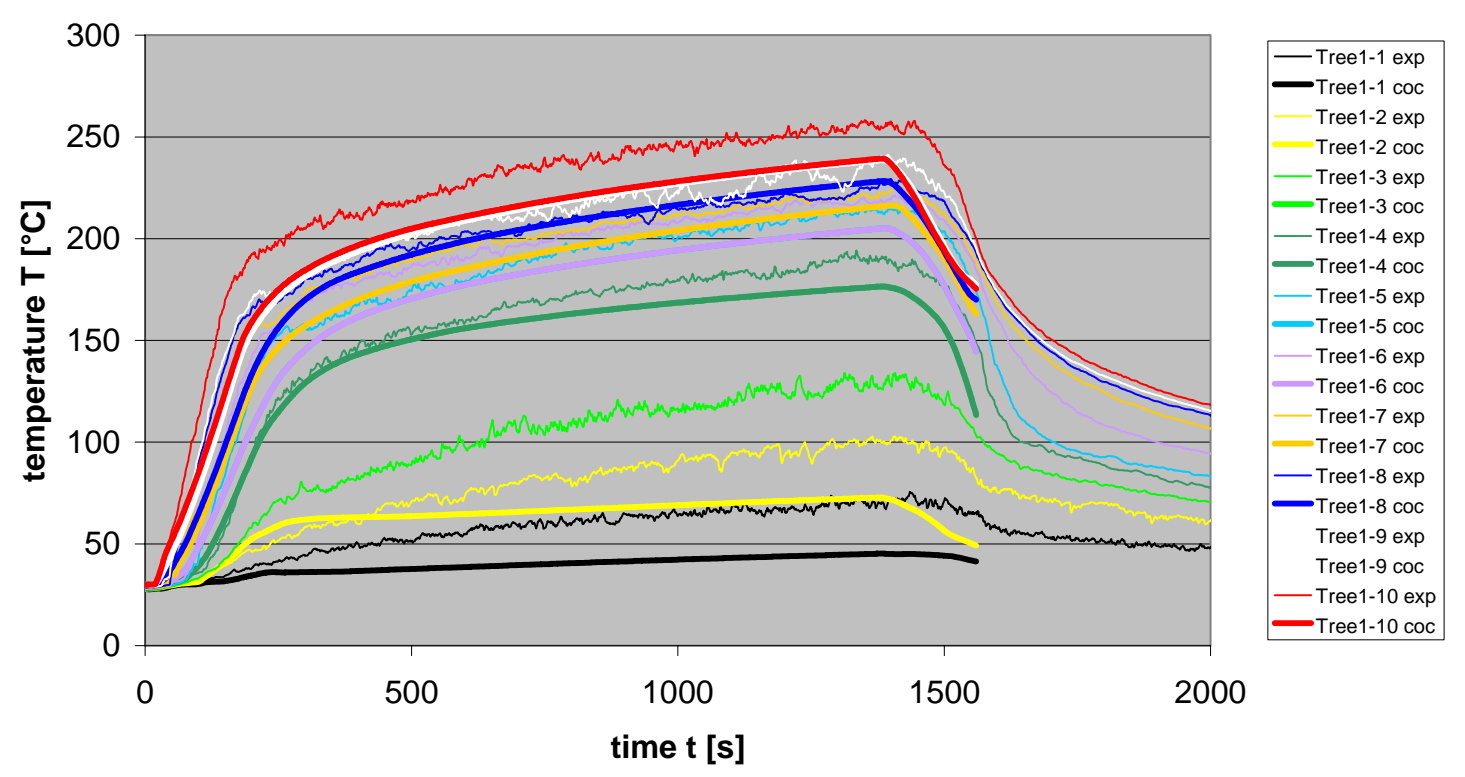

Fig. 5-7 Test 3: Temperatures at Tree 1 
Test 4 (blind) :Temperature Tree 1

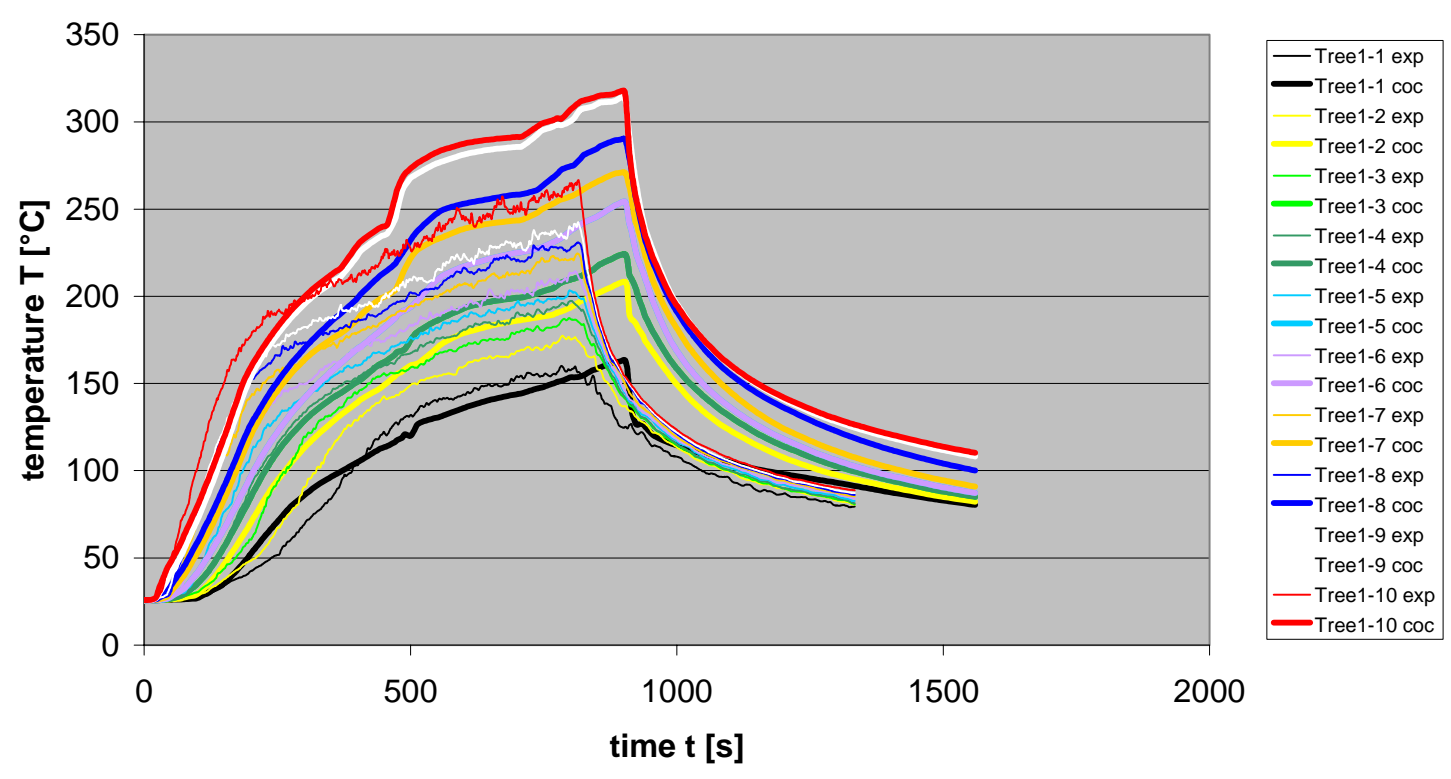

Fig. 5-8 Test 4: Temperatures at Tree 1

Test 2 (blind) : Tree temperature profiles

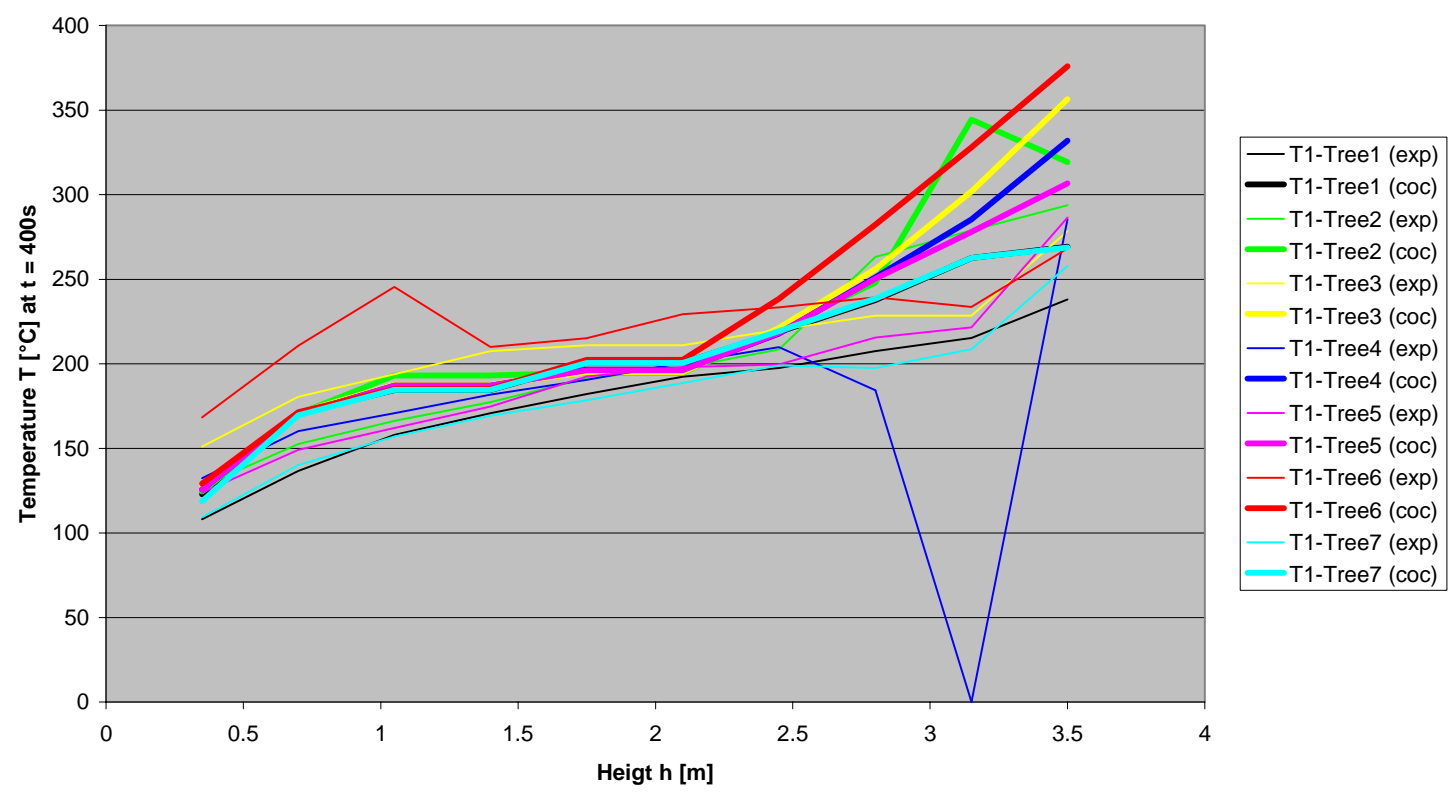

Fig. 5-9 Test 2: Temperature profiles at $400 \mathrm{~s}$ 
Test 3 (blind) : Tree temperature profiles
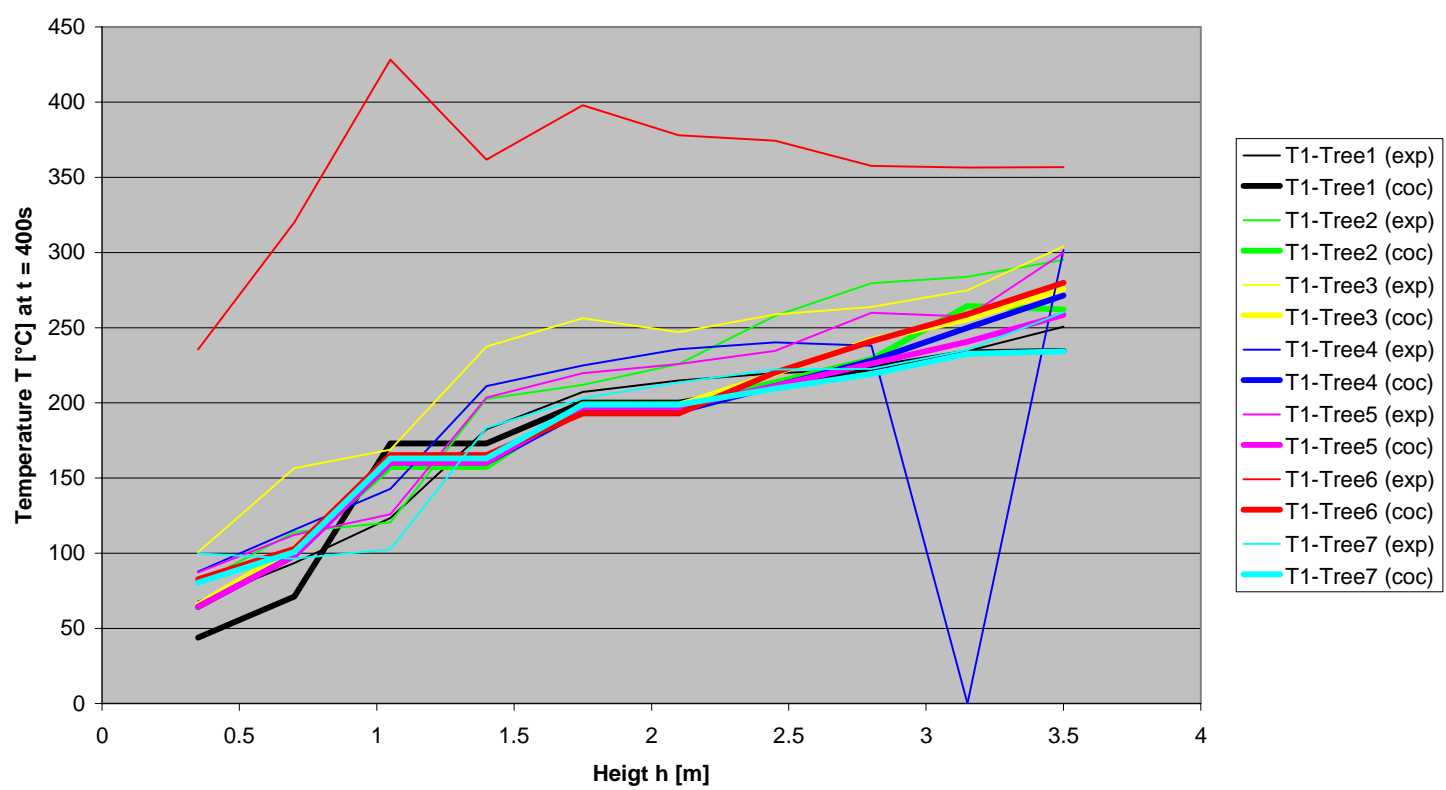

Fig. 5-10 Test 3: Temperature profiles

Test 4 (blind) : Tree temperature profiles

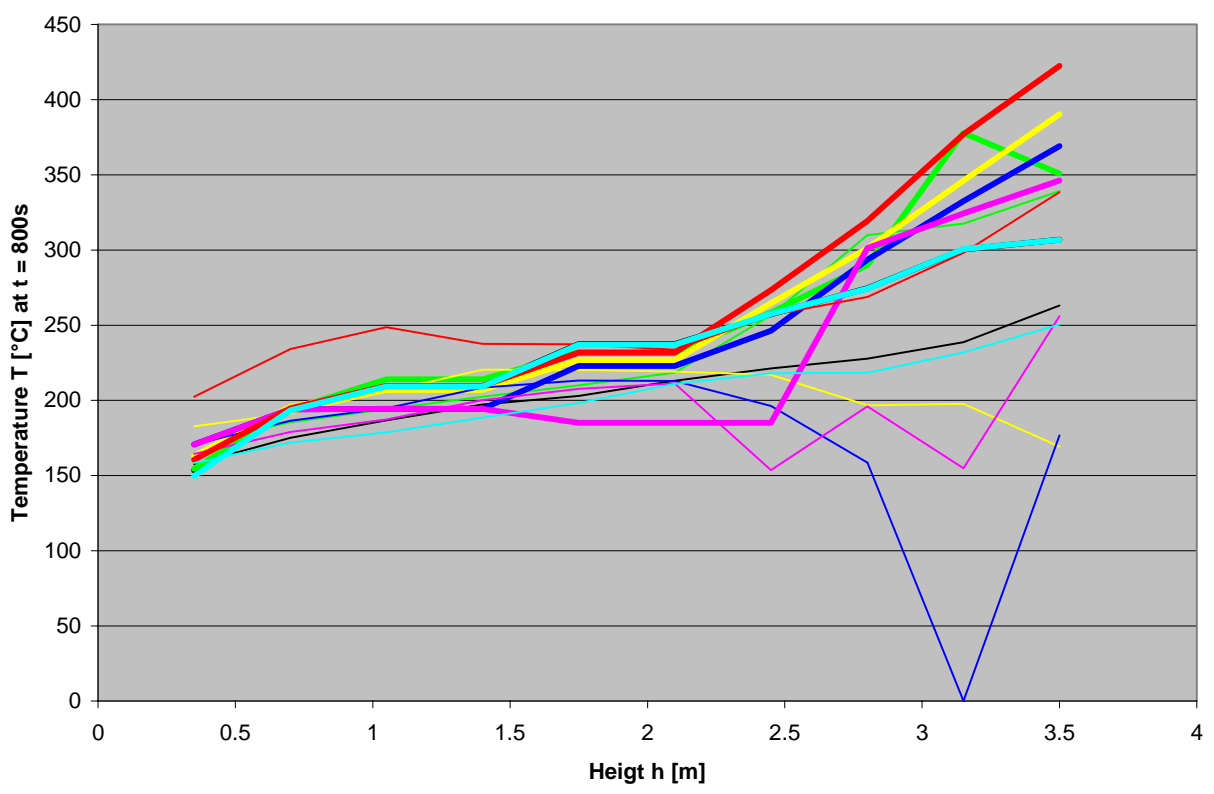

- T1-Tree1 (exp)

-T1-Tree1 (coc)

- T1-Tree2 (exp)

- T1-Tree2 (coc)

T1-Tree3 (exp)

-T1-Tree3 (coc)

-T1-Tree4 (exp)

-T1-Tree4 (coc)

- T1-Tree5 (exp)

-T1-Tree5 (coc)

- T1-Tree6 (exp)

-T1-Tree6 (coc)

- T1-Tree7 (exp)

-T1-Tree7 (coc)

Fig. 5-11 Test 4 : Temperature profiles 
Test 2 (blind) : Inner Floor temperatures
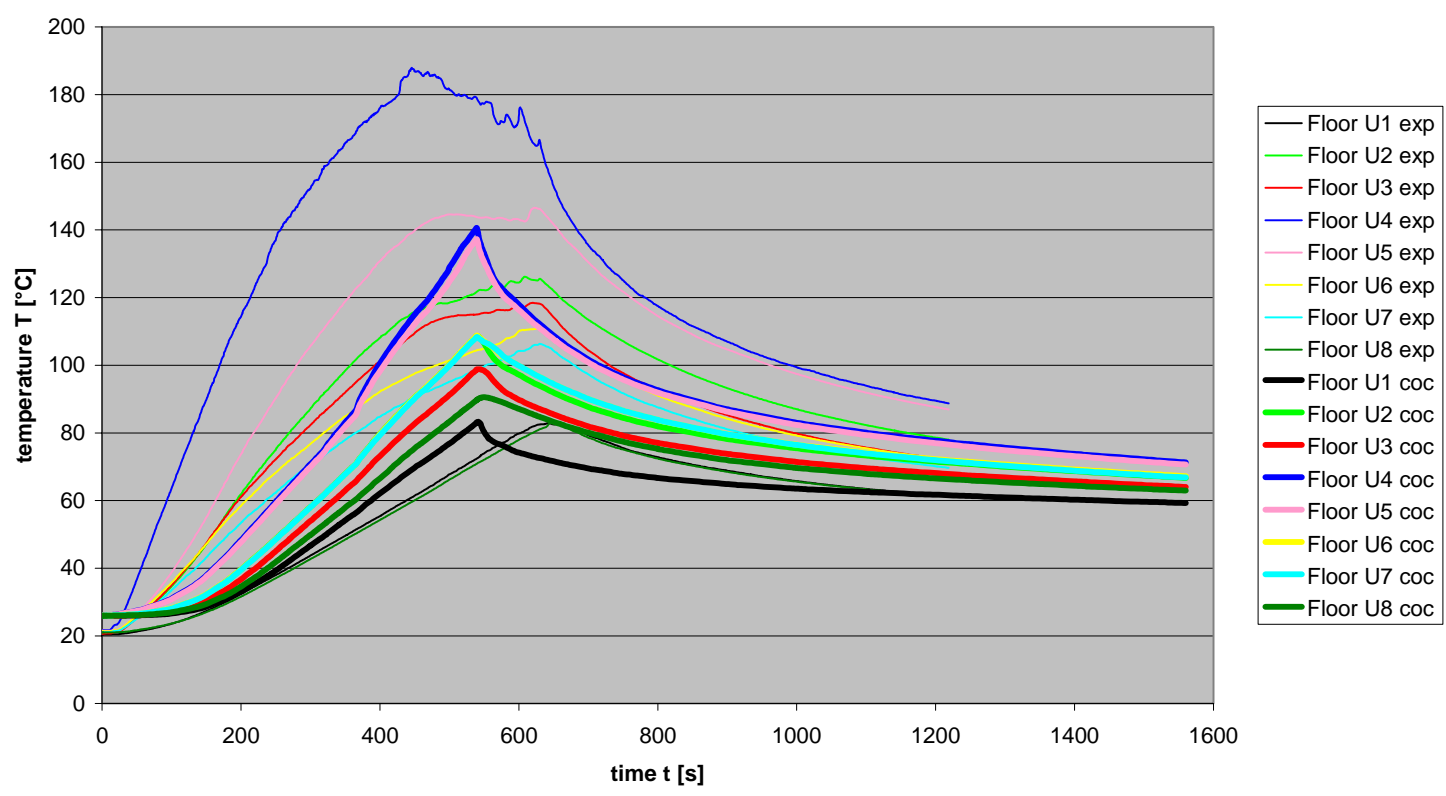

Fig. 5-12 Test 2: Floor wall temperatures

Test 3 (blind) : Inner Floor temperatures

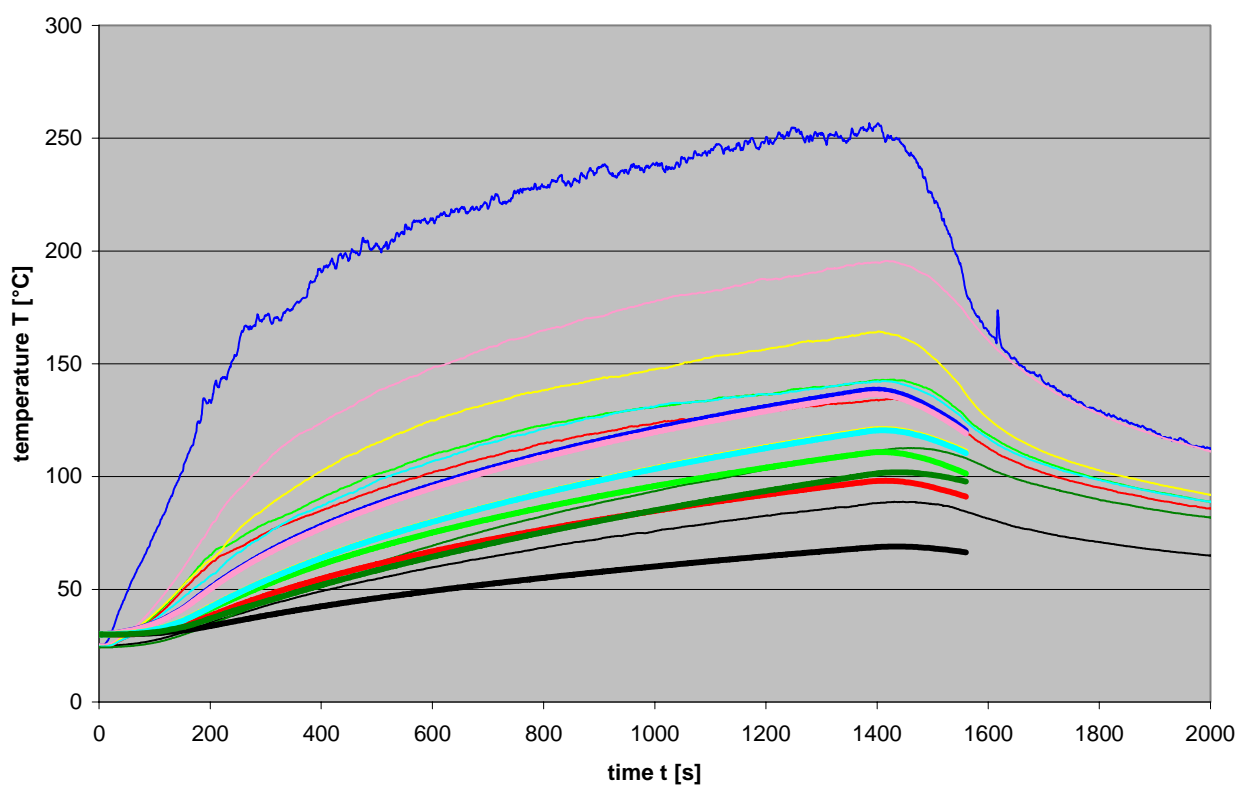

- Floor U1 exp - Floor U2 exp - Floor U3 exp - Floor U4 exp Floor U5 exp - Floor U6 exp - Floor U7 exp - Floor U8 exp - Floor U1 coc Floor U2 coc - Floor U3 coc - Floor U4 coc - Floor U5 coc - Floor U6 coc - Floor U7 coc -Floor U8 coc

Fig. 5-13 Test 3: Floor wall temperatures 
Test 4 (blind) : Inner Floor temperatures

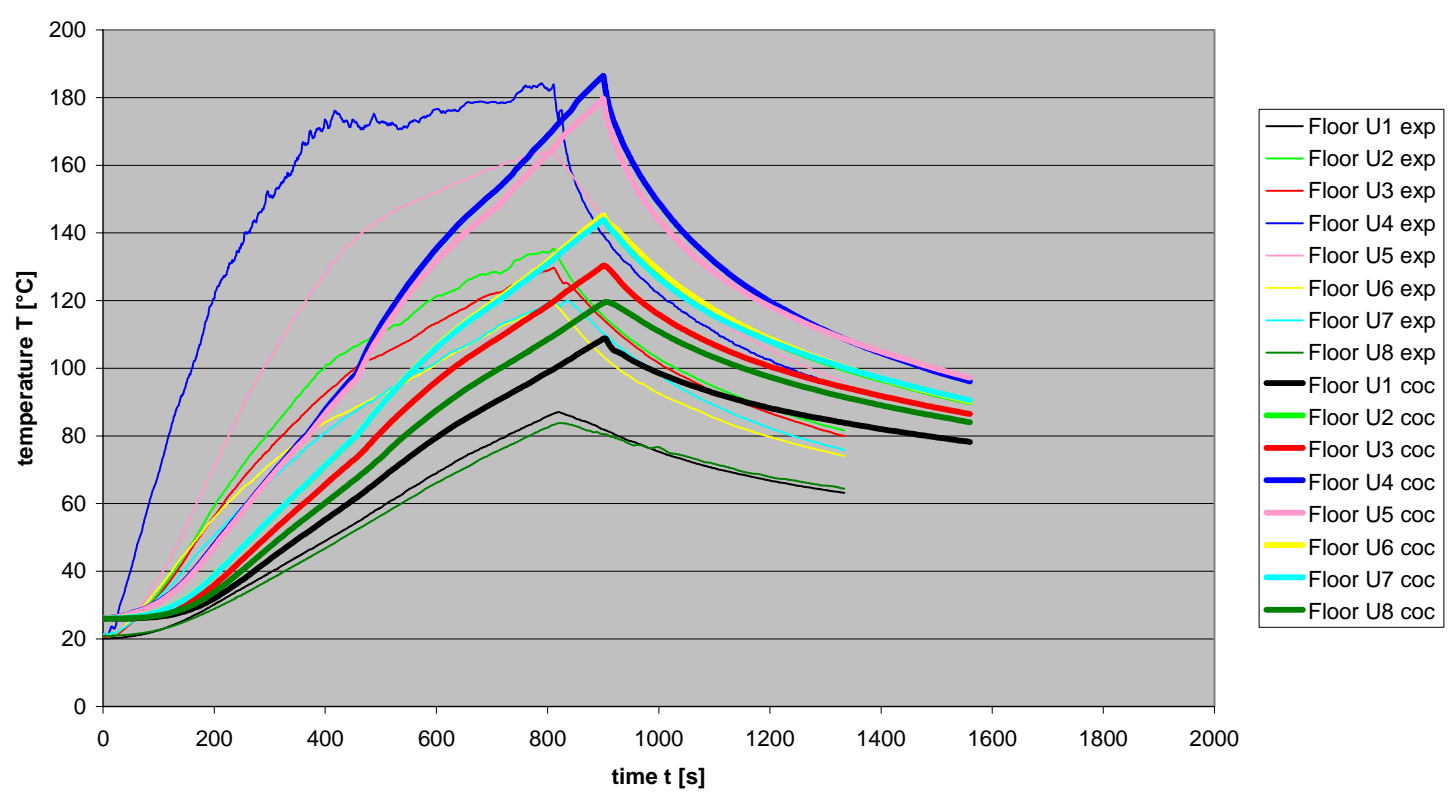

Fig. 5-14 Test 4: Floor wall temperatures

Test 2 (blind) : Inner west wall temperatures

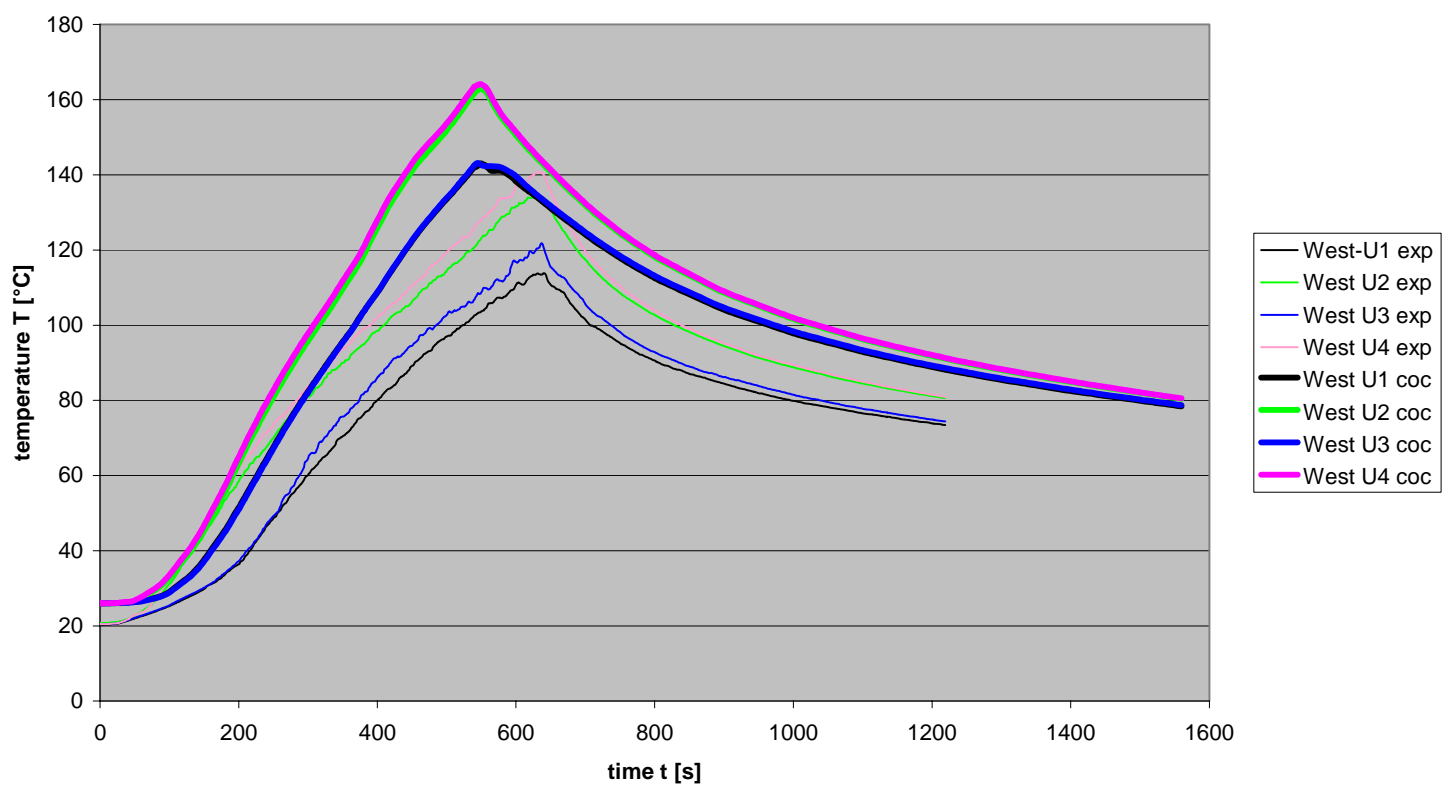

Fig. 5-15 Test 2: West wall temperatures 
Test 3 (blind) : Inner west wall temperatures

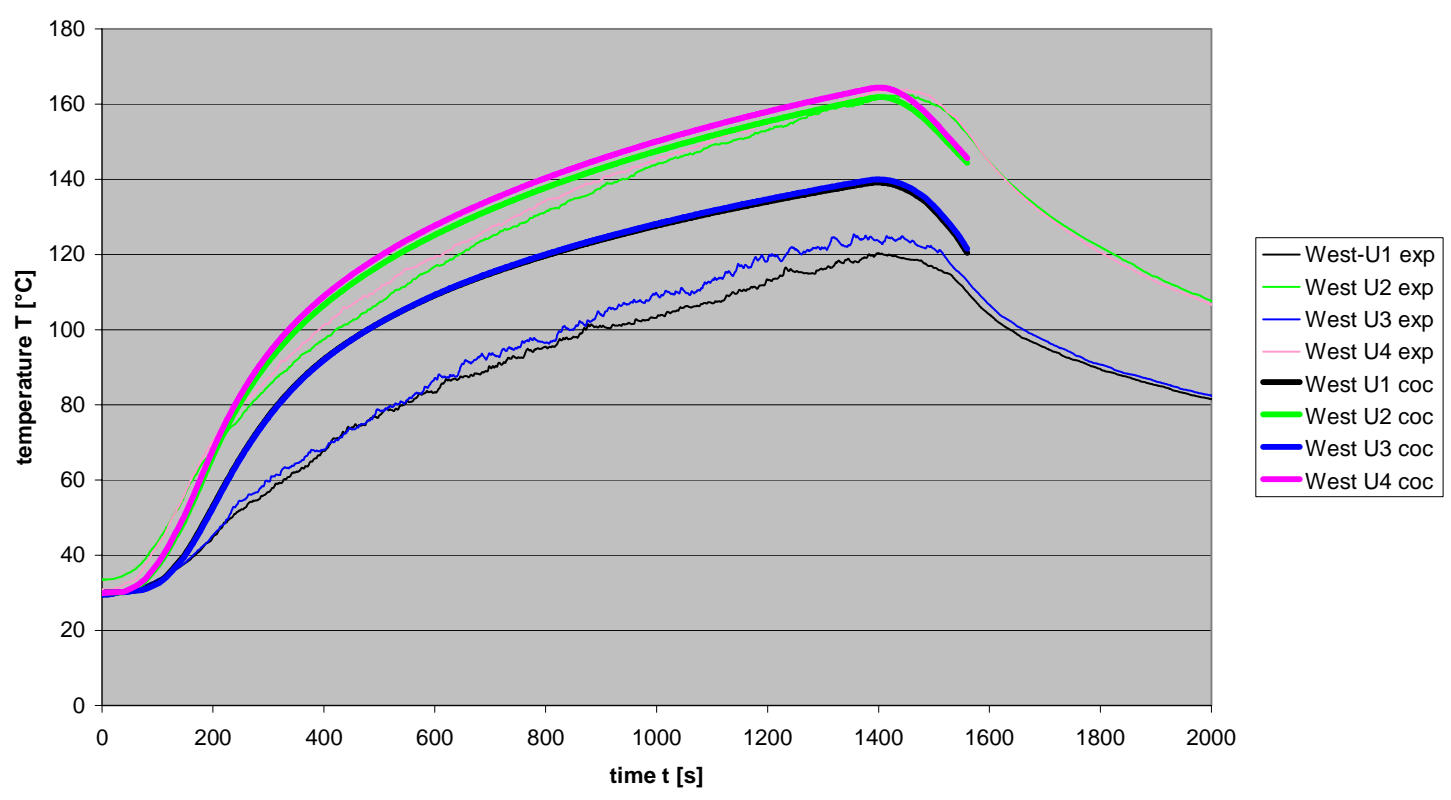

Fig. 5-16 Test 3: West wall temperatures

Test 4 (blind) : Inner west wall temperatures

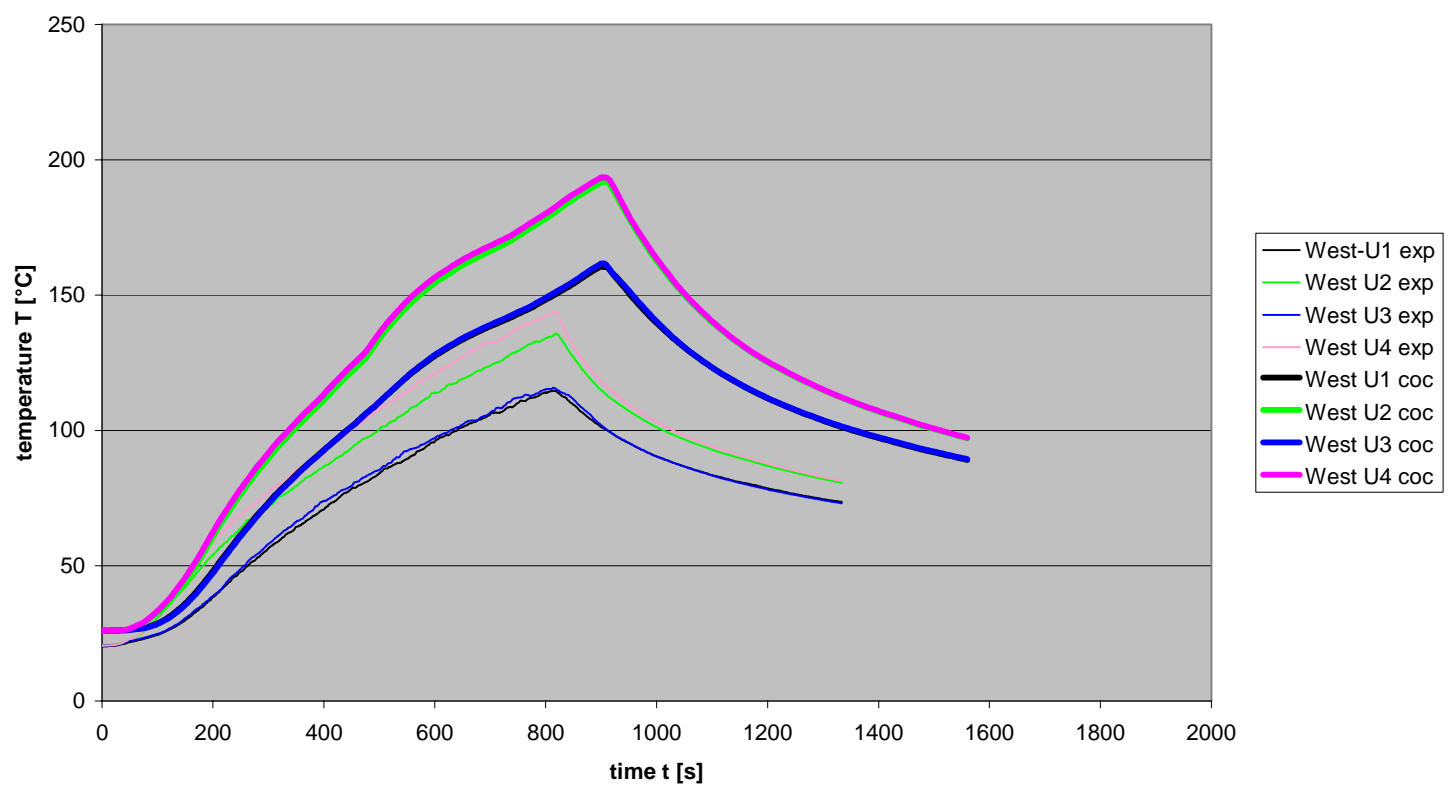

Fig. 5-17 Test 4: West wall temperatures 
Test 2 (blind) : Inner south wall temperatures

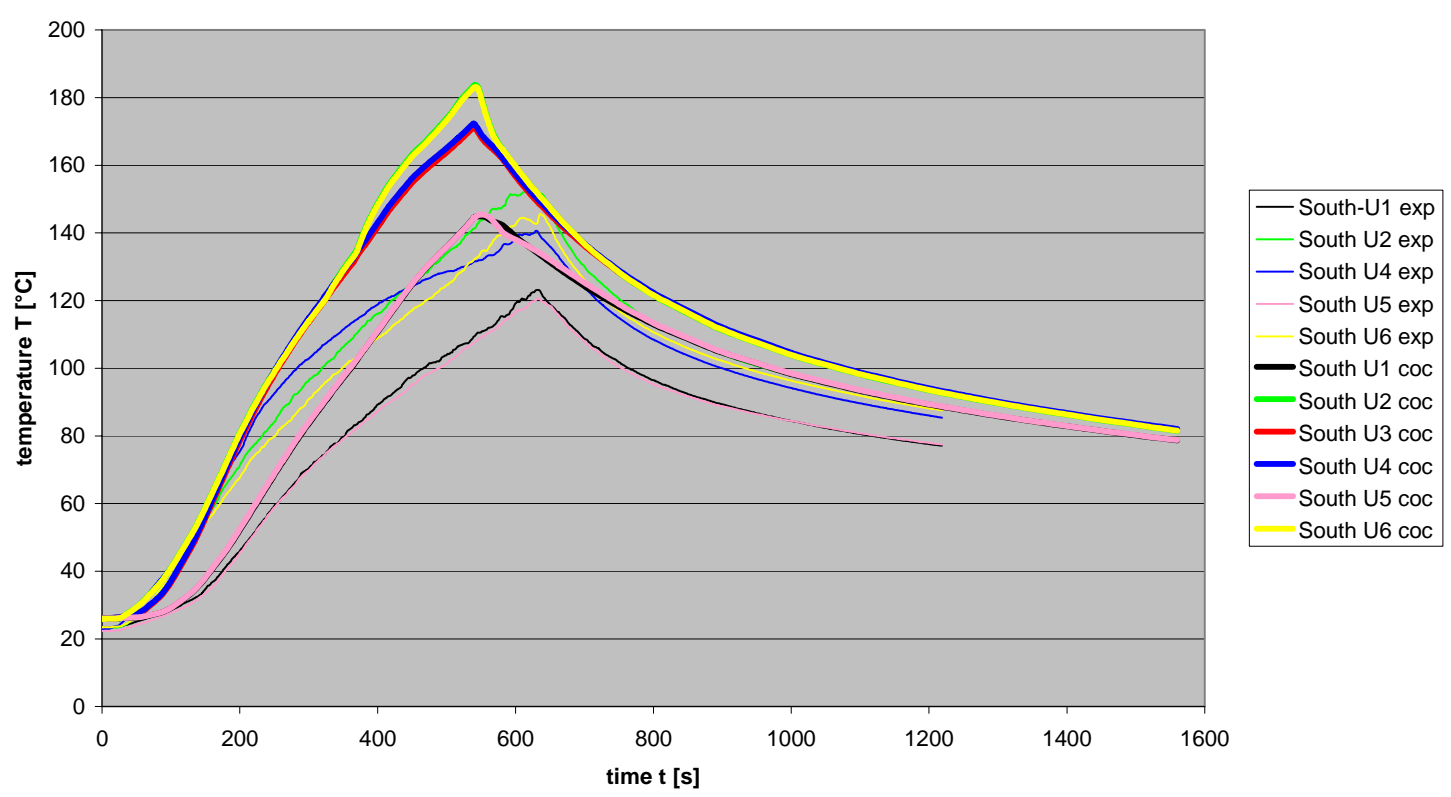

Fig. 5-18 Test 2: South wall temperatures

Test 3 (blind) : Inner south wall temperatures

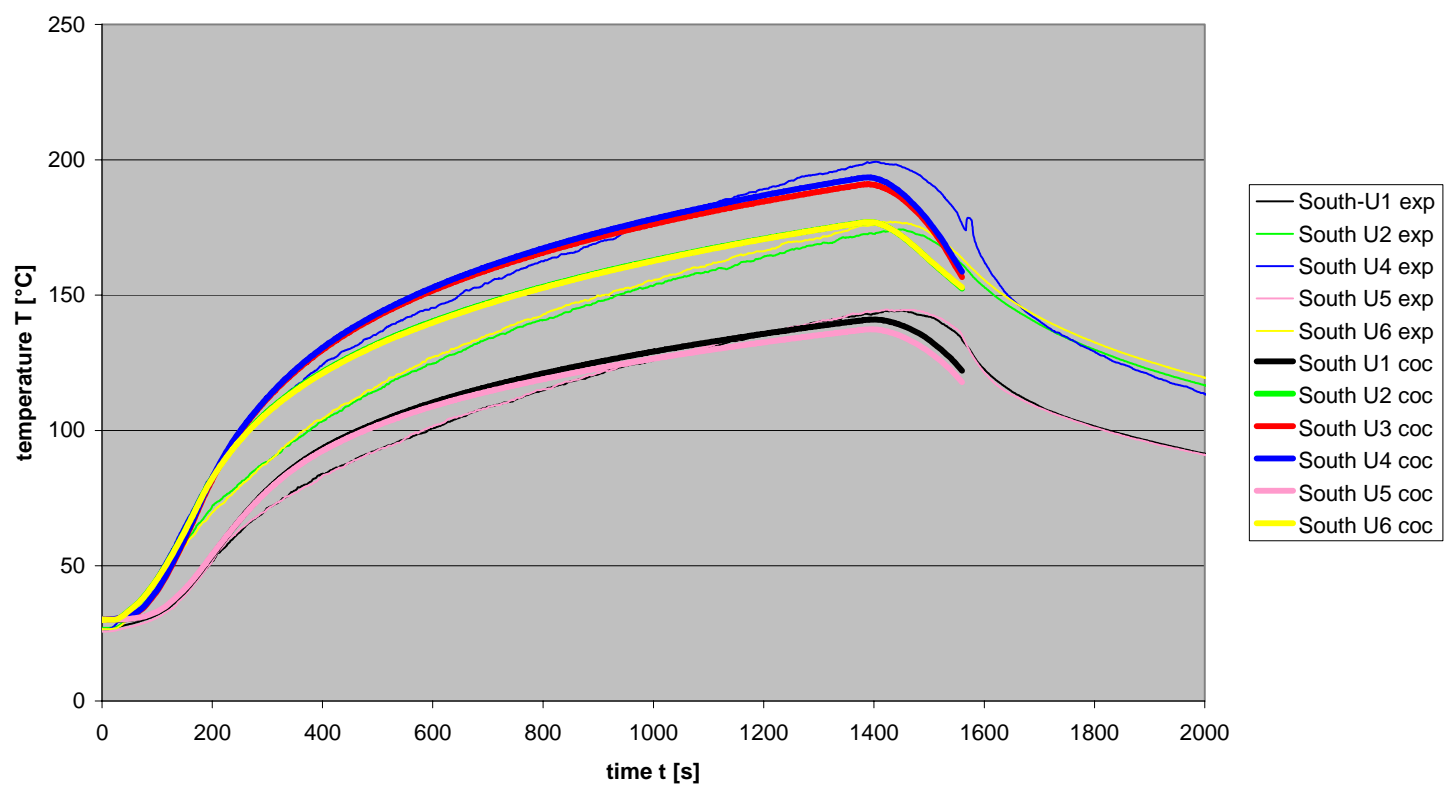

Fig. 5-19 Test 3: South wall temperatures 
Test 4 (blind) : Inner south wall temperatures

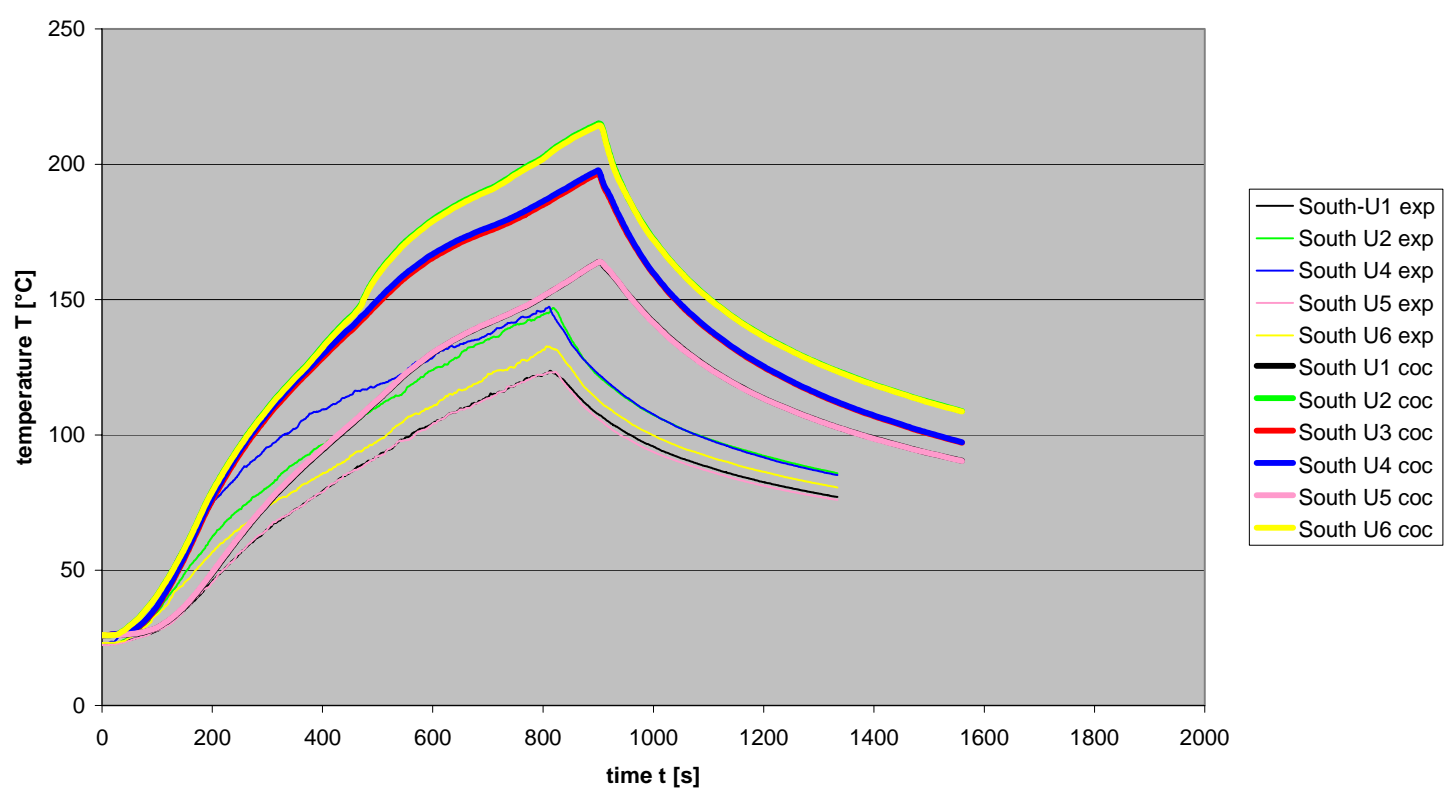

Fig. 5-20 Test 4: South wall temperatures

Test 2 (blind) : Inner ceiling temperatures

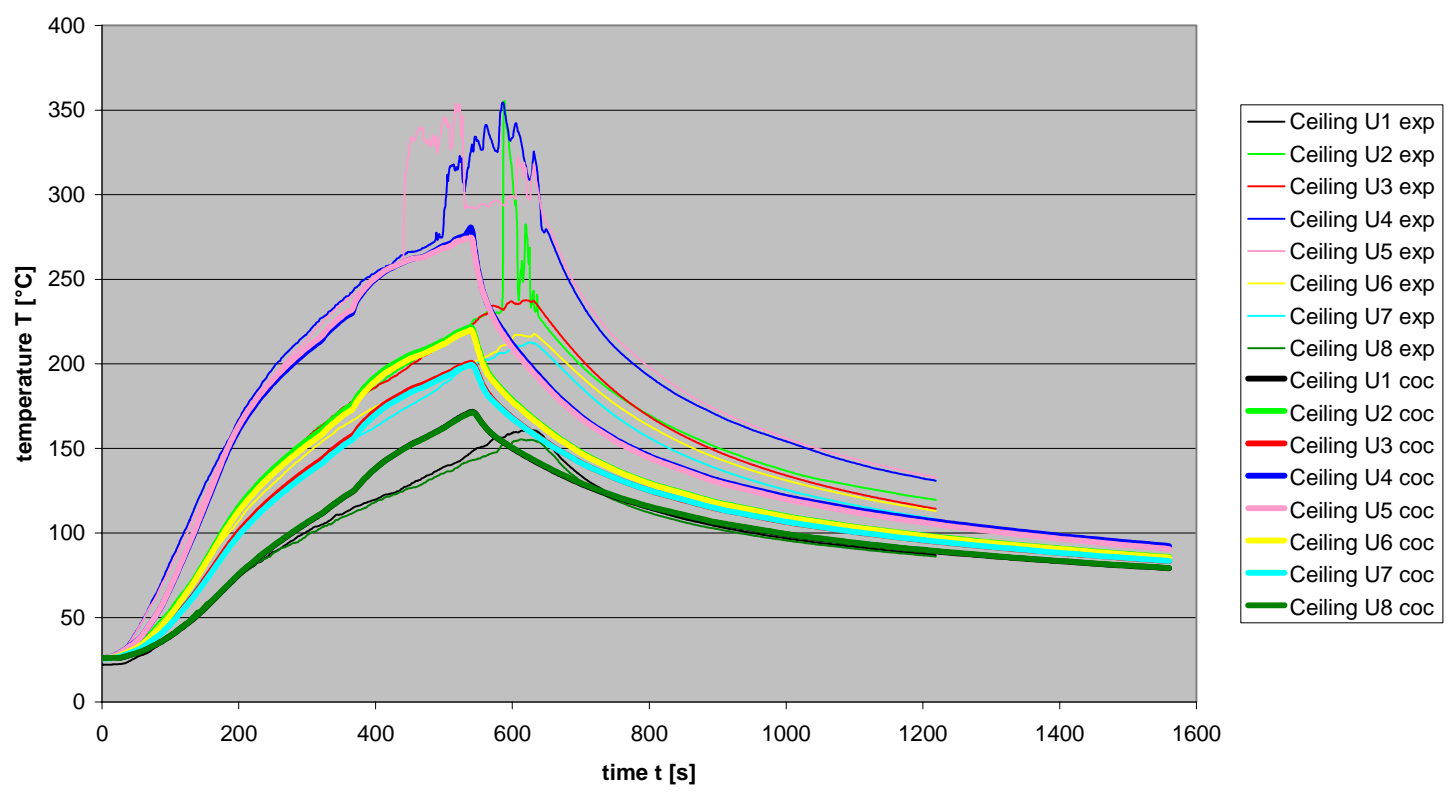

Fig. 5-21 Test 2: Ceiling temperatures 
Test 3 (blind) : Inner ceiling temperatures
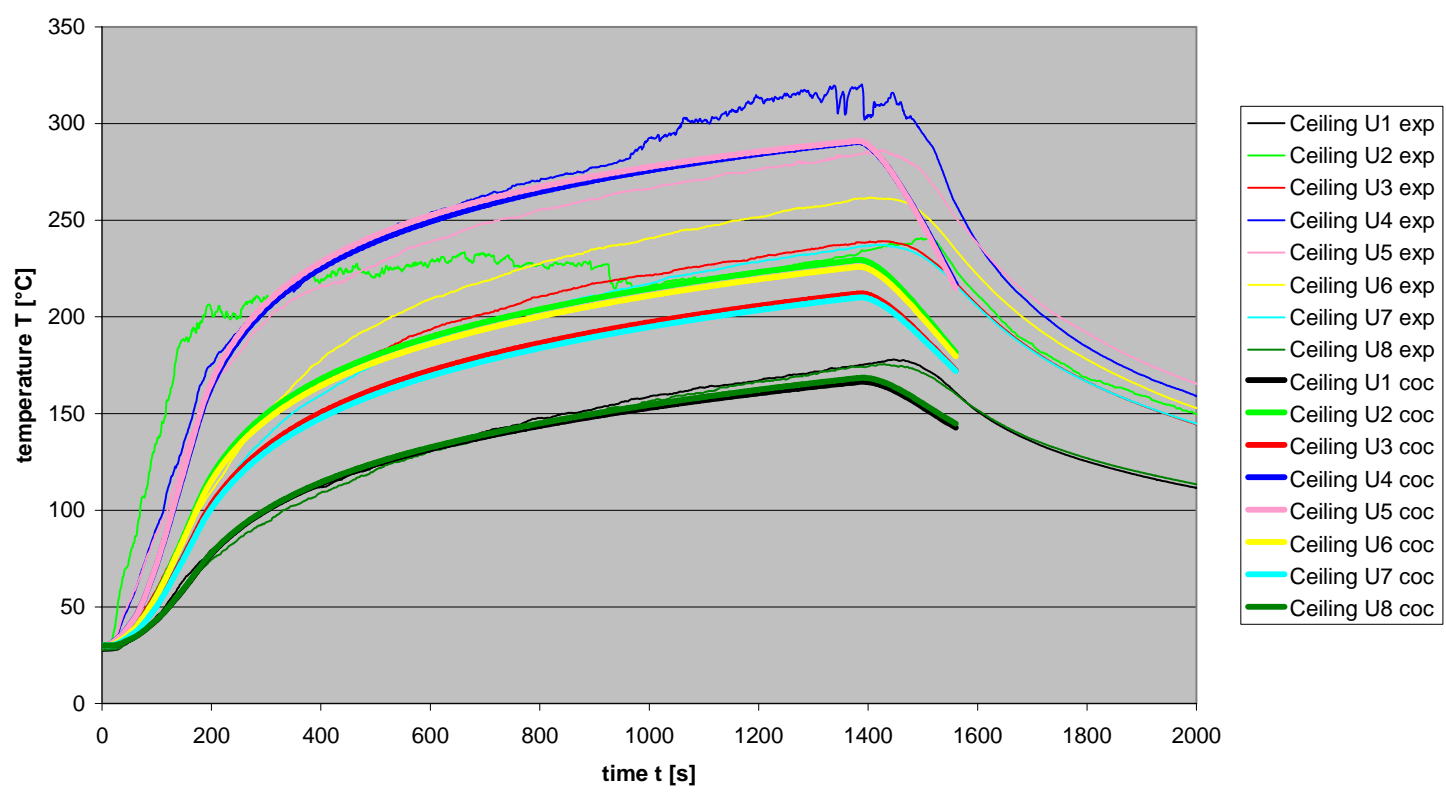

Fig. 5-22 Test 3: Ceiling temperatures

Test 4 (blind) : Inner ceiling temperatures

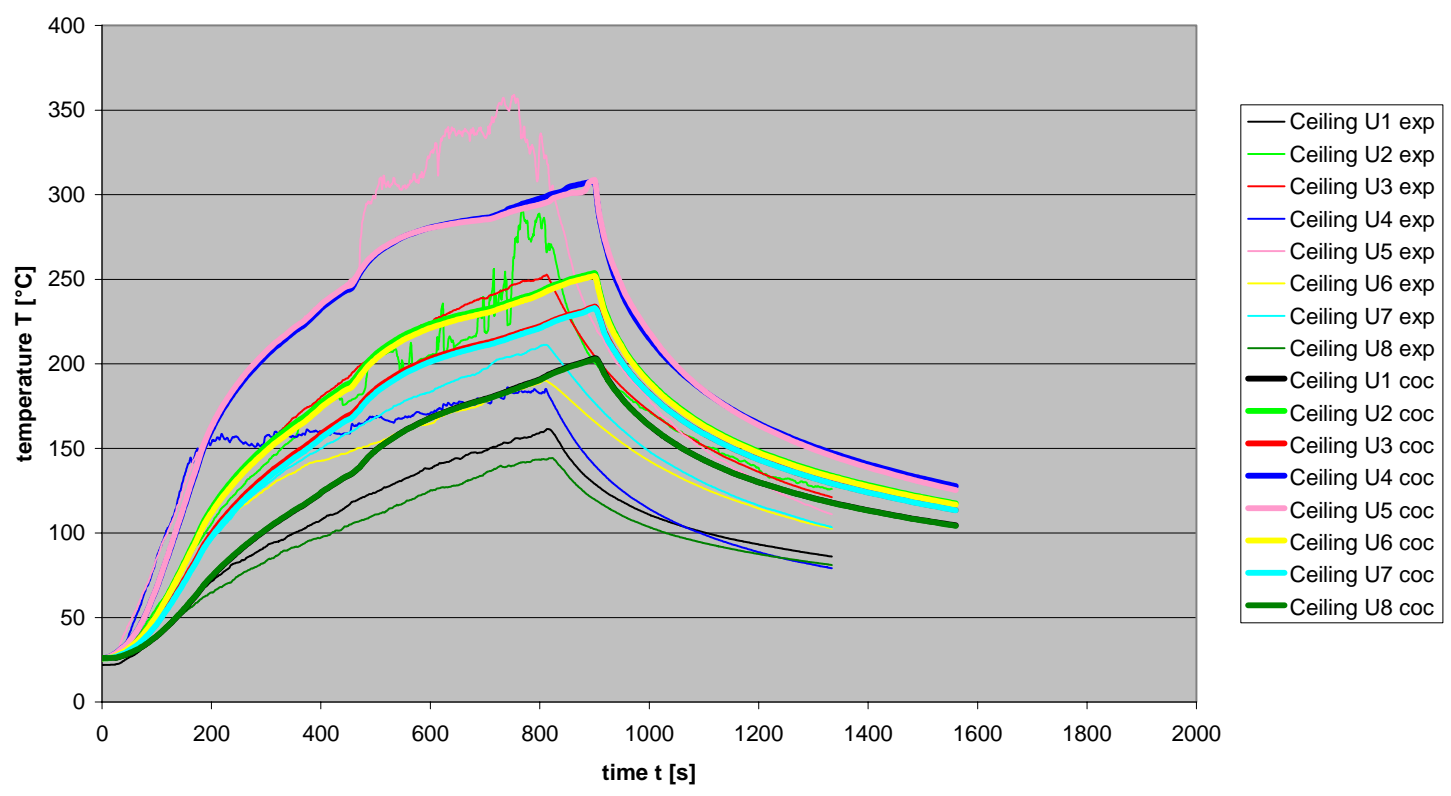

Fig. 5-23 Test 4: Ceiling temperatures 
Test 2 (blind) : Gas concentrations

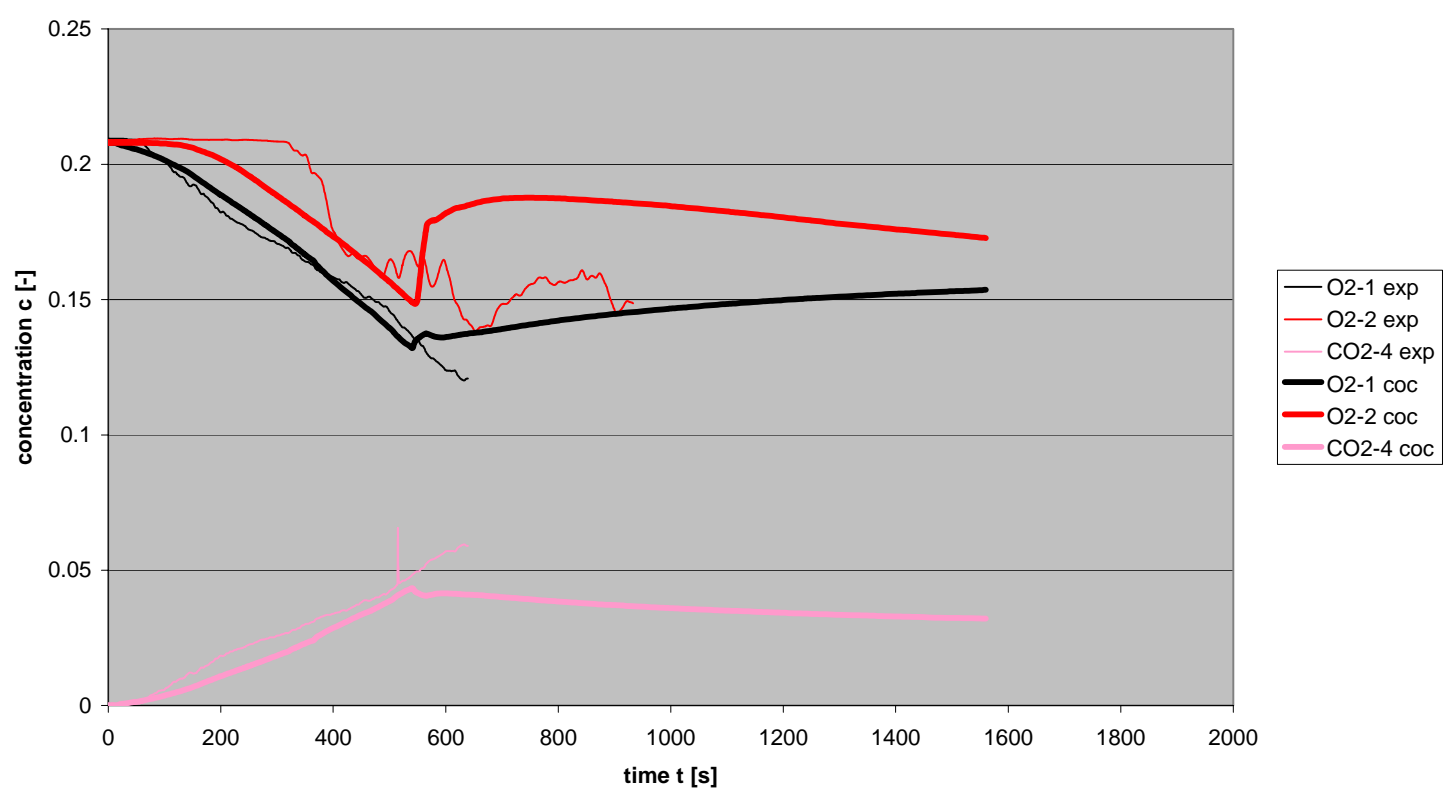

Fig. 5-24 Test 2: Concentrations

Test 3 (blind) : Gas concentrations

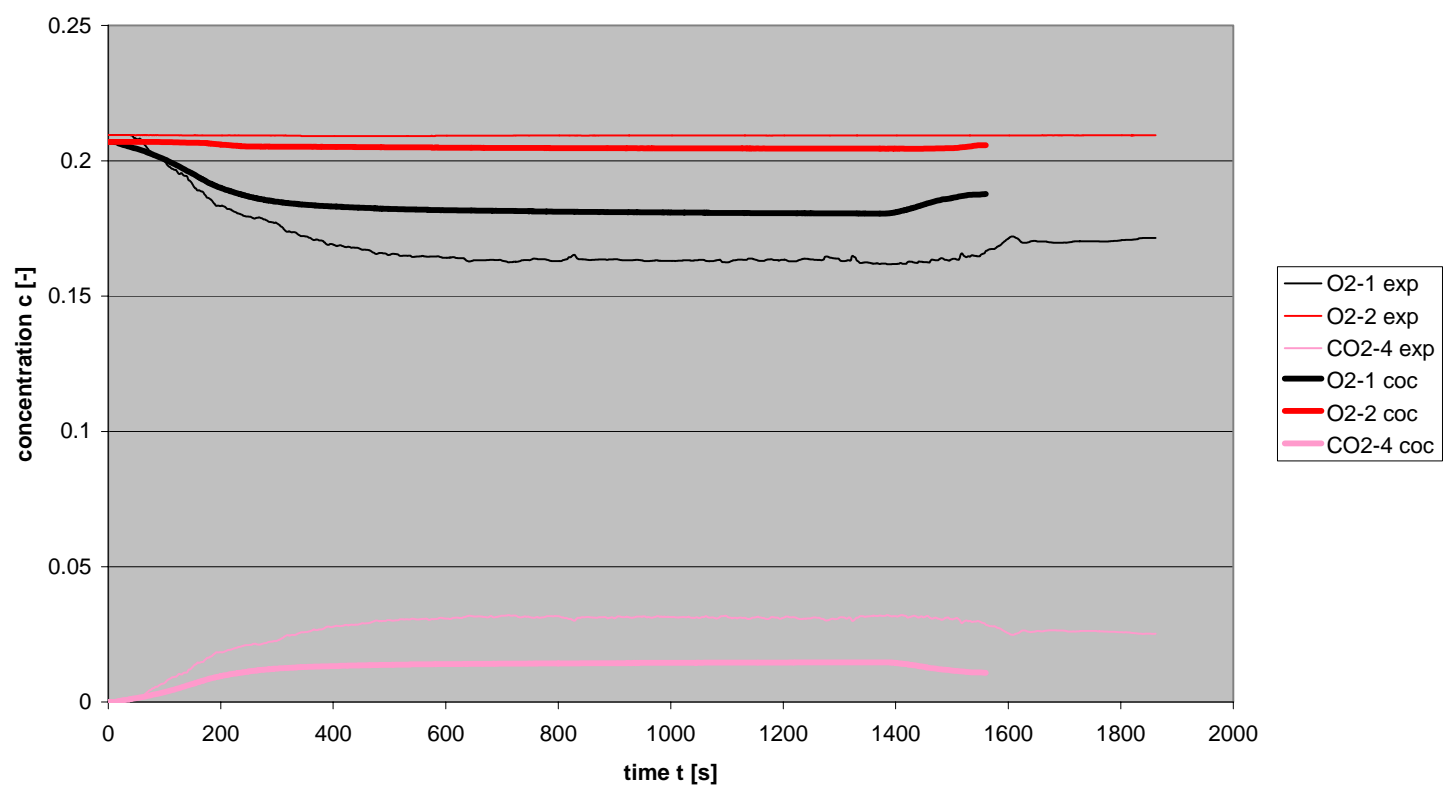

Fig. 5-25 Test 3: Concentrations 
Test 4 (blind) : Gas concentrations

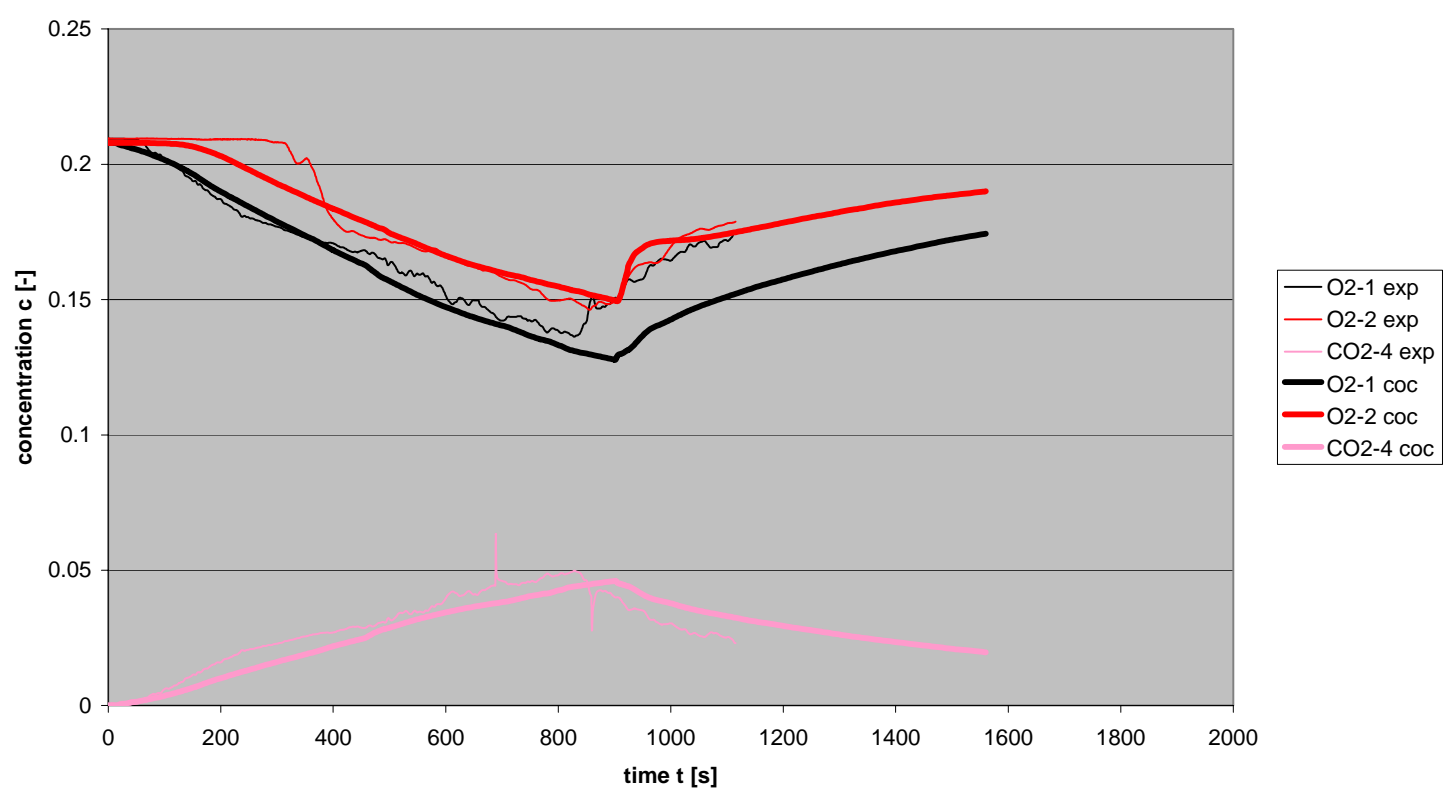

Fig. 5-26 Test 4: Concentrations

Test 2 (blind) : Tray temperatures

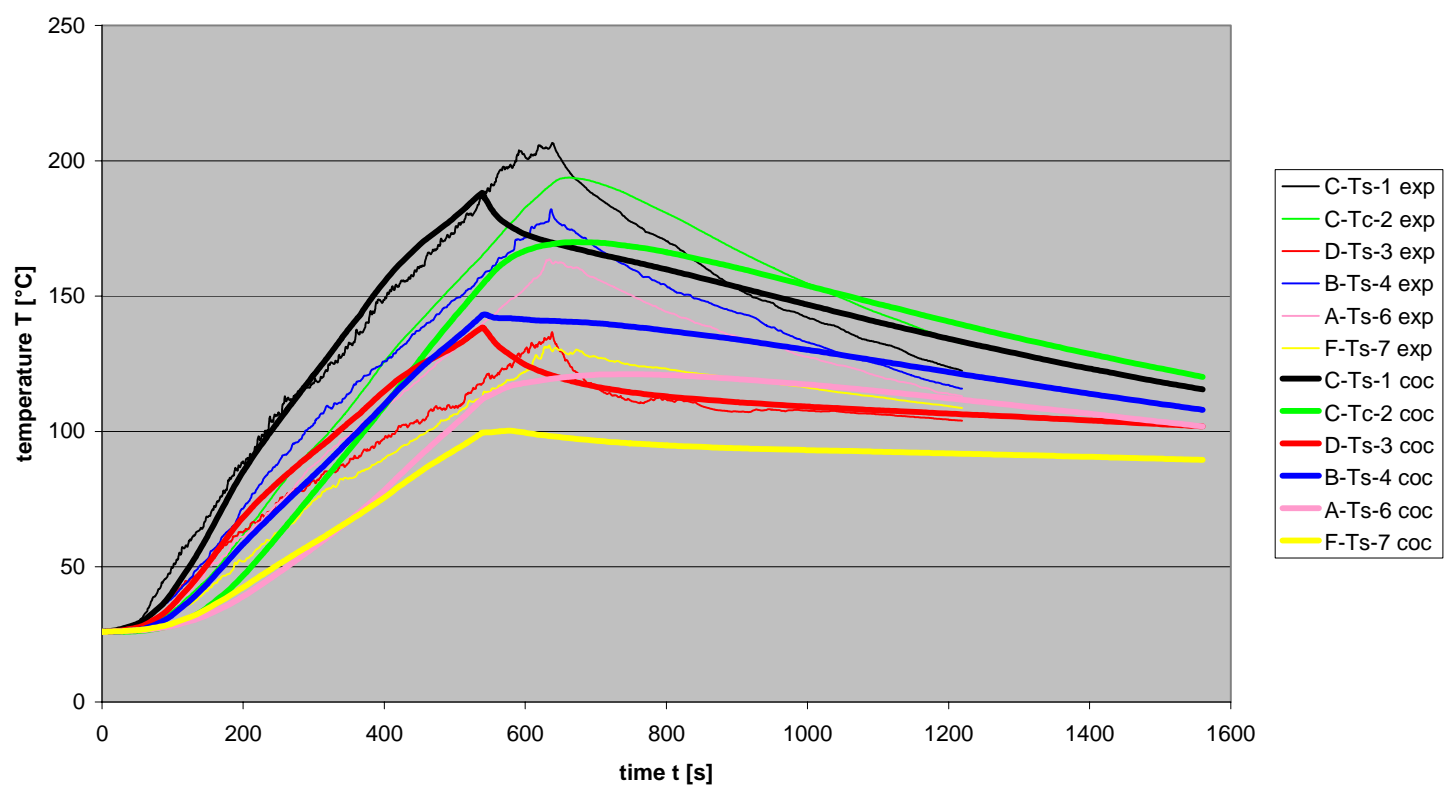

Fig. 5-27 Test 2: tray temperatures 1 to 7 
Test 2 (blind) : Tray temperatures

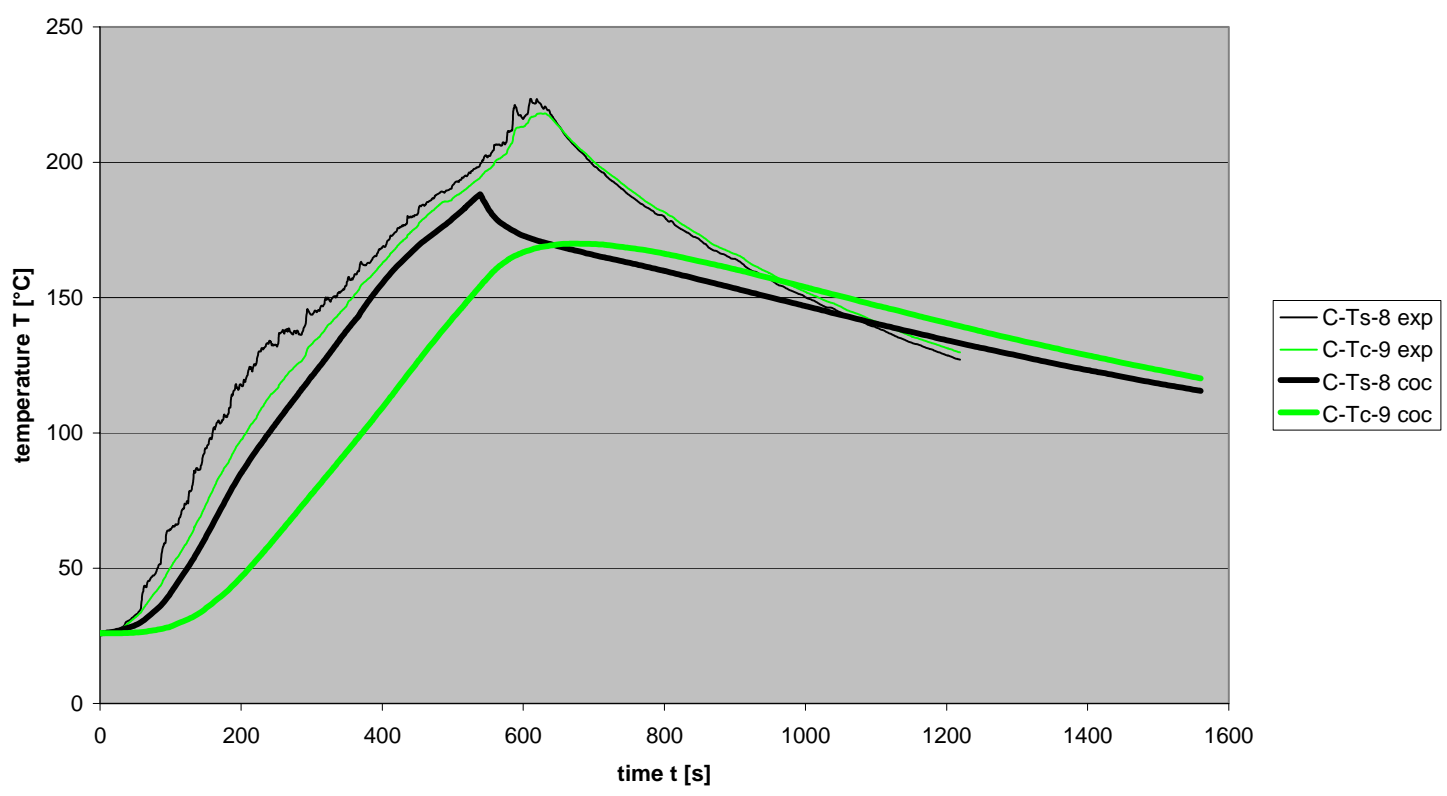

Fig. 5-28 Test 2: tray temperatures 8 to 9

Test 2 (blind) : Tray temperatures

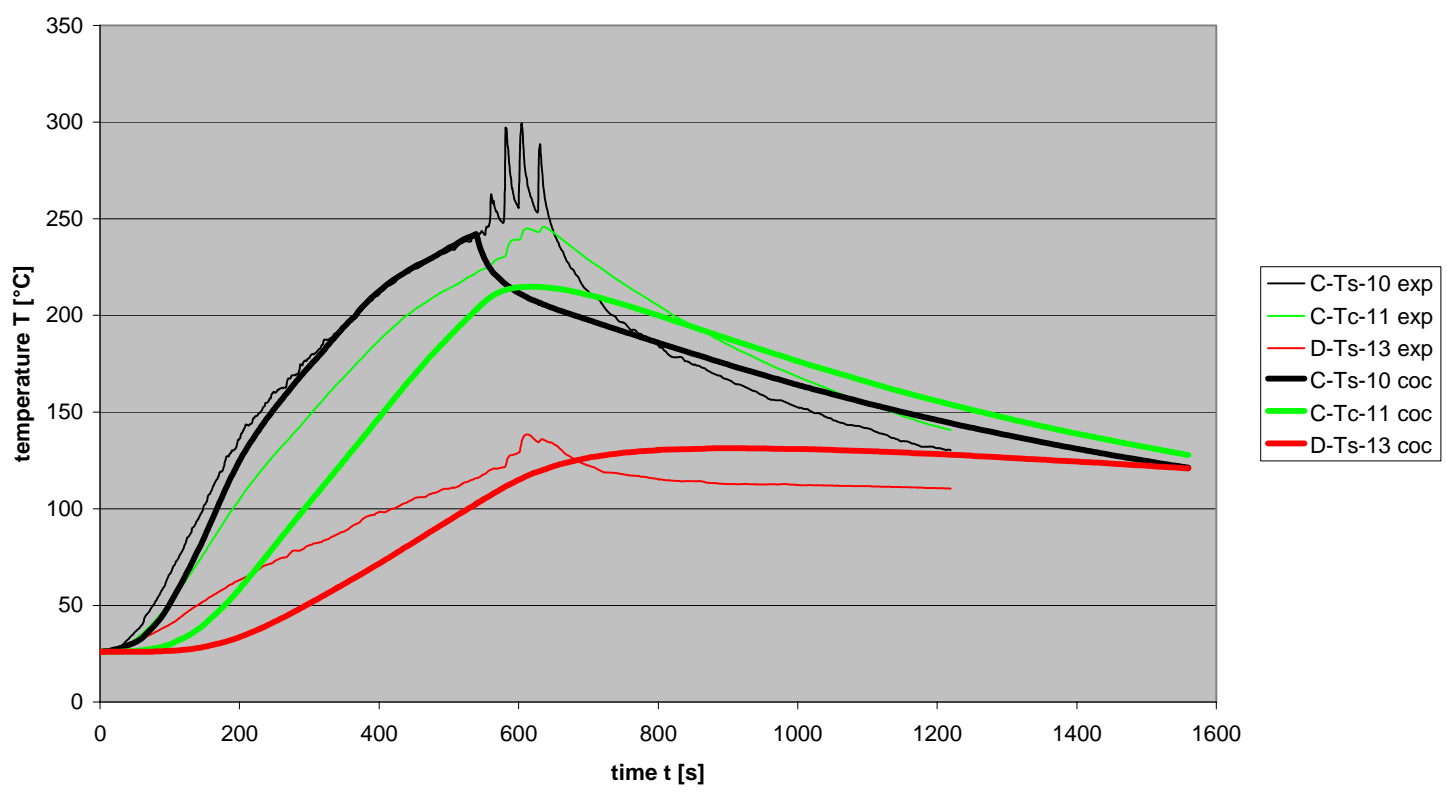

Fig. 5-29 Test 2: tray temperatures 10 to 13 
Test 2 (blind) : Tray temperatures

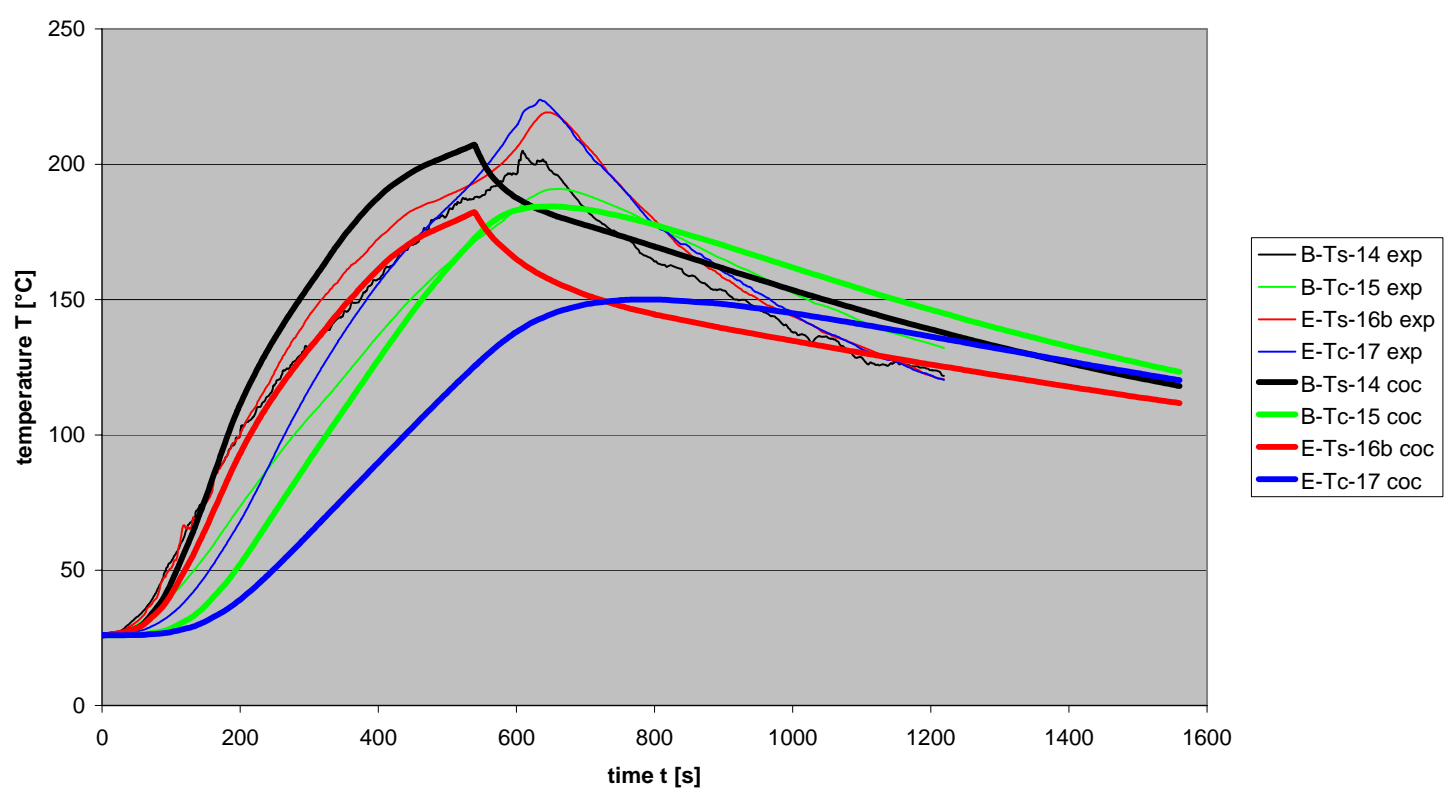

Fig. 5-30 Test 2: tray temperatures 14 to 17

Test 2 (blind) : Tray temperatures

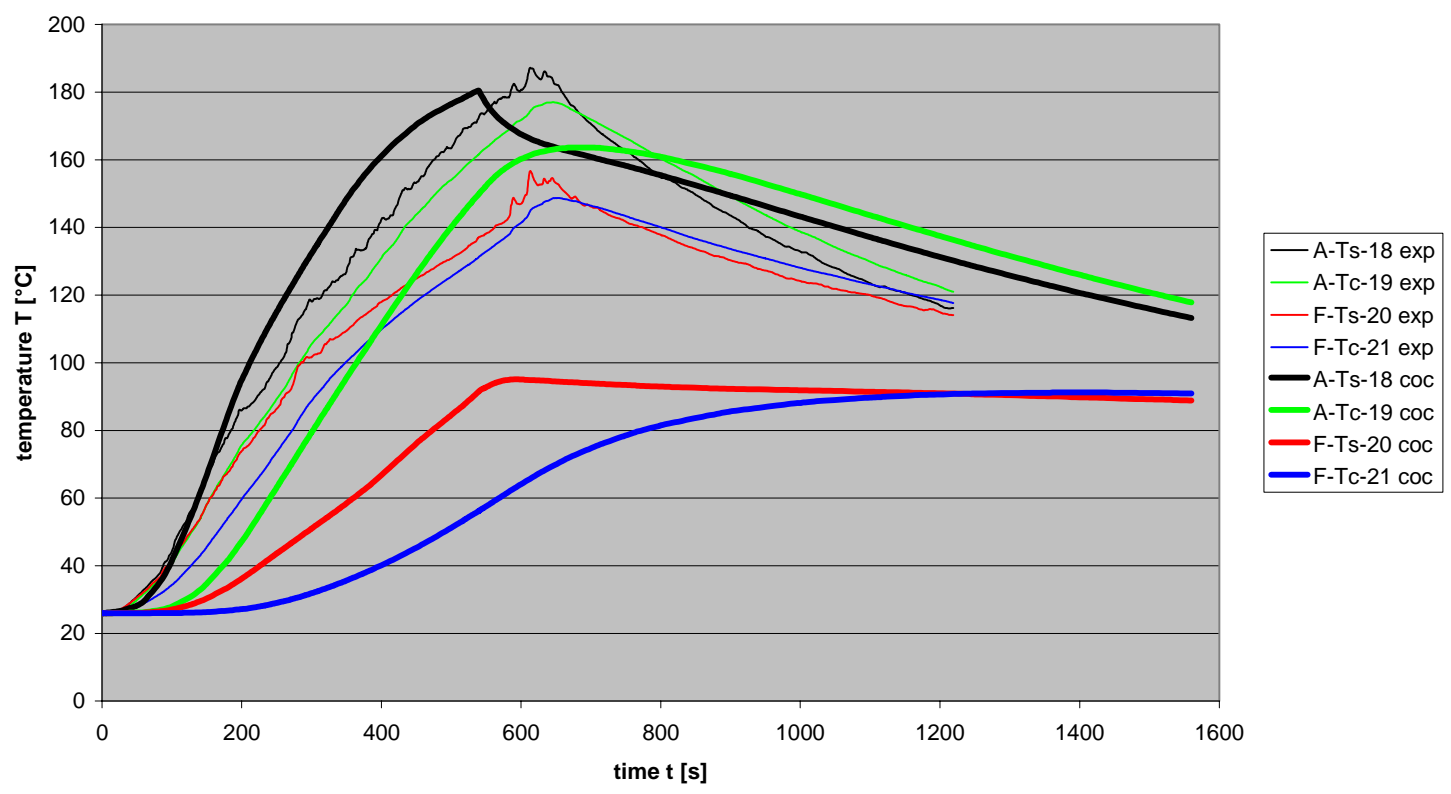

Fig. 5-31 Test 2: tray temperatures 18 to 21 
Test 2 (blind) : Tray temperatures

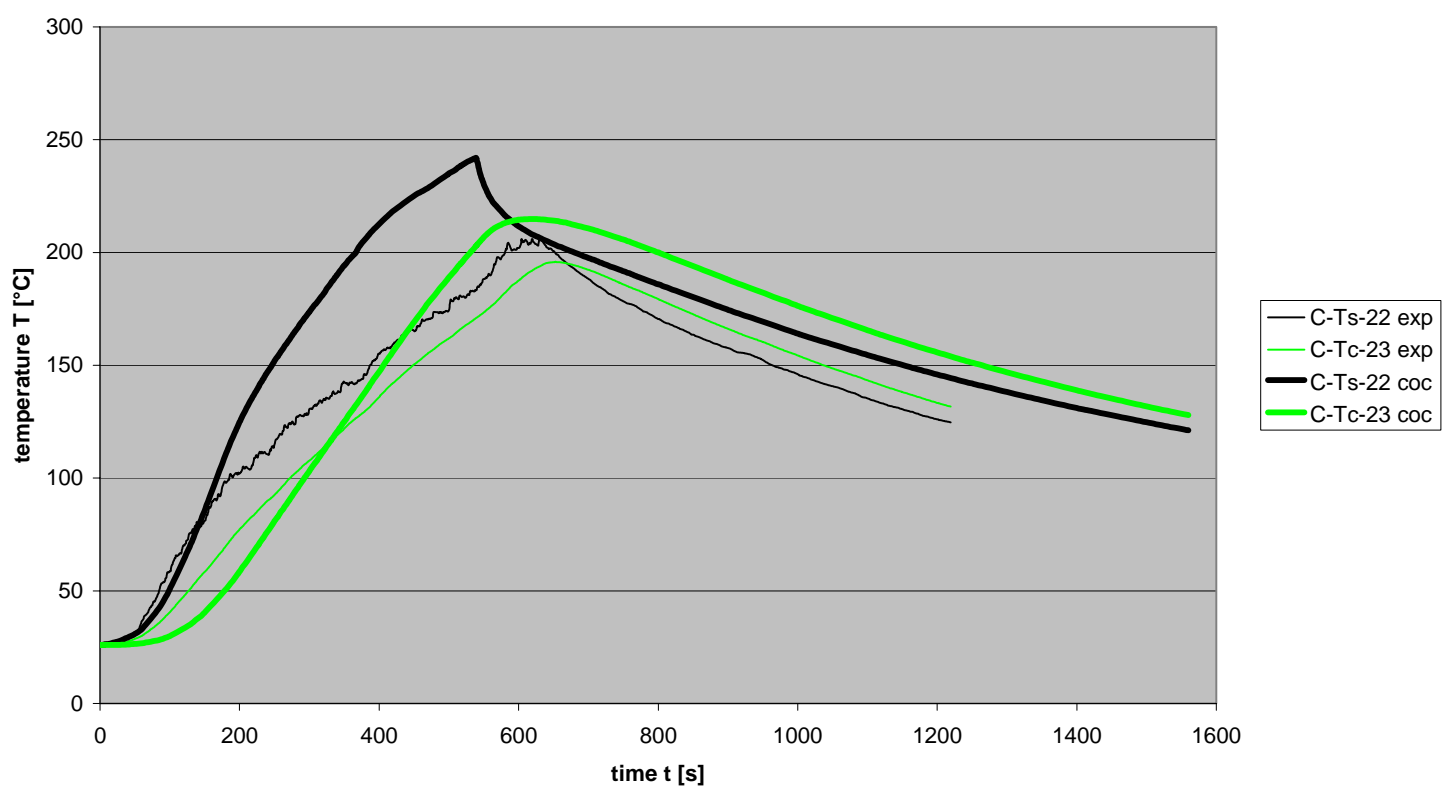

Fig. 5-32 Test 2: tray temperatures 22 to 23

Test 2 (blind) : Tray temperatures

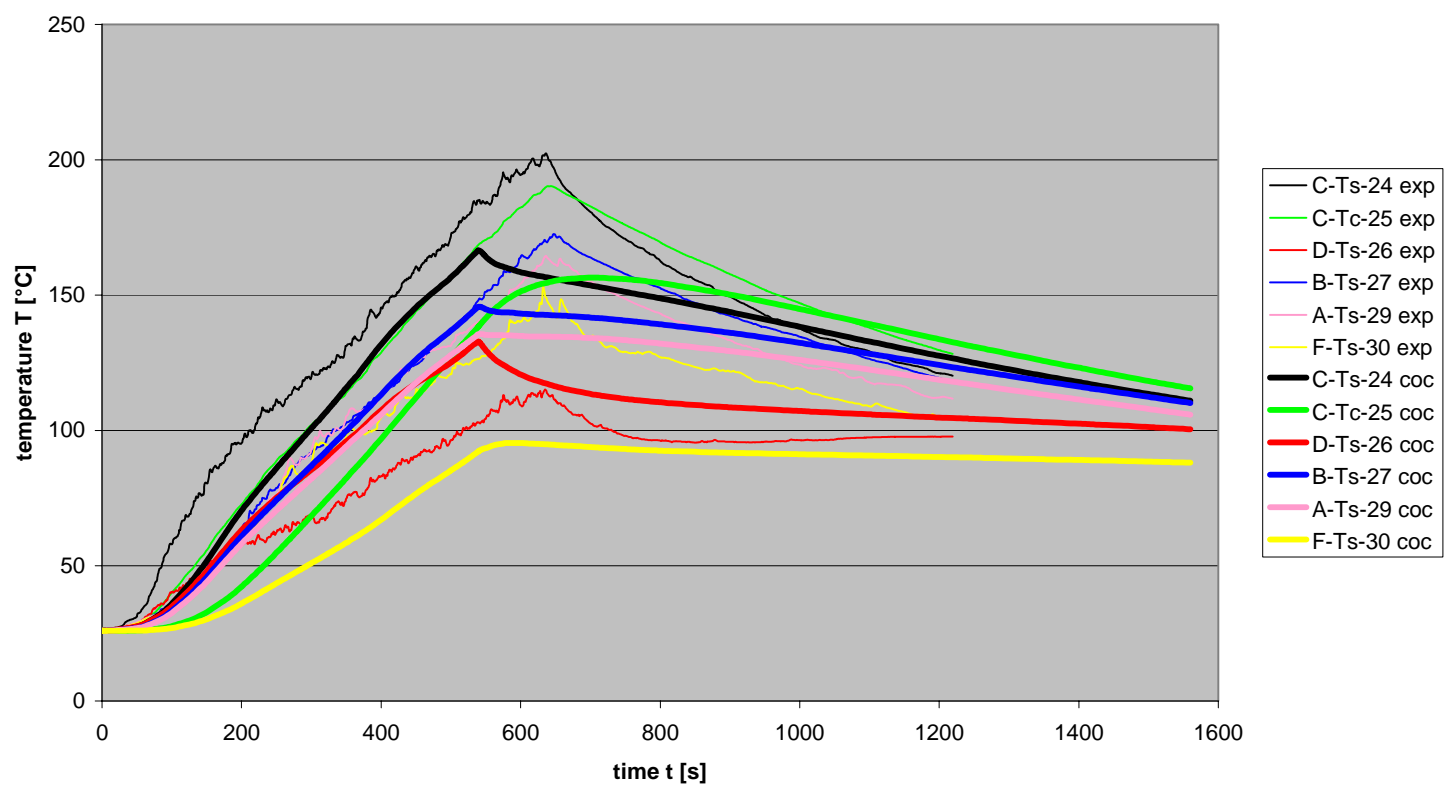

Fig. 5-33 Test 2: tray temperatures 24 to 30 
Test 2 (blind) : Vertical tray temperatures

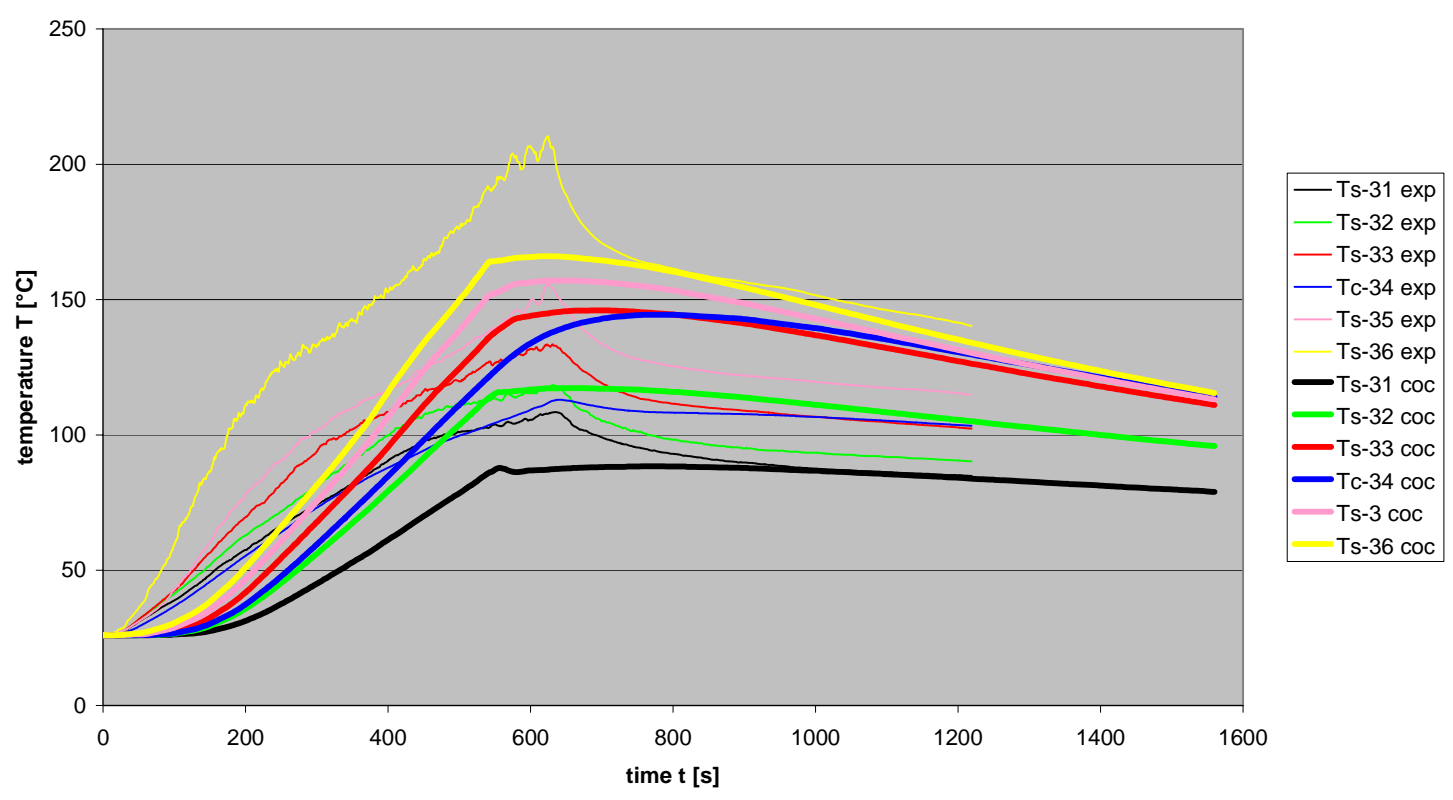

Fig. 5-34 Test 2: tray temperatures 31 to 36

Test 3 (blind) : Tray temperatures

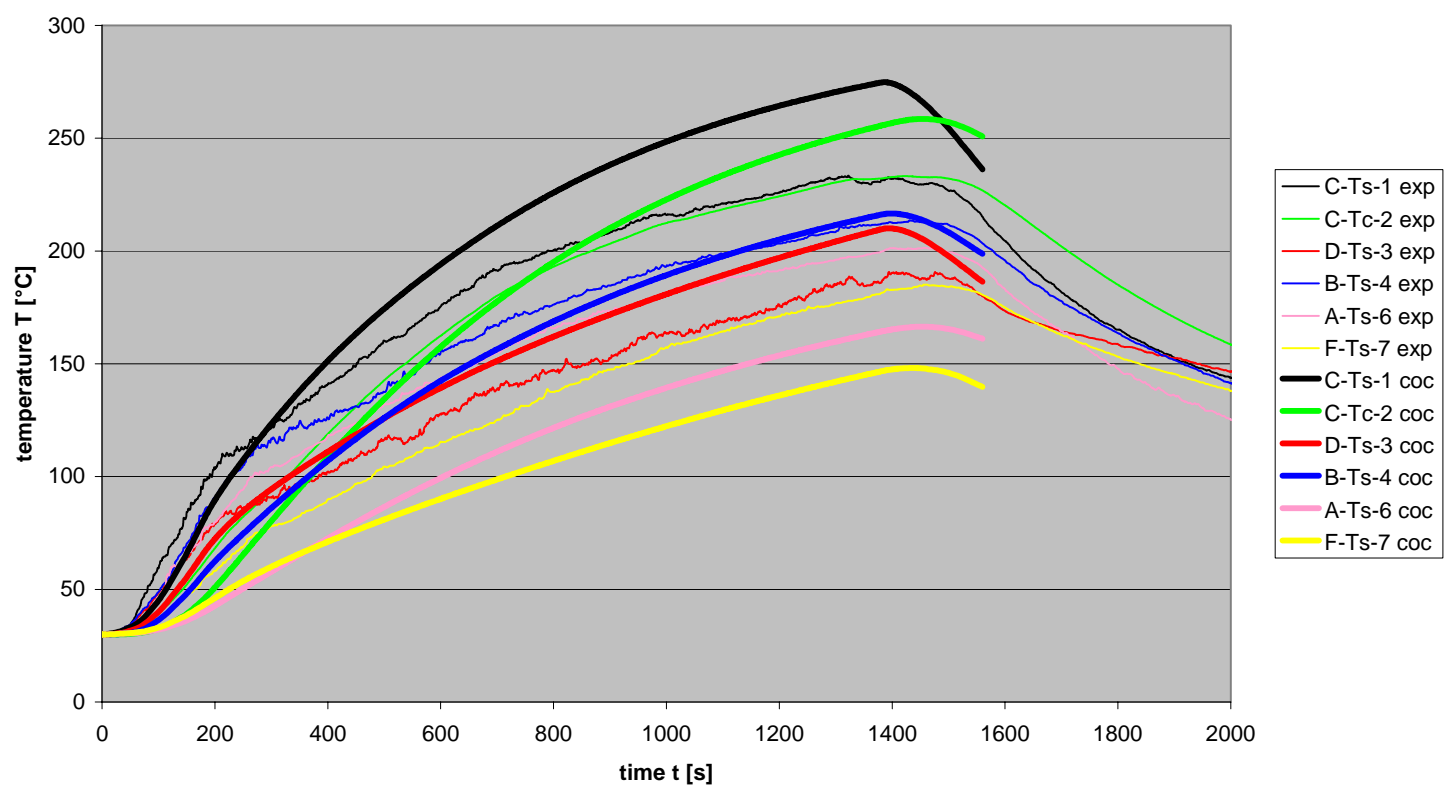

Fig. 5-35 Test 3: tray temperatures 1 to 7 
Test 3 (blind) : Tray temperatures

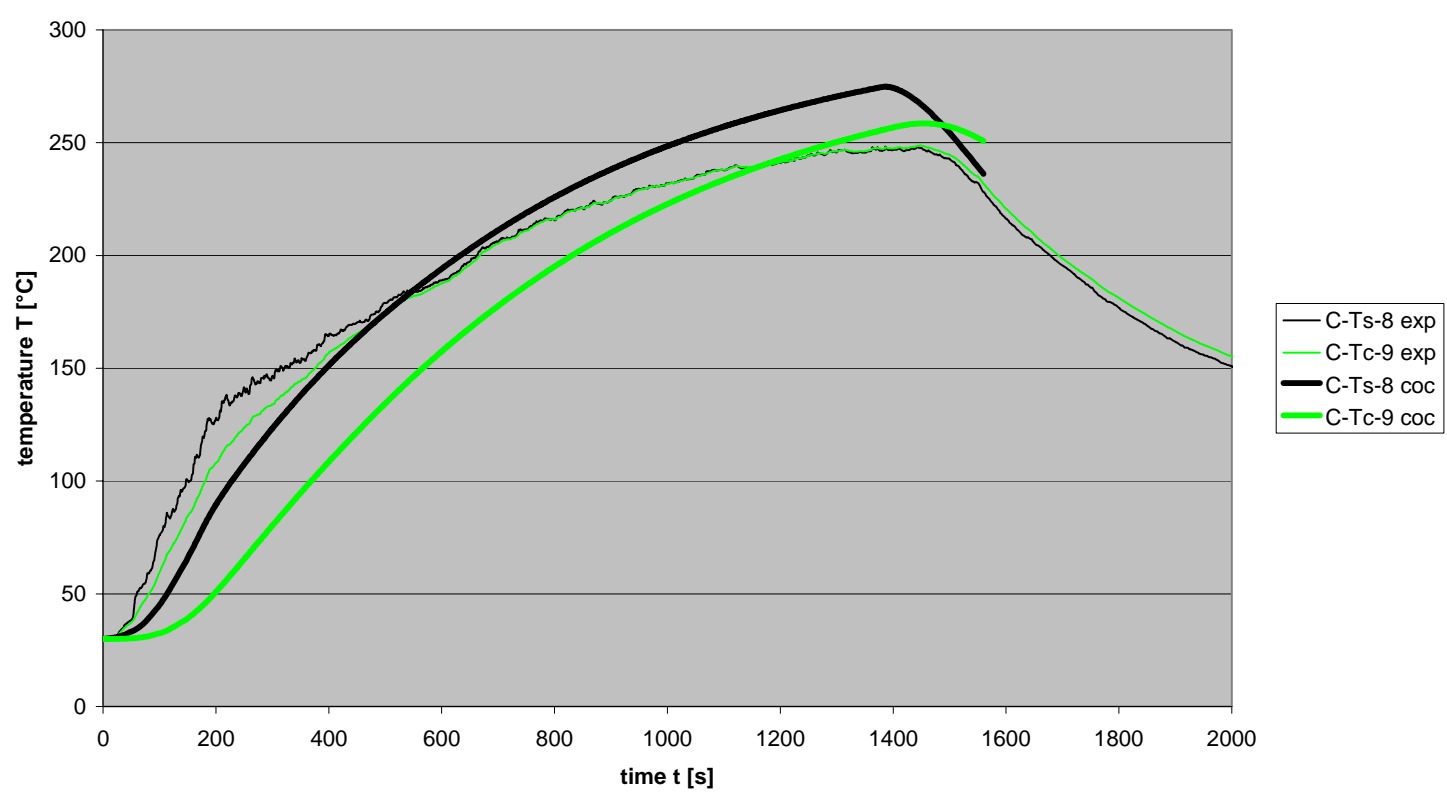

Fig. 5-36 Test 3: tray temperatures 8 to 9

Test 3 (blind) : Tray temperatures

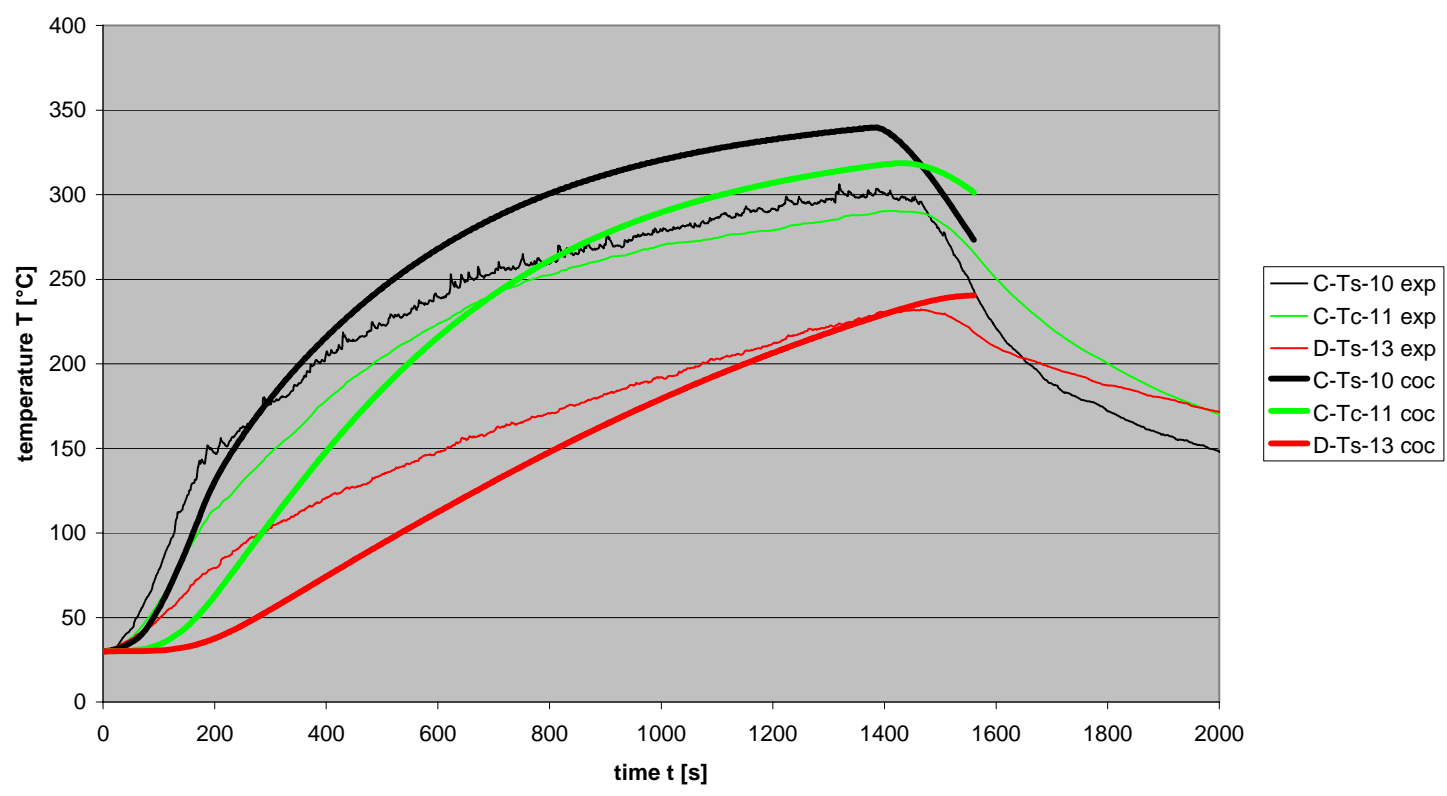

Fig. 5-37 Test 3: tray temperatures 10 to 13 
Test 3 (blind) : Tray temperatures

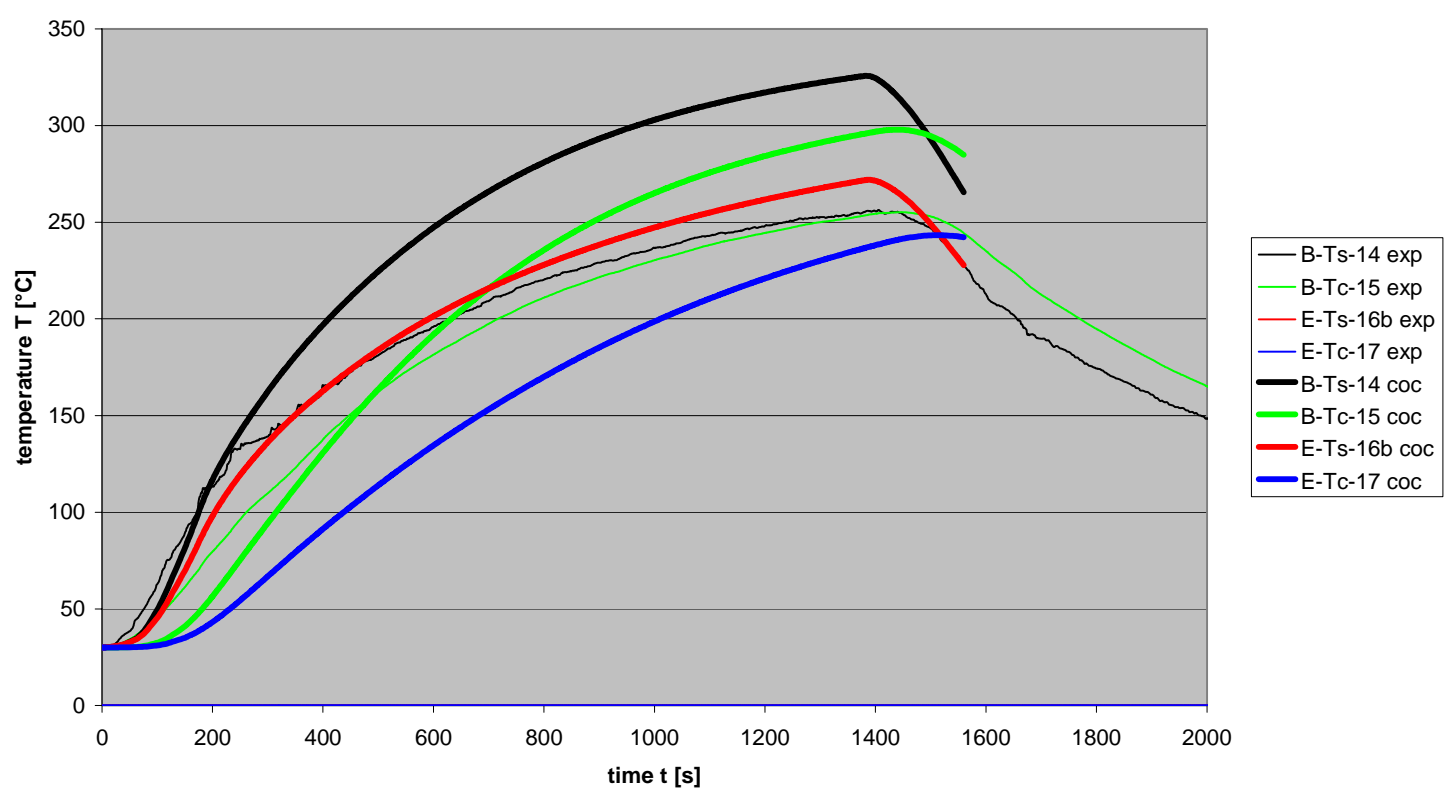

Fig. 5-38 Test 3: tray temperatures 14 to 17

Test 3 (blind) : Tray temperatures

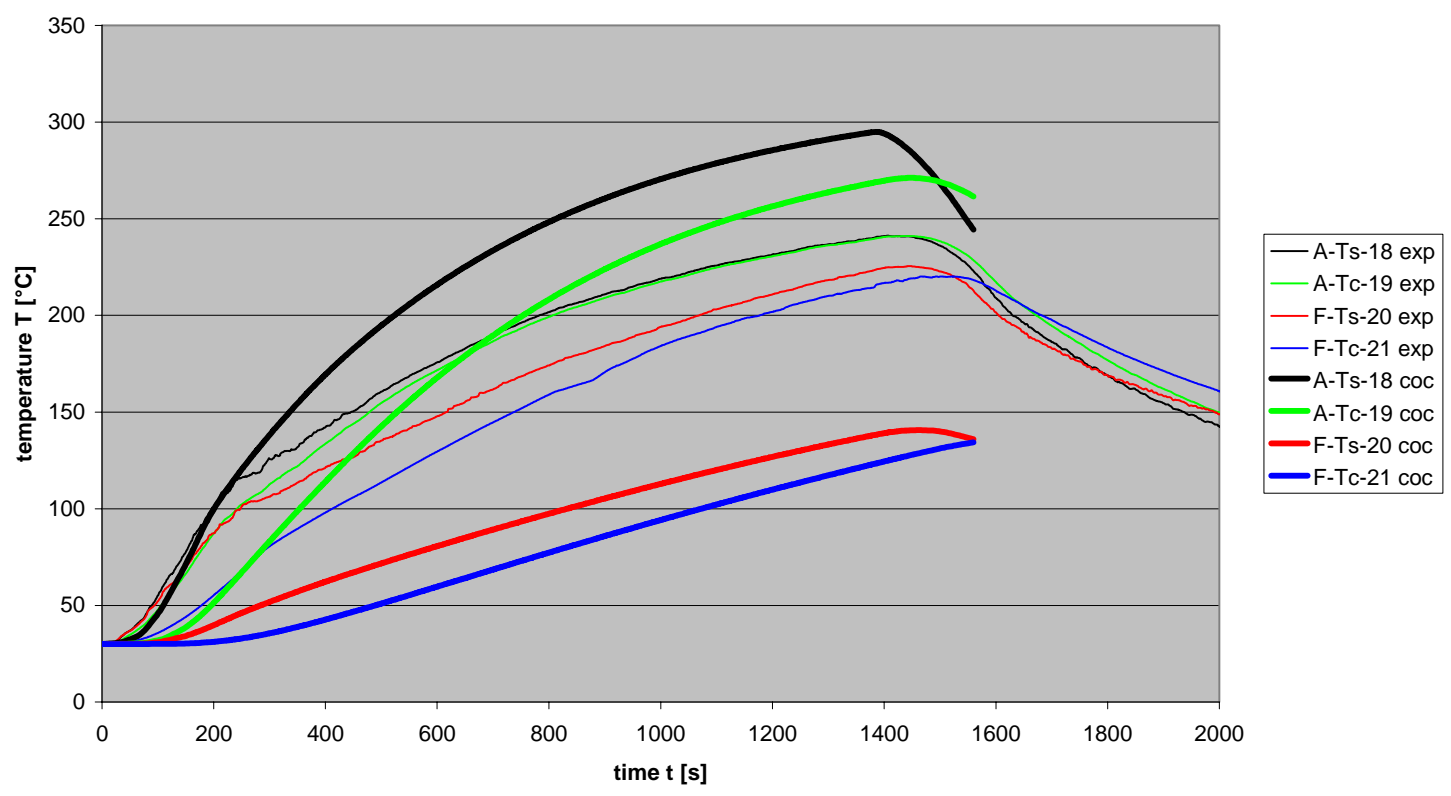

Fig. 5-39 Test 3: tray temperatures 18 to 21 
Test 3 (blind) : Tray temperatures

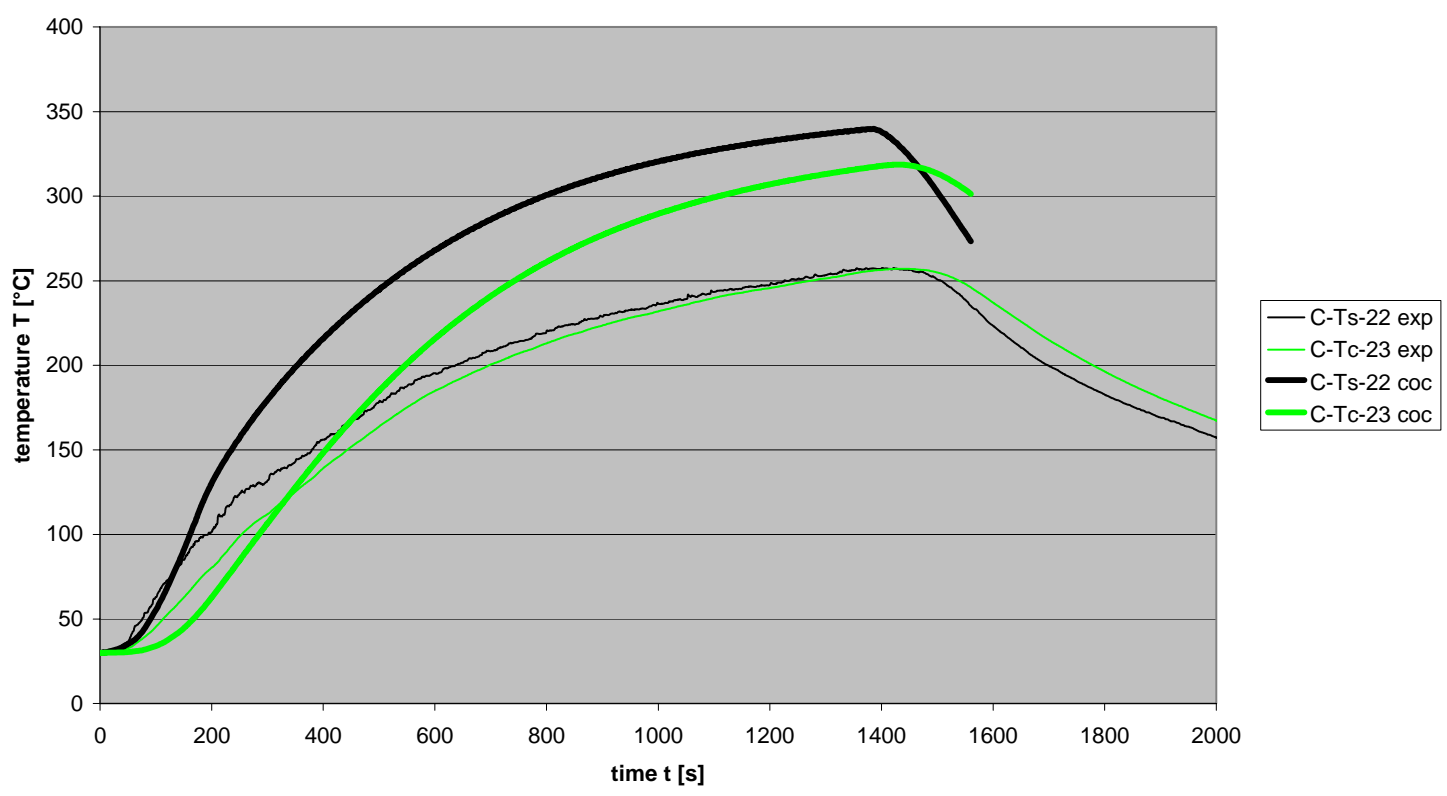

Fig. 5-40 Test 3: tray temperatures 22 to 23

Test 3 (blind) : Tray temperatures

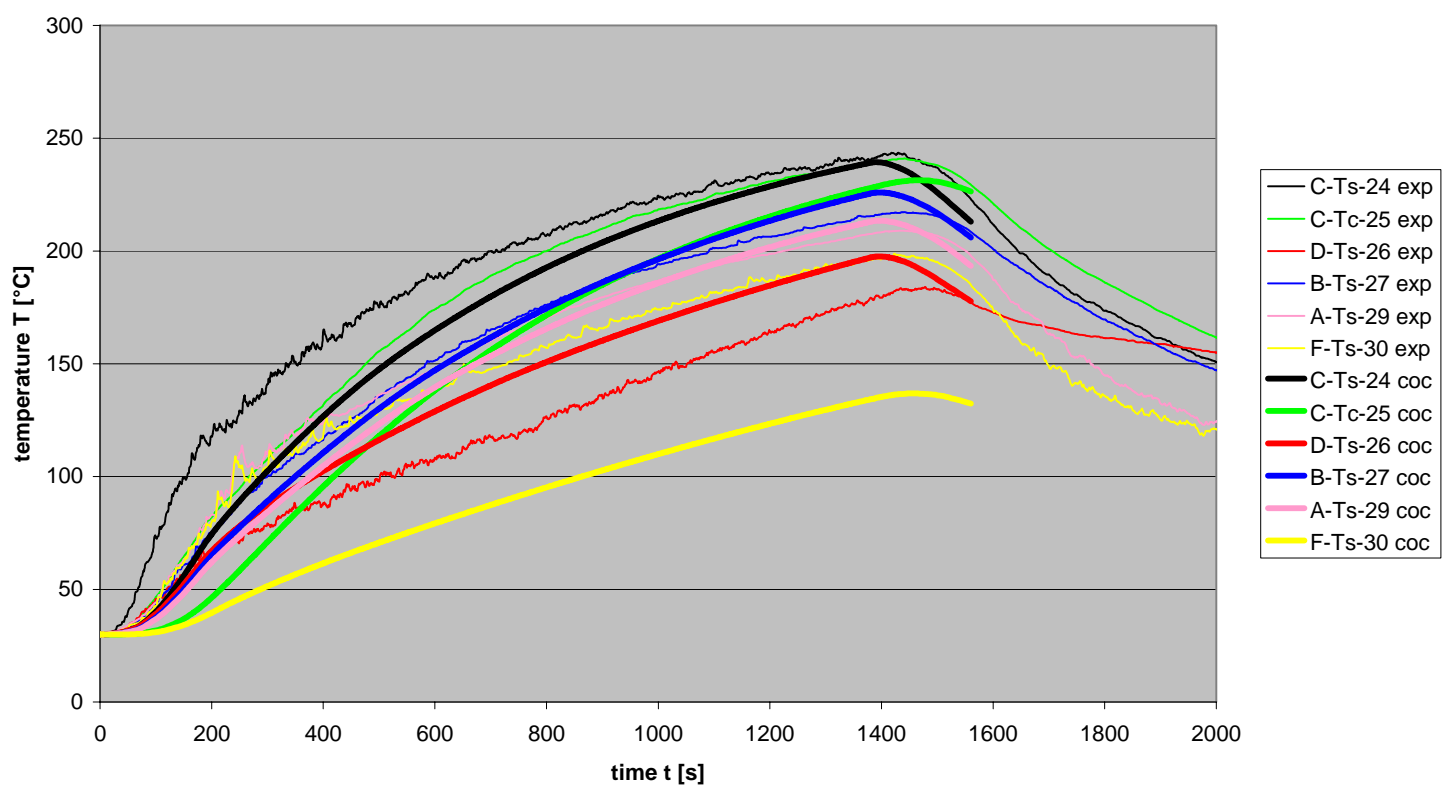

Fig. 5-41 Test 3: tray temperatures 24 to 30 
Test 3 (blind) : Vertical tray temperatures

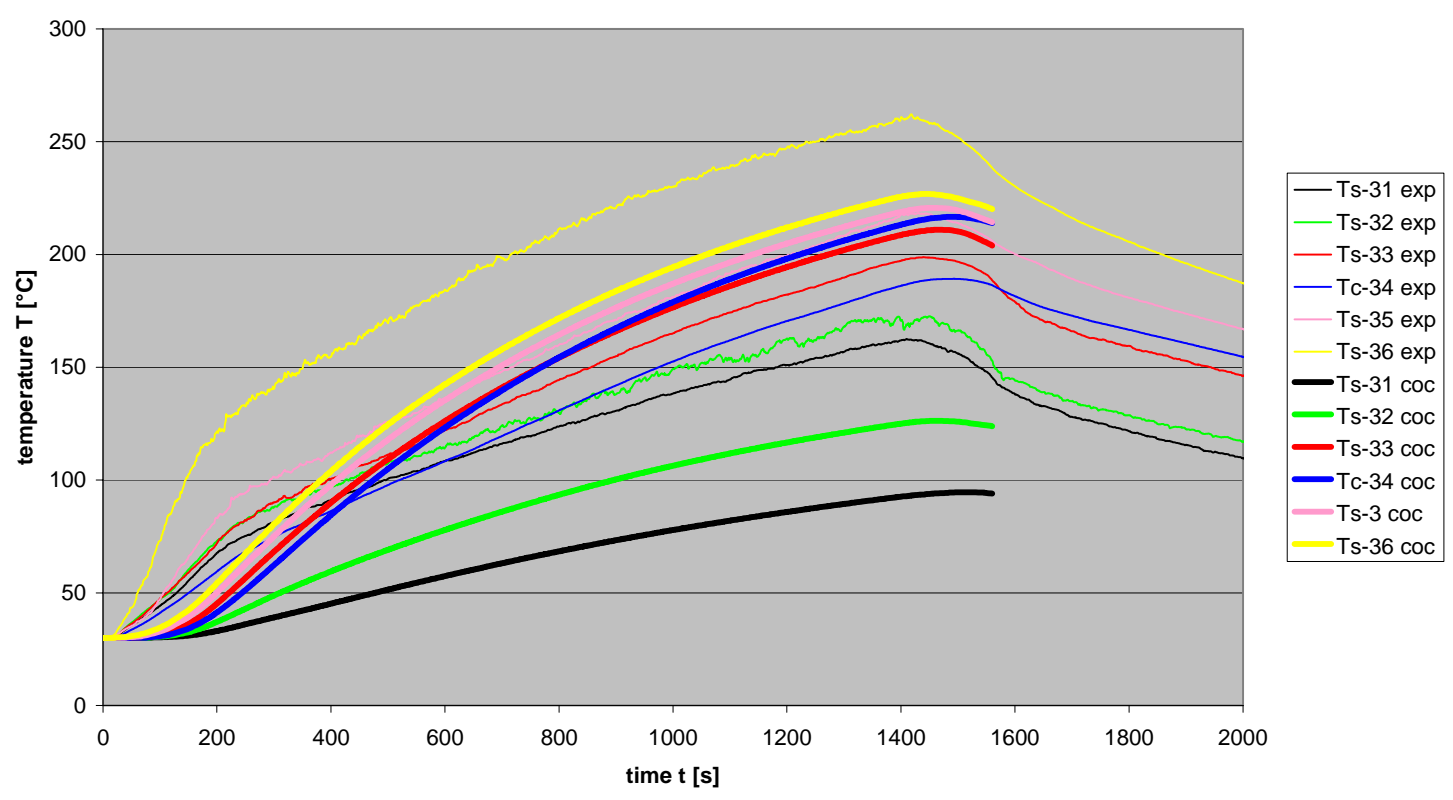

Fig. 5-42 Test 3: tray temperatures 31 to 36

Test 4 (blind) : Tray temperatures

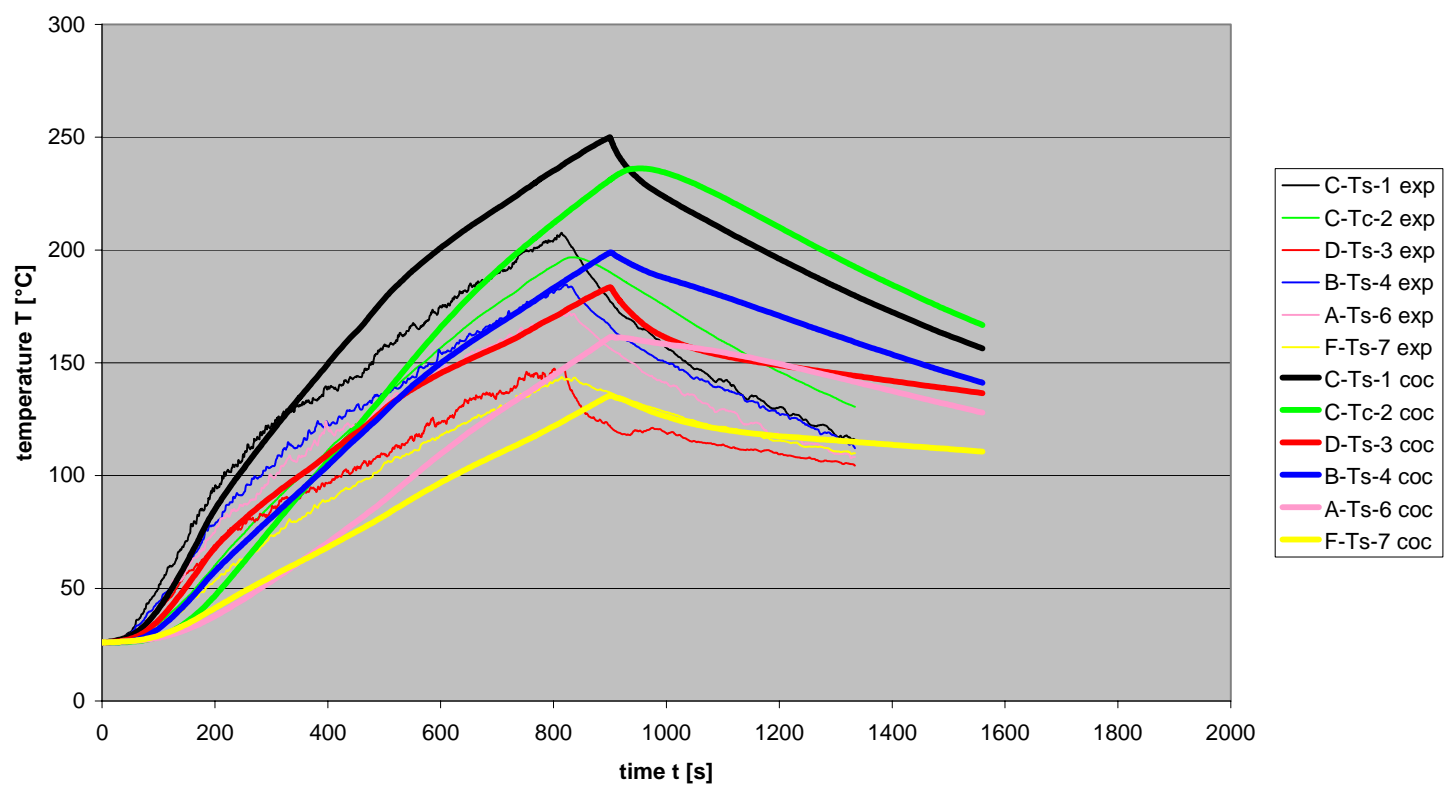

Fig. 5-43 Test 4: tray temperatures 1 to 7 
Test 4 (blind) : Tray temperatures

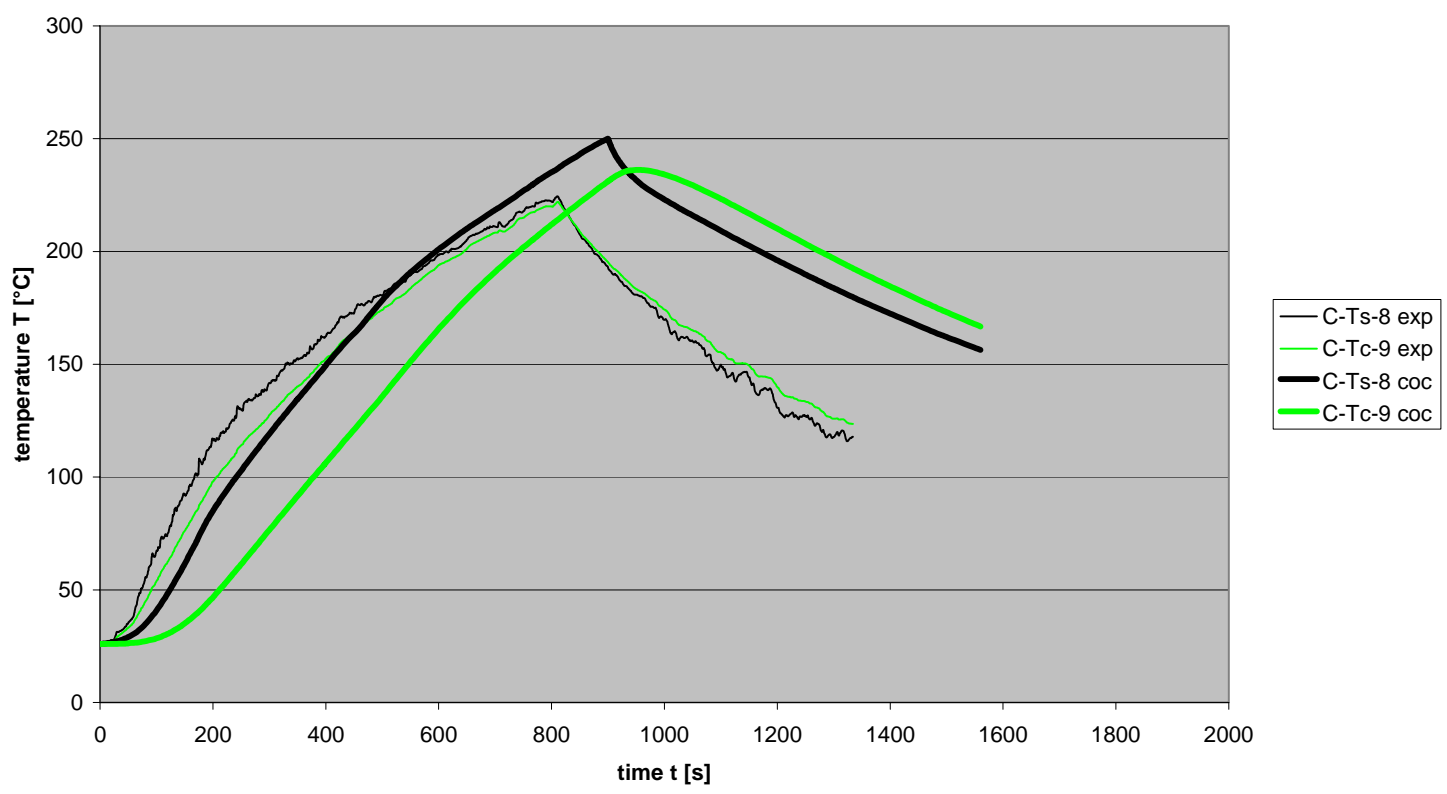

Fig. 5-44 Test 4: tray temperatures 8 to 9

Test 4 (blind) : Tray temperatures

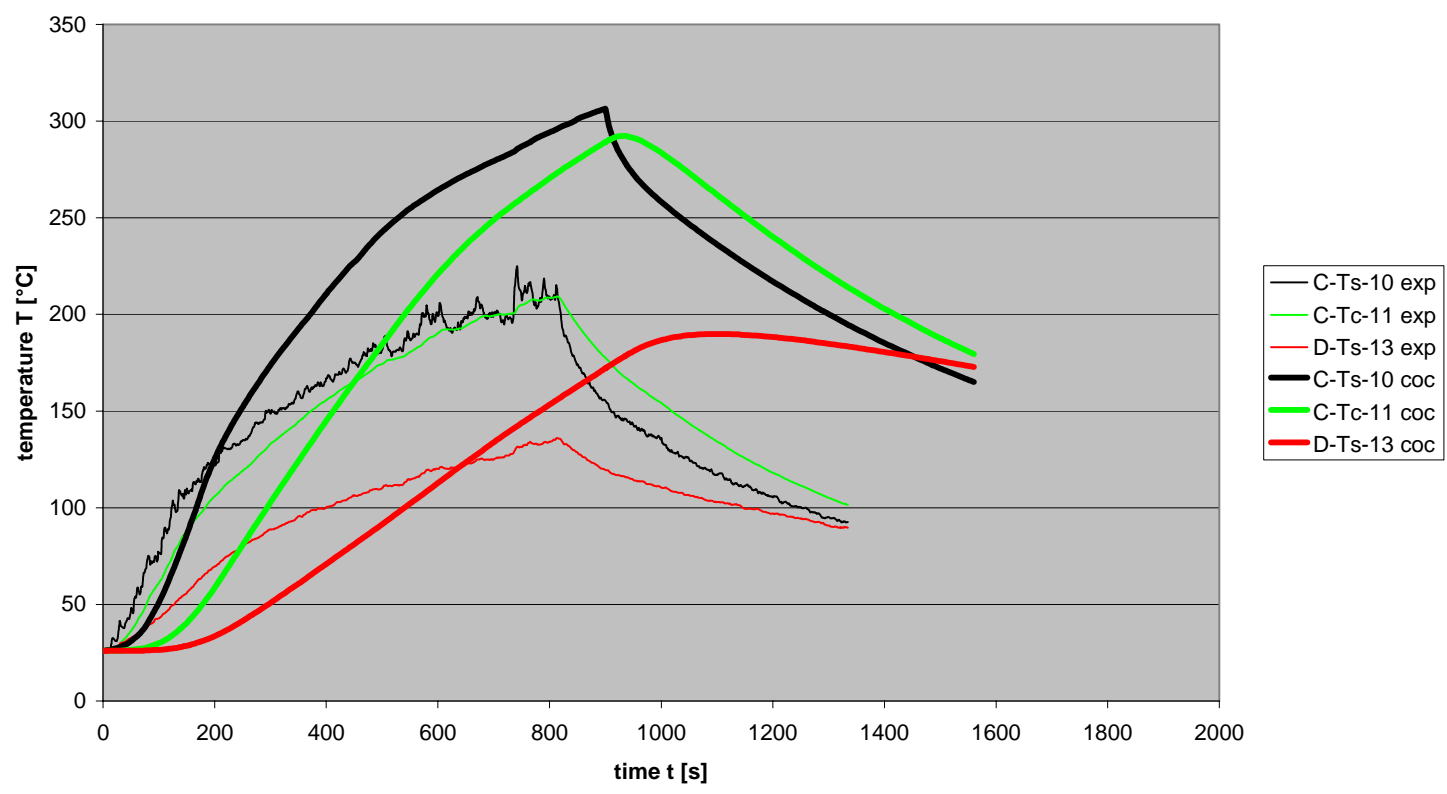

Fig. 5-45 Test 4: tray temperatures 10 to 13 
Test 4 (blind) : Tray temperatures

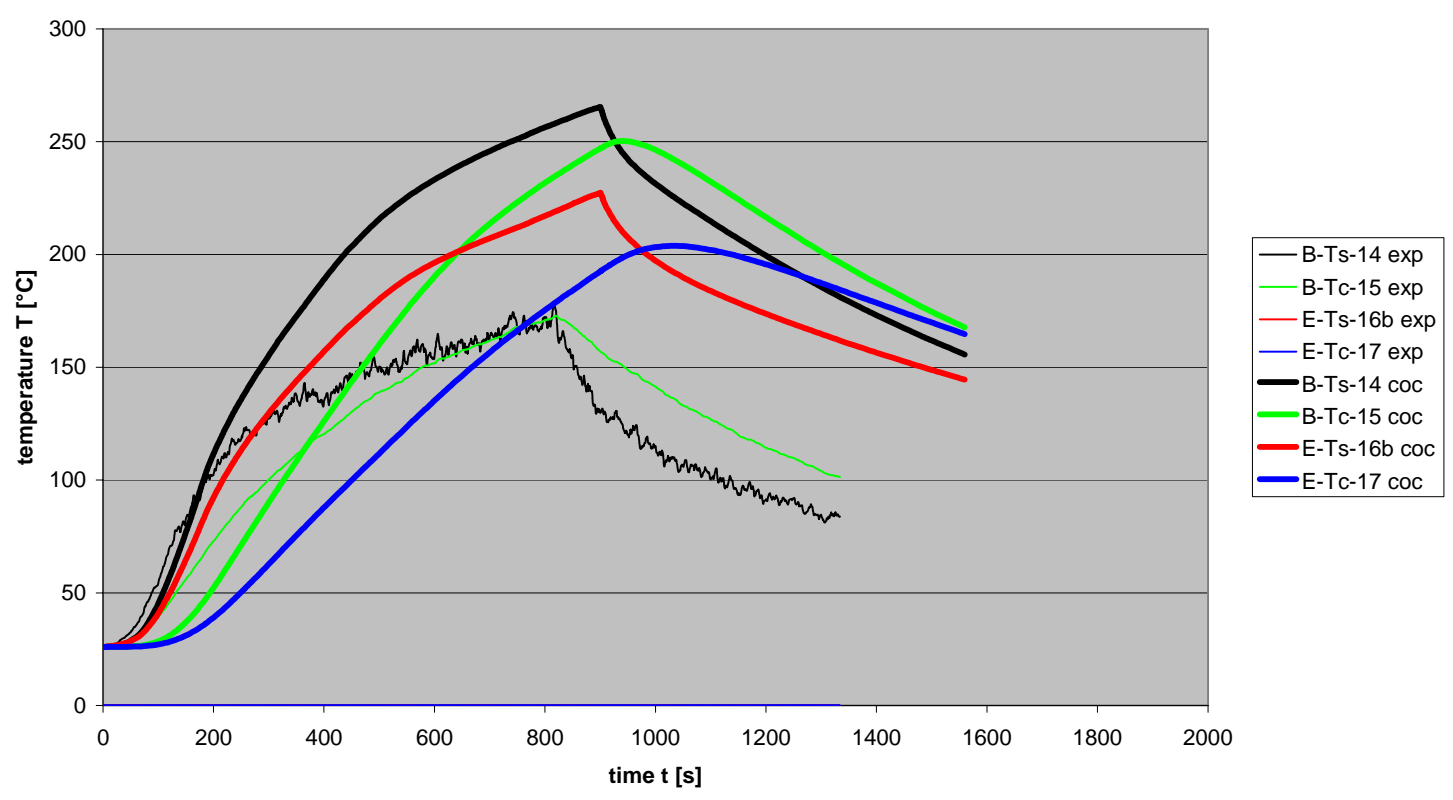

Fig. 5-46 Test 4: tray temperatures 14 to 17

Test 4 (blind) : Tray temperatures

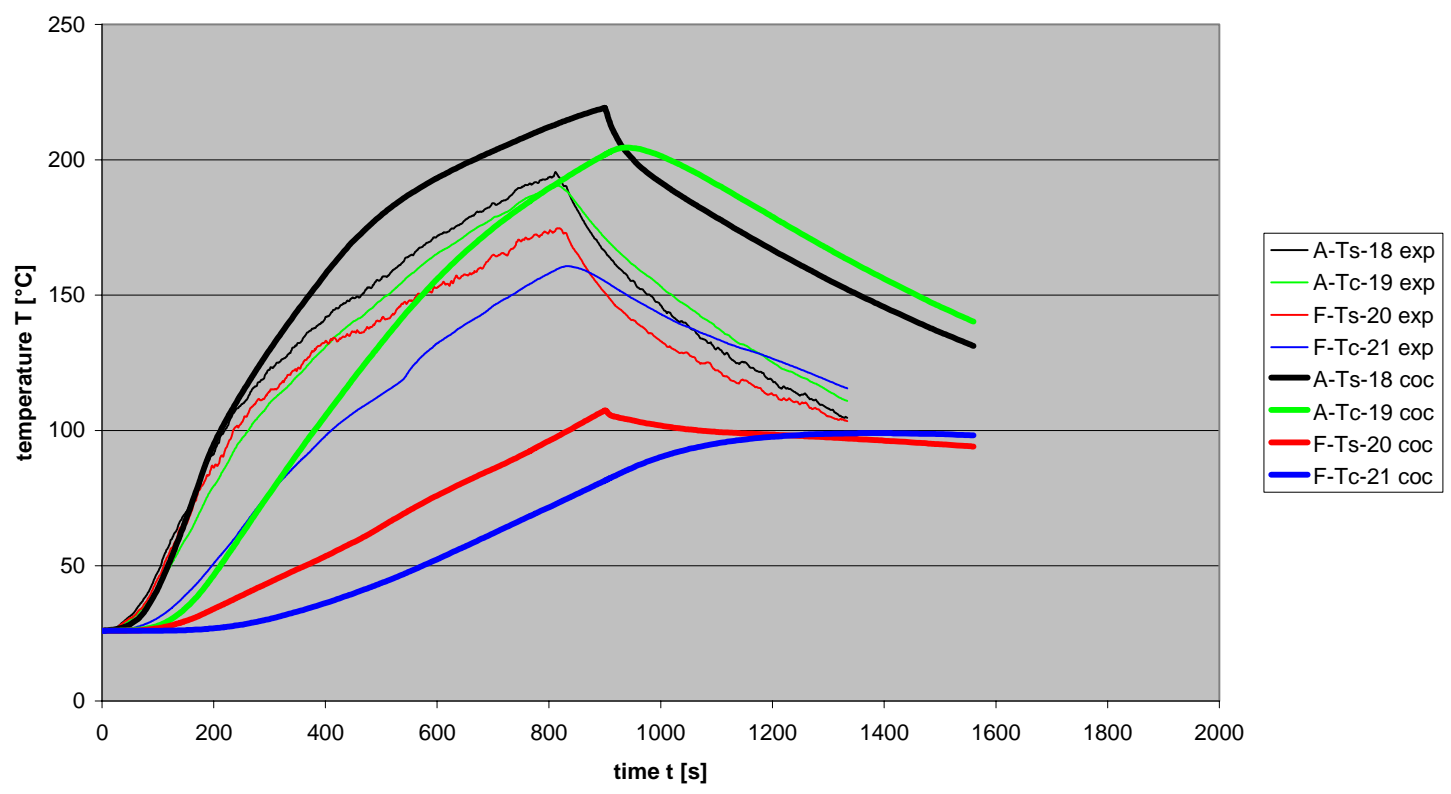

Fig. 5-47 Test 4: tray temperatures 18 to 21 
Test 4 (blind) : Tray temperatures

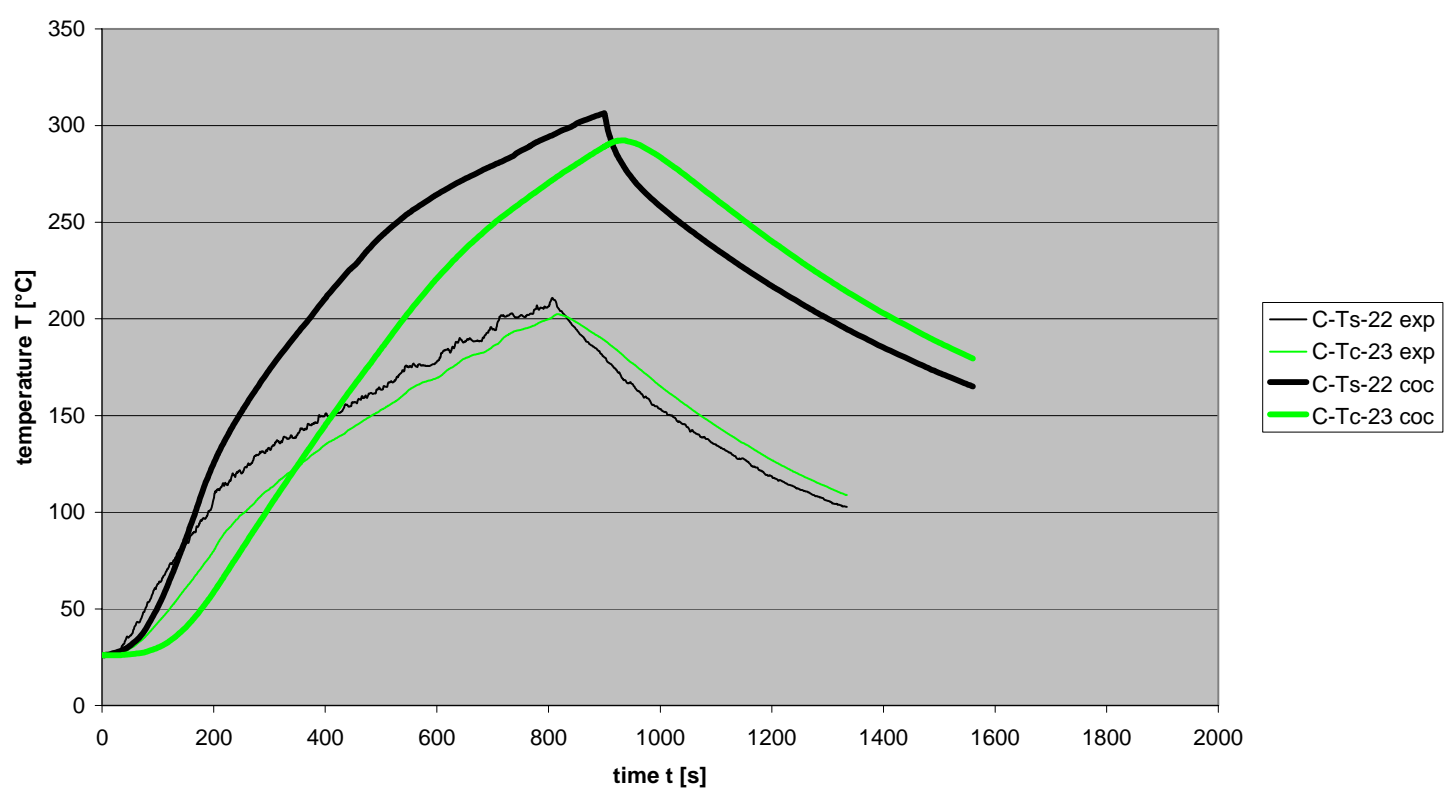

Fig. 5-48 Test 4: tray temperatures 22 to 23

Test 4 (blind) : Tray temperatures

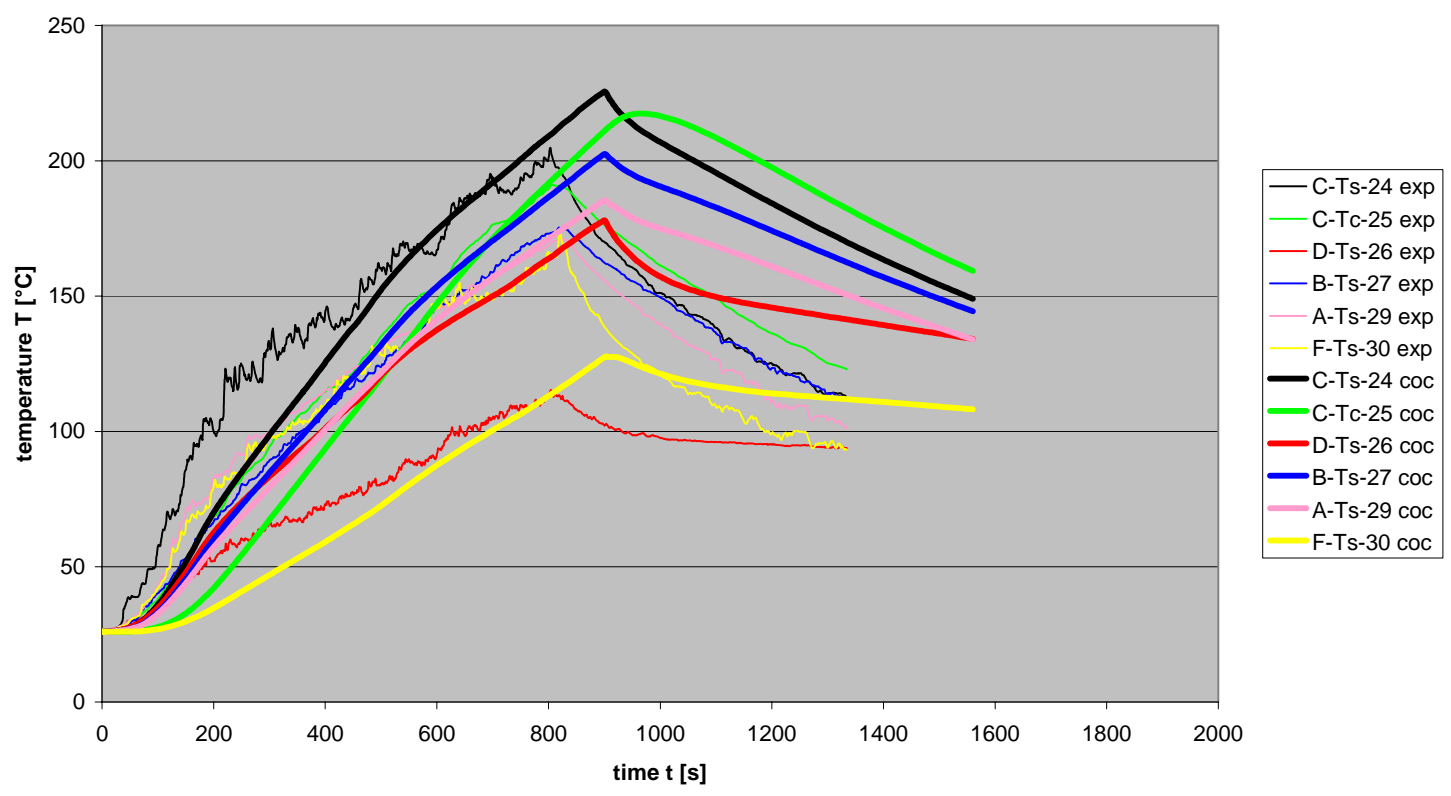

Fig. 5-49 Test 4: tray temperatures 24 to 30 
Test 4 (blind) : Vertical tray temperatures

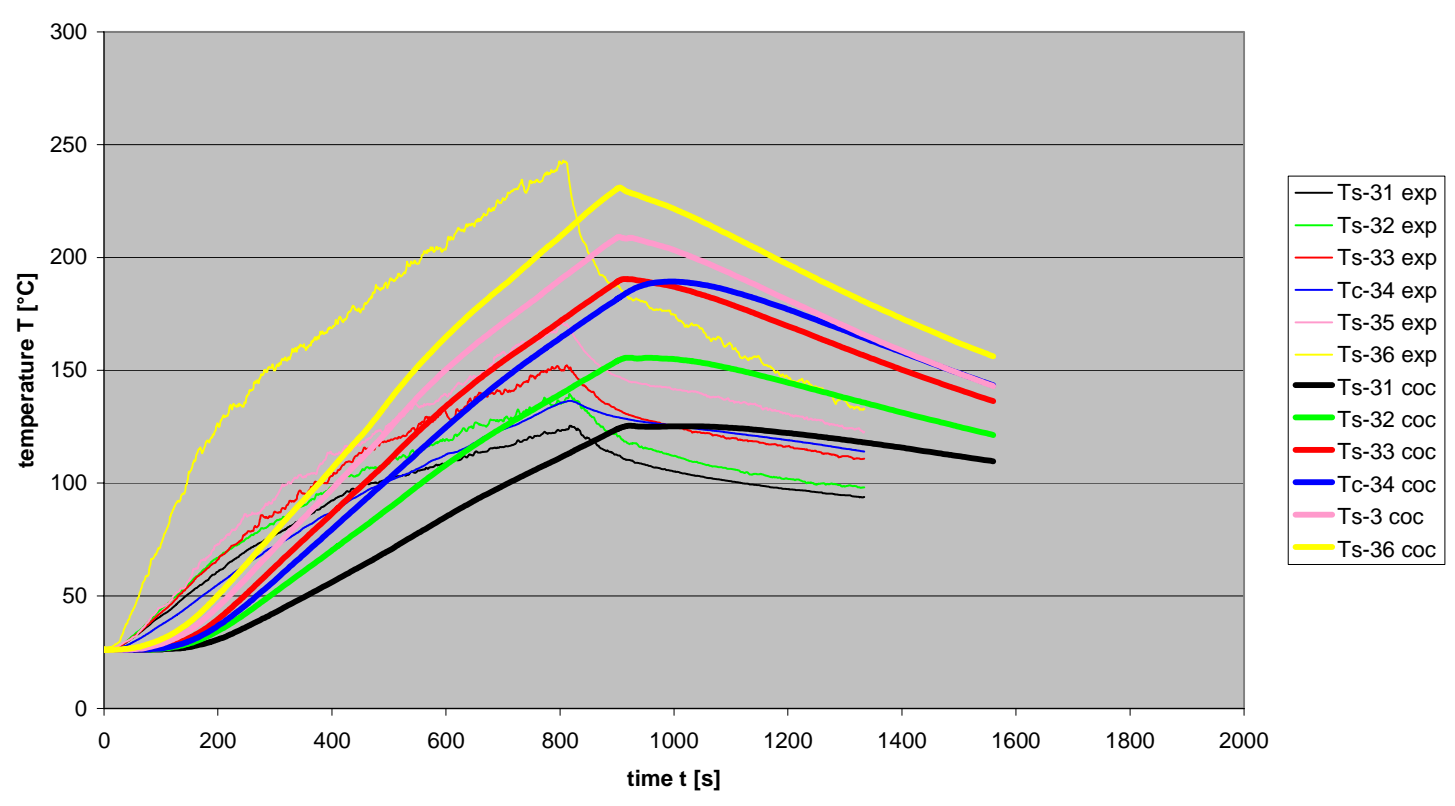

Fig. 5-50 Test 4: tray temperatures 31 to 36

Test 2 (blind) : Junction box temperatures

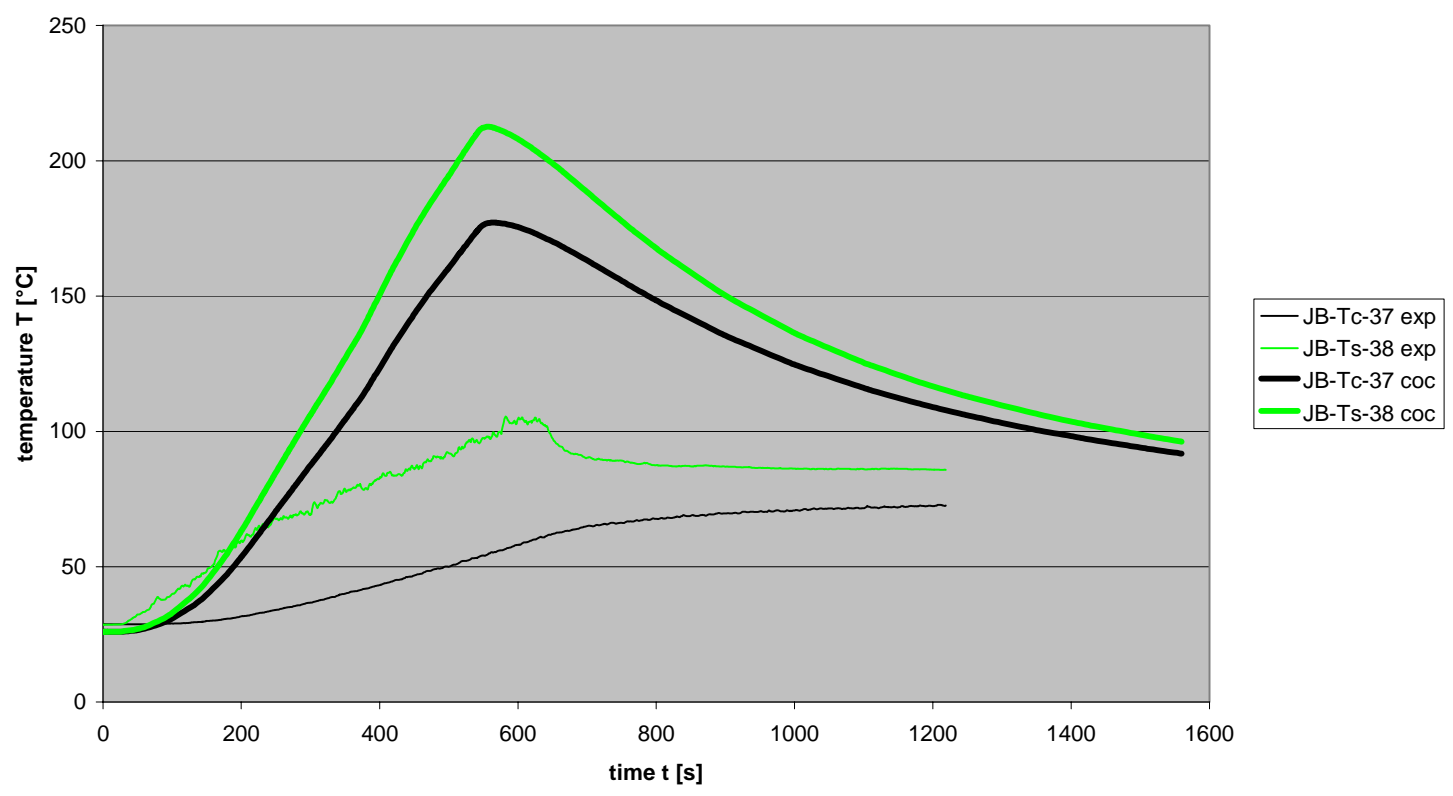

Fig. 5-51 Test 2: Temperatures at junction box 
Test 3 (blind) : Junction box temperatures

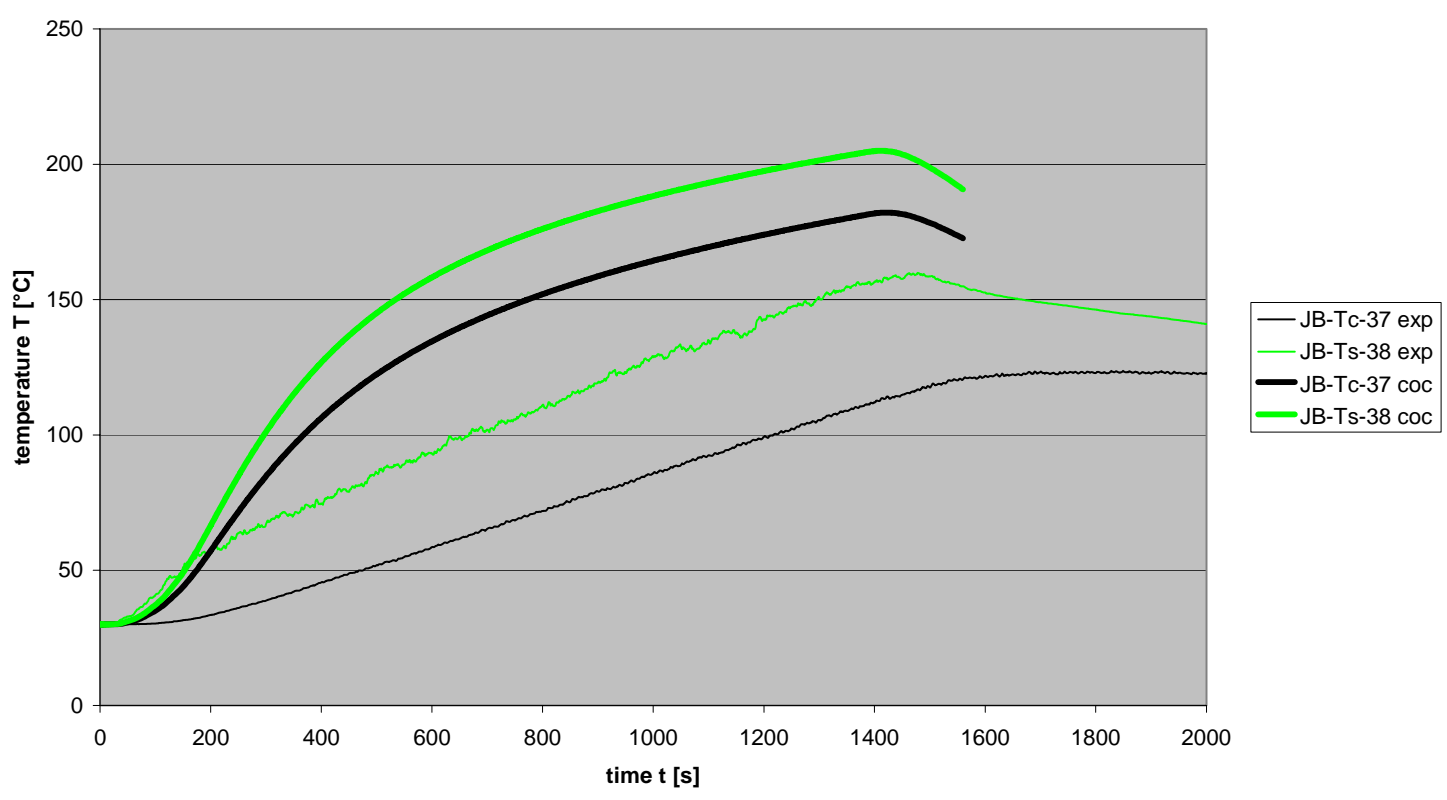

Fig. 5-52 Test 3: Temperatures at junction box

Test 4 (blind) : Junction box temperatures

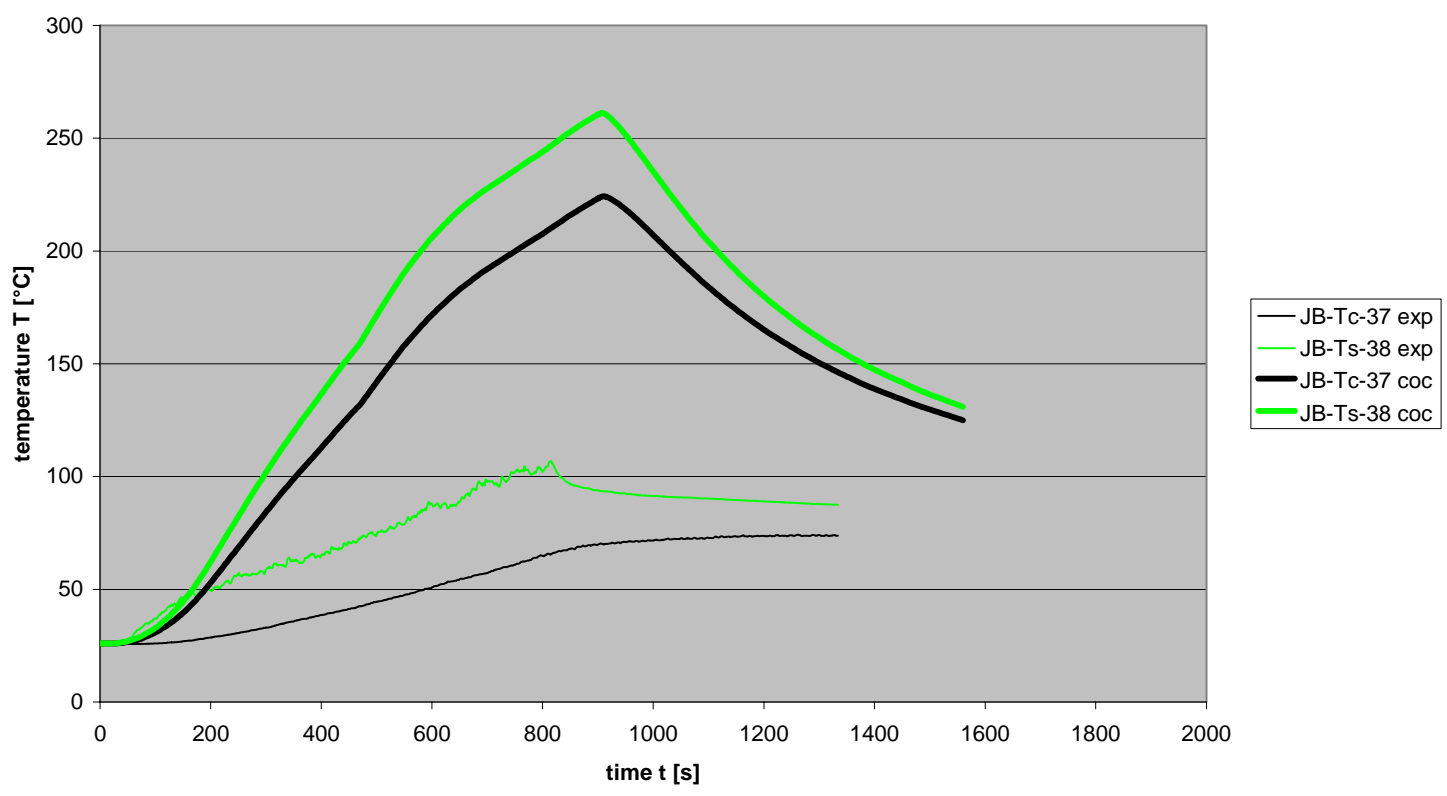

Fig. 5-53 Test 4: Temperatures at junction box 
Test 3 (blind) : Temperatures at door

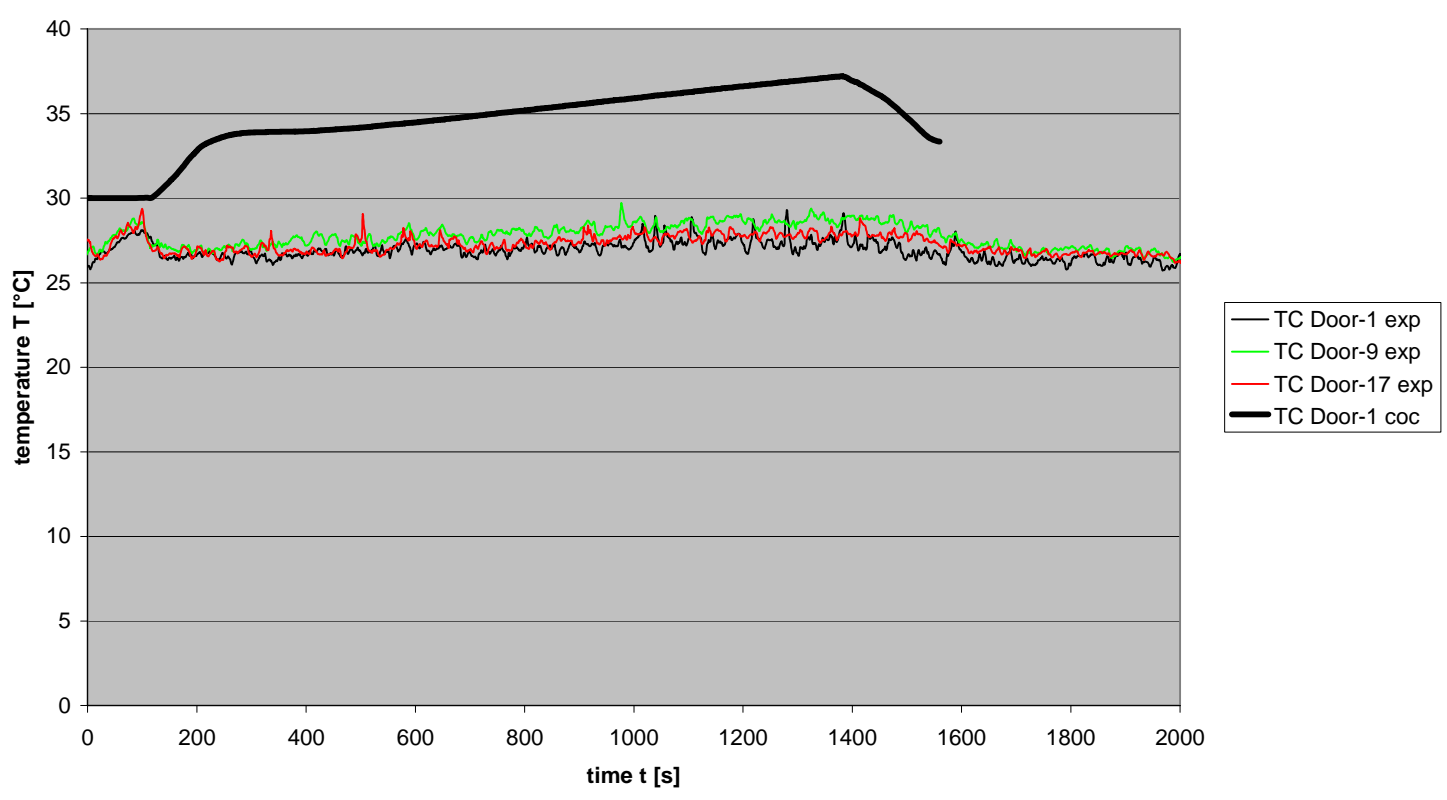

Fig. 5-54 Test 3: Temperature at door (level 1)

Test 3 (blind) : Temperatures at door

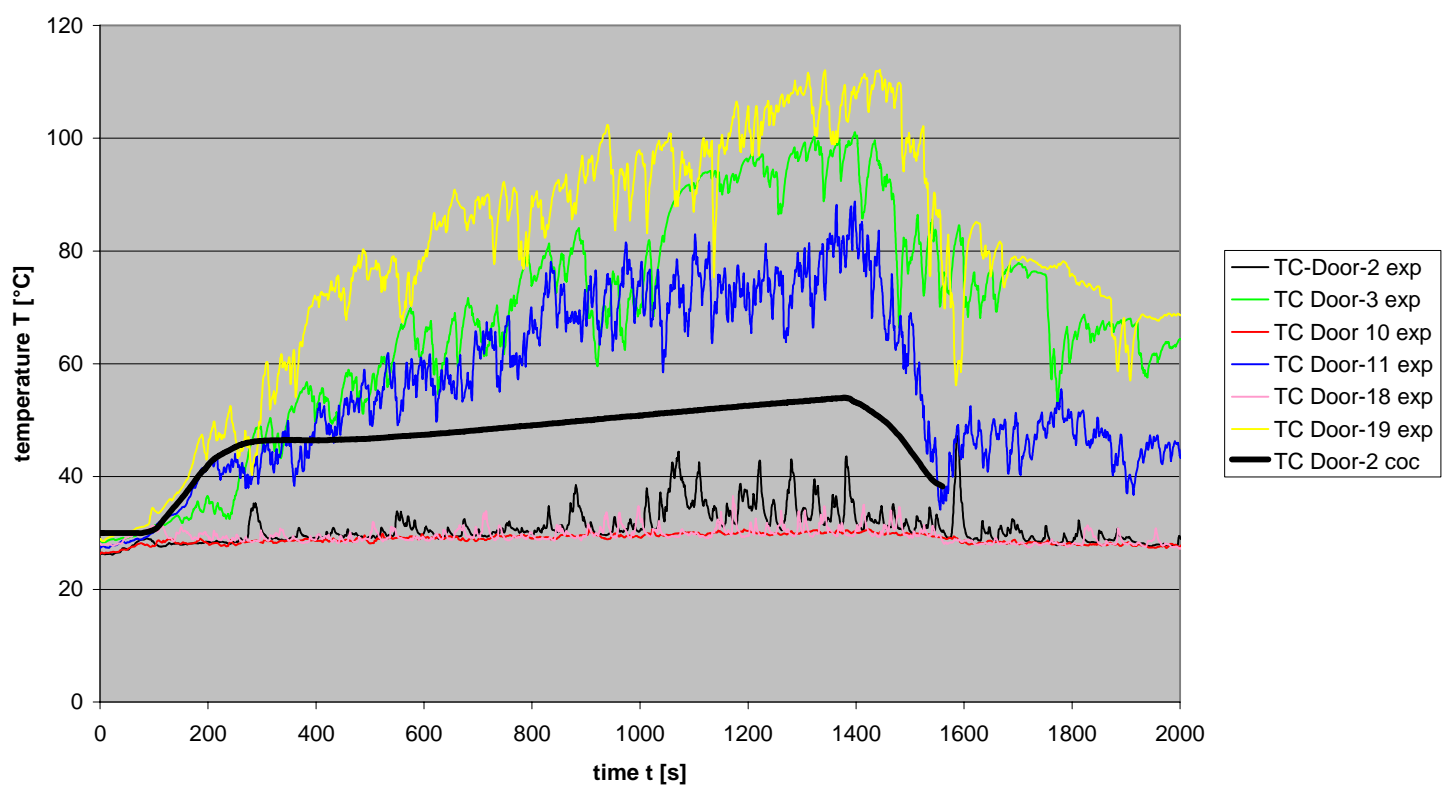

Fig. 5-55 Test 3: Temperature at door (level 2) 
Test 3 (blind) : Temperatures at door

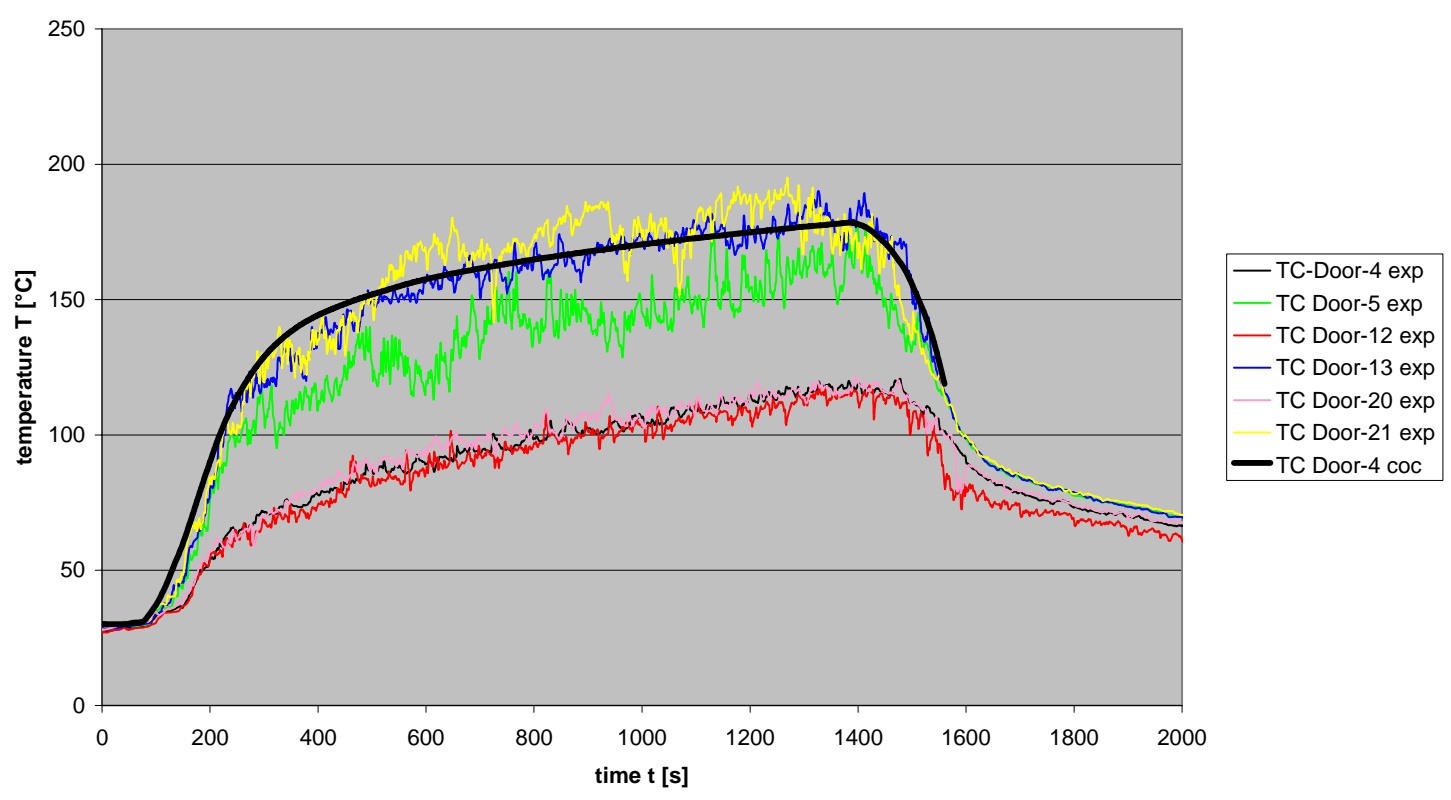

Fig. 5-56 Test 3: Temperature at door (level 3)

Test 3 (blind) : Temperatures at door

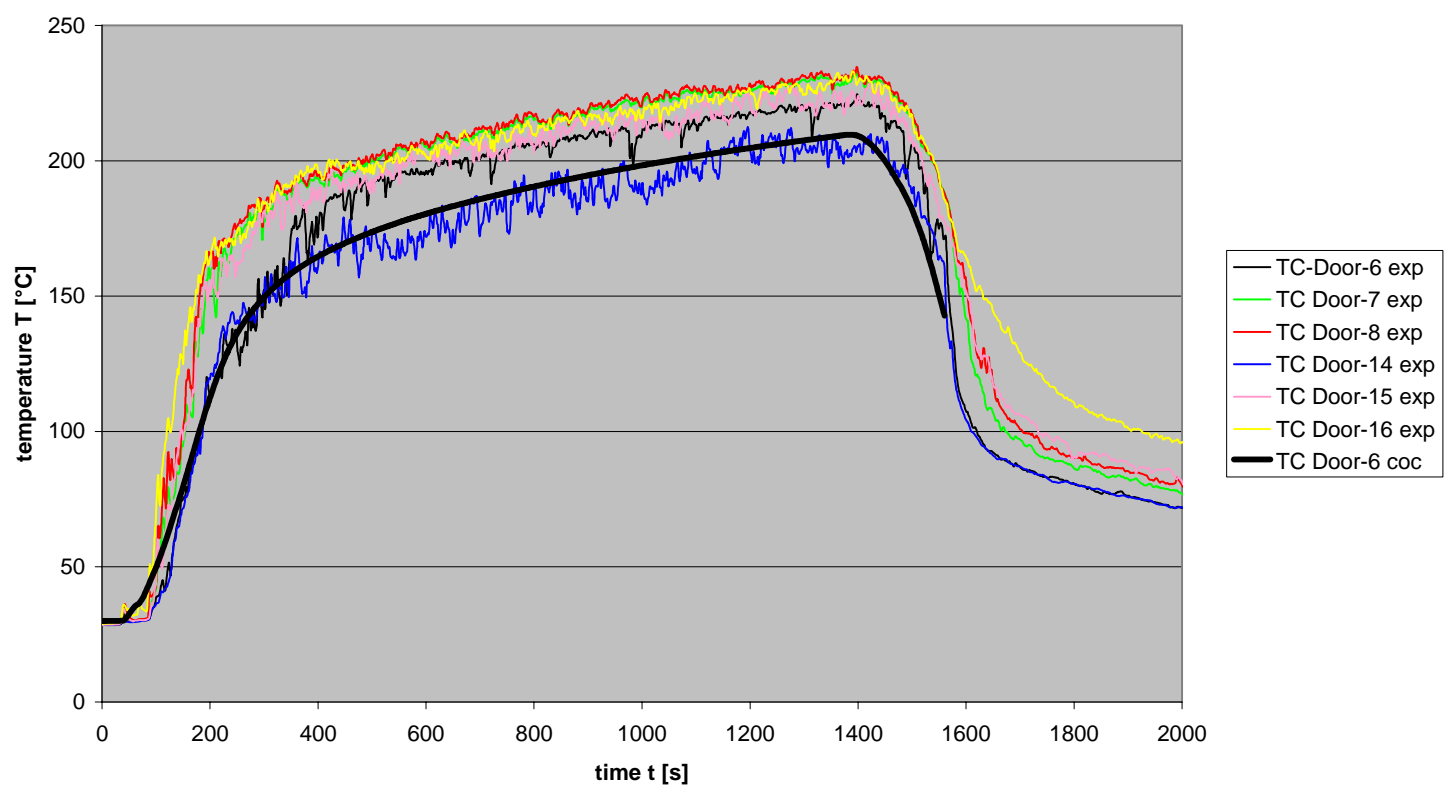

Fig. 5-57 Test 3: Temperature at door (level 4) 
Test 3 (blind) : Velocity at door

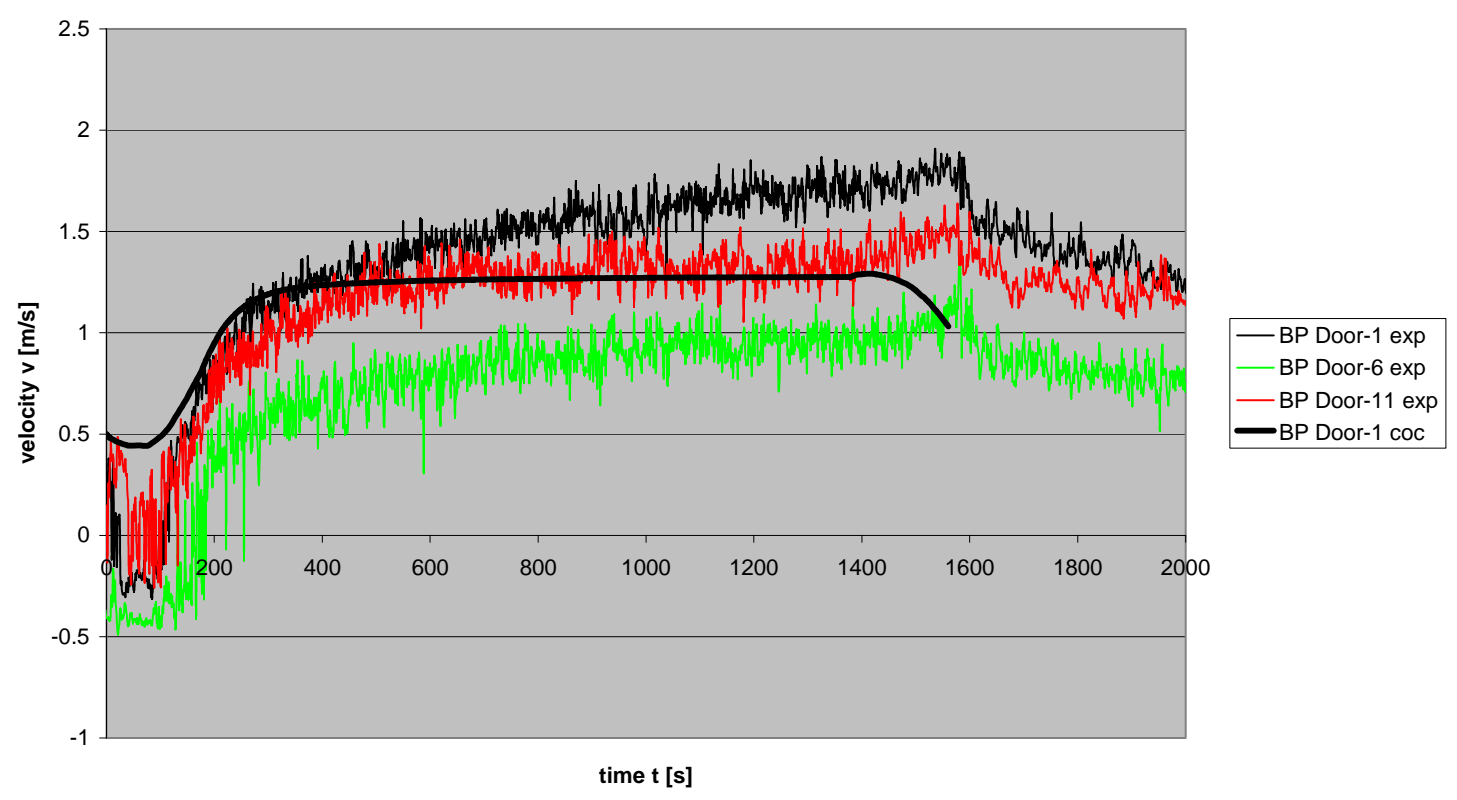

Fig. 5-58 Test 3: Velocity at door (level 1)

Test 3 (blind) : Velocity at door

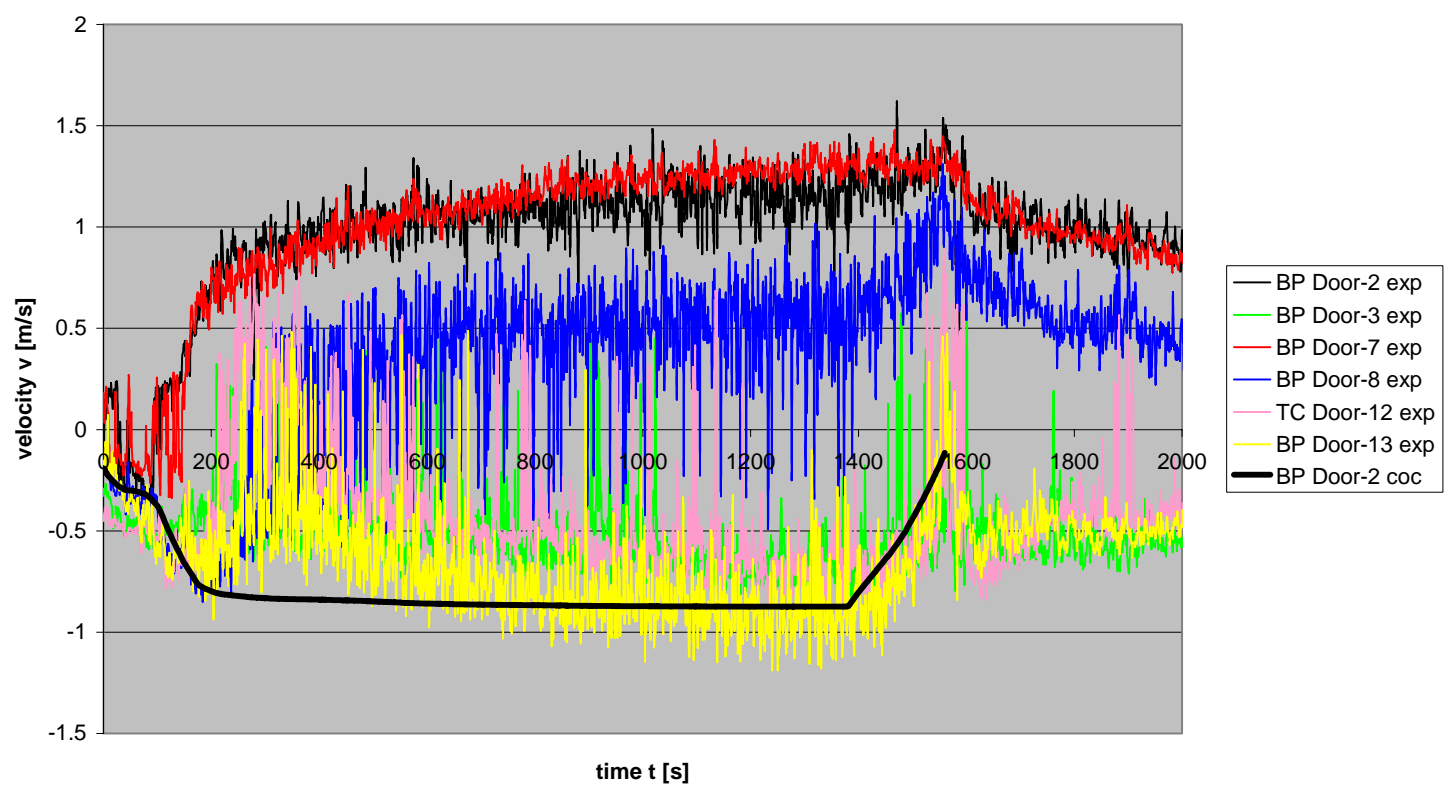

Fig. 5-59 Test 3: Velocity at door (level 2) 
Test 3 (blind) : Velocity at door

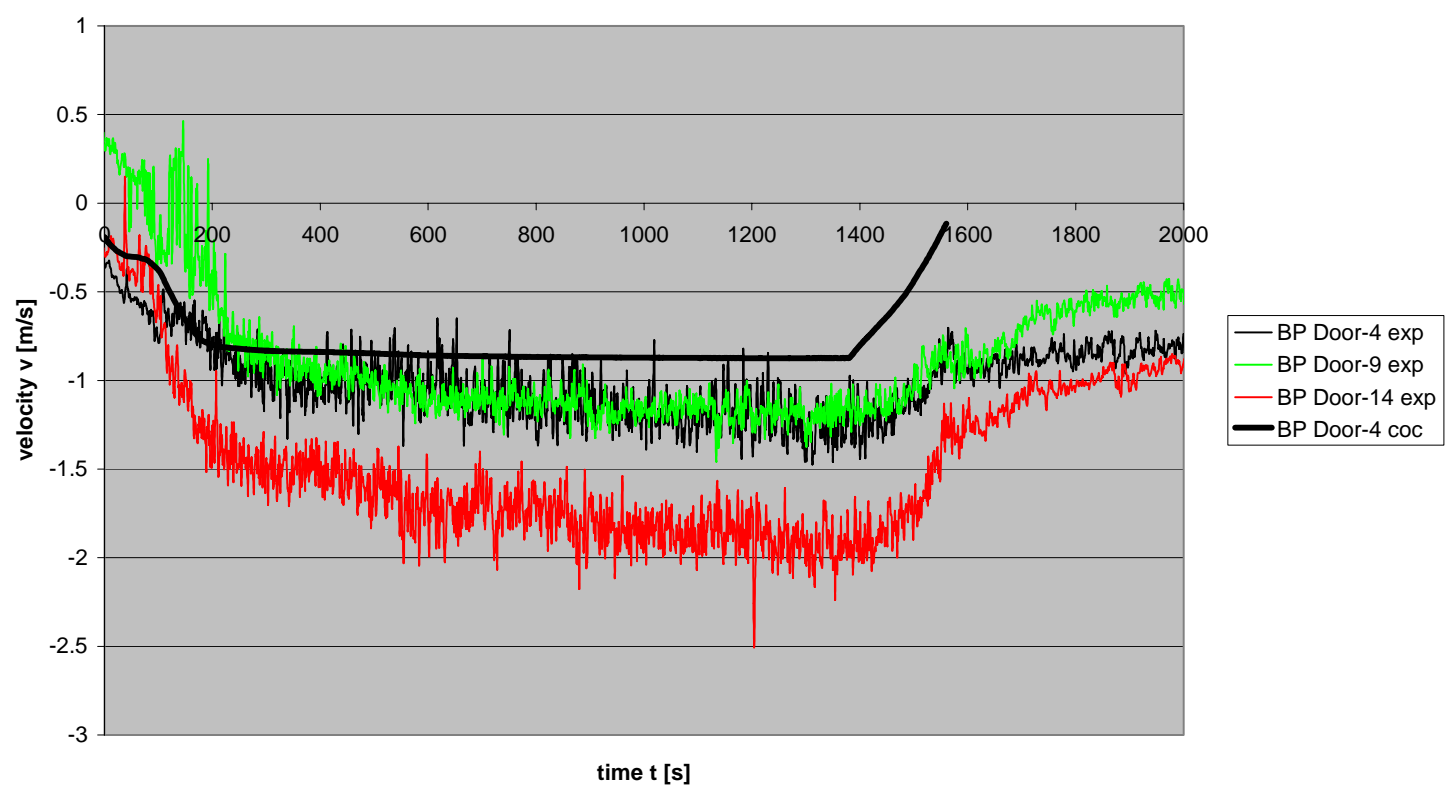

Fig. 5-60 Test 3: Velocity at door (level 3)

Test 3 (blind) : Velocity at door

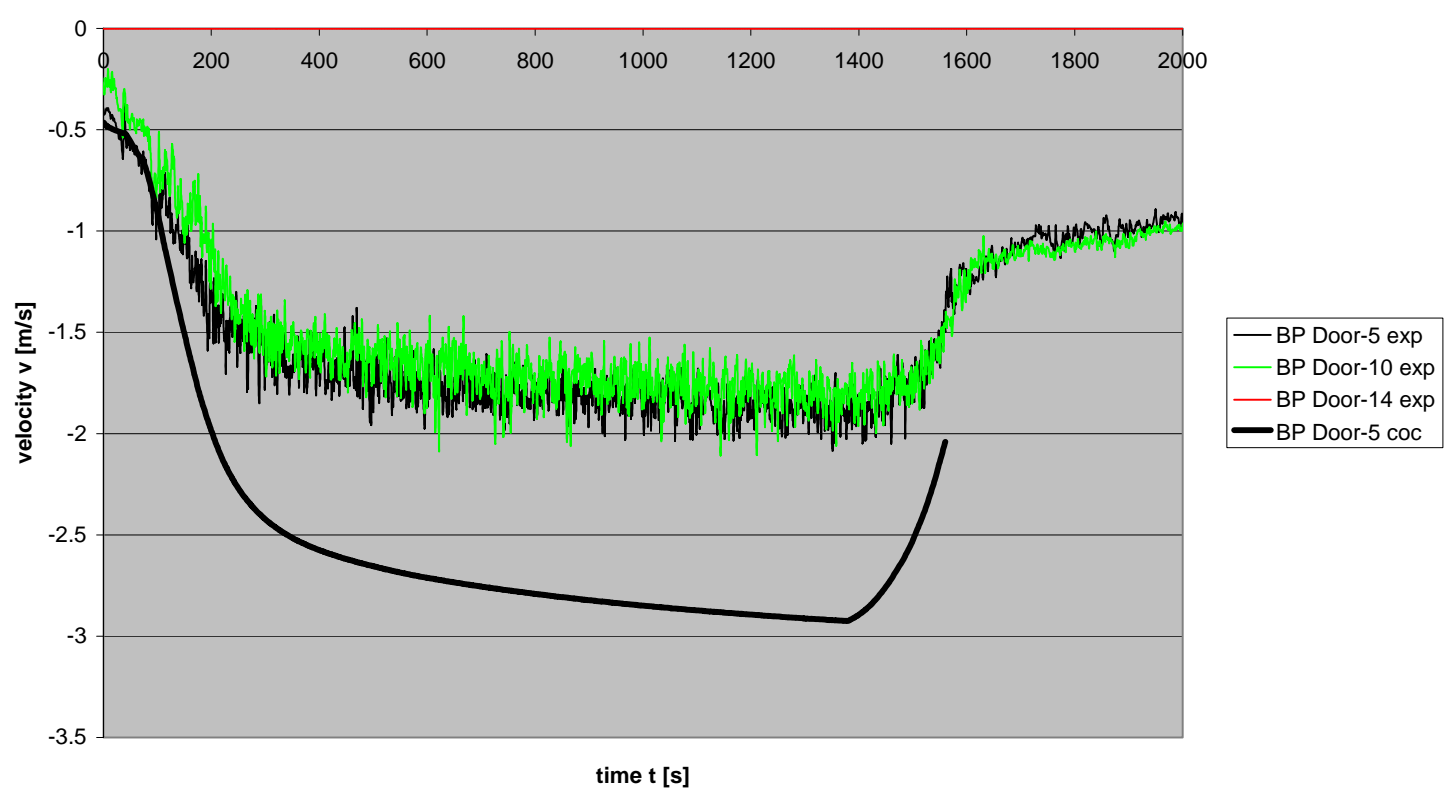

Fig. 5-61 Test 3: Velocity at door (level 4) 
Test 4 (blind) : Ventilation

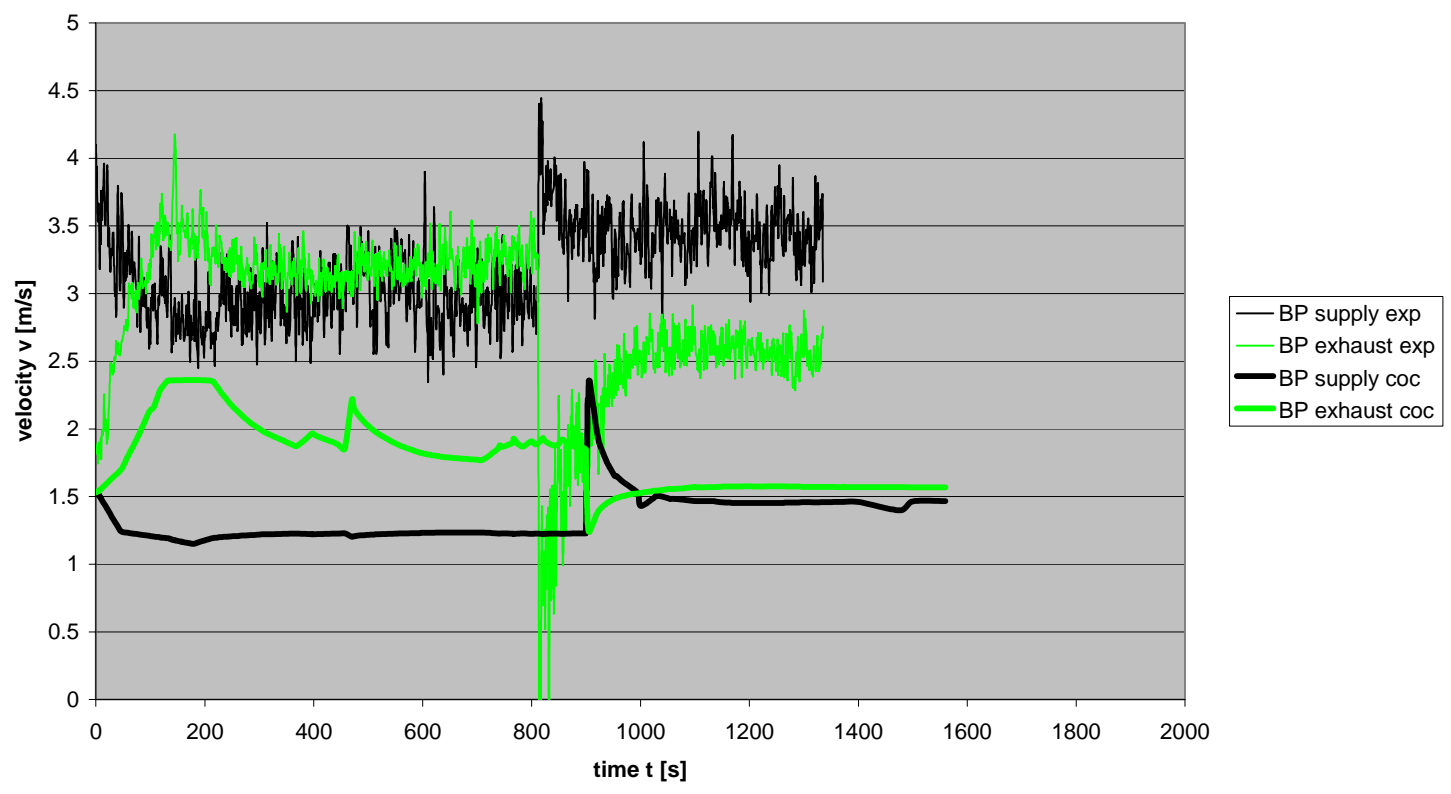

Fig. 5-62 Test 4: Velocity at ventilation 


\subsubsection{Open calculation of test 2, 3 and 4}

The calculations of tests 2, 3 and 4 were repeated after the release of the experimental data. As far as possible, the given experimental boundary conditions were used. In the following only some part of the results are discussed, especially the differences from the blind calculations.

Fig. 5-63 to Fig. 5-65 show the temperature results at tree 1 for the three tests. Compared to the blind calculation the results are now better. This is valid especially for the test 3 and for test 4 with the improved ventilation flow rate. Only in the lower positions the deviations are somewhat larger.

The quality of the results is very similar to that of the blind calculations. Larger changes could be observed for test 4 . For example the calculated temperatures on the floor area is now too low (Fig. 5-66). The increase of the radiation fraction could not compensate the increased volume exchange via the ventilation. Additionally the calculated oxygen concentrations are now too high in this case. In case of an automatic simulation of the stop of fuel supply, the fuel supply wouldn't have stopped. The calculated $\mathrm{CO}_{2}$ concentration has been underestimated. These results show that the heat release and oxygen consumption are somewhat inconsistent in this calculation. Further tests are needed for the estimation of volume flow through the ventilation.

Fig. 5-68 to Fig. 5-72 show some results for the tray temperatures. Due to the higher radiation fraction the calculated values are somewhat higher. This effect is stronger for temperature positions close to the fire. The deviations in test 4 close to the ventilation are still rather high (Fig. 5-72). This problem results from the missing momentum balance in the COCOSYS simulation, so that cold jets close to the ventilation could not directly be simulated. One possible solution is the distribution of the air supply volume flow into several control volumes around the system.

Fig. 5-73 shows the comparison of the improved ventilation flow in test 4 . The exhaust velocity follows now more or less the experimental data. Larger deviations exist still for the supply velocity. But the difference in the velocity at the beginning should be clarified first. 
Test 2 (open) :Temperature Tree 1

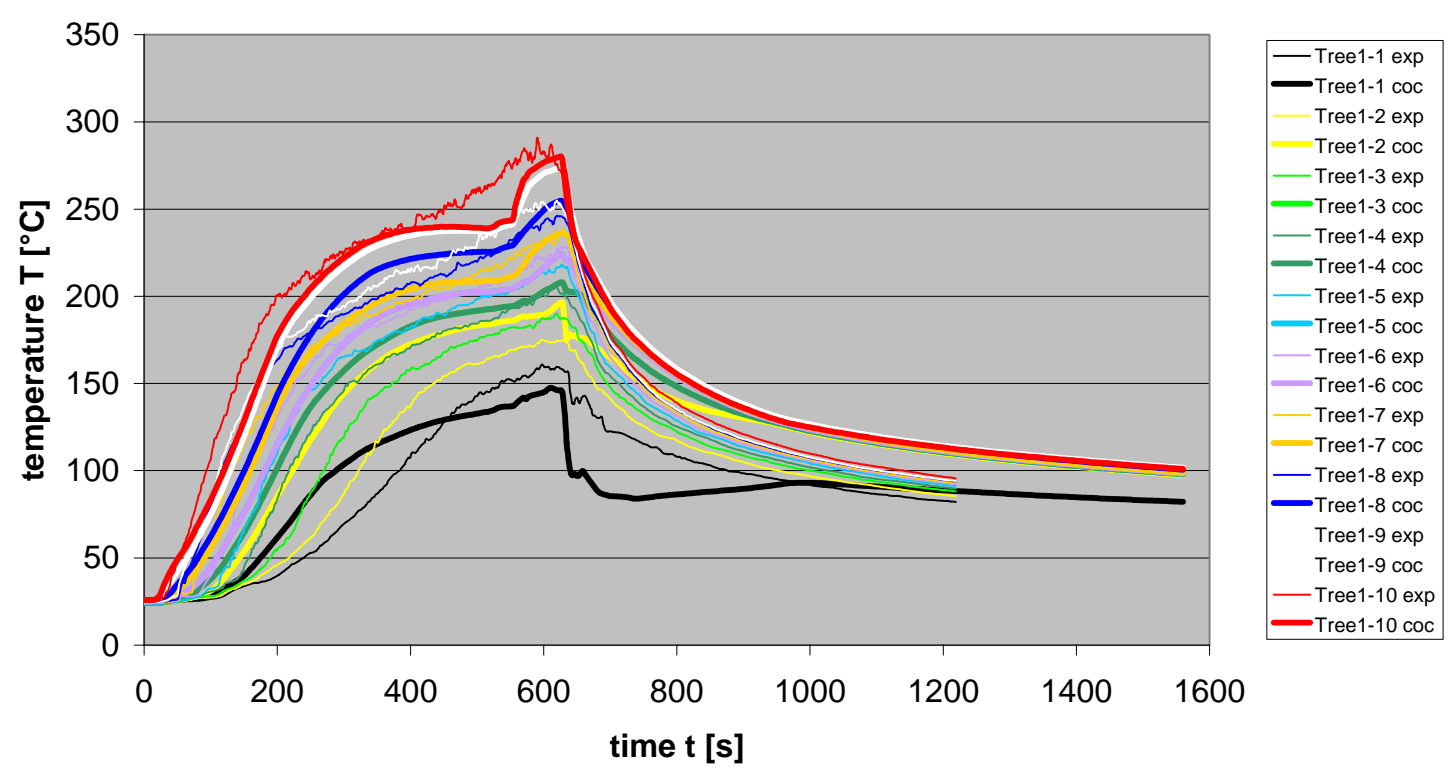

Fig. 5-63 Test 2 (open) : Temperatures at tree 1

Test 3 (open) :Temperature Tree 1

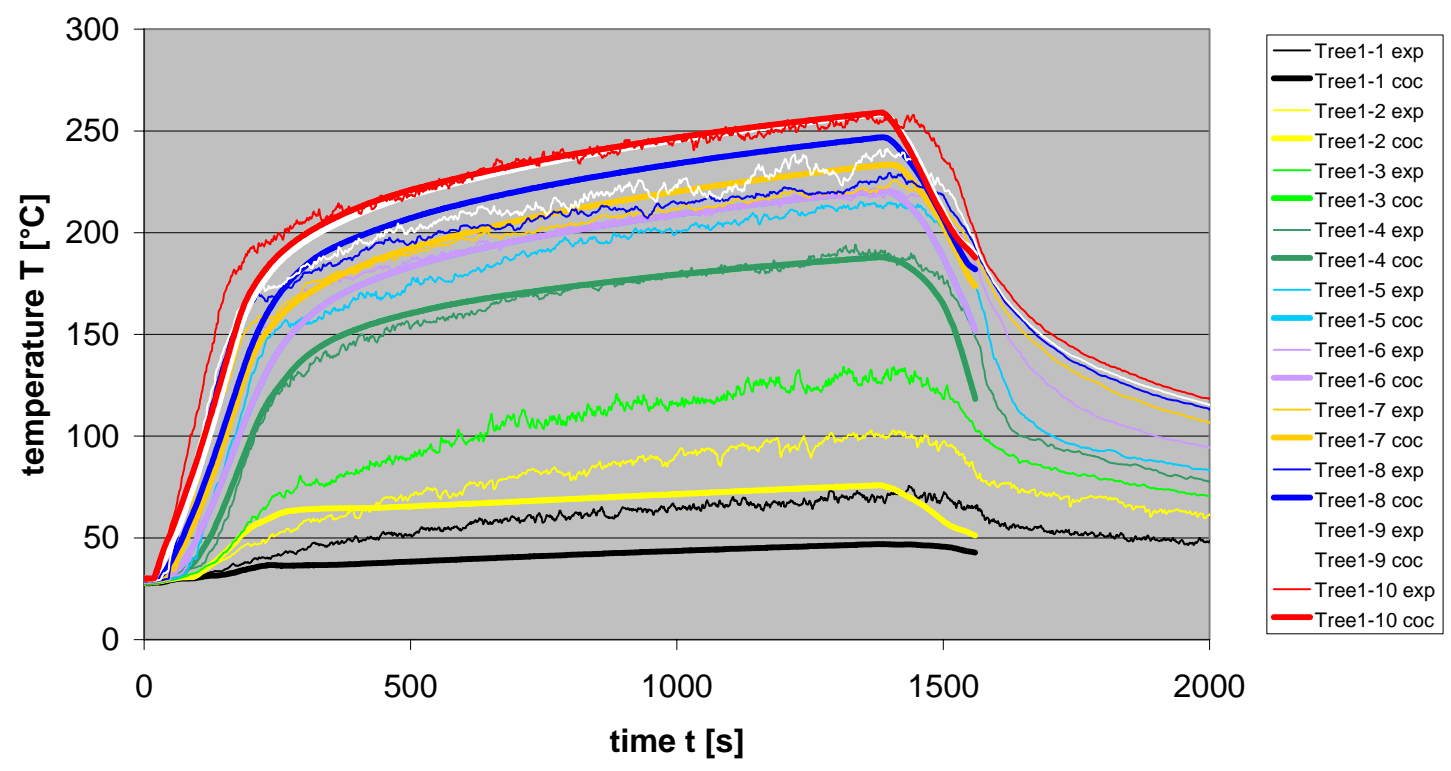

Fig. 5-64 Test 3 (open) : Temperatures at tree 1 
Test 4 (open) :Temperature Tree 1

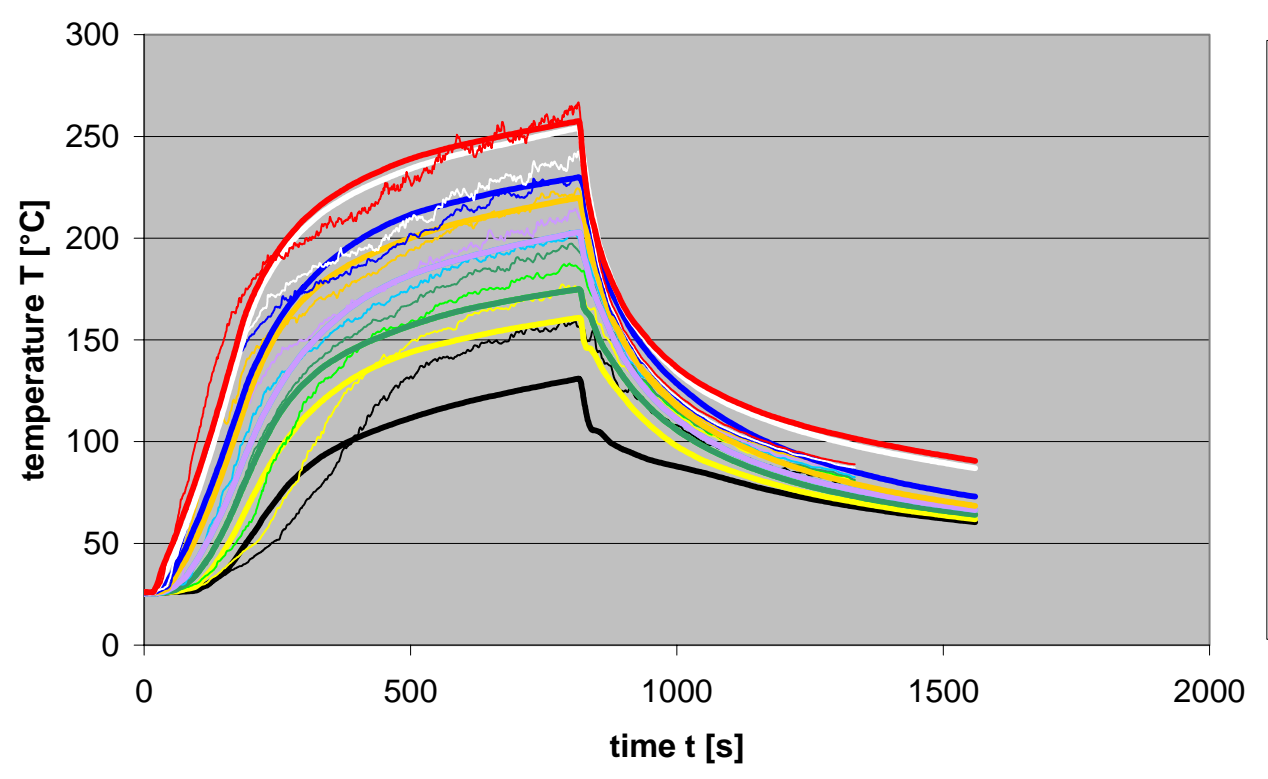

- Tree1-1 exp Tree1-1 coc Tree1-2 exp Tree1-2 coc -Tree1-3 exp Tree1-3 coc - Tree1-4 exp Tree1-4 coc - Tree1-5 exp -Tree1-5 coc - Tree1-6 exp Tree1-6 coc - Tree1-7 exp -Tree1-7 coc - Tree1-8 exp -Tree1-8 coc

Tree1-9 exp Tree1-9 coc - Tree1-10 exp -Tree1-10 coc

Fig. 5-65 Test 4 (open) : Temperatures at tree 1

Test 4 (open) : Inner Floor temperatures

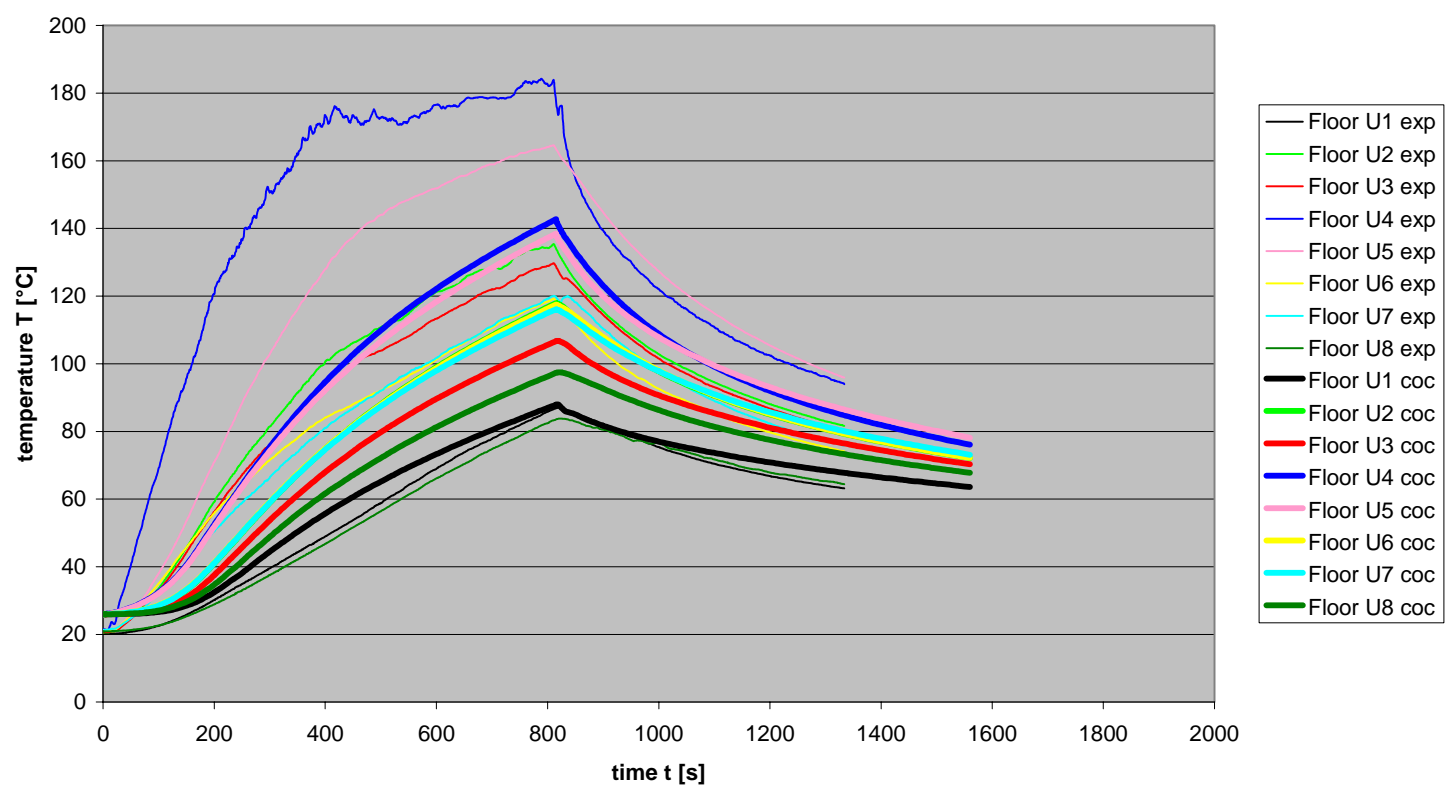

Fig. 5-66 Test 4 (open) : Temperatures at floor 
Test 4 (open) : Gas concentrations

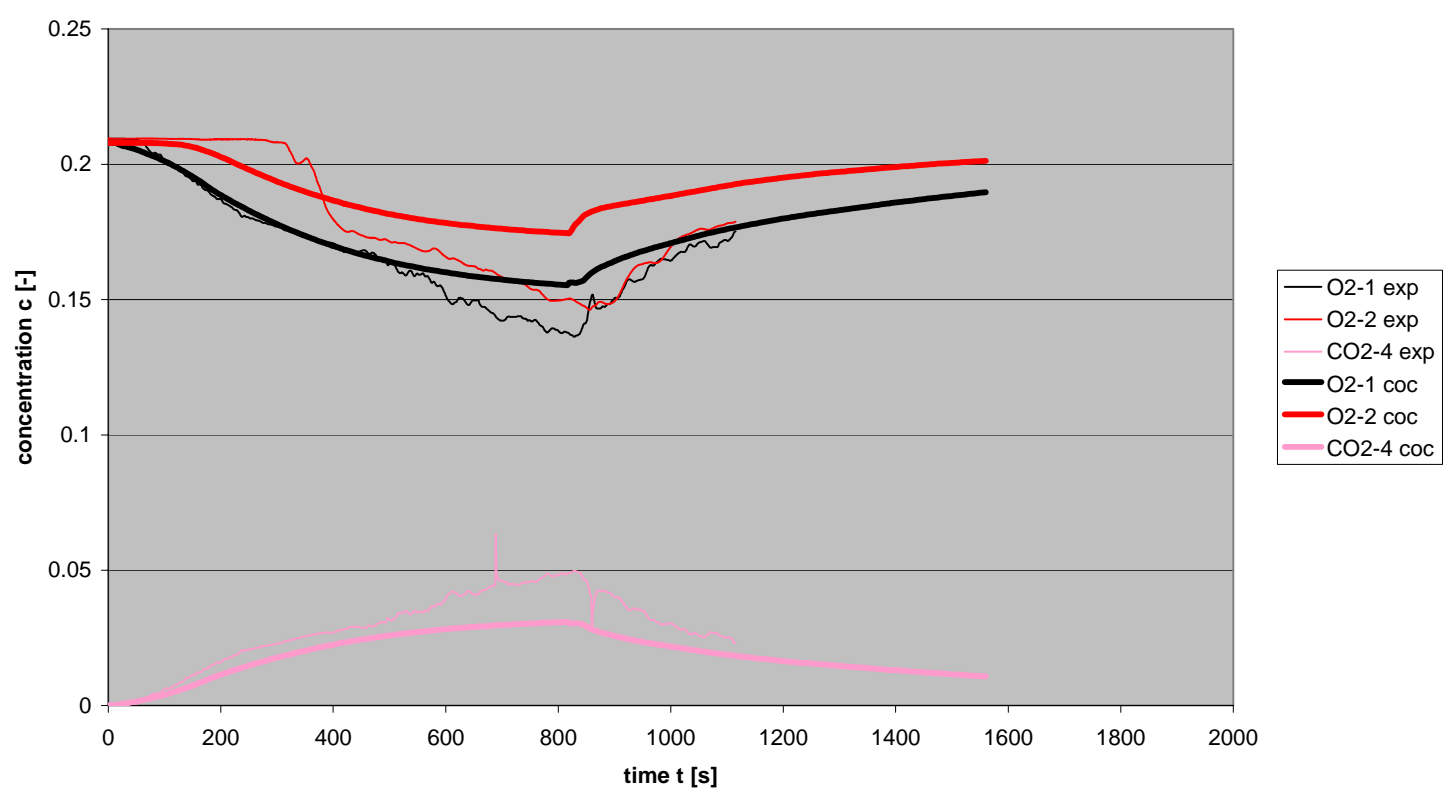

Fig. 5-67 Test 4 (open) : Gas concentrations

Test 2 (open) : Tray temperatures

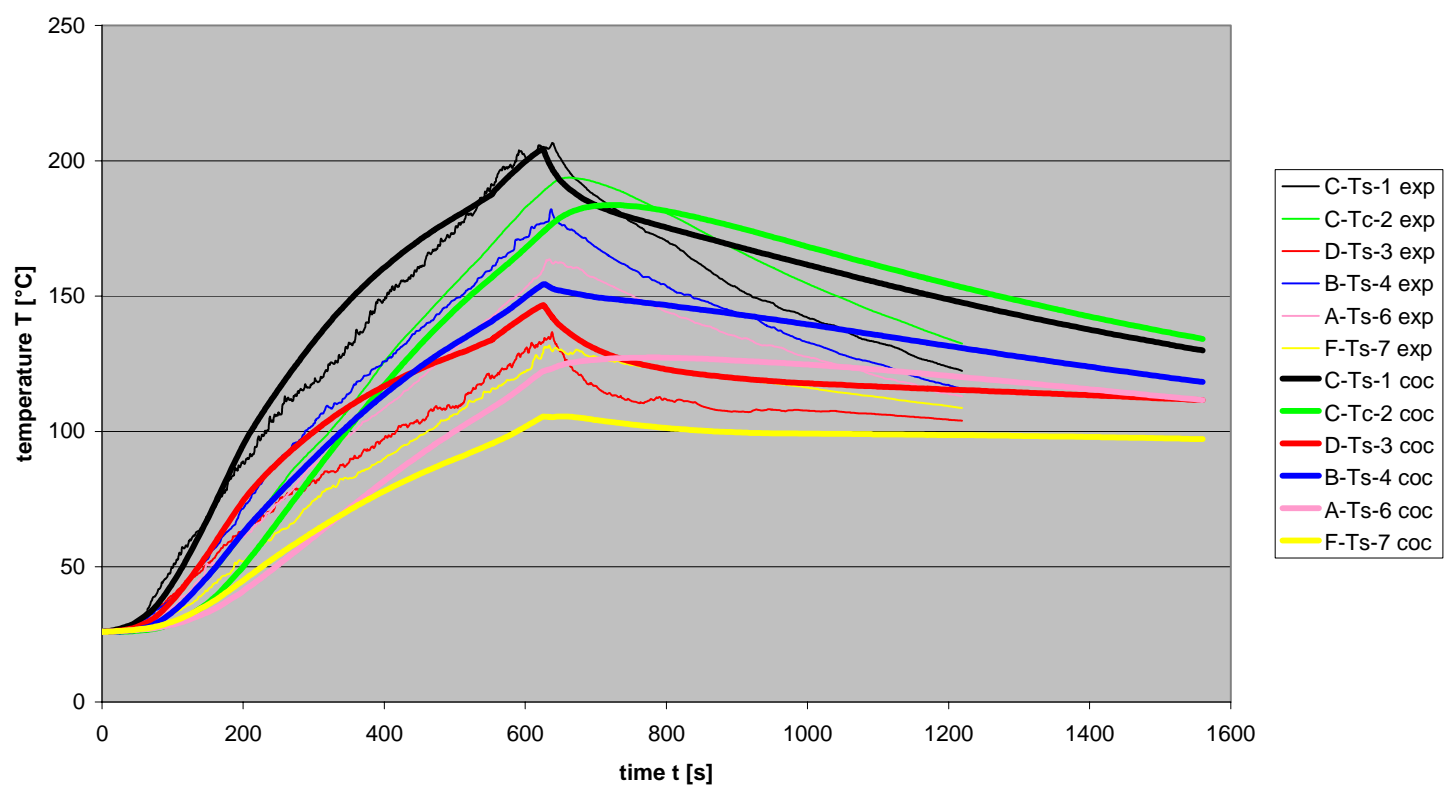

Fig. 5-68 Test 2 (open) : Tray temperatures at 1 to 7 
Test 2 (open) : Tray temperatures

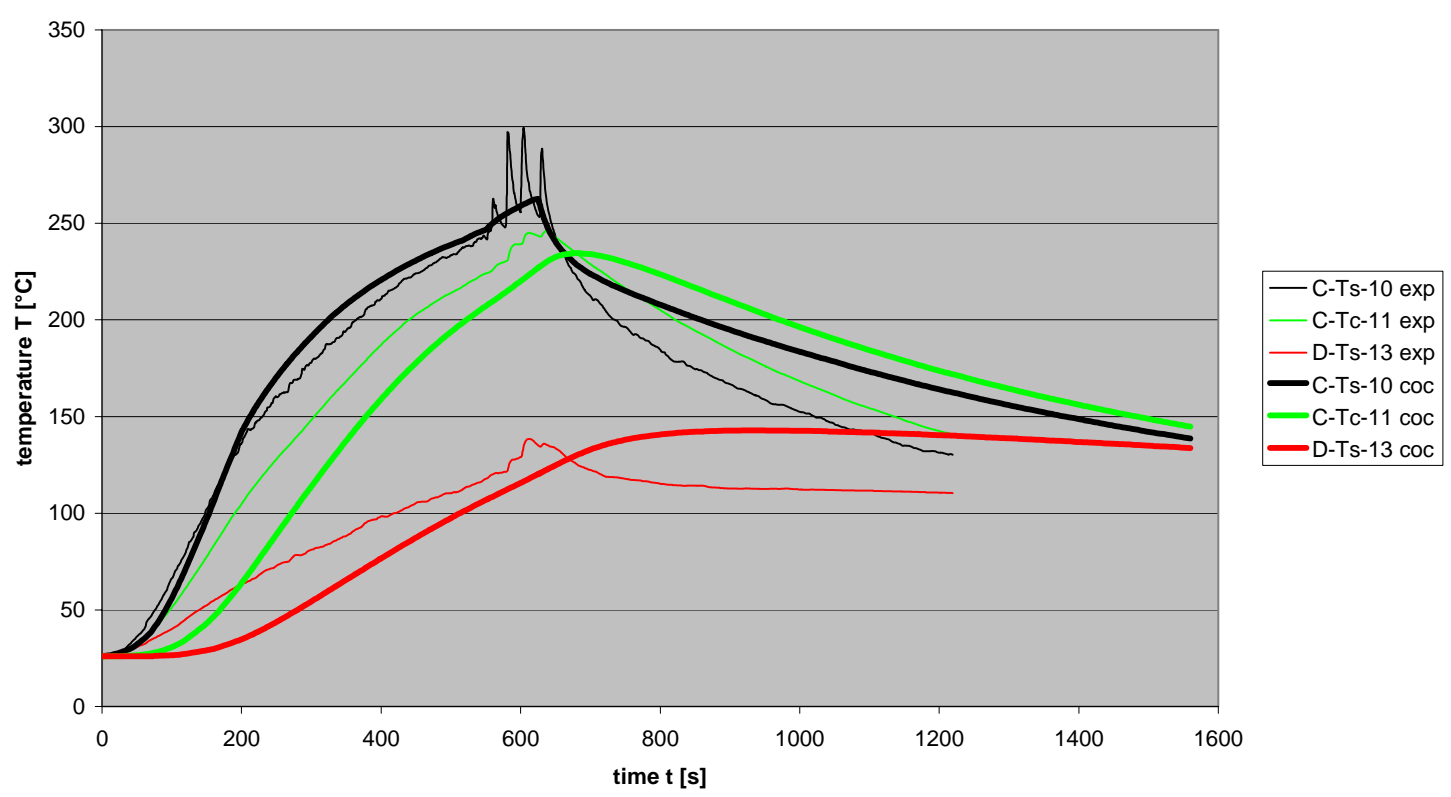

Fig. 5-69 Test 2 (open) : Tray temperatures at 10 to 13

Test 3 (open) : Tray temperatures

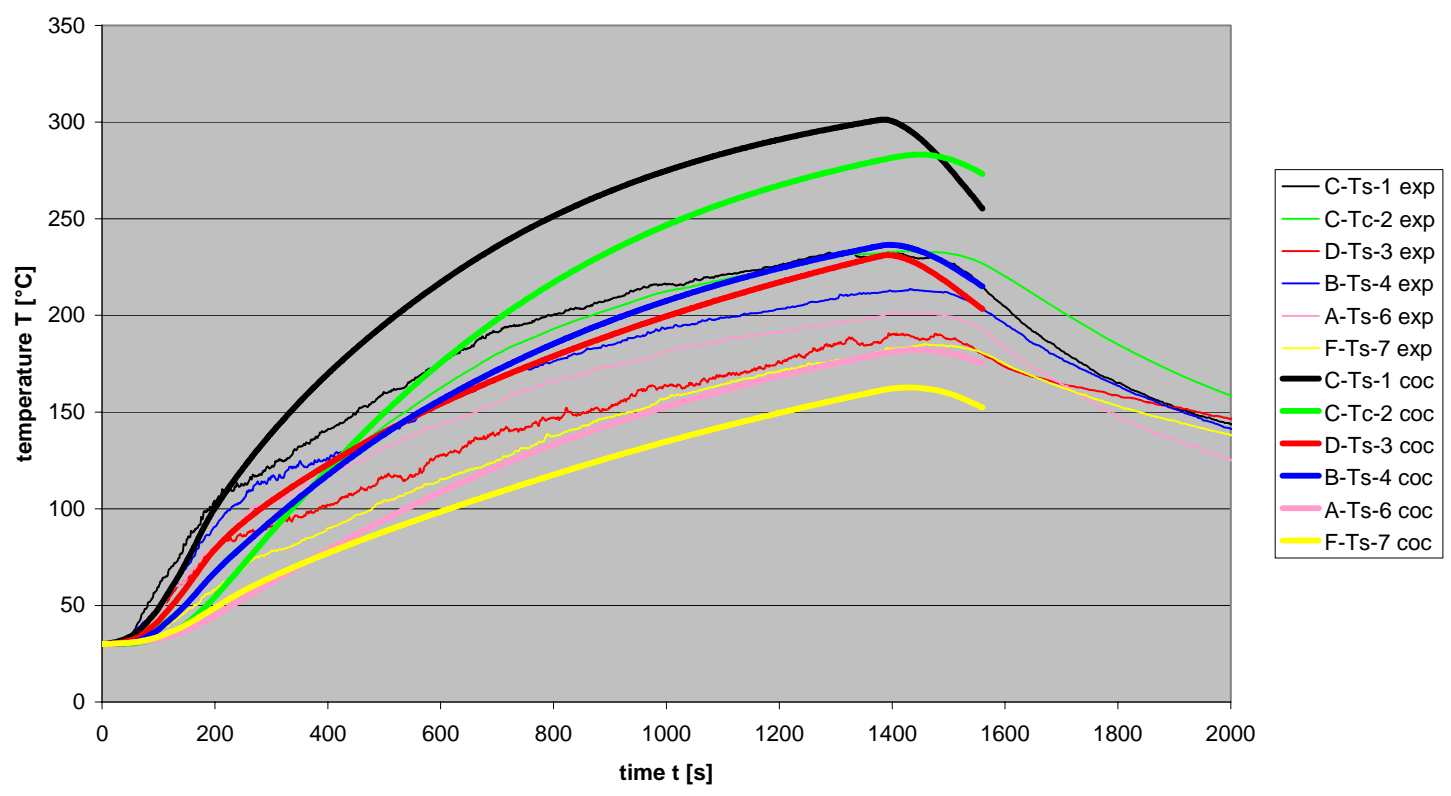

Fig. 5-70 Test 3 (open): Tray temperatures at 1 to 7 
Test 4 (open) : Tray temperatures

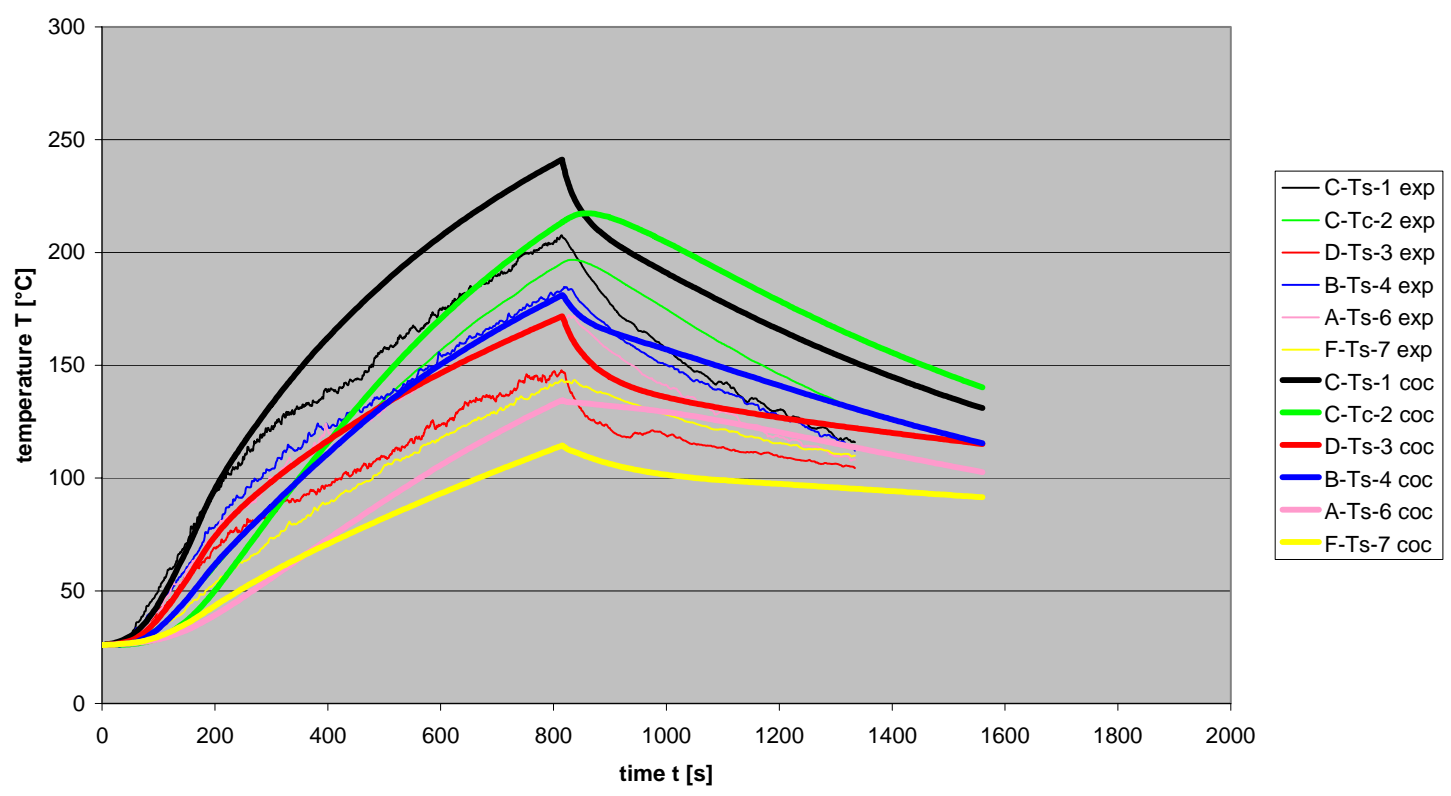

Fig. 5-71 Test 4 (open): Tray temperatures at 1 to 7

Test 4 (open) : Tray temperatures

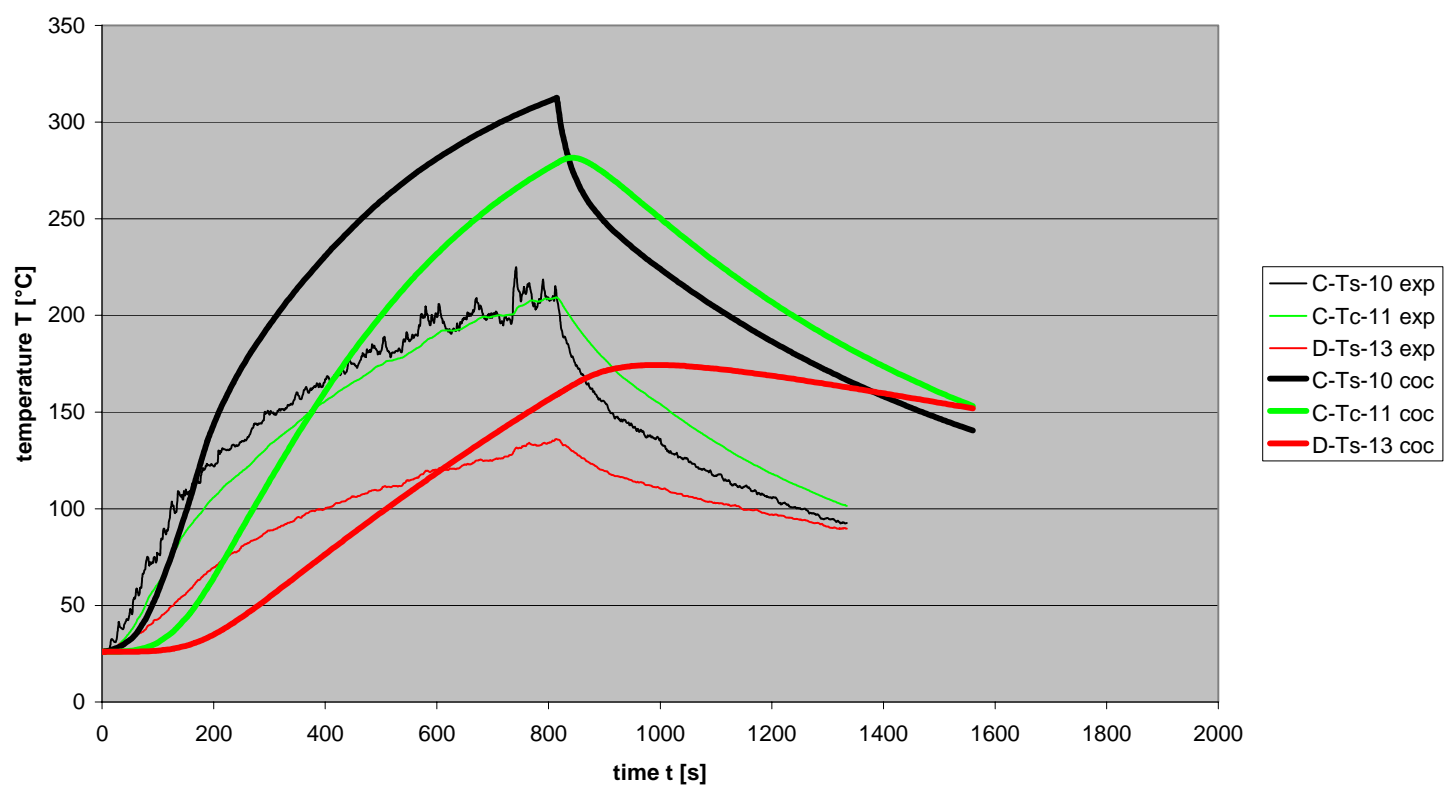

Fig. 5-72 Test 4 (open) : Tray temperatures 10 to 13 (close to ventilation) 
Test 4 (blind) : Ventilation

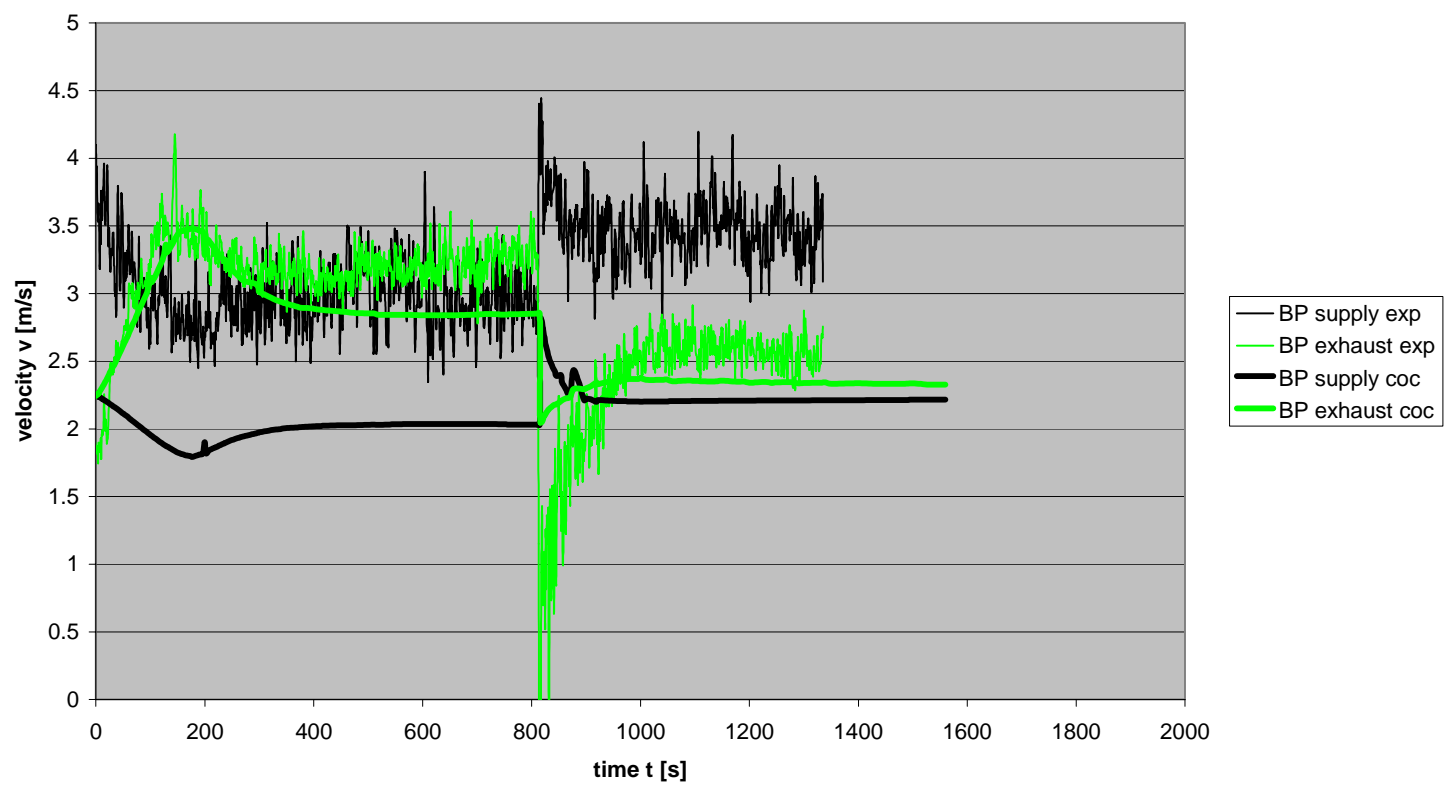

Fig. 5-73 Test 4 (open) : Velocity at ventilation openings 


\subsubsection{Further blind calculations}

In this section, the blind calculations of the remaining tests are discussed.

\section{Test 1}

The test 1 is similar to test 2 with a reduced heat release of about $0.35 \mathrm{MW}$. The calculated temperatures at tree 1 are quite similar to the experimental values (Fig. 5-74). Only in the lower regions the calculated temperatures are overestimated by about $40 \mathrm{~K}$ (position 2), which may be caused by numerical diffusion. The picture concerning to the temperature loads on the cable is quite similar to other tests (Fig. 5-75). The temperature load on control cables are overestimated by $20 \mathrm{~K}$. The calculated temperature on the power cable is quite good. But is has to be considered that the atmospheric temperature is about $20 \mathrm{~K}$ too high at this location. The simulation of oxygen and carbondioxid concentration is quite good. Only the lower oxygen concentration is underestimated by a few percent. This corresponds to the too high temperatures at lower positions (numerical diffusion).

\section{Test 5}

In test 5 a $1 \mathrm{MW}$ fire was used with open doors and running ventilation system. In this simulation the upper temperatures at tree 1 are overestimated by about $30 \mathrm{~K}$. The lower temperatures are calculated quite well (Fig. 5-77). Due to the open door these temperatures are lower than the temperatures far inside the fire compartment. Therefore the maximum calculated temperature load on the cables (like position 1 to 7 ) is larger and reaches values up to $350{ }^{\circ} \mathrm{C}$ (at position 10). Concerning the temperature load especially close the ventilation system the deviations to the experiment are rather large and reaches about $150 \mathrm{~K}$ for the single control cable (position 10) and about $100 \mathrm{~K}$ for the cable tray $\mathrm{D}$ (position 13). These deviations close to the ventilation system could be observed in the comparison of the temperature profile also (Fig. 5-78). Especially the temperatures at the upper positions of tree 3, 4 and 5 are overestimated by COCOSYS. The experimental values at tree 6 are rather high, especially at about $1 \mathrm{~m}$. This indicates a defection of the flame to the east side. In COCOSYS the plume is not really modeled. Due to the missing momentum balance a possible deflection could not be calculated also. The gas concentrations are simulated quite well (Fig. 5-81). Some results concerning velocity at door are shown in Fig. 5-82 and Fig. 5-83. In the lowest level the calculated results are quite similar to the experiment. One level higher 
the counter current effects occur in the range of $50 \mathrm{~cm}$. This indicates a sharp layer separation in the atmospheric flow.

\section{Test 7}

Test 7 is a replicate test to test 1 . Only the cable material has been changed from XPE to PVC. Therefore the calculated results are similar to test 1 . This is valid for the experimental data too, show the reproducibility of the tests. In the experiment the target temperatures are slightly higher for PVC material (Fig. 5-85). This could not be reproduced in the COCOSYS calculation. Here the results for XPE are higher compared to PVC. The reason may be the used thickness of the material layers, because all other input parameters are exactly the same. Here further clarification is necessary. Further the difference between calculated surface and center temperature is much larger compared to the experiment. The delivered results for the center temperature belongs to the real center of the cable. But the measured location is below the insulation jacket.

\section{Test 10}

Test 10 is a replicate test to test 4 . In this blind calculation the fuel stop is estimated by the calculated oxygen concentration. Unfortunately the calculated values are somewhat to high, so that the fuel stop is calculated about $600 \mathrm{~s}$ too late. Therefore the temperature at tree 1 and gas concentrations are presented only.

\section{Test 13}

Test 13 has been performed with closed fire compartment and a heat release rate of about 2 MW. Fig. 5-88 shows the comparison of temperatures at tree 1 . The deviations are about 50 $\mathrm{K}$ with a slight over-estimation in the upper part and an under-estimation in the lower part. The temperature decrease after the fuel stop is simulated too slowly. This indicates that the heat loss is higher than assumed in the COCOSYS calculation. Fig. 5-89 presents the temperature profile at $300 \mathrm{~s}$. As before the measured temperatures at tree 6 are much higher than those calculated by COCOSYS. These temperatures may be influenced by the spray fire directly. Otherwise the temperature profile is calculated quite well, with some larger deviations for tree 3 (also relatively close to the fire) and some larger deviations in the upper part. The temperature load is simulated quite well, with some underestimation of the maximum temperatures. Fig. 5-90 presents the tray temperatures at position 1 to 7 . The temperature increase of the surface temperature of the control cable $C$ (highest position) is simulated quite well. Corresponding to the atmospheric temperatures the temperature de- 
crease is underestimated. The temperature increase of the control cable $A$ and $B$ is underestimated, although the atmospheric temperature is simulated quite well at these locations. As in other cases the surface temperature of the power cable is underestimated. Fig. 5-91 and Fig. 5-92 present the comparison for the west wall and the ceiling temperatures. The deviations for the west wall are rather high, especially for the upper positions $U 2$ and $U 4$. Here the experimental values are not correct, because these have lower temperatures compared to the lower measurement positions. The ceiling temperatures are underestimated by about $100 \mathrm{~K}$ for the locations close to the fire plume. Here the measured values at position U1 and U8 are about $60{ }^{\circ} \mathrm{C}$ and much lower compared to test 2 with a lower heat release rate. The gas concentrations are simulated quite well (Fig. 5-93).

\section{Test 16}

The test 16 can be compared to test 13 with an additional active ventilation. The volume flow rate is quite important. This may be the explanation for the deviations in the gas concentrations (Fig. 5-95) which are higher compared to test 13. The deviations lead to a delay in the fuel supply stop. Up to the time point of the fuel stop in the experiment the temperature results are quite well (e. g. tree 1 in Fig. 5-94). The velocities at the air supply and exhaust system show a similar behavior as in the other tests. Here some additional clarification is needed, especially again for the initial values at $t=0 \mathrm{~s}$. The results for the junction box are bad as in all cases. Fig. 5-99 shows the comparison for the vertical tray temperatures. These are somewhat underestimated in the COCOSYS calculation, although the south wall temperatures are quite similar to the experiment. Therefore it may be necessary to improve the set up of cable tray materials (size and thickness) in the COCOSYS input data.

\section{Test 17}

Test 17 is the only test using toluene instead of heptane. Due to the high soot production the fuel supply was stopped earlier, before the threshold value of $15 \%$ oxygen was reached. This explains the large deviations between experiment and calculation. Up to the time point of fuel supply stop the increase of atmospheric temperature is simulated quite well. Only the ceiling temperatures are underestimated.

\section{Test 18}

In this test a $1 \mathrm{MW}$ was used at a different location and with open door. The measured atmospheric temperature increase is quite similar to test 3 . In COCOSYS the results are somewhat lower (Fig. 5-103). It seems that the nodalization around the fire plume is not 
good enough in this case. This shows that the nodalization may have an influence on the temperature development in the fire compartment. This effect has to be considered in the evaluation of the calculated results. For the results of ceiling temperatures it is interesting to compare the positions of U4 (close to fire) and U5. In COCOSYS the value at U4 is quite good, but the value at U5 is underestimated by about $50 \mathrm{~K}$. Larger deviations (also compared to test 3 ) could be observed for position U6. The higher value could be explained by the 'long' control volumes leading to a stronger heat distribution to the side. Surprisingly the experimental values for $\mathrm{U} 2$ and $\mathrm{U} 6$ are quite different. It looks that the experimental value at U6 is too low. The fire source is now much closer to the south wall leading to an increased wall temperature there (position U4). This has not been reproduced in the COCOSYS calculation so far. Here the differences are about $110 \mathrm{~K}$. This seems to be the results of the missing plume model in COCOSYS. The vertical tray temperatures and the gas concentration is simulated quite good and the quality is similar to that of test 3 calculation. 
Test 1 - Temperature Tree 1

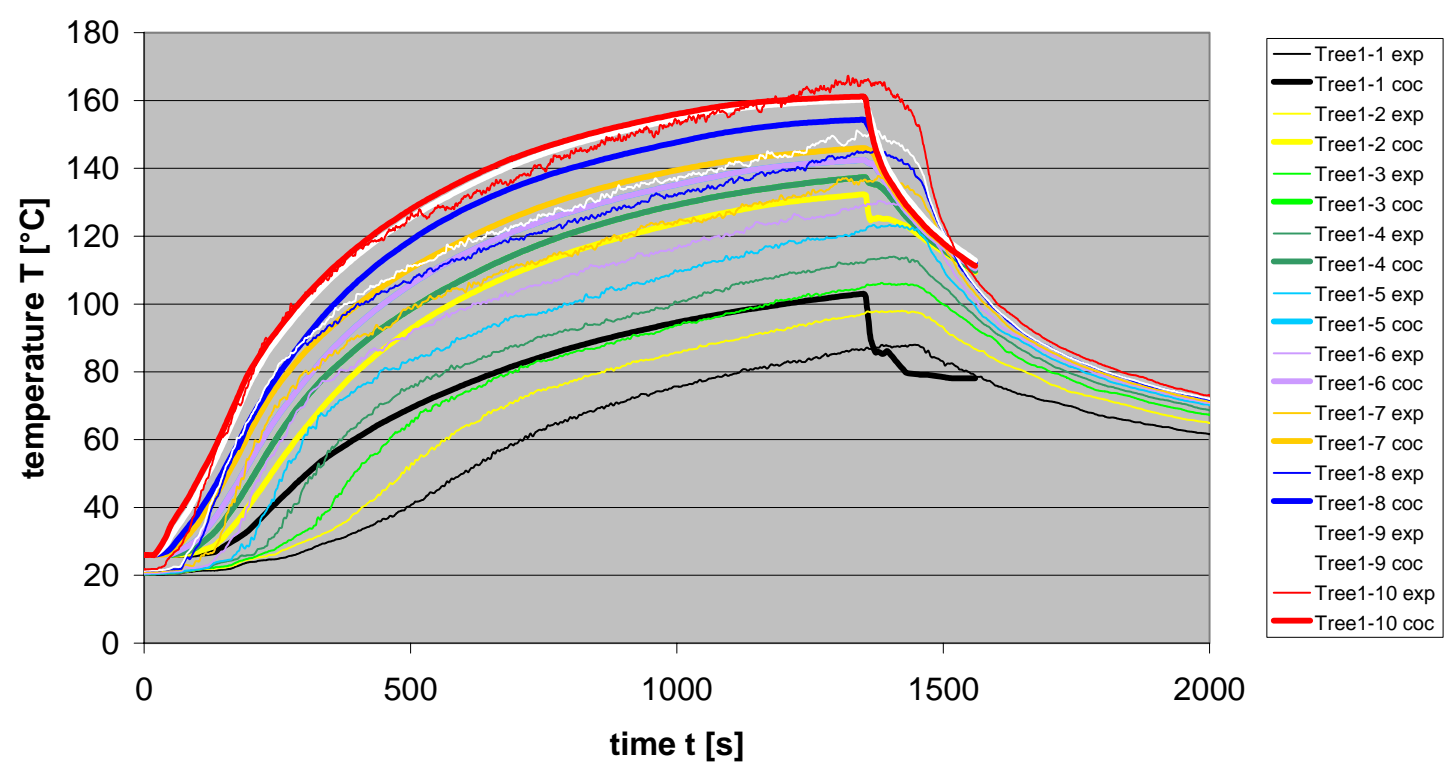

Fig. 5-74 Test 1 : Temperatures at tree 1

Test 1 (blind) : Tray temperatures

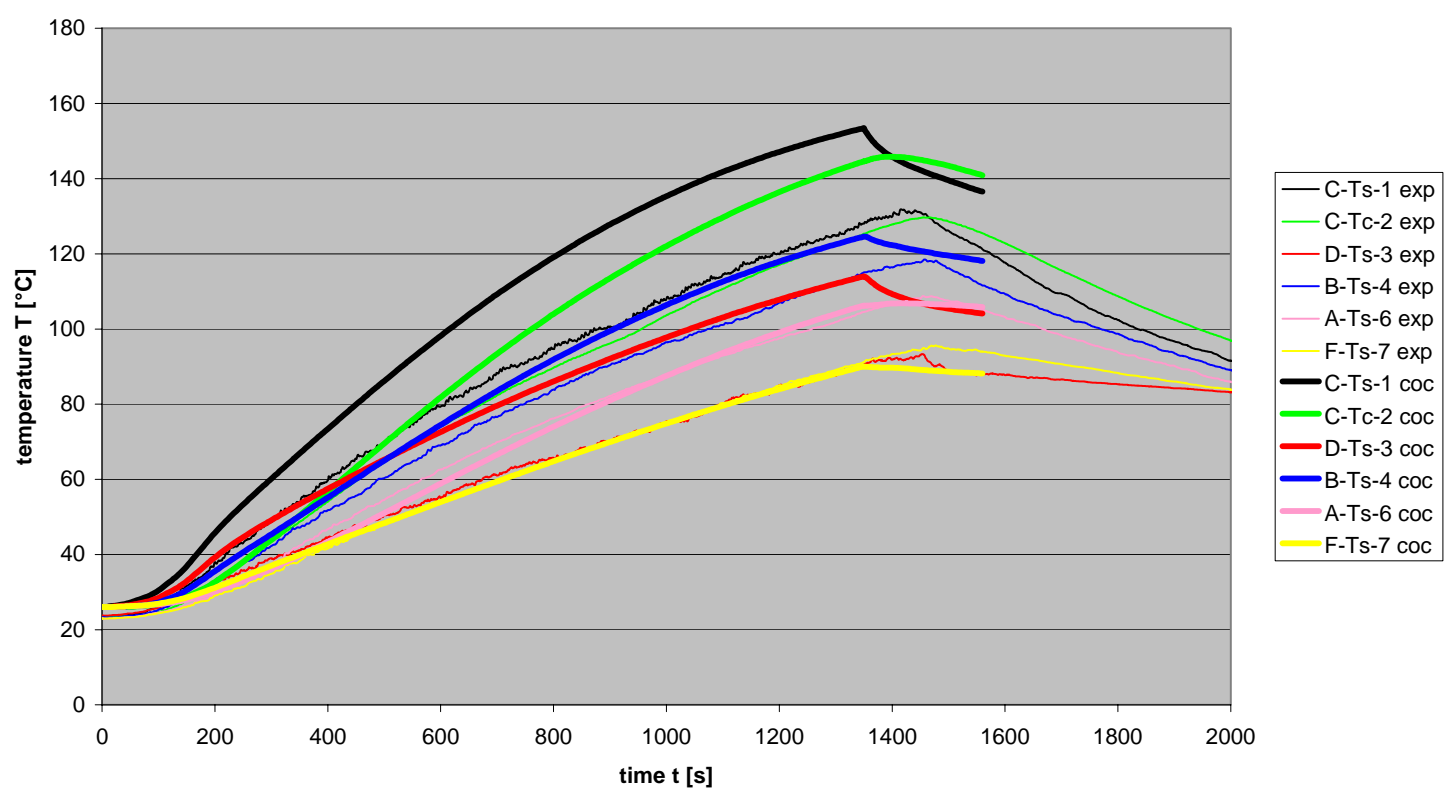

Fig. 5-75 Test 1: Tray temperatures at 1 to 7 
Test 1 (blind): Concentrations

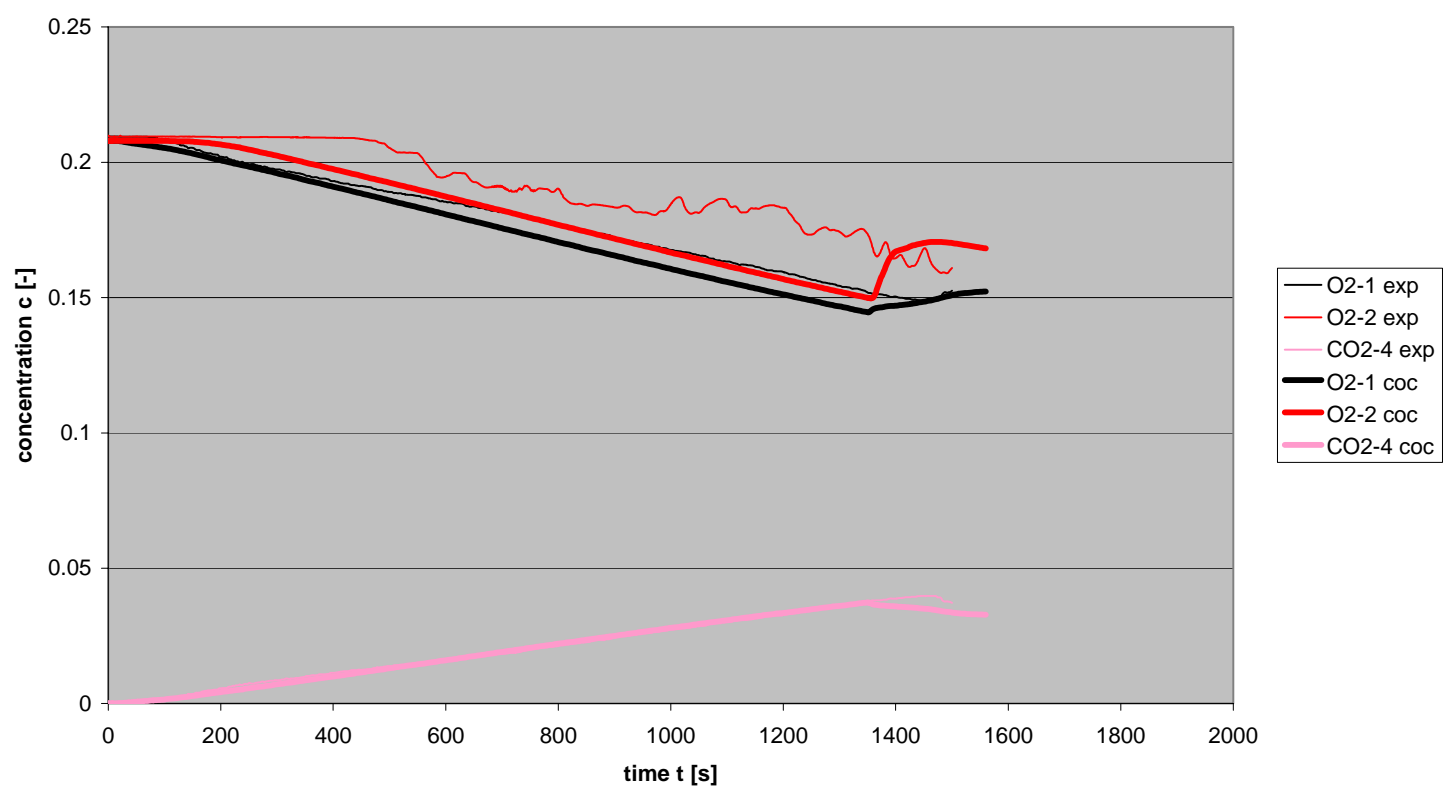

Fig. 5-76 Test 1: Gas concentrations

Test 5 (blind) :Temperature Tree 1

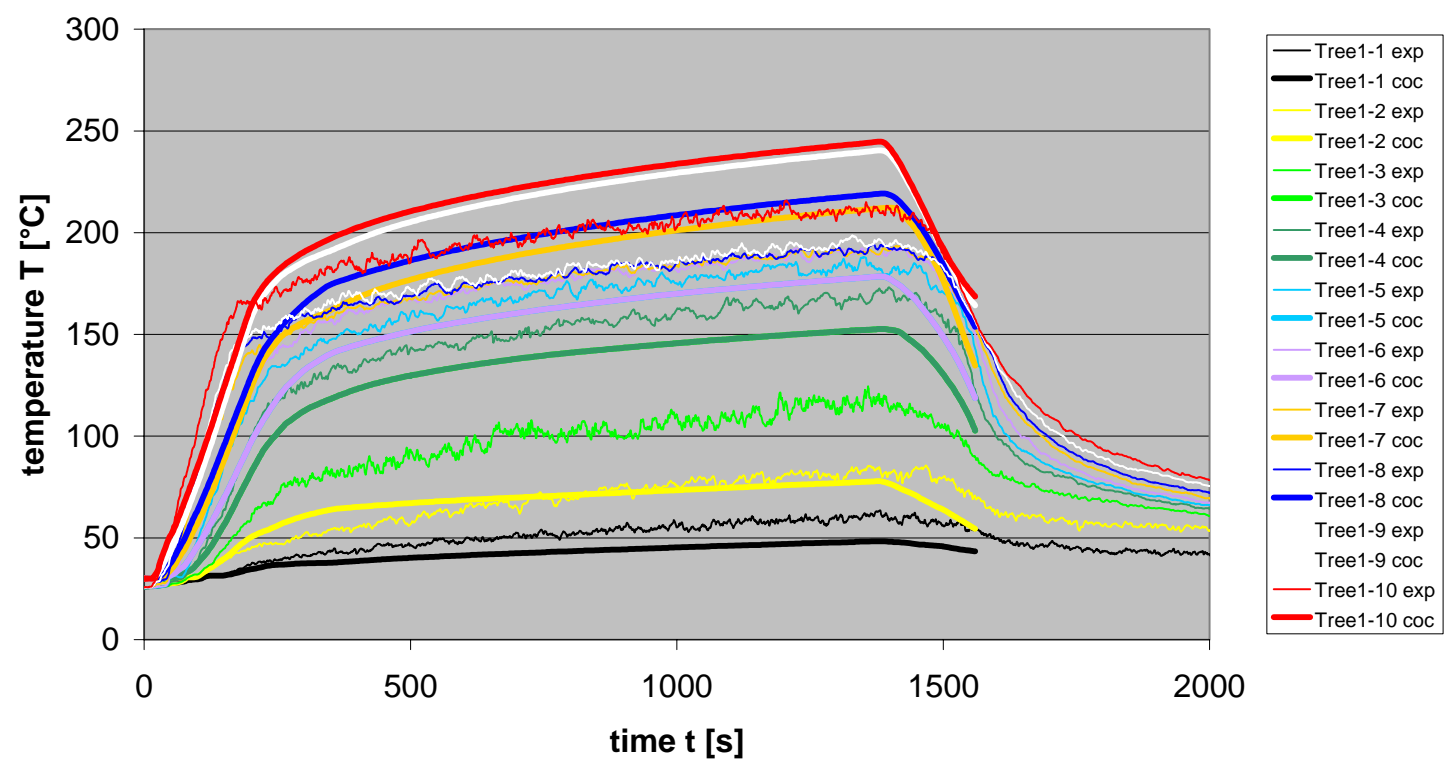

Fig. 5-77 Test 5: Temperatures at tree 1 
Test 5 (blind) : Tree temperature profiles

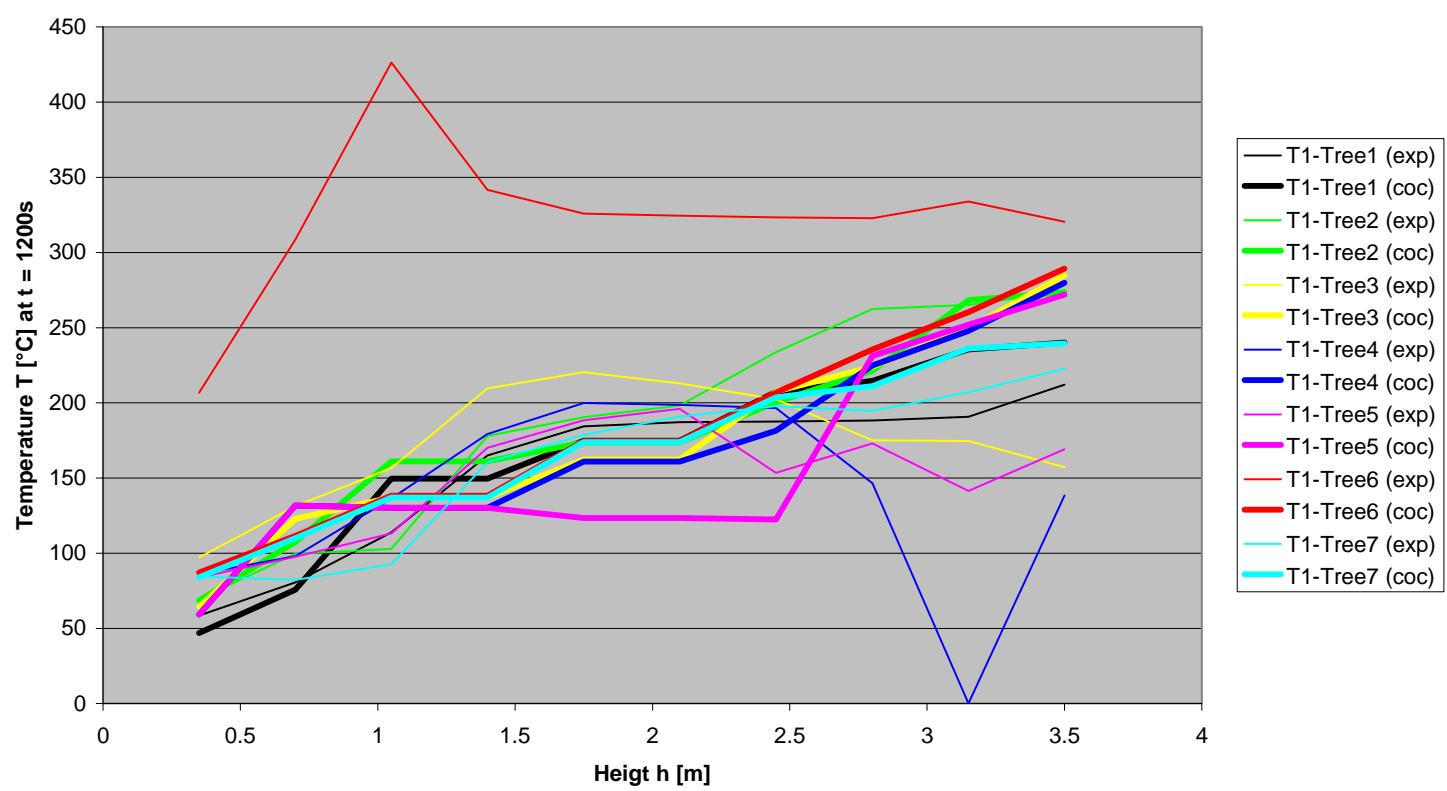

Fig. 5-78 Test 5: Temperature profile at $1200 \mathrm{~s}$

Test 5 (blind) : Tray temperatures

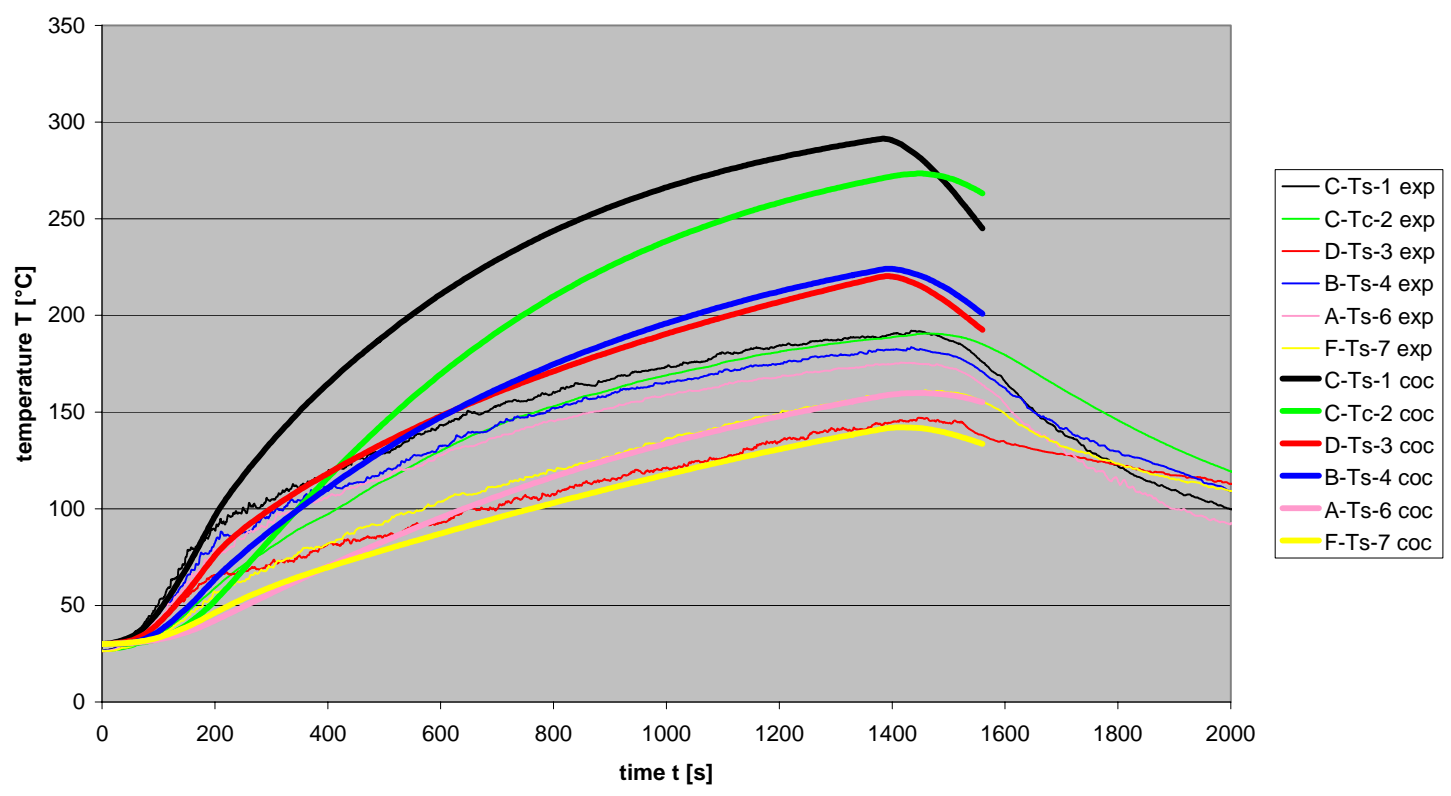

Fig. 5-79 Test 5: Tray temperatures at position 1 to 7 
Test 5 (blind) : Tray temperatures

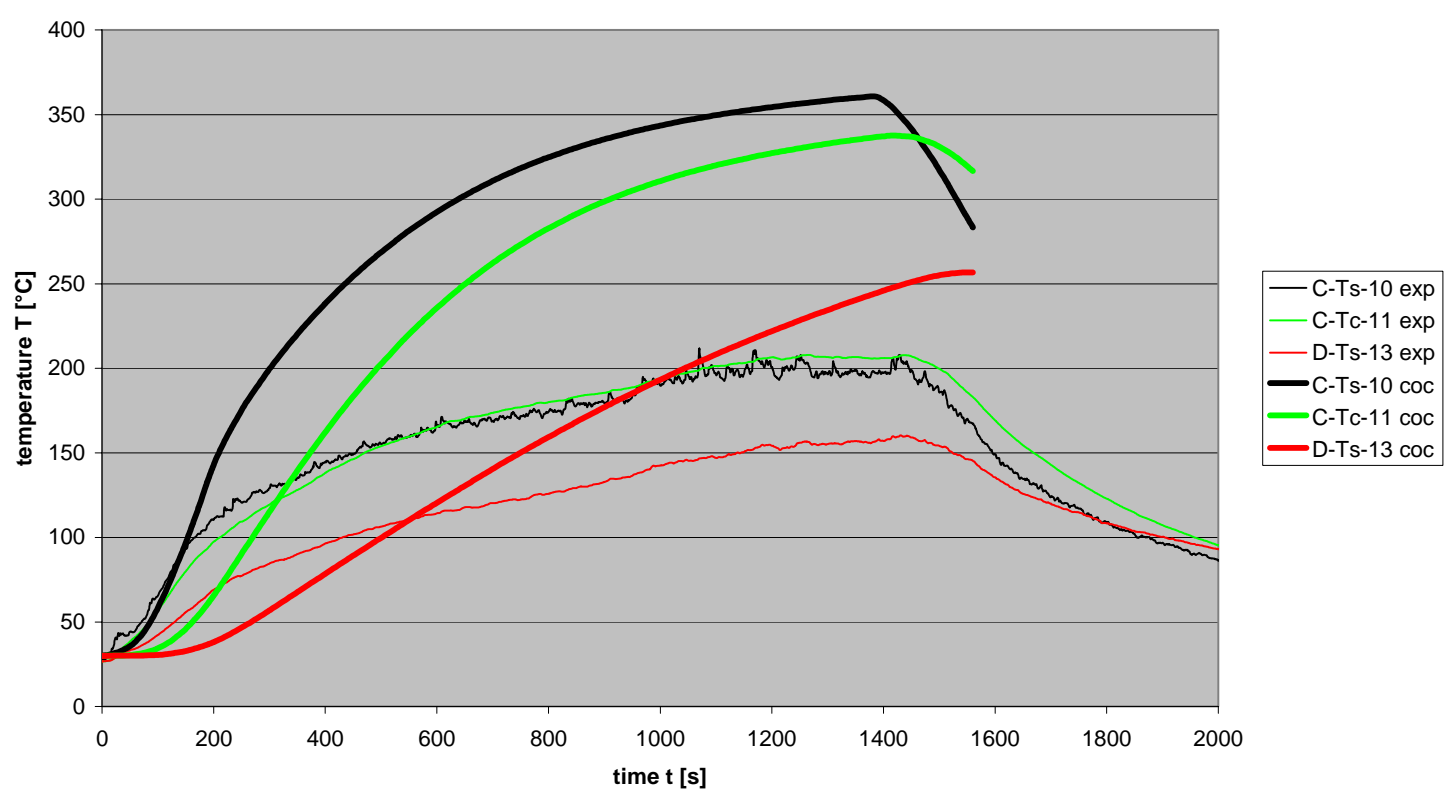

Fig. 5-80 Tray temperatures at position 10 to 13

Test 5 (blind) : Gas concentrations

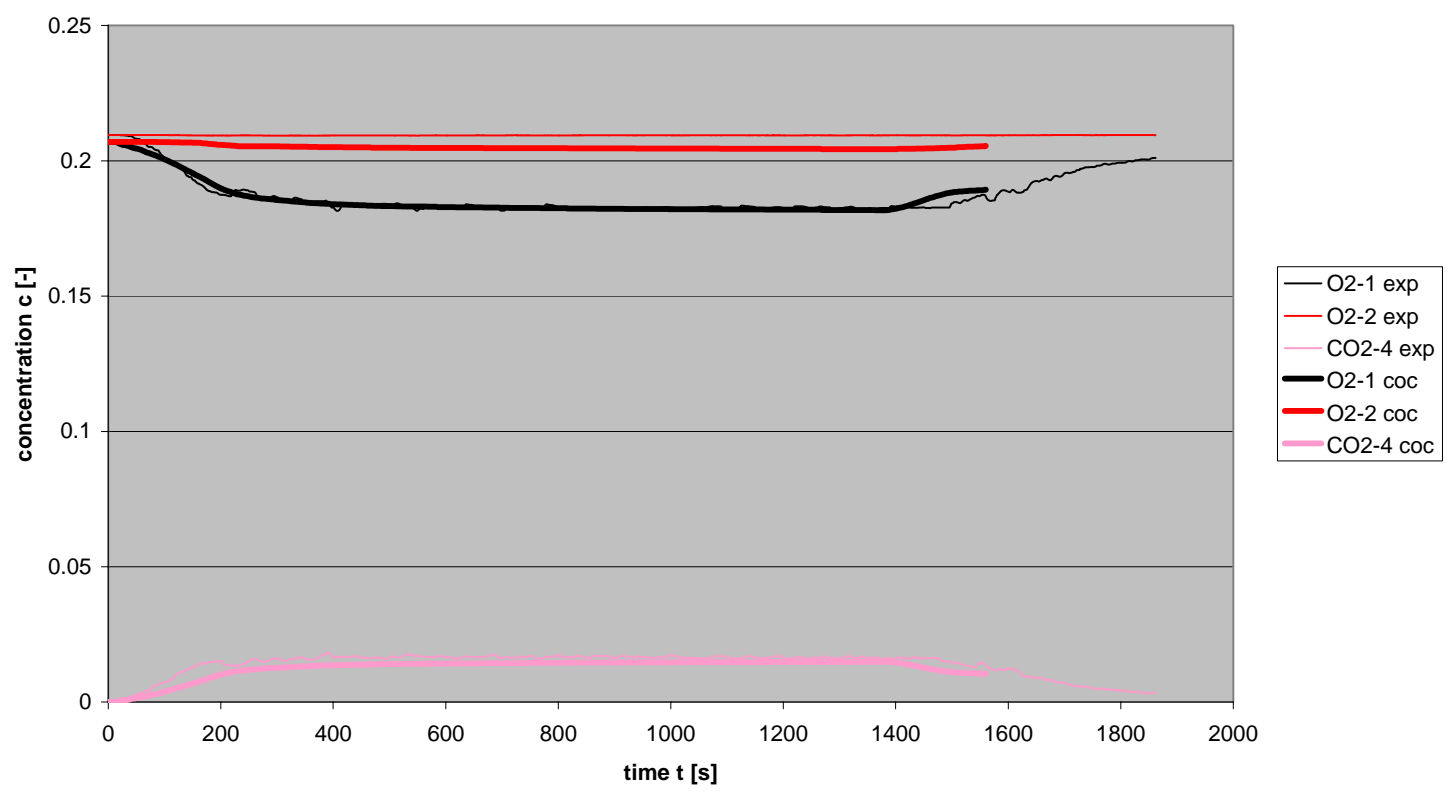

Fig. 5-81 Test 5: Gas concentrations 
Test 5 (blind) : Velocity at door

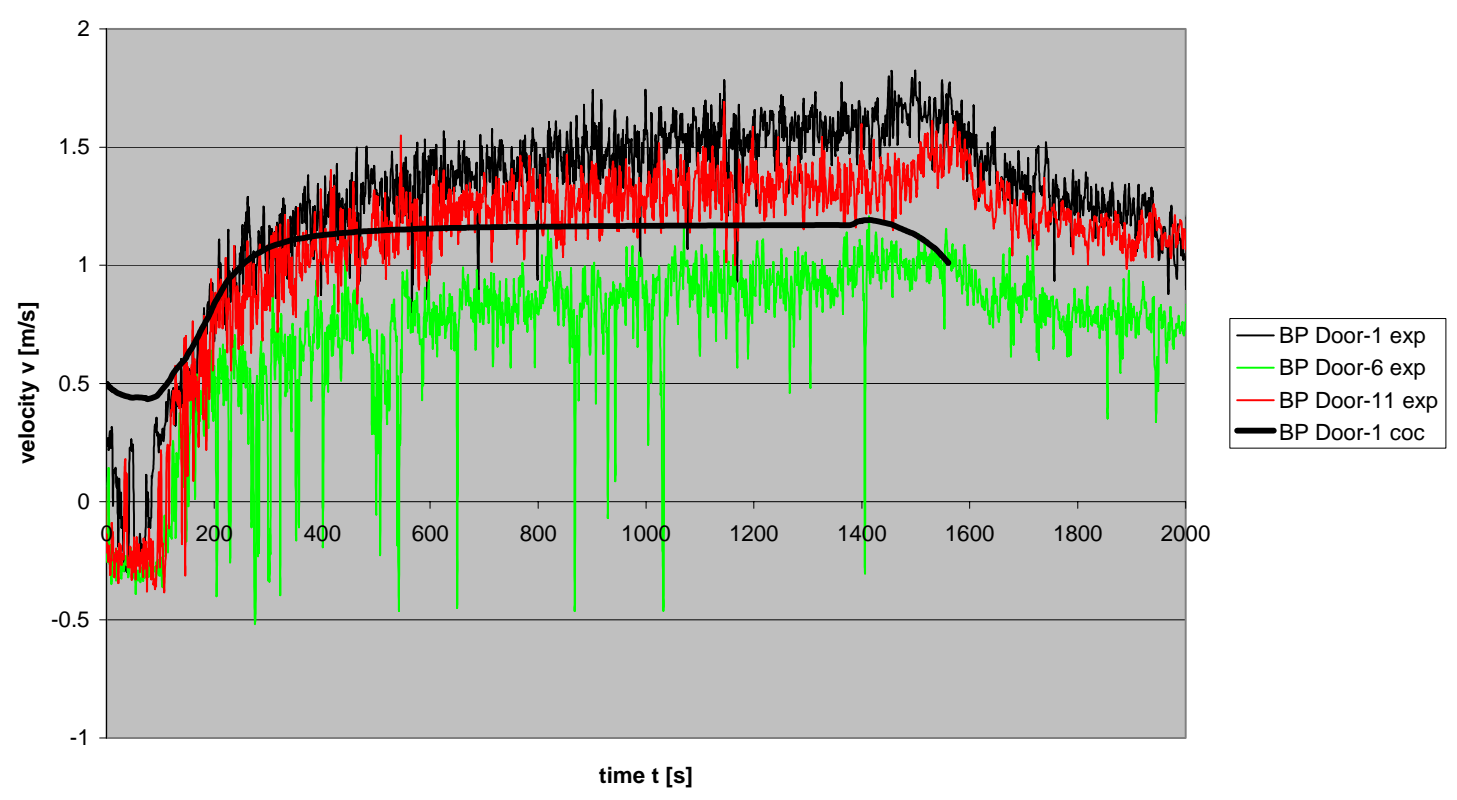

Fig. 5-82 Test 5: Velocity at door (level 1)

Test 5 (blind) : Velocity at door

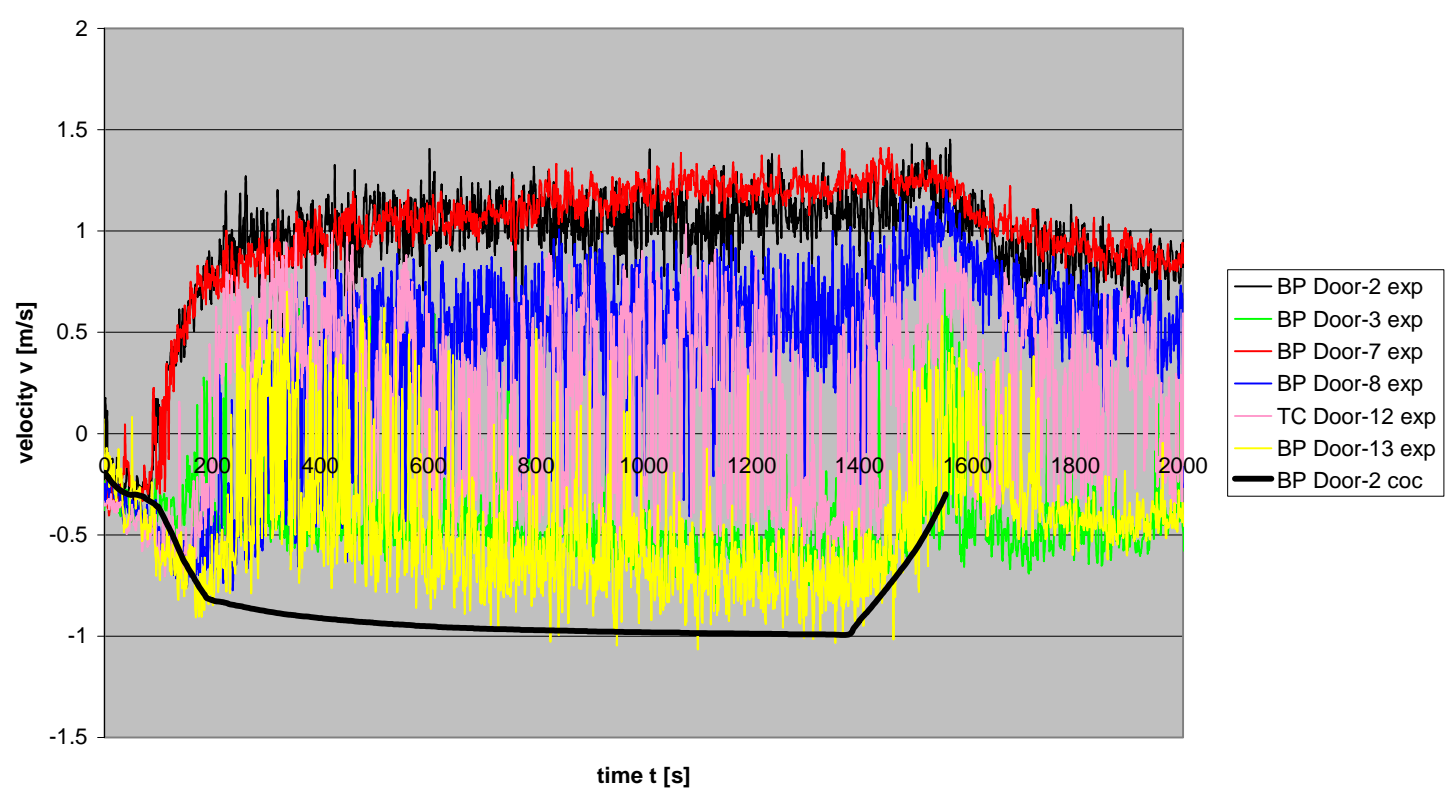

Fig. 5-83 Test 5: Velocity at door (level 2) 
Test 7 (blind) :Temperature Tree 1

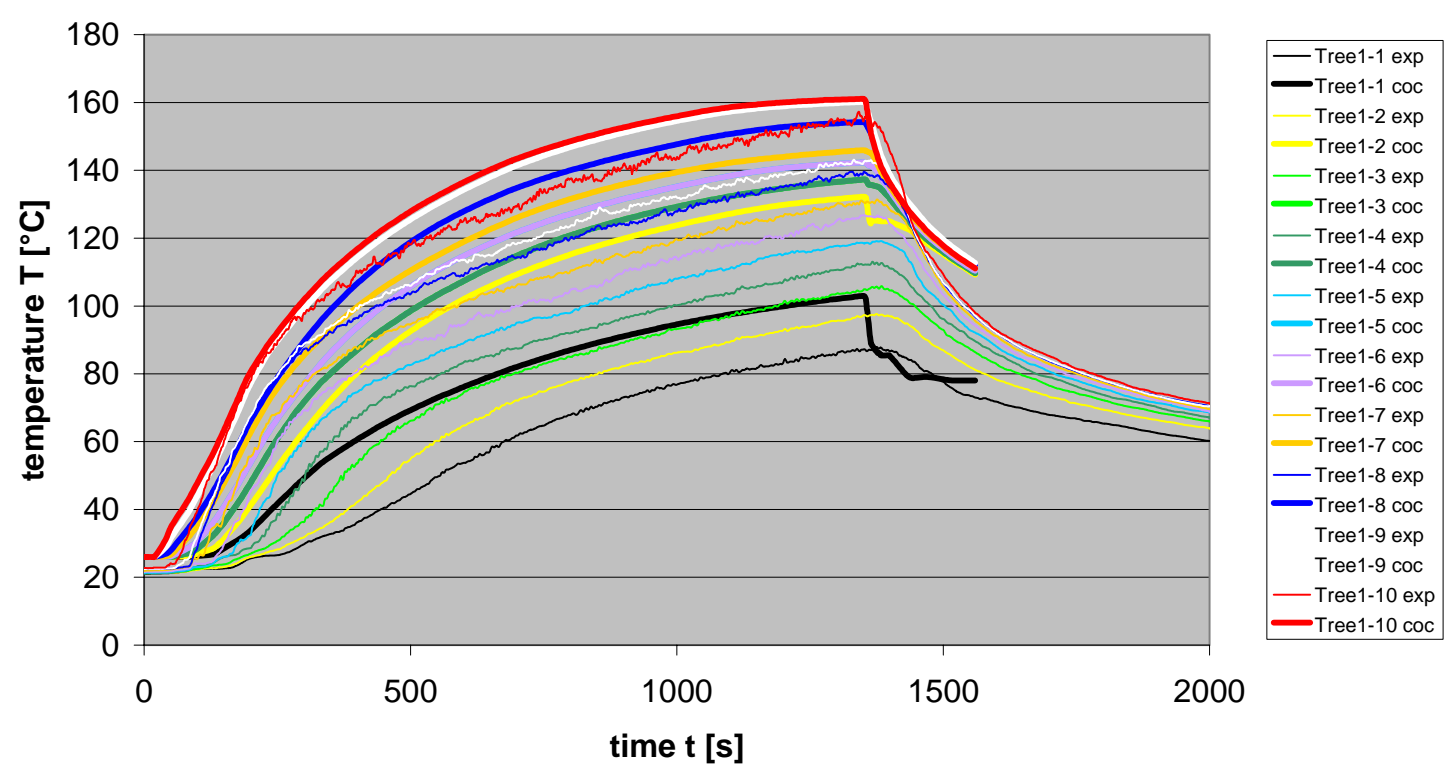

Fig. 5-84 Test 7: Temperature at tree 1

Test 7 (blind) : Tray temperatures

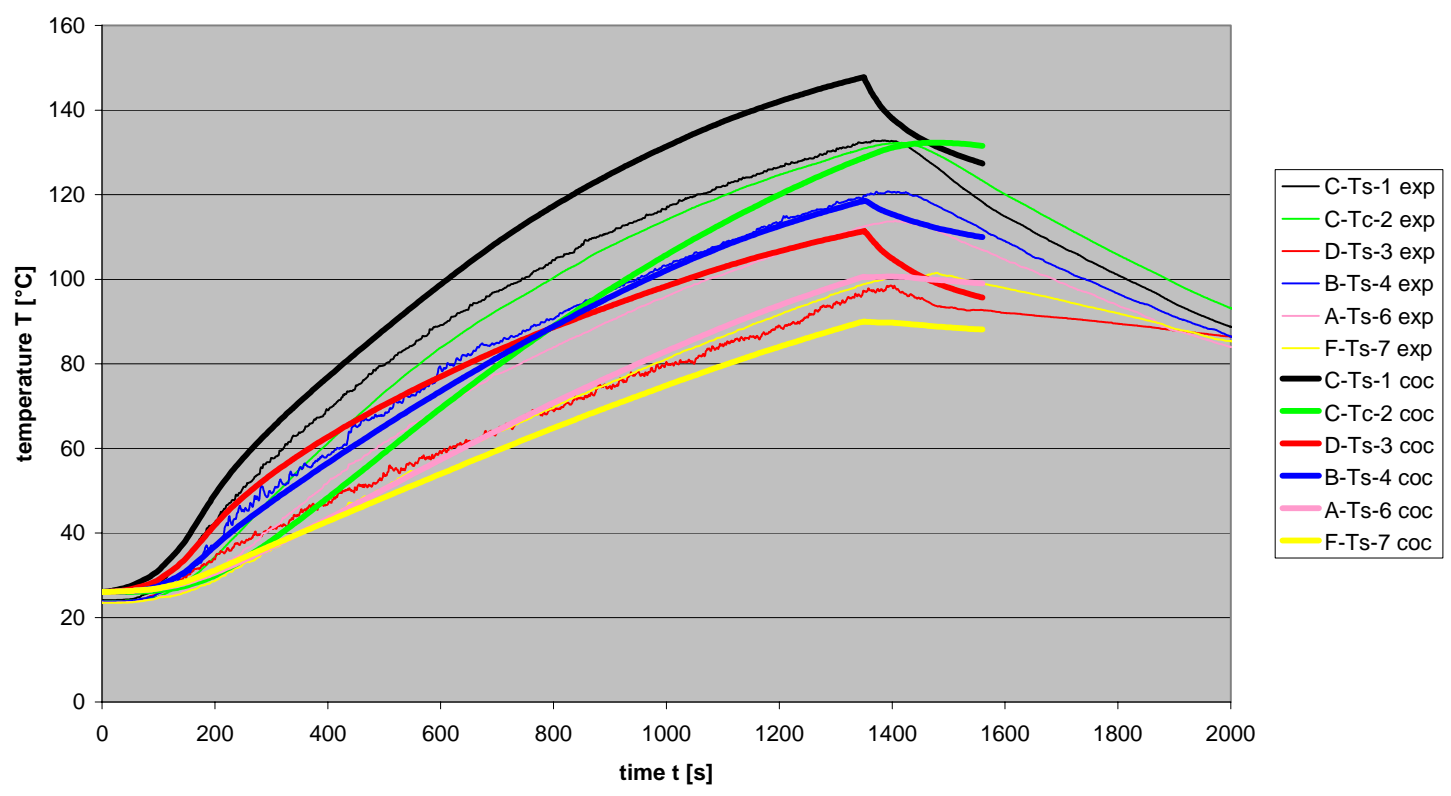

Fig. 5-85 Test 7: Tray temperatures at position 1 to 7 
Test 10 (blind) :Temperature Tree 1

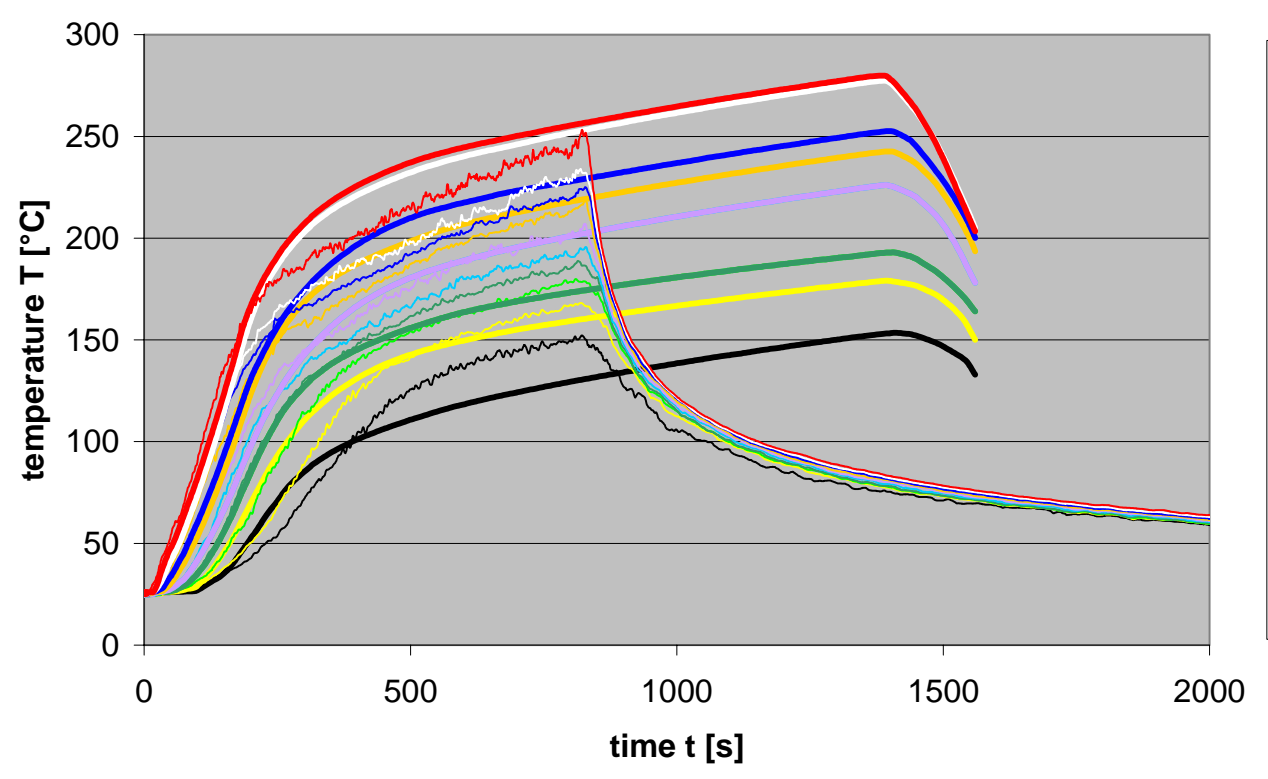

- Tree1-1 exp Tree1-1 coc -Tree1-2 exp Tree1-2 coc - Tree1-3 exp Tree1-3 coc - Tree1-4 exp Tree1-4 coc - Tree1-5 exp -Tree1-5 coc - Tree1-6 exp Tree1-6 coc - Tree1-7 exp -Tree1-7 coc - Tree1-8 exp Tree1-8 coc

Tree1-9 exp Tree1-9 coc - Tree1-10 exp -Tree1-10 coc

Fig. 5-86 Test 10: Temperatures at tree 1

Test 10 (blind) : Gas concentrations

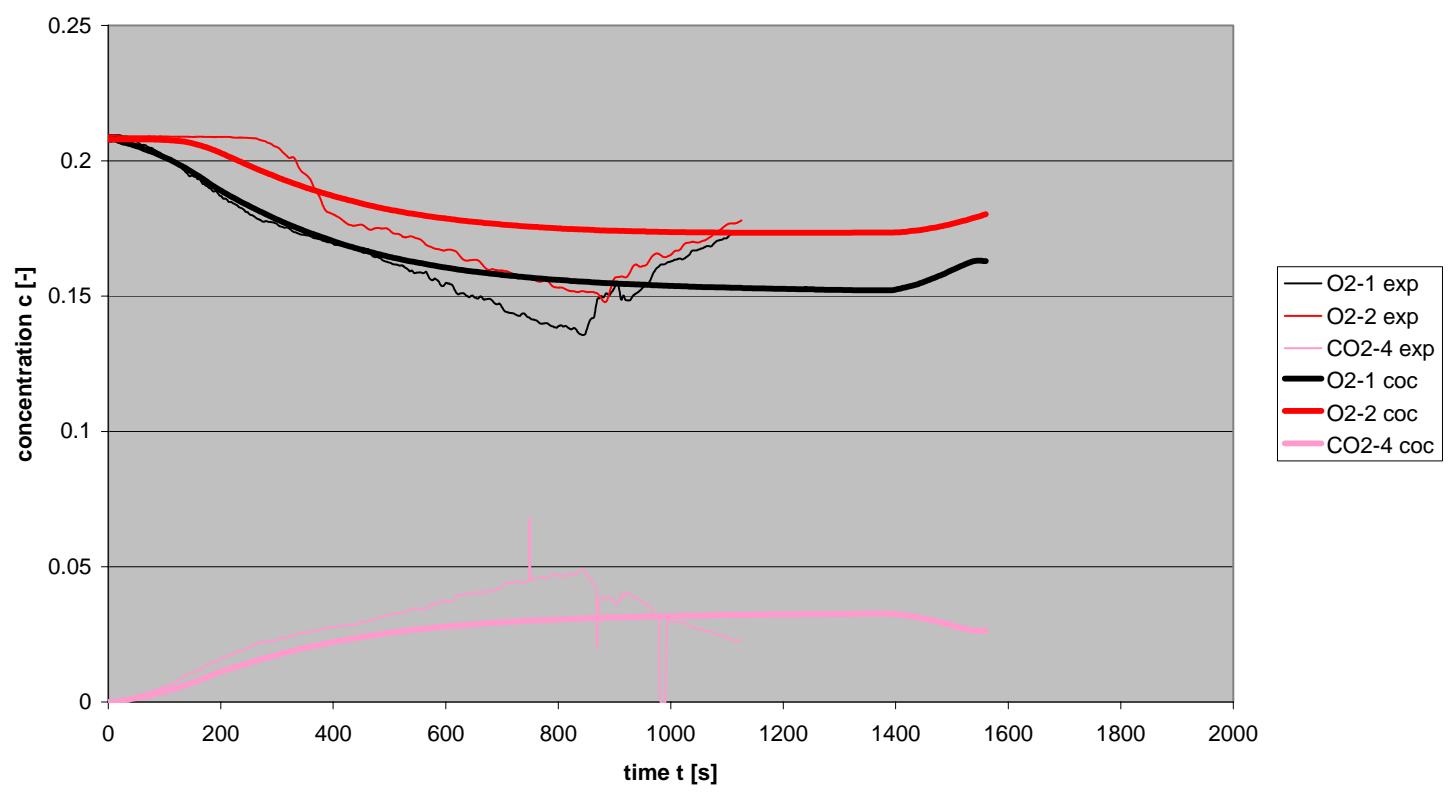

Fig. 5-87 Test 10: Gas concentrations 
Test 13 (blind) :Temperature Tree 1

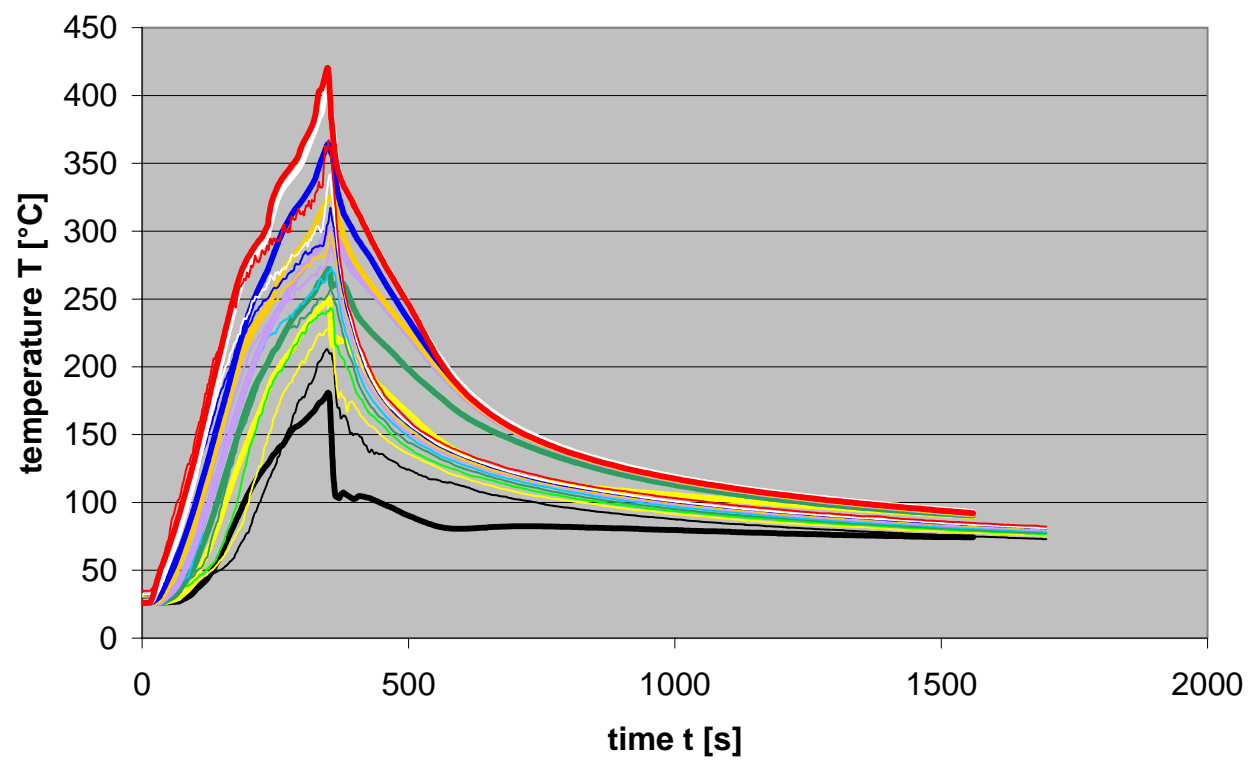

- Tree1-1 exp Tree1-1 coc Tree1-2 exp Tree1-2 coc - Tree1-3 exp Tree1-3 coc - Tree1-4 exp -Tree1-4 coc - Tree1-5 exp Tree1-5 coc - Tree1-6 exp Tree1-6 coc -Tree1-7 exp Tree1-7 coc -Tree1-8 exp -Tree1-8 coc

Tree1-9 exp Tree1-9 coc - Tree1-10 exp -Tree1-10 coc

Fig. 5-88 Test 13: Temperatures at tree 1

Test 13 (blind) : Tree temperature profiles

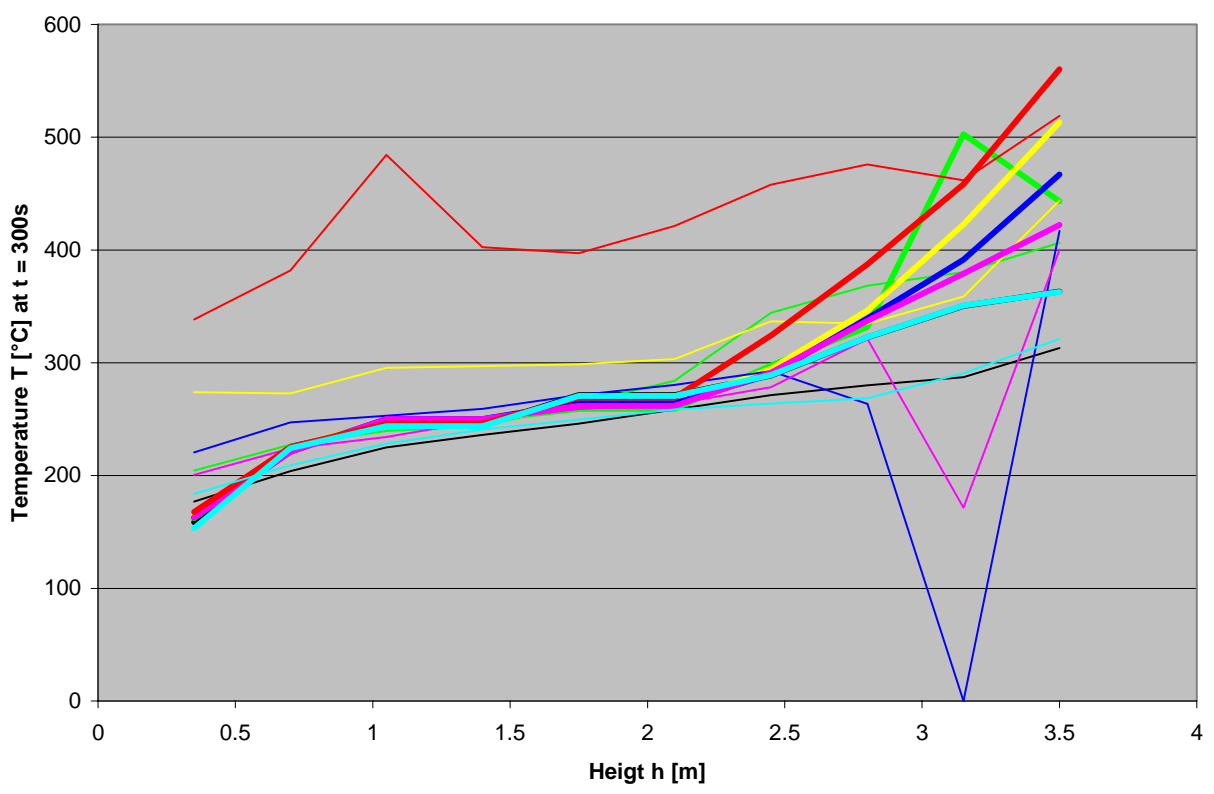

- T1-Tree1 (exp)

-T1-Tree1 (coc)

-T1-Tree2 (exp)

T1-Tree2 (coc)

T1-Tree3 (exp)

T1-Tree3 (coc)

- T1-Tree4 (exp)

T1-Tree4 (coc)

- T1-Tree5 (exp)

T1-Tree5 (coc)

— T1-Tree6 (exp)

-T1-Tree6 (coc)

- T1-Tree7 (exp)

T1-Tree7 (coc)

Fig. 5-89 Test 13: Temperature profile at $300 \mathrm{~s}$ 
Test 13 (blind) : Tray temperatures

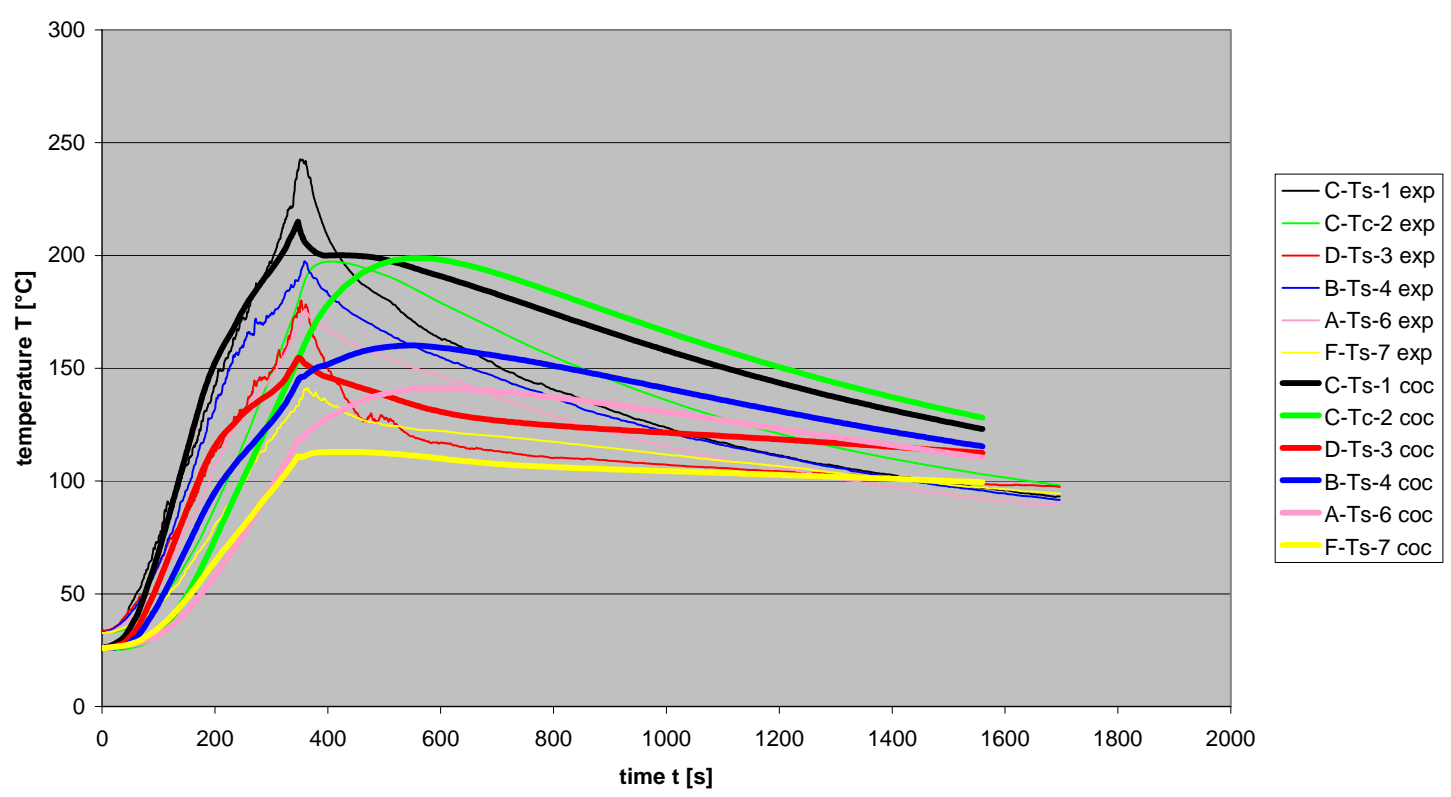

Fig. 5-90 Test 13: Tray temperatures at position 1 to 7

Test 13 (blind) : Inner west wall temperatures

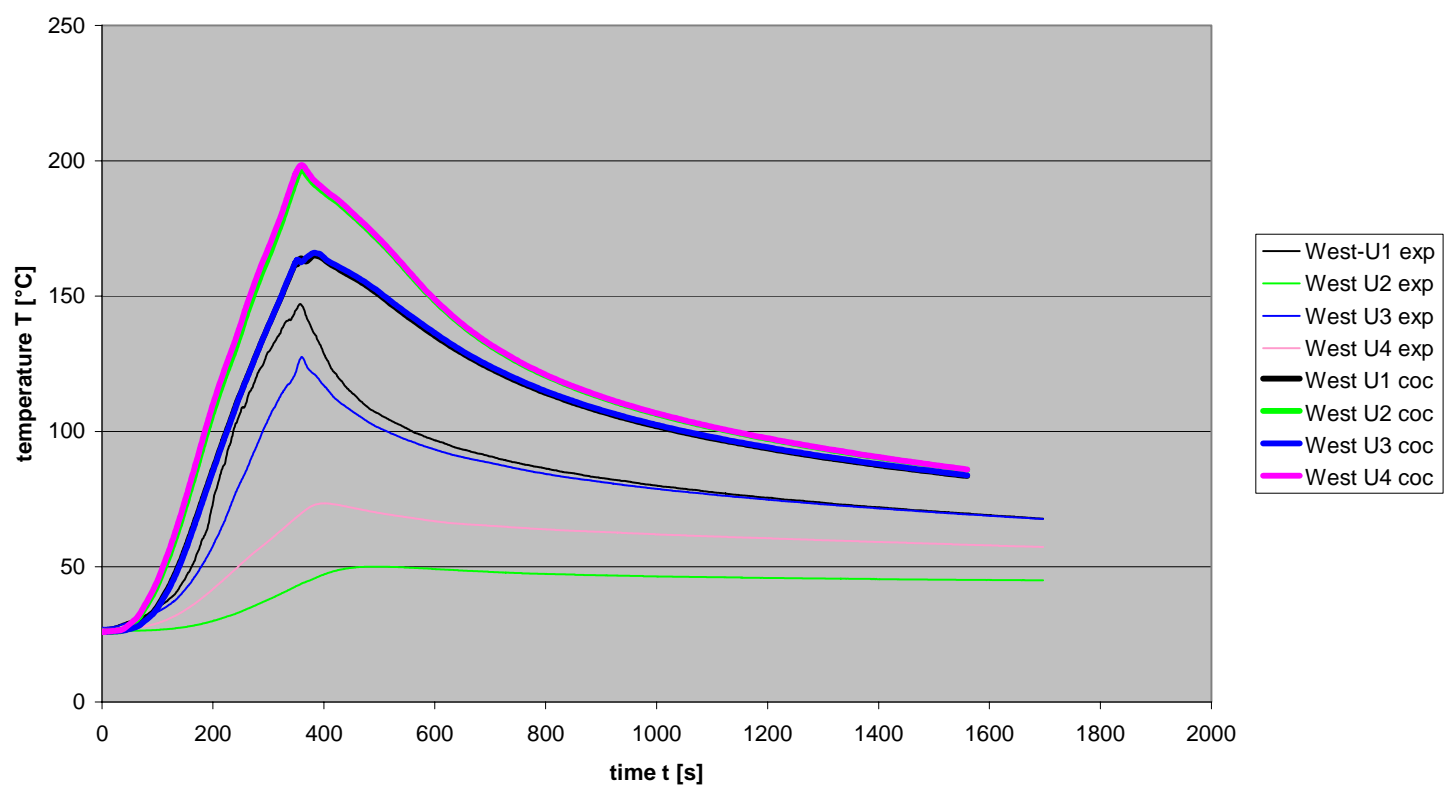

Fig. 5-91 Test 13: West wall temperatures 
Test 13 (blind) : Inner ceiling temperatures
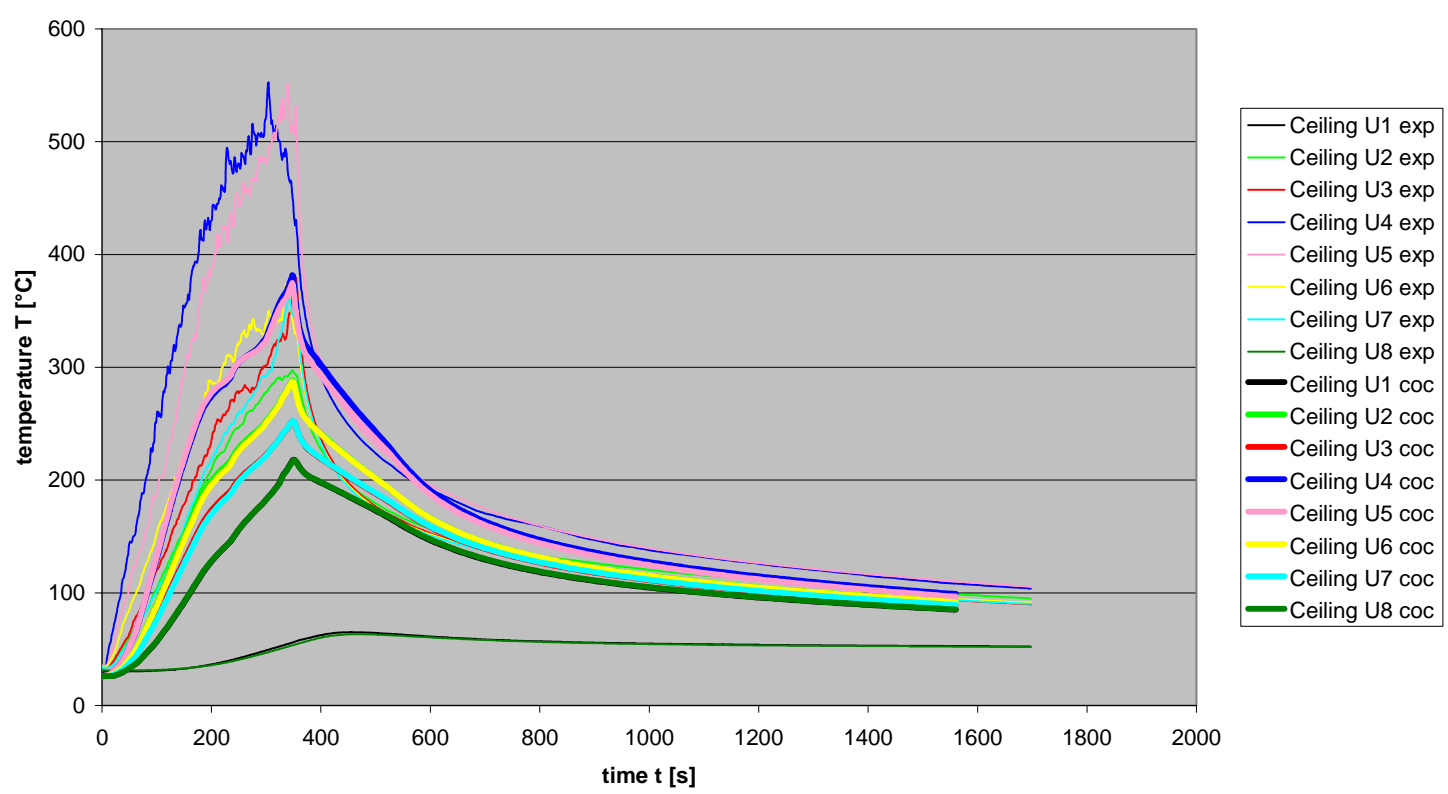

Fig. 5-92 Test 13: Ceiling temperatures

Test 13 (blind) : Gas concentrations

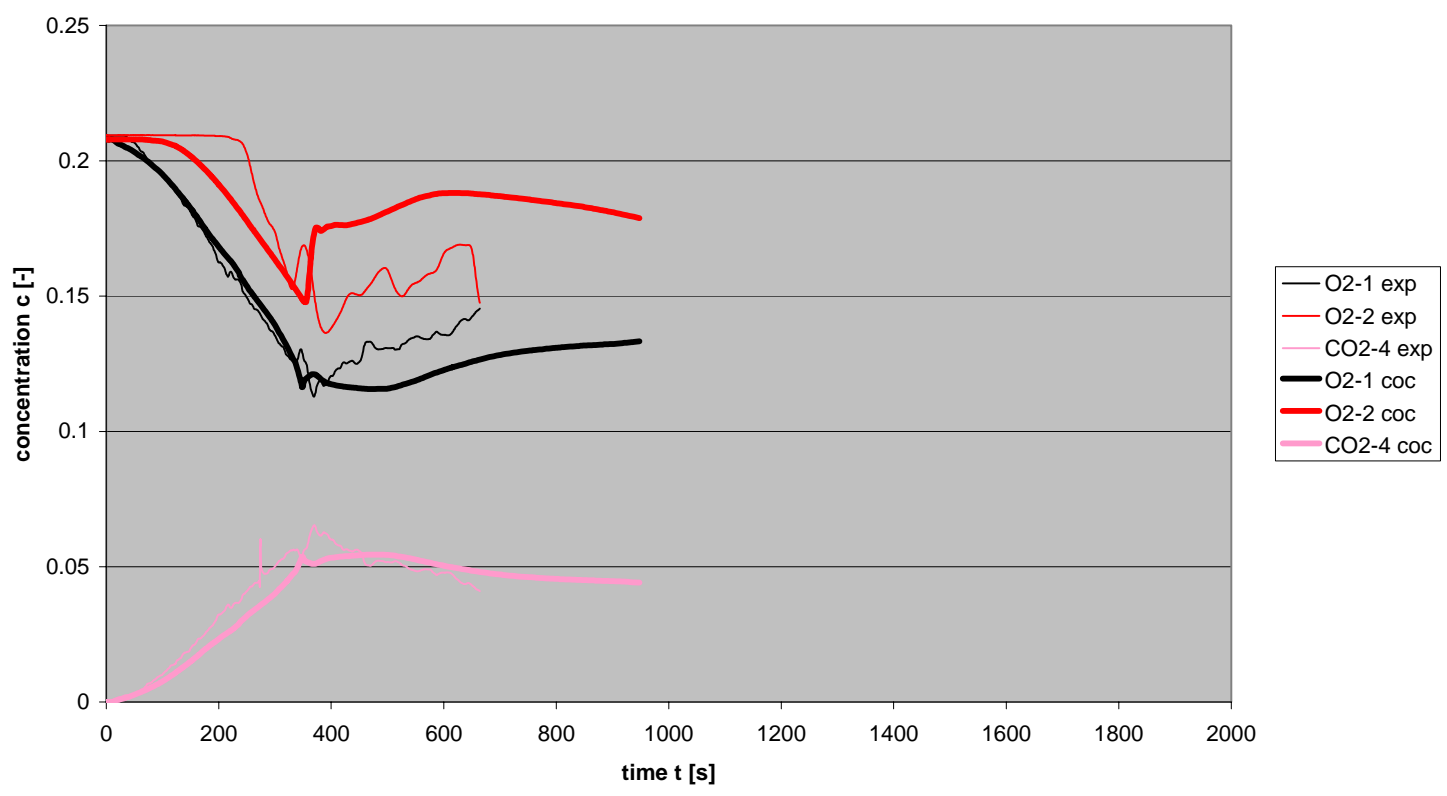

Fig. 5-93 Test 13: Gas concentrations 
Test 16 (blind) :Temperature Tree 1

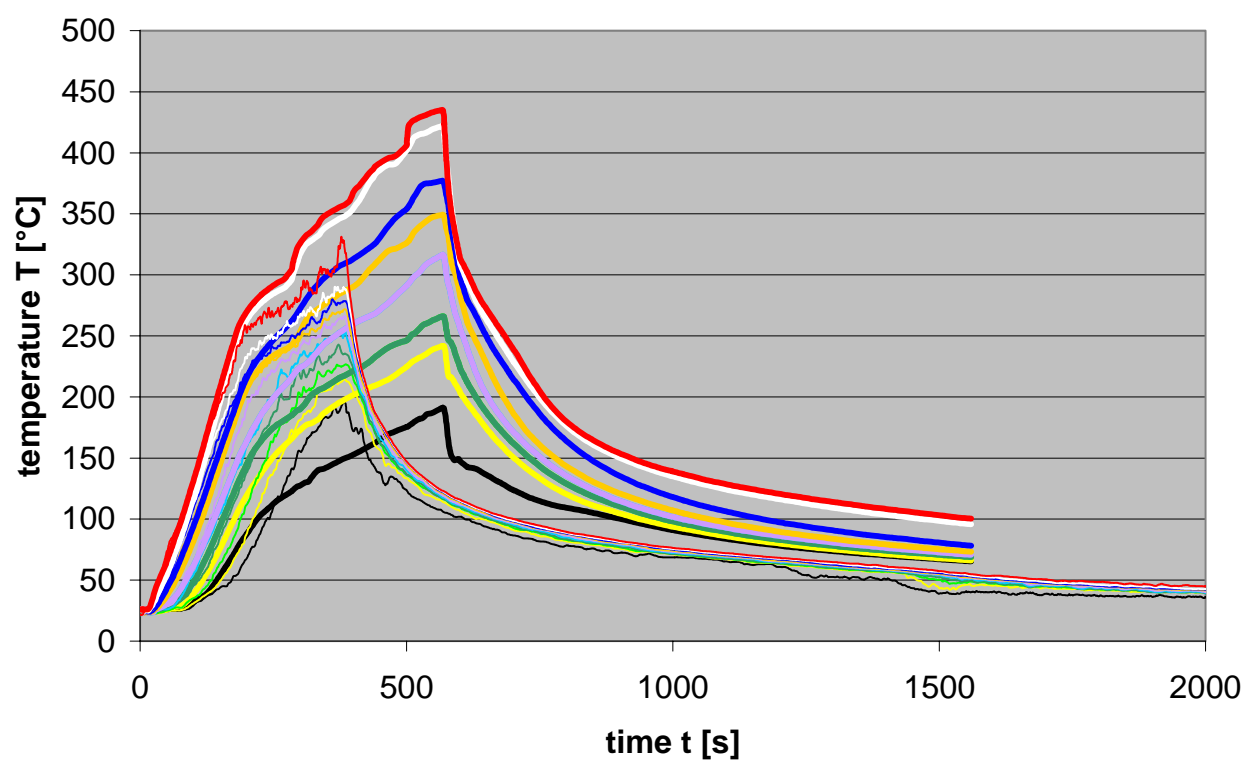

- Tree1-1 exp Tree1-1 coc Tree1-2 exp Tree1-2 coc

- Tree1-3 exp Tree1-3 coc - Tree1-4 exp -Tree1-4 coc - Tree1-5 exp -Tree1-5 coc - Tree1-6 exp Tree1-6 coc - Tree1-7 exp Tree1-7 coc - Tree1-8 exp -Tree1-8 coc

Tree1-9 exp Tree1-9 coc - Tree1-10 exp -Tree1-10 coc

Fig. 5-94 Test 16: Temperatures at tree 1

Test 16 (blind) : Gas concentrations

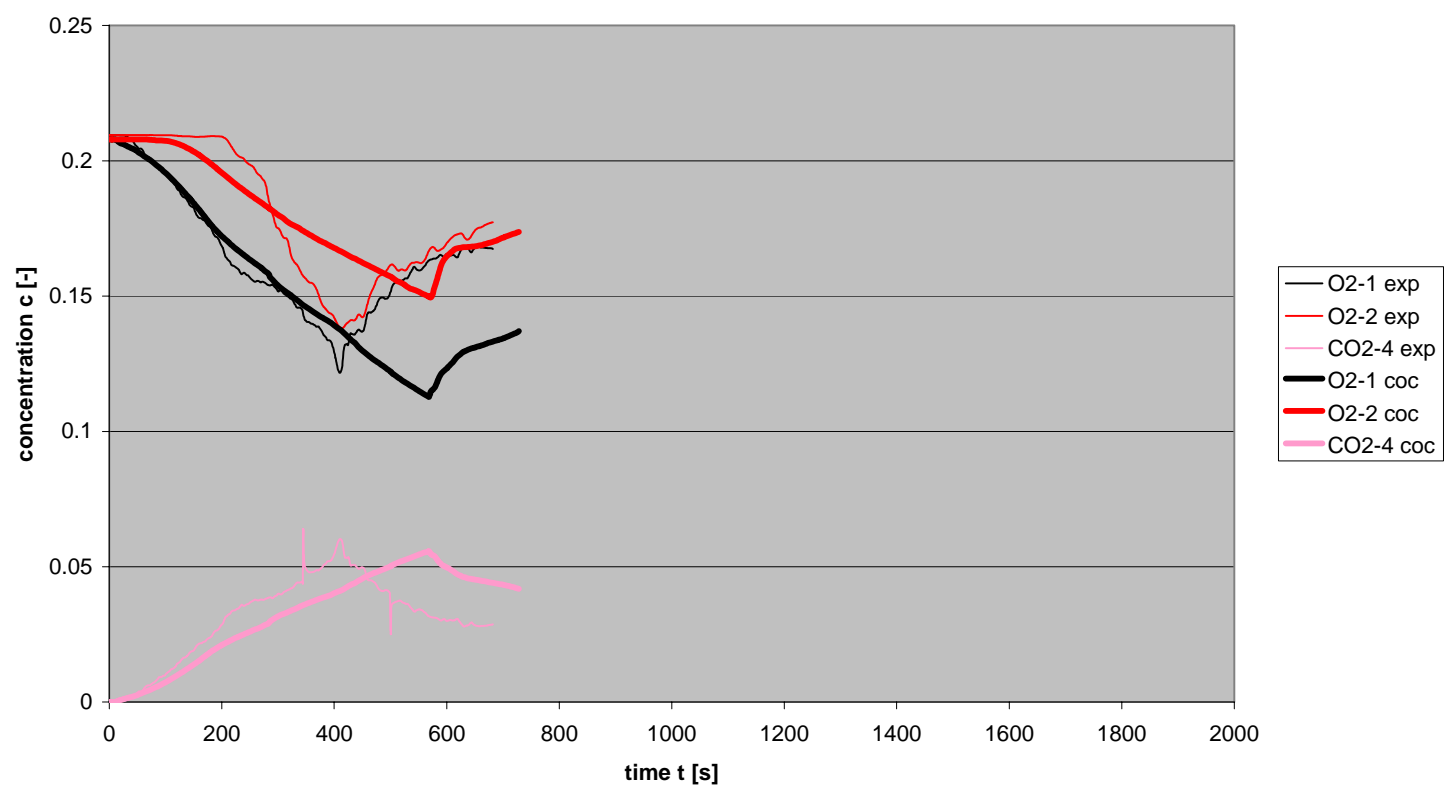

Fig. 5-95 Test 16: Gas concentrations 
Test 16 (blind) : Ventilation

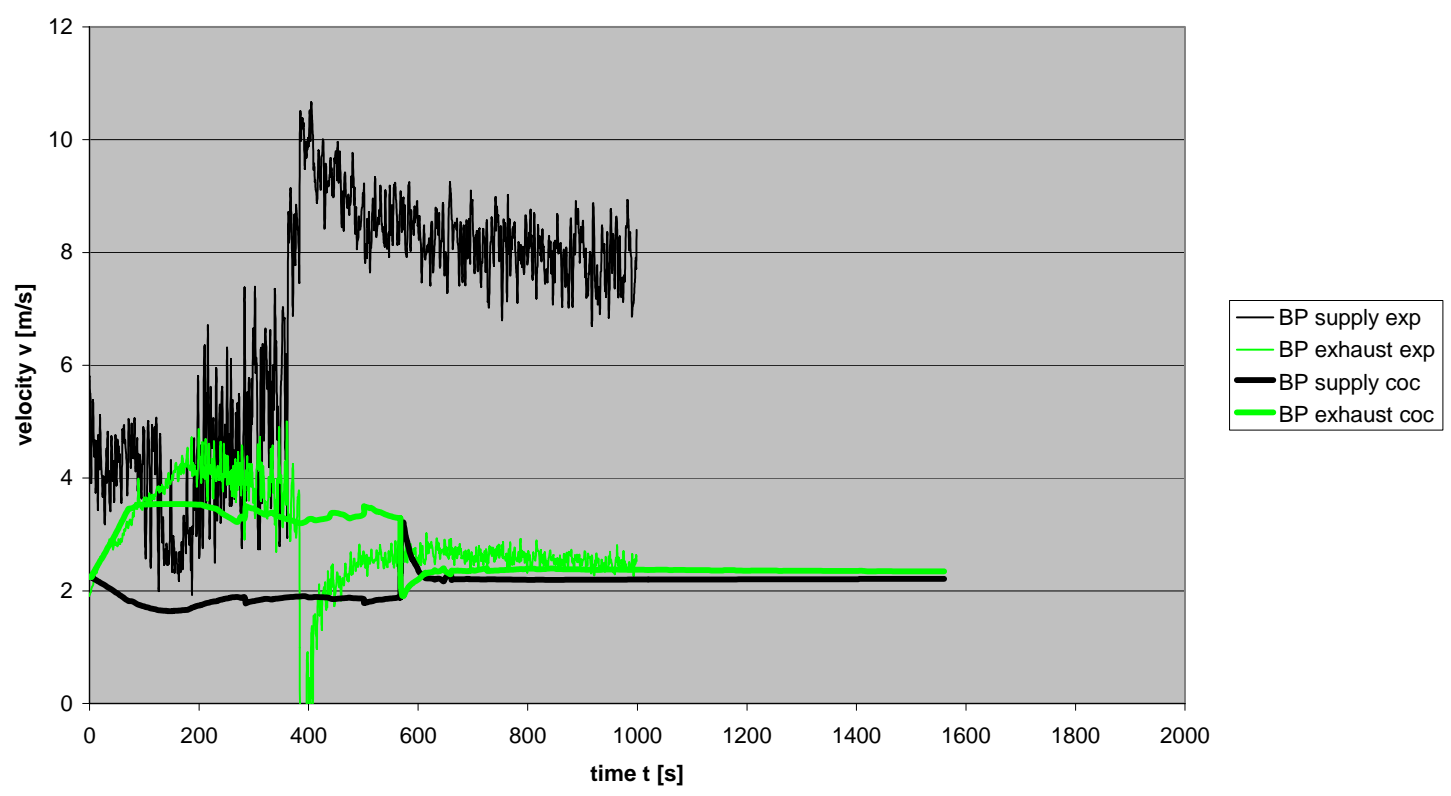

Fig. 5-96 Test 16: Velocities at ventilation systems

Test 16 (blind) : Junction Box

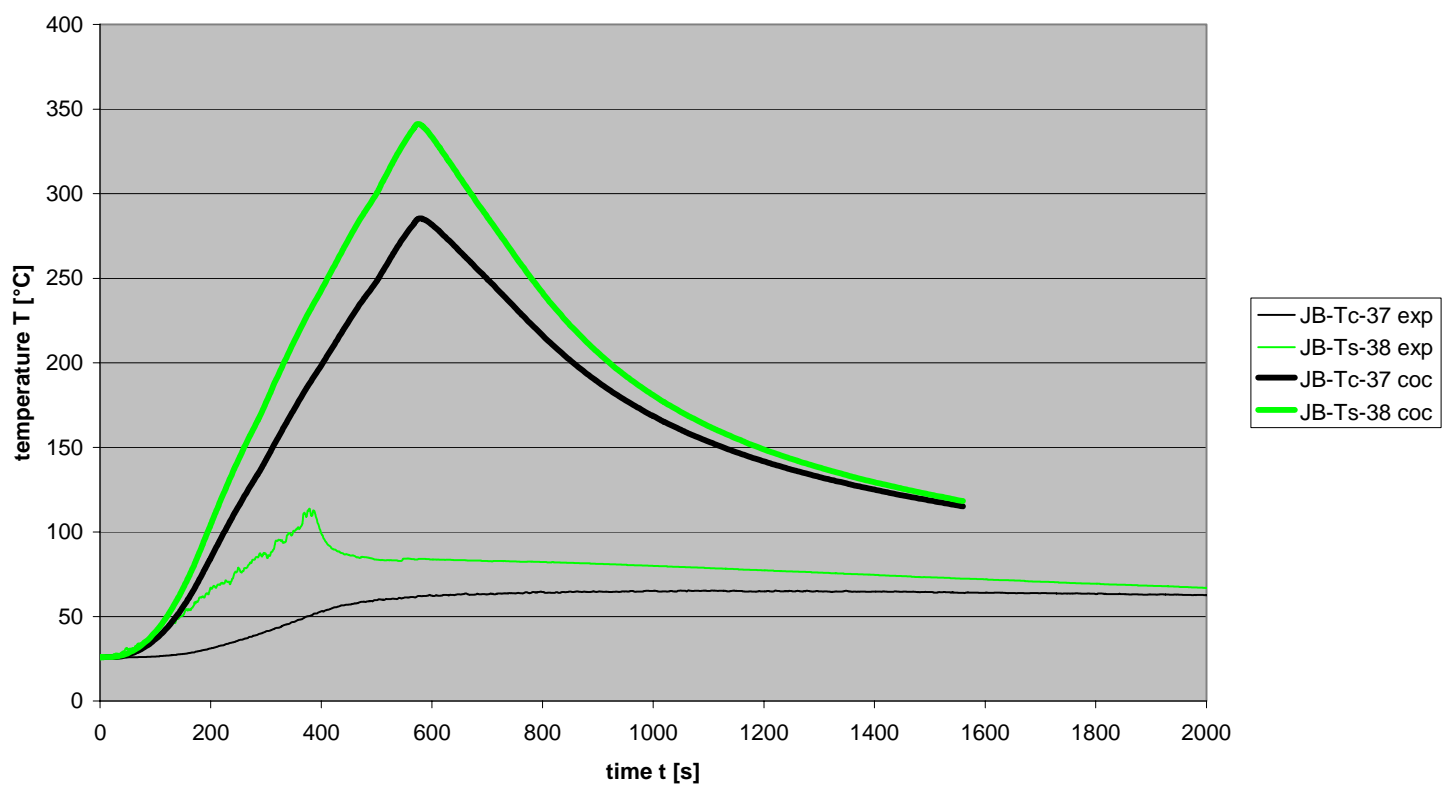

Fig. 5-97 Test 16: Temperatures at junction box 
Test 16 (blind) : Inner south wall temperatures

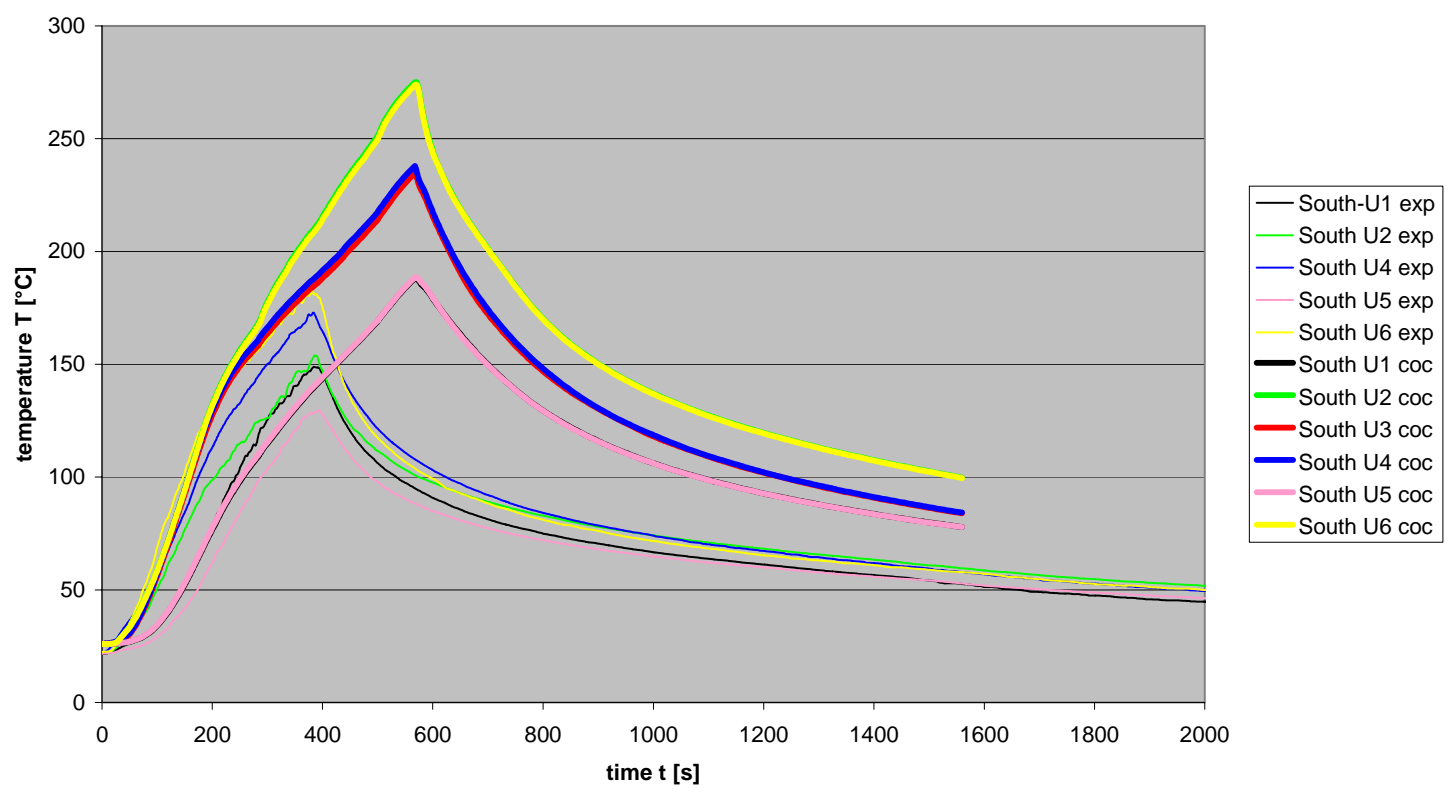

Fig. 5-98 Test 16: South wall temperatures

Test 16 (blind) : Vertical tray temperatures

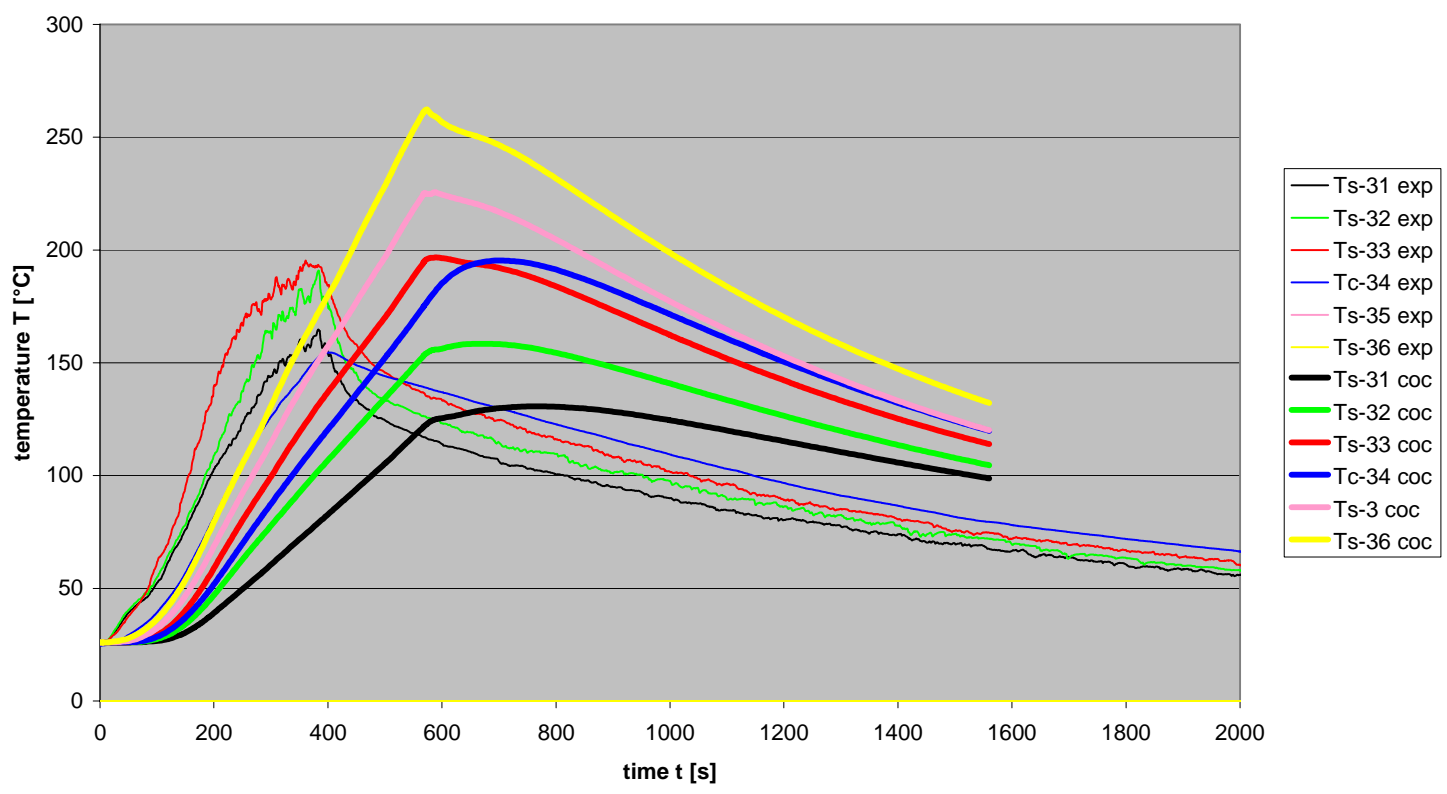

Fig. 5-99 Test 16: Vertical tray temperatures 
Test 17 (blind) :Temperature Tree 1

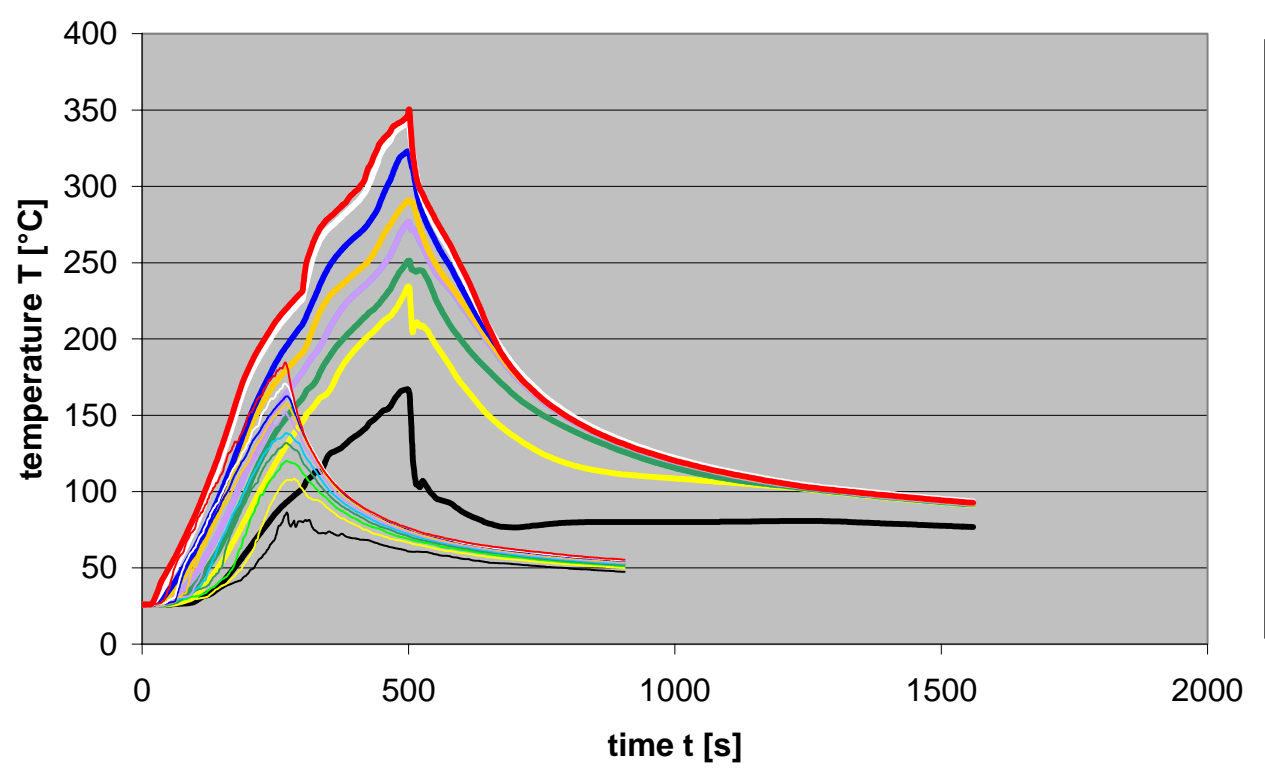

- Tree1-1 exp Tree1-1 coc Tree1-2 exp Tree1-2 coc - Tree1-3 exp -Tree1-3 coc - Tree1-4 exp Tree1-4 coc - Tree1-5 exp -Tree1-5 coc - Tree1-6 exp Tree1-6 coc - Tree1-7 exp Tree1-7 coc - Tree1-8 exp -Tree1-8 coc

Tree1-9 exp Tree1-9 coc - Tree1-10 exp -Tree1-10 coc

Fig. 5-100 Test 17: Temperatures at tree 1

Test 17 (blind) : Gas concentrations

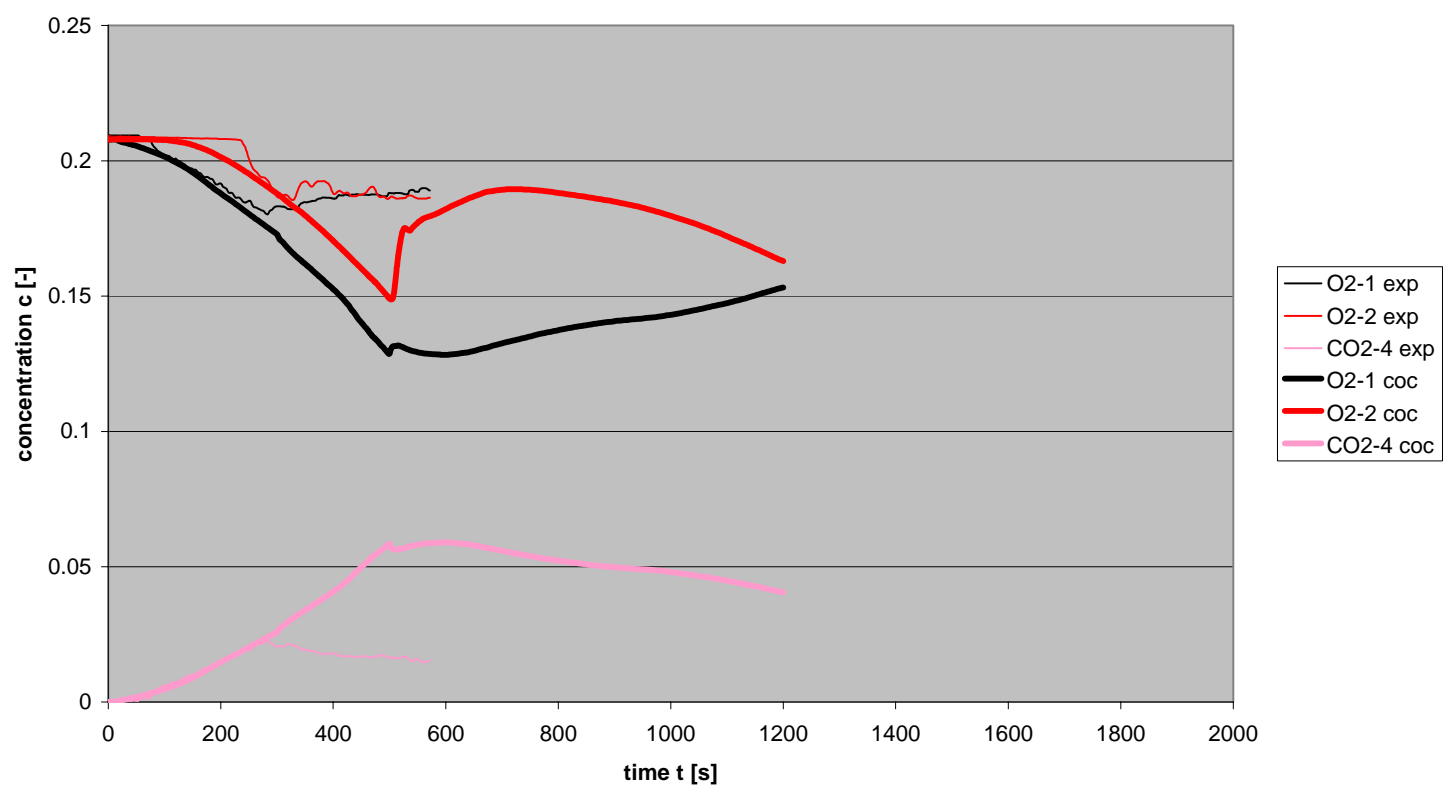

Fig. 5-101 Test 17: Gas concentration 
Test 17 (blind) : Inner ceiling temperatures

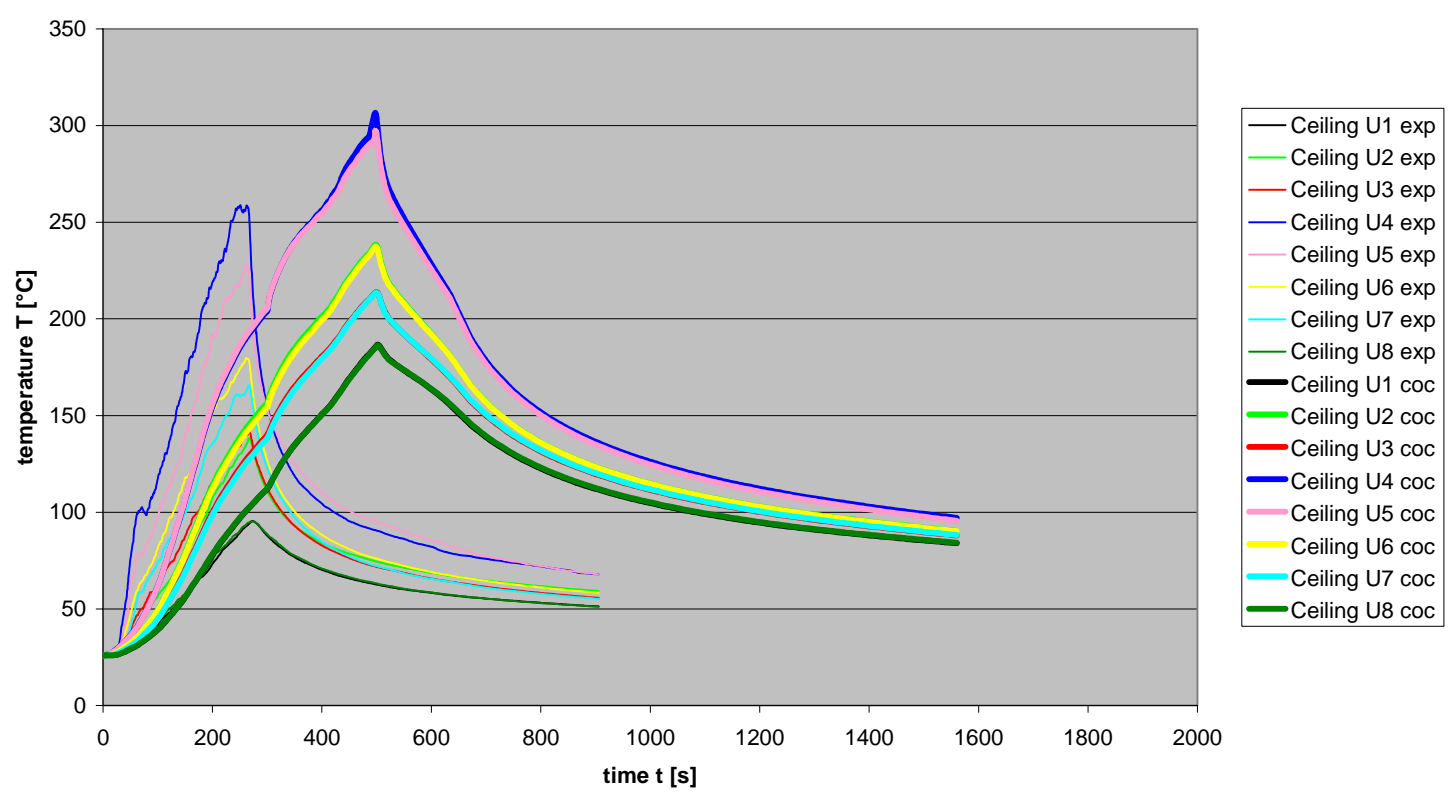

Fig. 5-102 Test 17: Ceiling temperatures

Test 18 (blind) :Temperature Tree 1

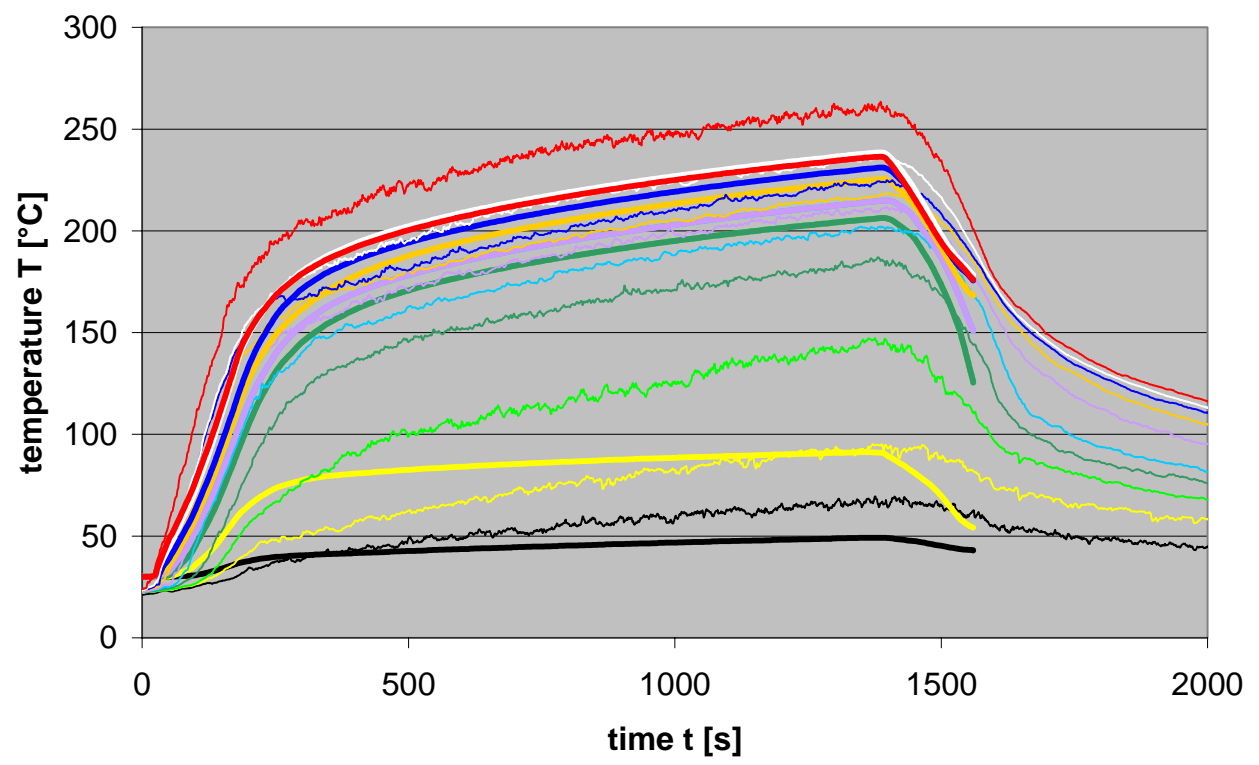

- Tree1-1 exp Tree1-1 coc

Tree1-2 exp

Tree1-2 coc

-Tree1-3 exp Tree1-3 coc

- Tree1-4 exp

Tree1-4 coc

- Tree1-5 exp

Tree1-5 coc

- Tree1-6 exp

Tree1-6 coc

- Tree1-7 exp

-Tree1-7 coc

- Tree1-8 exp

-Tree1-8 coc

Tree1-9 exp Tree1-9 coc

time $\mathbf{t}$ [s]

Fig. 5-103 Test 18: Temperatures at tree 1 
Test 18 (blind) : Inner ceiling temperatures
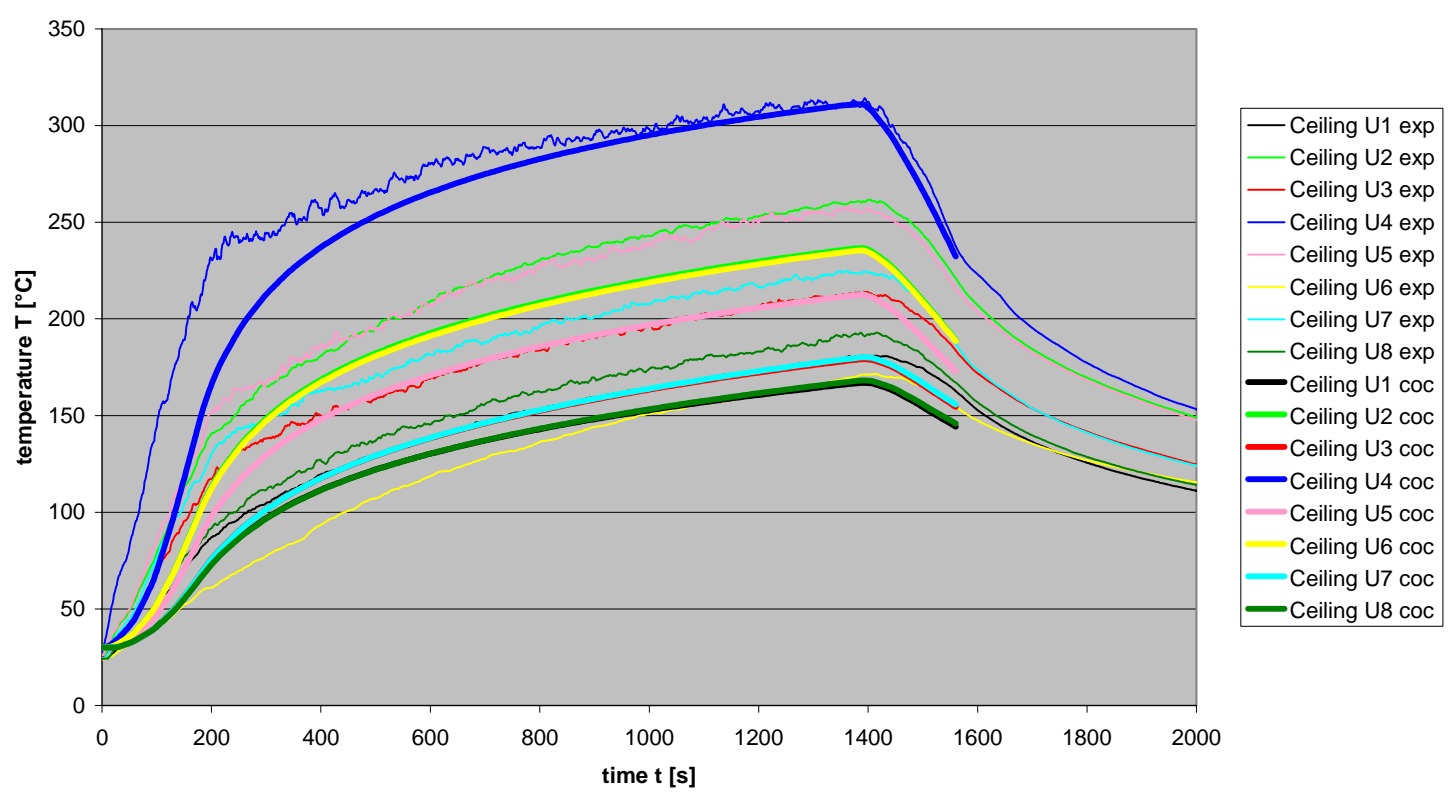

Fig. 5-104 Test 18: Ceiling temperatures

Test 18 (blind) : Inner south wall temperatures

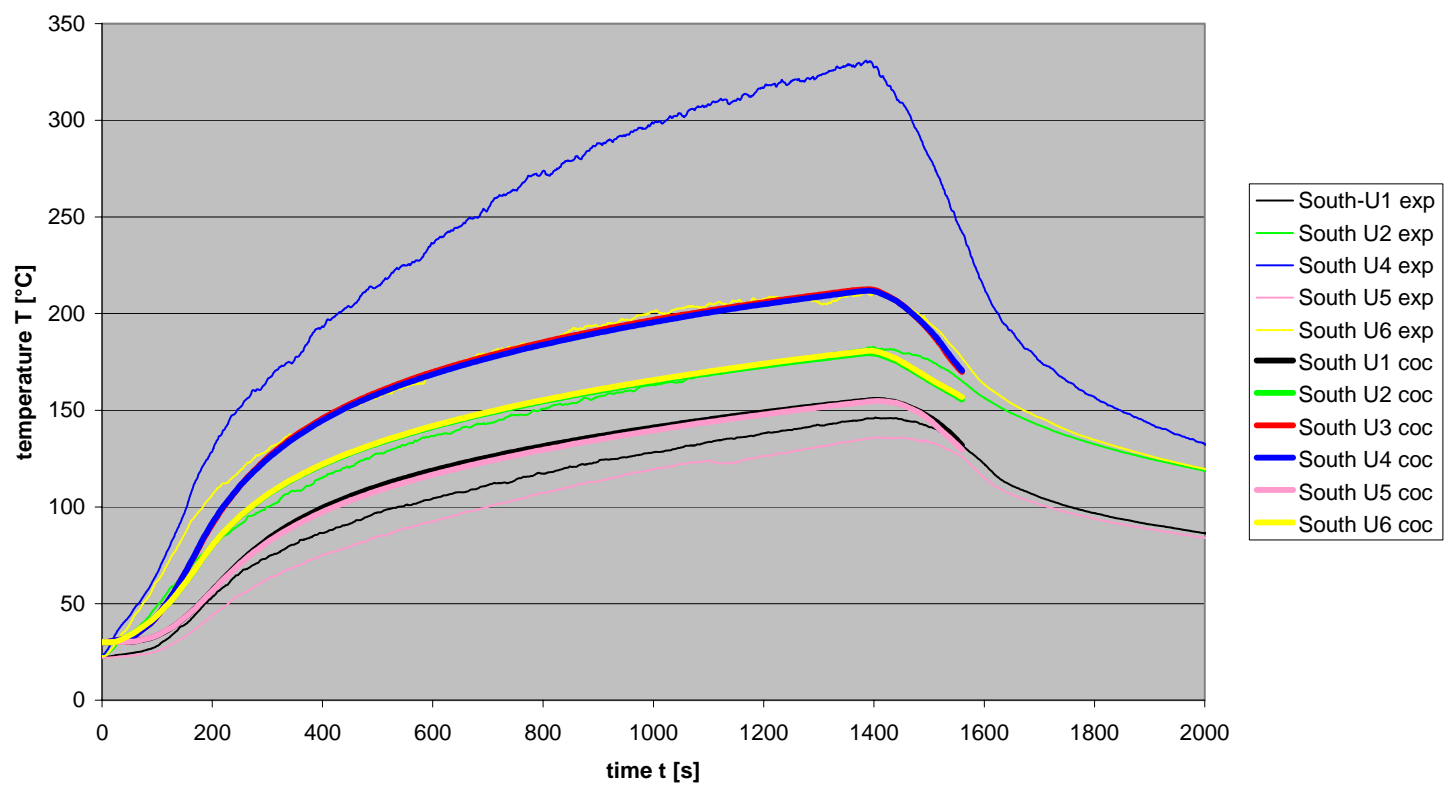

Fig. 5-105 Test 18: South wall temperatures 
Test 18 (blind) : Vertical tray temperatures

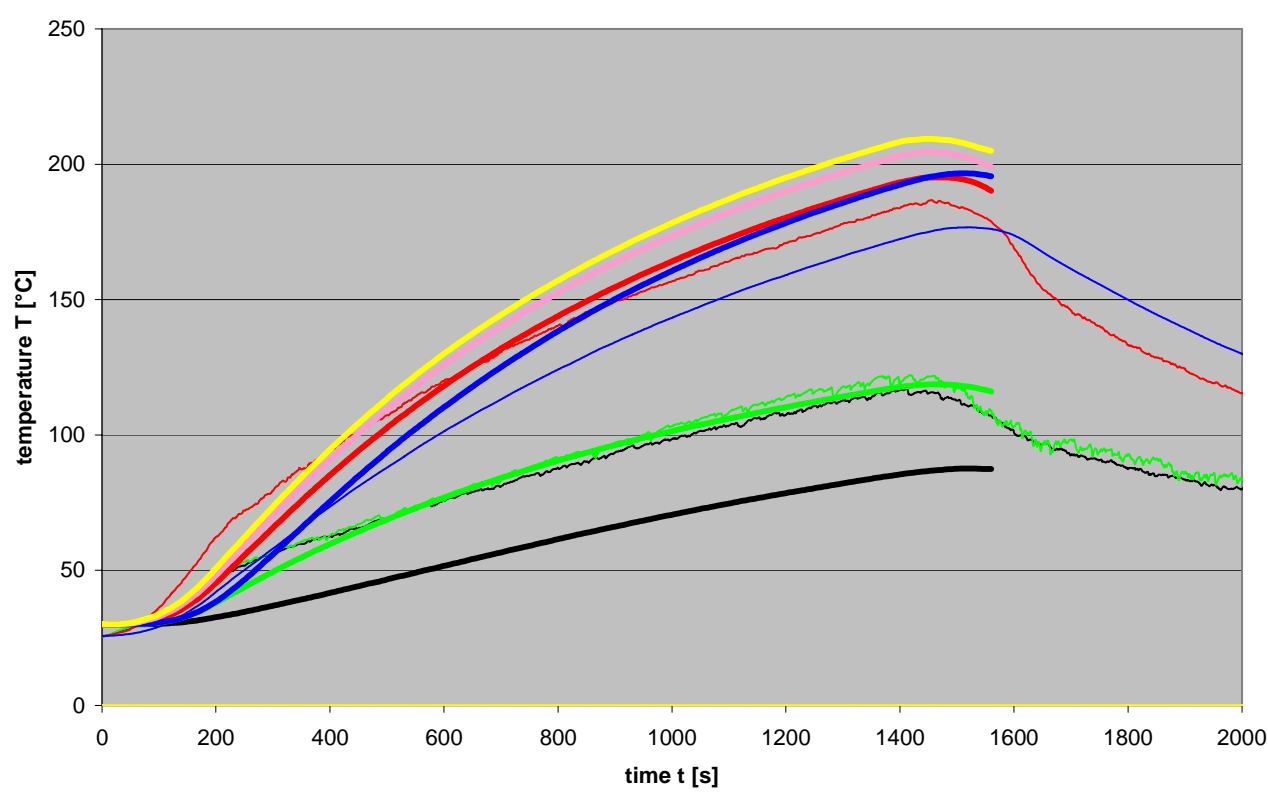

\begin{tabular}{|c|}
\hline Ts-31 exp \\
- Ts-32 exp \\
- Ts-33 exp \\
- Tc-34 exp \\
Ts-35 exp \\
Ts-36 exp \\
Ts-31 coc \\
\hline Ts-32 coc \\
Ts-33 coc \\
Tc-34 coc \\
Ts-3 coc \\
Ts-36 coc
\end{tabular}

Fig. 5-106 Test 18: Temperatures at vertical tray

Test 18 (blind) : Gas concentrations

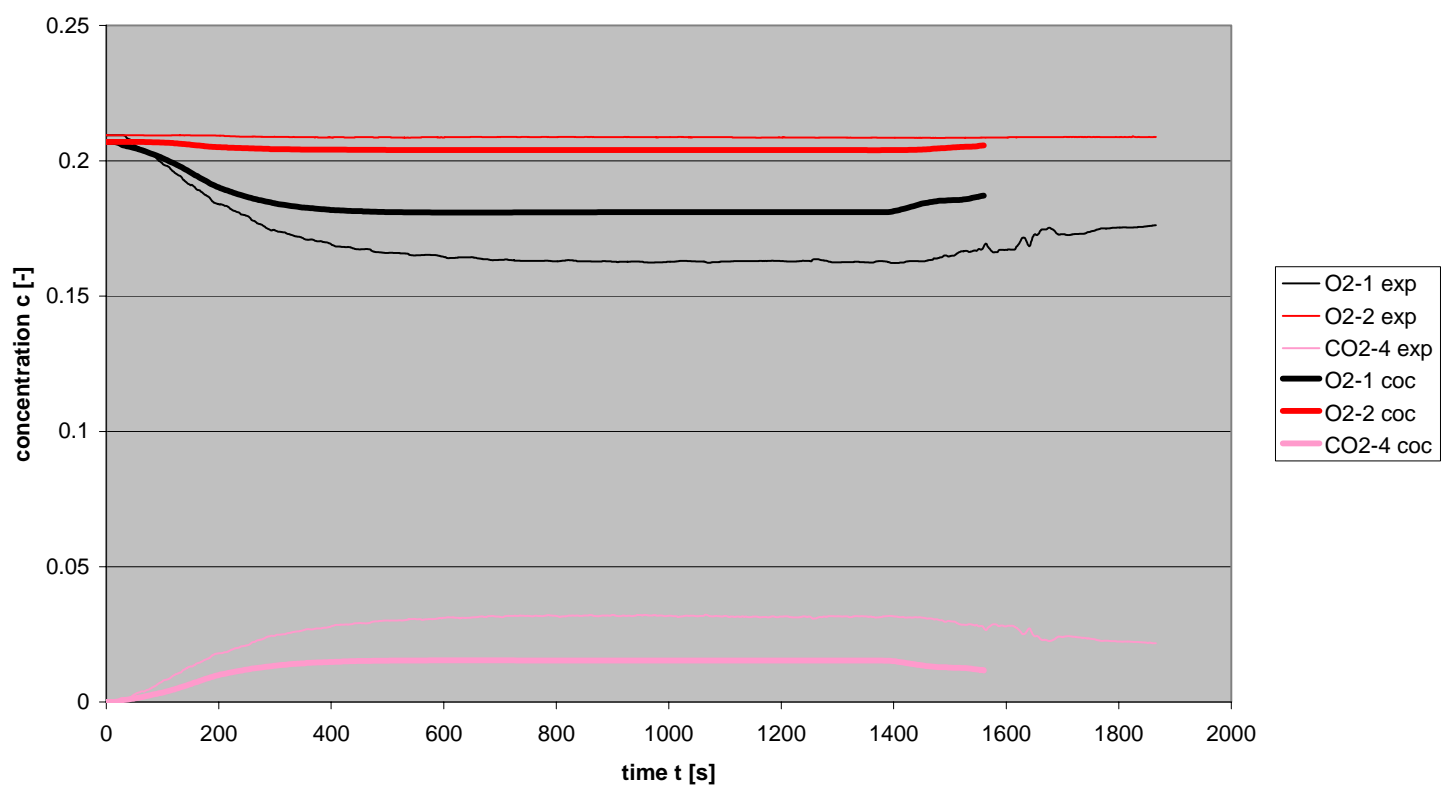

Fig. 5-107 Test 18: Gas concentration 


\subsection{Conclusions and Recommendations for COCOSYS}

Within the framework of ICFMP benchmark \#3 several blind and open calculations have been performed using COCOSYS. The pyrolysis model in COCOSYS is quite new. Therefore the possibility to perform a set of blind calculations was a good opportunity to check the quality of the models inside COcOSYS. The calculations have been performed in three steps: first blind calculations of test 2, 3 and 4 followed by corresponding open calculations. For these open calculations additional information (like fuel flow rate, heat release) was given. Further the radiation fraction has been specified in more detail. As far as possible COCOSYS has used all these specified material properties. The overall quality of the results is quite good, although due to some restriction inside the lumped-parameter concept of COCOSYS and missing models some deviations could be observed. In the following these are discussed in more detail:

- In the lumped-parameter concept it is possible to get more local information compared to zone models, but on the other side the momentum balance is not solved. This leads to some deviations mostly closed to the fire plume and close to possible ventilation jets. This could be observed especially for tests with running ventilation, although the volume flow rate should be clarified in more detail.

- Up to now COCOSYS did not have a fire plume model. Therefore test 18 shows some effects due to the less appropriate nodalization around the fire source. The fire source has been simulated by an injection on a 'pan' (structure surface). No spraying a somewhat higher location has been used. This may explain the deviations of the floor temperatures close to the fire.

- The targets were simulated by plate-type wall structures. The surface corresponds to the vertical projection of the tray or cable. The thickness to the material density. Some consistent deviation could be observed between control (mostly over-estimated temperatures) and power cable (mostly under-estimated temperatures). Here some rules are needed for the future, how to set up these kinds of targets.

- The velocity and temperature close to the door show the strong separation between the hot and cold gas layer. In COCOSYS eight layers of control volumes have been used. Using a 'fixed grid' some numerical diffusion will occur. Therefore the temperature profile is usually smoother in COCOSYS compared to the experiment. 
- The results for the junction box are completely wrong. Here some additional clarification is necessary.

In the future further model improvements are planned. This test series has provided information on what has to be improved. Further, the knowledge concerning interpretations of results has been increased. 
For the simulation of selected tests from Benchmark \#3, the code CFX 5.7 was used. CFX is a general purpose CFD code, which is not specially developed for fire simulation, offers however most of the features and models needed. Only for special results like sub-layer height is additional coding necessary. For this purpose a Fortran interface is provided to add or modify code models.

Between Benchmark \#2 and \#3 the code was completely rewritten and changed to a different internal structure. In this light the application to Benchmark \#3 experiments involves something like a test phase for CFX 5. Taking into account the higher computational effort compared with other codes, it was not possible to carry out parametric studies to improve results if necessary. The results presented in the following chapters were calculated with the code options valid also for other benchmarks. A number of results do not exhibit a satisfactory agreement with data. Work on improvement of results is continuing.

From the available test matrix the tests 2, 3 and 4 were simulated. These tests represent basic situations and are therefore of general interest.

\subsection{CFX Input Parameters and Assumptions}

The basic model developed for CFX is shown in Fig. 6-1. The model varies slightly for tests 2,3 and 4.

For all cases simulated some assumptions were made. In view of large compartments or arrangements of compartments to model, the surrounding walls were not included in the mesh explicitly. Instead, an extra model to simulate one-dimensional heat conduction was used as a boundary condition for CFX. With the intention to really save computing effort, the ceiling, the side walls and the floor are represented by a single solid temperature each. This may include some deficiencies in the representation of wall temperatures in the simulations.

A problem was detected concerning the location of the vertical ladder tray relative to the exhaust vent. In the specification report, the location of the ladder tray $G$ is indicated to be in the center of the north wall and is $0.3 \mathrm{~m}$ wide. This means, it extends from $10.68 \mathrm{~m}$ to 
$10.98 \mathrm{~m}$. The exhaust vent however, was specified to be located between $10.87 \mathrm{~m}$ and $11.57 \mathrm{~m}$. This would imply that tray and vent overlap. But this was not intended. The tray was consequently moved to a position close to the exhaust opening. As a result, the specified monitor locations for tray $G$ do no longer match. This involves some uncertainties for simulated temperatures.

In Fig. 6-1 the leakage area of the test room in the west wall is shown in green.

Not all targets specified were included in the mesh. Only the targets A, D and G are represented. The junction box was not included in the mesh.

The mesh is almost identical for all three simulated tests. Two cuts through the mesh are shown in Fig. 6-2. There are a total of 163411 cells. The mesh was developed as a mixture of structured and unstructured cells. Unstructured cells were grouped around the tiny cable targets and at some distance transferred into structured cells. This hybrid approach offers more flexibility in combining different length scales. However, the number of cells increases rapidly if unstructured cells are used. Although these cells represent only a small portion of the total volume, the ratio of structured to unstructured cells is 50649 to 112762 .

The mixture of air and fuel is modeled by the individual species, which are heptane (tests 2 to 4), oxygen, nitrogen, carbon dioxide and water vapor. Soot is also created according to the Magnussen soot model implemented in CFX. In this model a number of constants are used, which were not further investigated in the given context. Nitrogen represents a background gas, not participating in any reaction. The chemical reaction of heptane and oxygen $\left(\mathrm{C}_{7} \mathrm{H}_{16}+11 \mathrm{O}_{2}->7 \mathrm{CO}_{2}+8 \mathrm{H}_{2} \mathrm{O}\right)$ is represented by a single-step mixing controlled reaction within the Eddy Dissipation model. A predefined share of the reaction heat (44\%) is emitted from the fire tray as radiation flux and distributed by the P1 radiation model in CFX. This radiation model solves an extra transport equation and assumes direction independent radiation transport. It allows heating of the fluid due to radiation from a boundary (here the fuel pan). The fuel flow from the fire pan is prescribed according to the measured fuel consumption. The uncertainty in the measured heat release rate and the radiative fraction were not considered; instead the nominal values were used. The chemical reaction happens according to the mixing intensity in the fluid domain and the local availability of oxygen. For test 4 the flow through the supply opening was set according to the recorded test results. 
Instead of using the $15 \%$ oxygen fire termination threshold as for the tests, it was decided to use the recorded heptane consumption curves. This was done to avoid uncertainties in the heat release release.

\subsection{Comparison of CFX Predictions with Measurements}

\subsubsection{Test 2}

In Fig. 6-3 the heptane mass flow entered to the code is displayed. It follows the specification in the beginning section of the experiment and decreases to zero when the test is terminated because of oxygen decrease. Fig. 6-4 through Fig. 6-6 shows gas temperatures for trees 1,5 and 7 . There is a general under-estimation for all values. The calculated values do not include any radiative influence; the measured temperatures, however, are subject to this. Another influence on the calculation of gas temperatures may be the approximated temperature of the surrounding walls. The calculated total heat flow (convection and radiation) to the ceiling corresponds well with measured data, as Fig. 6-7 shows. A similar comparison for the floor heat flow in Fig. 6-8 reveals much less correspondence to the data. In the calculation considerably less energy flows to the floor. The radiative fraction of the heat release rate is emitted from the tray surface in the CFX model. In reality radiation is released from the flame above the fire tray, and more radiation is emitted towards the ground. In the model this is not possible as the tray surface has no direct view path to the floor.

Fig. 6-9 shows the $\mathrm{CO}_{2}$ history in the test compartment. For a specific time $600 \mathrm{~s}$ after initiation of the fire the distribution of $\mathrm{CO}_{2}$ in the test facility is depicted in Fig. 6-10. All along the floor there is a layer with very low $\mathrm{CO}_{2}$. The oxygen history in Fig. 6-11 reveals that this species decreases too fast. The spatial distribution at $600 \mathrm{~s}$ is shown in Fig. 6-12. Lowest values are found directly below the ceiling and highest close to the floor. It may be that the boundary between high and low oxygen in the simulation is slightly displaced compared with the measurement, resulting in a lower calculated values. The time $600 \mathrm{~s}$ belongs to the period when the fire is still at full power. Shortly after at $700 \mathrm{~s}$ the fire is extinguished because of low oxygen. Then the leak flow reverses and through the leakage opening fresh air enters the compartment. This is displayed in Fig. 6-13. The leakage mass flow over time (Fig. 6-14) exhibits the same tendency. With the extinction of the fire the heat source collapses, the pressure decreases and sucks fresh air from outside into the fire lab. 
Fig. 6-15 depicts the flow distribution in the facility short before termination of the experiment. Close to the ceiling there is an expansion flow away from the fire pan, in the lower section it is directed towards the fire. Underneath the expansion flow from the fire along the ceiling there is a layer of gas flowing backwards to the fire.

\subsubsection{Test 3}

Calculation results for test 3 show more severe disagreements with measurement but they presented for completeness. Work for improvement is continuing concerning radiation modelling and wall representation.

Test 3 uses ventilation through an open door. In order to realistically calculate the flow across this opening, an extra block was added to the model of the fire lab. The connection to environment by means of a pressure boundary is thereby moved away from the door, where uniform pressure is more realistic. The model together with cuts through the mesh is shown in Fig. 6-17 and Fig. 6-18. The leakage opening right from the door in the same wall is kept open as for the other tests. If this affects the flow in and out of the door was not investigated.

The heptane release and consumption which was input to the code is shown in Fig. 6-19. This curve corresponds to a nominal fire power of $1150 \mathrm{~kW}$.

A temperature distribution in the fire lab at $1000 \mathrm{~s}$ is displayed in Fig. 6-20. The simulation time at $1000 \mathrm{~s}$ has been selected because it is well placed in the long steady state period developed for this experiment and will also be used for other 2D distributions. Temperatures along trees 1, 5 and 7 are shown in Fig. 6-21 through Fig. 6-23. Unlike simulations for tests 2 and 4 temperatures are over-predicted by CFX. This behavior is still under investigation. It is possible that under fully ventilated conditions (with enough oxygen in all locations) the simulated reaction rate is higher than measured and under locally reduced oxygen availability it is too low. The oxygen history at probe location 02-2 is depicted in Fig. 6-24. The simulation predicts a slight decrease which was not measured. The 2D distribution for oxygen in Fig. 6-25 indicates quite uniform availability. A slight enrichment is found close to the floor due to inflow from the door. The opposite behavior as for oxygen is found for the $\mathrm{CO}_{2}$ production at the available probe location $\mathrm{CO}_{2}-4$ (Fig. 6-26). Here the simulation calculates, at least at the given probe location, a lower molar fraction. 
The door flow can be characterized by the flow velocity size and orientation. Selected velocity probes in the middle plane of the door are displayed and compared with CFX predictions in Fig. 6-27. In the simulation the inwards directed flow (low elevation, blue) is calculated at a higher speed than measured. In the middle location $(z=1 \mathrm{~m})$ CFX calculates a weak outflow whereas from the measurement inflow can be seen. There may be some uncertainty in the location of the boundary between positive and negative velocities, which is quite sensitive to predict. The outflow velocity at $1.79 \mathrm{~m}$ is well predicted. A qualitative velocity distribution of flow velocities can be seen in Fig. 6-28. The net mass flow through the door is close to zero for most of the time. This is also predicted by CFX (Fig. 6-29), although the initial outflow peak is almost completely missing. It should be mentioned, that flow through the neighboring leak opening is also enabled for this experiment. It may have some effect on the door flow.

\subsubsection{Test 4}

The test 4 model is shown in Fig. 6-30. The supply and exhaust openings in the model were extruded outwards. This eases the formation of a stable flow profile in the supply opening and results in a more uniform pressure distribution in the exhaust opening. This corresponds better to the pressure condition available in CFX for openings. Two cuts through the computational mesh are shown in Fig. 6-31. Some sections around the horizontal trays are meshed with unstructured cells, most parts of the test facility contain structured cells.

The heptane flow released for reaction in the gas space is shown in Fig. 6-32. After about $814 \mathrm{~s}$ the fire is stopped because of oxygen consumption below $15 \%$ at location $02-2$. Fig. 6-33 through Fig. 6-37 display gas temperatures at three trees. Similar to the simulations of tests 2 and 3 gas temperatures are under-predicted. This may be due to the fact that in the simulation only convection contributes to the gas heat up. Thermocouples instead are affected by radiation. In Fig. 6-34 temperatures from tree 5 are depicted. The temperature in position 5 (red) is very low. This is due to cold air coming through the supply opening. The corresponding flow pattern and temperature distribution in the whole test facility is shown in Fig. 6-35 at time $700 \mathrm{~s}$. A vertical temperature profile along tree 5 is also added to this figure. The measurement however shows the characteristic decrease due to cold air inflow at a more elevated location. Fig. 6-36 compares calculated and measured temperature profiles. The experimental profile has the cold air temperature minimum above $2.5 \mathrm{~m}$ whereas the calculation predicts it at about $2 \mathrm{~m}$. This difference may be related to the lower calculated gas temperatures in general. A smaller difference to the environmental temperature means less buoyancy and consequently a stronger fall-down of the incoming air flow. 
The calculated oxygen decrease in the test lab is compared to available test data in Fig. 6-38. Fig. 6-39 was added to illustrate the oxygen distribution at different 2D planes. The selected time (700 s) is representative for most of the experiment and shows a continuous increase towards the floor. In some sections of the test facility more oxygen is found on the side of the supply opening. A comparison of the $\mathrm{CO}_{2}$ formation at location $\mathrm{CO}_{2}-4$ can be found in Fig. 6-40. Oxygen and $\mathrm{CO}_{2}$ behave in a consistent way: Slightly less oxygen is consumed and less $\mathrm{CO}_{2}$ is built up.

Fig. 6-41 and Fig. 6-42 summarize the total energy flows to ceiling and floor. Radiation plays with $44 \%$ of the total energy release an important role. Radiation is emitted from the plane fire tray surface and behaves therefore different from reality. The ceiling receives the correct energy flow (Fig. 6-41). Side walls and in particular the floor, however, do not receive enough radiation. This is reflected in Fig. 6-42.

The mass flow into the test lab through the supply opening was specified according to the recorded data from the test. The exhaust flow was calculated. Fig. 6-43 compares both flows. The exhaust flow compares qualitatively well with the data but under-estimates the values slightly. An illustration of the flow distribution at selected planes is shown in Fig. 6-44. Highest speeds can be found along the ceiling. There is also a layer of higher flow speed in the middle of the test lab. 


\subsection{Conclusions and Recommendations for CFX}

For the simulated test cases (2, 3 and 4) the agreement with measured data is worse compared with other benchmark exercises of the ICFMP. A main reason is seen in the way the radiative fraction of the reaction heat is released. For simplicity this was done from the surface of the fire tray. This location involves incorrect radiative fluxes to side walls and floor. Lower wall temperatures are the result. In turn this involves higher convective heat fluxes from the gas and lower gas temperatures. Also, the surrounding walls were not included in the mesh in order to limit the computing effort. Instead, a one-dimensional algebraic heat conduction model was applied but only to larger patches. An improvement can be achieved by calculating extra wall (surface and inner sections) temperatures for each individual fluid cell.

In a further step, the data obtained from the experiments can be used to validate constants to be set in the Magnussen soot model available in CFX.

\section{Acknowledgement}

The presented work was funded by the German Ministry of Environmental Protection and Radiological Protection (BMU), which is gratefully acknowledged. 
BM3: Heptane Fire in a Compartment
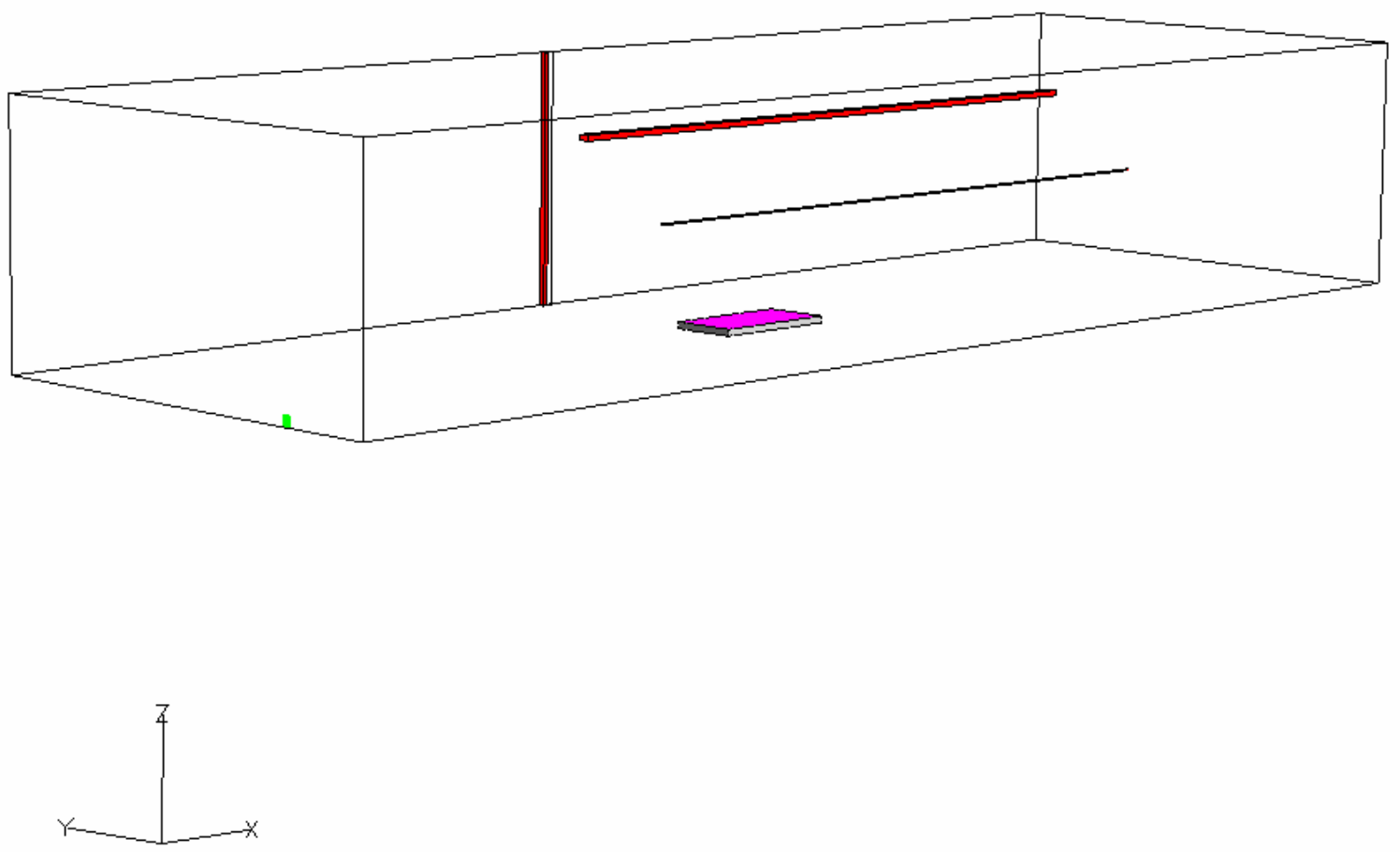

Case 2

Fig. 6-1 CFX model for test 2 
BM3: Heptane Fire in a Compartment
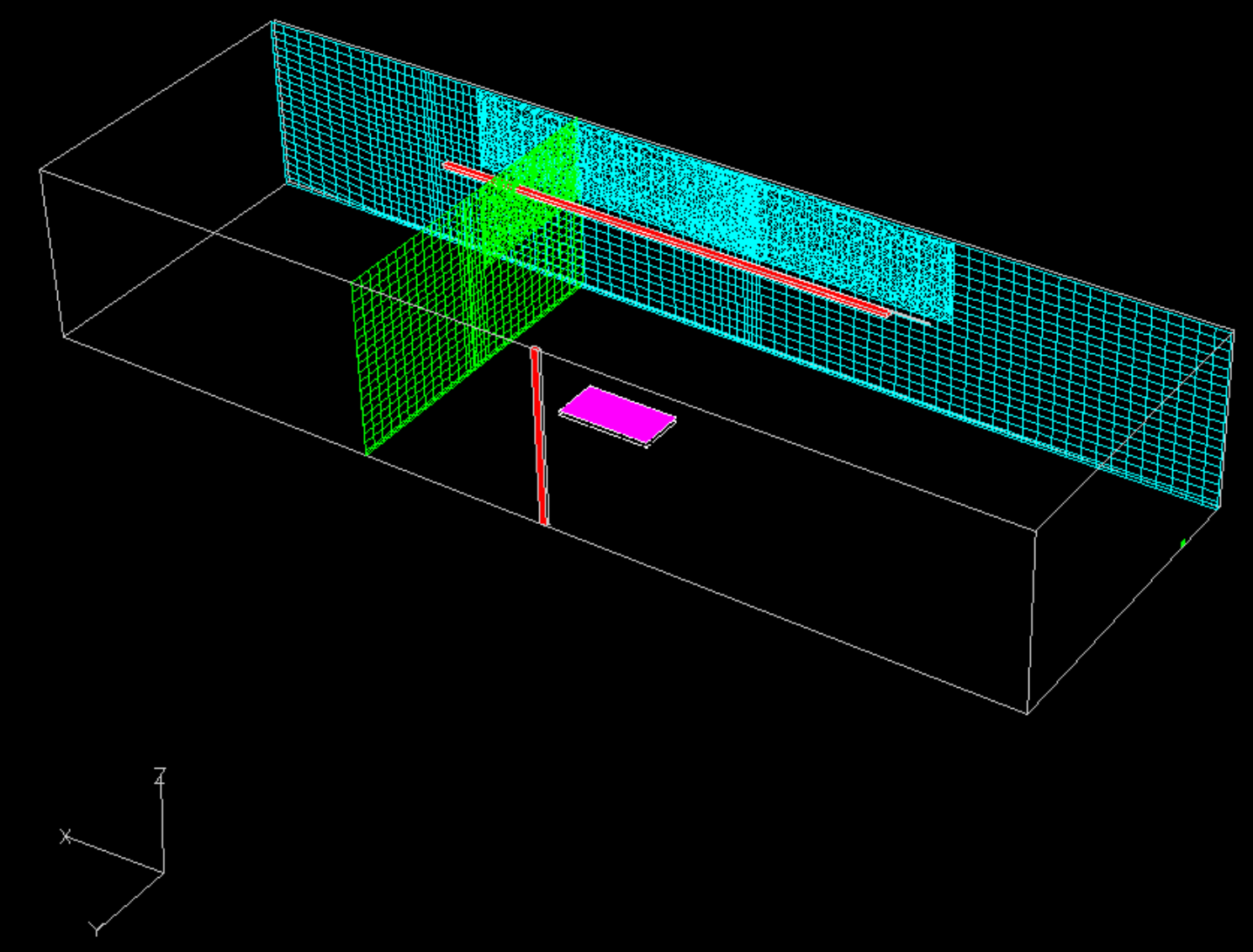

Case 2

Fig. 6-2 Distribution of cells within the test facility 


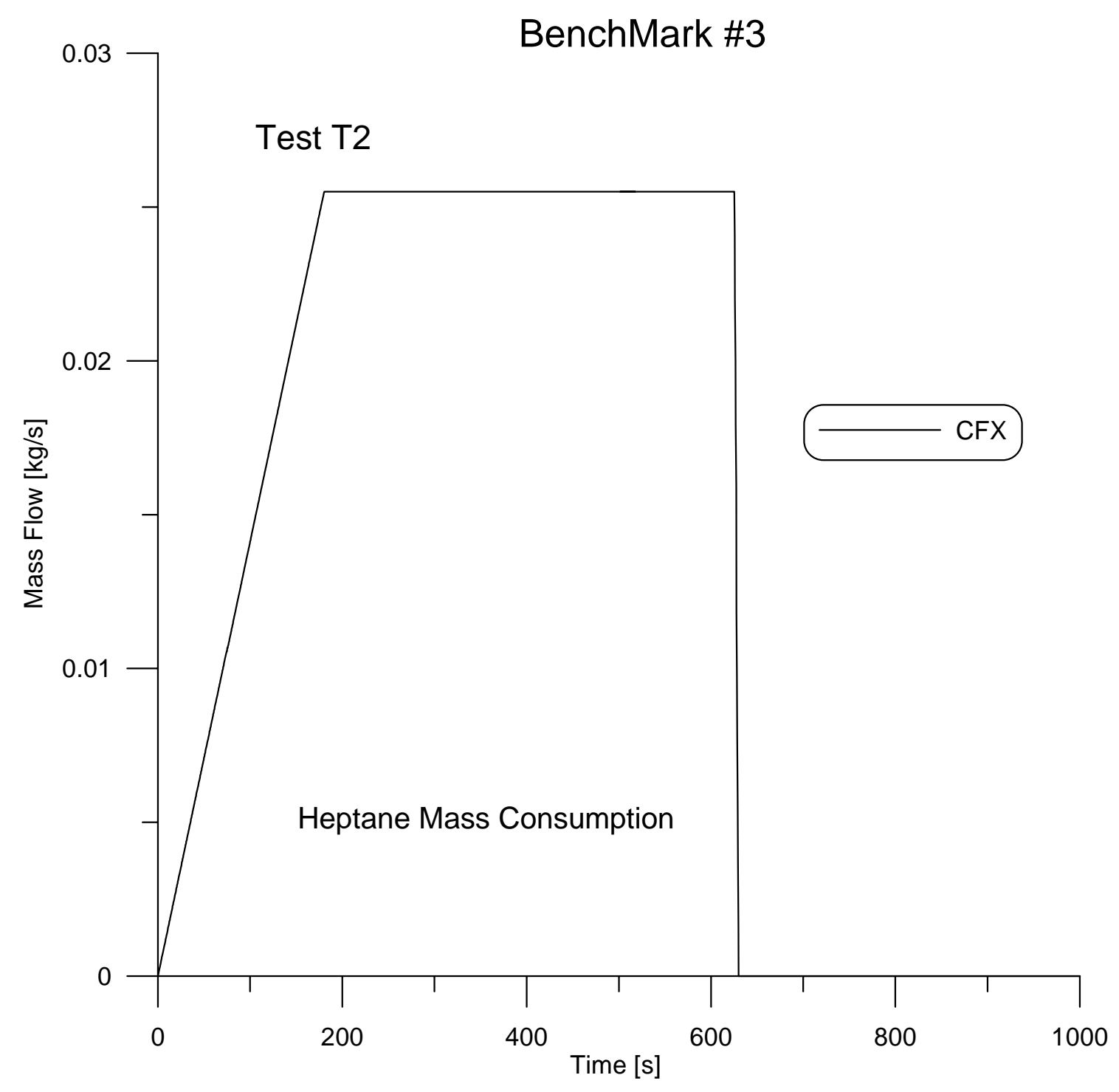

Fig. 6-3 Heptane mass release (test 2) 


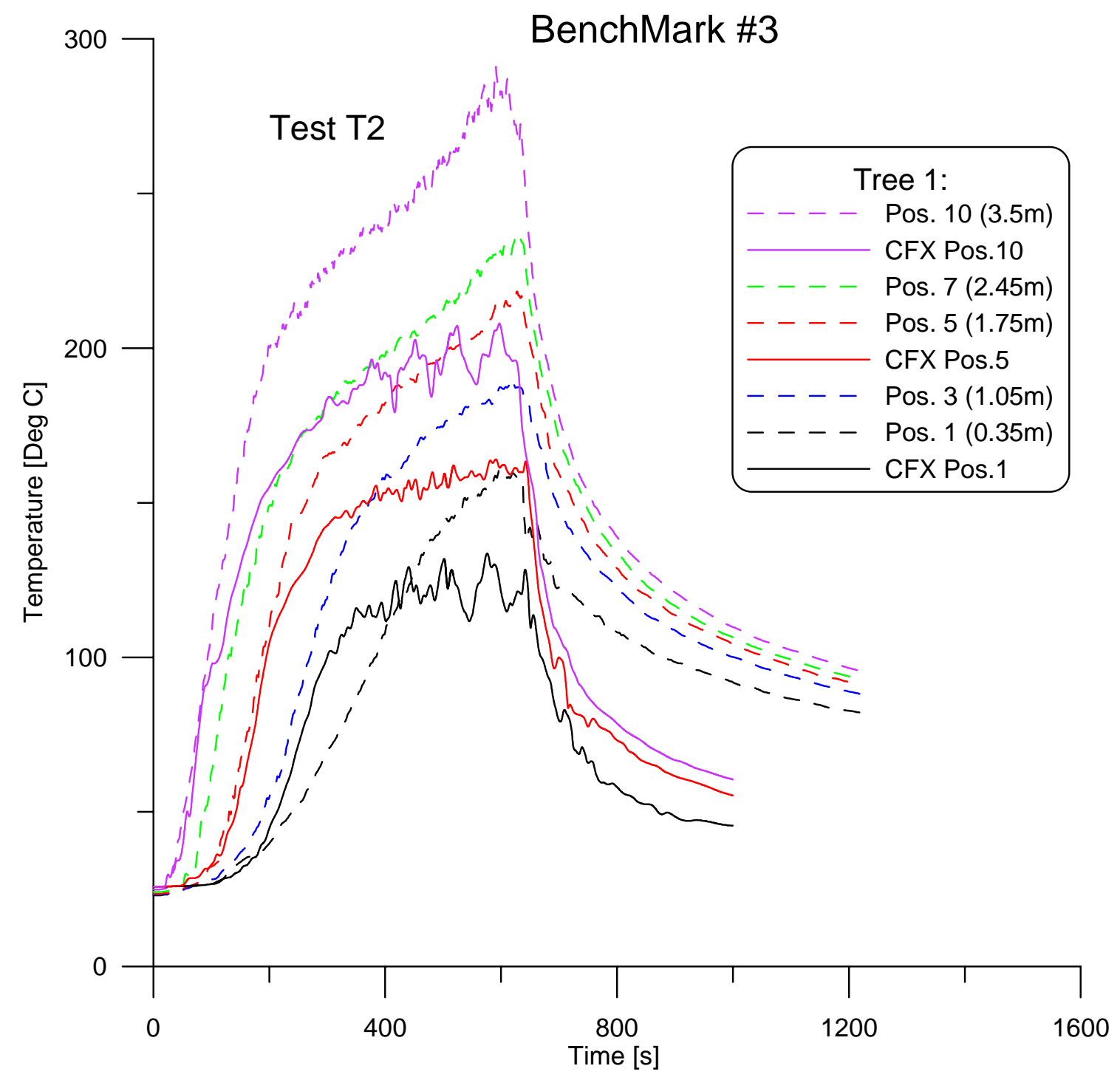

Fig. 6-4 Selected temperatures in tree 1 (test 2) 


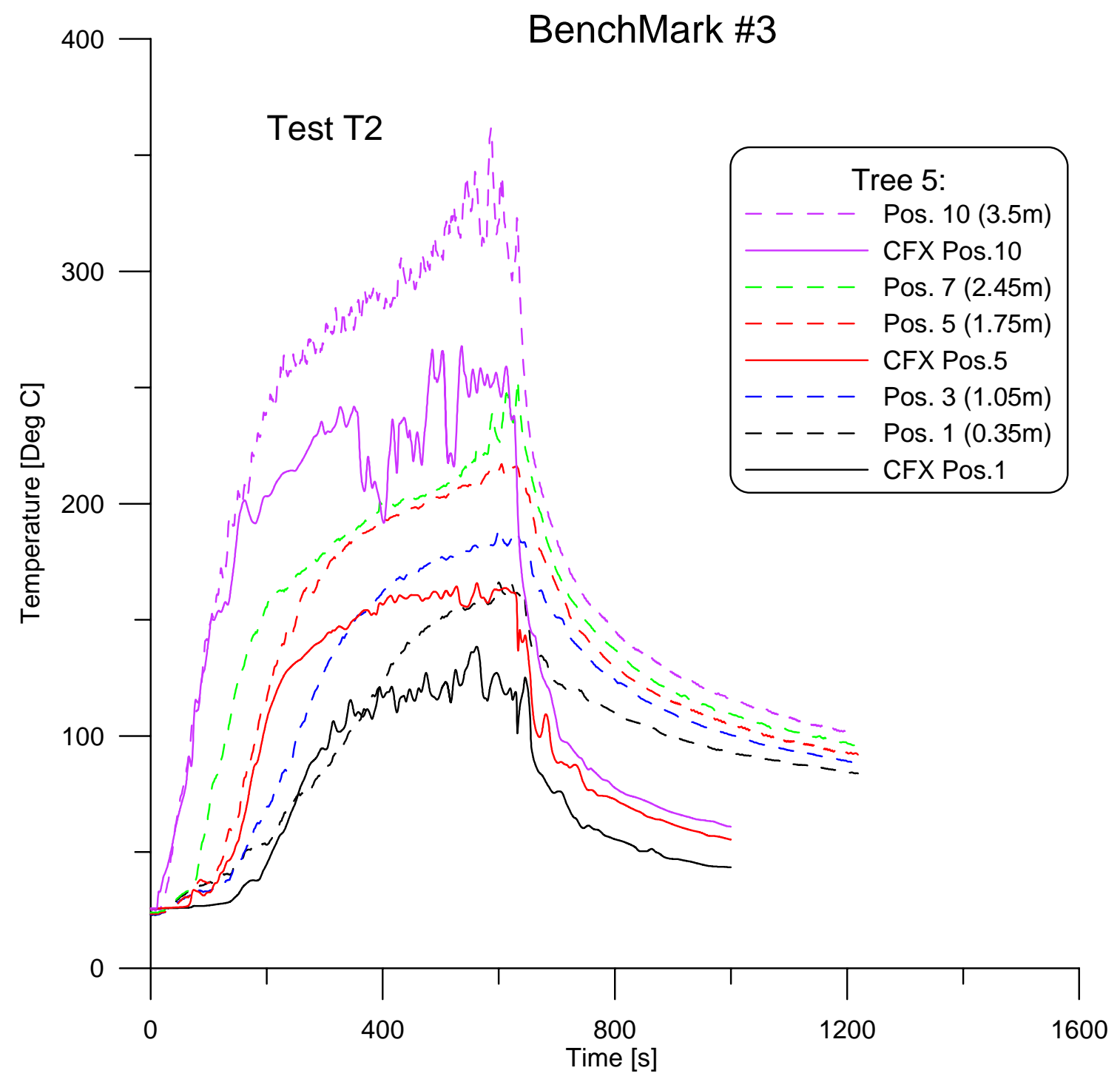

Fig. 6-5 Selected temperatures in tree 5 (test 2) 


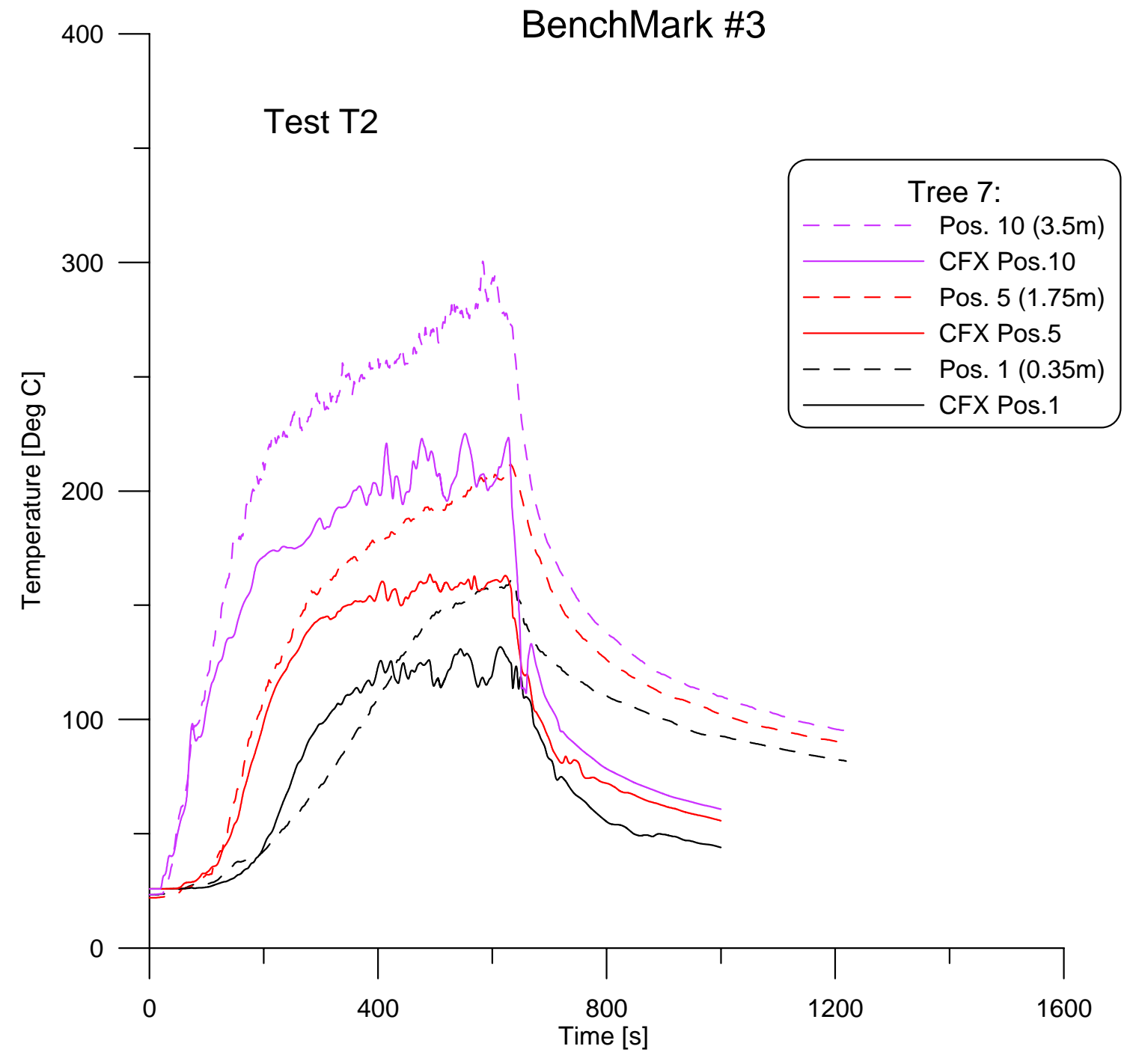

Fig. 6-6 Selected temperatures in tree 7 (test 2) 


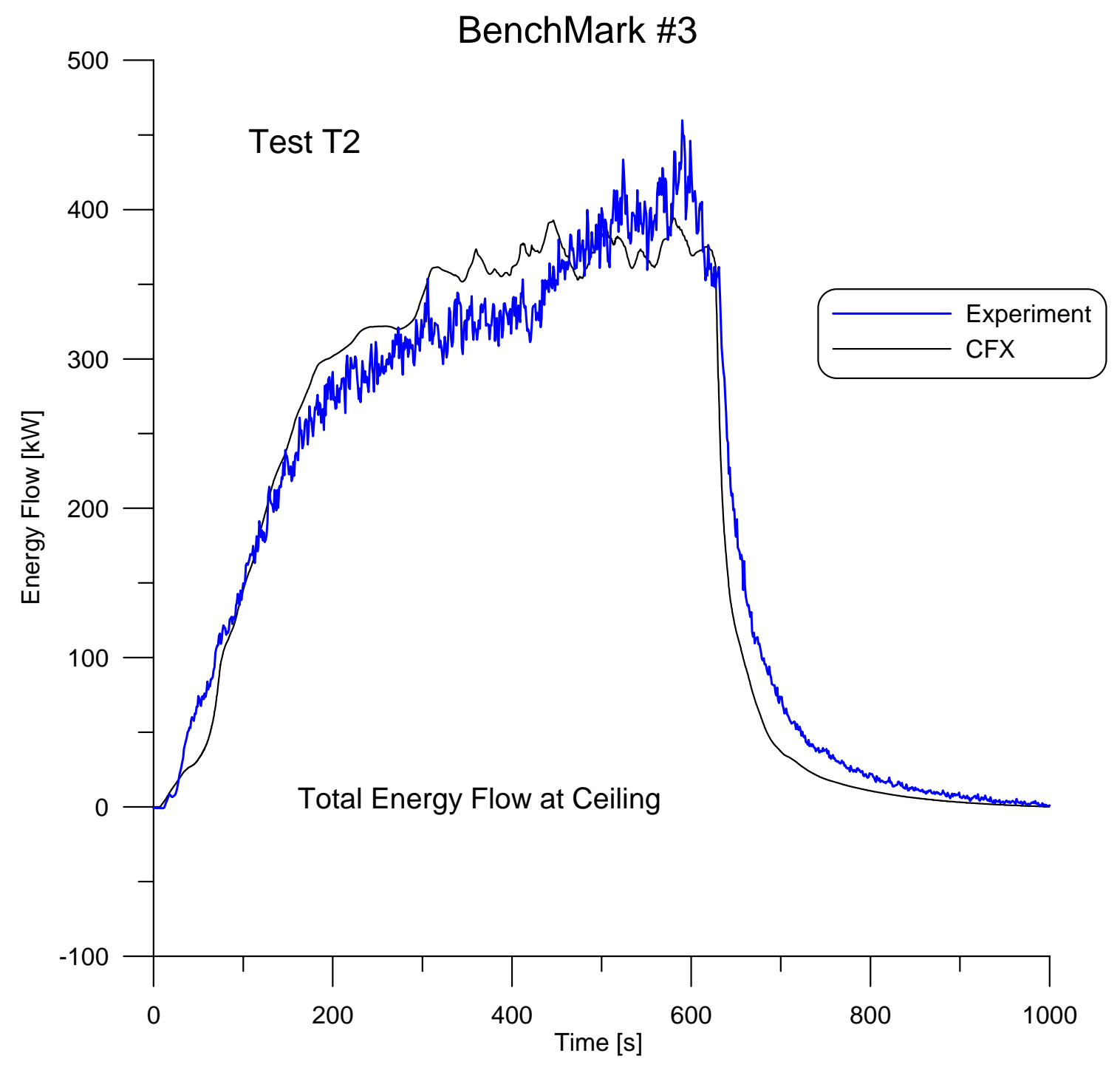

Fig. 6-7 Total energy flow to ceiling (test 2) 


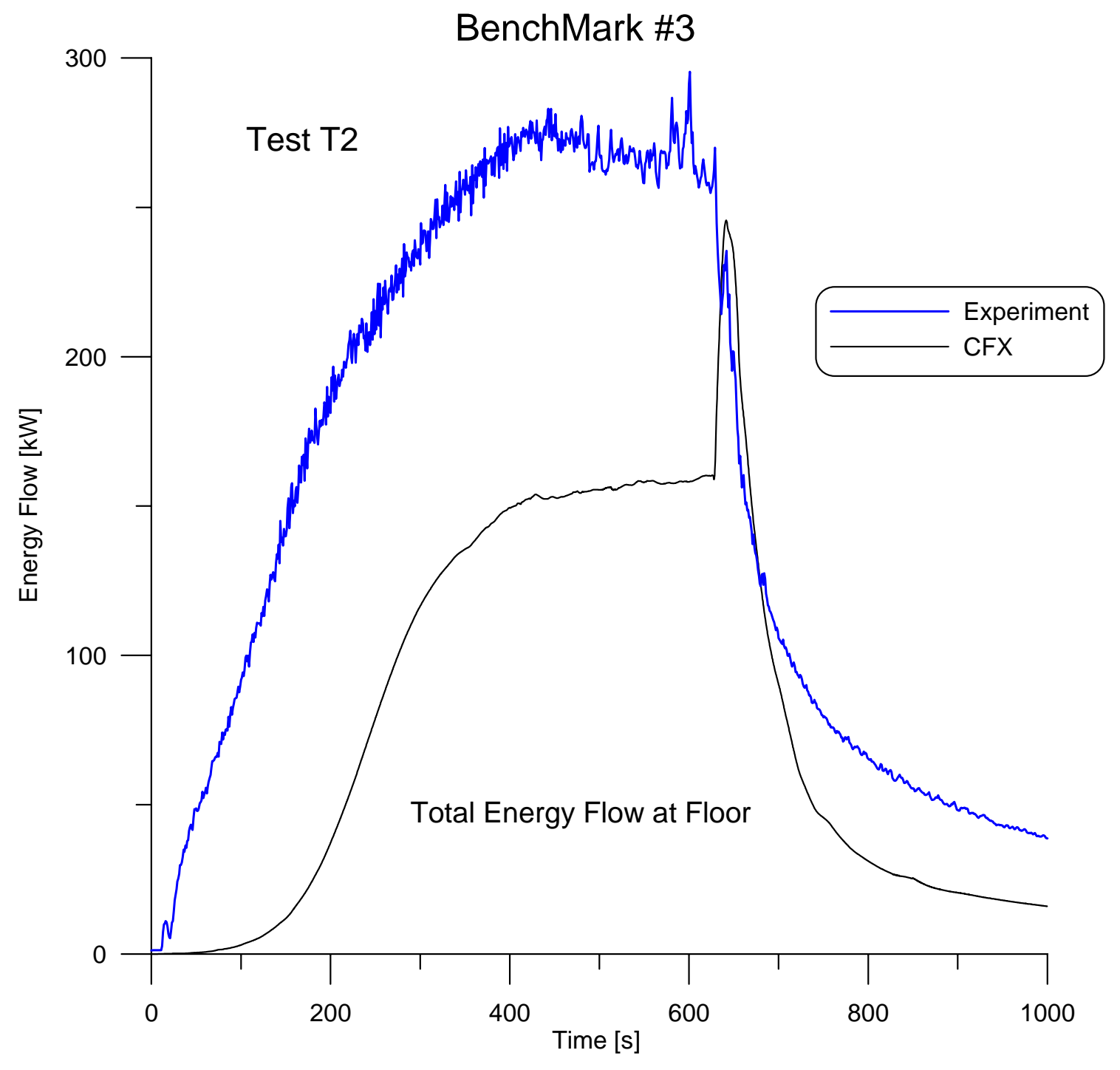

Fig. 6-8 Total energy flow to floor (test 2) 


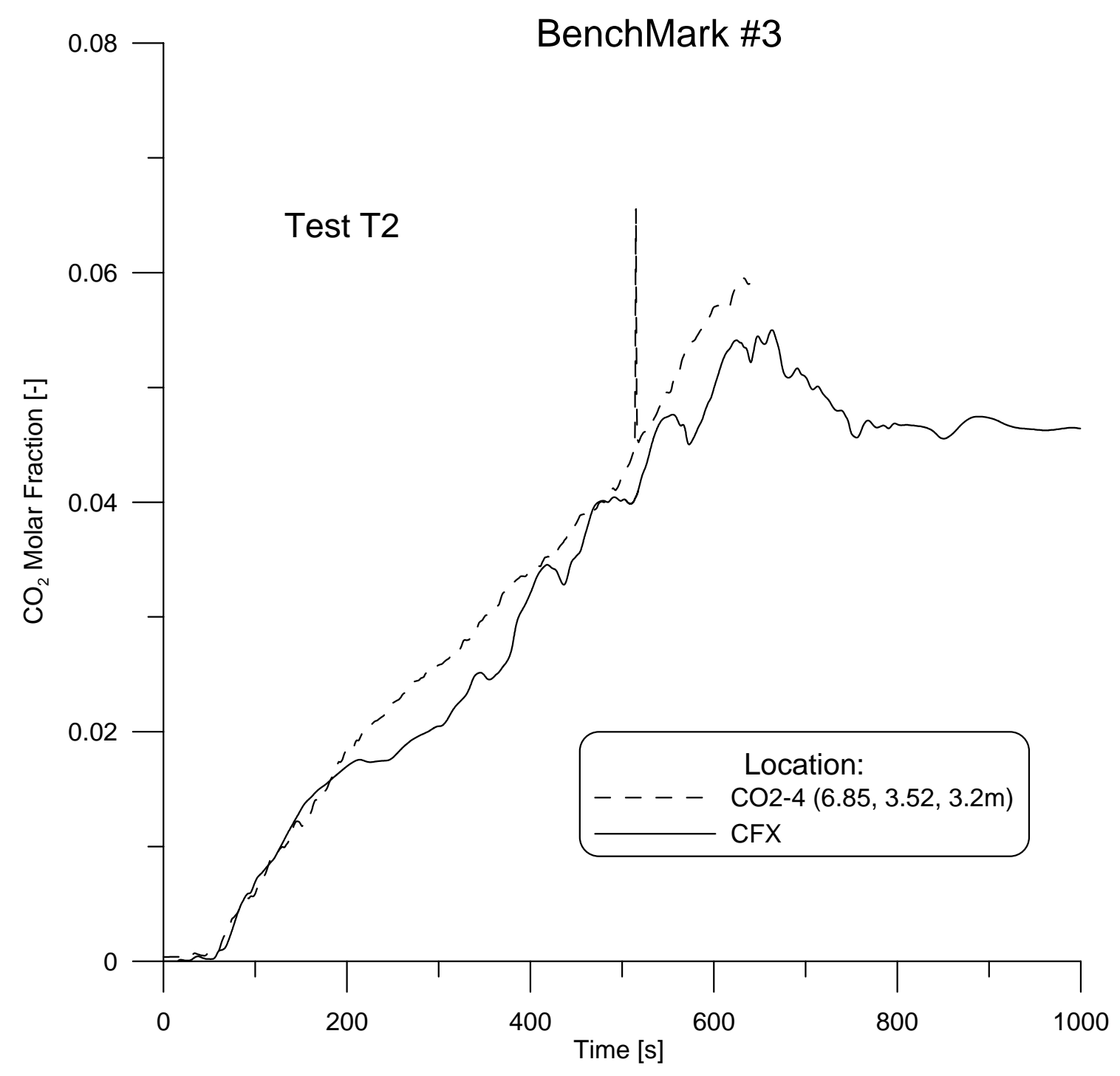

Fig. 6-9 $\quad \mathrm{CO}_{2}$ history at probe location 4 (test 2) 
BM3: Heptane Fire in a Compartment

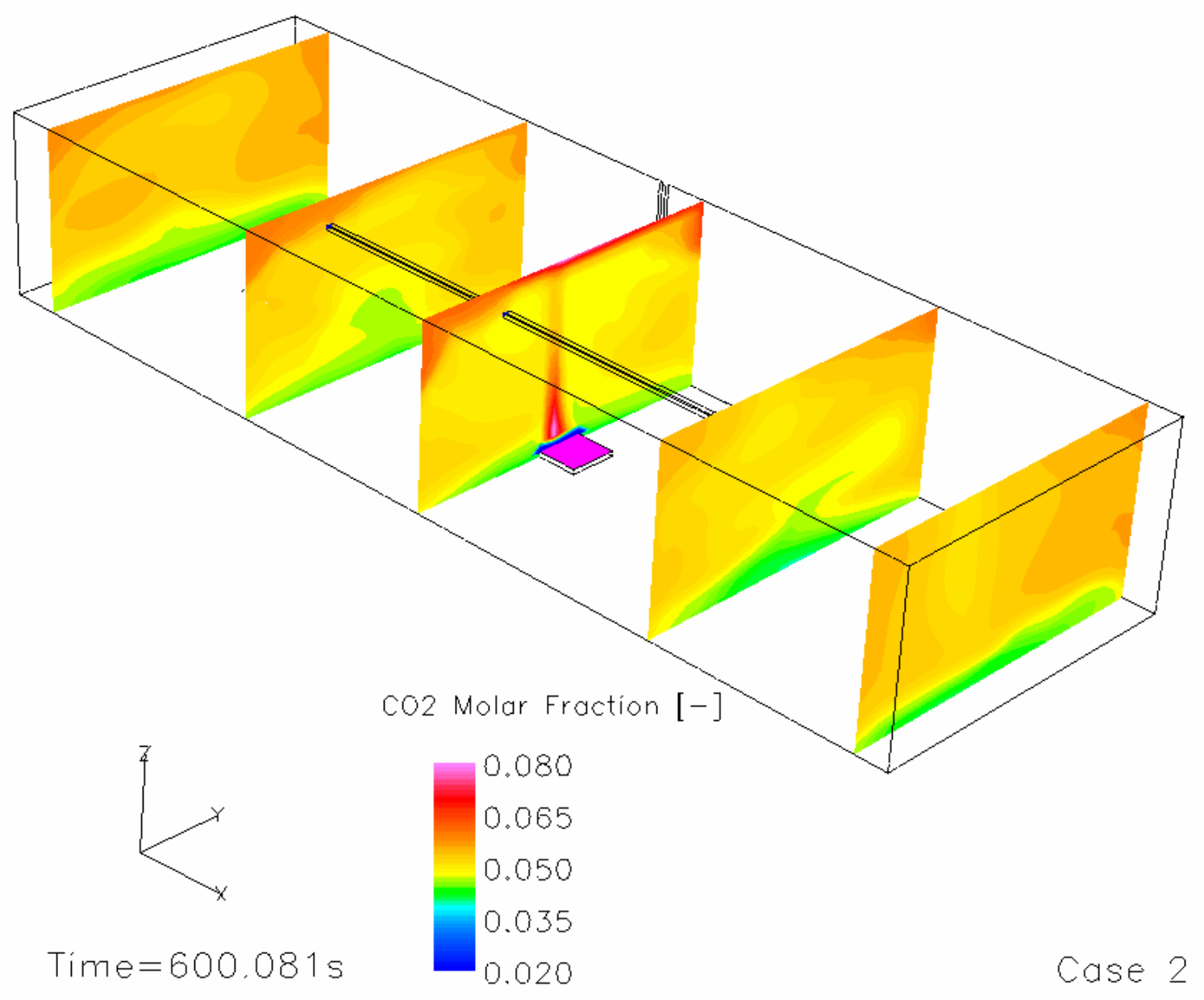

Fig. 6-10 $\mathrm{CO}_{2}$ distribution at different locations after $600 \mathrm{~s}$ (test 2) 


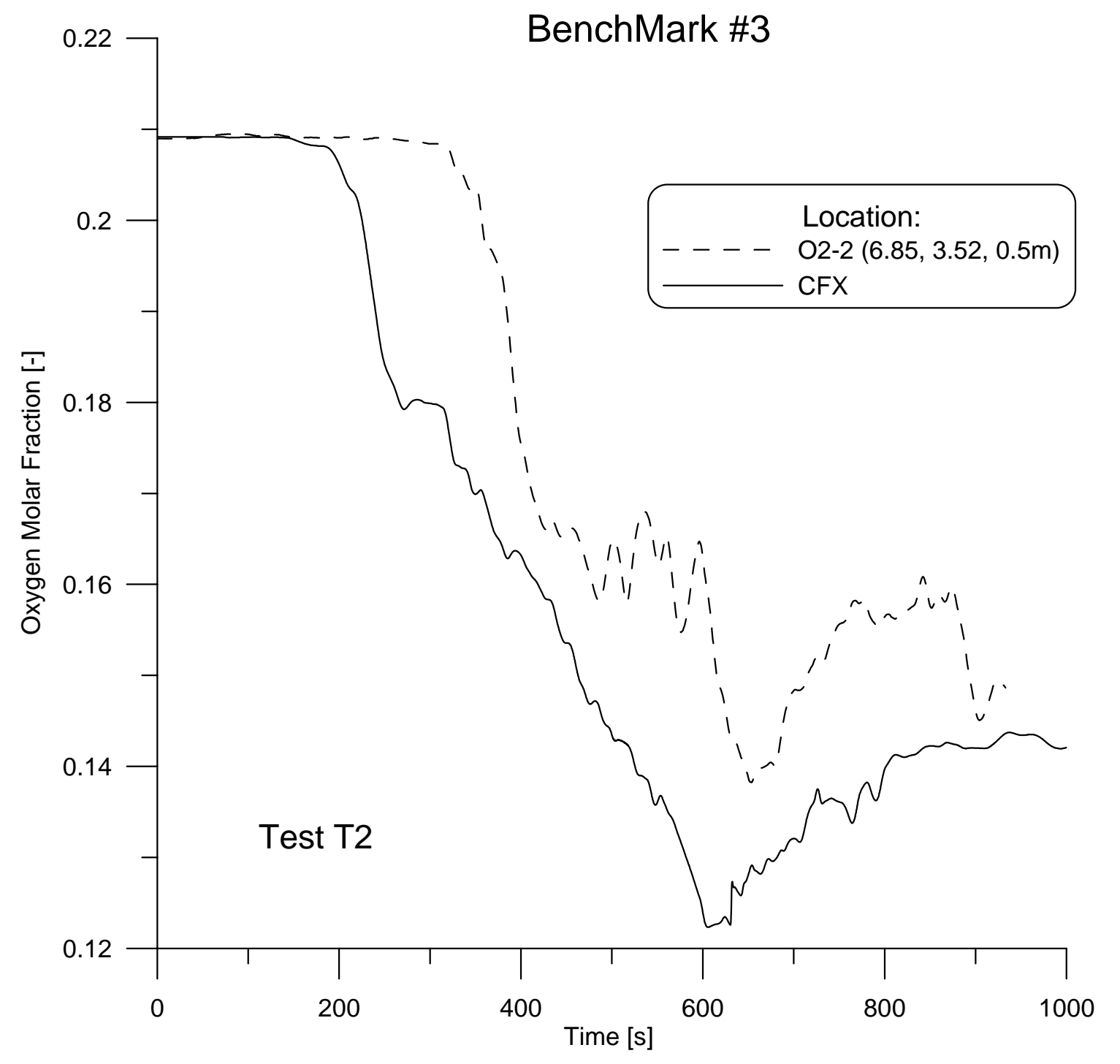

Fig. 6-11 Oxygen history at lower probe location (test 2) 
BM3: Heptane Fire in a Compartment

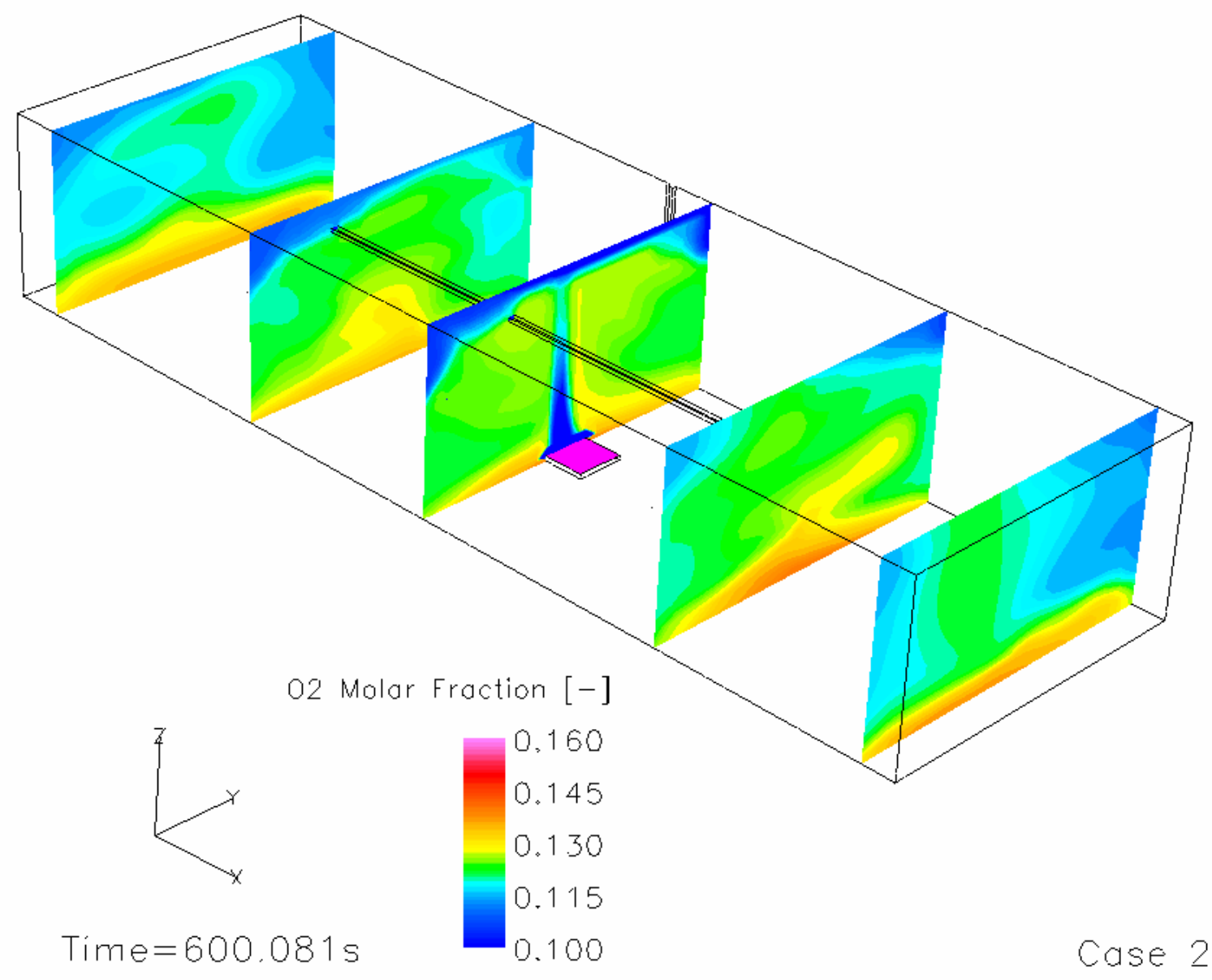

Fig. 6-12 Oxygen distribution at different locations after $600 \mathrm{~s}$ (test 2) 
BM3: Heptane Fire in a Compartment

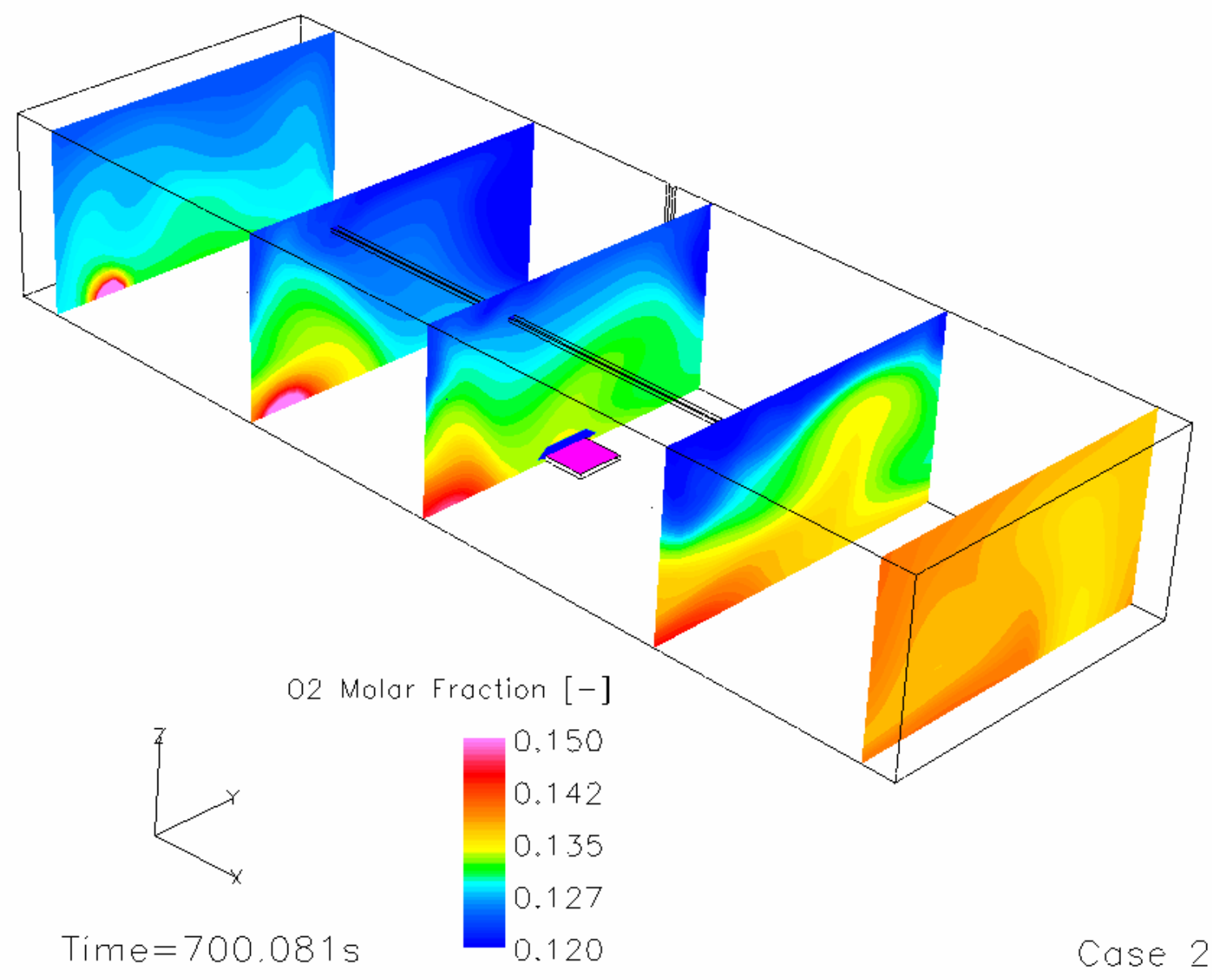

Fig. 6-13 Oxygen distribution at different locations after $700 \mathrm{~s}$ (test 2) 


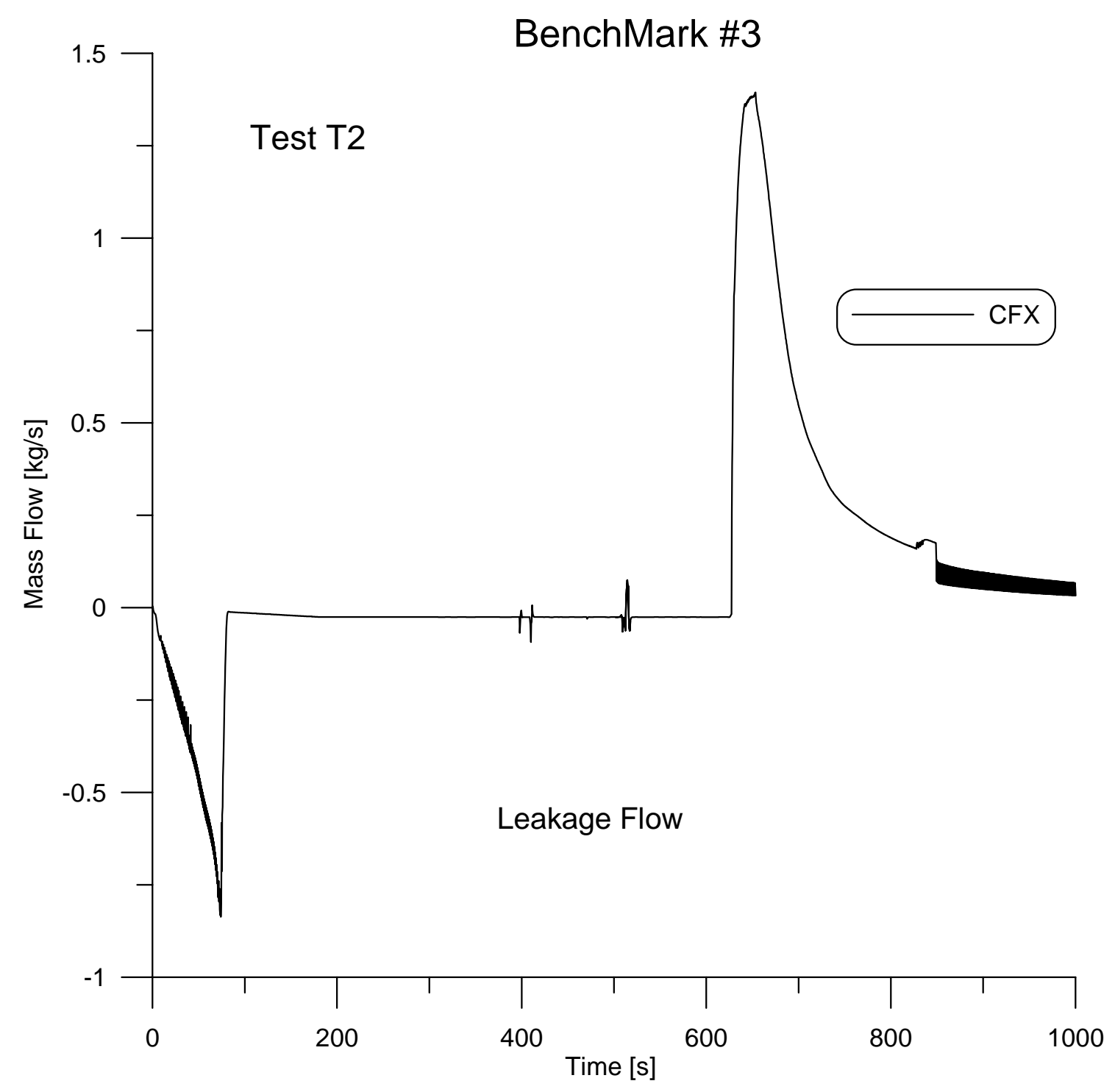

Fig. 6-14 Mass flow through leakage opening (test 2) 
BM3: Heptane Fire in a Compartment

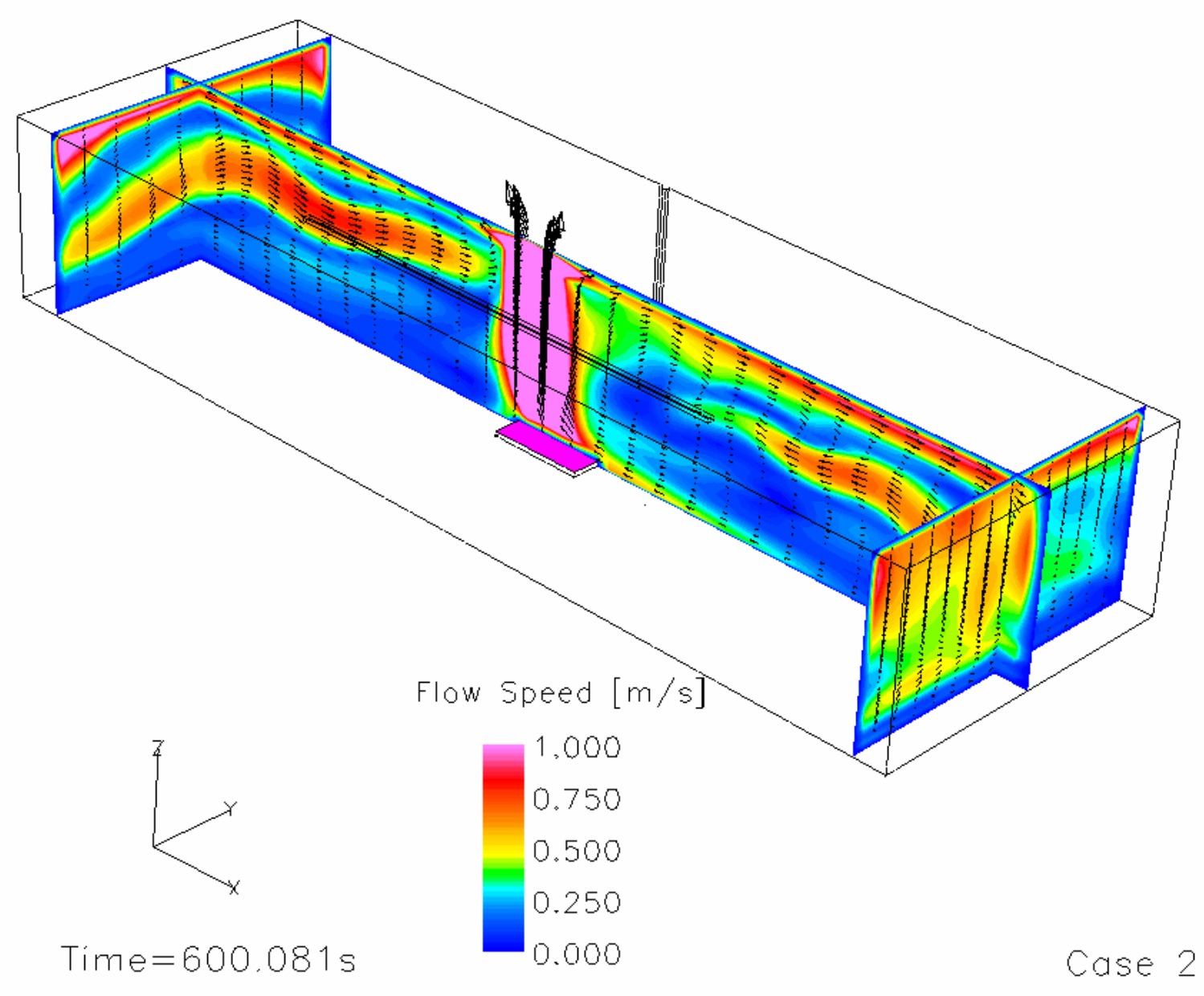

Fig. 6-15 Flow speed distribution in the test facility after $600 \mathrm{~s}$ (test 2) 


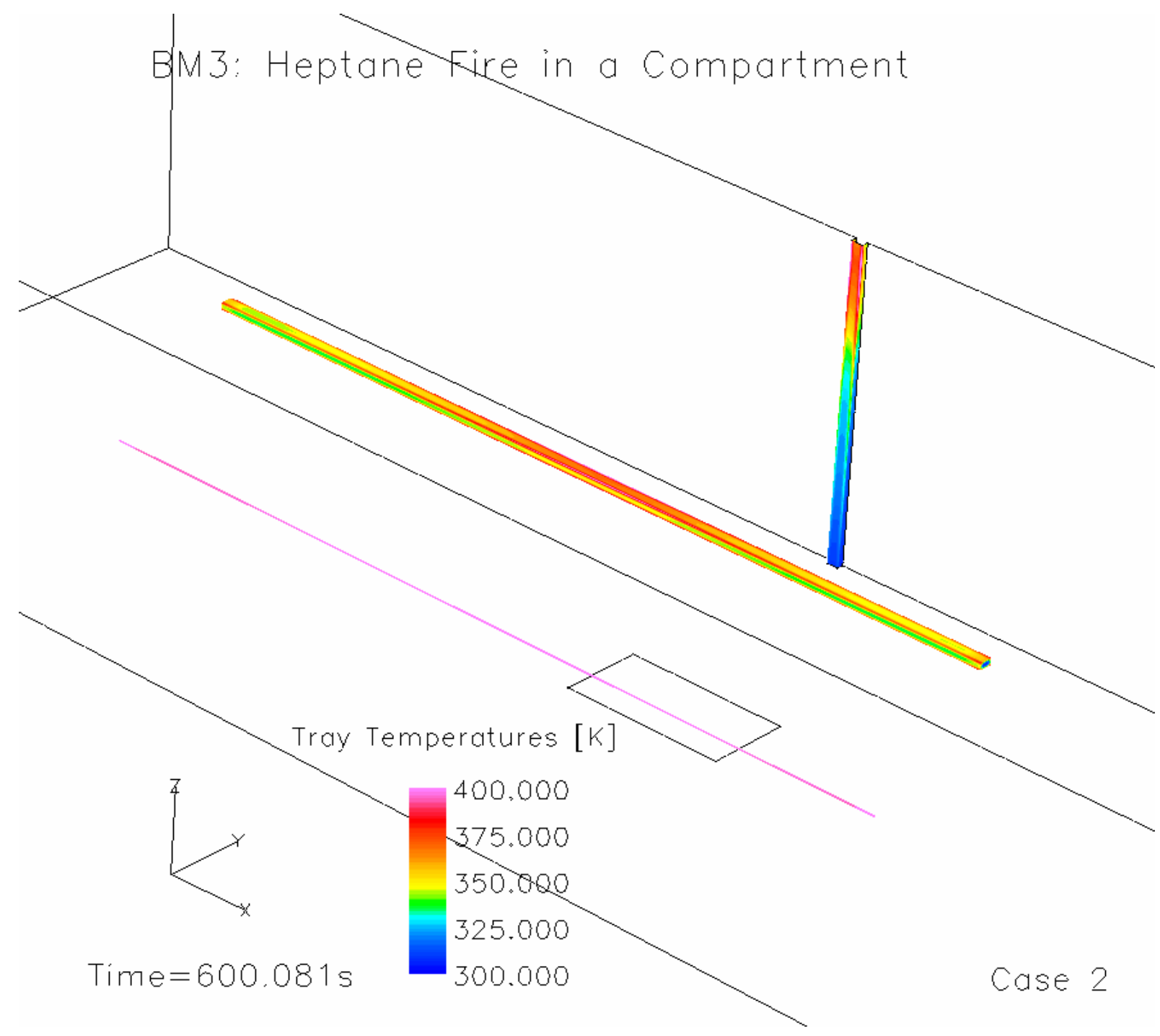

Fig. 6-16 Temperature distribution along trays D, F and G (test 2) 
BM3: Heptane Fire in a Compartment
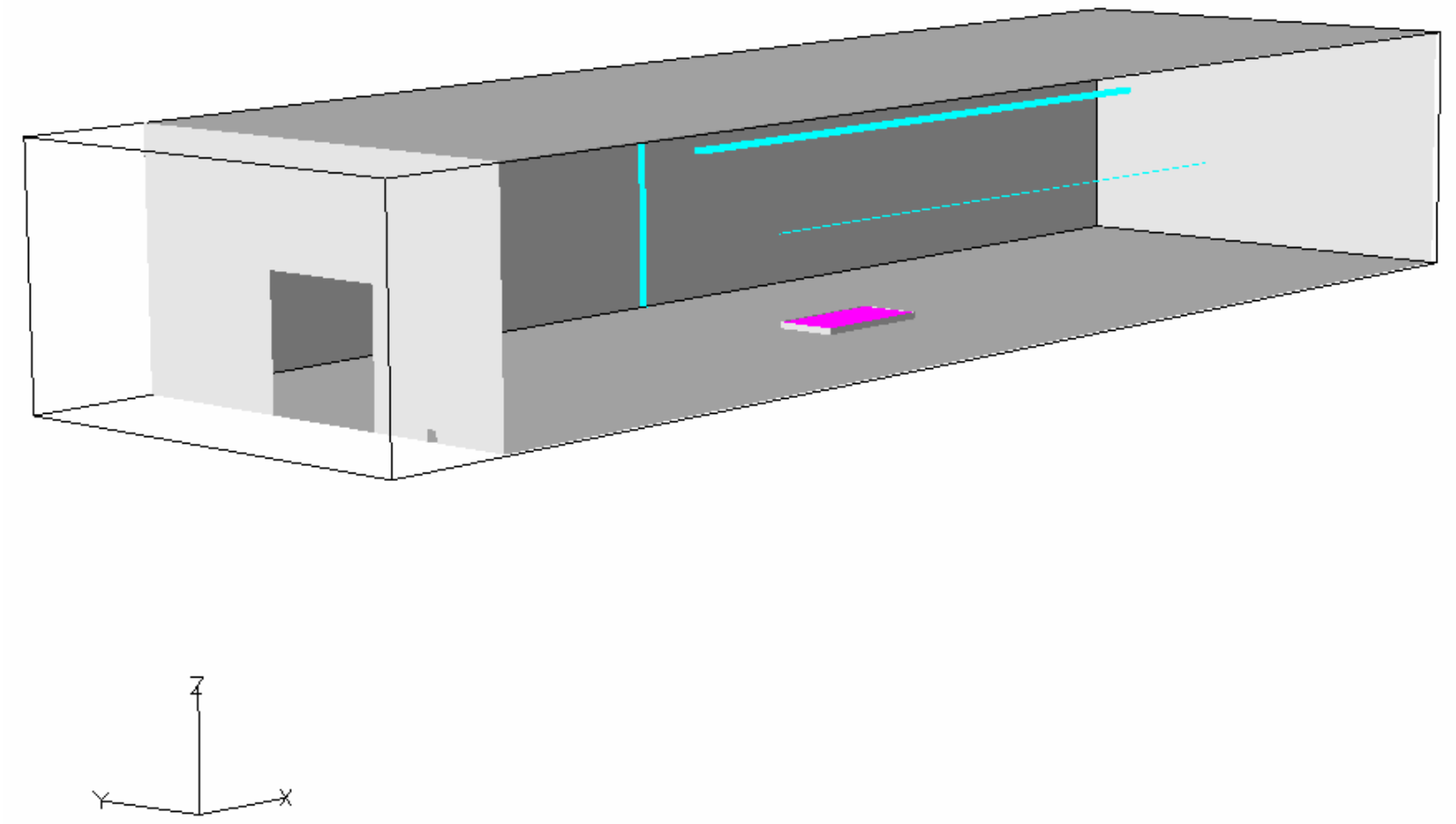

Test $\mathrm{C} 3$

Fig. 6-17 CFX model for test 3 
BM3: Heptane Fire in a Compartment

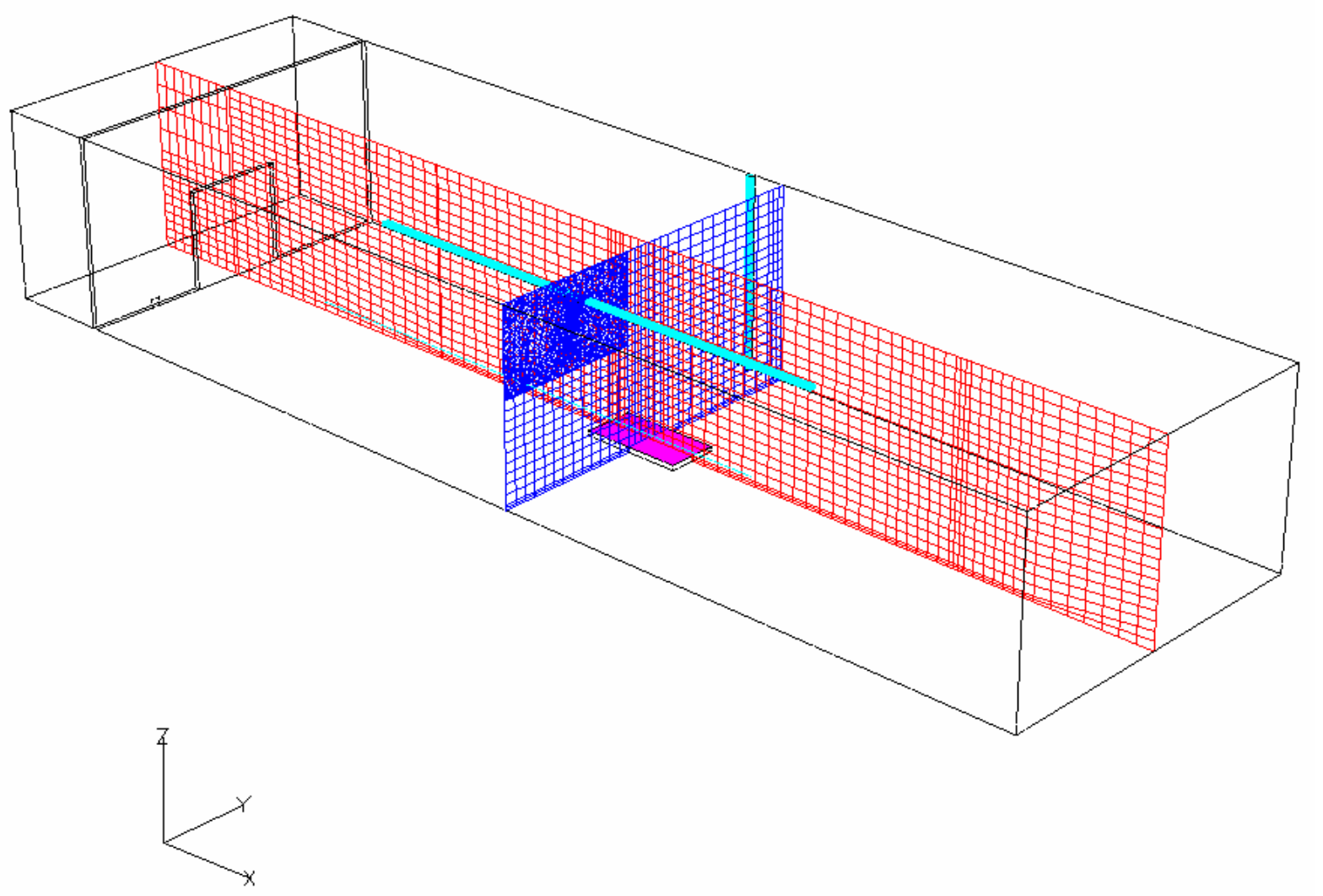

Test $\mathrm{C} 3$

Fig. 6-18 Cuts showing the mesh for test 3 


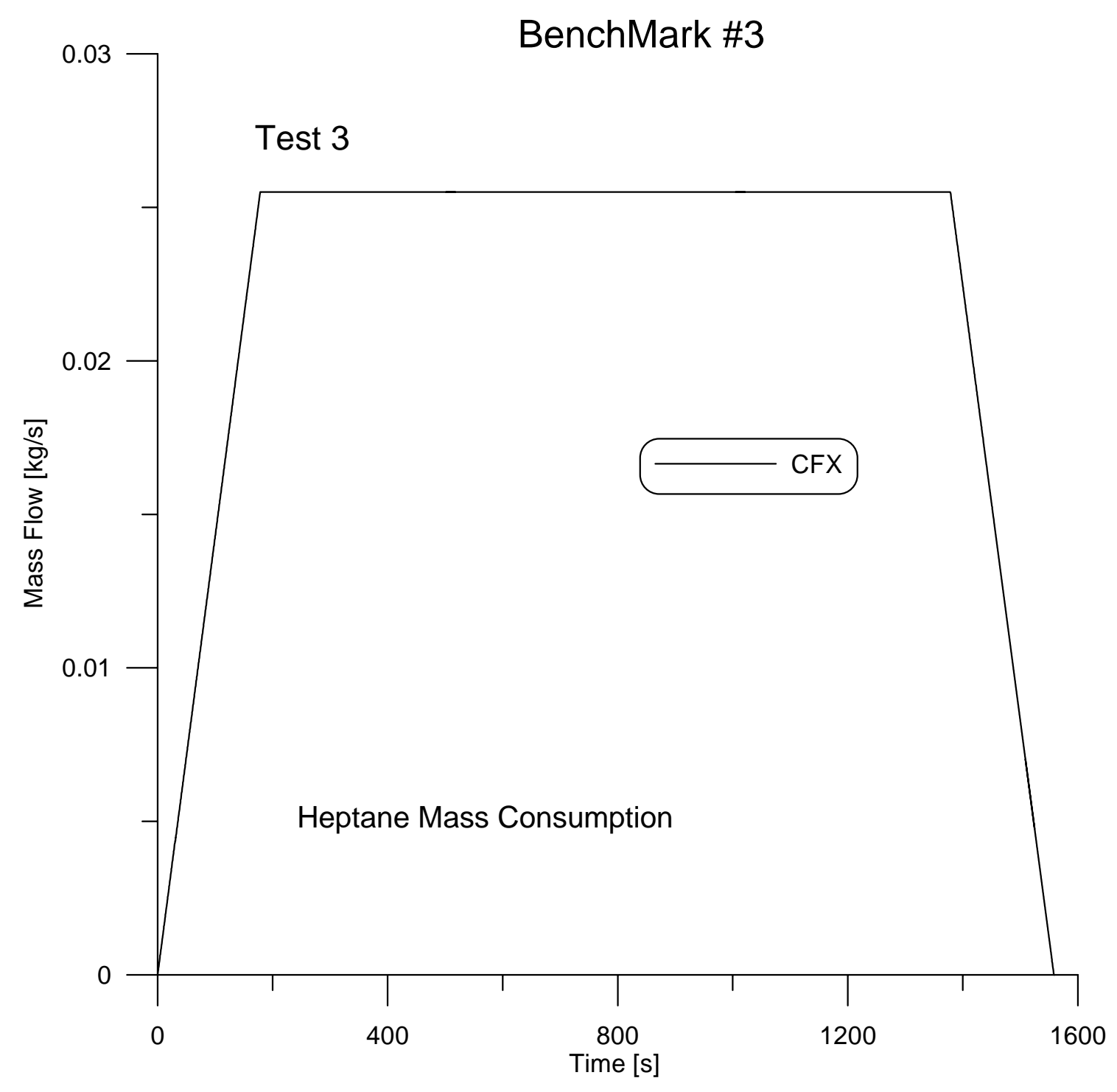

Fig. 6-19 Heptane release during test 3 
BM3: Heptane Fire in a Compartment Test c3
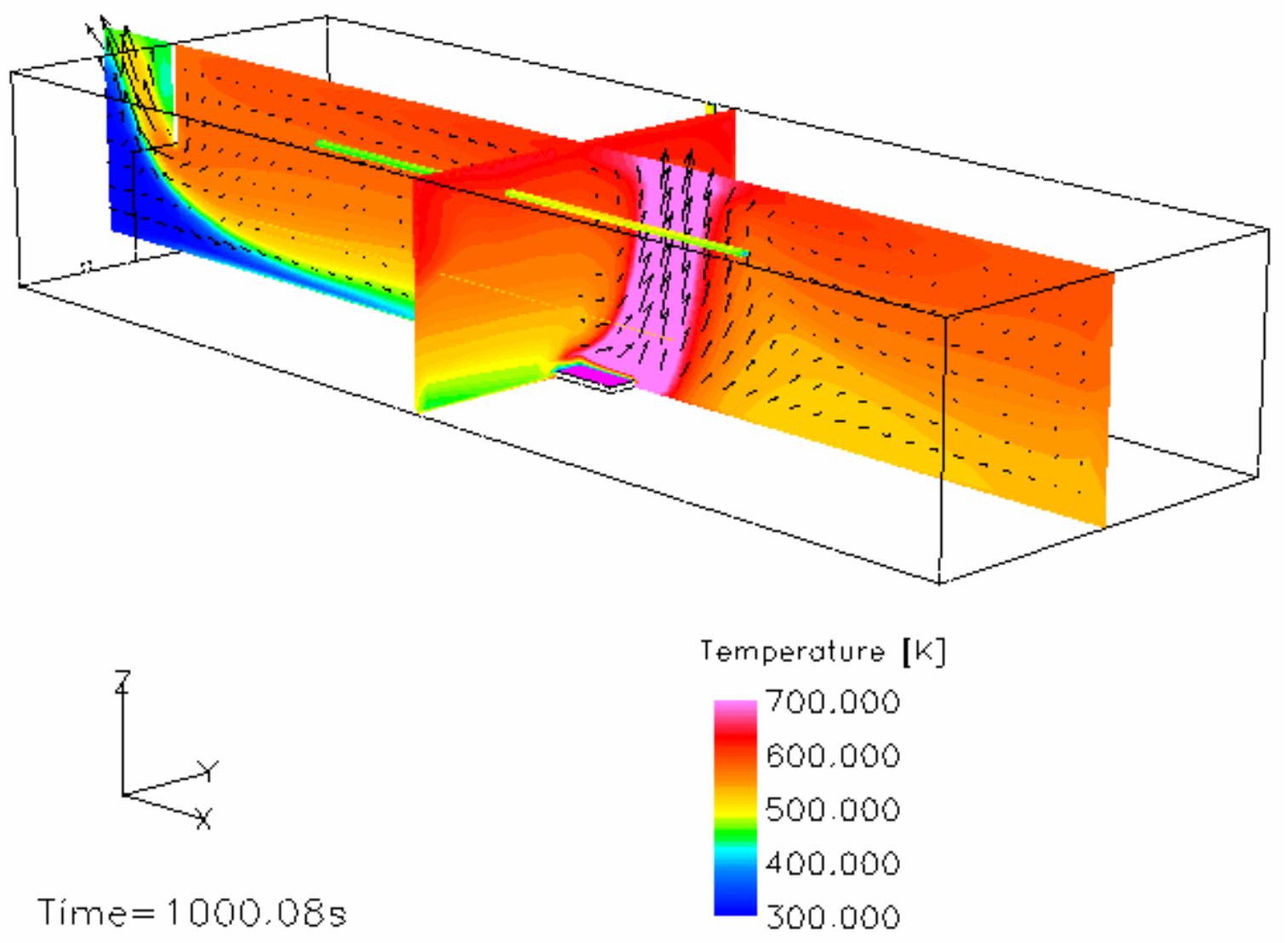

Fig. 6-20 Temperature distribution at $1000 \mathrm{~s}$ (test 3) 


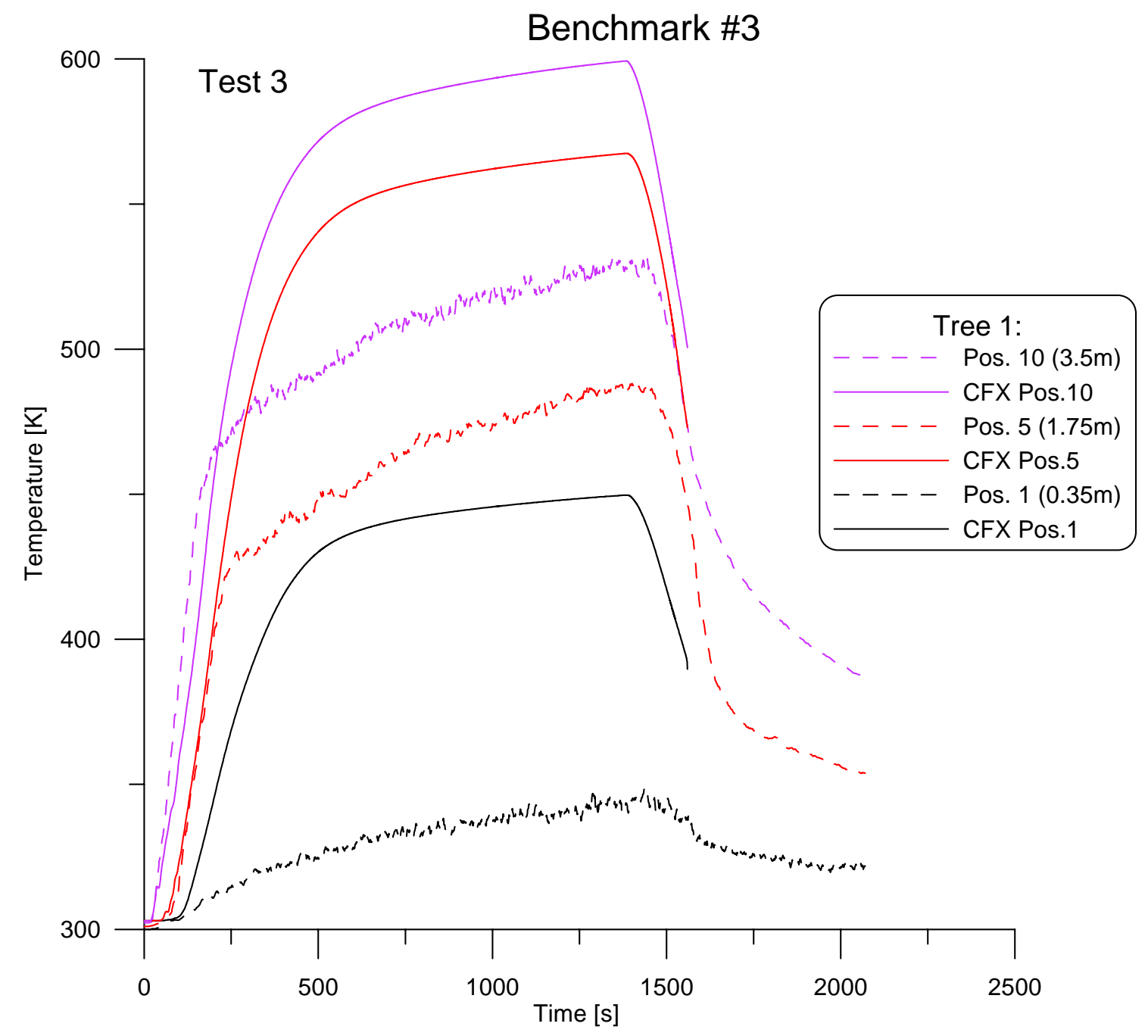

Fig. 6-21 Tree 1 temperatures (test 3) 


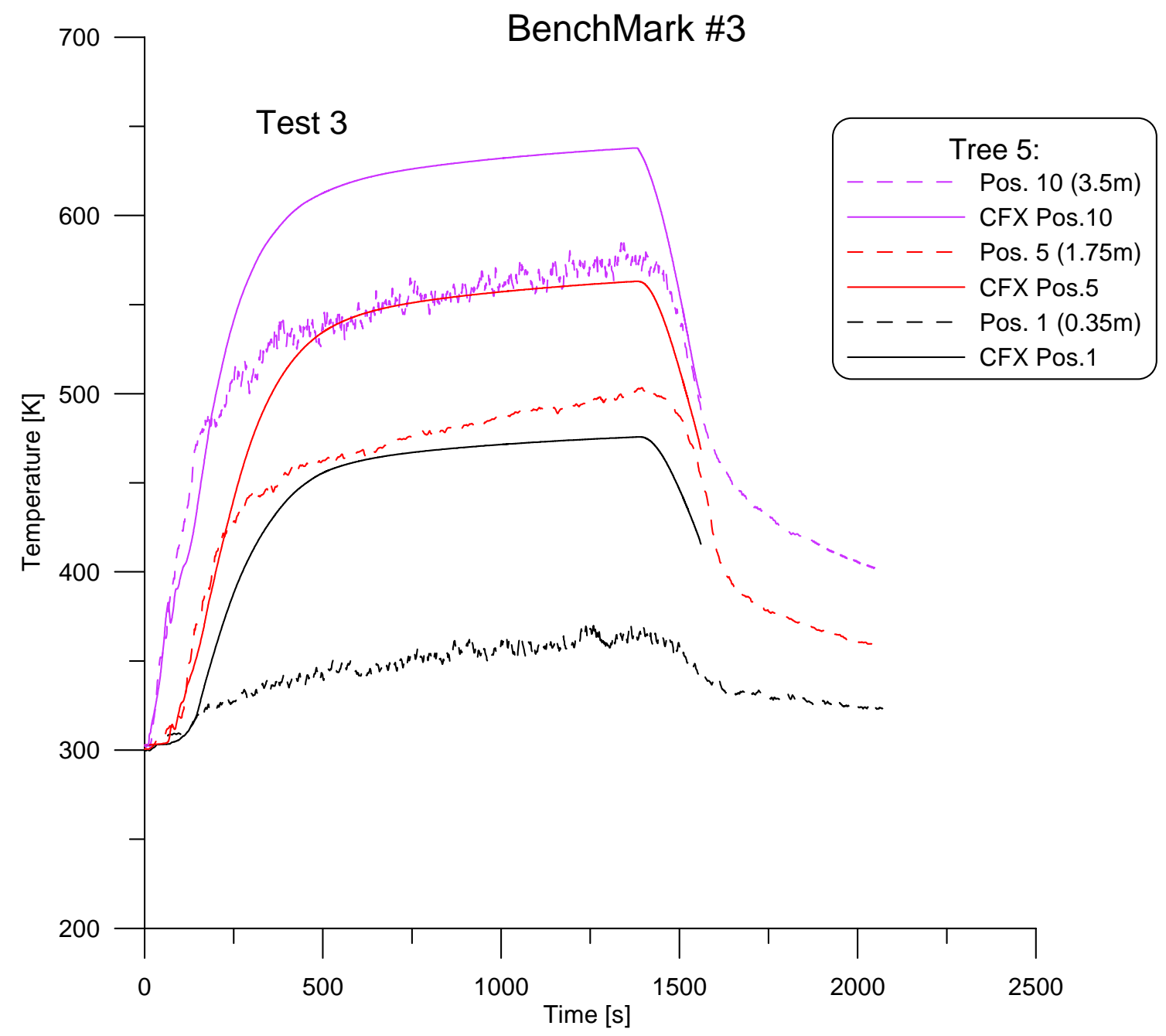

Fig. 6-22 Tree 5 temperatures (test 3) 


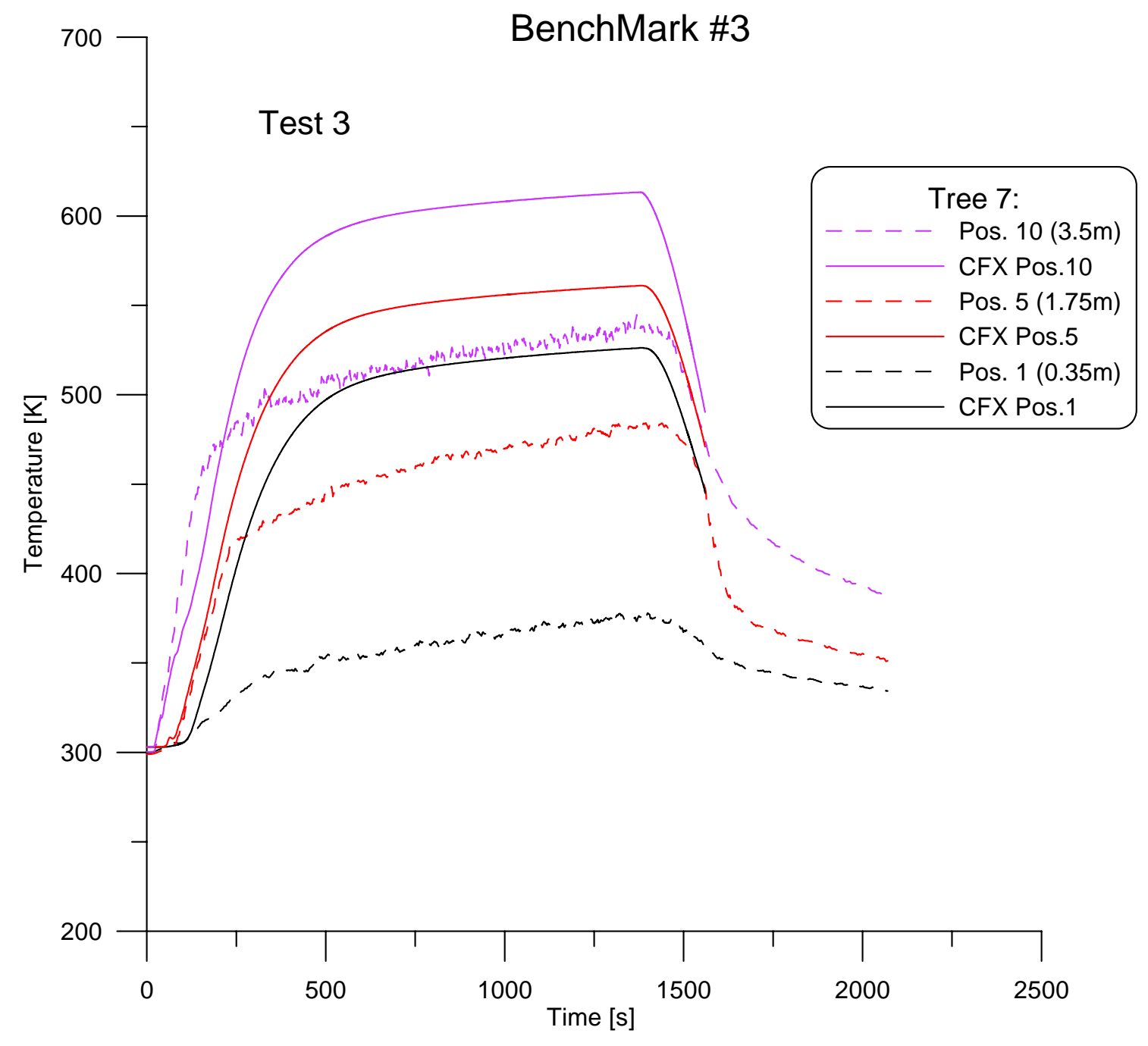

Fig. 6-23 Tree 7 temperatures (test 3) 


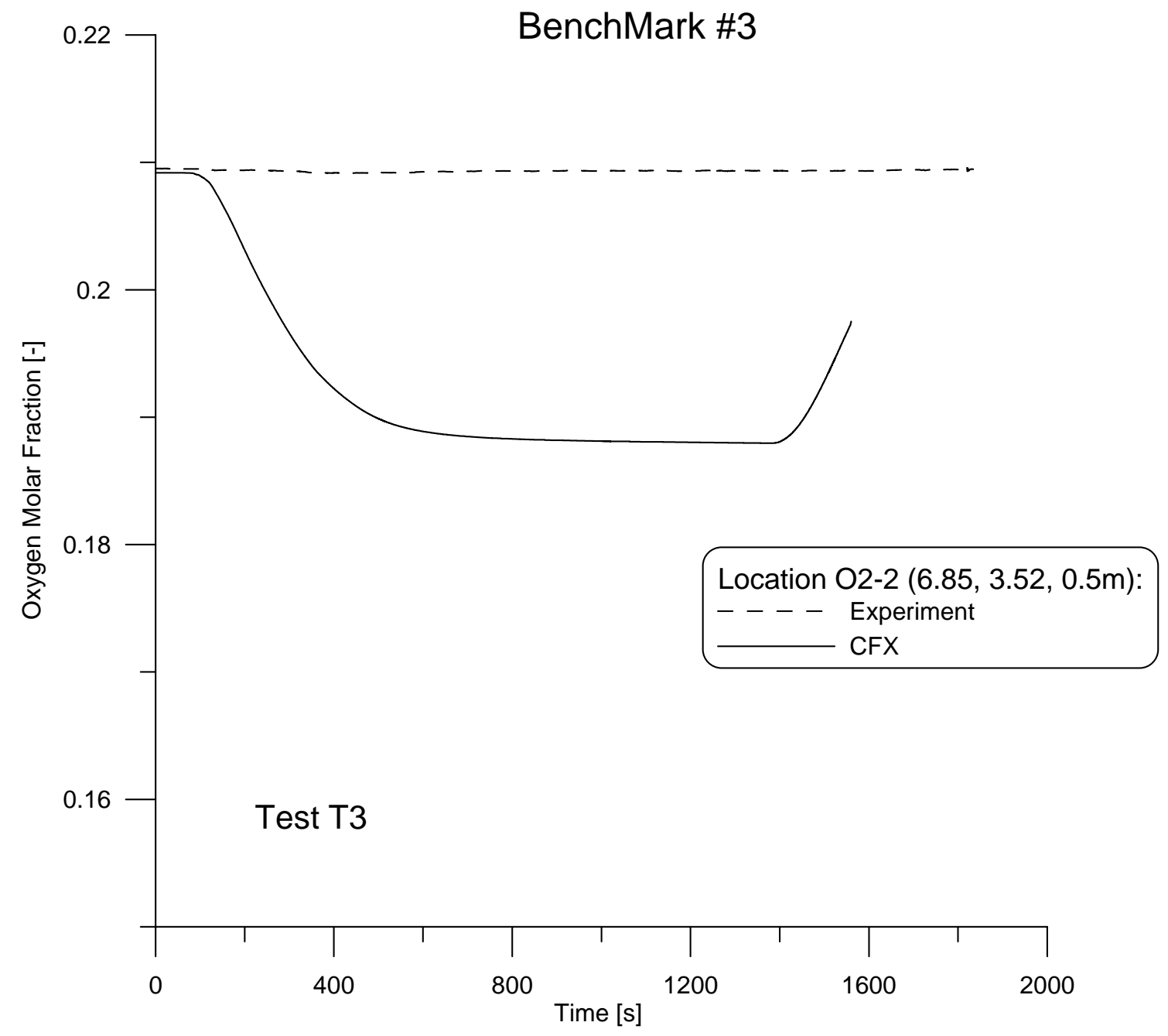

Fig. 6-24 Oxygen behaviour at location O2-2 (test 3) 
BM3: Heptane Fire in a Compartment

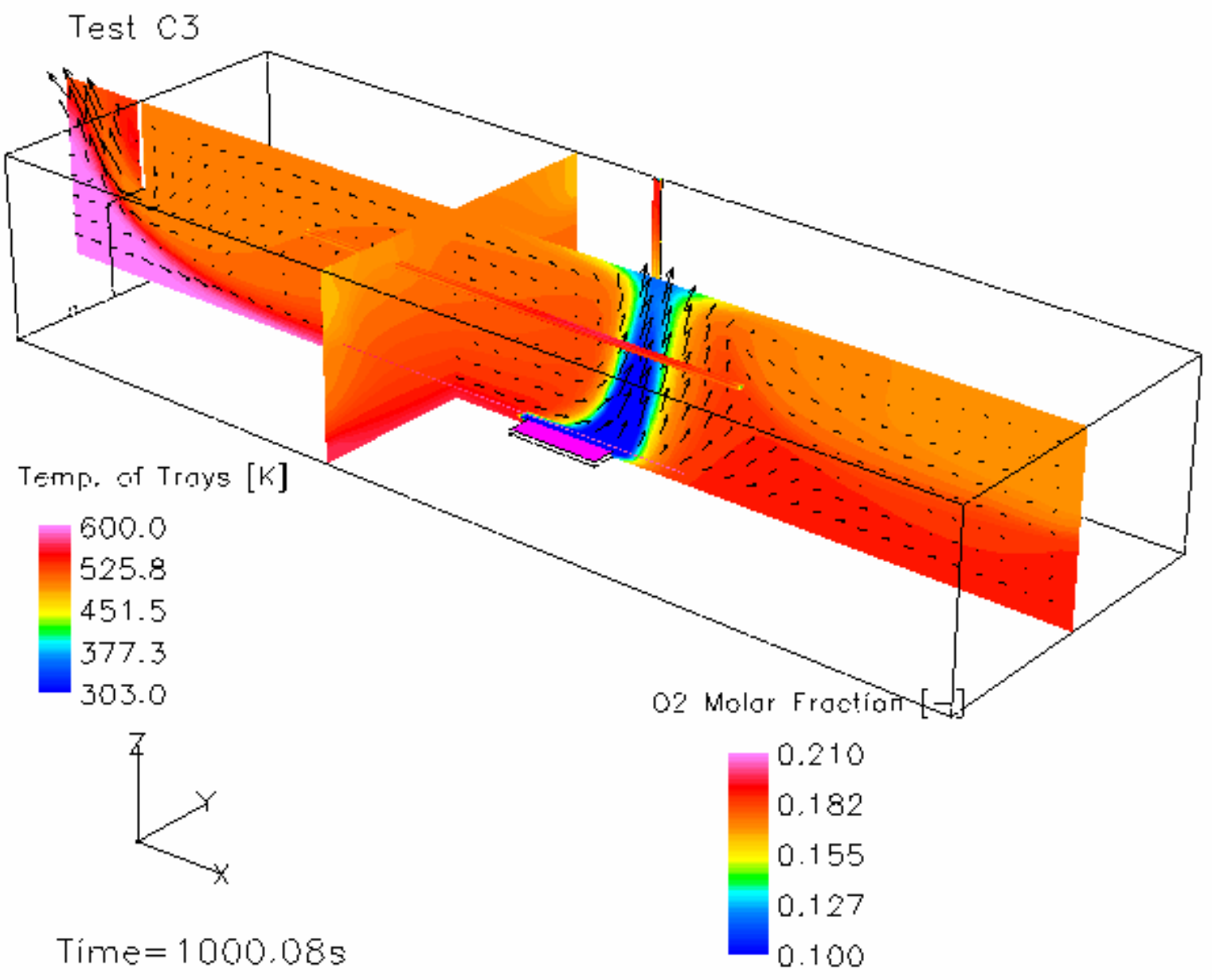

Fig. 6-25 Oxygen distribution at $1000 \mathrm{~s}$ (test 3) 


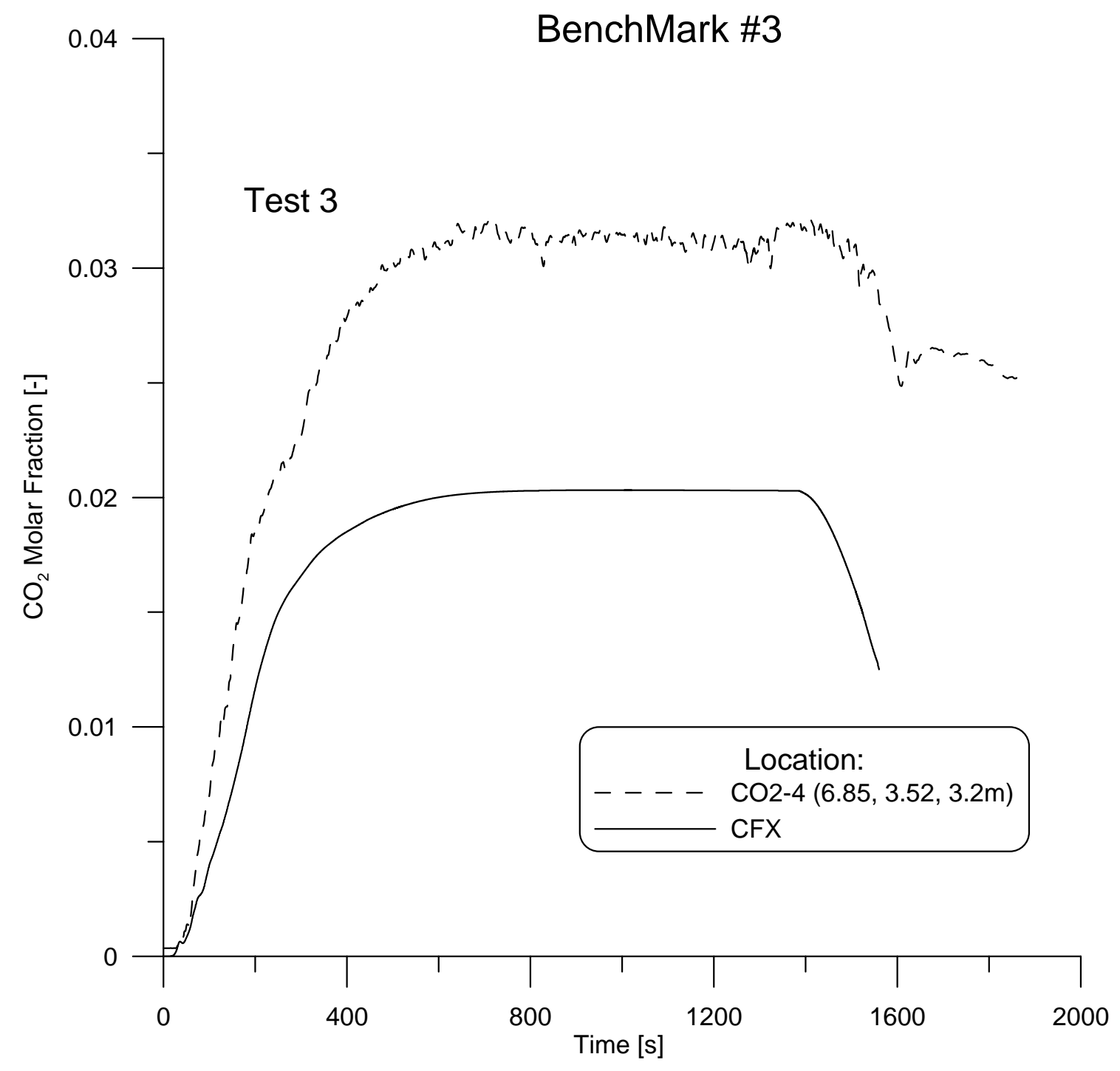

Fig. 6-26 $\mathrm{CO}_{2}$ concentration at location $\mathrm{CO}_{2}-4$ (test 3) 


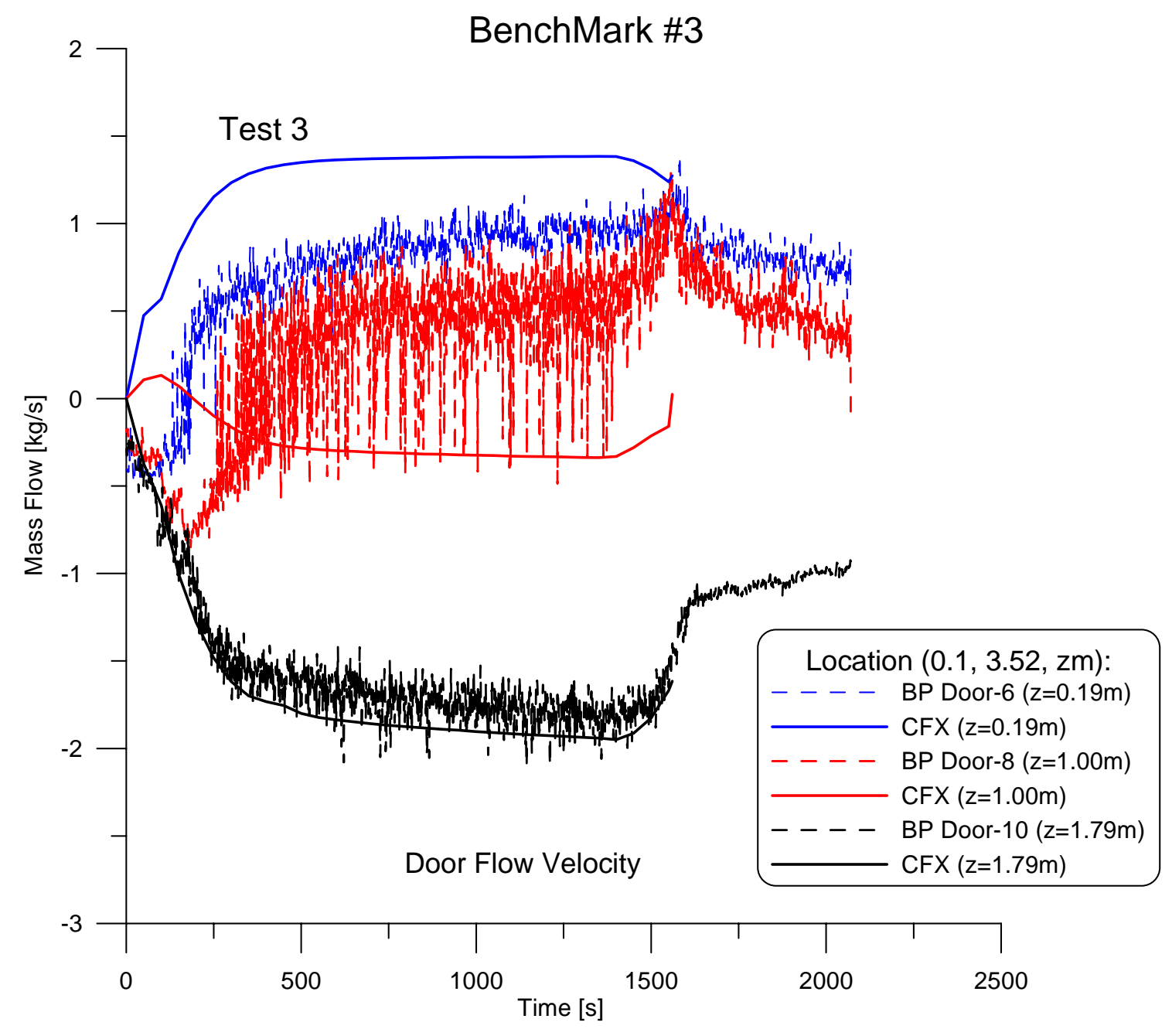

Fig. 6-27 Selected flow velocities in the door opening (test 3) 


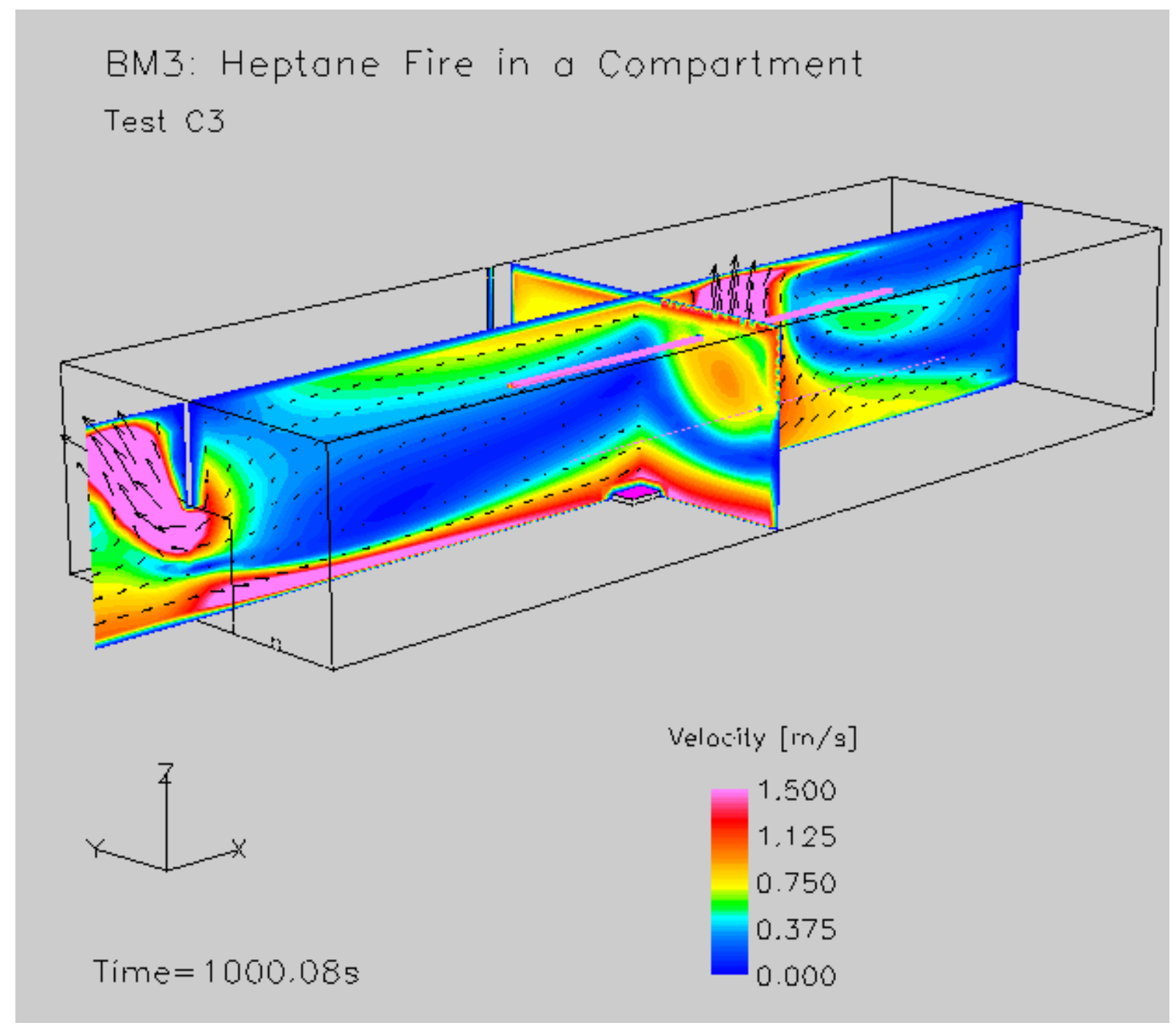

Fig. 6-28 Flow speed distribution at door and in the fire compartment at $1000 \mathrm{~s}$ (Test 3) 


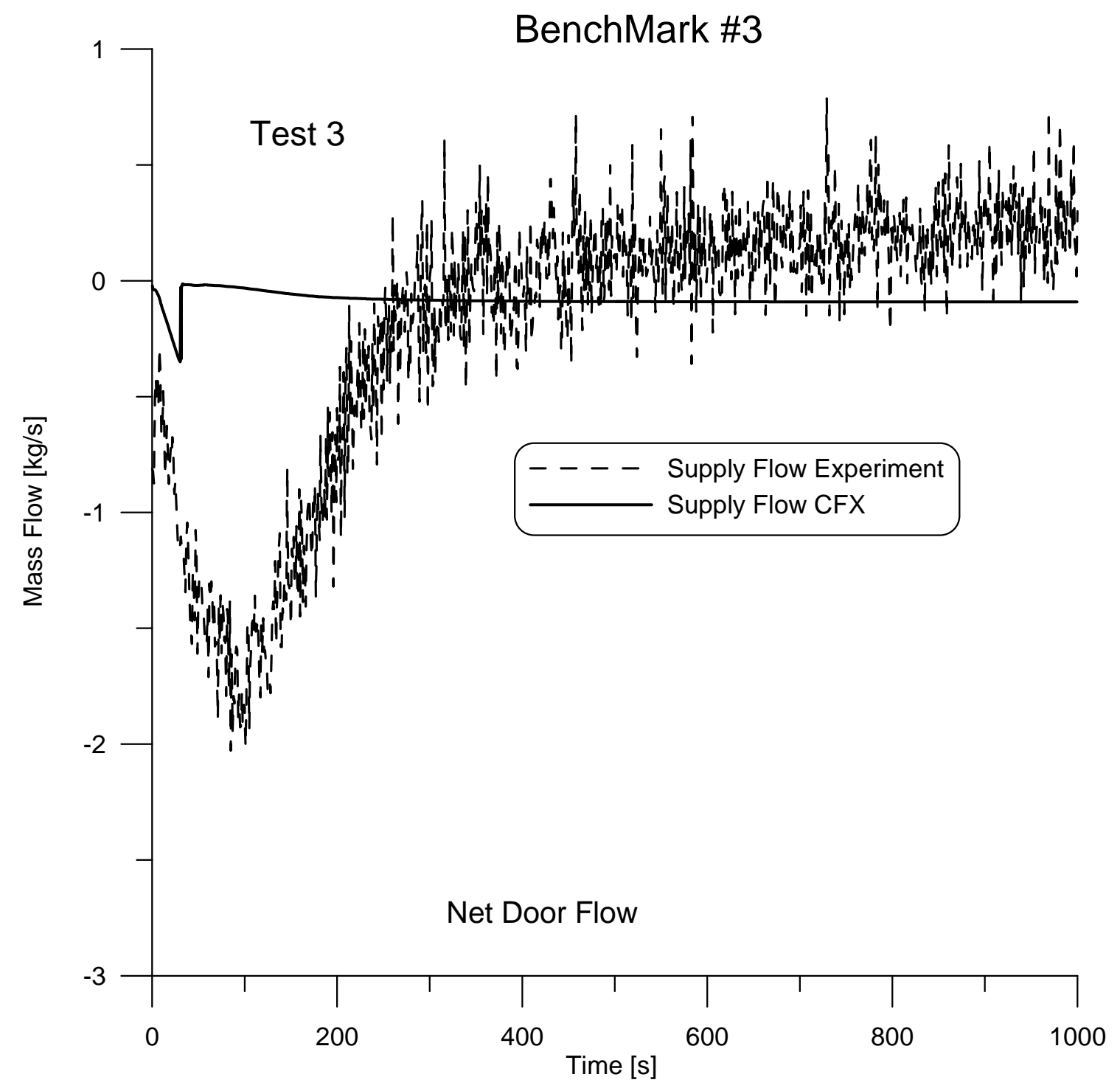

Fig. 6-29 Net mass flow through door (test 3) 
BM3: Heptane Fire in a Compartment

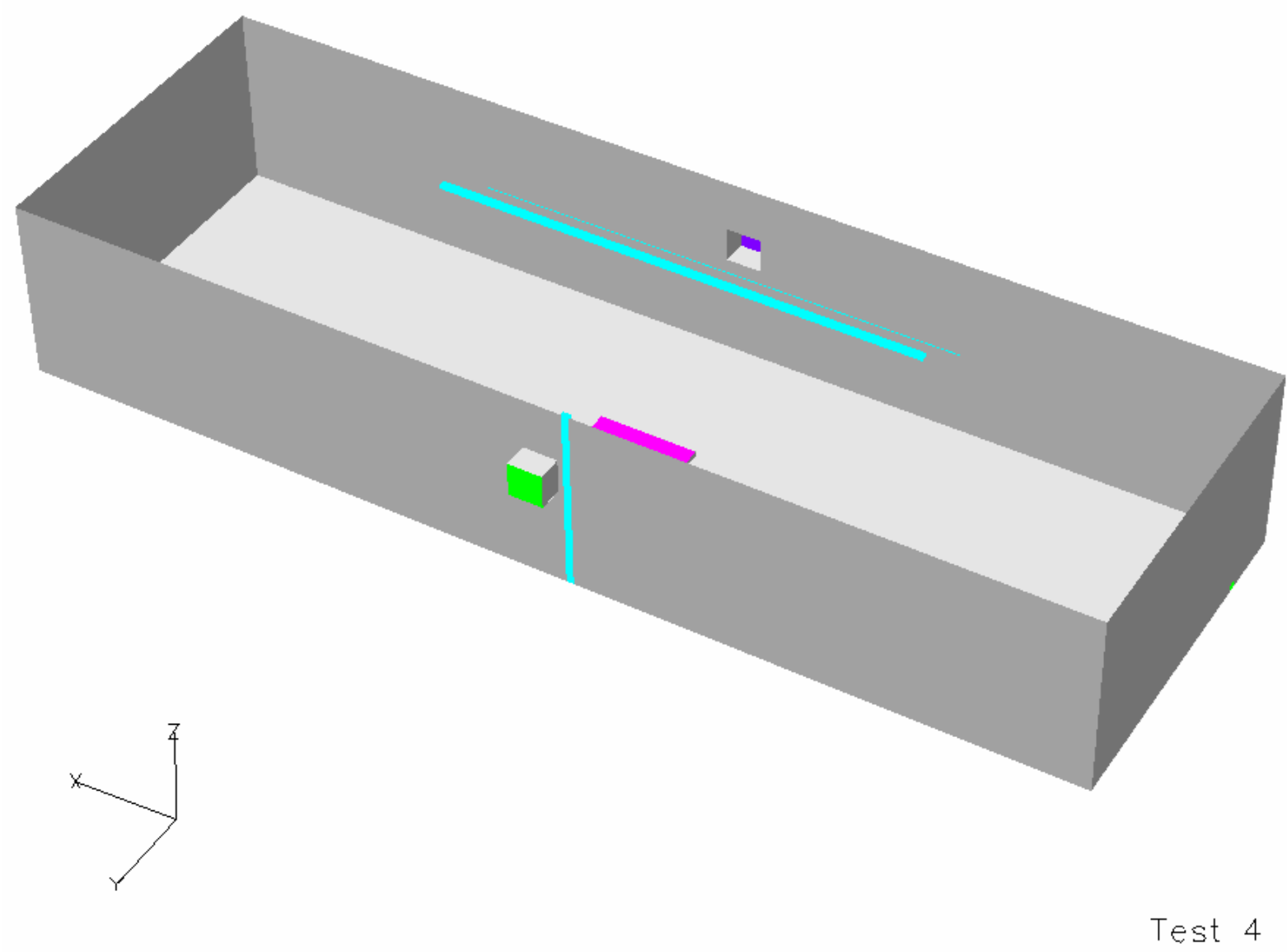

Fig. 6-30 Configuration of the test facility for test 4 
BM3: Heptane Fire in a Compartment

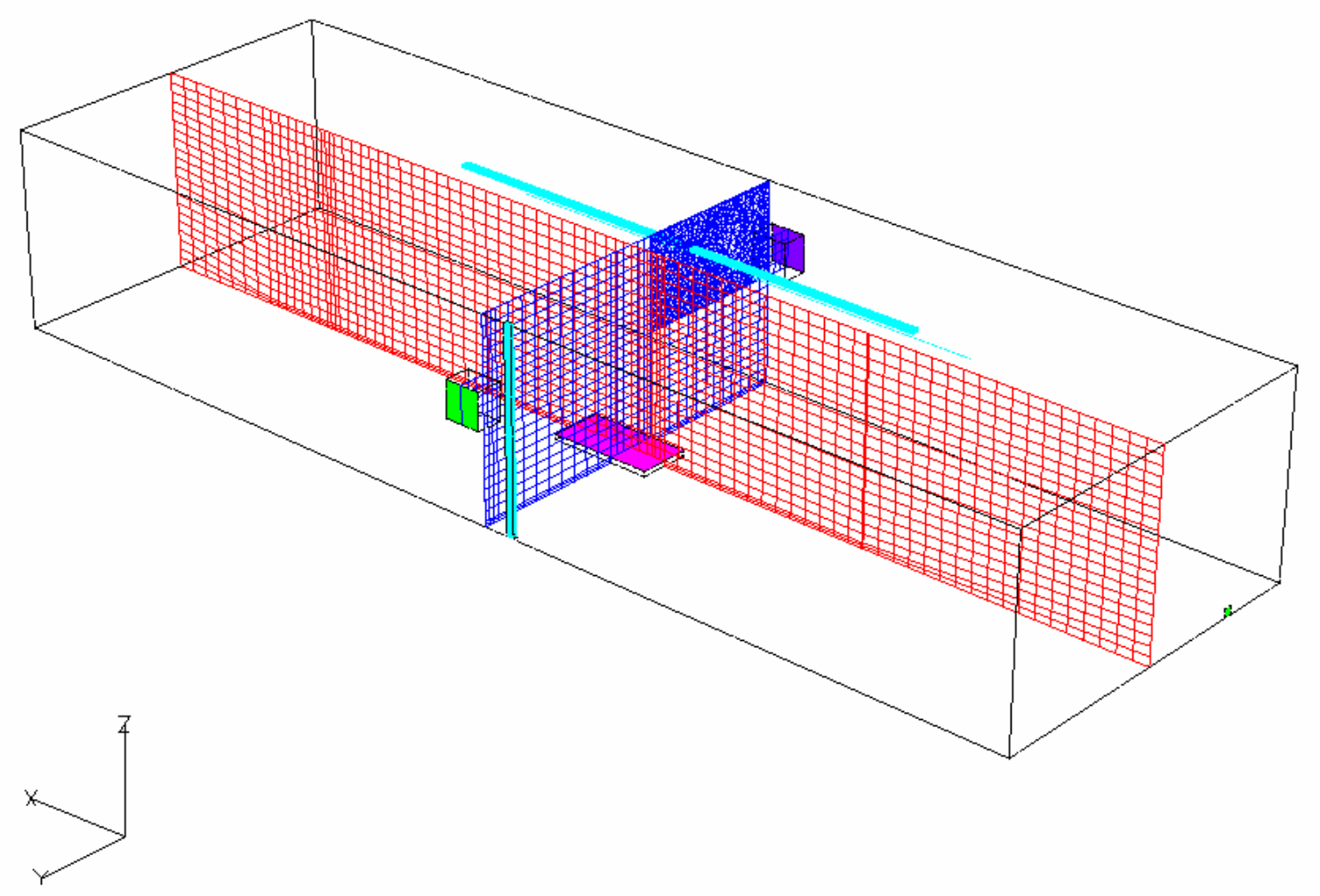

Test 4

Fig. 6-31 Mesh and main components of test configuration 4 


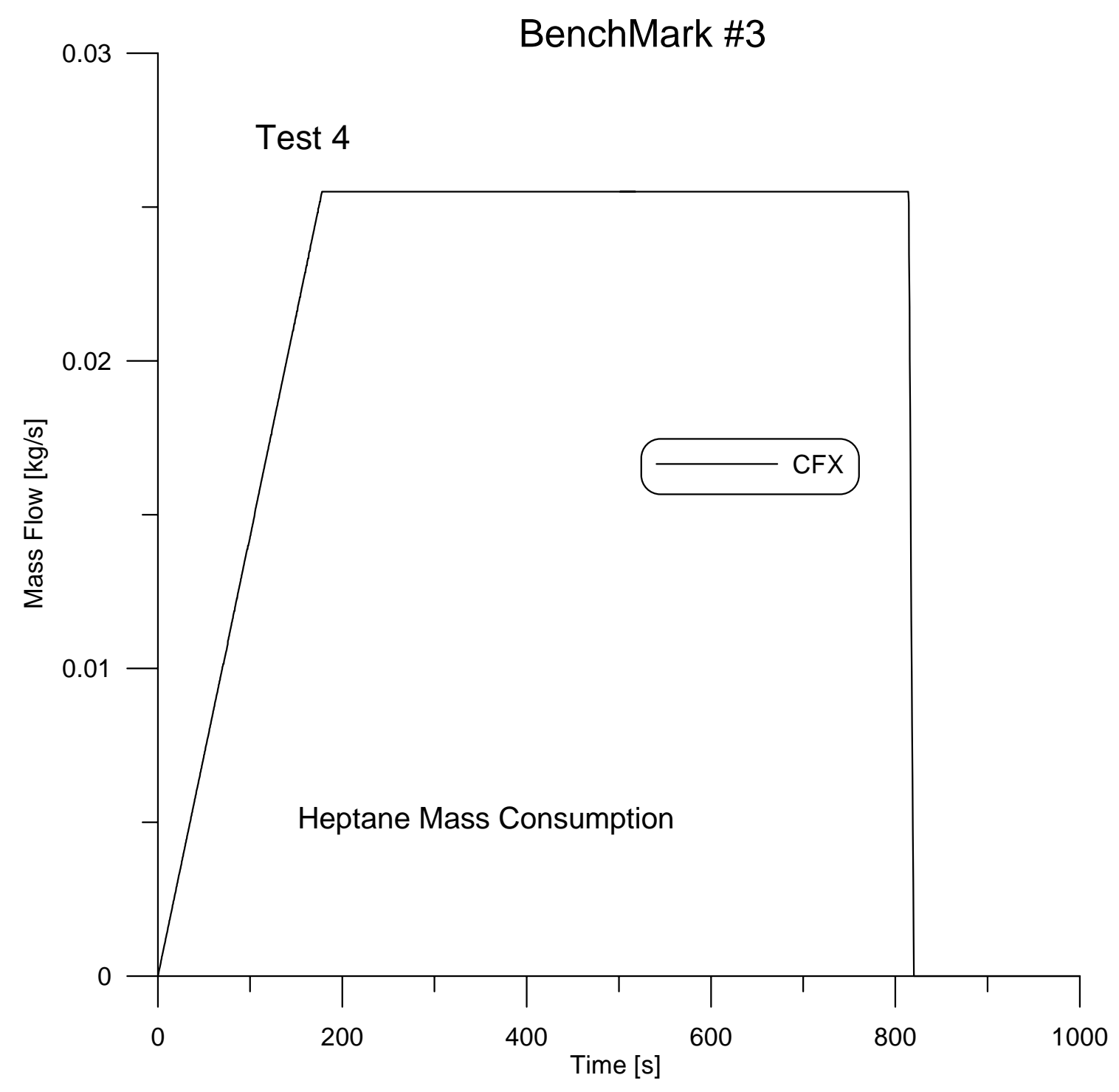

Fig. 6-32 Heptane mass consumption (test 4) 


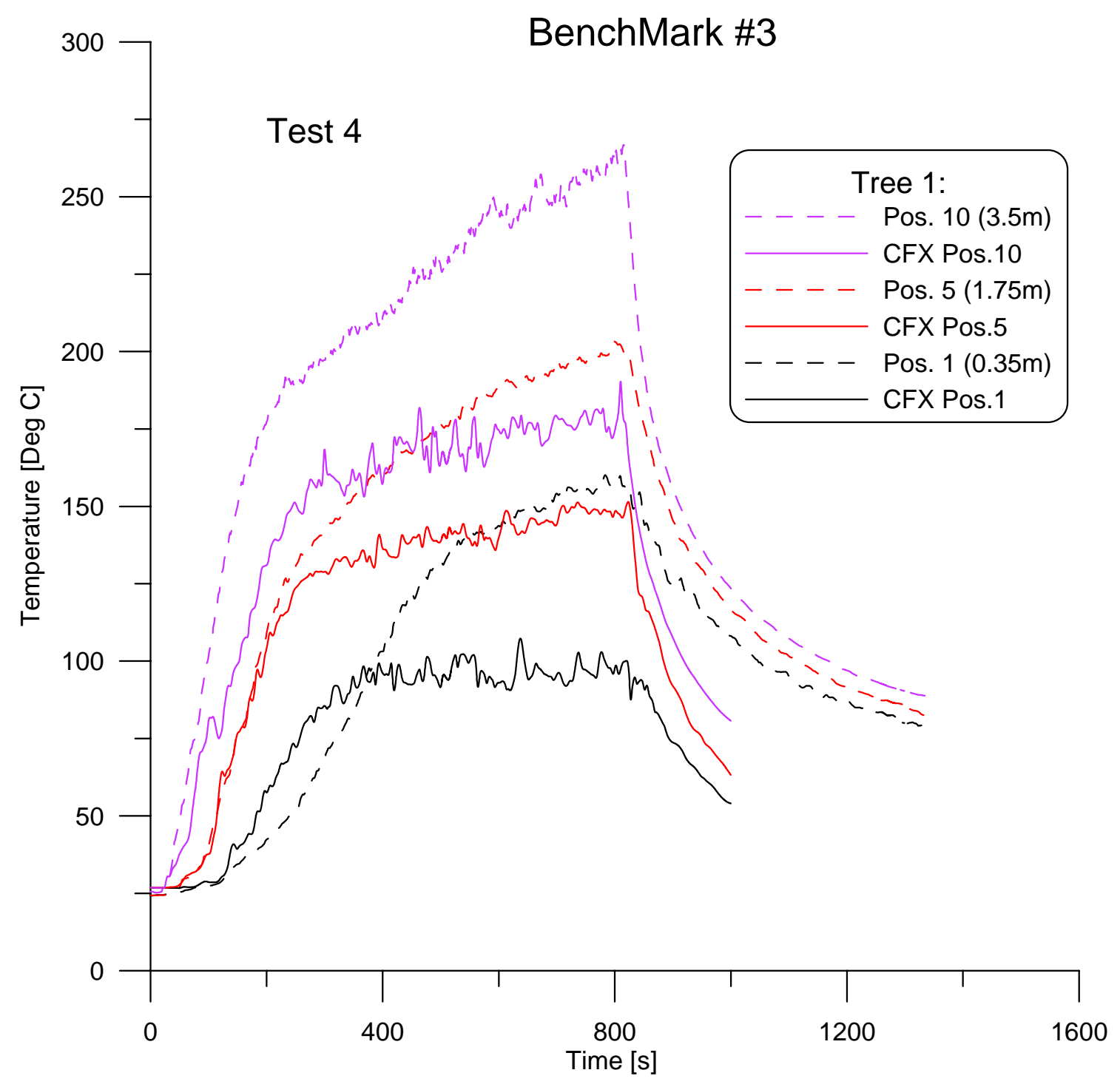

Fig. 6-33 Selected temperatures on tree 1 (test 4) 


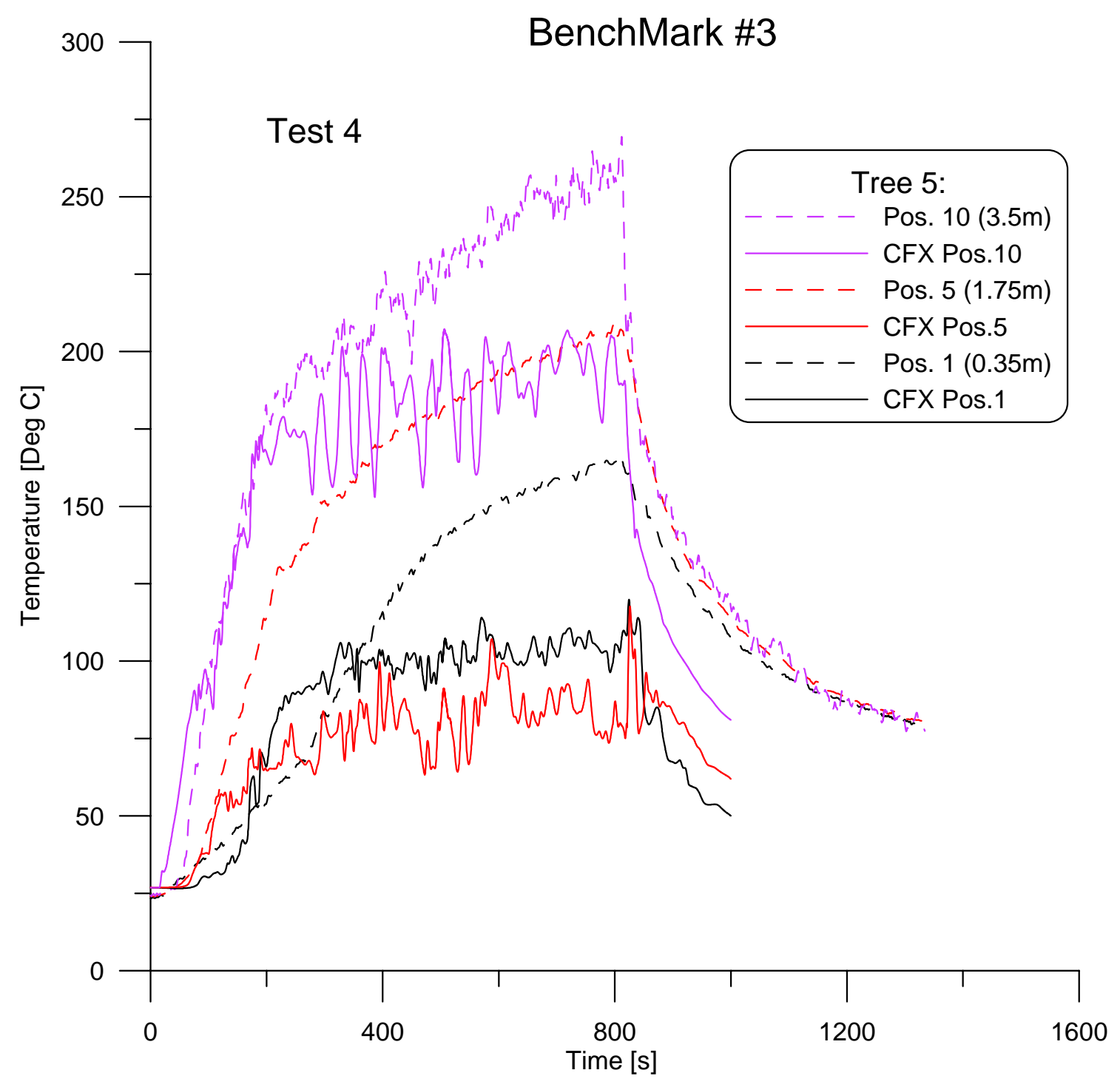

Fig. 6-34 Selected temperatures on tree 5 (test 4) 
BM3: Heptane Fire in a Compartment

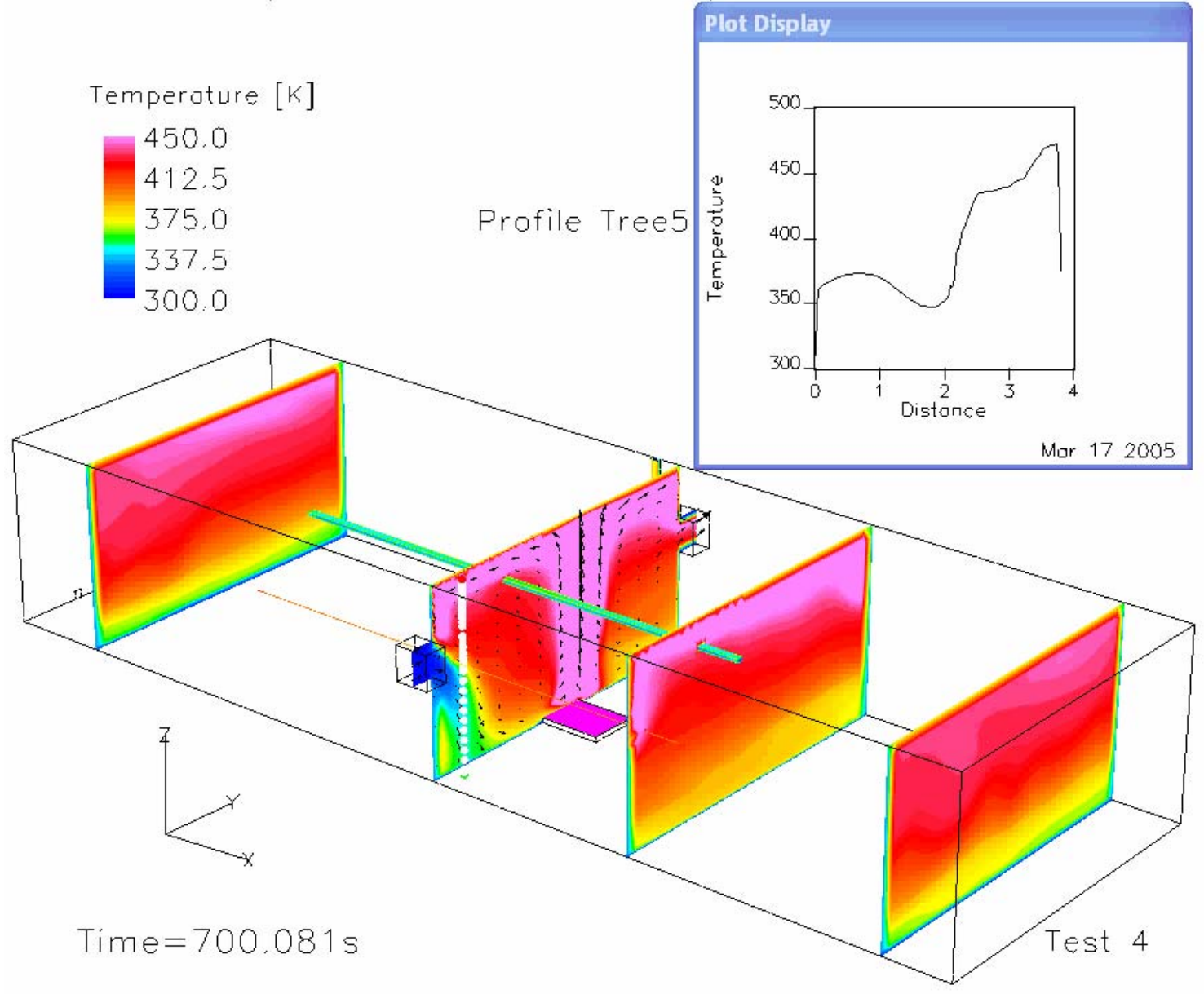

Fig. 6-35 Temperature distribution and tree 5 profile at $700 \mathrm{~s}$ (test 4) 


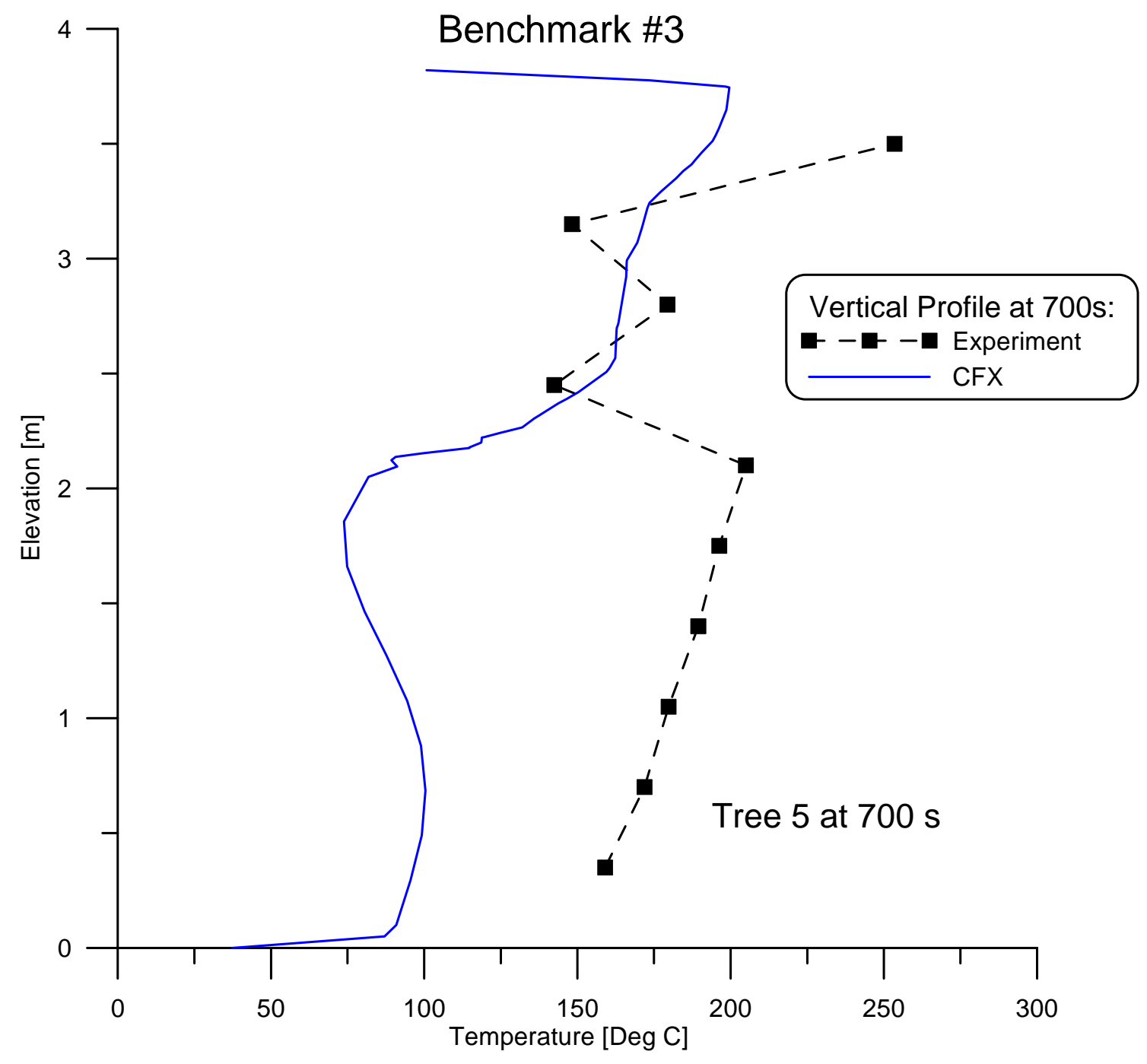

Fig. 6-36 Vertical temperature profile at $700 \mathrm{~s}$ (test 4) 


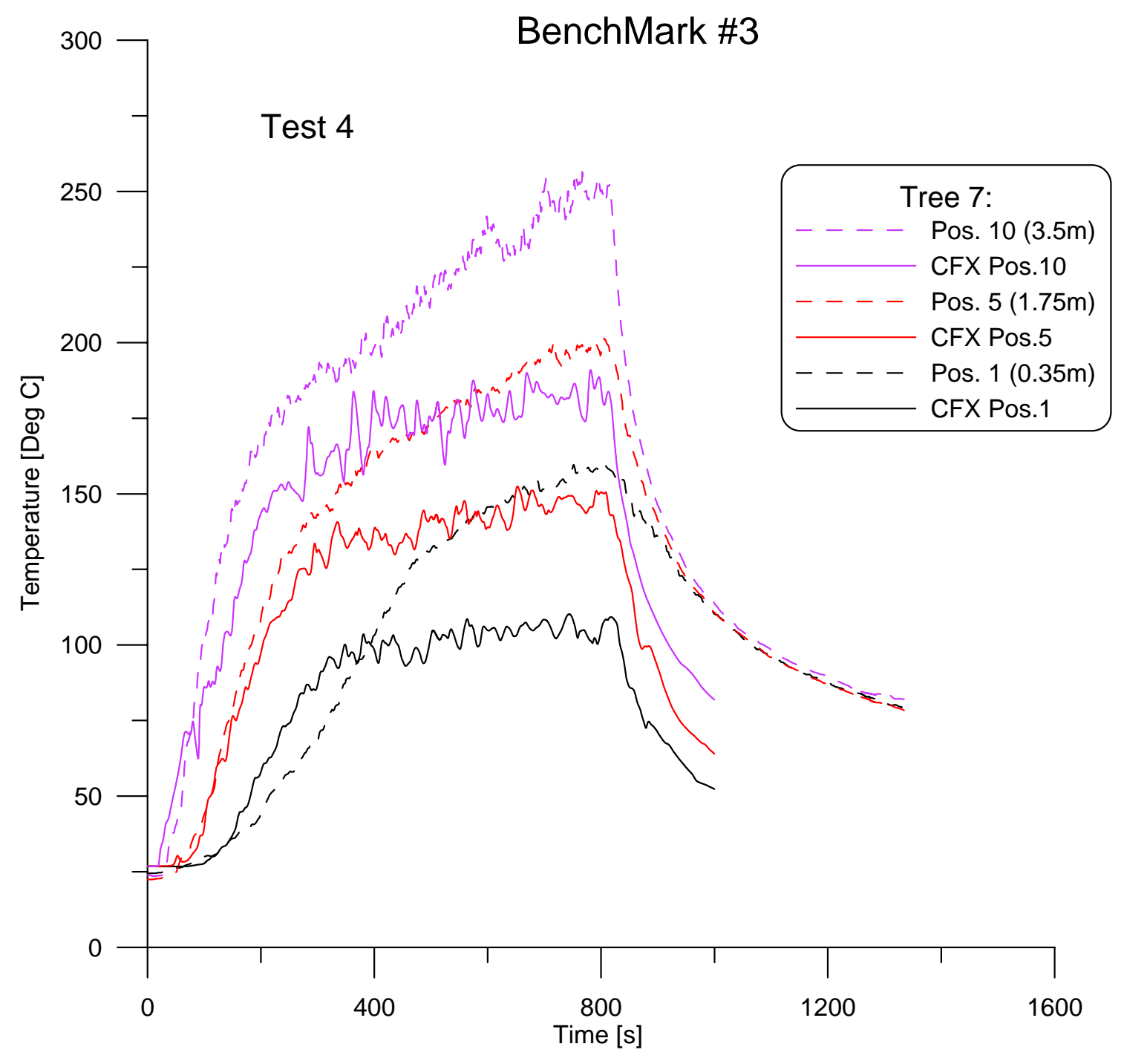

Fig. 6-37 Selected temperatures on tree 7 (test 4) 


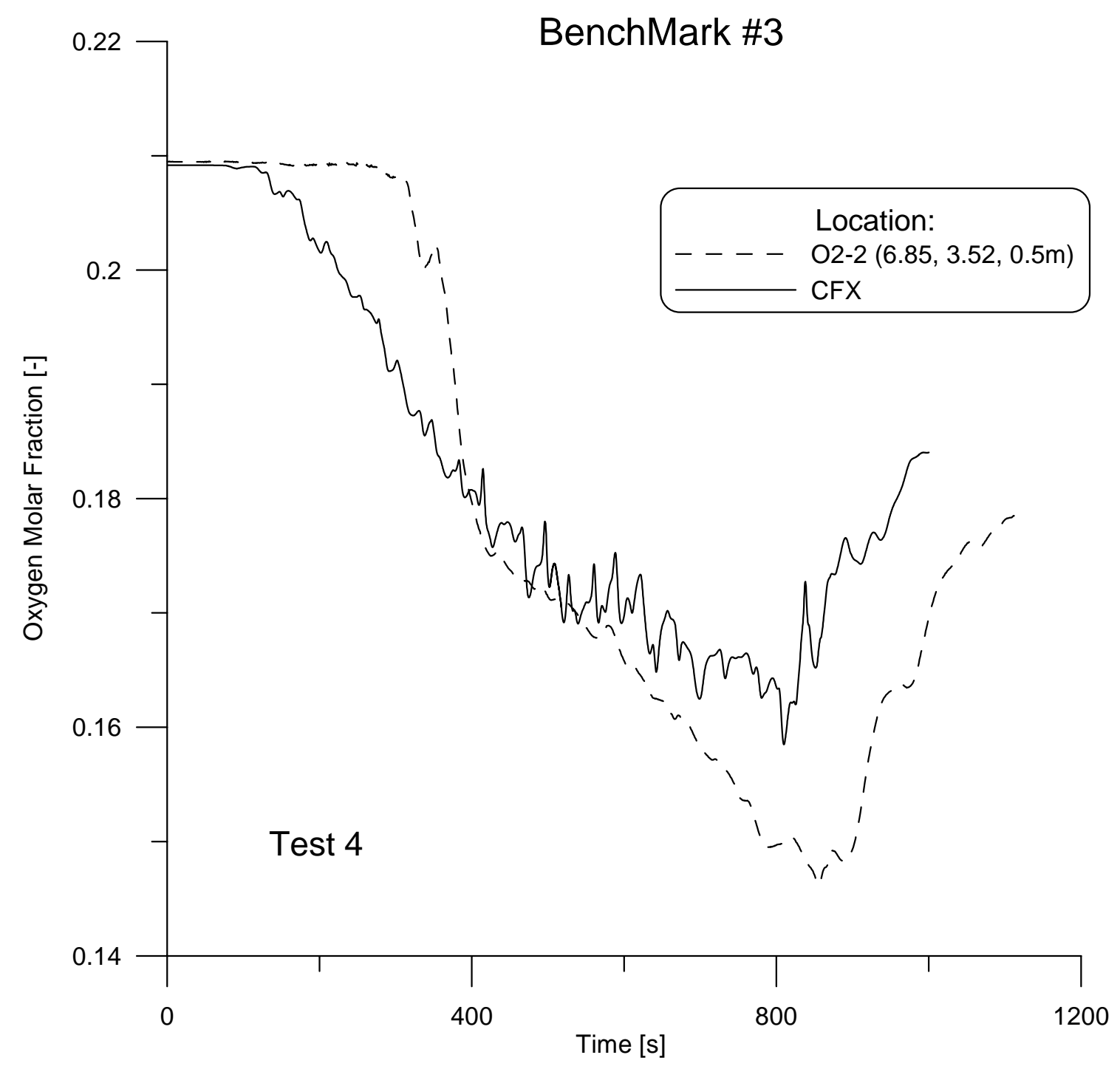

Fig. 6-38 Oxygen behaviour at position O2-2 (test 4) 


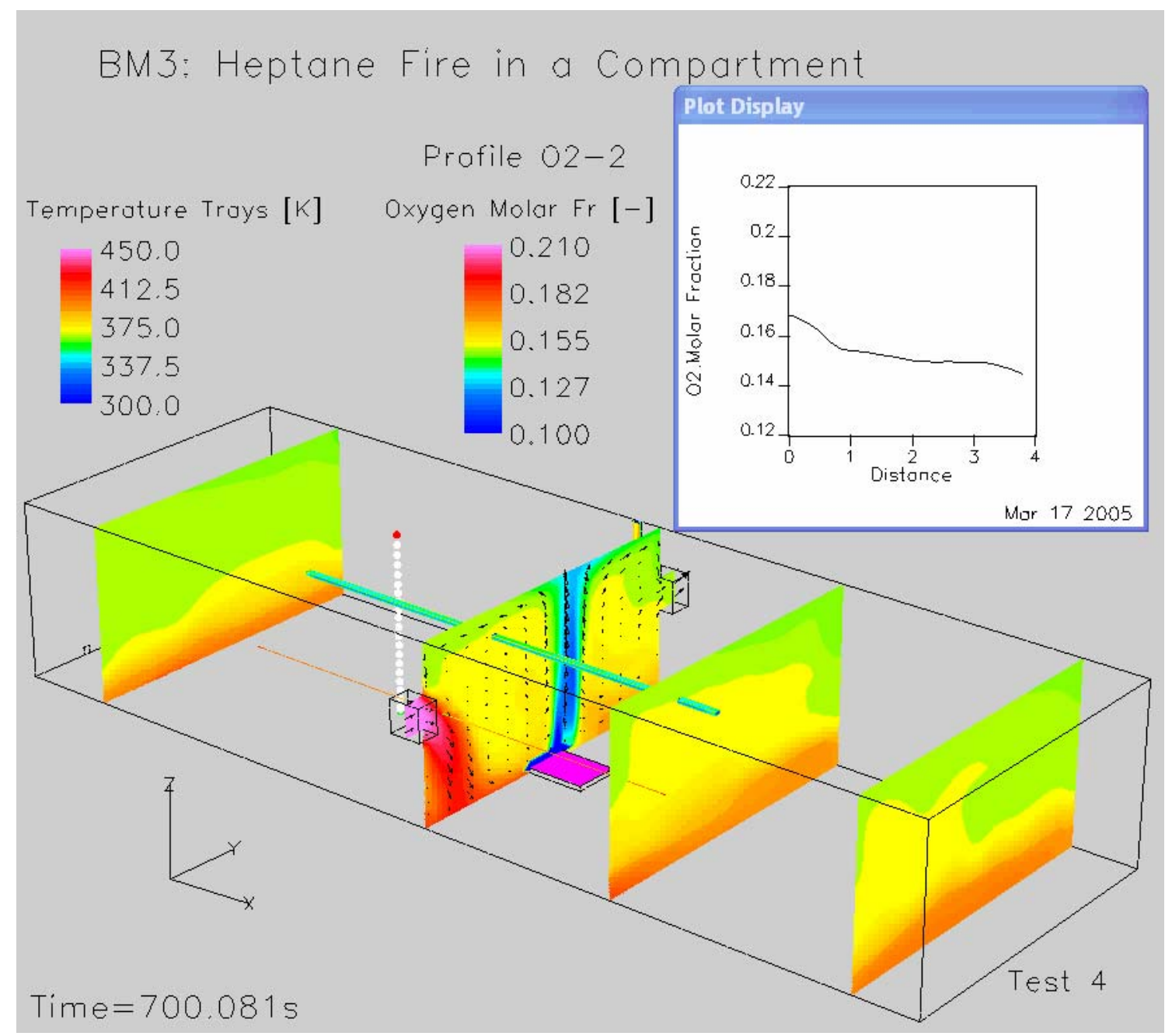

Fig. 6-39 Oxygen in the fire lab after $700 \mathrm{~s}$ (test 4) 


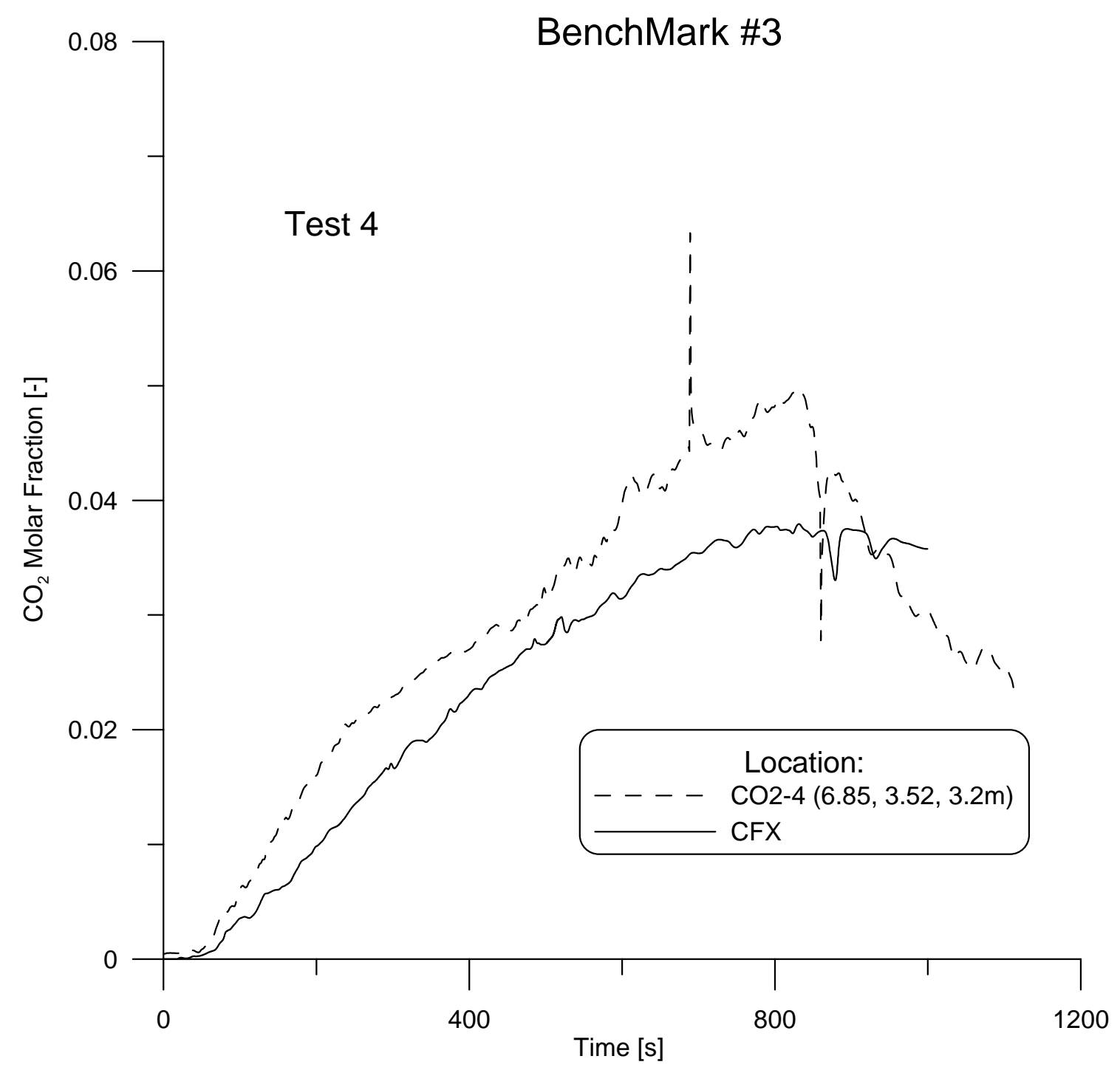

Fig. 6-40 $\mathrm{CO}_{2}$ behaviour at position $\mathrm{CO} 2-4$ (test 4) 


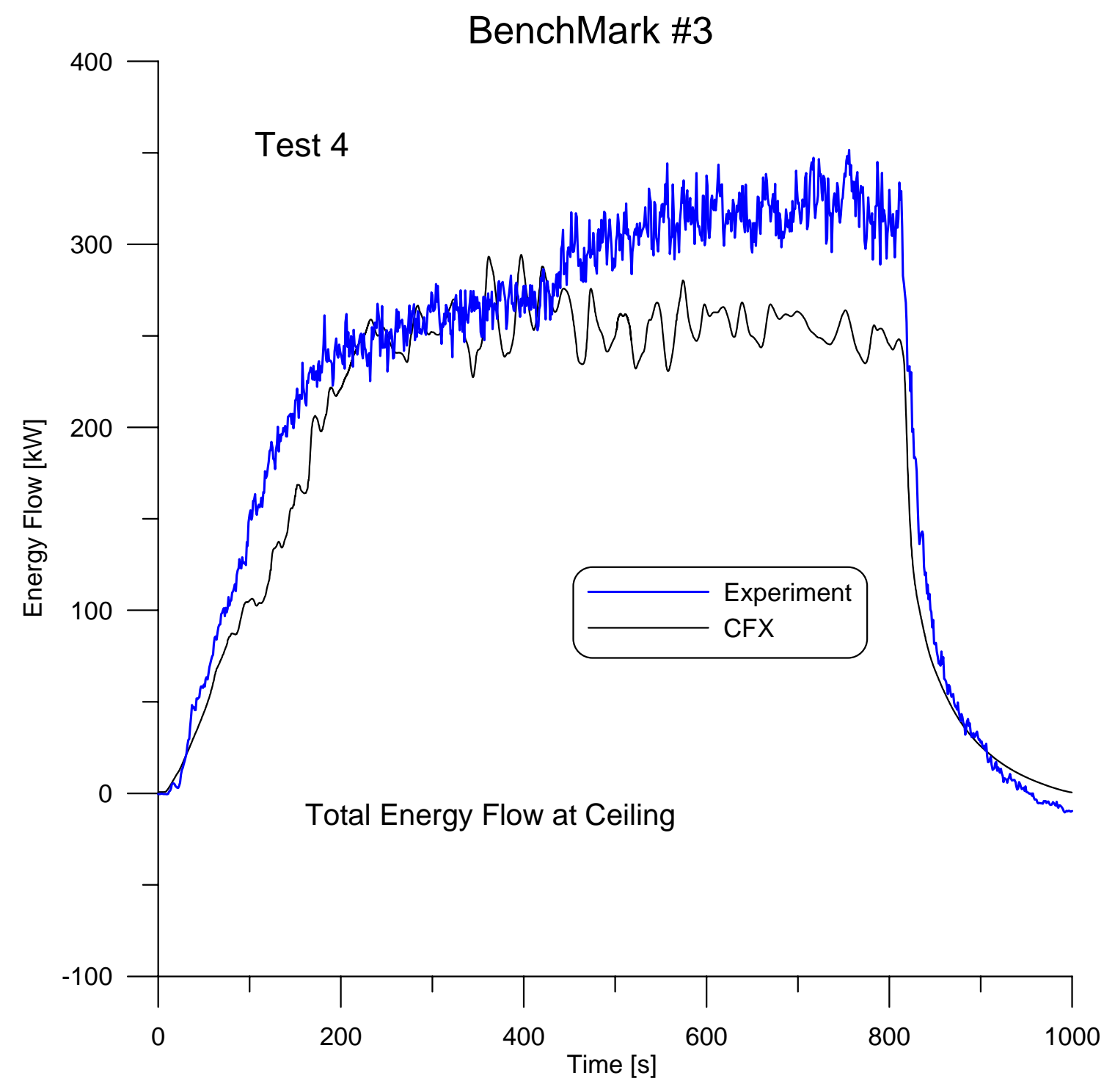

Fig. 6-41 Total energy flow to the ceiling (test 4) 


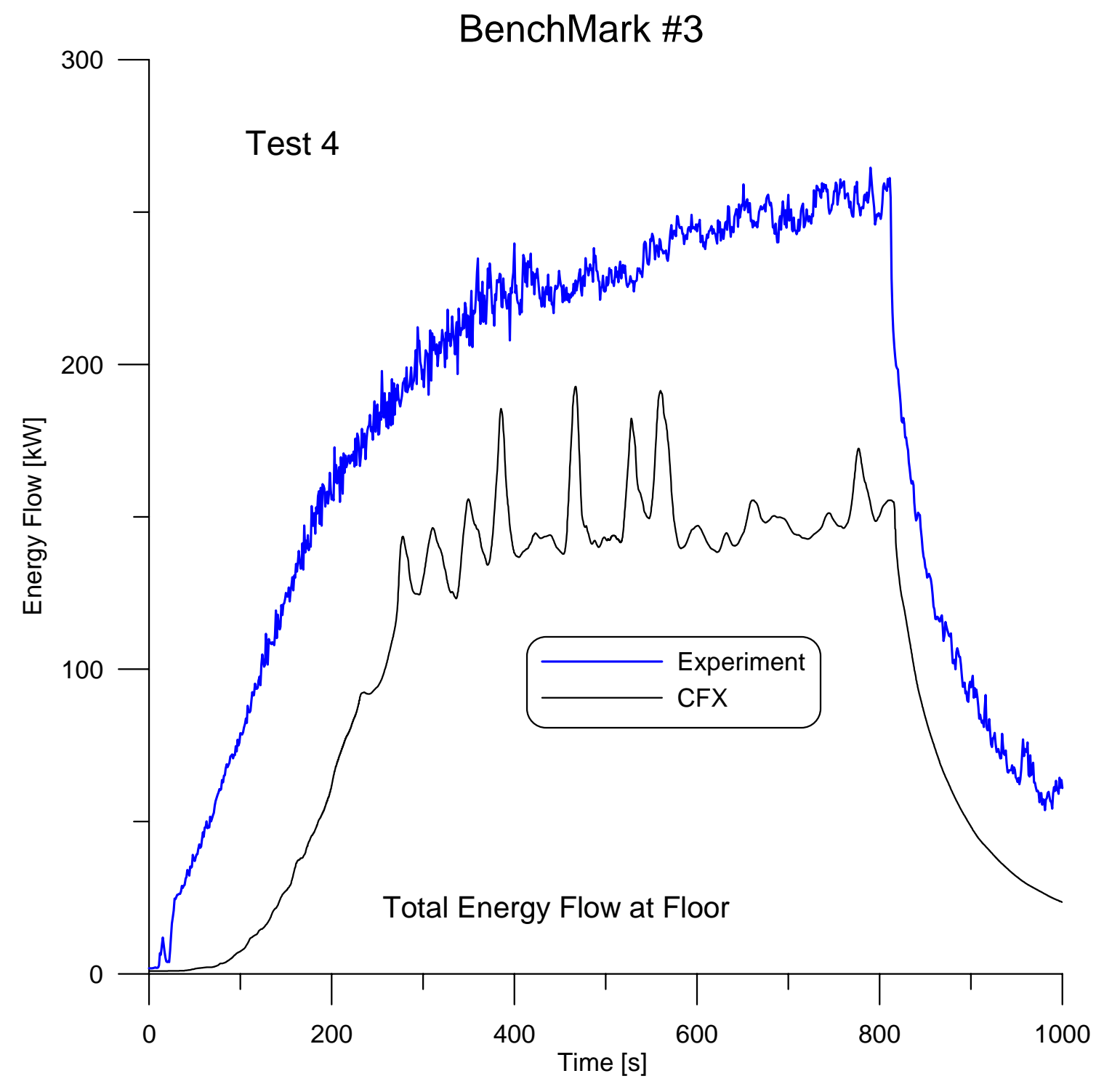

Fig. 6-42 Total energy flow to the floor (test 4) 


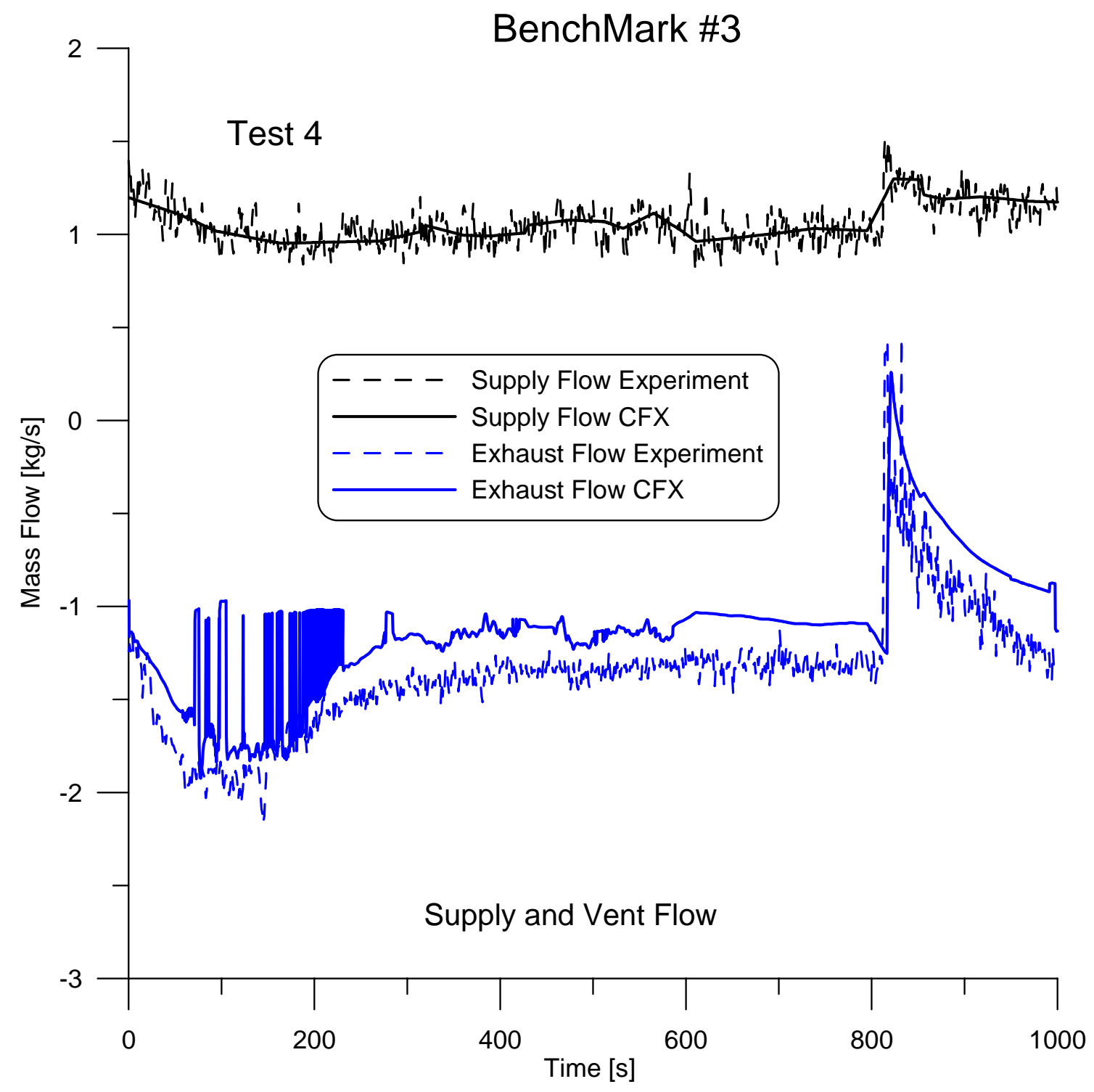

Fig. 6-43 Mass flows through supply and exhaust (test 4) 
BM3: Heptane Fire in a Compartment

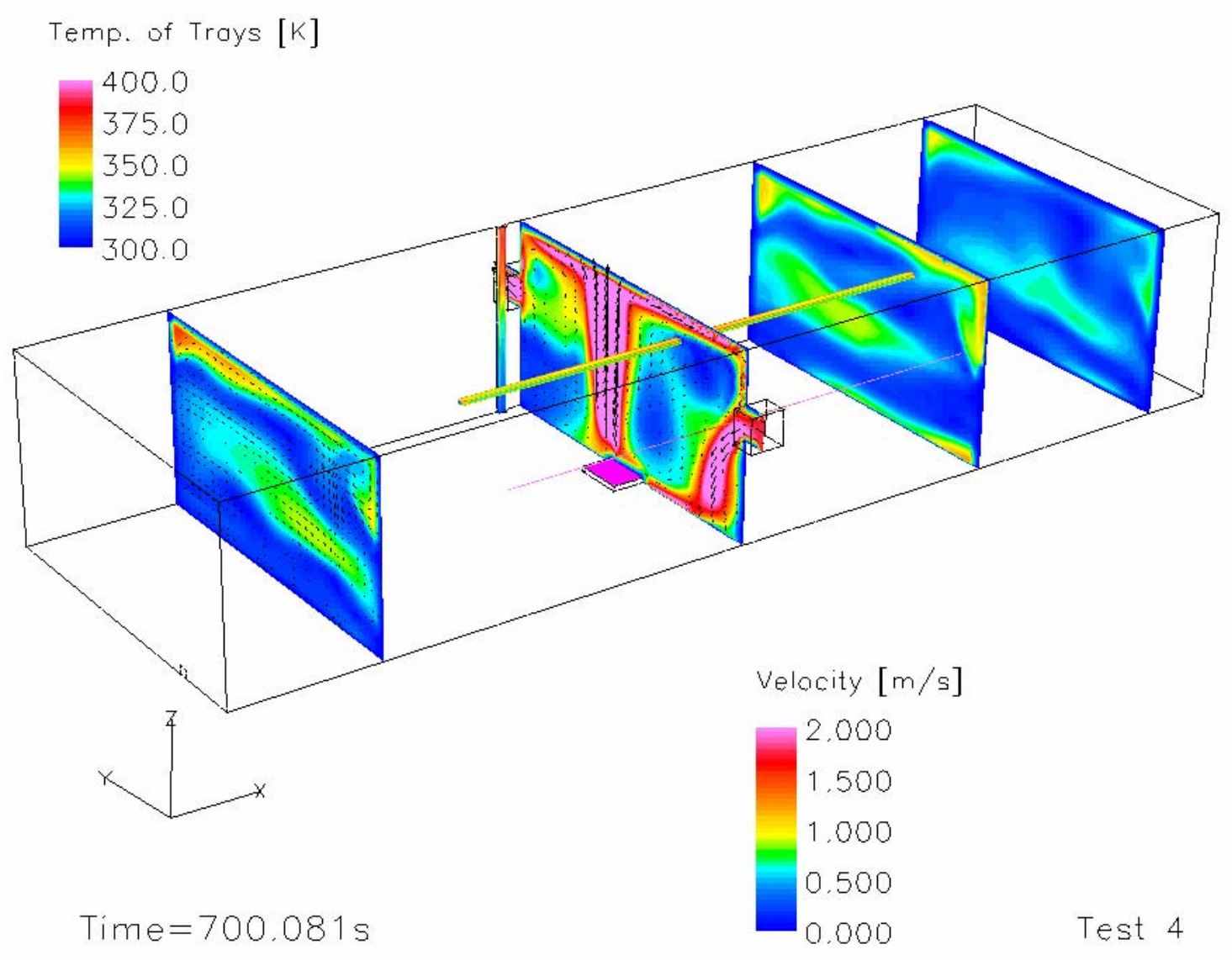

Fig. 6-44 Flow speed distribution after $700 \mathrm{~s}$ (test 4) 
Benchmark Exercise 3 was used to assess the accuracy of the Fire Dynamics Simulator (FDS) in calculating the transport of energy and combustion products from a fire of known heat release rate. The intent of the exercise was not to predict the heat release rate, but rather the transport of the fire's exhaust products throughout a fairly large compartment. The compartment was heavily instrumented so that all of the energy from the fire could be accounted for and reported in terms of conductive losses to walls, convective flux through openings, etc. With the large number of measurements, it was possible to go beyond the traditional point by point comparison and discover why the model either over-predicted or under-predicted a given measurement. It was also possible to compare the transport of energy, starting with the combustion of fuel, and ending with effluent exiting into a large hood. Based on these integrated quantities, discrepancies in heat flux and gas concentration predictions could be tied to errors in the overall energy budget allowing us to assess the accuracy of various components within the model.

\subsection{FDS Input Parameters and Assumptions}

In cooperation with the fire protection engineering community, a computational fire model, Fire Dynamics Simulator (FDS), has been developed at the National Institute of Standards and Technology (NIST) in the USA to study fire behavior and to evaluate the performance of fire protection systems in buildings. The software was released into the public domain in 2000 , and since then has been used for a wide variety of analyses by fire protection engineers. A complete description of the model can be found in McGrattan (2004). Briefly, FDS is a computational fluid dynamics code that solves the Navier-Stokes equations in low Mach number, or thermally-expandable, form. The transport algorithm is based on large eddy simulation techniques, radiation is modeled using a gray-gas approximation and a finitevolume method is used to solve the radiation transport equation. Combustion is modeled using a mixture fraction approach, in which a single transport equation is solved for a scalar variable representing the fraction of gas originating in the fuel stream.

In this section, the most important features of the simulations are described, with emphasis placed on those features that were most important for this particular application. 


\subsubsection{Compartment Geometry}

The geometry of the compartment was relatively simple. The overall enclosure was rectangular, as were the vents and most of the obstructions. A single, rectilinear grid spanned the interior of the compartment. The dimensions of the grid were $100 \times 36 \times 32$, and the cells were shrunk in the horizontal directions to create cells nominally $10 \mathrm{~cm}$ in size in the vicinity of the fire pan. The decision to use a $10 \mathrm{~cm}$ grid was based on the observation that the ratio of the fire's characteristic diameter, $D^{*}$, to the size of the grid cell, $d x$, is an indicator of the degree of resolution achieved by the simulation. $D^{*}$ is given by the expression $\left(\dot{Q} / \rho_{\infty} c_{p} T_{\infty} \sqrt{g}\right)^{2 / 5}$, and was about $1 \mathrm{~m}$ for this series of fires. In short, the greater the ratio $D * / d x$, the more the fire dynamics are resolved directly, and the more accurate the simulation. Past experience has shown that a ratio of 10 produces favorable results at a moderate computational cost (McGrattan et al. 2003).

The vent and door of the compartment conformed to the local gas phase grid. Both were rectangular. The vent was given a prescribed volume flow rate, and the door (when open) was taken as a constant-pressure boundary. In the closed door tests, the compartment was assumed to leak via a small uniform flow spread over the walls and ceiling. The leakage rate was determined from the prescribed leak area and the computed compartment overpressure.

FDS performs a one-dimensional heat transfer calculation into an assumed homogenous material of given thickness and (temperature-dependent) thermal properties. Solid obstructions within the computational domain must conform to the underlying gas phase grid, but the assumed thickness of the solid is not tied to the gas phase grid. Thus, the solid phase heat transfer calculation is entirely decoupled from the gas phase except for the transfer of mass and heat at the gas-solid interface. The compartment walls and ceiling were made of $2.54 \mathrm{~cm}$ thick Marinite I, a product of BNZ Materials, Inc. The manufacturer provided the thermal properties of the material used in the calculation: density $737 \mathrm{~kg} / \mathrm{m}^{3}$, conductivity $0.12 \mathrm{~W} / \mathrm{m} / \mathrm{K}$, specific heat $1.17 \mathrm{~kJ} / \mathrm{kg} / \mathrm{K}$ at $93^{\circ} \mathrm{C}$ ramping linearly to $1.42 \mathrm{~kJ} / \mathrm{kg} / \mathrm{K}$ at $425^{\circ} \mathrm{C}$. A testing laboratory also provided thermal properties, but the results were relatively insensitive to the differences, as will be shown below.

The cable trays were modeled as rectangular solids with cross sectional areas conforming to the local computational grid. The sides of the solids were assumed to be made of thin steel, the top and bottom slabs of plastic (PVC or XLP) whose properties were provided. No at- 
tempt was made to model the individual cables. Rather, the collections of cables were taken as solid slabs with a thickness equal to their actual diameter. Lateral heat conduction along the metal conductors within the cables was not modeled. There are no specific sub-models in FDS to address the thermal response of bundled power cables other than the assumptions just discussed. Thus, for this type of fire scenario, the physical description of the heat transfer and ultimately the burning of the cables is the most likely cause of "user effects". FDS is designed to produce an accurate prediction of the gas temperature and heat flux to objects in the compartment. It does not have detailed algorithms to model the thermal response of a complicated object, but rather that of a simplified object of equivalent thermal behavior. The user must describe using a limited set of parameters how this simpler object is to be represented in the model, and little guidance is provided in the model documentation as there are simply too many objects to consider.

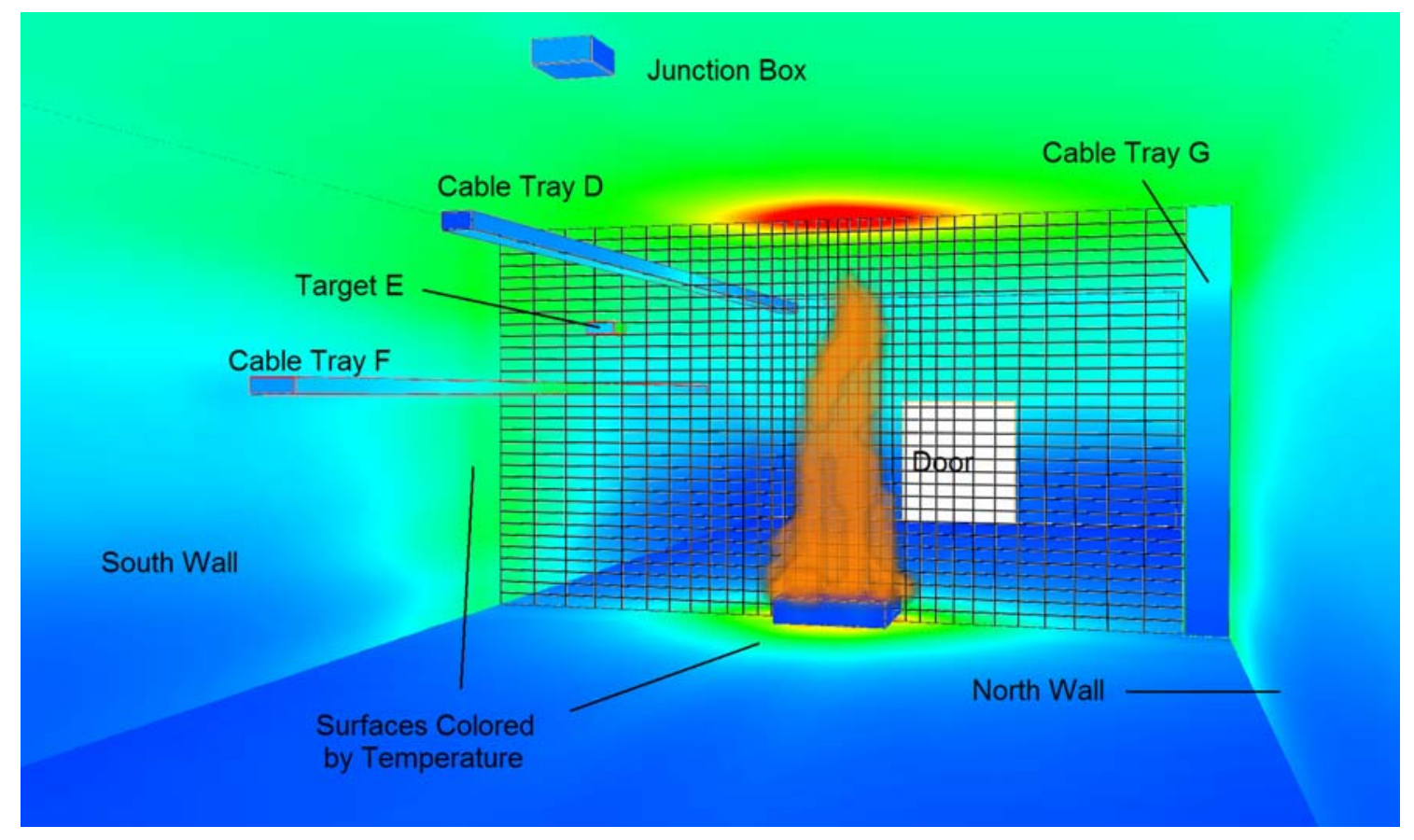

Figure 7-1. View of Test 3 simulation from the rear of the compartment.

\subsubsection{Fire}

The properties of the liquid fuels used in the tests were provided from measurements made on a series of unconfined burns (Hamins 2003). Most of the tests were run with a blend of heptane isomers, primarily $\mathrm{C}_{7} \mathrm{H}_{16}$. Its soot yield was prescribed a constant value of $1.5 \%$.; the $\mathrm{CO}$ yield a constant value of $0.6 \%$. The present version of FDS does not adjust the soot 
or $\mathrm{CO}$ yield as a consequence of reduced compartment ventilation or combustion efficiency. In one test (Test 17) the fuel used was toluene, $\mathrm{C}_{7} \mathrm{H}_{8}$, with a soot yield of $16 \%$ and a $\mathrm{CO}$ yield of $7 \%^{2}$. The reported radiative fractions of the test fires was not used directly in the calculations. Rather, FDS assumed that the radiative fraction from the fire was the larger of two values: $35 \%$ or that which was calculated directly by the radiation solver. Details can by found in McGrattan (2004).

The heat release rate of the simulated burner was set to that which was measured in the experiments. No attempt was made to model the spray burner. The pan was simply prescribed as a solid object out of which the given fuel flowed uniformly at a rate necessary to achieve the specified heat release rate. The only change made was to make the pan $1 \mathrm{~m}$ by $1 \mathrm{~m}$ in the model, to account for the fact that the fire did not engulf the entire pan area, but roughly half. This change was made after the series of blind calculations. It was an easy change to make, and produced only a slight improvement in results.

In almost all fire simulations, the heat release rate (HRR) is the most important parameter. The mass and energy introduced into the compartment are proportional to the HRR, and the measured quantities are all nearly linear functions of the HRR. While the simulations of the fire tests involved dozens of user-prescribed parameters, none was more important than the HRR. The specified HRR was not achieved in the experiments, and as a result the calculations that were re-done after the blind series were modified with a roughly $15 \%$ increase in the HRR.

\subsubsection{Radiation}

FDS uses a finite volume method to solve the radiation transport equation in the gray gas limit. By default, the radiation from the fire and hot gases is tracked in 100 directions. While this is adequate to predict the radiation heat flux to nearby targets (a few fire diameters away), it is not always adequate to predict the flux to distant targets (greater than three diameters, roughly). Because part of the exercise was to predict the heat flux to targets greater than $3 \mathrm{~m}$ from the fire, the number of radiation angles used by the solver in FDS was increased to 200. To compensate for the increased computational cost of doubling the num-

\footnotetext{
${ }^{2}$ The test specification listed the soot yield of the toluene as $19.5 \% \pm 5.2 \%$. In the simulations, $16 \%$ was used simply because it was left over from another study.
} 
ber of angles, the frequency at which the radiation field was updated was roughly halved. The loss in temporal fidelity was not considered a problem because the fire was not spreading or changing rapidly in size. Given the time step used in the simulations, the radiation field was fully updated about every $0.2 \mathrm{~s}$.

Figure 7-2 demonstrates the "spotting" by the radiation solver early in the simulation of Test 3 (40 s). Note that the east wall (left side of figure) shows evidence of the finite number of angles with which the radiation solver uses to calculate the transport of radiation from the fire. Slightly later, at $90 \mathrm{~s}$, the spotting is less pronounced because the source of the thermal radiation is no longer the fire alone, but rather the hot upper layer forming over the entire length and width of the compartment. The sensitivity of the results to the choice of radiation angles is discussed below. 


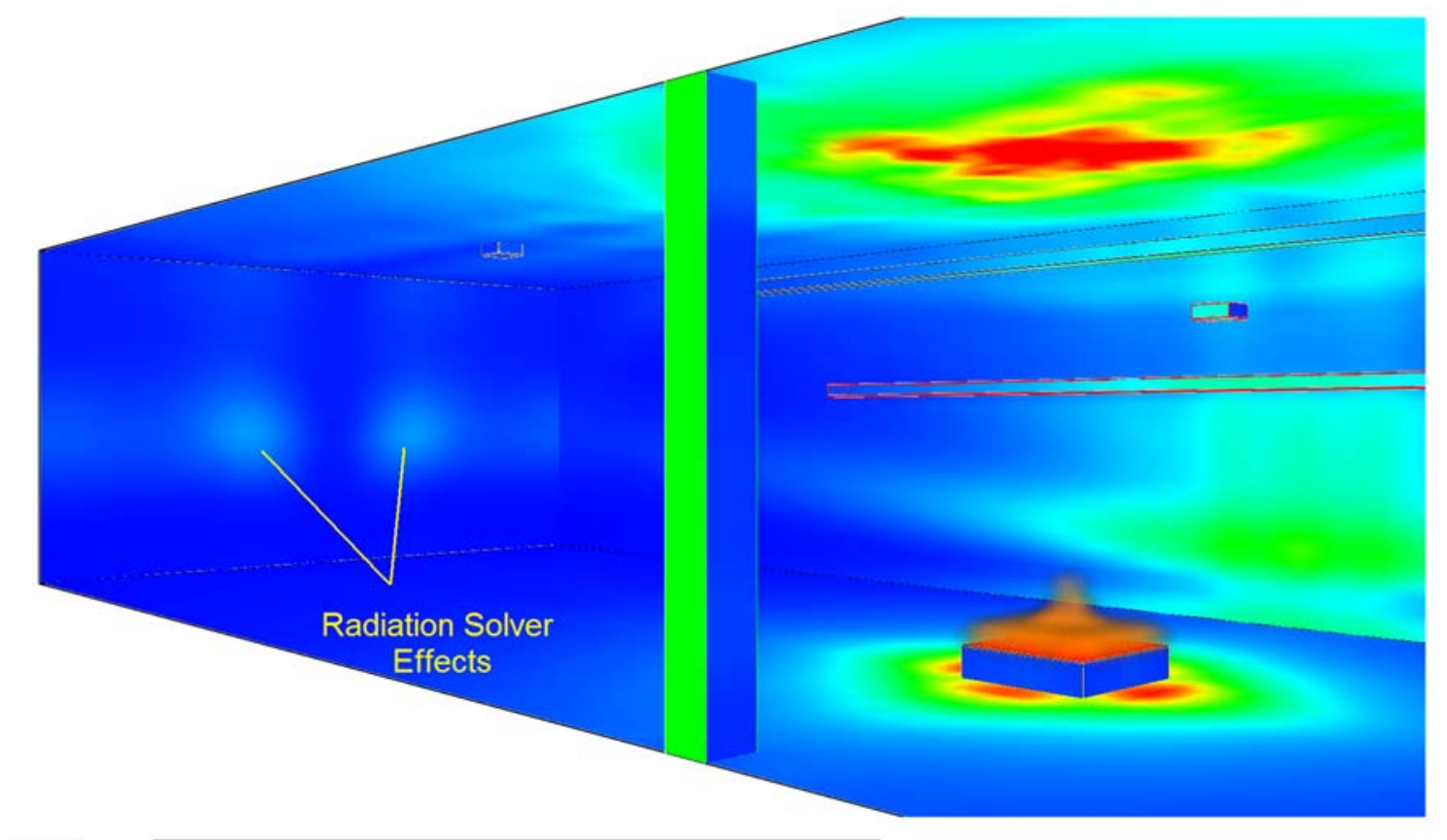

Time: 40.0

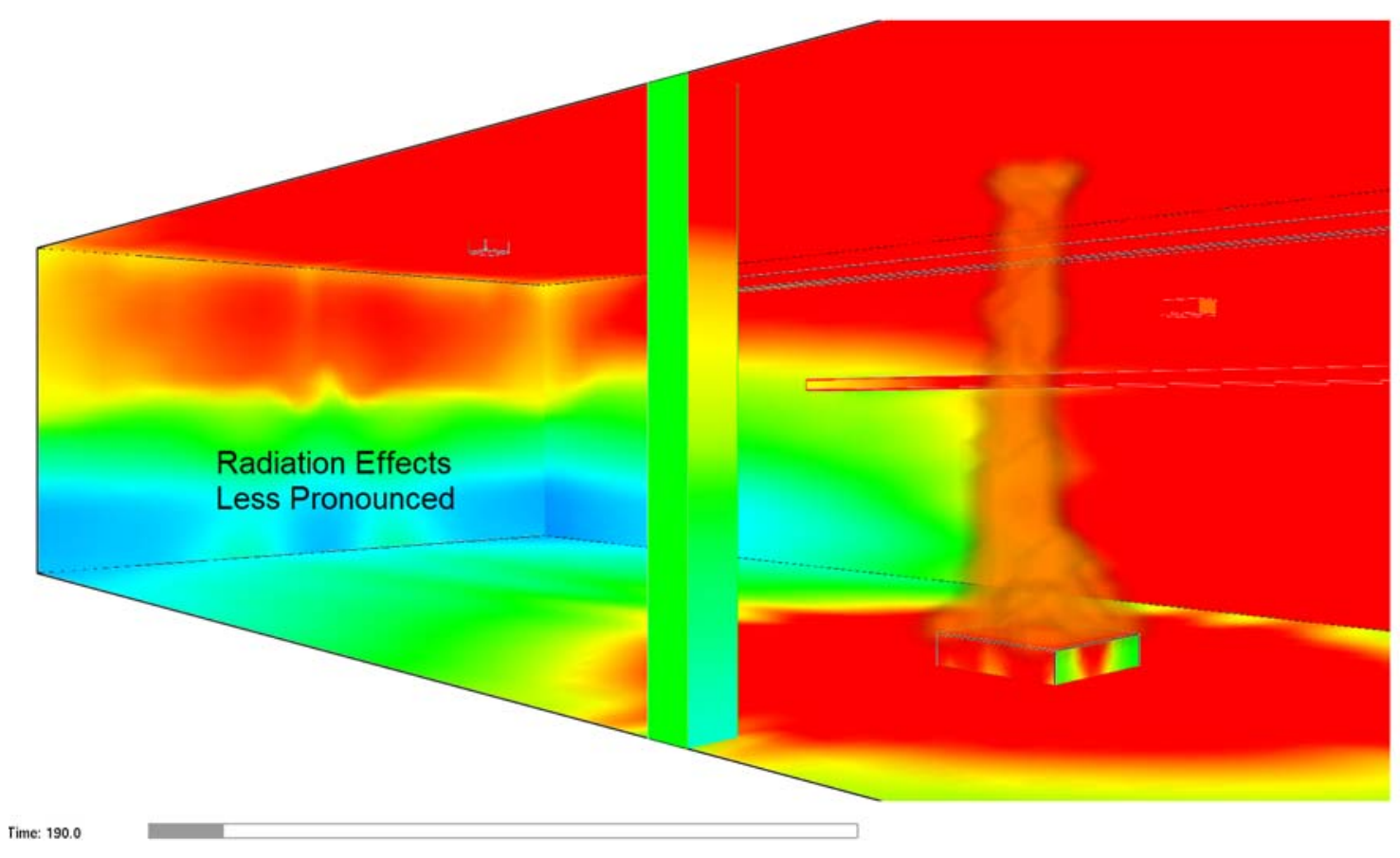

Figure 7-2. Heat flux to compartment surfaces, highlighting numerical effects. 


\subsubsection{Output}

During the simulation, values of temperature, heat flux and gas species concentrations, etc., were reported as $5 \mathrm{~s}$ averages. Linear interpolation was used to approximate values between the $10 \mathrm{~cm}$ grid cells. The results were saved in a text file and compared with the measurements.

Note that the measured and predicted gas temperatures that are reported here are the barebead thermocouple temperatures. FDS has an option of estimating the temperature of the thermocouple bead itself, rather than the true gas temperature (Welsh and Rubini 1997; McGrattan and Forney 2004). Often the reported gas temperature is actually the temperature of the thermocouple bead itself. The distinction usually only matters for the lower layer bare-bead thermocouples that are subjected to significant heat fluxes from the nearby fire and/or hot smoke layer. The original predictions of gas temperature under-predicted the lowest thermocouple temperatures by $20 \%$ to $30 \%$. When recomputed as thermocouple temperatures, the agreement was significantly improved ${ }^{3}$.

Also, note that the heat flux to the compartment walls was reported by the experimentalists as a net flux. The uncooled wall flux gauges consisted of essentially a thermocouple preened to the back side of a small steel plate. On the other hand, the heat flux gauges mounted near the cable trays were cooled and measured the total heat flux. To make both sets of heat flux measurements/predictions consistent, the measured net heat flux to the compartment walls, ceiling and floor were corrected (by the analyst, not the experimentalists) using the formula:

$\dot{q}_{\text {total }}^{\prime \prime}=q_{n e t}^{\prime \prime}+\sigma\left(T_{s}^{4}-T_{\infty}^{4}\right)+h\left(T_{s}-T_{\infty}\right)$

where $T_{s}$ is the surface temperature, $T_{\infty}$ is the ambient temperature, and $h$ is the heat transfer coefficient between the gas and the wall. The total heat flux was predicted directly by FDS. The heat transfer coefficient used in the conversion of the measured fluxes was the same correlation that was used by FDS (McGrattan 2004).

\footnotetext{
${ }^{3}$ When correcting the predicted gas temperatures, it was assumed that the bead diameter was $1 \mathrm{~mm}$ and the emissivity of the bead was 0.85 , the default settings in FDS.
} 
Whether one compares the total or net heat flux, there are problems of interpretation. The net heat flux is dependent on the surface temperature of the target, which is another quantity that the model is attempting to predict. The total heat flux is the heat flux to a "virtual" target whose temperature remains at ambient. This is a preferable quantity to use for comparison, but it is a more expensive measurement. Converting net to total or vis verse also introduces additional uncertainty. Thus, for the purpose of evaluating the heat flux predictions to the walls, the better comparison is that of wall surface temperature itself. No corrections were made to either measurement or prediction of wall surface temperature. In some sense, the wall itself acted as a type of flux gauge since its thermal properties were known to a fairly high degree of certainty. The heat flux measurements merely served as a "second opinion," suggesting why a particular wall temperature might have been over or under-predicted.

\subsubsection{Summary}

For Benchmark Exercise 3, the principal developer of FDS (McGrattan) observed the experiments and performed simulations before and after they were conducted. There is a tremendous advantage in understanding the subtleties of both. Thus, for the final calculations that are presented in the next section, care was taken to set up the input files in a way that could be expected of most engineers using the model. Nevertheless, some features were used that are often overlooked or not understood by the practicing engineer, even though they are documented in the User's Guide. Following is a list of those features:

- $\quad$ Outputting the thermocouple, as opposed to the gas, temperature.

- Increasing the number of angles in the radiation calculation.

- $\quad$ Stretching and shrinking the grid to increase spatial resolution near the fire.

- Choosing the geometric parameters for the modeled cable trays.

- Adjusting the burn area of the fire to conform to observation. 


\subsection{Comparison of FDS Open Predictions with Measurements}

Fifteen fire experiments were conducted as part of Benchmark Exercise 3. Of these, four were considered replicates. Thus, in this section, only the results of the 11 non-replicates are presented (Tests 1-5 and 13-18).

Wherever possible, multiple measurements at a given location in the compartment are presented on the same page to enable easier comparison. Experimental results are always plotted with solid lines, simulation with dotted lines. The labels included in the legends for each figure are the original labels found in the experimental data files and the simulation input files.

\subsubsection{Gas Temperatures}

Gas temperatures were measured in the experiments using seven floor-to-ceiling thermocouple arrays (or "trees") distributed throughout the compartment. Figure 7-3 through Figure 7-13 display the time histories of both measured and predicted compartment gas temperatures. Three measurement points were chosen from each tree: \#10 (green) which was $30 \mathrm{~cm}$ below the ceiling, \#5 (red) which was about halfway between floor and ceiling; and \#1 (black) which was $35 \mathrm{~cm}$ above the floor. In addition, several measurement locations were chosen in the doorway of the open-door tests: \#16 (black) which was $10 \mathrm{~cm}$ below the top of the door; \#11 (red) which was halfway between door top and bottom; and \#9 (green) which was $20 \mathrm{~cm}$ above the floor.

A means of assessing the accuracy of the predicted temperatures is to first consider the accuracy of the prescribed heat release rate. According to an empirical correlation by McCaffrey, Quintiere and Harkleroad (Walton and Thomas 2003), the increase above ambient in the upper layer gas temperature is proportional to the HRR raised to the $2 / 3$ power. The reported uncertainty in the HRR measurement was $15 \%$ (two standard deviations). This uncertainty led to a $2 / 3 \times 15 \%=10 \%$ uncertainty in the temperature rise. The difference between measured and predicted compartment temperature for most of the measurement locations was less than $10 \%$, i.e. within experimental uncertainty, with some notable exceptions that are discussed below. Errors due to the FDS computation of the thermocouple, as opposed to the gas, temperatures, plus errors in the thermocouple measurements themselves, were far less than the uncertainty associated with the measured HRR. 
Notable disagreement with experimental measurement of compartment temperatures occurred for two reasons. First, in Tests 4, 5 and 16, a fan blew air into the compartment through a vent in the south wall. The measured velocity profiles were input into FDS, but in Tests 4 and 5, the observed cooling of the upper layer temperatures at Tree 3, 4 and 5 was not replicated in the model (In the figures, this discrepancy is denoted by the phrase "Ventilation Effects"). In Test 16, the cooling effect was not observed in either the experiments or the predictions. A possible reason for the discrepancy in Tests 4 and 5 may have been that the grid resolution at the fan was fairly coarse. The grid was shrunk near the burner, but stretched elsewhere. Also, the velocity profile at the supply duct was not uniform, with the bulk of the air blowing from the lower third of the duct, and some recirculation occurring above.

The second notable source of disagreement between measured and predicted temperatures was the near-field behavior of the fire. The lower thermocouple predictions for Tree 6 were often in poor agreement with measurement (In the figures, this discrepancy is denoted by the phrase "Near-Field Effects"). Tree 6 was $10 \mathrm{~cm}$ east of the fuel pan, but in the simulations, the pan was shortened because the burning fuel was observed to have been restricted mainly to the center of the pan. Nevertheless, the shifting of the fire's "footprint" in the model effectively moved the lower thermocouples on Tree 6 further from the fire, leading to lower predictions of their temperature. The situation could have been improved somewhat by experimenting with different "footprints", but such "tweaking" would have given the impression that the model was more accurate in the near-field than it actually was. Remember that the grid cells were $10 \mathrm{~cm}$, and moving the fire by increments of $10 \mathrm{~cm}$ would have led to noticeable variations in the near-field predictions. 

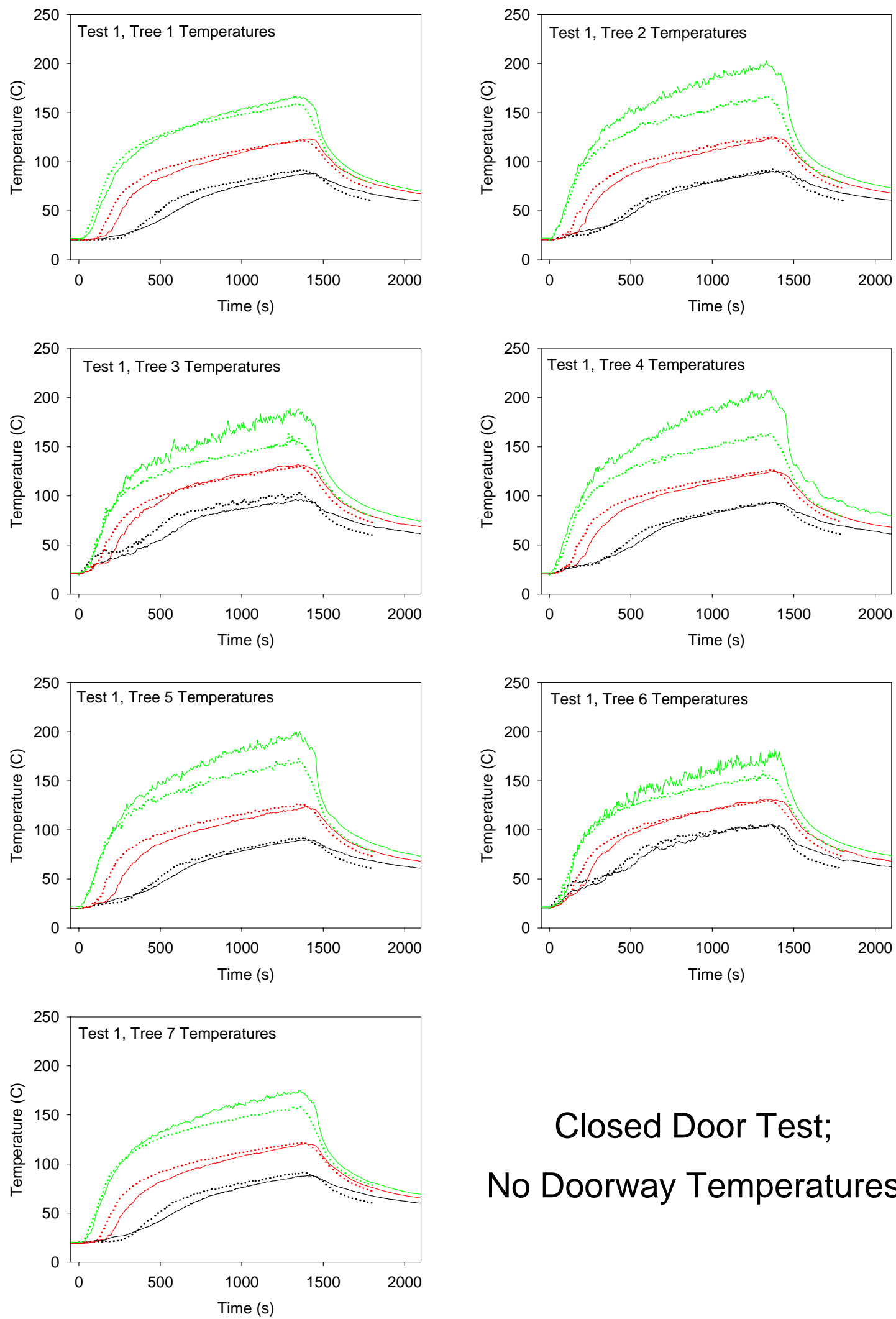

\section{Closed Door Test; No Doorway Temperatures}

Figure 7-3. Gas temperature comparison for Test 1. 

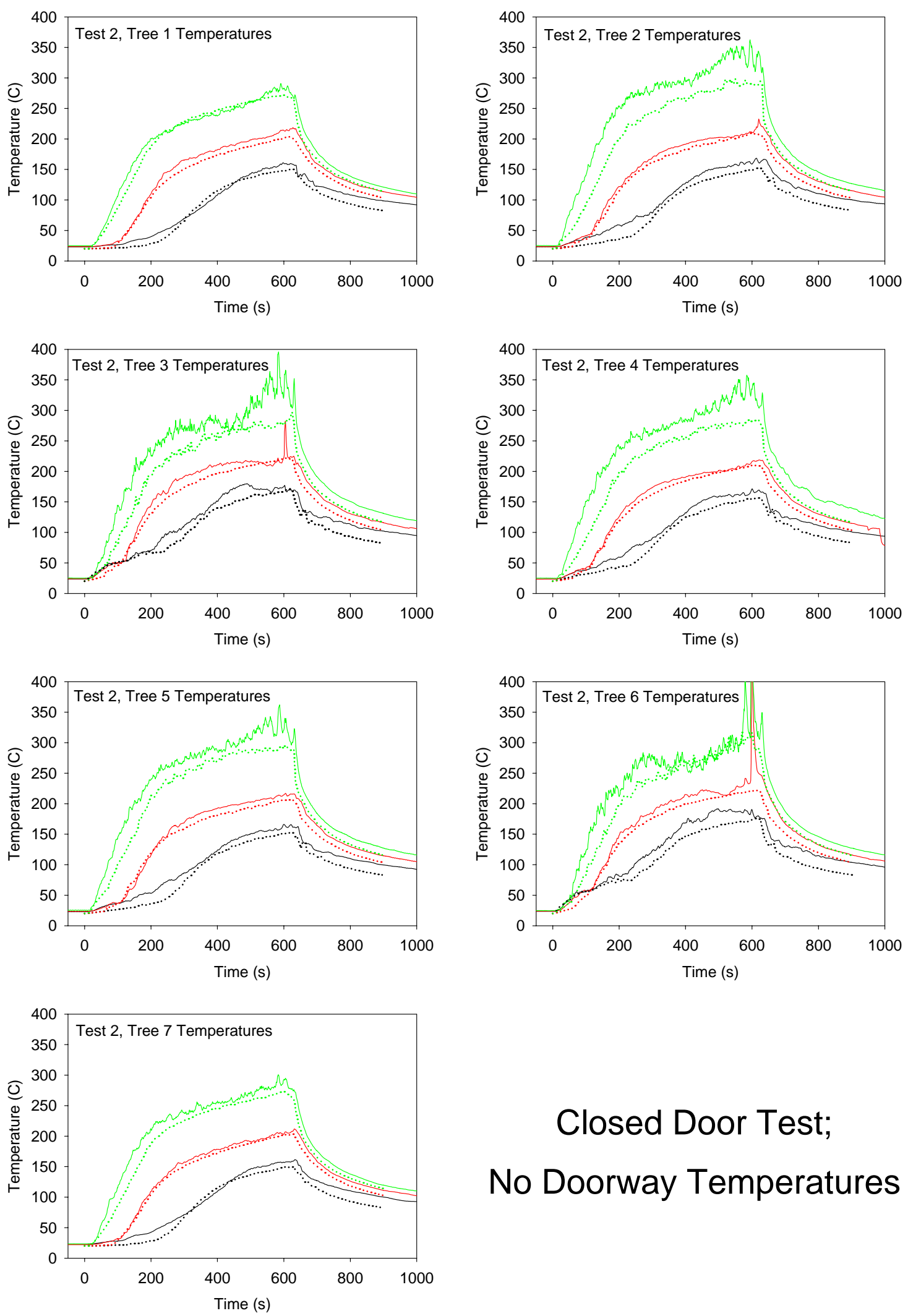

\section{Closed Door Test;}

No Doorway Temperatures

Figure 7-4. Gas temperature comparison for Test 2. 

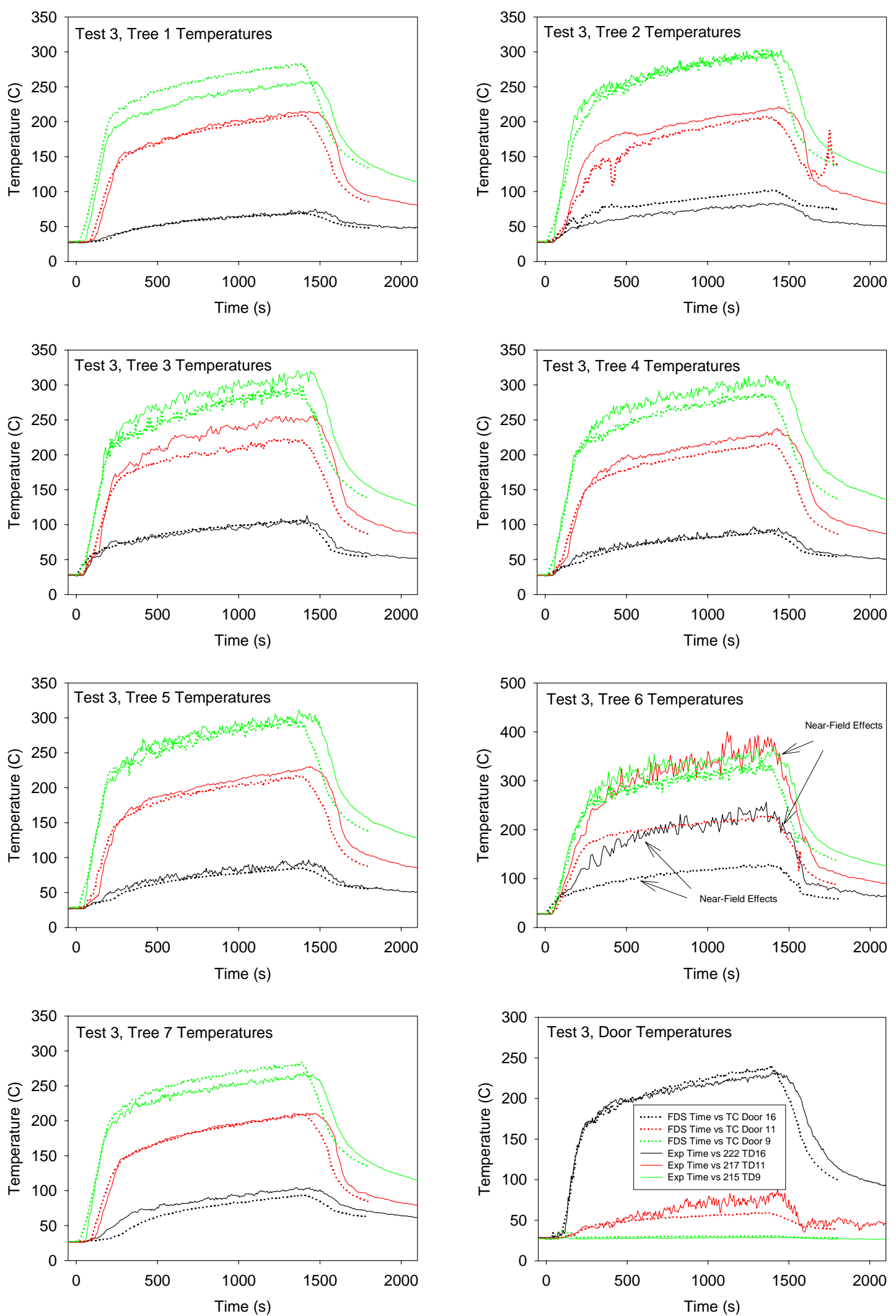

Figure 7-5. Gas temperature comparison for Test 3. 

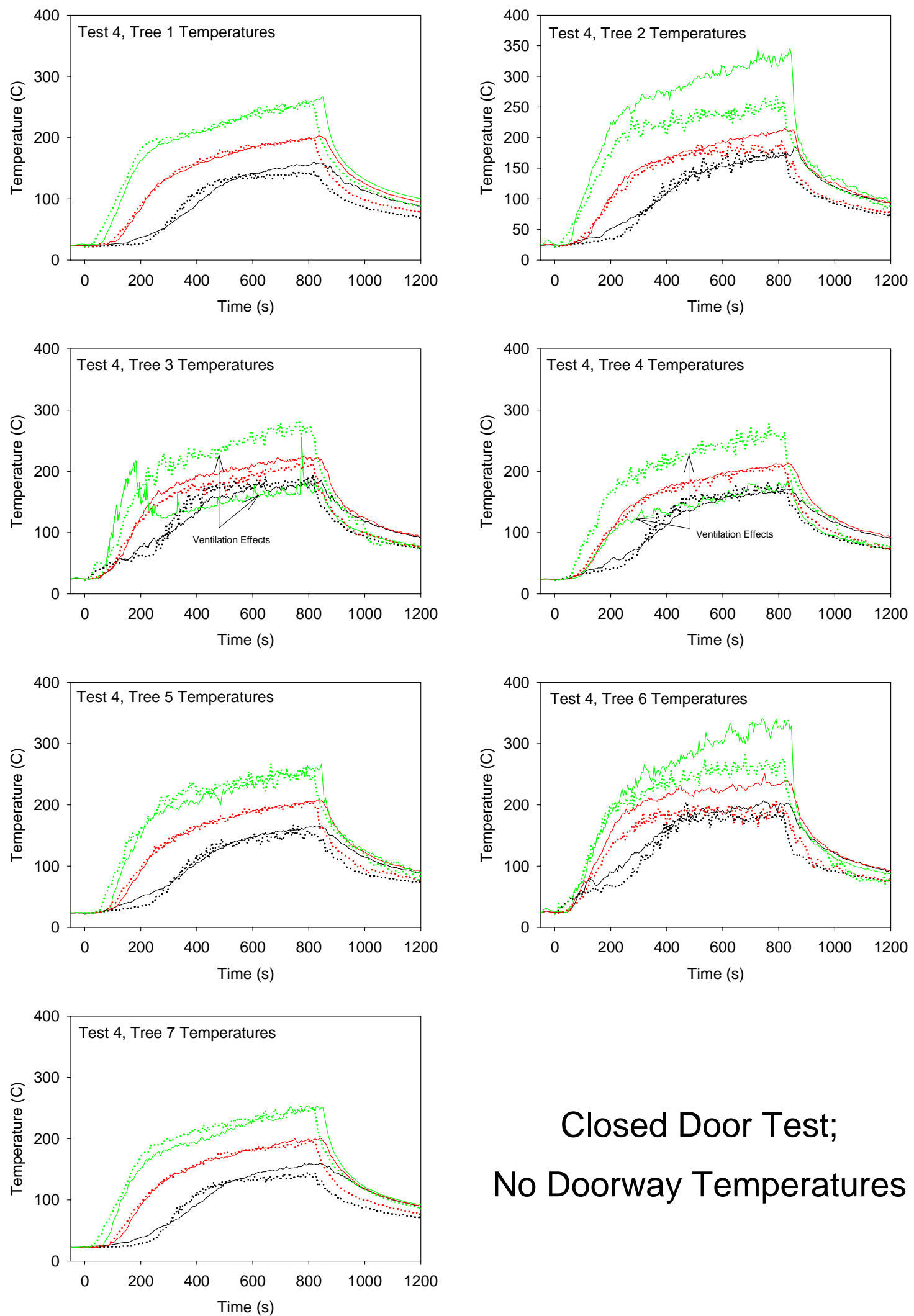

\section{Closed Door Test;}

No Doorway Temperatures

Figure 7-6. Gas temperature comparison for Test 4. 

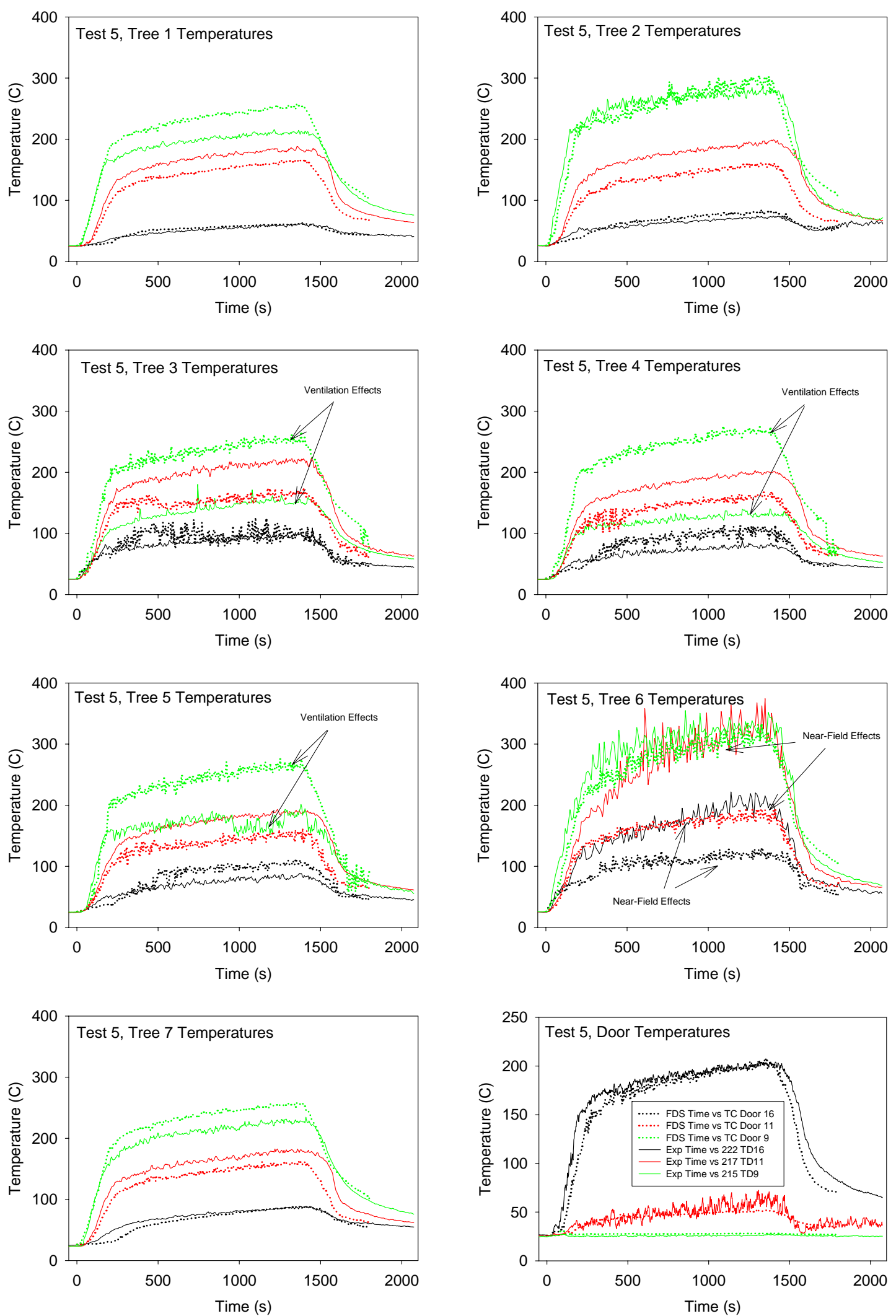

Figure 7-7. Gas temperature comparison for Test 5. 

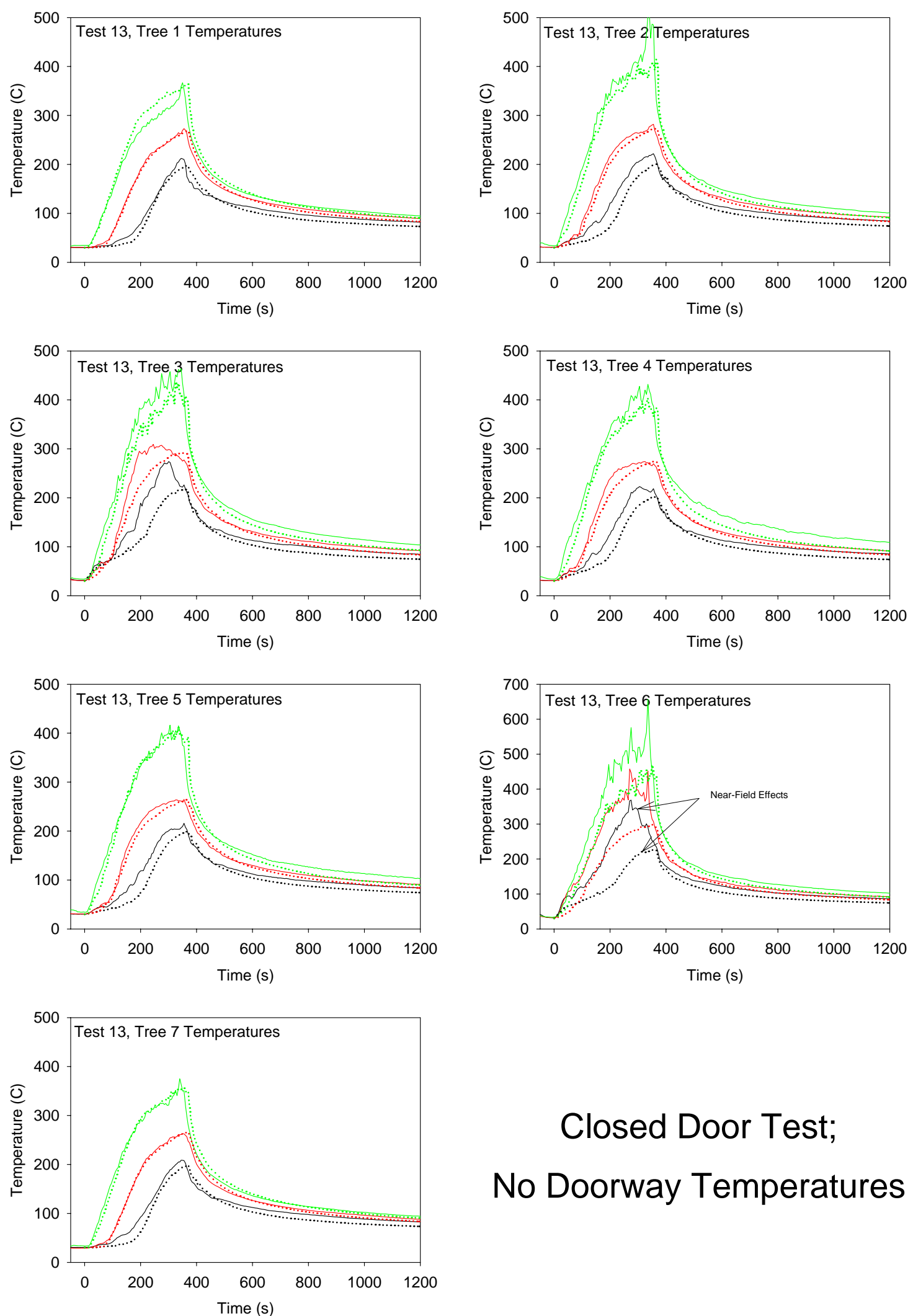

Closed Door Test; No Doorway Temperatures

Figure 7-8. Gas temperature comparison for Test 13. 

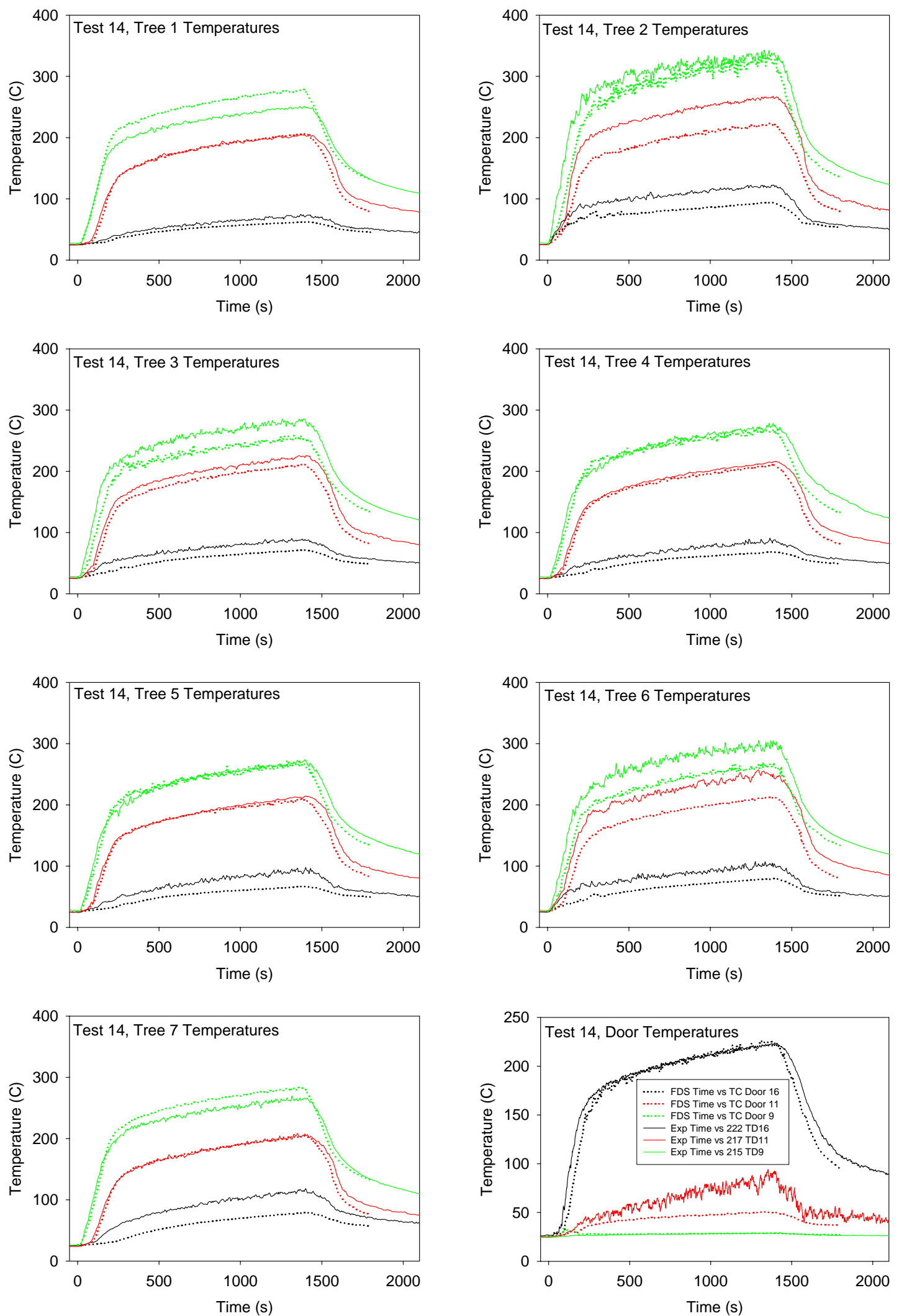

Figure 7-9. Gas temperature comparison for Test 14. 

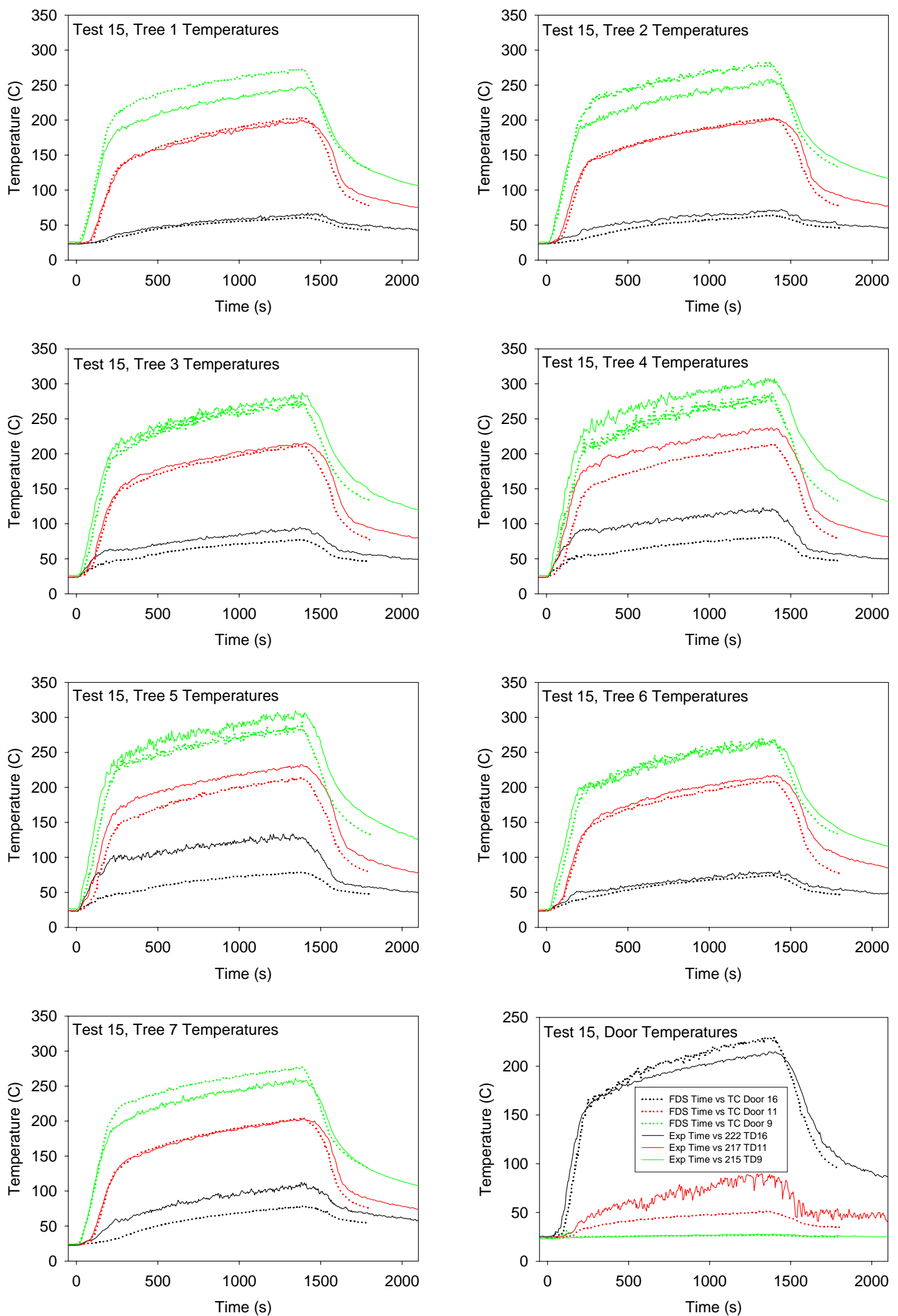

Figure 7-10. Gas temperature comparison for Test 15. 

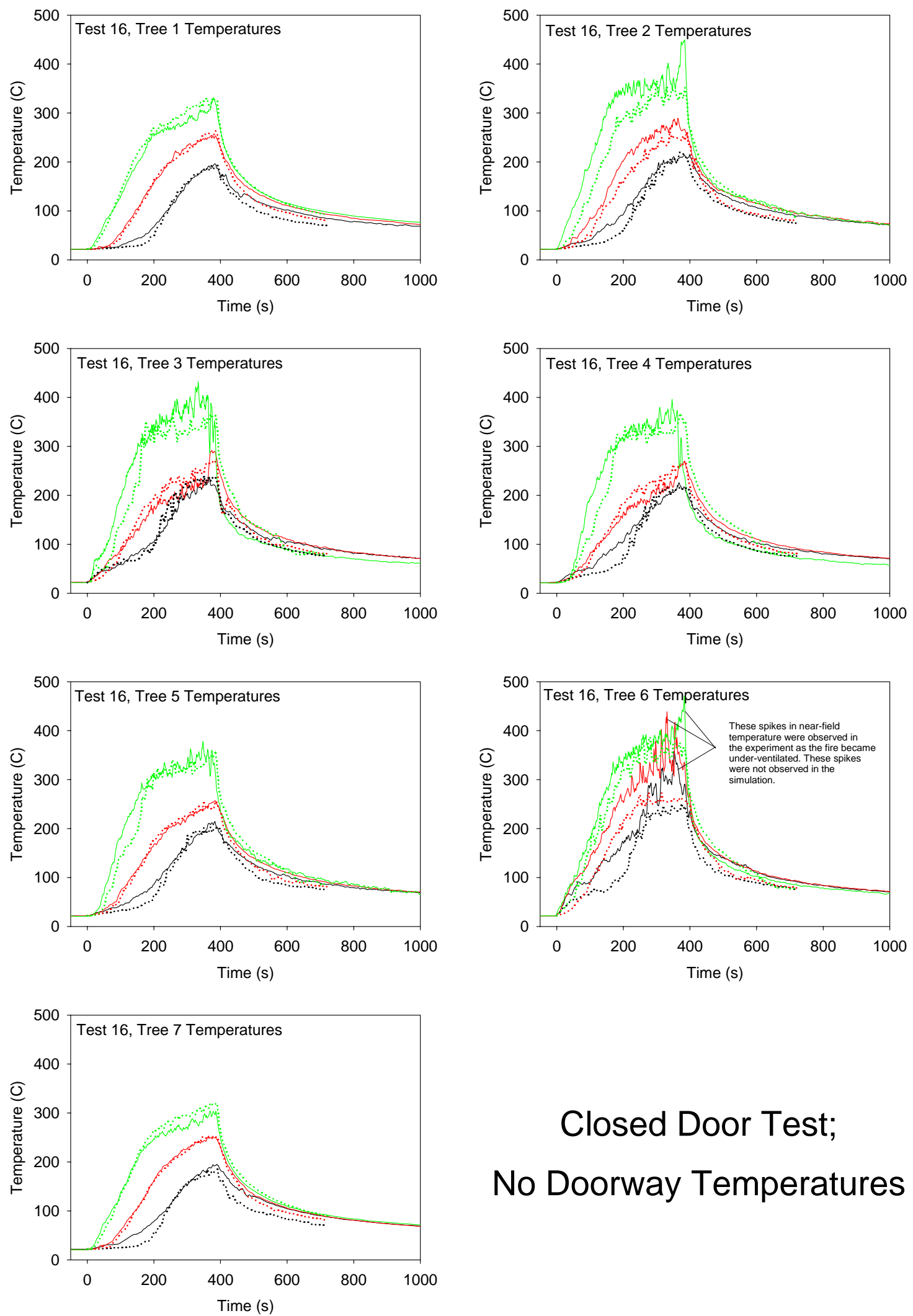

Closed Door Test;

No Doorway Temperatures

Figure 7-11. Gas temperature comparison for Test 16. 

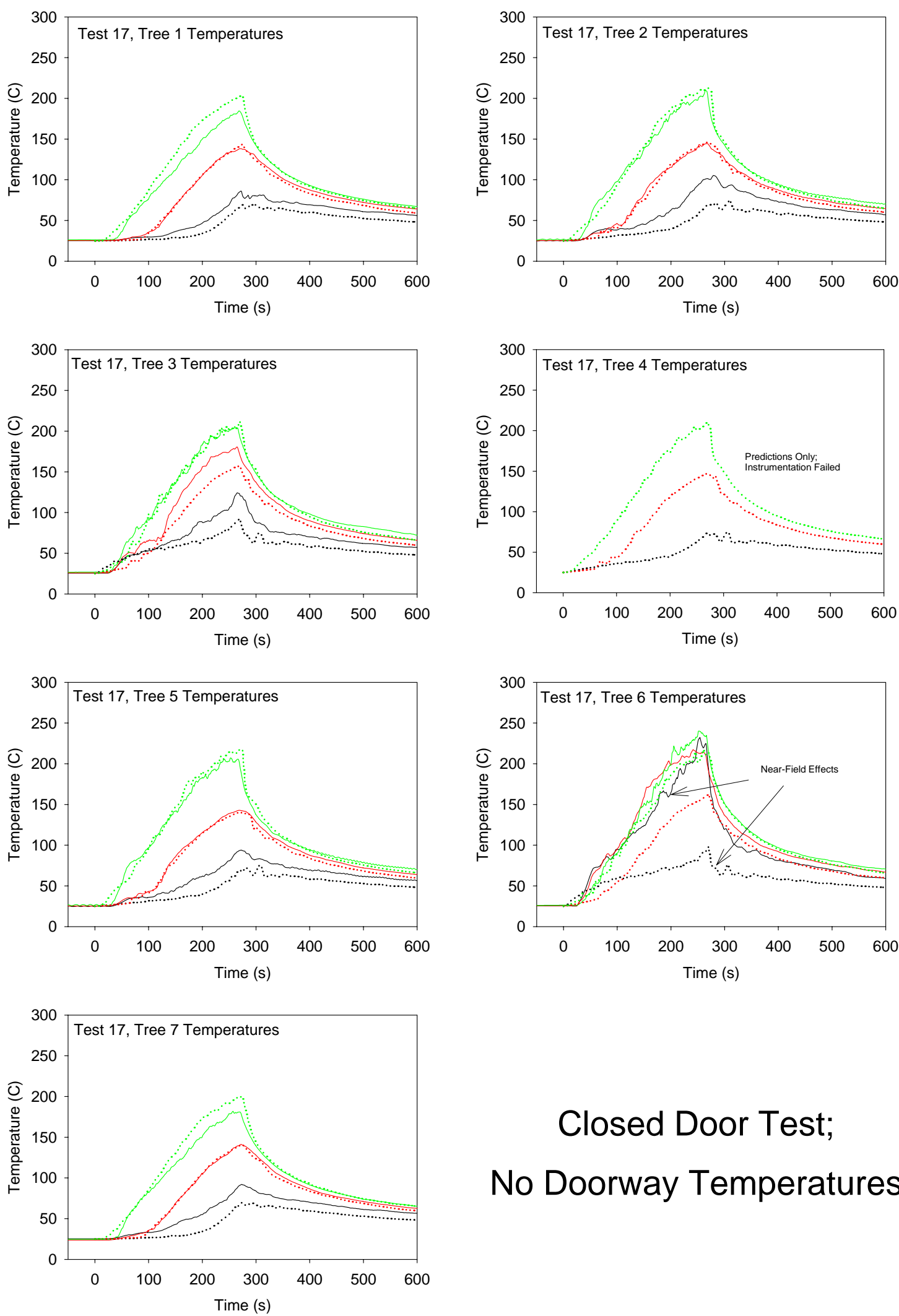

\section{Closed Door Test; No Doorway Temperatures}

Figure 7-12. Gas temperature comparison for Test 17. 

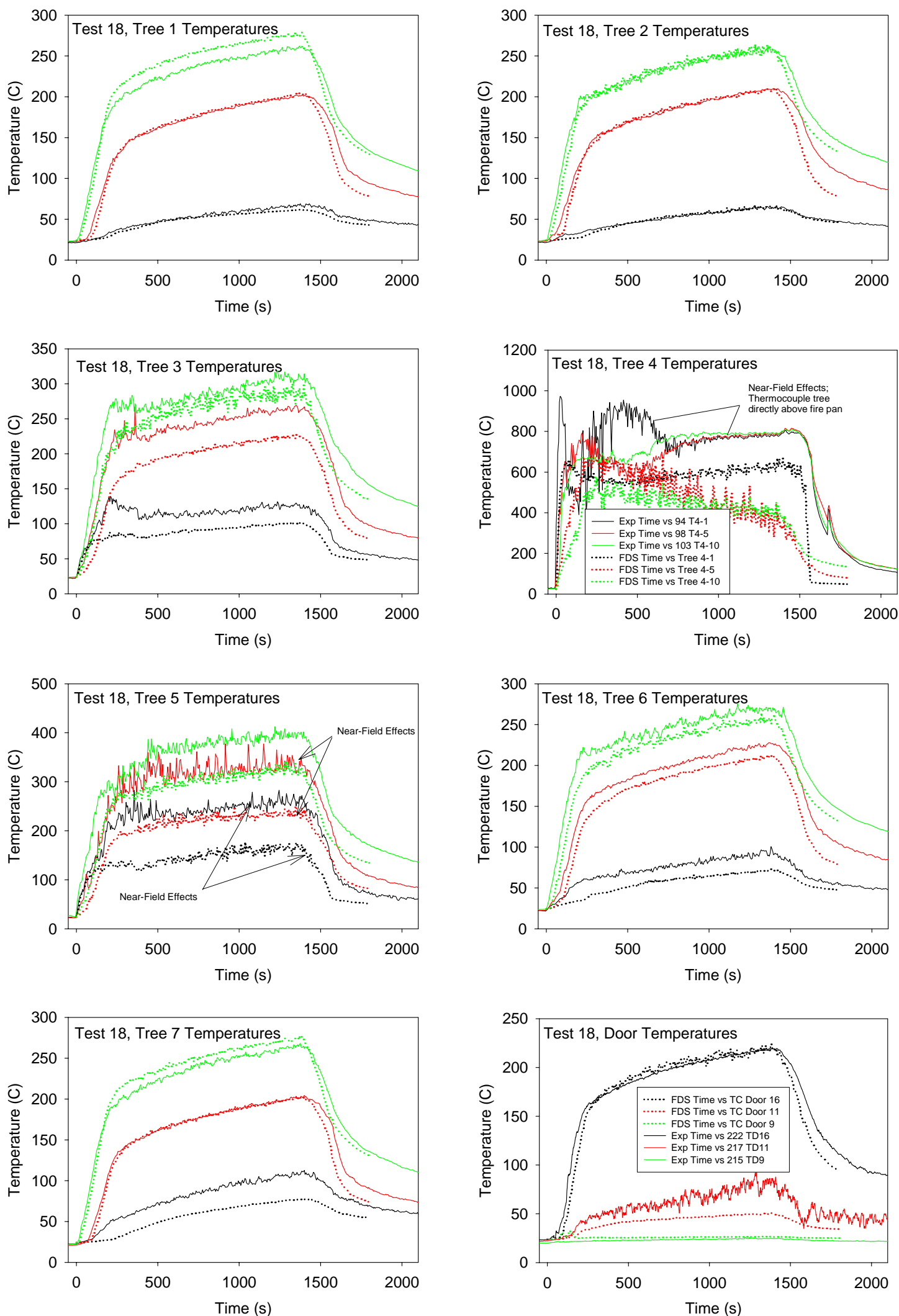

Figure 7-13. Gas temperature comparison for Test 18. 


\subsubsection{Heat Flux and Surface Temperature at the Walls, Ceiling and Floor}

Heat flux gauges were positioned at various locations on all four walls of the compartment, plus the ceiling and floor. Comparisons between measured and predicted heat fluxes and surface temperatures are shown in Figure 7-14 through Figure 7-24. Only half of the measurement points are shown because the points not shown were in roughly the same relative location to the fire and hence the measurements and predictions were similar. For example, only data for the east and north walls are shown because the data from the south and west walls are comparable.

Overall, the compartment surface temperature predictions were very accurate because away from the immediate vicinity of the fire, these surfaces were heated primarily by the hot smoke layer whose temperature was also accurately predicted. The compartment was lined with a material (Marinite) whose thermal properties were well-characterized, there were no steep gradients in the gas temperature field, the convective heat transfer to the wall was based on a fairly reliable experimental correlation, and the radiative flux was easy to calculate as the upper layer gases were of fairly uniform composition and temperature.

Overall, the compartment surface temperature predictions were within $10 \%$ of the measurements, except at locations near the fire where deviations were sometimes larger. Some discrepancies could not be explained, which is why there are many measurement points included in the figures to provide a reasonable sampling of the results. 

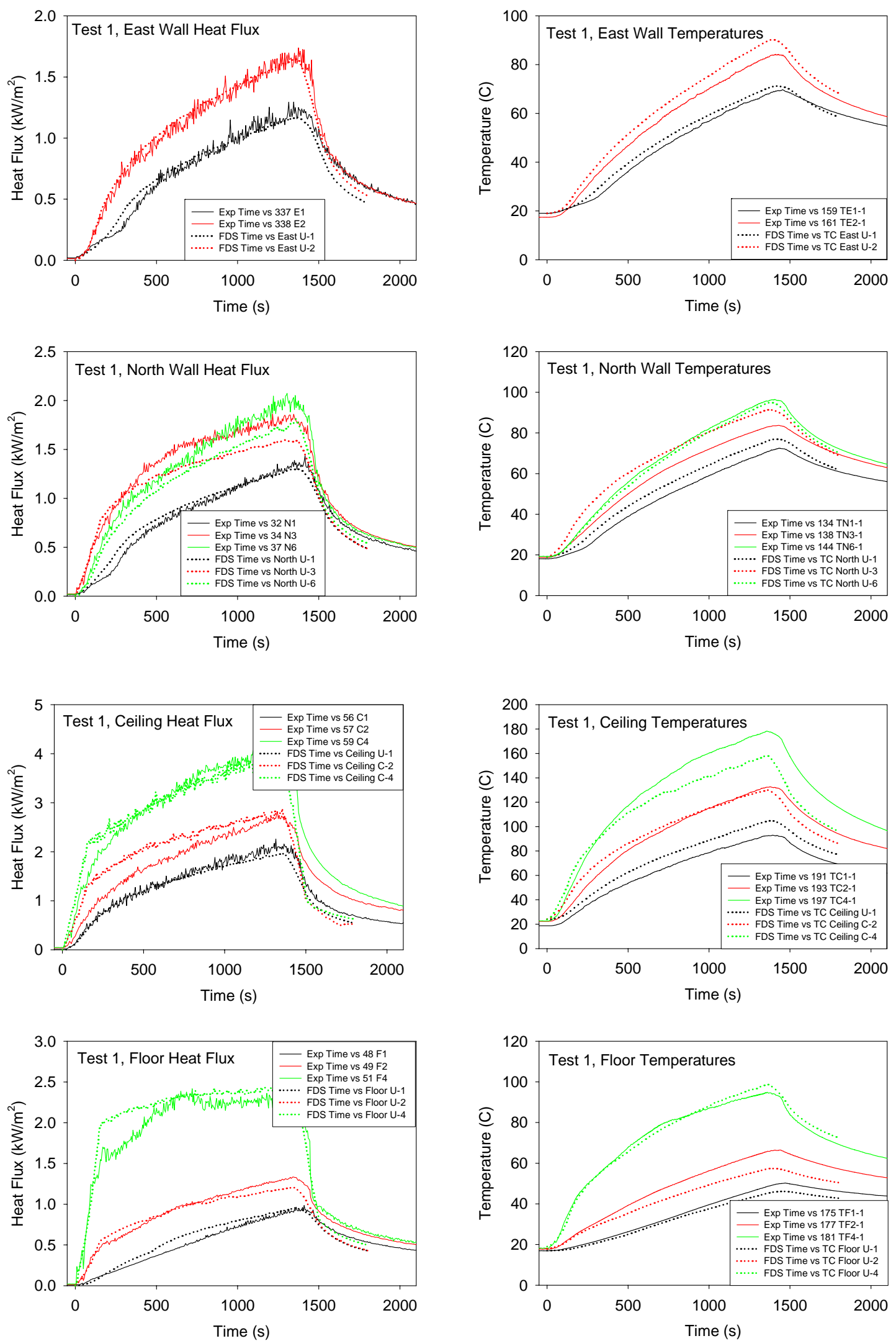

Figure 7-14. Heat flux and temperature at select points on the walls, Test 1. 

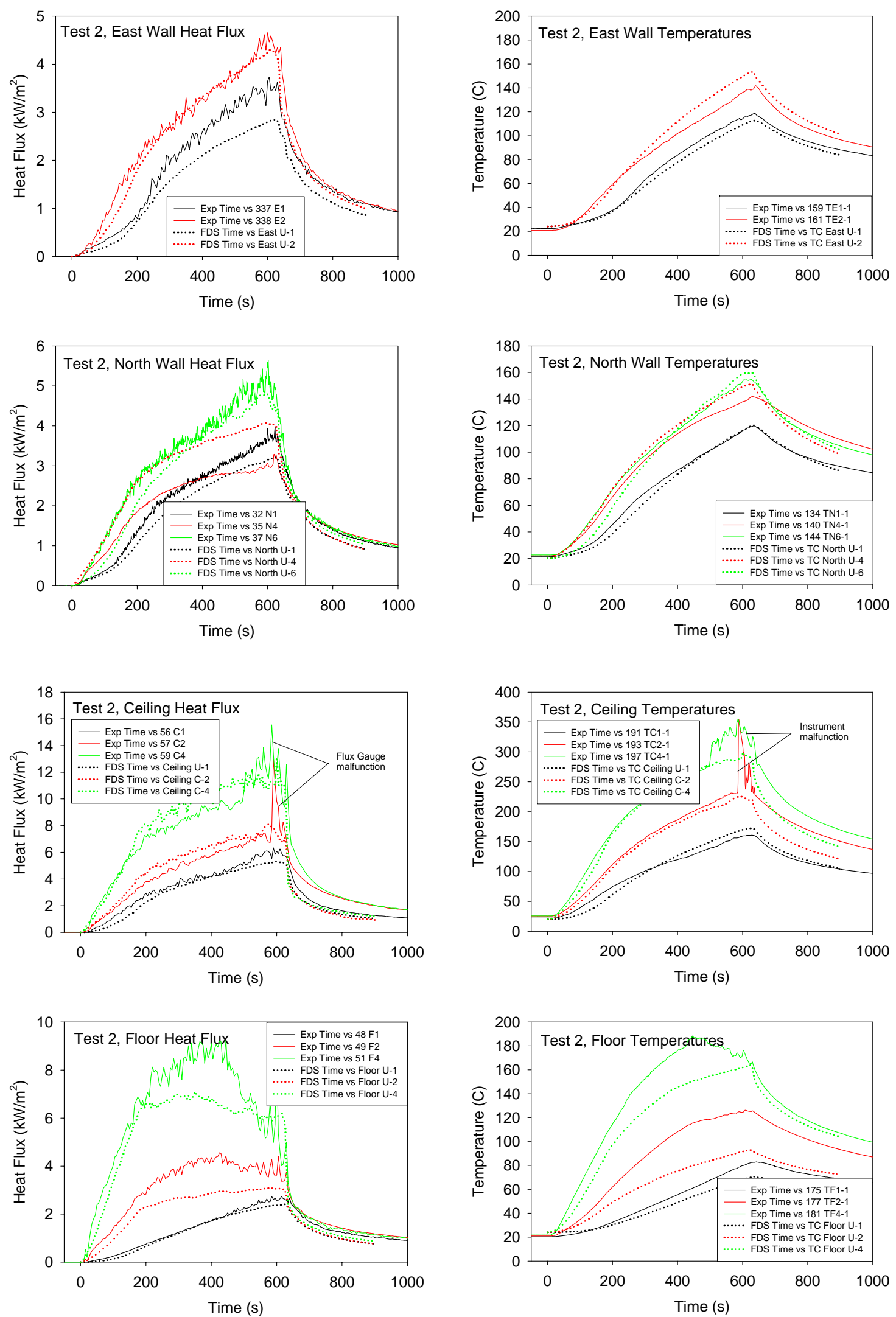

Figure 7-15. Heat flux and temperature at select points on the walls, Test 2. 

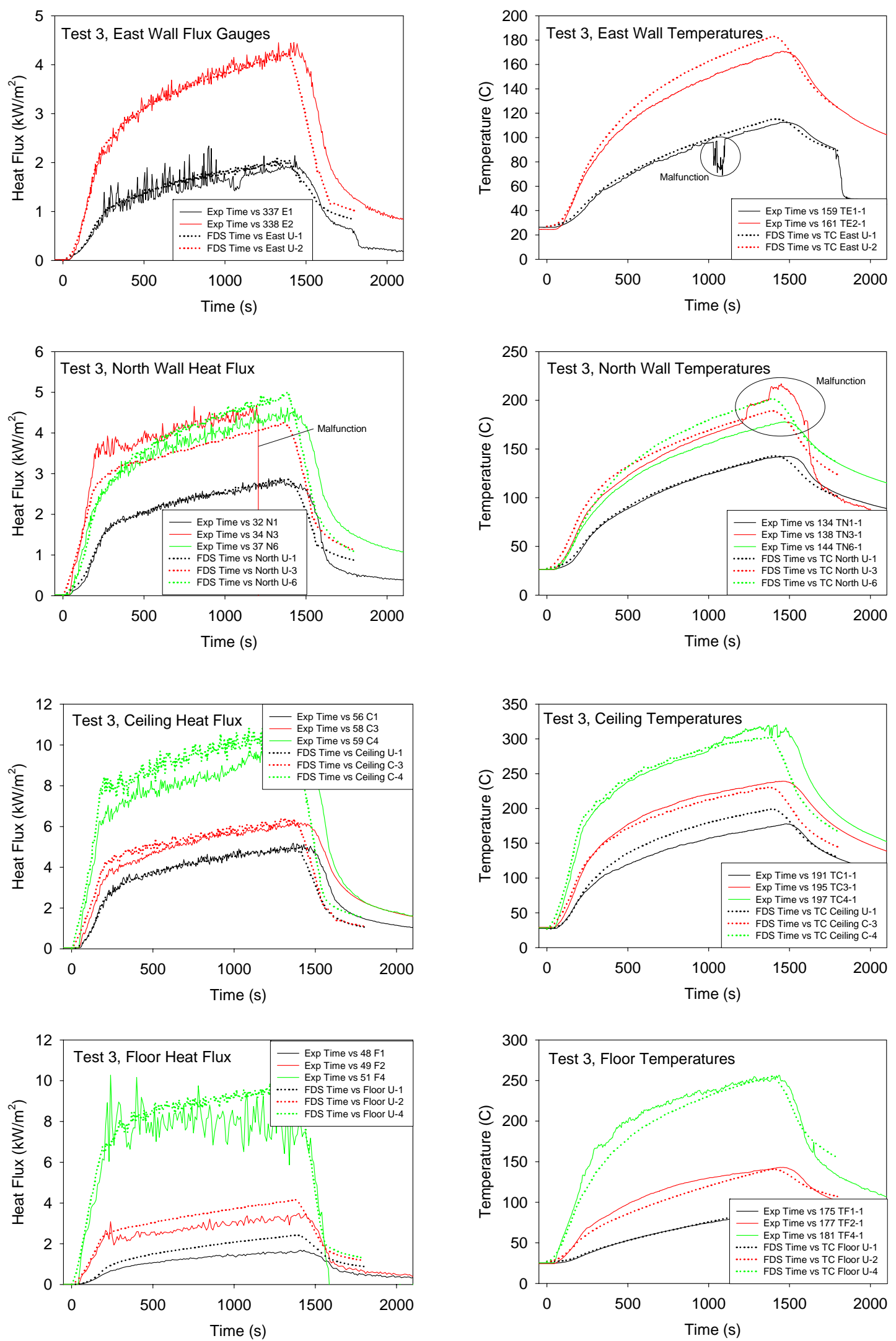

Figure 7-16. Heat flux and temperature at select points on the walls, Test 3. 

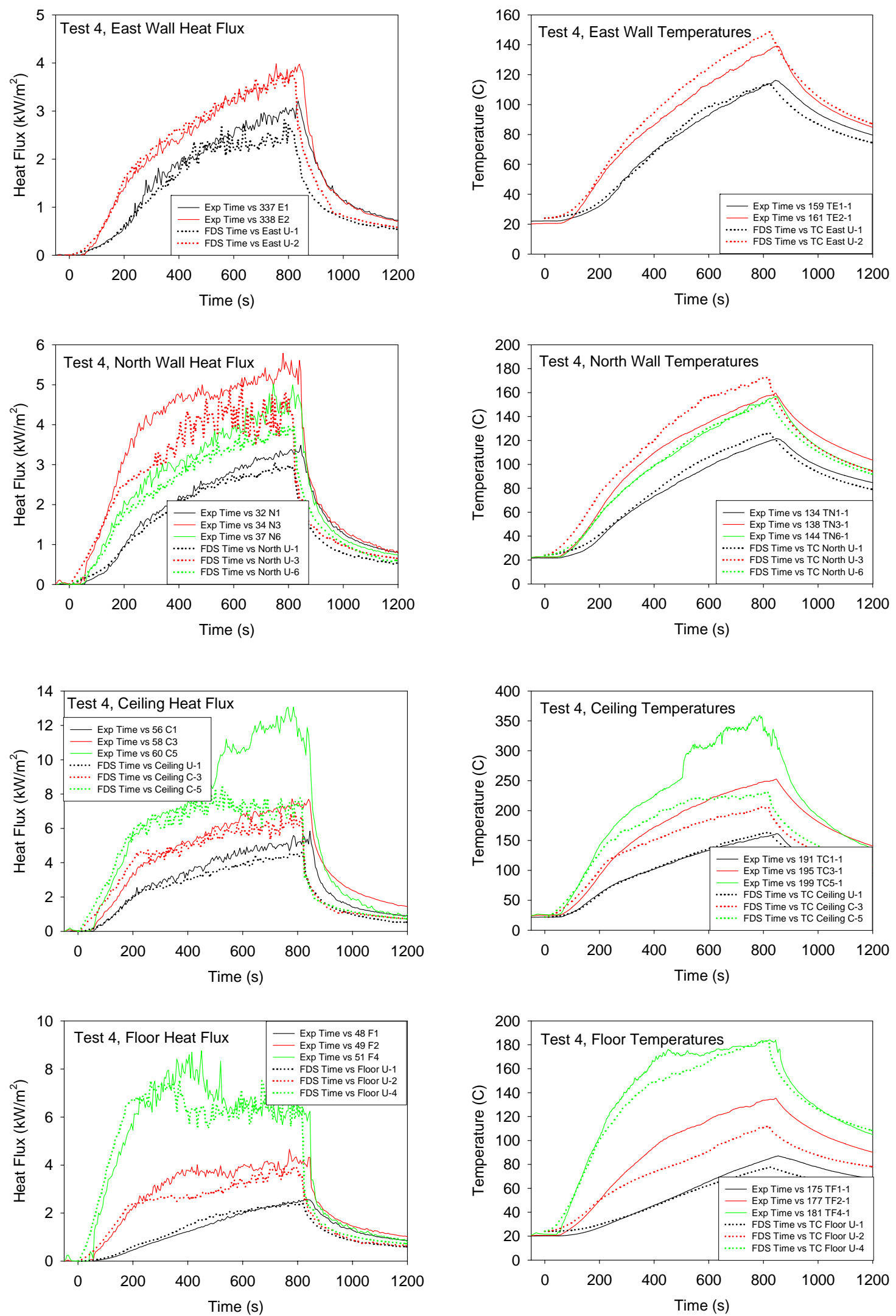

Figure 7-17. Heat flux and temperature at select points on the walls, Test 4. 

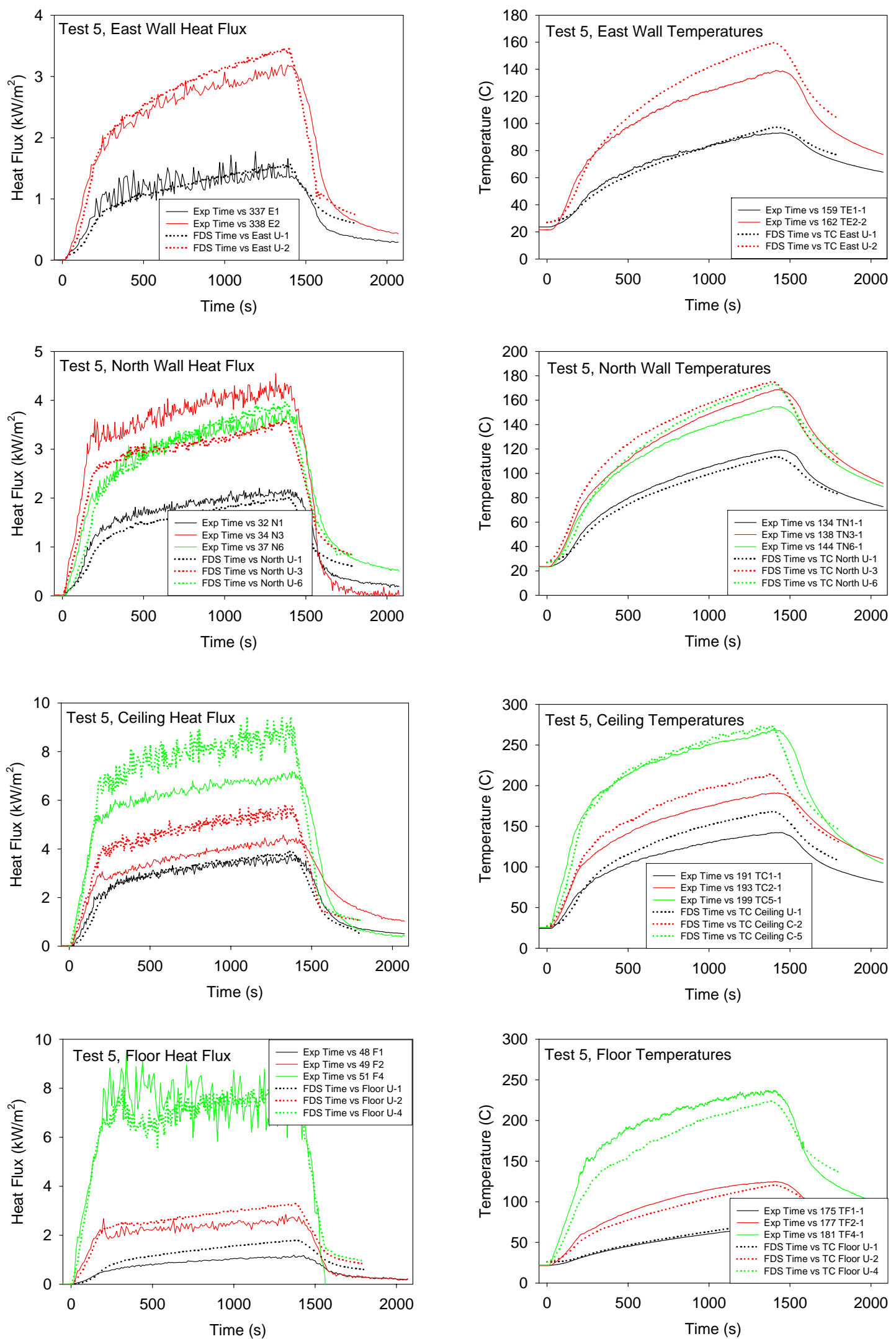

Figure 7-18. Heat flux and temperature at select points on the walls, Test 5. 

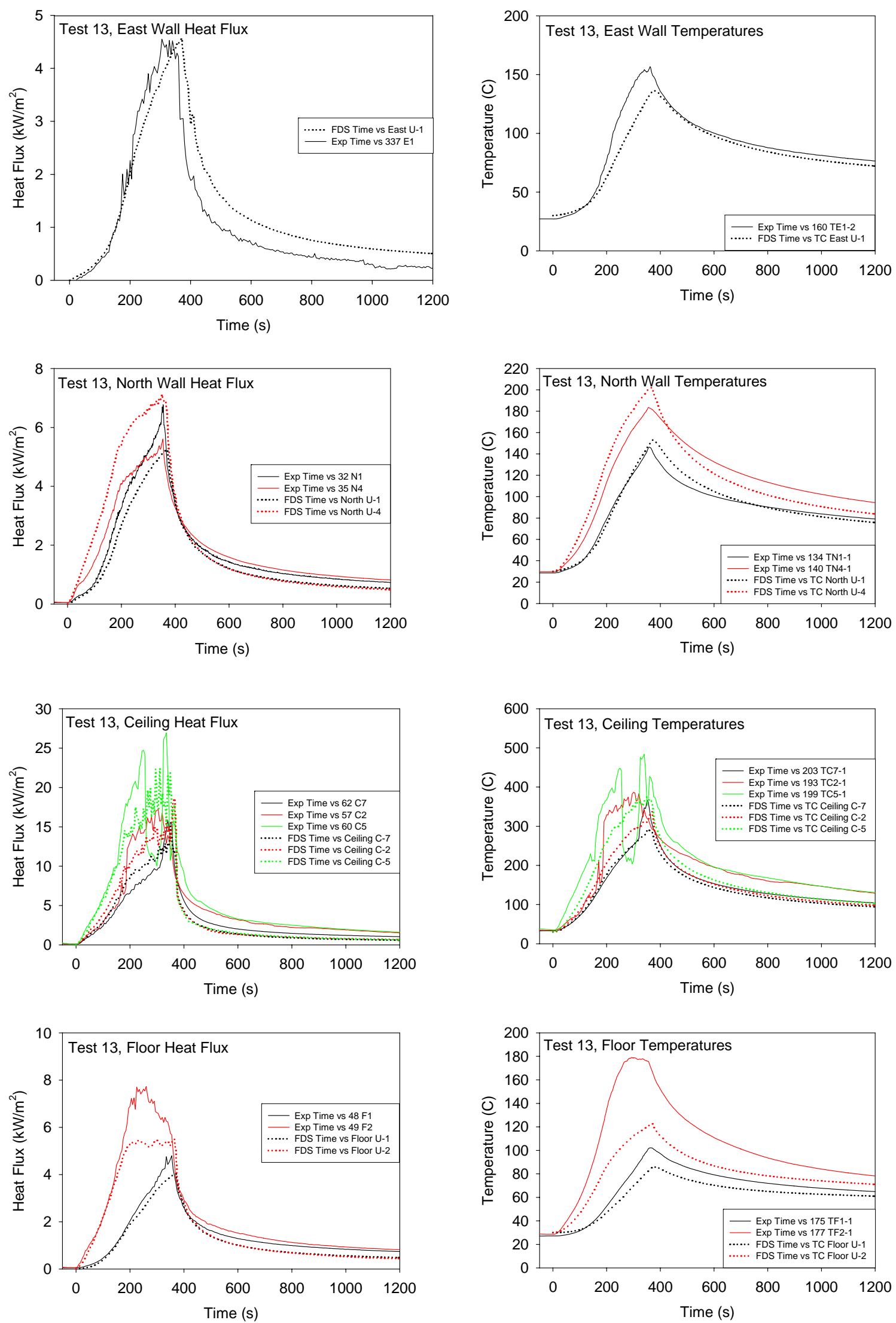

Figure 7-19. Heat flux and temperature at select points on the walls, Test 13. 

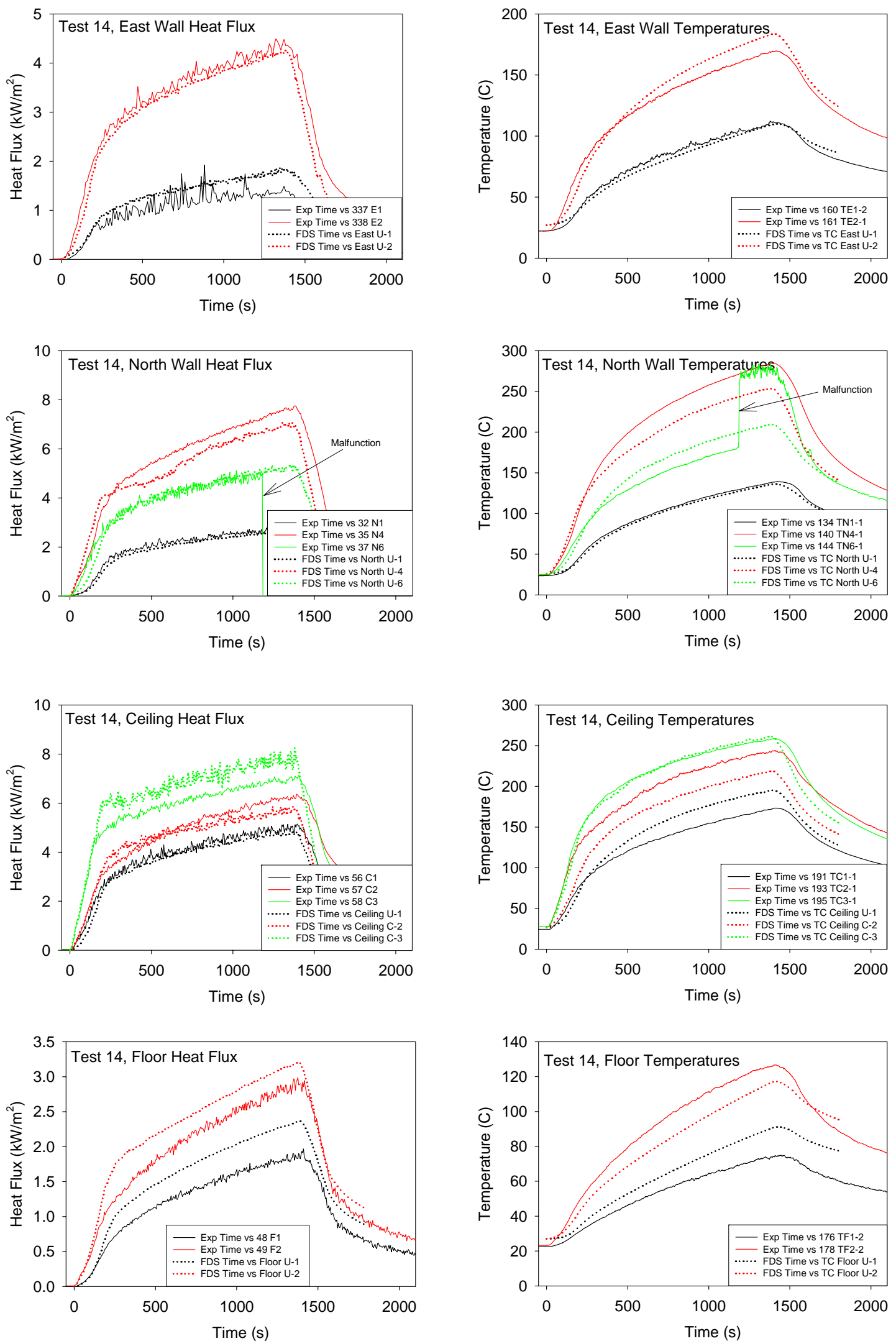

Figure 7-20. Heat flux and temperature at select points on the walls, Test 14. 

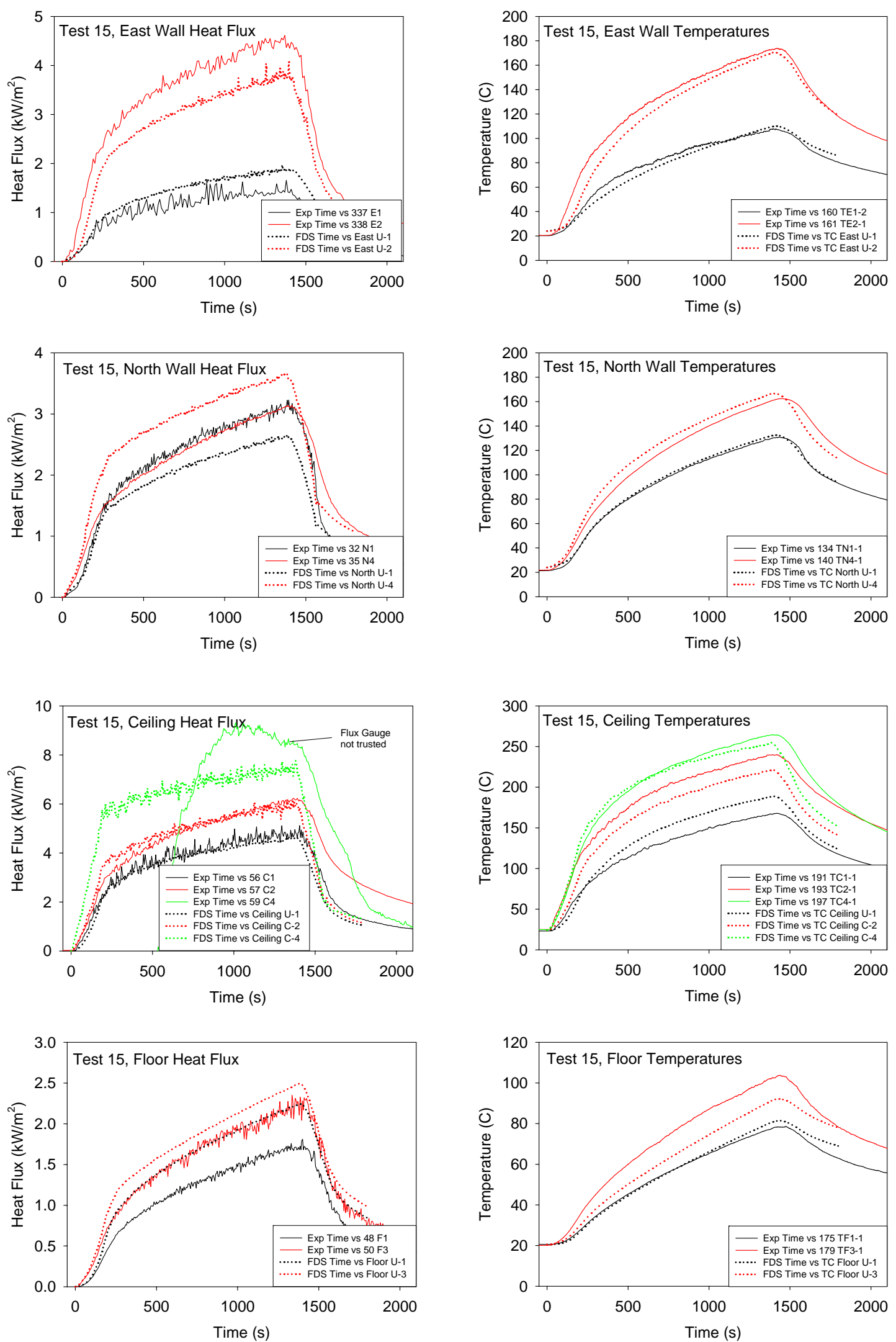

Figure 7-21. Heat flux and temperature at select points on the walls, Test 15. 

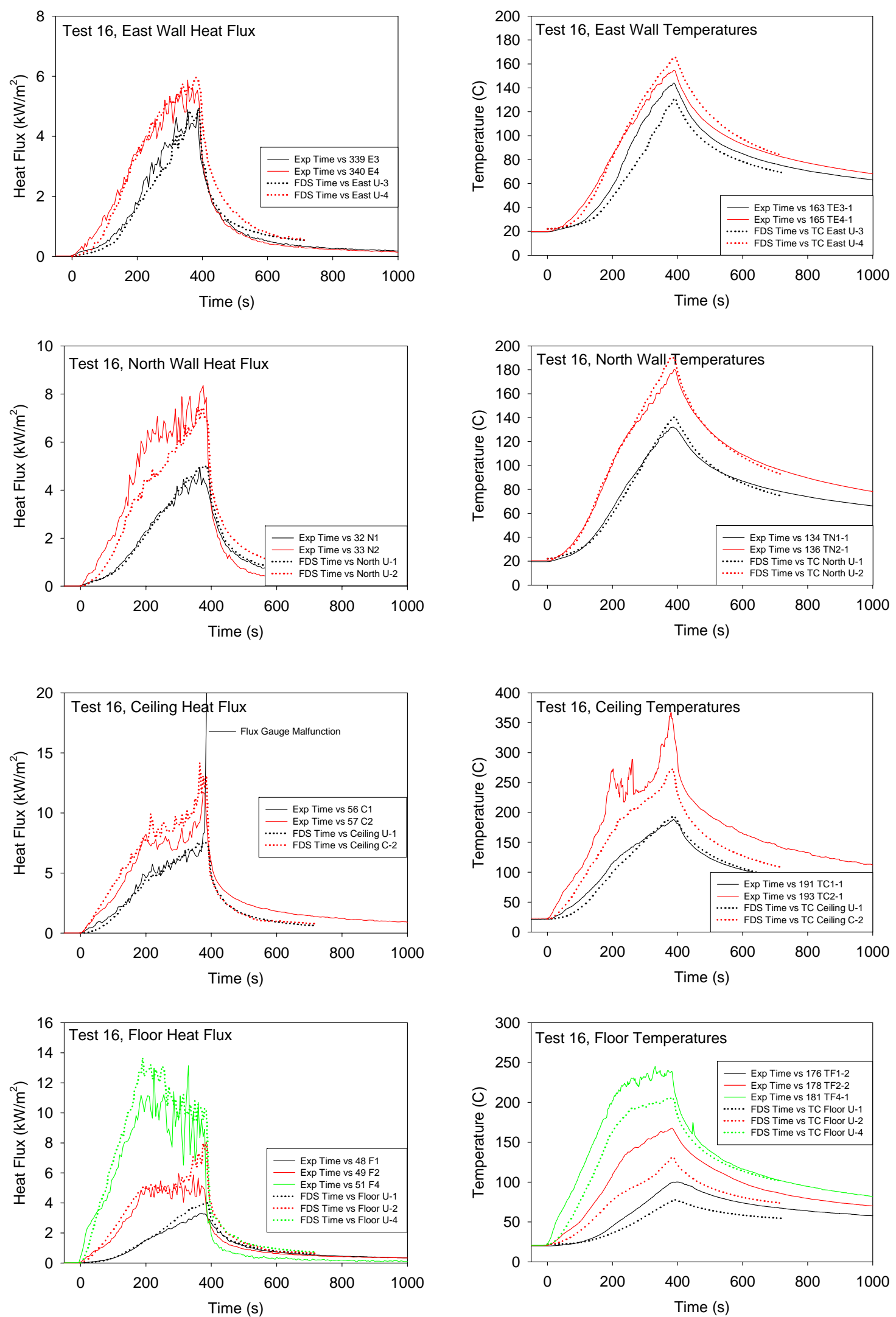

Figure 7-22. Heat flux and temperature at select points on the walls, Test 16. 

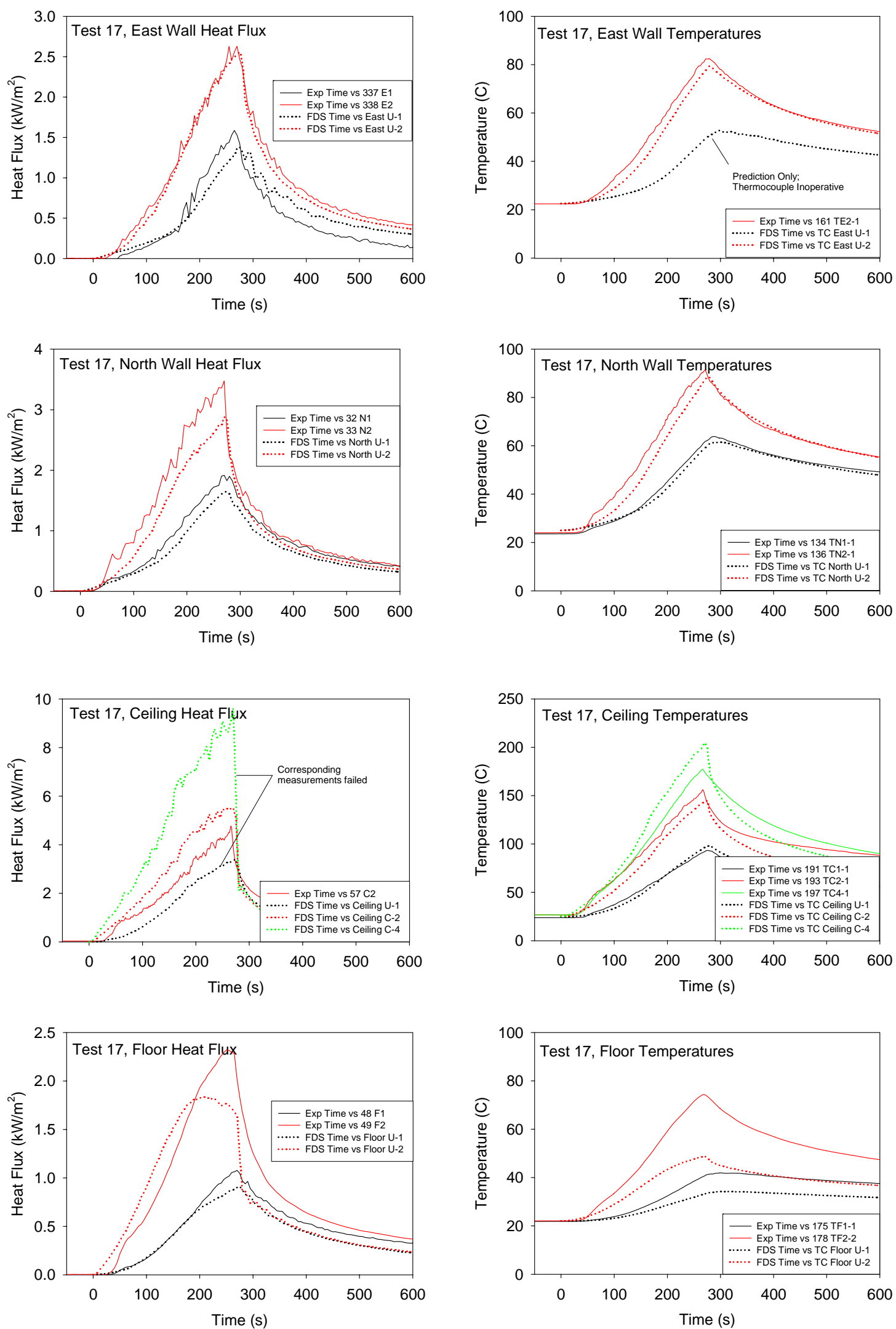

Figure 7-23. Heat flux and temperature at select points on the walls, Test 17. 

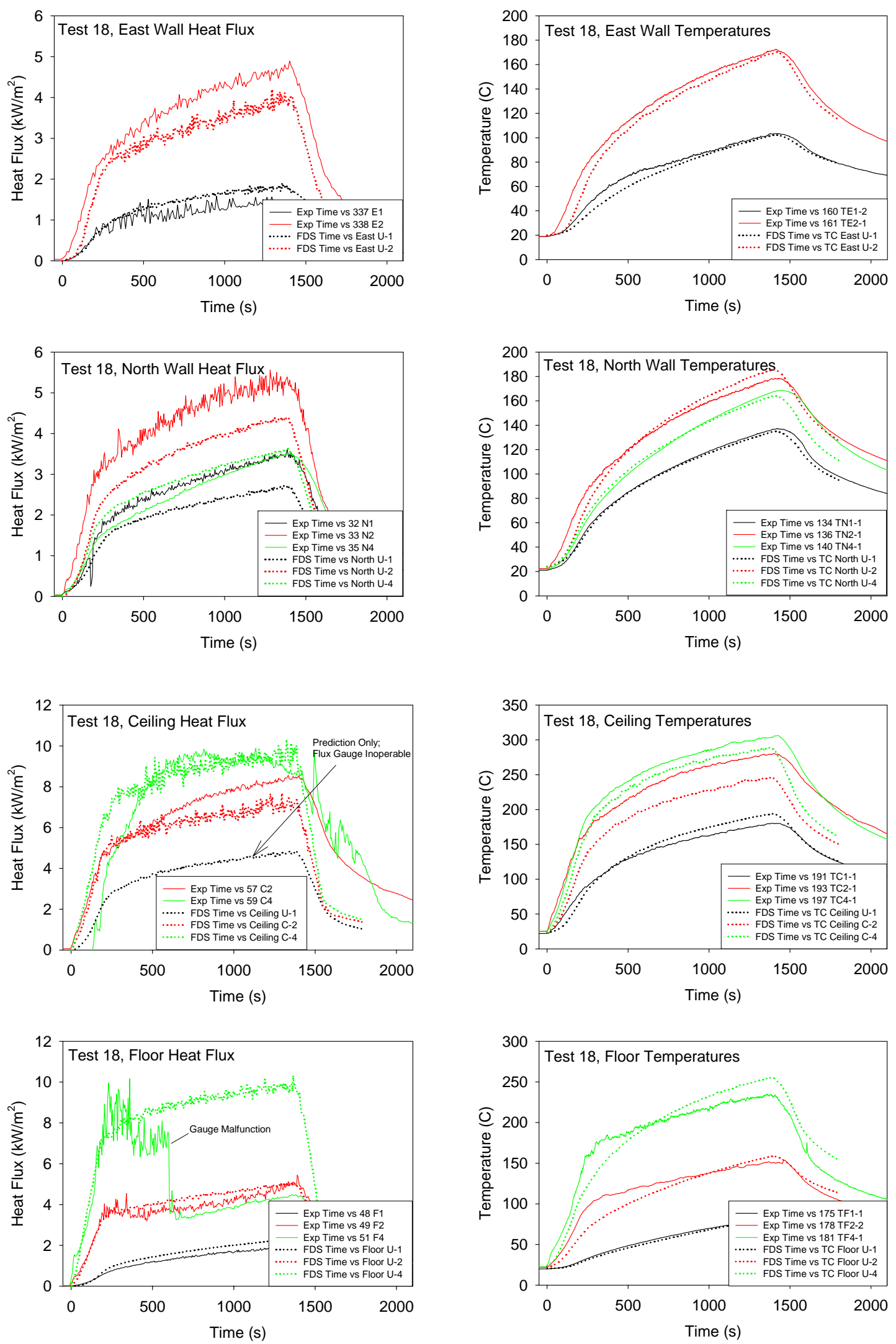

Figure 7-24. Heat flux and temperature at select points on the walls, Test 18. 


\subsubsection{Heat Flux and Surface Temperature at Cable Targets}

Each of the cable trays were instrumented with thermocouples and heat flux gauges, plus a thermocouple tree was positioned near to each one. For the purpose of model validation, the central measurement location for each tray was the most valuable because at this point, the gas temperature, heat flux and surface temperature could be analyzed together. Shown in Figure 7-25 through Figure 7-46 are the gas temperatures, heat flux and cable surface temperatures for Trays/Targets D, E, F and G. The individual cables A, B and C could not be included in the simulation because of their small size relative to the numerical grid. Nevertheless, the data for Trays/Targets D, E, F and G demonstrate the model's ability to characterize the thermal environment in the vicinity of relatively small objects.

Note that only the inner temperature of "slab" E is reported. FDS does not have a detailed solid phase model that can account for the heat transfer within the bundled, cylindrical, nonhomogenous cables. At best, it calculates the surface temperature of the outermost cable in the bundle assuming that it is a solid, homogenous mass of similar thickness to the width of the bundle. Only the solid rectangular stick of plastic (Target E) could be modeled both inside and out even though the predicted surface and inner temperature of this $1 \mathrm{~cm} \times 1 \mathrm{~cm}$ stick were in greatest disagreement with measurement. The reason is that FDS only performs a one-dimensional heat transfer calculation normal to the surface, assuming that the surface area greatly exceeds the depth. In reality, the heat penetrated the plastic stick in all directions, leading to a consistently higher measured surface and inner temperature than the corresponding predictions.

The superposition of gas temperature, heat flux and surface temperature in the figures on the following pages was a very valuable exercise. Favorable or unfavorable predictions of cable surface temperatures could usually be explained in terms of comparable errors in the prediction of the thermal environment in the vicinity of the cable tray. Regardless of the complexity of the target, the model must be able to predict the thermal insult to it. In most cases, the cable surface temperatures in trays $D, F$ and $G$ were predicted to within $10 \%$ of the measurements, with deviations due to a number of near-field effects, plus errors related to the simplification of the cable bundles as solid slabs of plastic. 

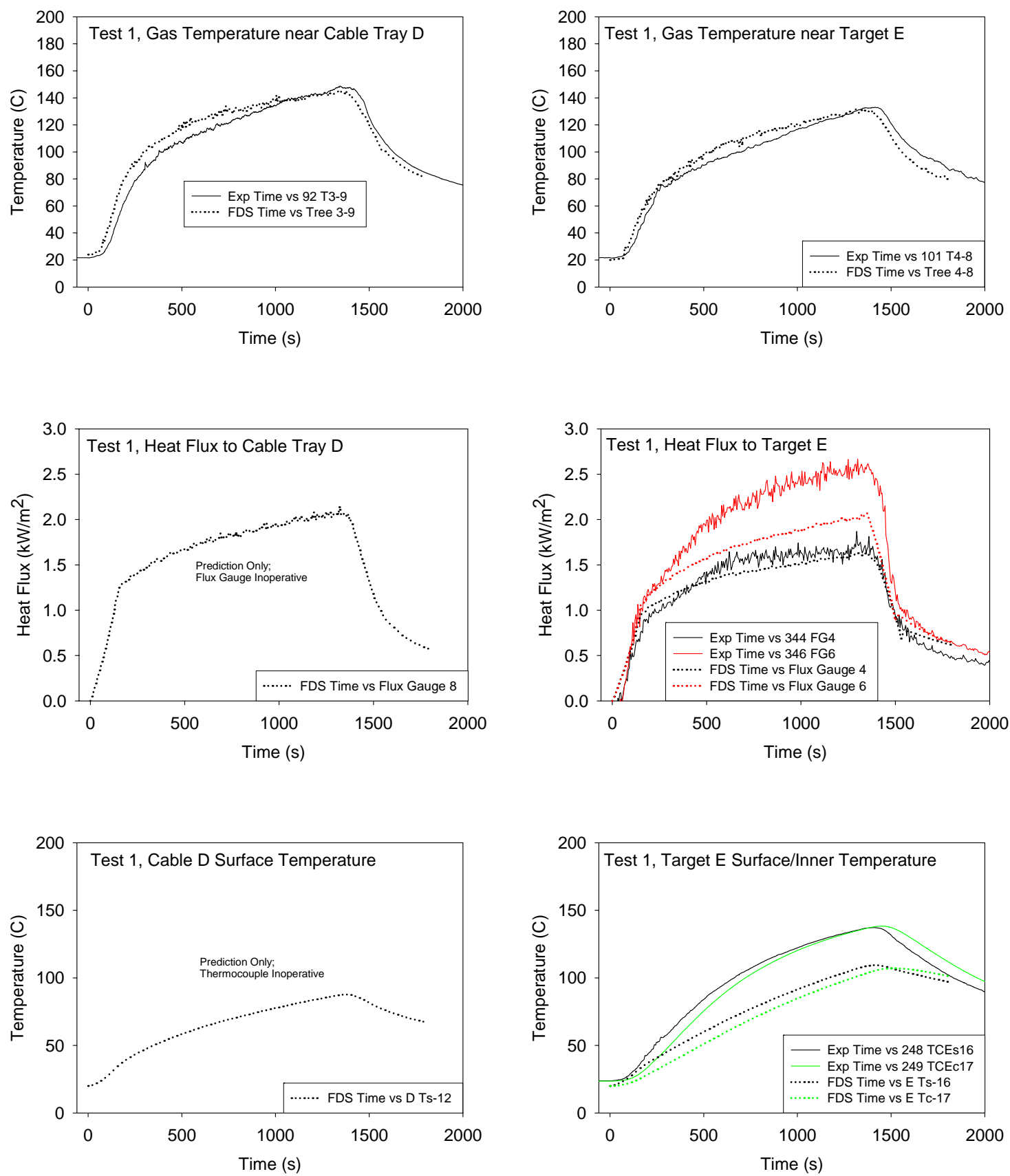

Figure 7-25. Thermal environment near Tray D and Target E, Test 1. 

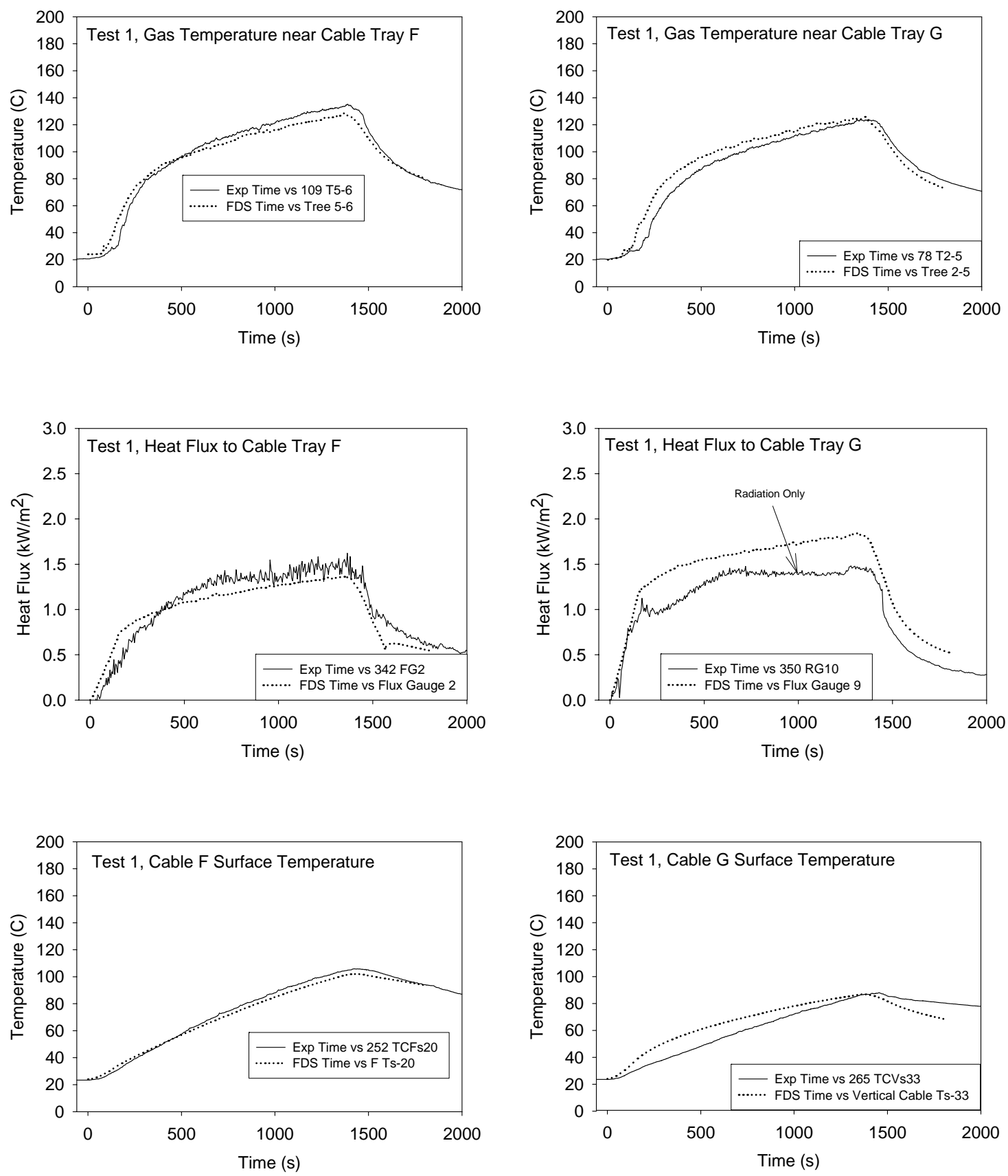

Figure 7-26. Thermal environment near Cable Trays F and G, Test 1. 

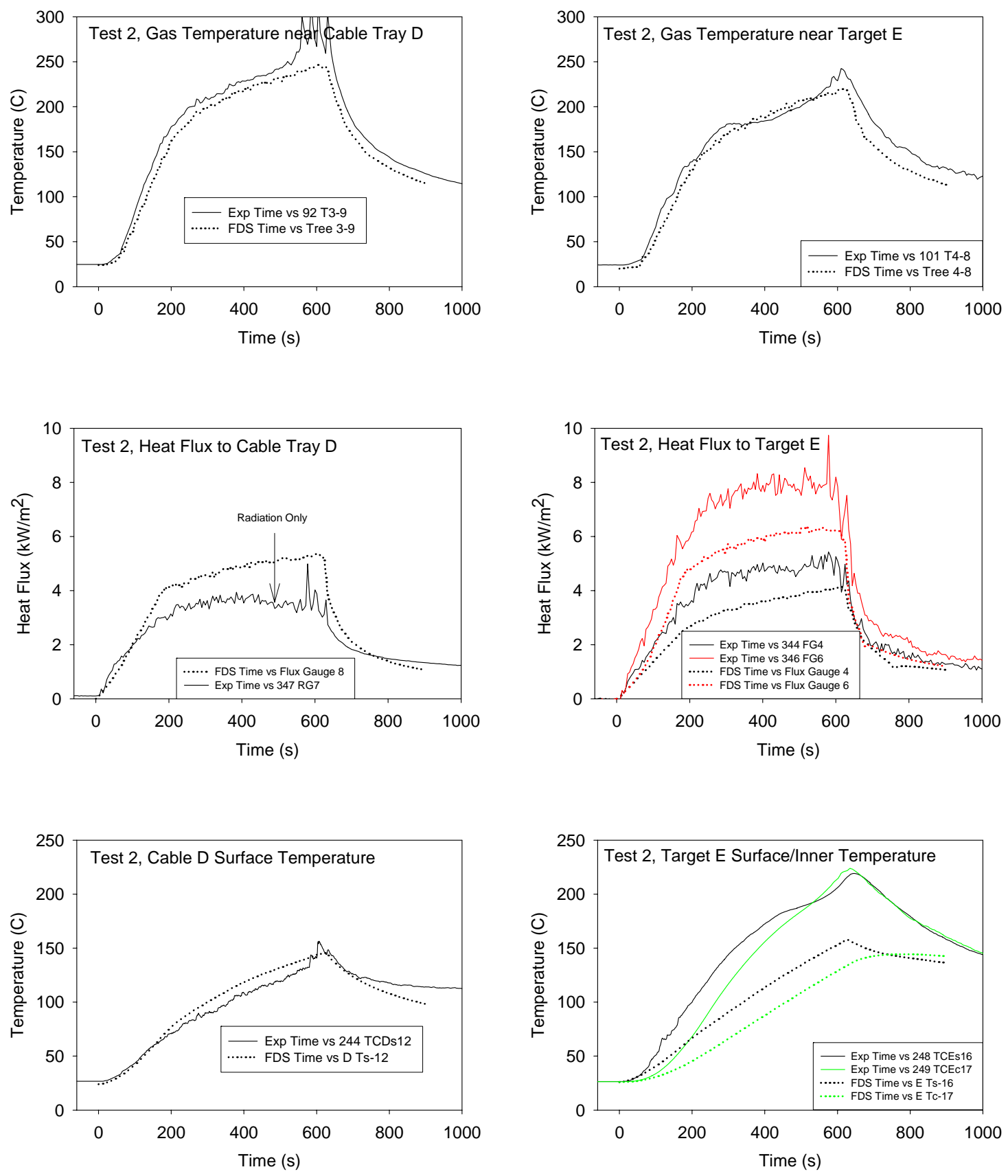

Figure 7-27. Thermal environment near Tray D and Target E, Test 2. 

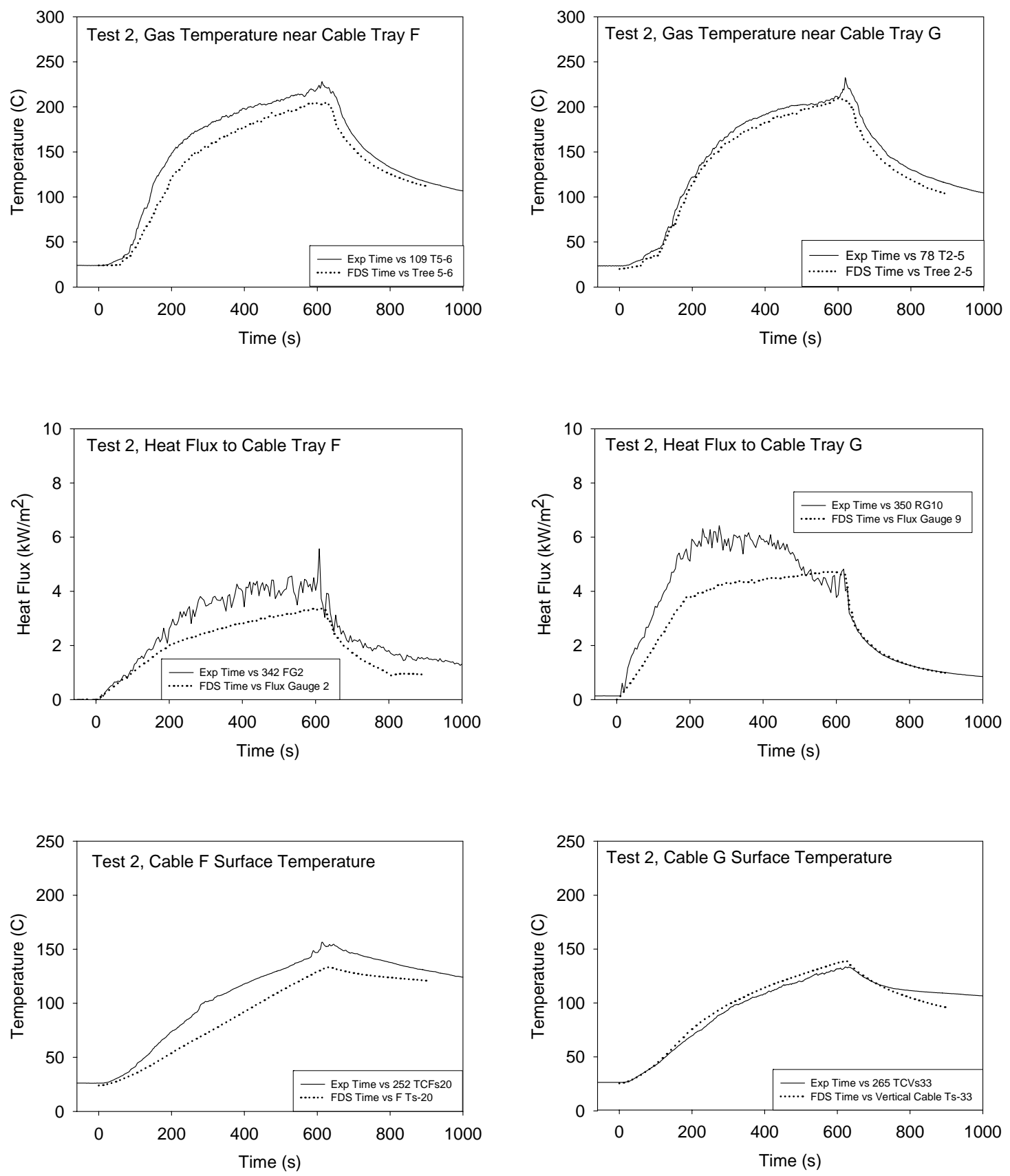

Figure 7-28. Thermal environment near Cable Trays F and G, Test 2. 

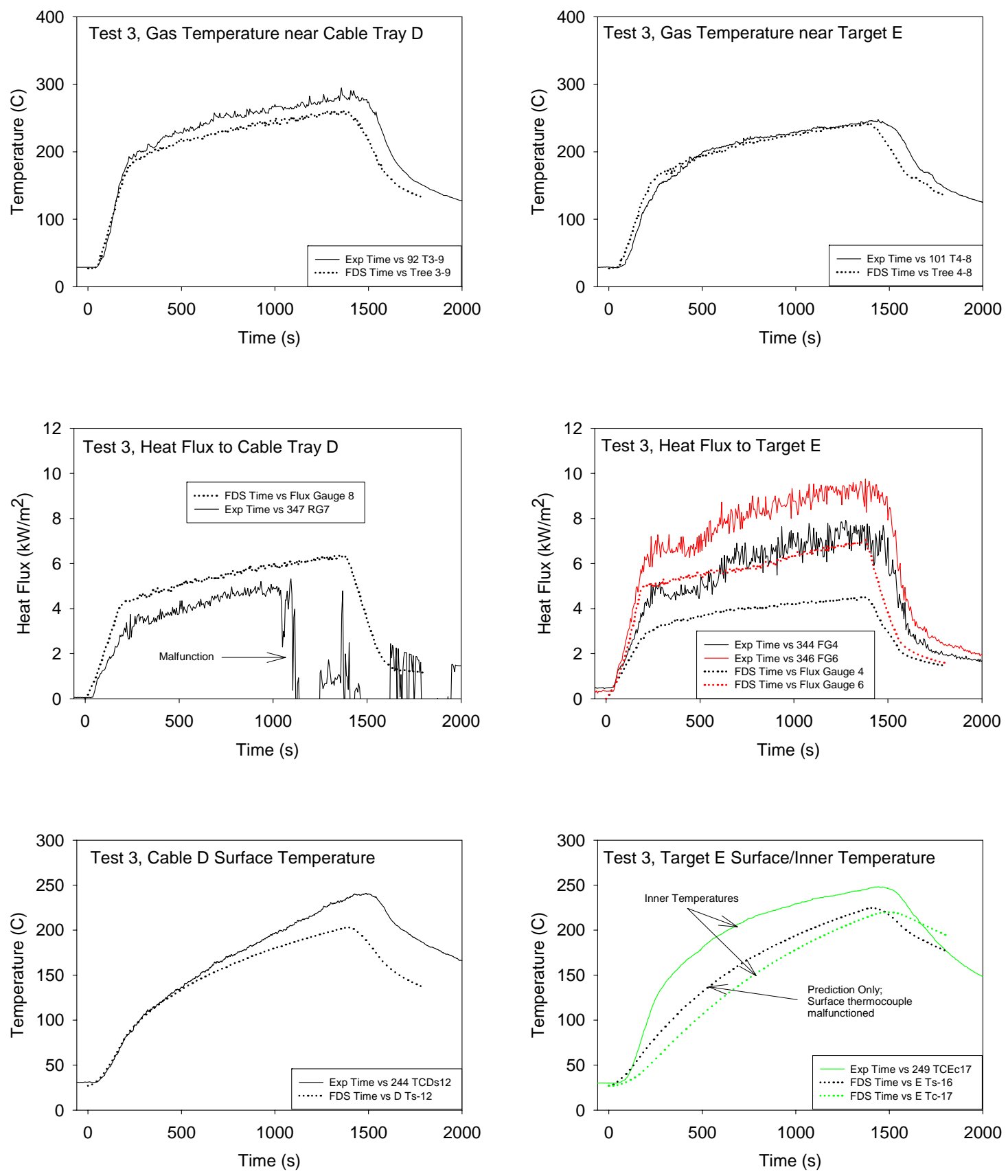

Figure 7-29. Thermal environment near Tray D and Target E, Test 3. 

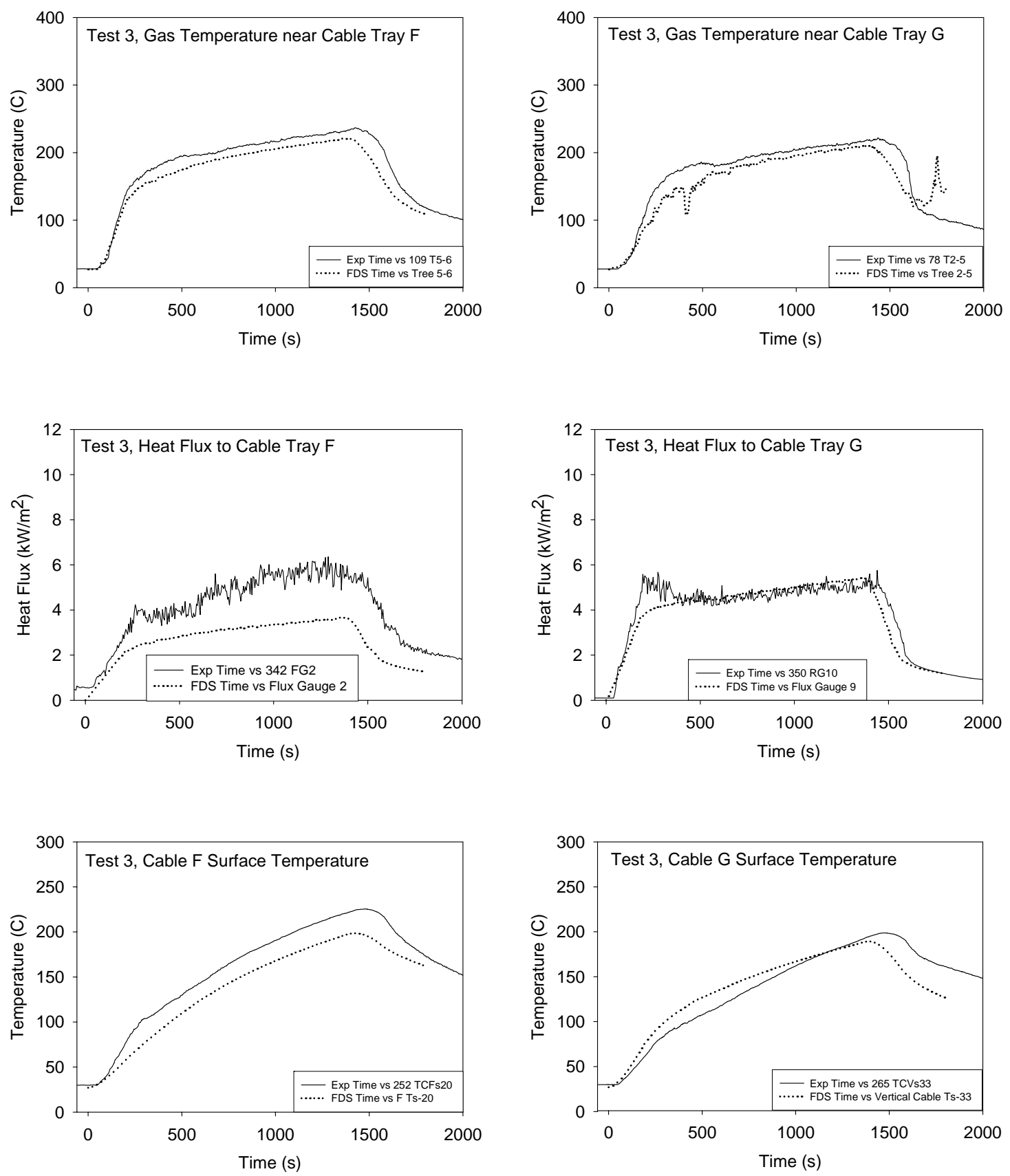

Figure 7-30. Thermal environment near Cable Trays F and G, Test 3. 

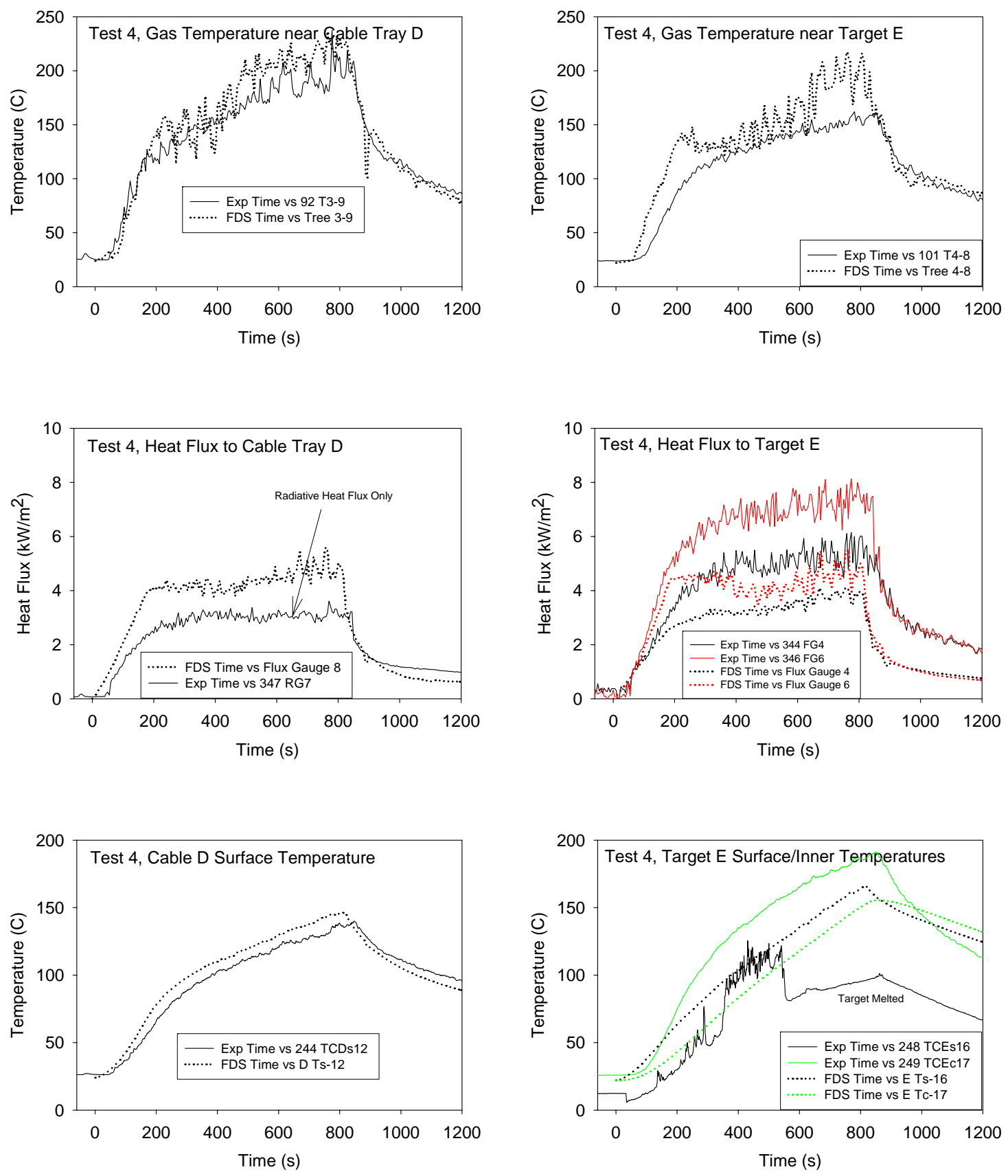

Figure 7-31. Thermal environment near Tray D and Target E, Test 4. 

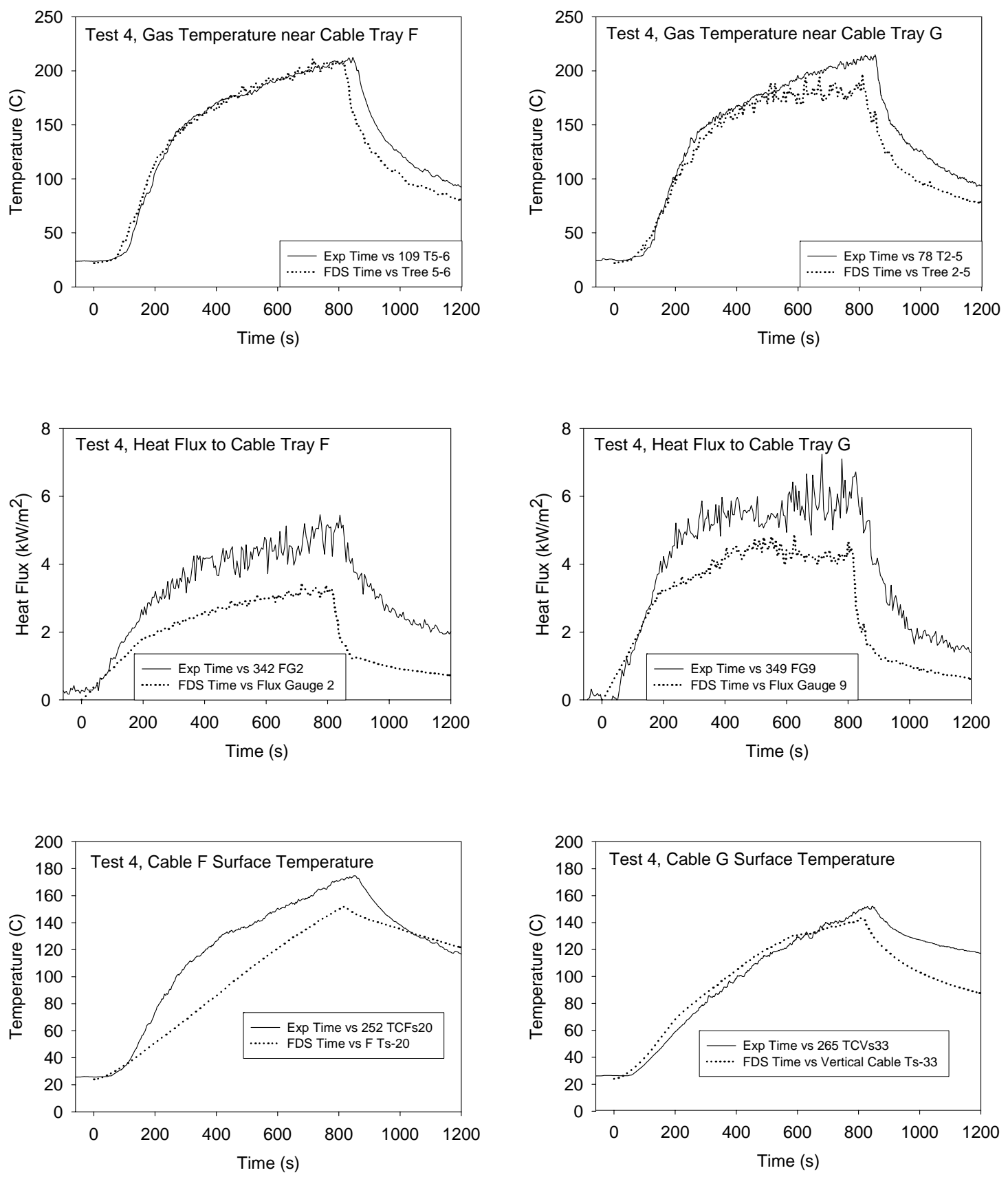

Figure 7-32. Thermal environment near Cable Trays F and G, Test 4. 

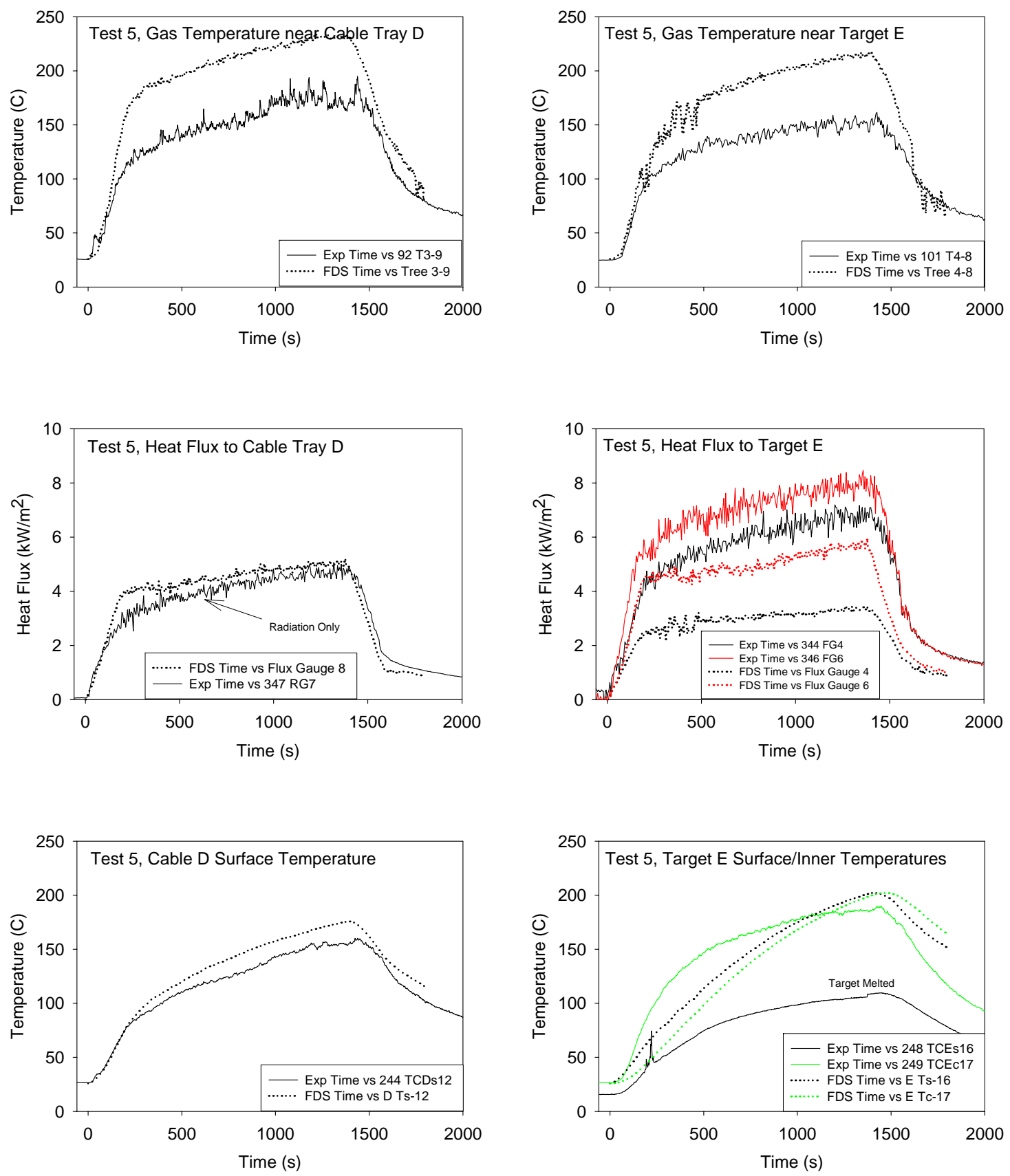

Figure 7-33. Thermal environment near Tray D and Target E, Test 5. 

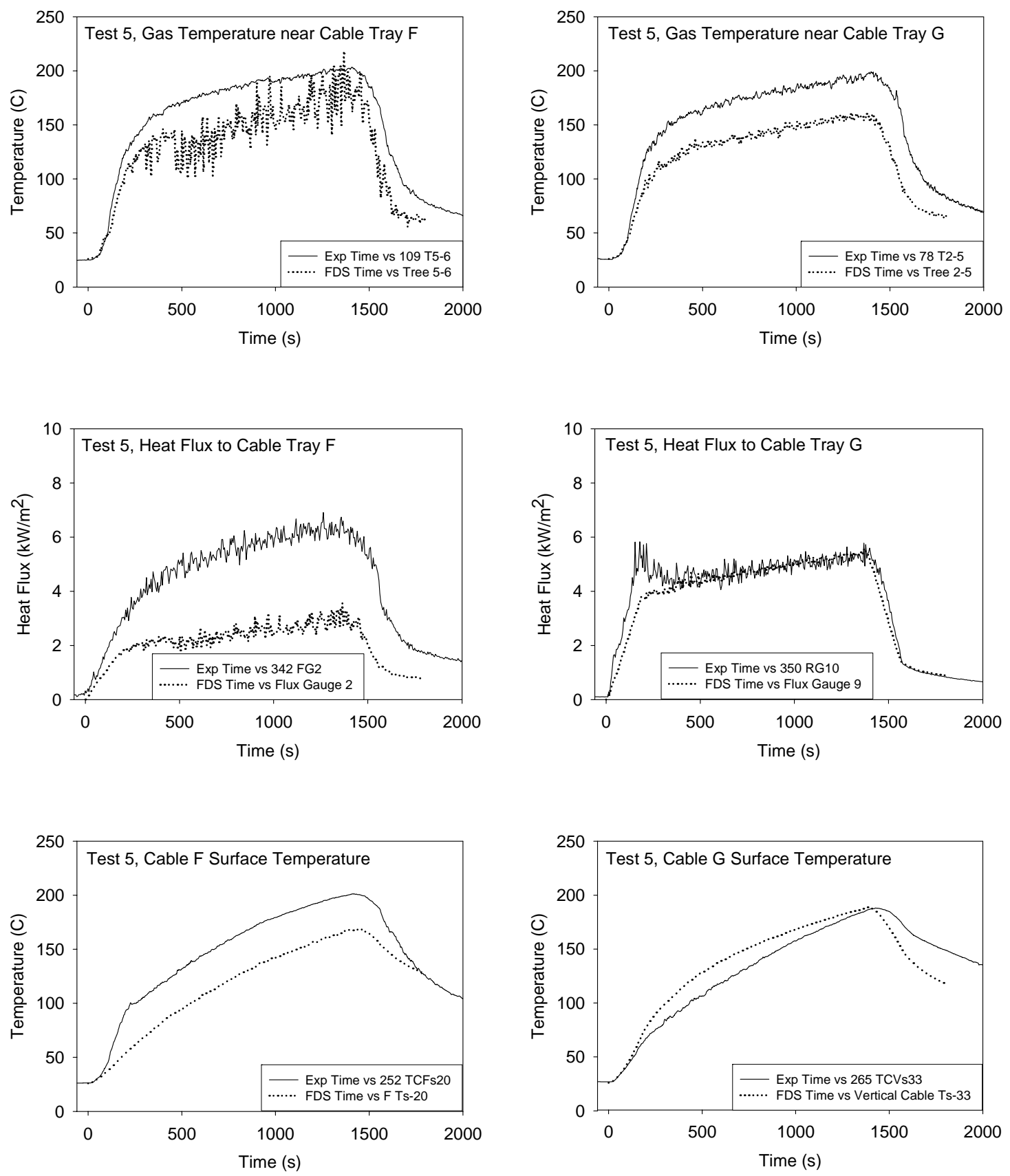

Figure 7-34. Thermal environment near Cable Trays F and G, Test 5. 

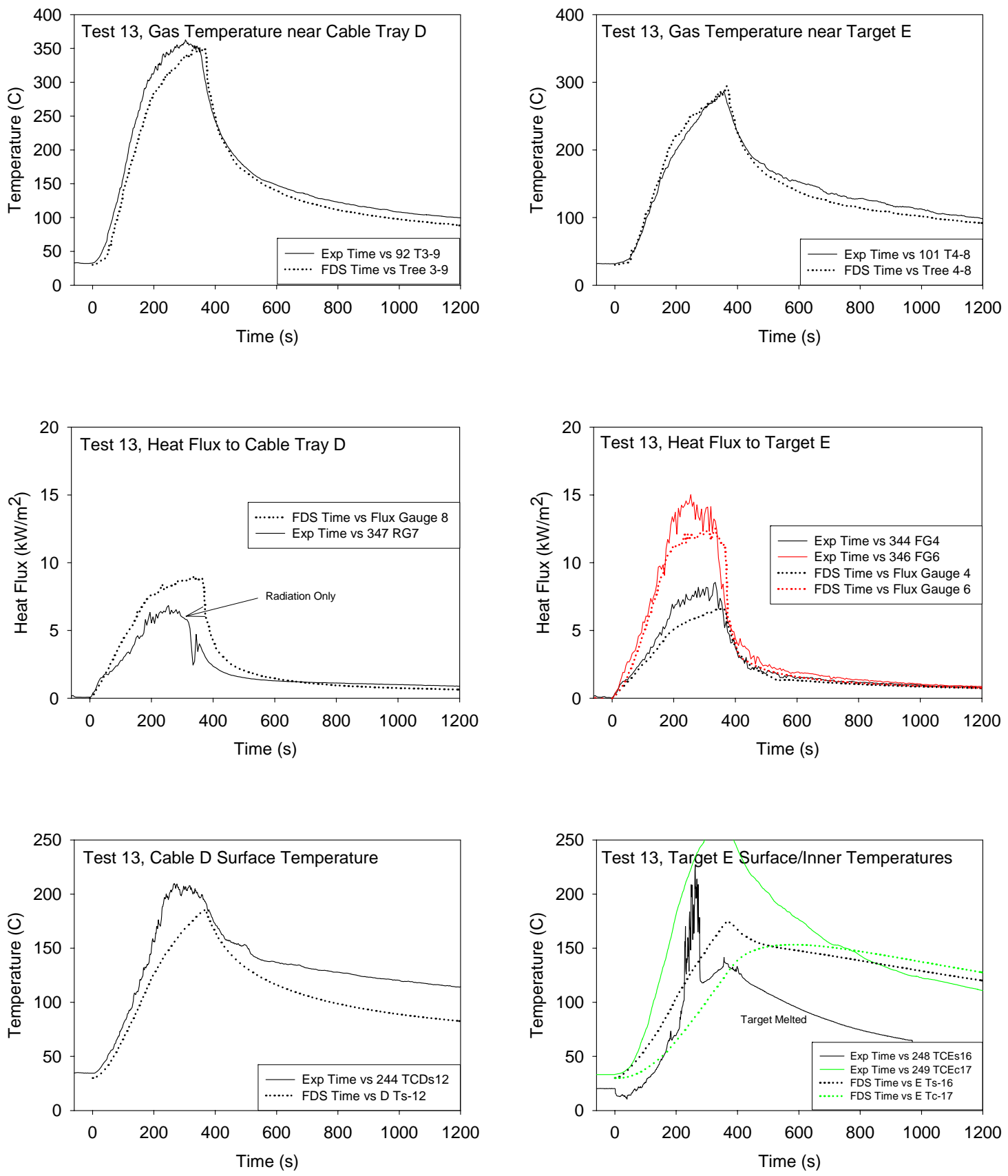

Figure 7-35. Thermal environment near Tray D and Target E, Test 13. 

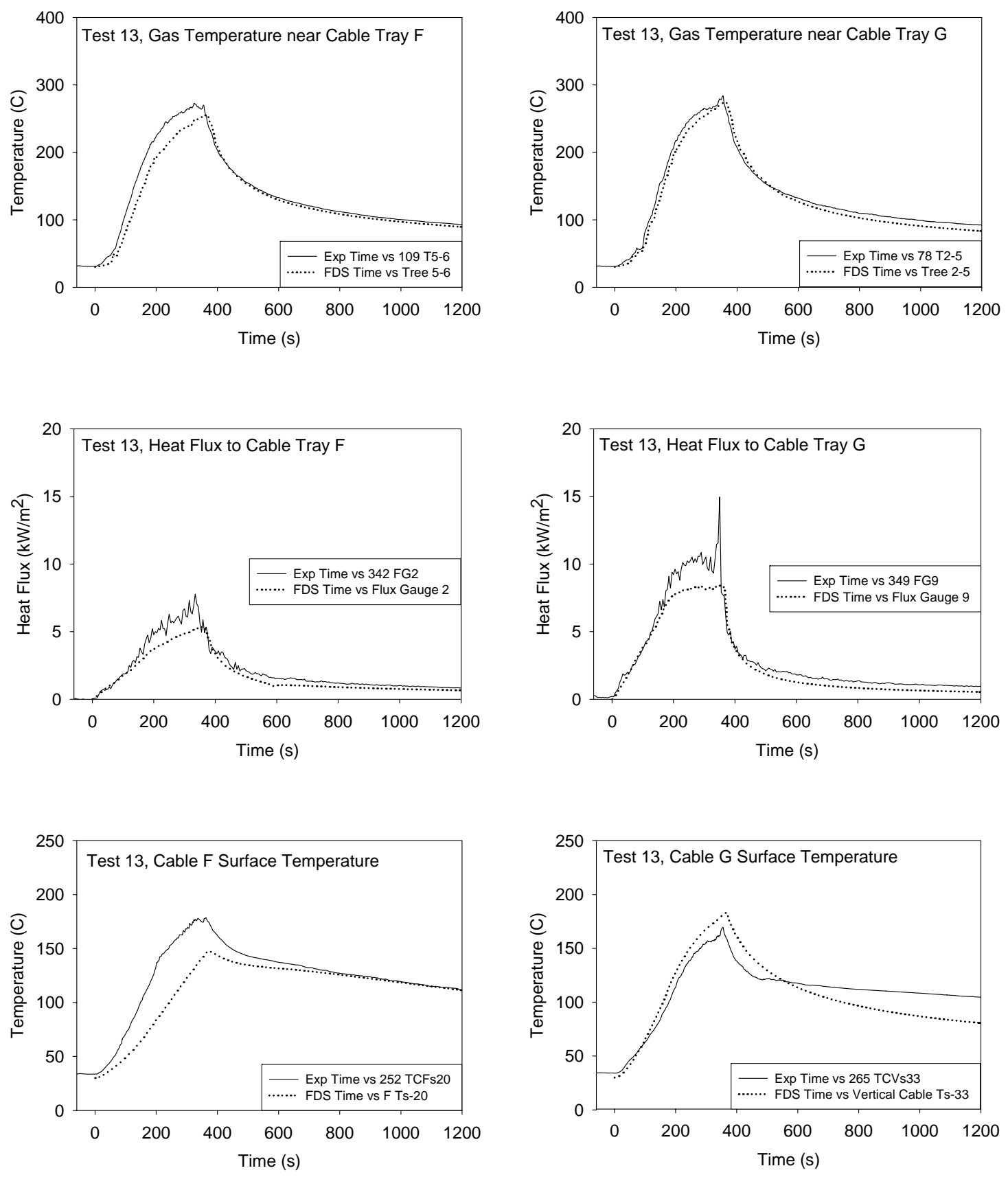

Figure 7-36. Thermal environment near Cable Trays F and G, Test 13. 

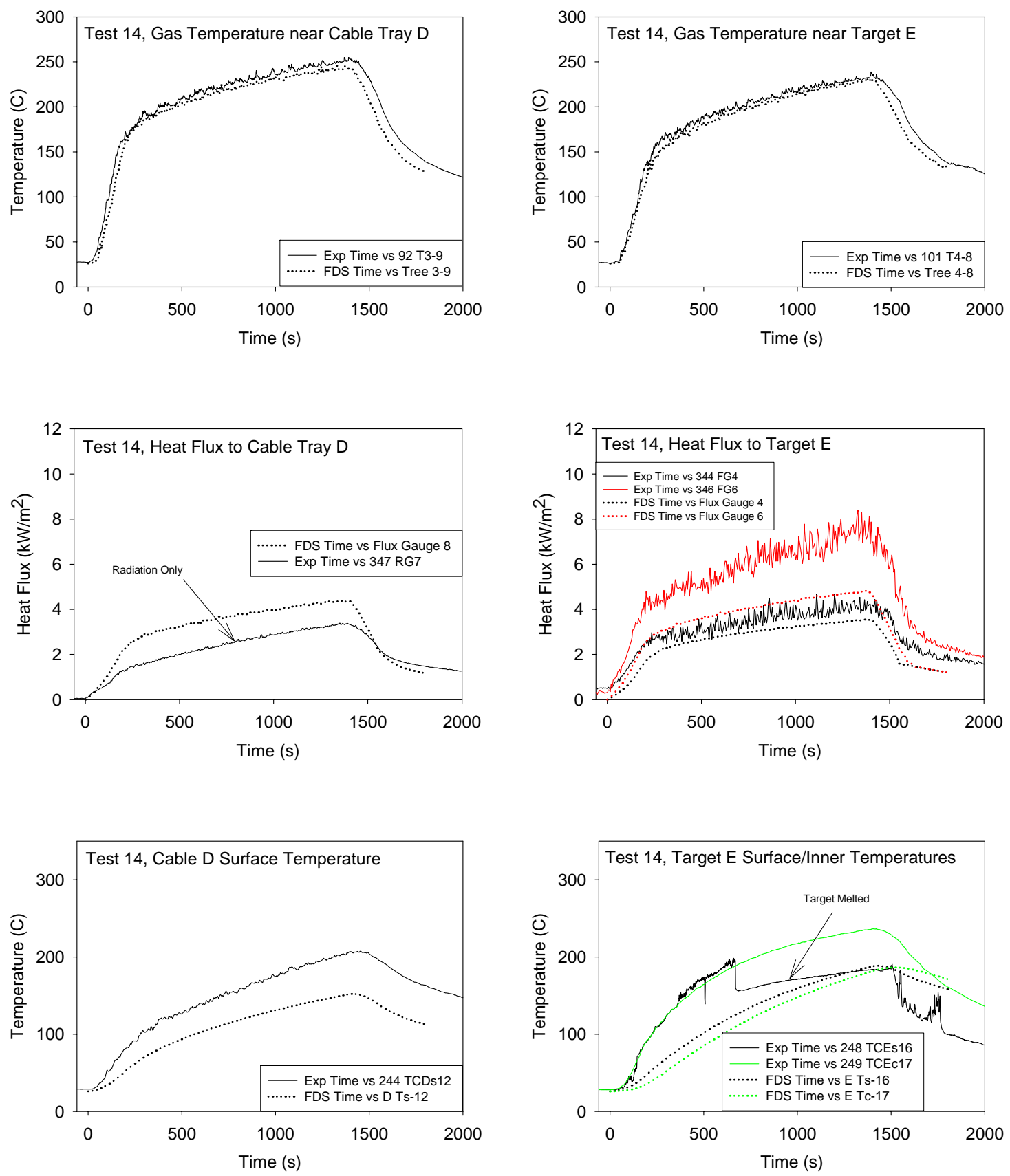

Figure 7-37. Thermal environment near Tray D and Target E, Test 14. 

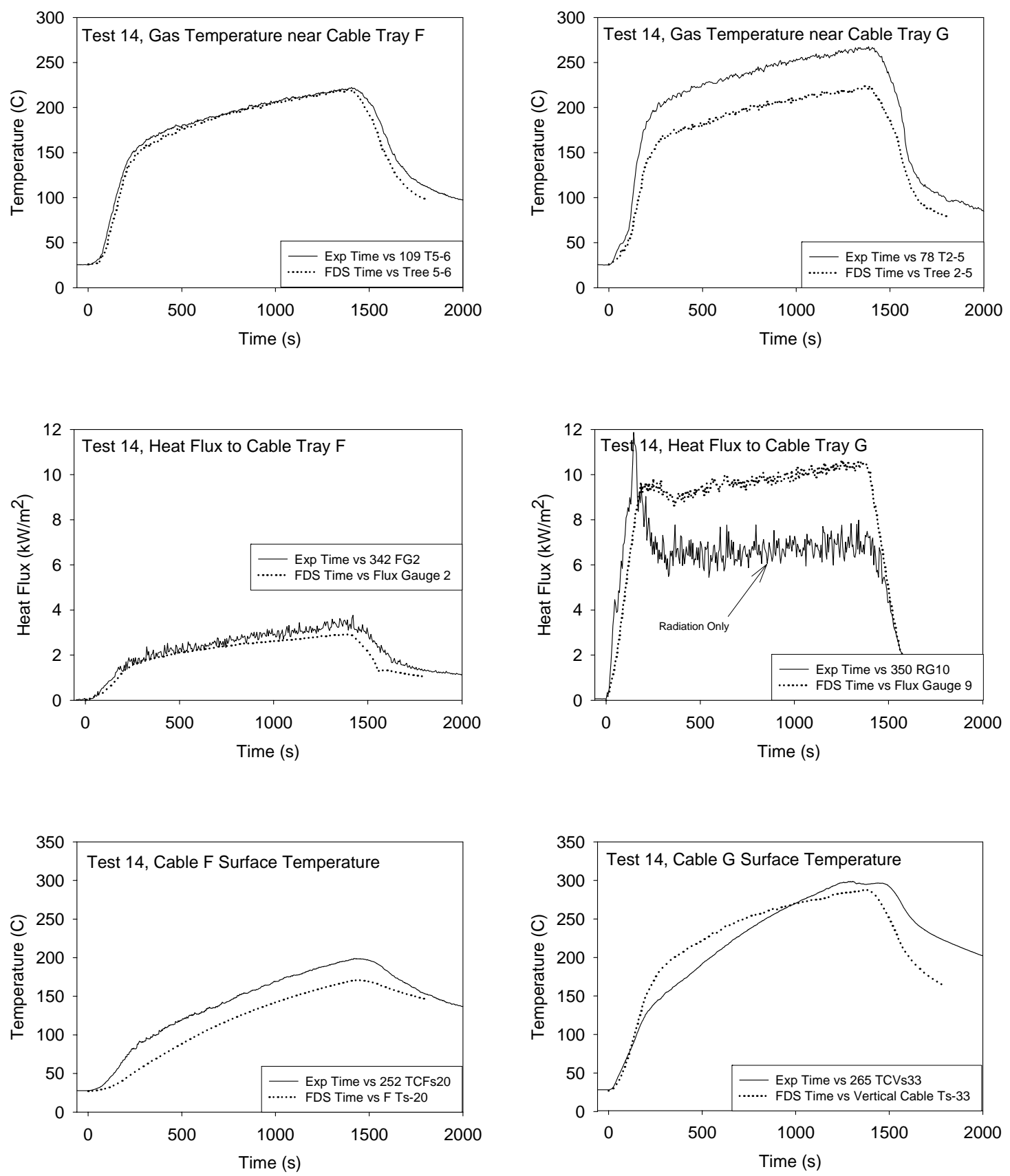

Figure 7-38. Thermal environment near Cable Trays F and G, Test 14. 

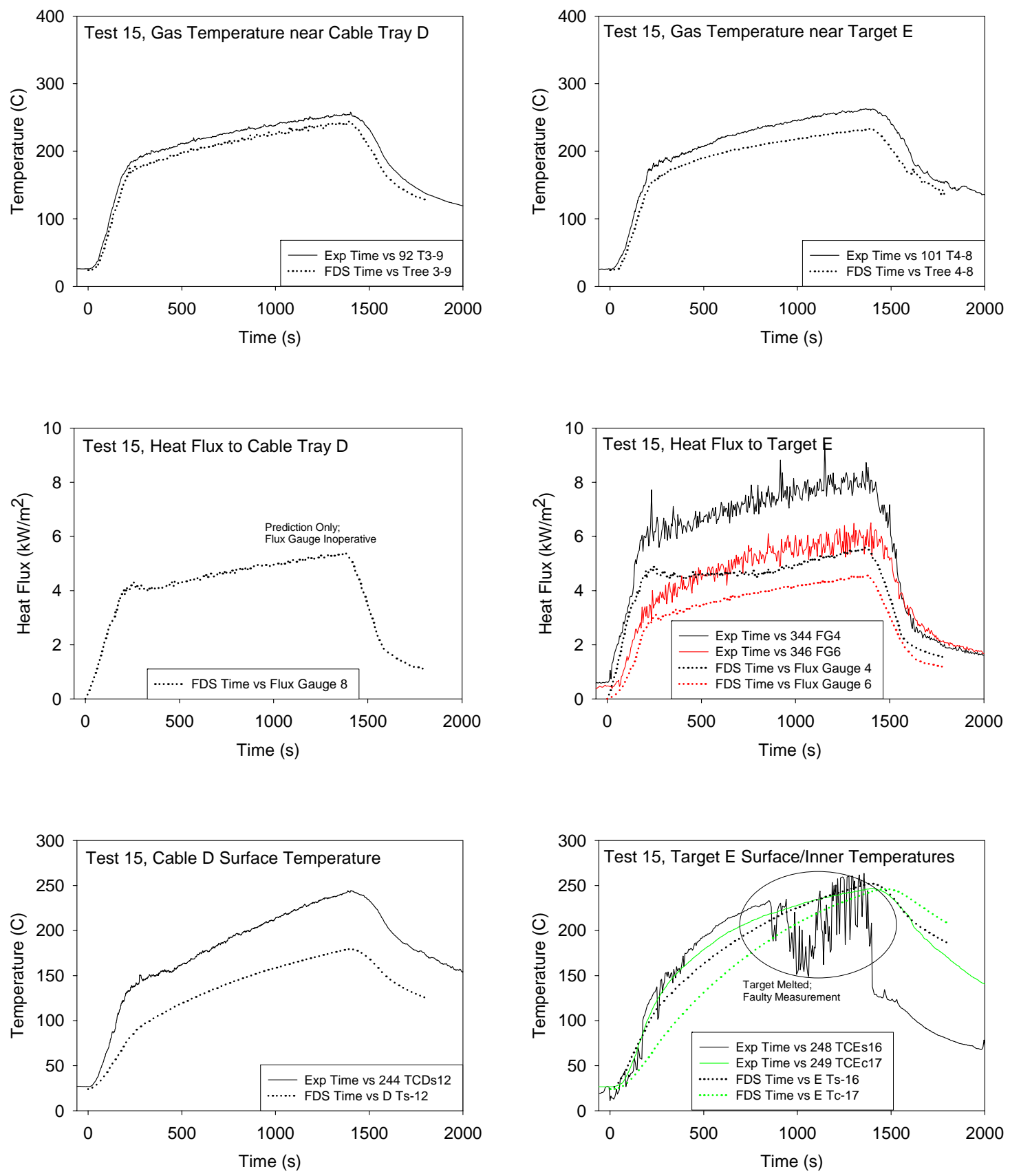

Figure 7-39. Thermal environment near Tray D and Target E, Test 15. 

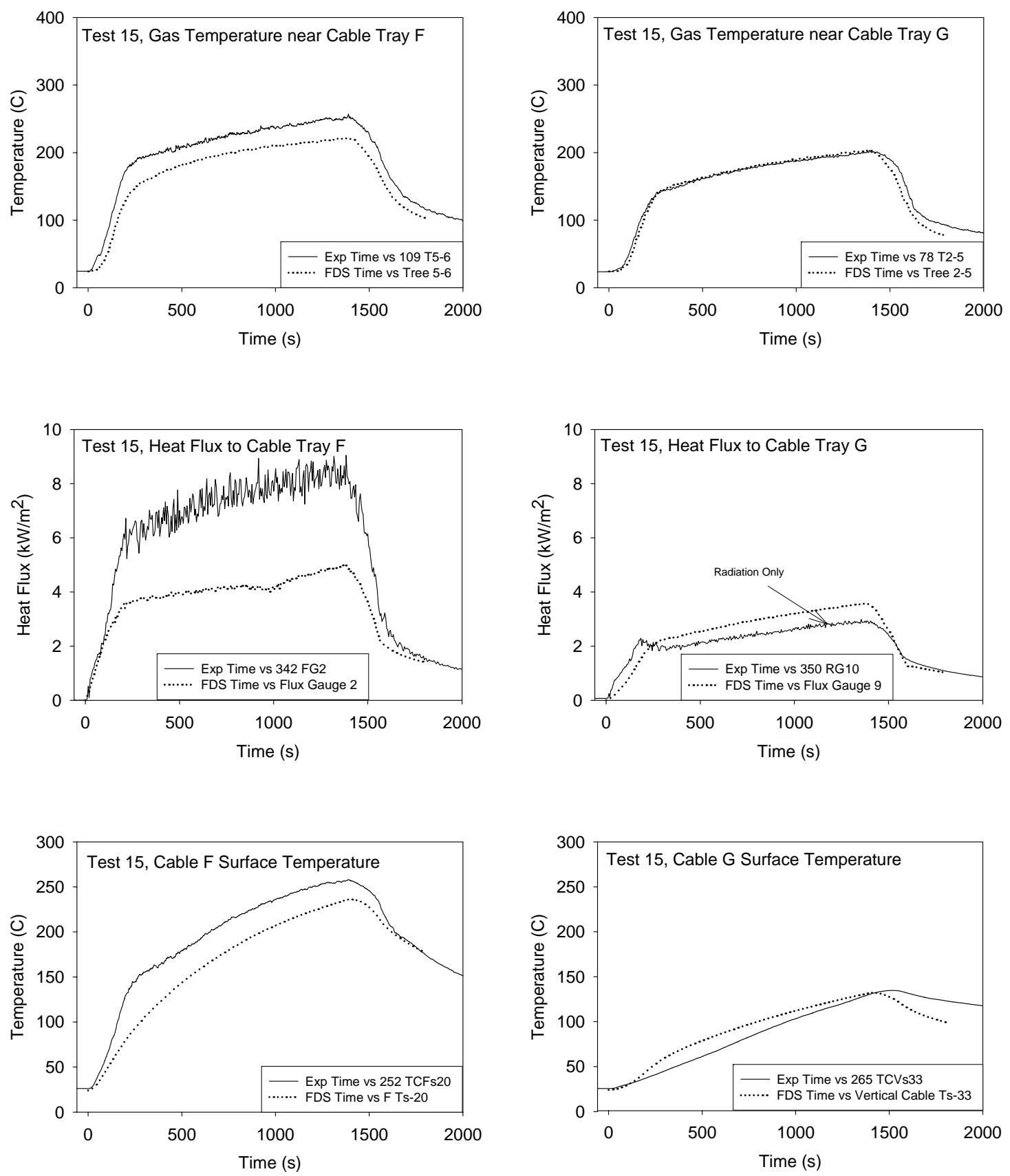

Figure 7-40. Thermal environment near Cable Trays F and G, Test 15. 

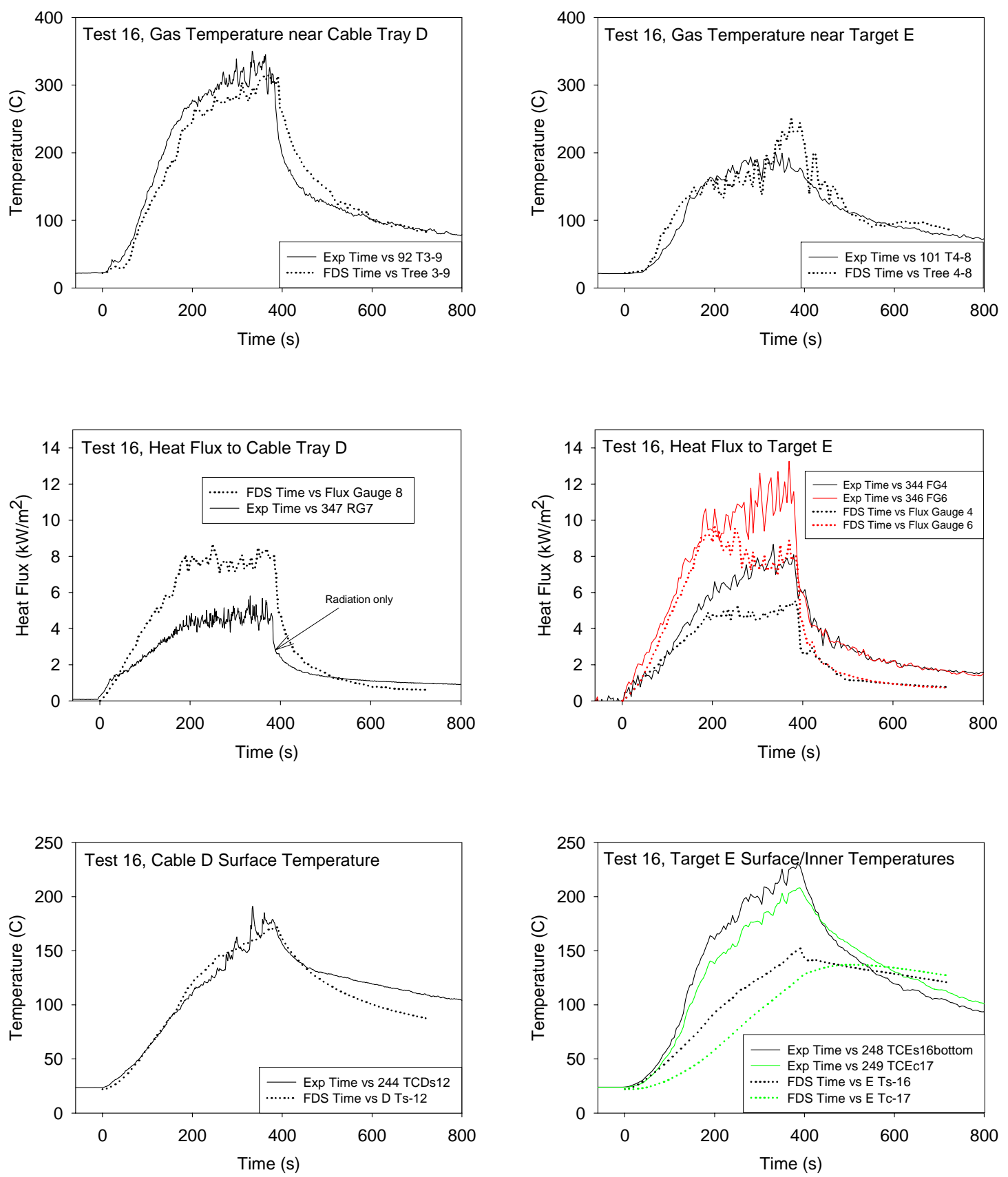

Figure 7-41. Thermal environment near Tray D and Target E, Test 16. 

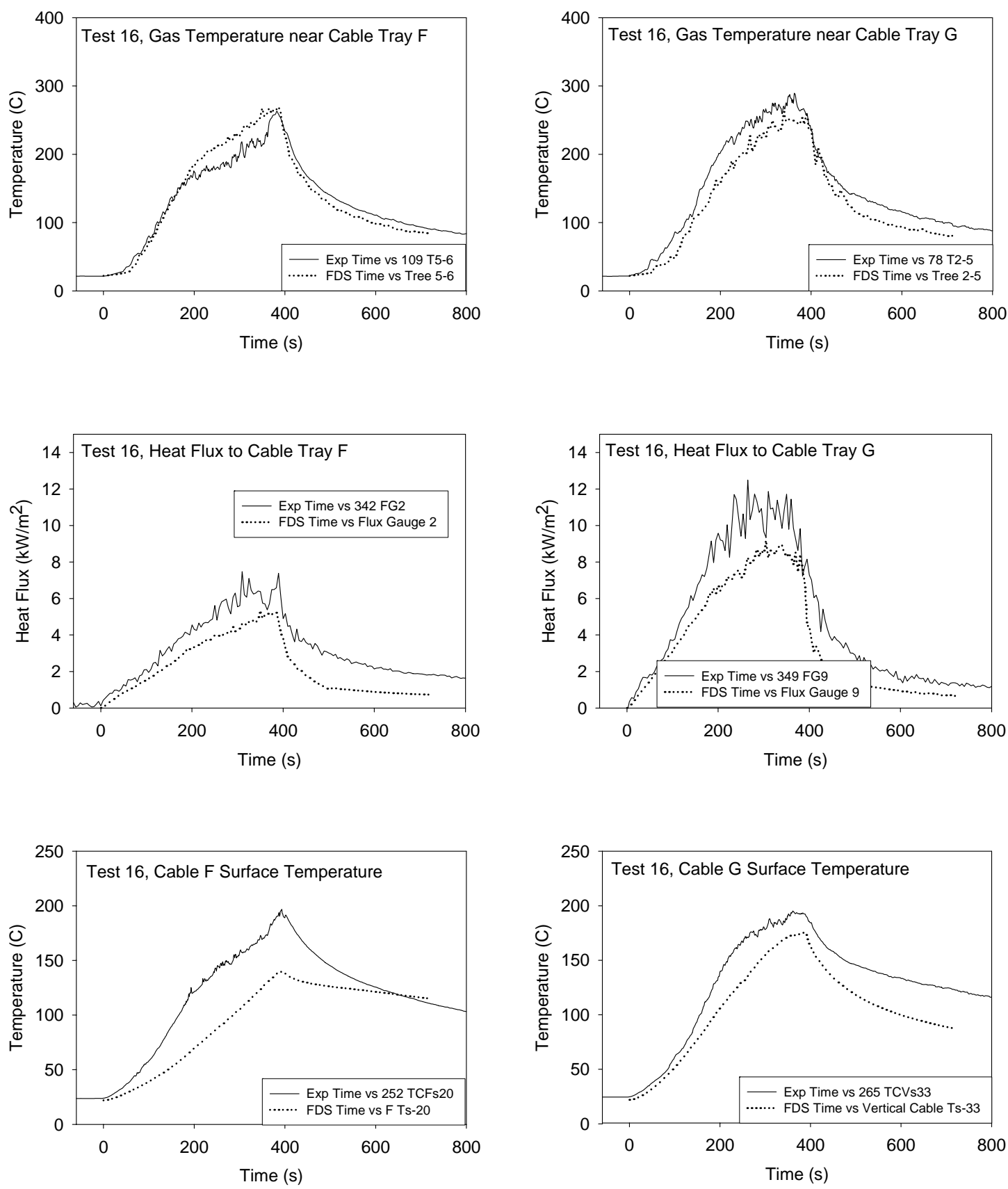

Figure 7-42. Thermal environment near Cable Trays F and G, Test 16. 

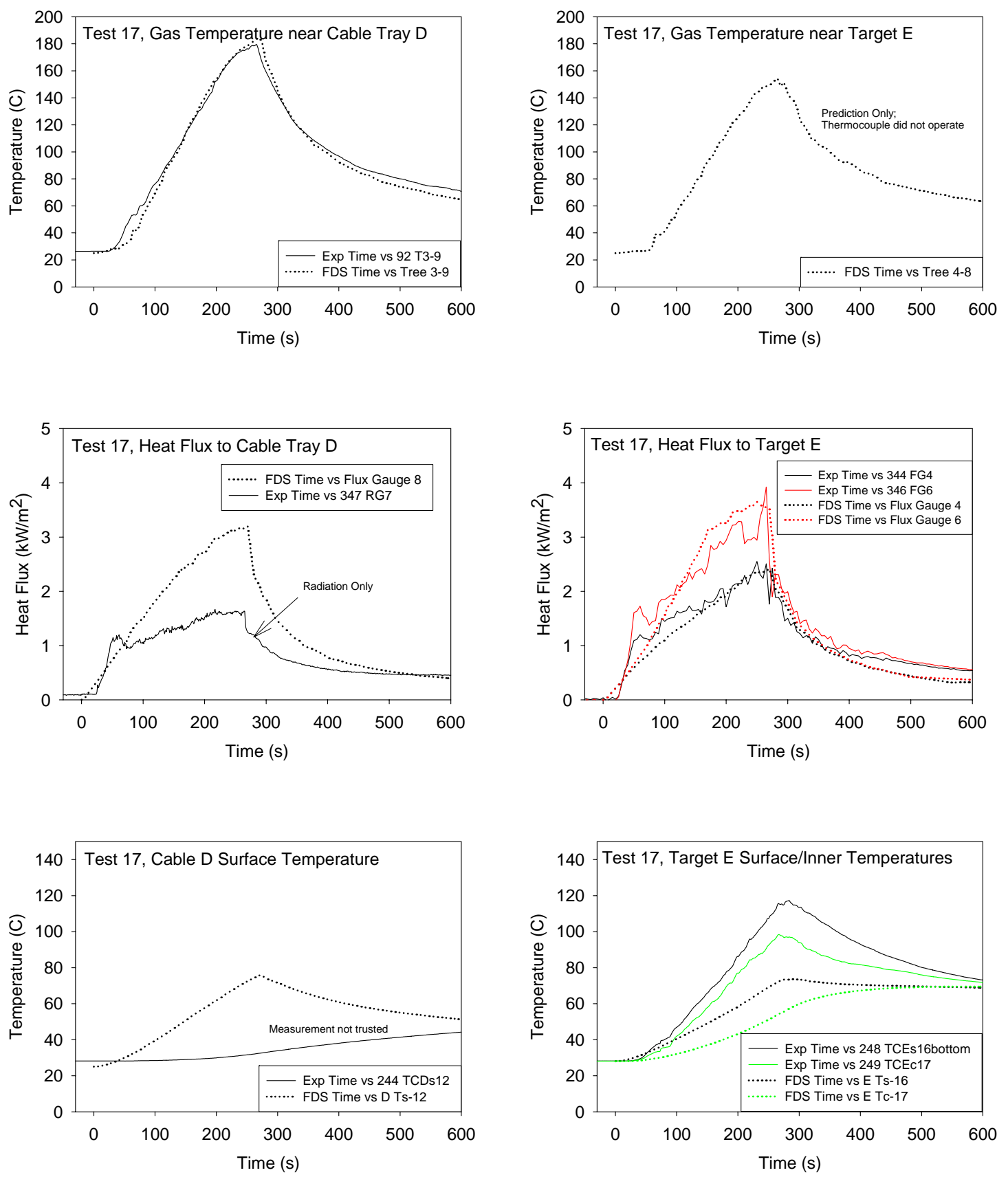

Figure 7-43. Thermal environment near Tray D and Target E, Test 17. 

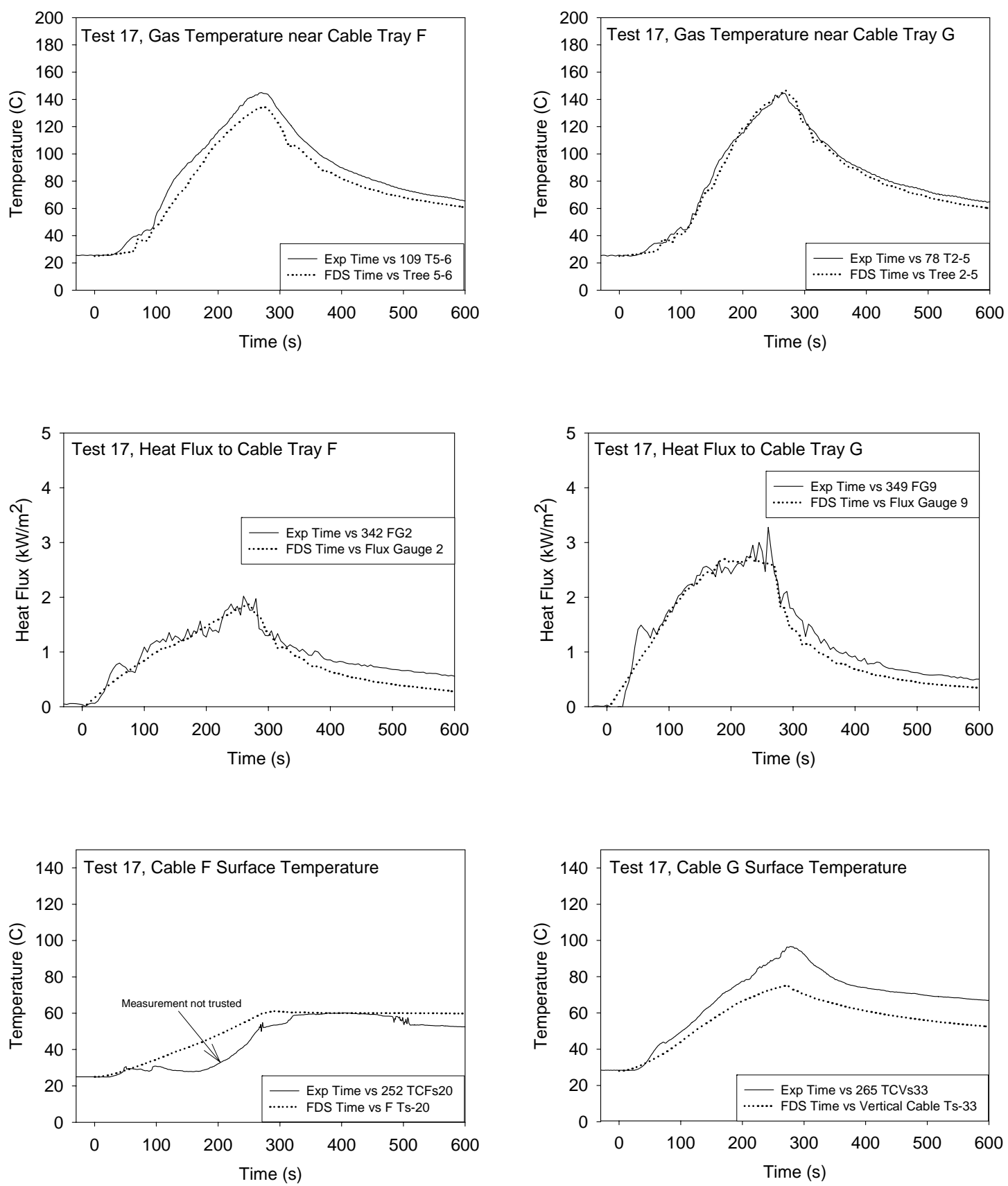

Figure 7-44. Thermal environment near Cable Trays F and G, Test 17. 

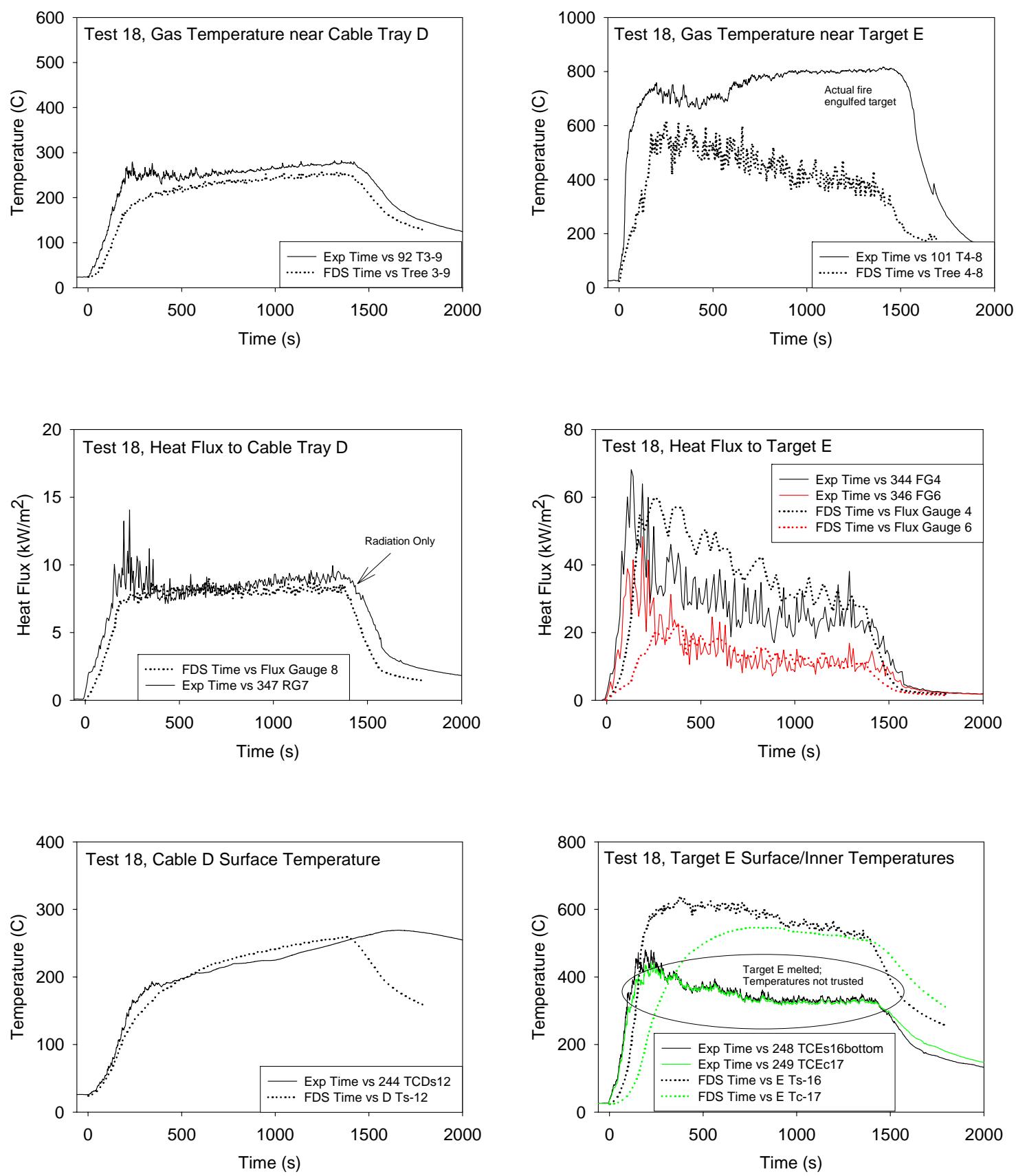

Figure 7-45. Thermal environment near Tray D and Target E, Test 18. 

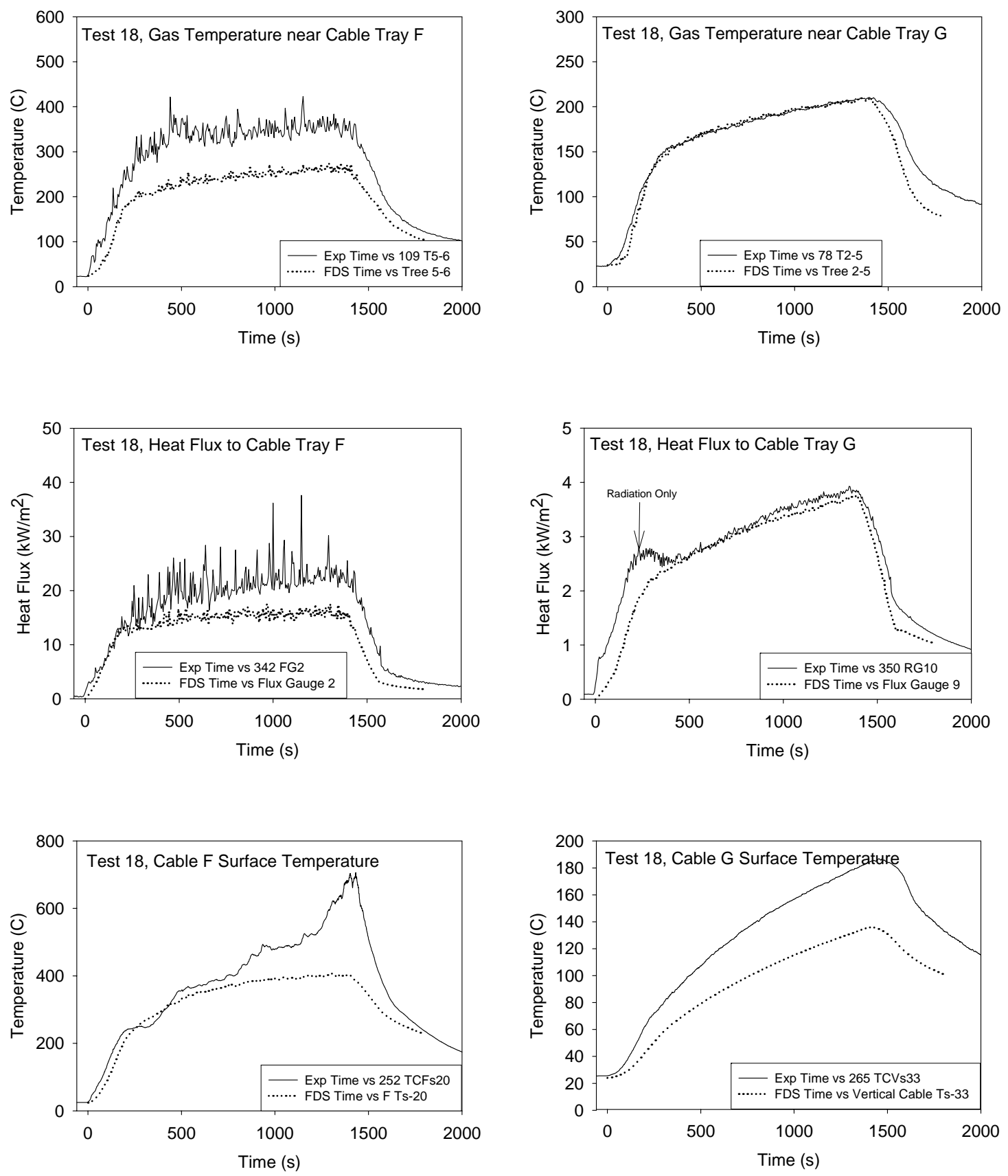

Figure 7-46. Thermal environment near Cable Trays F and G, Test 18. 


\subsubsection{Gas Species Concentrations}

FDS uses a mixture fraction combustion model, meaning that all gas species within the compartment are assumed to be functions of a single scalar variable. FDS solves only one transport equation for this variable, and reports gas concentrations at any given point at any given time by extracting its value from a pre-computed "look-up table." For the major species, like carbon dioxide and oxygen, the predictions are essentially an indicator of how well FDS is predicting the bulk transport of combustion products throughout the space. For minor species, like carbon monoxide, FDS at the present time does not account for changes in combustion efficiency, relying only on a fixed yield of $\mathrm{CO}$ from the combustion process. In reality, the generation rate of $\mathrm{CO}$ changes depending on the ventilation conditions in the compartment.

Figure 7-47 through Figure 7-49 present comparisons of oxygen and carbon dioxide concentration predictions with experiment. There were two oxygen measurements, one in the upper layer, one in the lower layer. There was only one carbon dioxide measurement in the upper layer. The agreement between measurement and prediction was about $10 \%$, consistent with the accuracy of the temperature predictions and confirming that FDS is handling the bulk transport of gases well.

The CO predictions are not shown because the measurements appeared flawed. Instead, the smoke, another combustion product with a fixed yield, is described in the next section. 

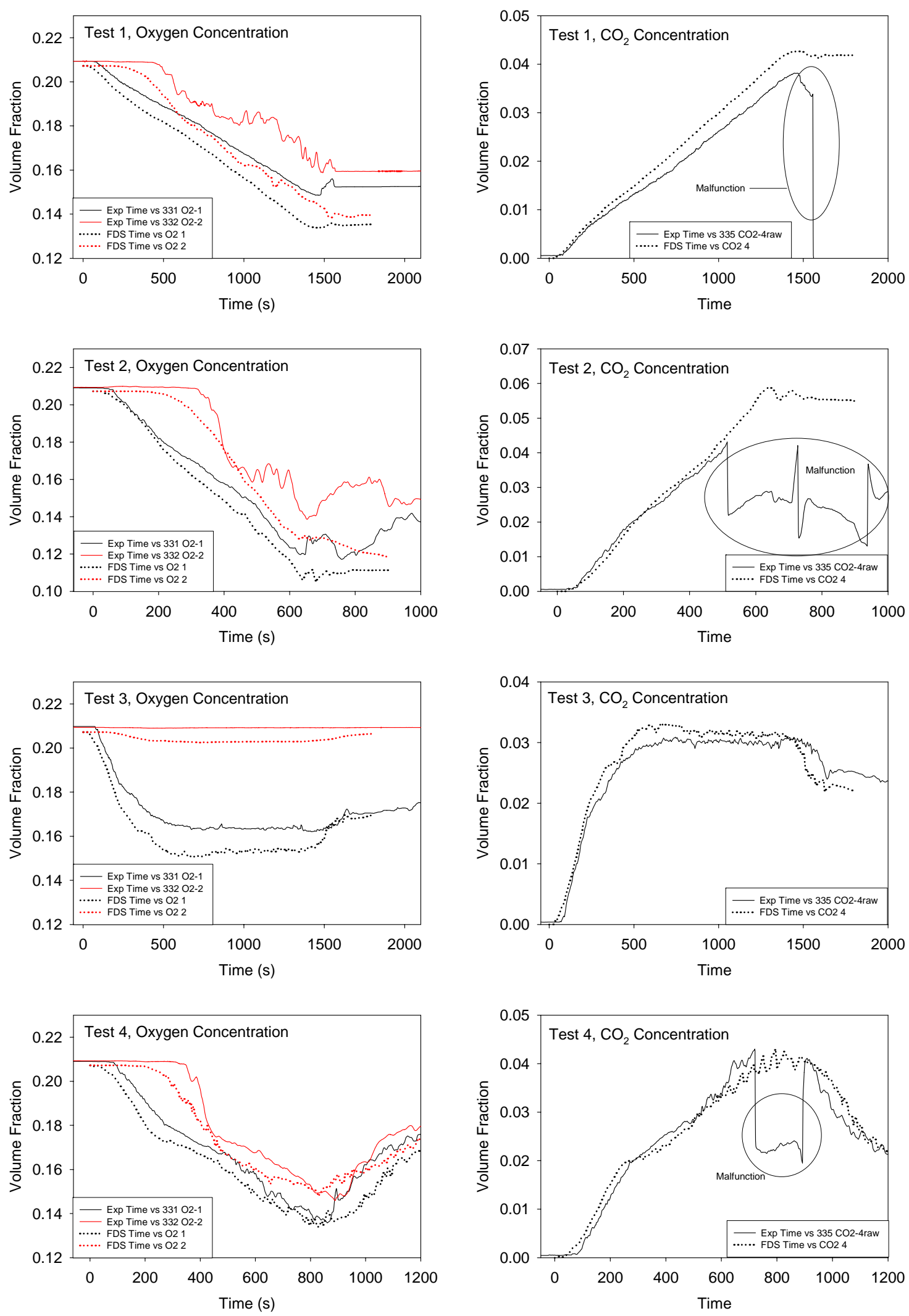

Figure 7-47. Oxygen and carbon dioxide concentration, Tests 1-4. 

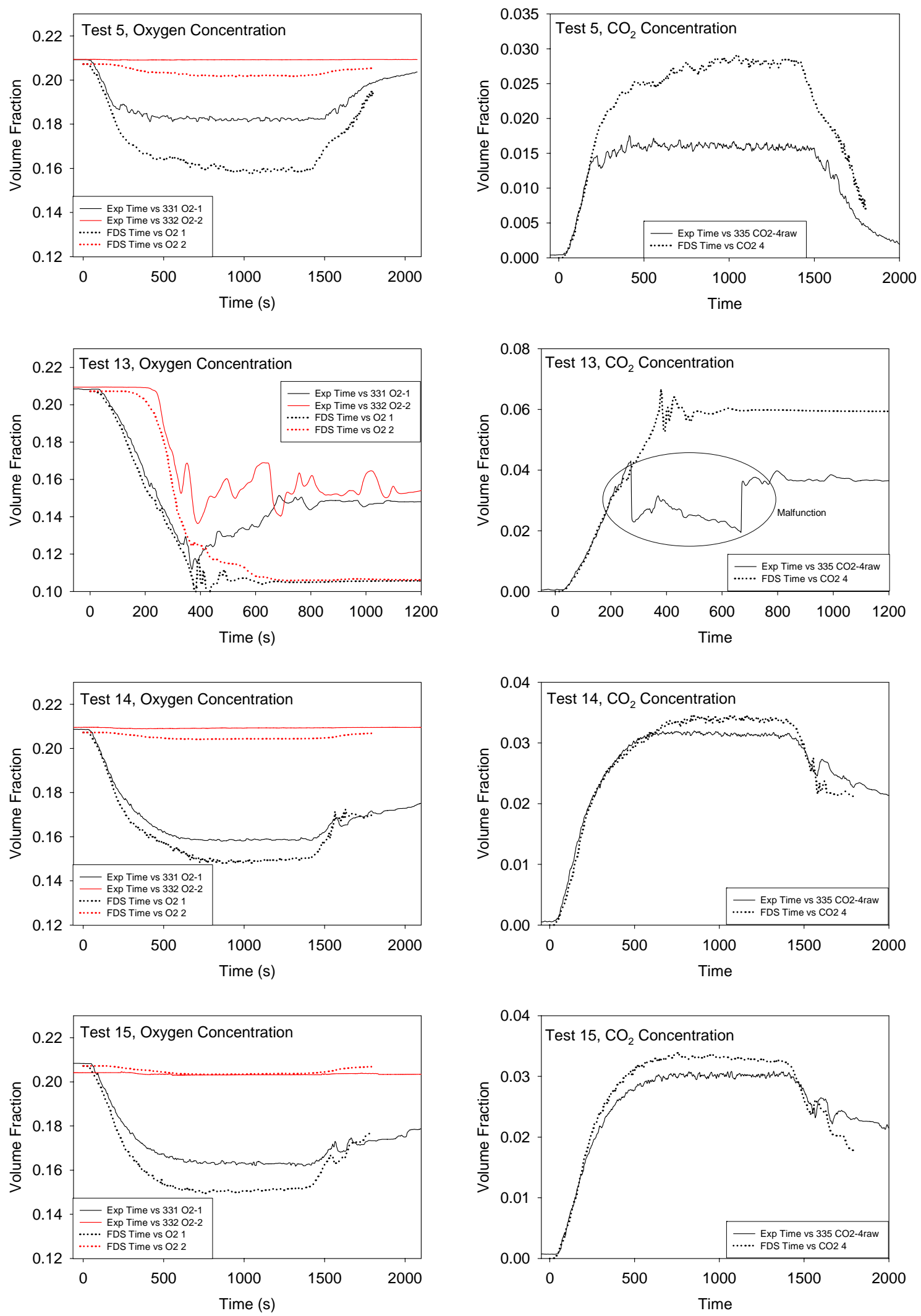

Figure 7-48. Oxygen and carbon dioxide concentration, Tests 5, 13-15. 

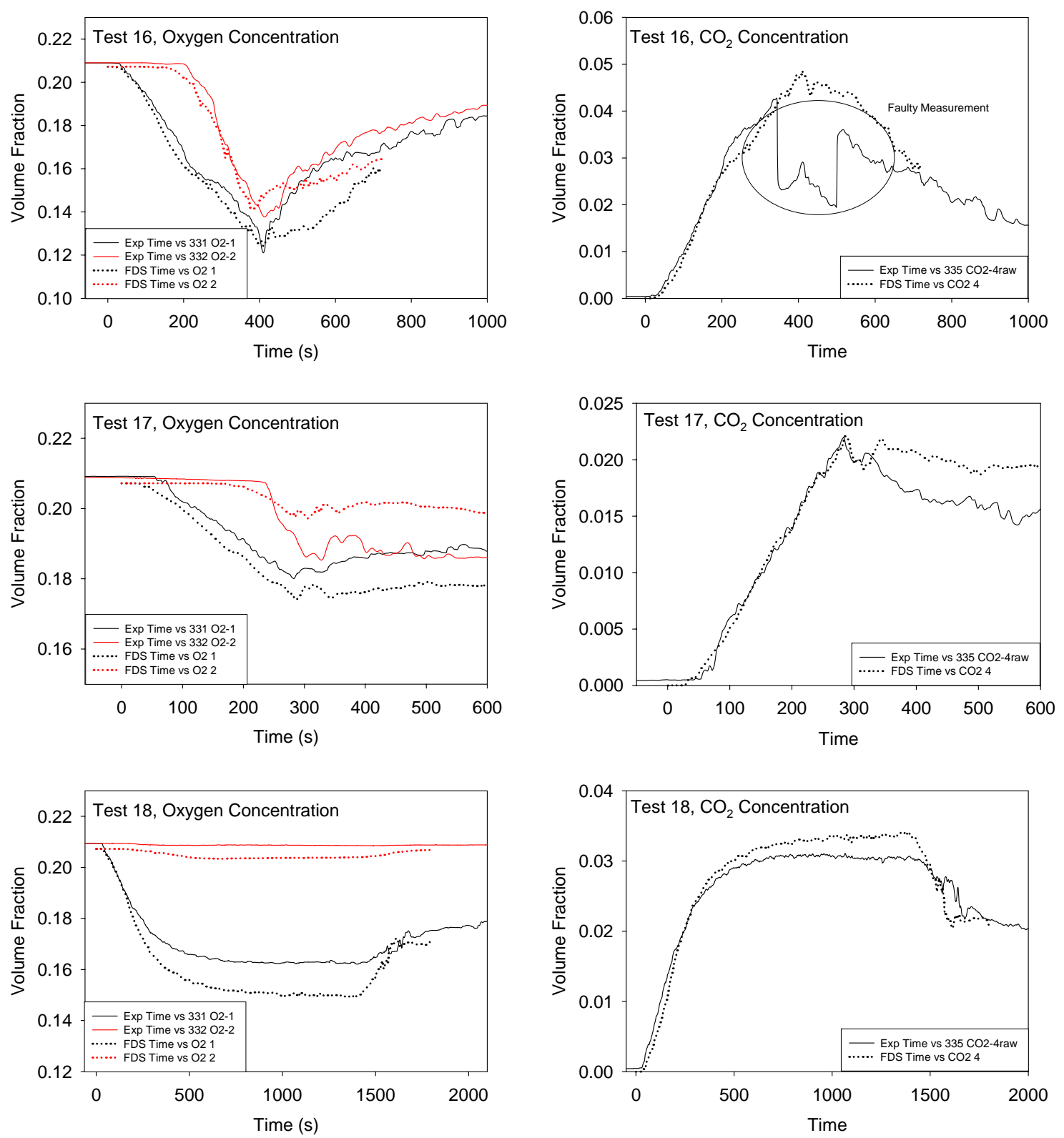

Figure 7-49. Oxygen and carbon dioxide concentration, Tests 16-18. 


\subsubsection{Smoke Concentration}

FDS treats smoke like all other combustion products, basically a tracer gas whose mass fraction is a function of the mixture fraction. To model smoke movement, the user need only prescribe the smoke yield, that is, the fraction of the fuel mass that is converted to smoke particulate. For $B E \# 3$, the smoke yield was specified as one of the test parameters.

Figure 7-50 and Figure 7-51 contain comparisons of measured and predicted smoke concentration at one measuring station in the upper layer. There are two obvious trends in the figures: first, the predicted concentrations were about $50 \%$ higher than the measured. Second, the predicted concentrations increased at the end of the closed door tests.

Consider the first issue. The reported mass concentration of smoke was computed using the following expression

$$
M_{s}=\frac{\ln \left(I_{0} / I\right)}{\phi_{s} L}
$$

Errors in the measurement were due to errors in the path length $L$, the light attenuation $I_{0} / I$, and the assumed specific extinction coefficient $\phi_{s}$. Hamins reported the expanded uncertainty of the measurement to be $18 \%$. In addition, the simulation was subject to error mainly from the prescribed soot yield. The soot yields were given as $1.5 \% \pm 0.3 \%$ (heptane) and $20 \% \pm 5 \%$ (toluene, Test 17). The combination of numerical and measurement error for the heptane tests was therefore $18 \%+20 \%=40 \%$, and for the toluene test $18 \%$ $+25 \%=45 \%$.

Assuming that the mixture fraction model is valid, at least for the open door tests, it can be assumed that virtually all of the carbon atoms in the fuel either ended up in the $\mathrm{CO}_{2}$ or the soot (with relatively small amounts going to $\mathrm{CO}$, unburned hydrocarbons, etc.). It can also be assumed that the soot (smoke) and $\mathrm{CO}_{2}$ were transported together with no significant separation or reaction. If these assumptions are true, there is no reason to expect the predicted smoke concentration shown in Figure 7-52 to be roughly $50 \%$ higher than the measured value unless the soot yield uncertainty and the measurement error combined to cause it. 

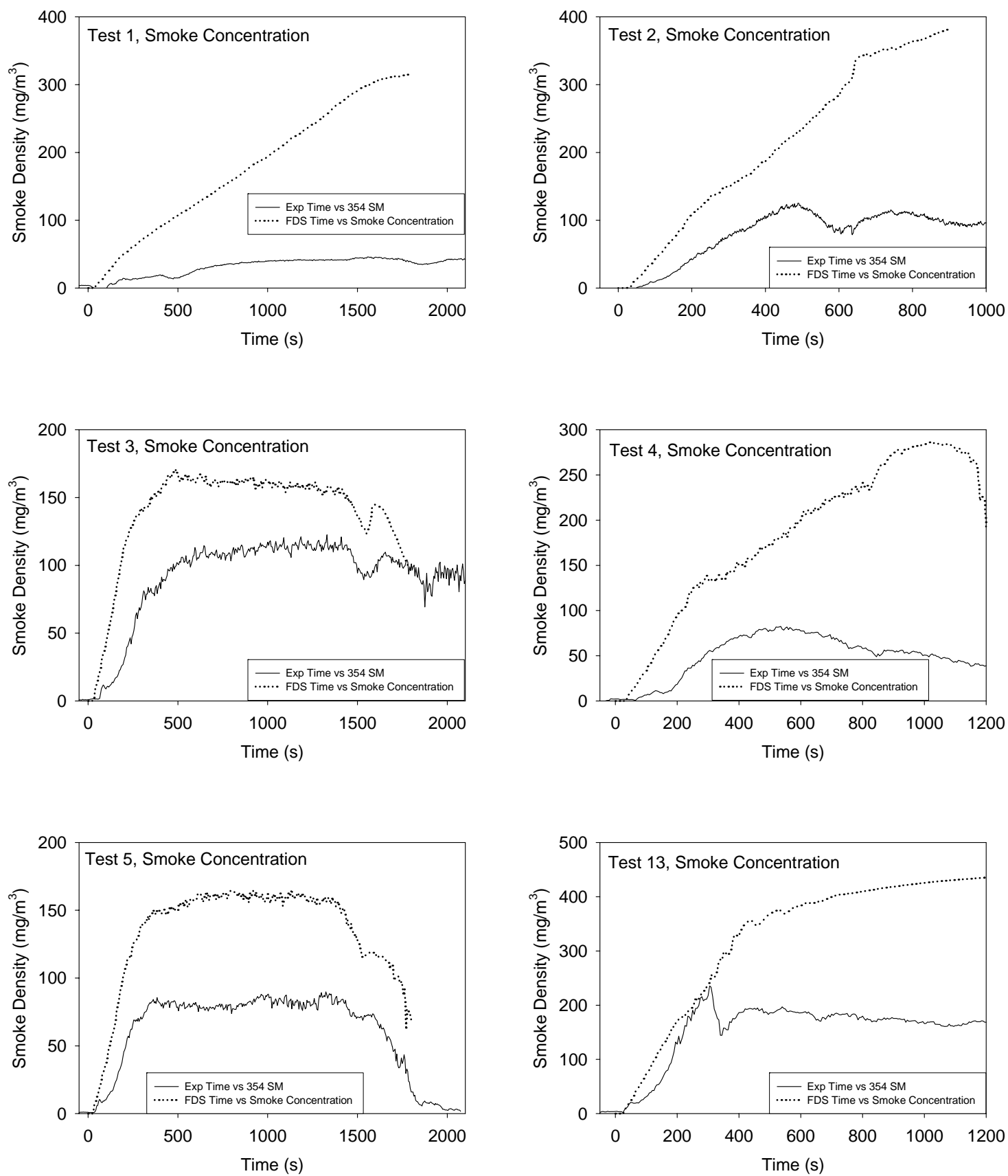

Figure 7-50. Smoke Concentration, Tests 1-5 and 13. 

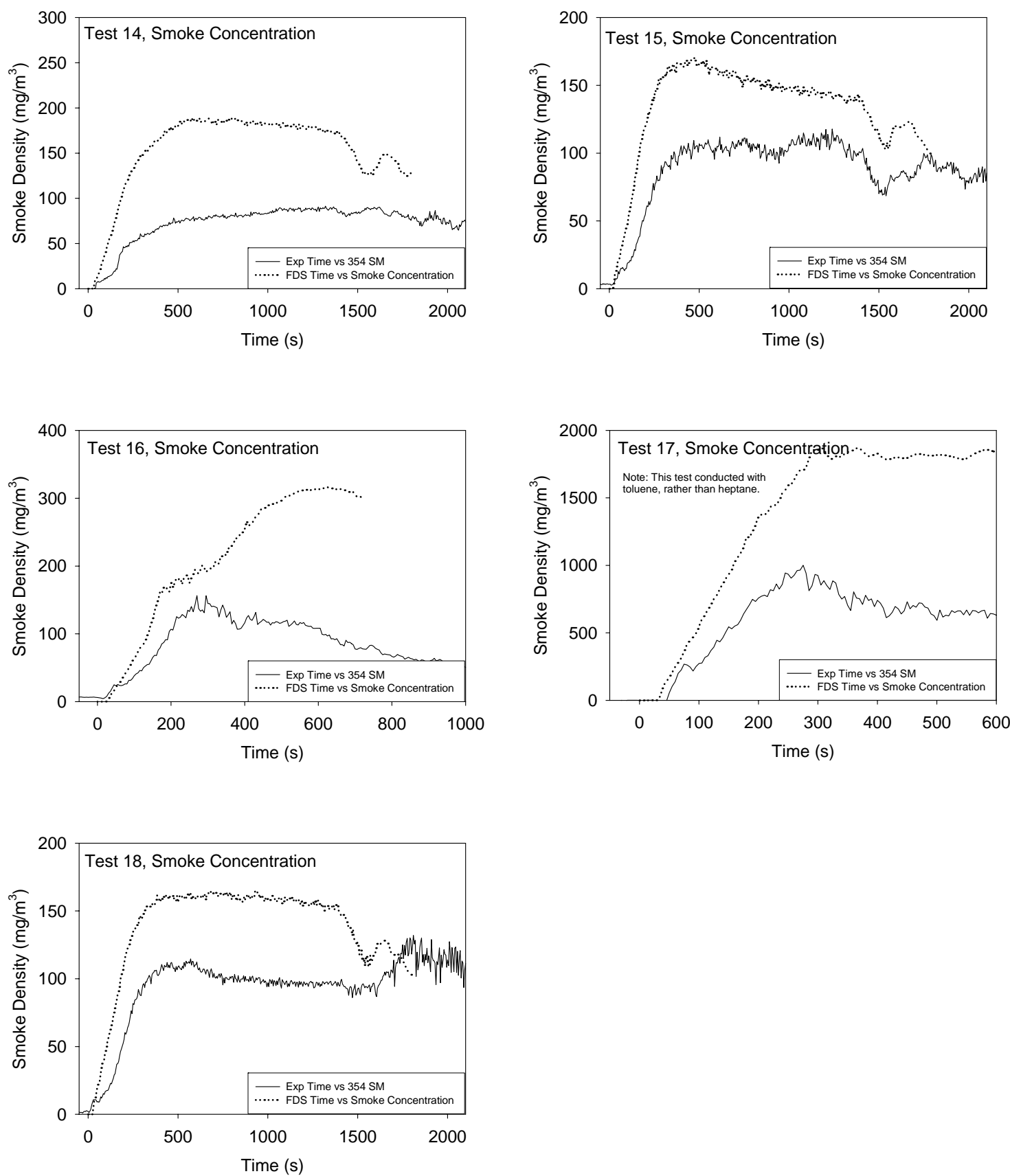

Figure 7-51. Smoke Concentration, Tests 14-18. 

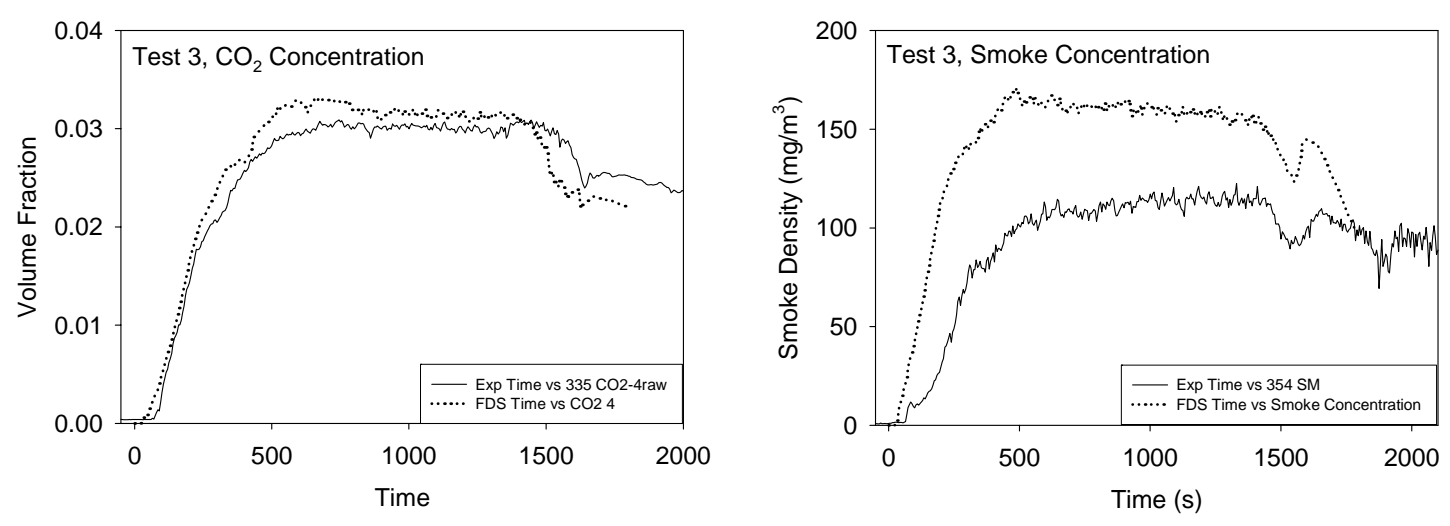

Figure 7-52. Tracking the carbon in Test 3.

The other notable difference between measured and predicted smoke concentration was the increase in predicted concentration after the closed door test fires were extinguished. In the model, the smoke concentration is the product of the mixture fraction $\times$ soot yield $\times$ gas density. As no doors were opened after the fires were extinguished, the mixture fraction at any given location did not change appreciably, but the density increased as the temperature decreased. Thus the predicted smoke concentration increased as the smoke layer shrank in volume. This effect was not observed in the measured smoke concentration histories.

\subsubsection{Compartment Pressure}

The pressure within the compartment was measured at a single point. Comparisons between measurement and prediction are shown in Figure 7-53 and Figure 7-54. For those tests in which the door to the compartment was open, the over-pressures were only a few Pascals, whereas when the door was closed, the over-pressures were several hundred Pascals.

In general, the predicted pressures were of comparable magnitude to the measured pressures, and in most cases differences could be explained using the reported uncertainties in the leakage area and the fact that the leakage area changed from test to test because of the thermal stress put on the compartment walls. The two notable exceptions were Tests 4 and 16. These were the only two experiments performed with the door closed and ventilation on. The measured mass flow through the exhaust duct exceeded the mass flow through the supply duct by roughly $40 \%$. The FDS simulations were performed with a fixed and equal 
volume (not mass) flow rate at the supply and exhaust ducts, which is why the FDS simulations over-predicted the peak compartment pressures in Tests 4 and 16. 

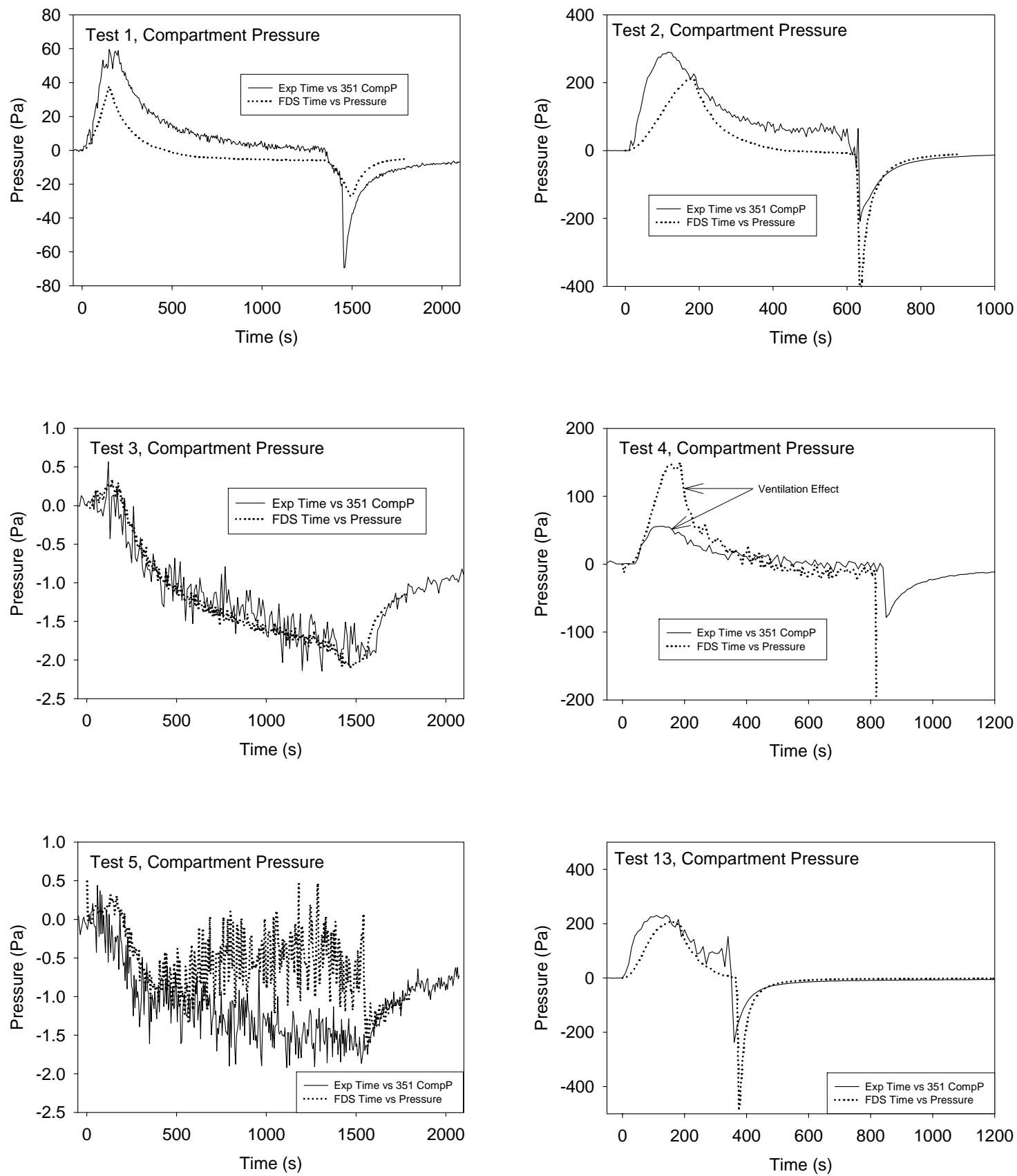

Figure 7-53. Compartment pressure, Tests 1-5 and 13. 

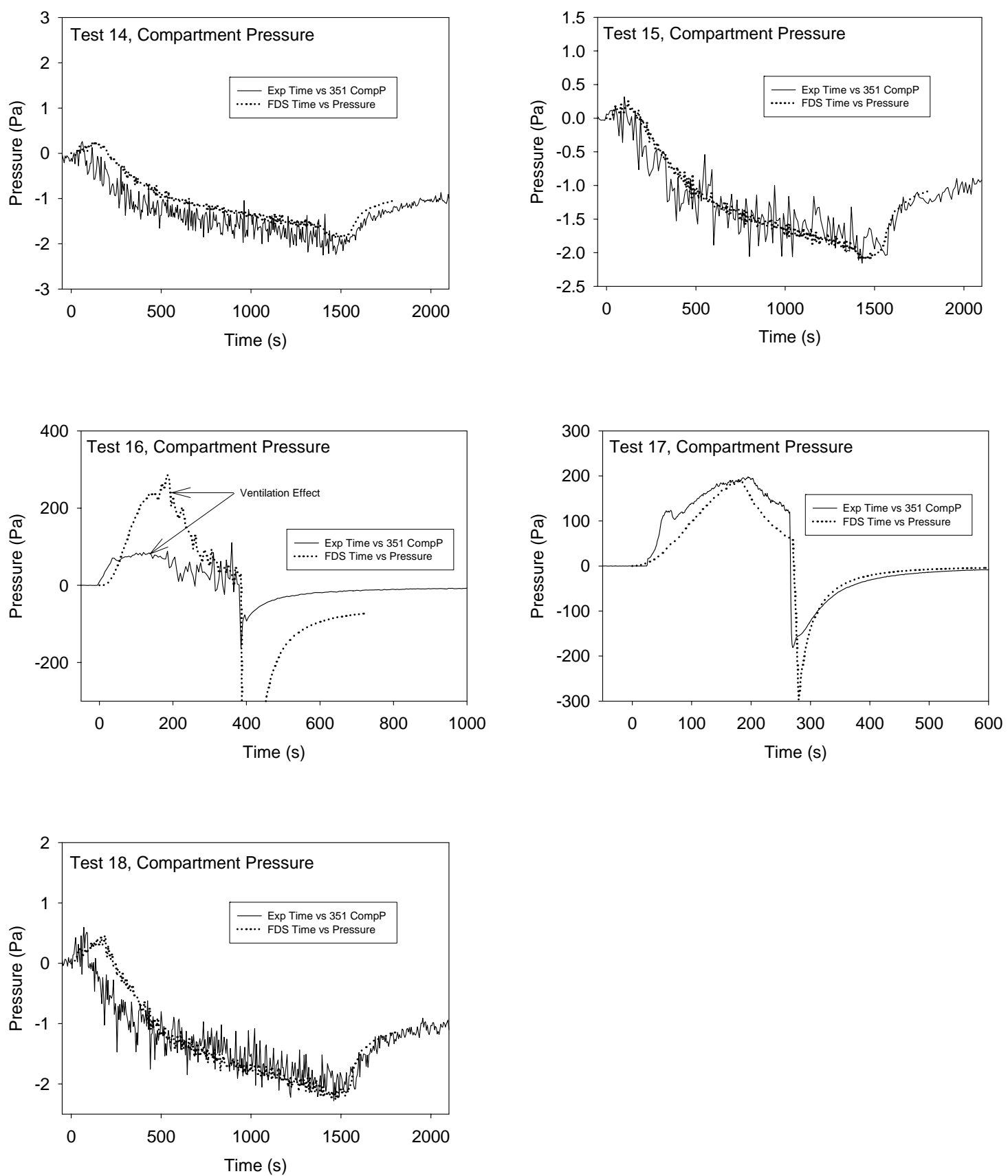

Figure 7-54. Compartment pressure, Tests 14-18. 


\subsubsection{Velocity}

Three vertical arrays of velocity (bi-directional) probes were placed in the $2 \mathrm{~m} \times 2 \mathrm{~m}$ doorway. Comparisons of experimental measurement and model prediction for the open-door tests $(3,5,14,15$ and 18) are shown in Figure 7-56. The data shown was taken from the array that was located nominally $50 \mathrm{~cm}$ south of the north edge of the doorway. Probe 15 (black) was located $20 \mathrm{~cm}$ below the top of the doorway. Probe 13 (red) was $1 \mathrm{~m}$ below, and Probe 11 (green) was $20 \mathrm{~m}$ above the floor. The results from the other two arrays of probes were similar to this one and are not included.

The comparison of inlet and outlet velocities reveals that the model is over-predicting the velocity near the top of the doorway. This is most likely a grid resolution effect. Near the top of the doorway, the jet of hot gases exiting the compartment could not be resolved adequately with a $10 \mathrm{~cm}$ grid (see Figure 7-55). This is not to say that the model inaccurately predicted the integrated mass or volume flux, but rather that it had difficulty predicting accurately the velocity at any given point in the doorway. Overall mass conservation was validated by the compartment pressure comparisons for open-door Tests 3, 5, 14, 15, and 18 . Also, the integrations associated with the global energy budget discussed below are a further check that the model is handling well the bulk transport of mass through the doorway.

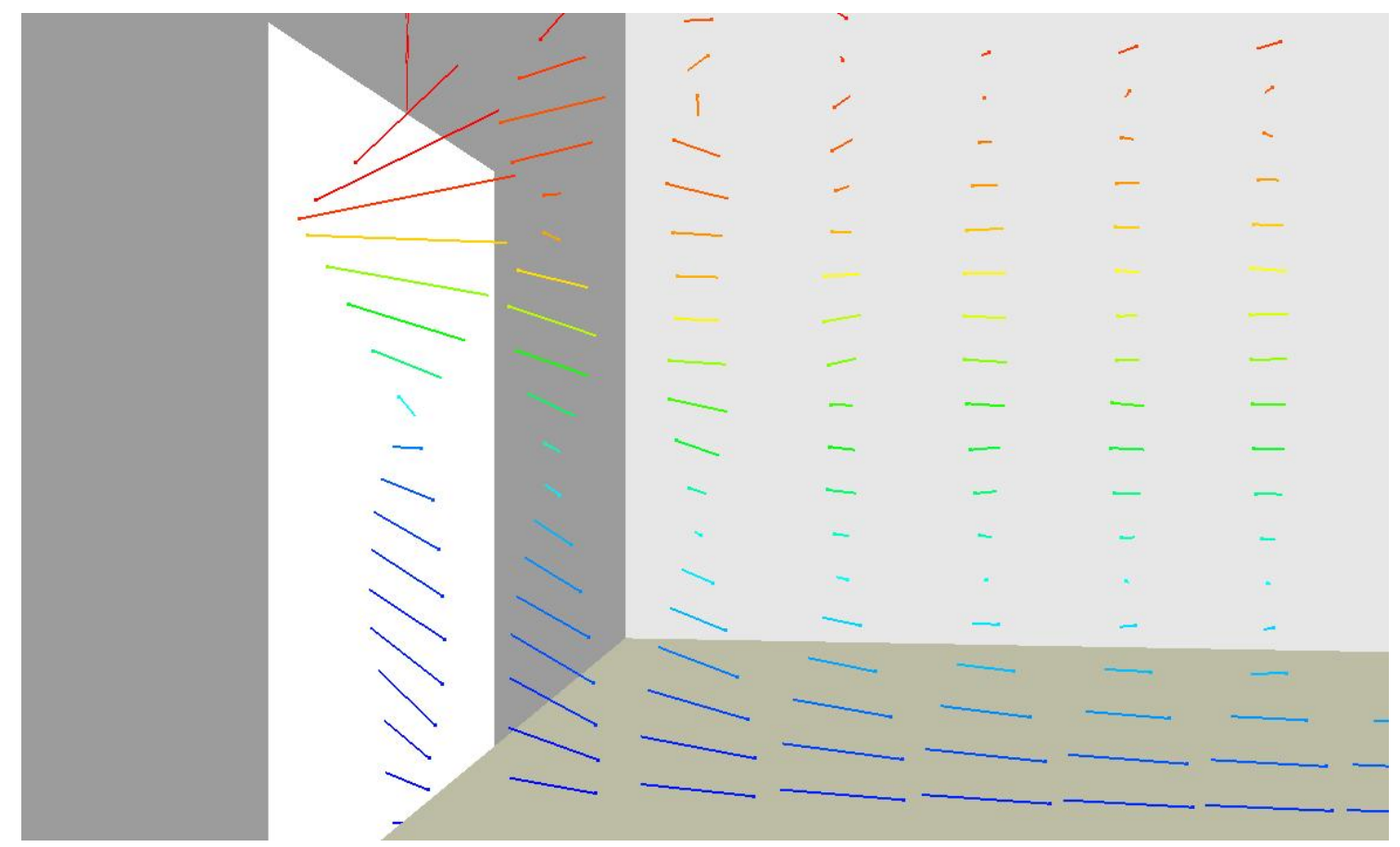

Figure 7-55. Flow vectors near the compartment doorway. 

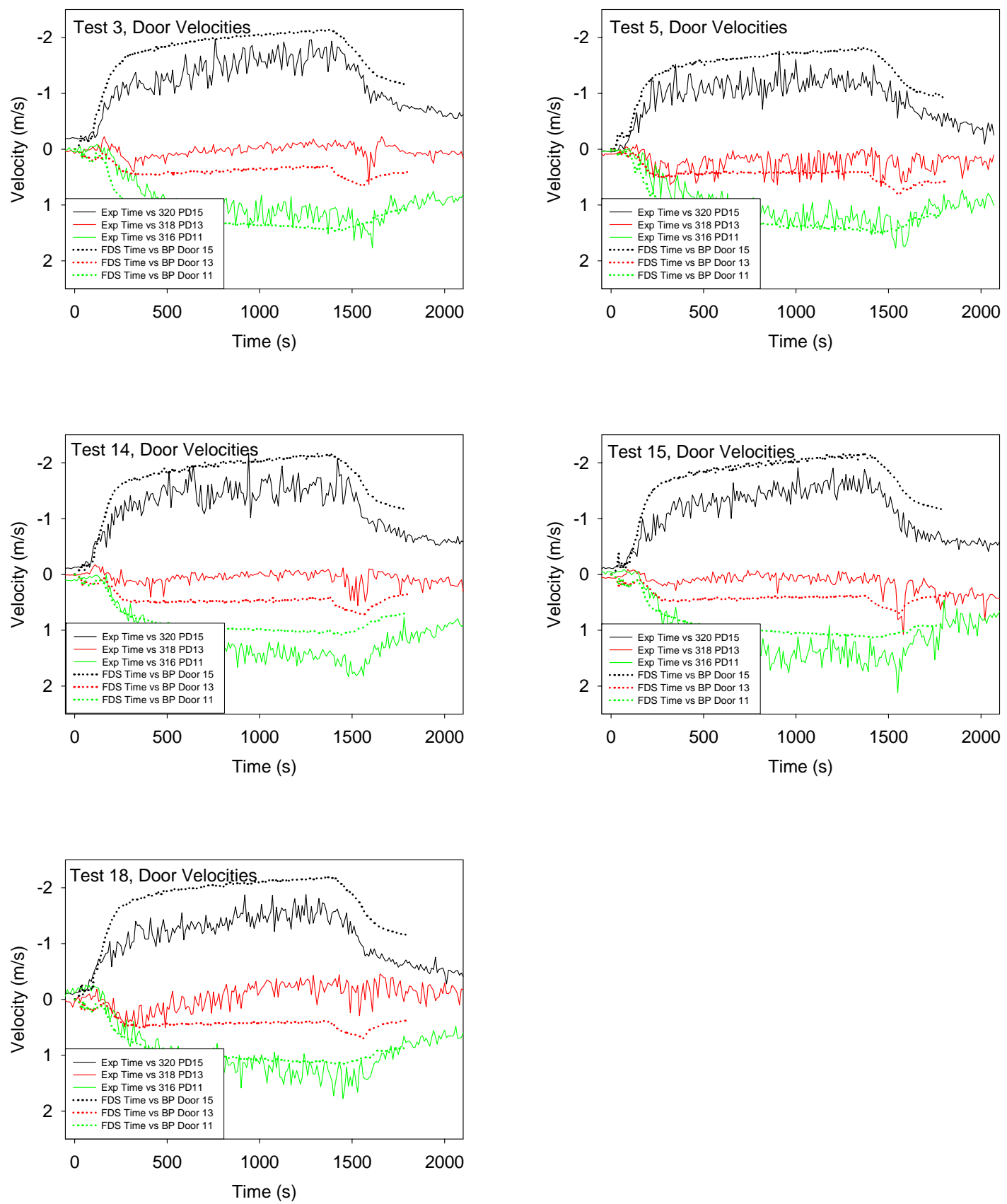

Figure 7-56. Door Velocities, Open Door Tests 3, 5, 14, 15, and 18. 


\subsubsection{Energy Budget}

There were enough temperature, heat flux and velocity measurements made in Benchmark Exercise 3 so that it was possible to account for all of the energy released by the fire, both in the experiment and in the simulation. As an example, consider the plot of the total energy budget shown in Figure 7-57. As the thermal environment of the compartment moves towards a steady state, the heat release rate of the fire should be balanced with the heat conducted to the walls and the heat flowing out the door. This is depicted graphically in the figure. The Total Loss (green), consisting of the rate of heat lost the walls (black) plus the rate of heat flowing out the door (red), should equal the heat release rate of the fire (pink). It is not perfect in either experiment or simulation because the compartment did not achieve steady-state, but it is close enough to confirm that the model conserves energy, and that the measurements are reasonably accurate and do account for the bulk of the heat transfer within the compartment.

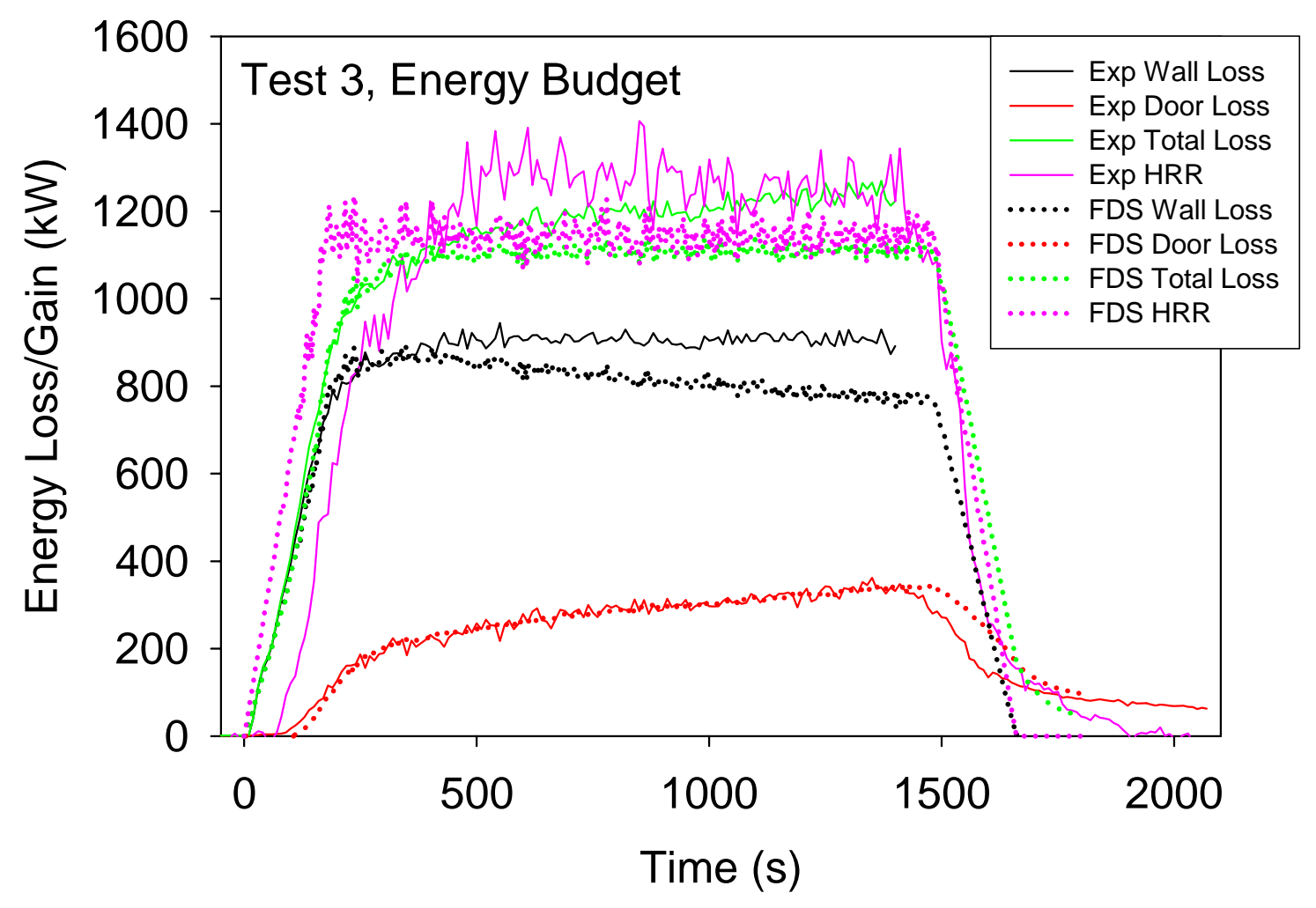

Figure 7-57. Energy budget for Test 3. 


\subsection{FDS Sensitivity Analysis}

There are two types of input parameters for any fire model: numerical and physical. Physical parameters describe the wall materials, fuel properties, reaction stoichiometry, etc. Numerical parameters control the mathematical solution of the governing equations, the most important of which is the size of the numerical grid. The most important physical parameter in Benchmark Exercise 3 was the prescribed heat release rate, and there was discussion above about its impact on all of the results. Included below is some discussion on the sensitivity of the simulations to the wall material properties.

\subsubsection{Grid Sensitivity}

The most important numerical parameter is the grid cell size. CFD models solve an approximate form of the conservation equations of mass, momentum and energy on a numerical grid. The error associated with the discretization of the partial derivatives is a function of the size of the grid cells and the type of differencing used. FDS uses second-order accurate approximations of both the temporal and spatial derivatives of the Navier-Stokes equations, meaning that the discretization error is proportional to the square of the cell size. In other words reducing the grid cell size by a factor of 2 reduces the discretization error by a factor of 4 . However, it also increases the computing time by a factor of 16 (a factor of 2 for the temporal and each spatial dimension). Clearly, there is a point of diminishing returns as one refines the numerical mesh. Determining what size grid cell to use in any given calculation is known as a grid sensitivity study.

A modest grid sensitivity study was performed for this exercise. Shown in Figure 7-58 are some results for Test 3 computed on the nominally $10 \mathrm{~cm}$ "fine" grid and a $20 \mathrm{~cm}$ "coarse" grid. (All of the results shown above were computed on a $10 \mathrm{~cm}$ grid.) The coarse simulation of Test 3 required a few hours to complete on a single $2.4 \mathrm{GHz}$ Pentium processor, whereas the fine required a few days. A quick glance at the sample results shows relatively little difference in the accuracy. This is not surprising because the basic conservation equations still ensure a reasonably good distribution of the energy from the fire on the coarse grid, and there are no steep gradients in the selected quantities that would have been more sensitive to the grid size.

So why bother with a multi-day calculation? Consider what is not shown in Figure 7-58. The cable trays and other targets, which were difficult to resolve even on the fine grid, were sim- 
ply too small to even include on the coarse grid. The door and vent were less accurately prescribed on the coarse grid, leading to greater error in the computation of the compartment pressure. Still worse, had the fire scenario required a prediction of the heat release rate, or flame spread along the cable trays, even the fine grid would not have been enough to handle it, never mind the coarse.

So the lesson to be learned is that coarse grid CFD, much like zone model calculations, can provide reasonable predictions of certain quantities, especially those that can be traced directly to conservation equations of mass and energy, like average temperatures and pressures. However, the user has to be aware that the results are generally less reliable than those obtained from a finer grid, and that certain results cannot be obtained at all.

\subsubsection{Sensitivity to Wall Material Properties}

The compartment walls and ceiling were made of $2.54 \mathrm{~cm}$ thick Marinite I, a product of BNZ Materials, Inc. The manufacturer provided the thermal properties of the material used in the calculation: density $737 \mathrm{~kg} / \mathrm{m}^{3}$, conductivity $0.12 \mathrm{~W} / \mathrm{m} / \mathrm{K}$, specific heat $1.17 \mathrm{~kJ} / \mathrm{kg} / \mathrm{K}$ at $93{ }^{\circ} \mathrm{C}$ ramping linearly to $1.42 \mathrm{~kJ} / \mathrm{kg} / \mathrm{K}$ at $425^{\circ} \mathrm{C}$. A testing laboratory also provided thermal properties, based on a sample provided by NIST: density $669 \mathrm{~kg} / \mathrm{m}^{3}$, conductivity $0.11 \mathrm{~W} / \mathrm{m} / \mathrm{K}$ at 23 ${ }^{\circ} \mathrm{C}$ ramping linearly to 0.20 at $650{ }^{\circ} \mathrm{C}$, specific heat $0.78 \mathrm{~kJ} / \mathrm{kg} / \mathrm{K}$ at $23{ }^{\circ} \mathrm{C}$ ramping to 1.22 $\mathrm{kJ} / \mathrm{kg} / \mathrm{K}$ at $650{ }^{\circ} \mathrm{C}$. The manufacturer's property data was used in the FDS calculations merely because simulations similar to BE \#3 had been performed at NIST as part of its investigation of the World Trade Center disaster, and these input files were modified for use in $B E \# 3$. To check the sensitivity of the simulations to the variations in the wall properties, a simulation was performed using the Marinite properties that were measured by the testing laboratory (and subsequently recommended for use by the participants of the benchmark exercise).

Figure 7-59 presents the predicted ceiling temperatures using the two sets of material properties for the Marinite. There was a slight increase in temperature with the material properties from the testing lab, roughly a few percent, most likely due to the lower density and specific heat. This difference is well within the uncertainty related to the measured HRR that was discussed above. 

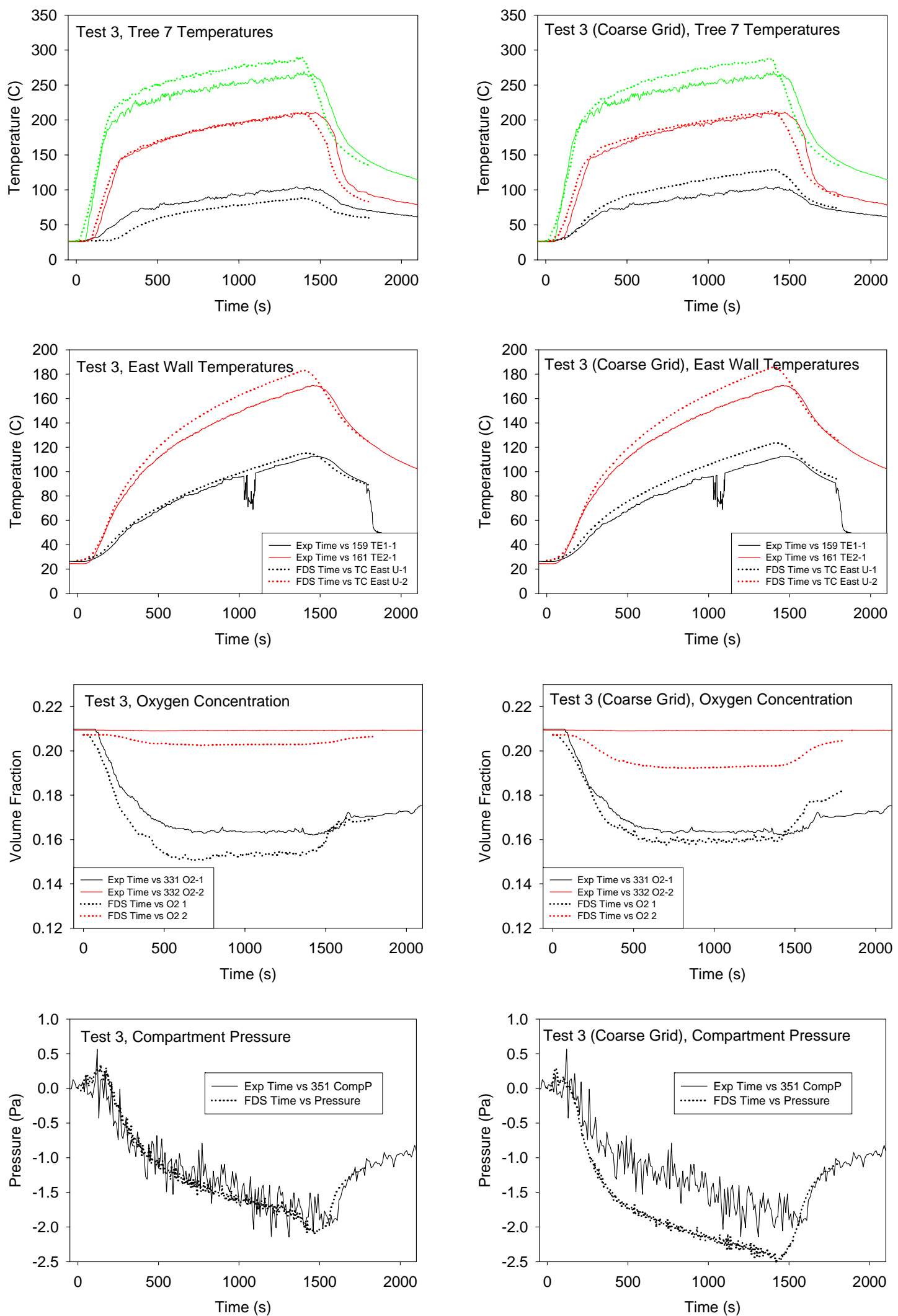

Figure 7-58. Comparison of fine (left) and coarse (right) results for Test 3. 

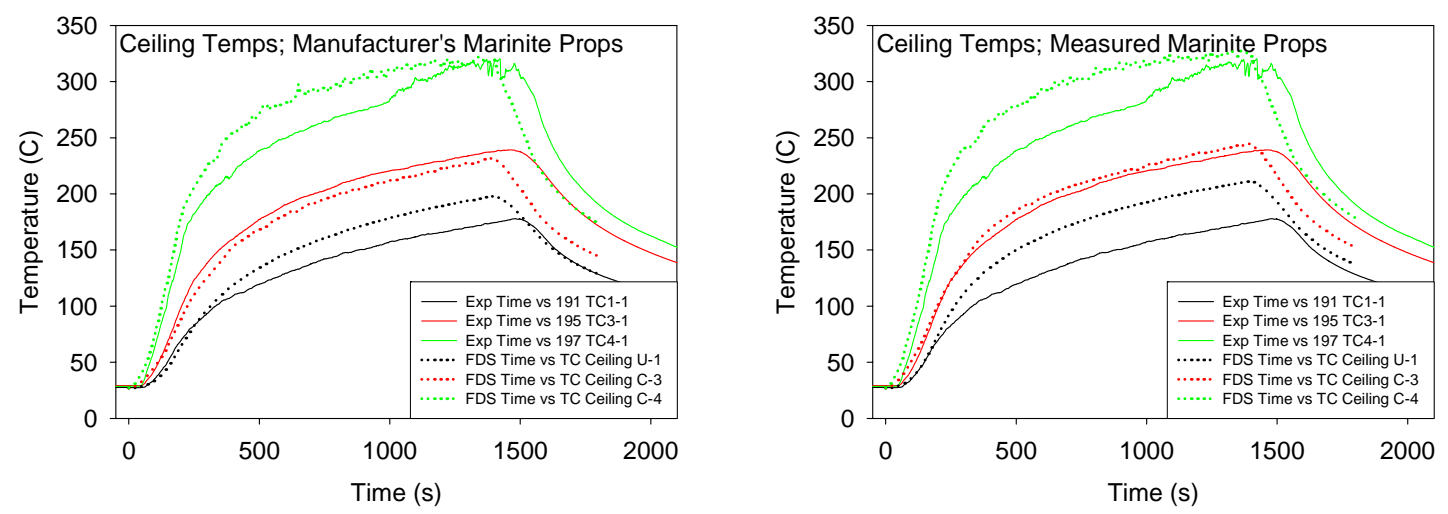

Figure 7-59. Test 3 results for two sets of wall material properties.

\subsubsection{Radiation Parameters}

Finally, because the final simulations were run using 200 radiation angles instead of the default 100, the coarse grid simulation of Test 3 was repeated with the default settings. There was no noticeable change in the results, confirming in this case that the default number of angles would have been adequate. However, this does not mean that the default settings are always appropriate. Sensitivity studies like the one performed here ought to be used to determine if and when to change the default settings.
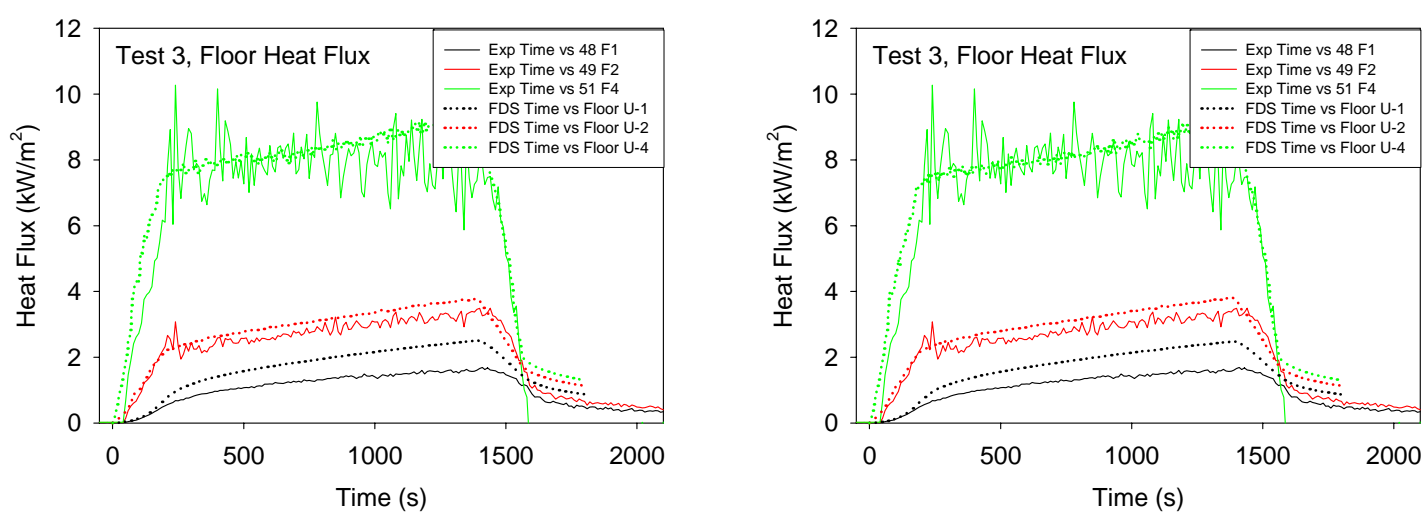

Figure 7-60. Test 3 results using 200 radiation angles (left) and 100 (right). 


\subsection{Conclusions and Recommendations for FDS}

Simulations of Benchmark Exercise 3 were performed with the NIST Fire Dynamics Simulator (FDS). Because the heat release rate of the fire was given to the participants as an input parameter, the focus of the exercise was to predict the transport of heat and combustion products through a fairly large enclosure. For most deterministic fire models, including FDS, smoke and heat transport is the primary objective.

The predicted gas temperatures were within $10 \%$ of the reported measurements, with some exceptions due to the effect of forced ventilation and near-field inaccuracies of the model. In the tests with forced ventilation, a combination of the uncertainty in the measured velocity profile and the coarseness of the FDS grid near the vent probably were responsible for the discrepancies. As for near-field effects, the exact prescription of the fire's "footprint" in the pan had a significant effect on the lower thermocouples of an array $10 \mathrm{~cm}$ away from the pan.

The predicted wall, ceiling and floor temperatures were also within about $10 \%$ of the measurements, with the larger deviations occurring at points closer to the fire, especially on the ceiling. The over or under-prediction of the wall temperature at a given point could not always be explained by a comparable error in the heat flux prediction. This was probably due to the fact that the wall temperature measurement was made with a single thermocouple, whereas the heat flux measurement required additional interpretation in its conversion from net to total.

The accuracy of the predicted cable surface temperatures varied between 10 and $20 \%$ depending on their location relative to the fire and the accuracy of the corresponding gas temperature and heat flux. However, as with the wall temperature predictions, it was not always possible to explain discrepancies solely in terms of the over or under-prediction of the local gas temperature and heat flux because of the effect of multiple off-setting or compounding errors.

The simplified description of the cable trays was a weakness in the FDS model. There were no physical algorithms in the model to describe the detailed heat transfer within the bundled cables, nor the lateral heat transfer along the metal conductors. Nevertheless, the model did predict accurately the thermal environment surrounding the cables, and to a reasonable degree of accuracy it predicted the surface temperature of the cables themselves. In some cases, the measured temperature inside the cable was nearly the same as the surface tem- 
perature, an effect captured by the model for the improvised "slab" Target E. However, more complicated heat transfer within the cables would not have been accounted for by the model, and any predictions of respectable accuracy would have simply been fortuitous.

Predictions of oxygen and carbon dioxide were within about $10 \%$ of the measurements in most cases. This level of accuracy is consistent with that of the transport of the heat from the fire, as both mass and energy are transported in the model with the same basis algorithm. The predicted smoke concentration was typically about $50 \%$ higher than the measured value, most likely due to an error in the prescribed soot yield in combination with measurement error. There is no reason why the smoke and carbon dioxide predictions should not have been of comparable accuracy given that their prescribed yields and their respective measurement techniques were of comparable accuracy.

The predictions of compartment pressure were within experimental uncertainty except in two tests where the door was closed but the ventilation system was turned on. In these cases, the model used prescribed volumetric flow rates and could not account for the variations in mass flow rate as a result of changing compartment temperature. A change in the model boundary conditions would have rectified this situation.

If Benchmark Exercise 3 were a design fire scenario, FDS, or any CFD model, would have been sufficiently accurate. Indeed, it was shown that a calculation performed on a grid whose cells were twice as large as those used in the study produced results of comparable accuracy, at least for the bulk temperatures and heat fluxes. However, if the exercise were part of a forensic reconstruction of a fire that erupted from an accidental fuel spill and then ignited power cables within steel trays, FDS would not have been accurate enough to produce a blind prediction of the heat release rate, heat flux to the cables, and fire spread along the trays. It could have produced a qualitative description of the events, but with nothing near the accuracy shown above.

There is no reason why a more detailed sub-model of the cables could not be included in FDS since the gas and solid phase need only exchange mass and energy. However, the fact that the predicted heat flux to the cable surface was at times only $20 \%$ accurate calls into question the added value of a more detailed heat transfer algorithm for the cables. It is not clear whether the extra work in model development, and the added complexity of model use and code maintenance, would benefit the larger community of users. 
The most valuable measurements were those that combined multiple measurements at a given location/target. This allowed for a better assessment of the model's performance. Often in predicting a target's temperature, a model can over-predict the gas temperature and under-predict the heat flux, or vice verse, resulting in a deceptively accurate prediction. It is best to make sure that each accurate prediction of a target temperature is "traceable", that is, that it can be shown that the thermal properties, local gas temperature, and heat flux are all accurate as well.

\subsection{References}

Hamins, A., A. Maranghides and G. Mulholland. 2003. The Global Combustion Behavior of 1 MW to 3 MW Hydrocarbon Spray Fires Burning in an Open Environment. NISTIR 7013. National Institute of Standards and Technology, Gaithersburg, Maryland, USA.

McGrattan, K., J. Floyd, G. Forney, H. Baum and S. Hostikka. 2003. Improved Radiation and Combustion Routines for a Large Eddy Simulation Fire Model. Fire Safety Science -- Proceedings of the Seventh International Symposium. International Association for Fire Safety Science.

McGrattan, K. (ed.) 2004. Fire Dynamics Simulator (Version 4) Technical Reference Guide. NIST Special Publication 1018. National Institute of Standards and Technology, Gaithersburg, Maryland, USA.

McGrattan, K. and G. Forney. 2004. Fire Dynamics Simulator (Version 4) User's Guide. NIST Special Publication 1019. National Institute of Standards and Technology, Gaithersburg, Maryland, USA.

Quintiere, J.G. 1998. Principles of Fire Behavior. Delmar Publishers, Washington, DC, USA.

Walton, W.D. and P.H. Thomas. 2003. Estimating Temperatures in Compartment Fires. Fire Protection Handbook, $3^{\text {rd }} \mathrm{Ed}$. National Fire Protection Association, Quincy MA.

Welsh, S. and P. Rubini. 1997. Three-Dimensional Simulation of a Fire-Resistance Furnace. Fire Safety Science - Proceedings of the Fifth International Symposium. International Association for Fire Safety Science. 


\section{$8 \quad$ Summary of Conclusions from Individual Contributions}

This final chapter summarizes the conclusions of the individual modelers who participated in the Benchmark Exercise. Note that it is difficult to summarize in a simple way the accuracy of the models, as each participant chose different quantities to analyze, some performed calculations before the test data was released, some after. All of the participants agreed that the approximate forms of the conservation equations for mass, momentum and energy in the various fire models evaluated provide a "reasonable" prediction of the hot gas layer temperature, depth and product concentration. Other issues are discussed individually below.

\subsection{Modeling the Fire}

In this exercise, the flow rate of fuel into a pan was specified as an input parameter, and a fixed heat of combustion was assumed. This is not unusual in fire model validation exercises, especially when the intent is not to predict the heat release rate, but rather the transport of hot gases throughout the compartment. However, the size and shape of the fire has an impact on the predicted radiative flux from the fire plume. A variety of techniques were used to model the fire, ranging from a point source in the zone models to simple combustion models in the field (CFD) models. Several participants did sensitivity studies to assess the effect of various modeling inputs: the radiative fraction, area of the fire "footprint" in the pan, point source vs surface radiation assumptions.

\subsection{Fire Extinction}

The fires in two of the fifteen tests (Test 13 and 16) self-extinguished due to lack of oxygen. The oxygen concentration measured near the fire close to the floor of the compartment was about $15 \%$ at the time of extinction, consistent with the lower oxygen limit (LOL) that is often included in the various fire models as an indicator of a fire's viability in an under-ventilated environment. Although the LOL concept is fairly crude and glosses over the complexity of the combustion processes that occur in these types of fires, it was sufficient in these two cases to predict extinction. In other words, an accurate prediction of the fire's extinction in Tests 13 and 16 is based on the appropriate LOL assumption and an accurate calculation of oxygen transport. 


\subsection{Heat Flux}

The heat flux to walls and targets is predicted by the different models using a variety of methods. The zone models typically use a point source model, in which the radiative energy from the fire is assumed to emanate from a single point, in combination with the summation of radiative contributions from the hot walls and hot gas layer. This method works so long as the radiative fraction of the fire is chosen appropriately and the temperature of the hot gas layer predicted accurately. It is important to note that the radiation heat flux from the hot gas layer is a function of the (absolute) temperature raised to the fourth power. Thus, the uncertainty in the heat flux prediction is expected to be larger (by roughly a factor of 2 ) than the uncertainty in the hot gas layer temperature.

Field models typically do not rely on point source radiation models, but rather compute solutions to the radiative transport equation. While this is potentially more accurate than the point source method, it is important to define the emission and absorption of the fire and the hot, smoke-laden gases.

\subsection{Target Model}

A detailed heat transfer model for cables bundled in a tray is fairly complex. The fire models used in this benchmark exercise did not have the capability of modeling such complex cable configurations. Most of the codes used for the benchmark exercise had a one-dimensional slab model for targets. Some had simple models, such as a plate-type model with a conductor blanketed with insulation, a cylindrical model composed of two insulating materials, or the cable tray was modeled as a homogenous slab of cable insulation. For models in which the target is represented as a rectangular slab, the orientation of the slab is an issue.

\subsection{Carbon Monoxide and Soot}

The fire models that were exercised in the benchmark exercise do not have the capability to model the production of soot or carbon monoxide in the combustion process. The models use a constant yield input to the codes for these combustion products. The yields of carbon monoxide and soot, products of incomplete combustion, vary depending on the extent of under ventilation of the fire, and the size of the fire. This limitation was evident in the comparison of predictions with experimental data for the under-ventilated tests. 


\subsection{Mechanical Ventilation}

Most models used did not include the coupling of the fire compartment with the mechanical ventilation system. This limitation led to discrepancies in the predictions of supply and exhaust flow rates. The accurate prediction of the direction of supply flow was another issue. The prediction of the mixing of the supply air with gases in the compartment for underventilated environments determines the oxygen levels in the compartment. Generally larger discrepancies were noted for predictions of oxygen concentrations for under ventilated environments with forced ventilation, even with CFD codes. Most models predicted less oxygen depletion than measured.

\subsection{Compartment Pressure}

The computation of compartment over pressure for closed door cases is highly dependent on the assumed leakage, as discussed in Chapter 4. The accuracy of the predictions of the over pressure varied, with several codes over-predicting the pressure increase. The computation of the over pressure had an impact on the convergence of solutions in some CFD codes, especially when the leakage was assumed to be from a hypothetical orifice. 


$$
\text { A - 8-4 }
$$

